\title{
TOXICOLOGICAL PROFILE FOR
} XYLENE

\author{
U.S. DEPARTMENT OF HEALTH AND HUMAN SERVICES \\ Public Health Service \\ Agency for Toxic Substances and Disease Registry
}

August 2007 
DISCLAIMER

The use of company or product name(s) is for identification only and does not imply endorsement by the Agency for Toxic Substances and Disease Registry. 


\section{UPDATE STATEMENT}

A Toxicological Profile for Xylene, Draft for Public Comment was released in September 2005. This edition supersedes any previously released draft or final profile.

Toxicological profiles are revised and republished as necessary. For information regarding the update status of previously released profiles, contact ATSDR at:

Agency for Toxic Substances and Disease Registry

Division of Toxicology and Environmental Medicine/Applied Toxicology Branch

1600 Clifton Road NE

Mailstop F-32

Atlanta, Georgia 30333 
This page is intentionally blank. 


\section{FOREWORD}

This toxicological profile is prepared in accordance with guidelines developed by the Agency for Toxic Substances and Disease Registry (ATSDR) and the Environmental Protection Agency (EPA). The original guidelines were published in the Federal Register on April 17, 1987. Each profile will be revised and republished as necessary.

The ATSDR toxicological profile succinctly characterizes the toxicologic and adverse health effects information for the hazardous substance described therein. Each peer-reviewed profile identifies and reviews the key literature that describes a hazardous substance's toxicologic properties. Other pertinent literature is also presented, but is described in less detail than the key studies. The profile is not intended to be an exhaustive document; however, more comprehensive sources of specialty information are referenced.

The focus of the profiles is on health and toxicologic information; therefore, each toxicological profile begins with a public health statement that describes, in nontechnical language, a substance's relevant toxicological properties. Following the public health statement is information concerning levels of significant human exposure and, where known, significant health effects. The adequacy of information to determine a substance's health effects is described in a health effects summary. Data needs that are of significance to protection of public health are identified by ATSDR and EPA.

Each profile includes the following:

(A) The examination, summary, and interpretation of available toxicologic information and epidemiologic evaluations on a hazardous substance to ascertain the levels of significant human exposure for the substance and the associated acute, subacute, and chronic health effects;

(B) A determination of whether adequate information on the health effects of each substance is available or in the process of development to determine levels of exposure that present a significant risk to human health of acute, subacute, and chronic health effects; and

(C) Where appropriate, identification of toxicologic testing needed to identify the types or levels of exposure that may present significant risk of adverse health effects in humans.

The principal audiences for the toxicological profiles are health professionals at the Federal, State, and local levels; interested private sector organizations and groups; and members of the public.

This profile reflects ATSDR's assessment of all relevant toxicologic testing and information that has been peer-reviewed. Staff of the Centers for Disease Control and Prevention and other Federal scientists have also reviewed the profile. In addition, this profile has been peer-reviewed by a nongovernmental panel and is being made available for public review. Final responsibility for the contents and views expressed in this toxicological profile resides with ATSDR.

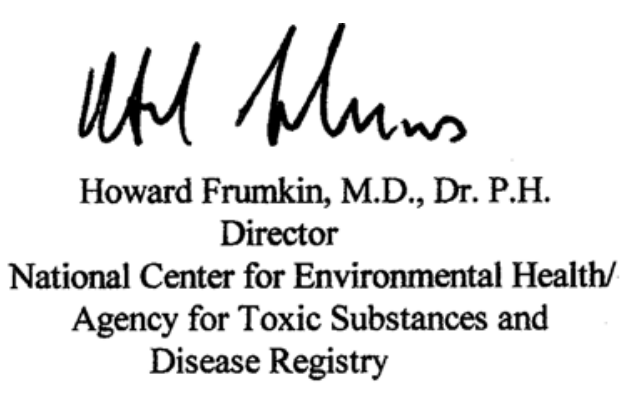

Howard Frumkin, M.D., Dr. P.H. Director National Center for Environmental Health/
Agency for Toxic Substances and
Disease Registry

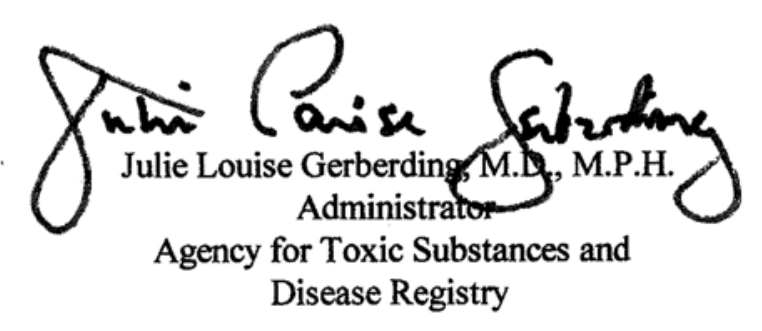


The toxicological profiles are developed in response to the Superfund Amendments and Reauthorization Act (SARA) of 1986 (Public Law 99-499) which amended the Comprehensive Environmental Response, Compensation, and Liability Act of 1980 (CERCLA or Superfund). This public law directed ATSDR to prepare toxicological profiles for hazardous substances most commonly found at facilities on the CERCLA National Priorities List and that pose the most significant potential threat to human health, as determined by ATSDR and the EPA. The availability of the revised priority list of 275 hazardous substances was announced in the Federal Register on December 7, 2005 (70 FR 72840). For prior versions of the list of substances, see Federal Register notices dated April 17, 1987 (52 FR 12866); October 20, 1988 (53 FR 41280); October 26, 1989 (54 FR 43619); October 17, 1990 (55 FR 42067); October 17, 1991 (56 FR 52166); October 28, 1992 (57 FR 48801); February 28, 1994 (59 FR 9486); April 29, 1996 (61 FR 18744); November 17, 1997 (62 FR 61332); October 21, 1999 (64 FR 56792); October 25, 2001 (66 FR 54014); and November 7, 2003 (68 FR 63098). Section 104(i)(3) of CERCLA, as amended, directs the Administrator of ATSDR to prepare a toxicological profile for each substance on the list. 


\section{QUICK REFERENCE FOR HEALTH CARE PROVIDERS}

Toxicological Profiles are a unique compilation of toxicological information on a given hazardous substance. Each profile reflects a comprehensive and extensive evaluation, summary, and interpretation of available toxicologic and epidemiologic information on a substance. Health care providers treating patients potentially exposed to hazardous substances will find the following information helpful for fast answers to often-asked questions.

\section{Primary Chapters/Sections of Interest}

Chapter 1: Public Health Statement: The Public Health Statement can be a useful tool for educating patients about possible exposure to a hazardous substance. It explains a substance's relevant toxicologic properties in a nontechnical, question-and-answer format, and it includes a review of the general health effects observed following exposure.

Chapter 2: Relevance to Public Health: The Relevance to Public Health Section evaluates, interprets, and assesses the significance of toxicity data to human health.

Chapter 3: Health Effects: Specific health effects of a given hazardous compound are reported by type of health effect (death, systemic, immunologic, reproductive), by route of exposure, and by length of exposure (acute, intermediate, and chronic). In addition, both human and animal studies are reported in this section.

NOTE: Not all health effects reported in this section are necessarily observed in the clinical setting. Please refer to the Public Health Statement to identify general health effects observed following exposure.

Pediatrics: Four new sections have been added to each Toxicological Profile to address child health issues:

\section{Section 1.6 How Can (Chemical X) Affect Children?}

Section 1.7 How Can Families Reduce the Risk of Exposure to (Chemical X)?

Section 3.7 Children's Susceptibility

Section 6.6 Exposures of Children

\section{Other Sections of Interest:}

Section 3.8 Biomarkers of Exposure and Effect

Section 3.11 Methods for Reducing Toxic Effects

\section{ATSDR Information Center \\ Phone: 1-800-CDC-INFO (800-232-4636) or 1-888-232-6348 (TTY) Fax: (770) 488-4178 \\ E-mail: cdcinfo@cdc.gov Internet: http://www.atsdr.cdc.gov}

The following additional material can be ordered through the ATSDR Information Center:

Case Studies in Environmental Medicine: Taking an Exposure History-The importance of taking an exposure history and how to conduct one are described, and an example of a thorough exposure history is provided. Other case studies of interest include Reproductive and Developmental Hazards; Skin Lesions and Environmental Exposures; Cholinesterase-Inhibiting Pesticide Toxicity; and numerous chemical-specific case studies. 
Managing Hazardous Materials Incidents is a three-volume set of recommendations for on-scene (prehospital) and hospital medical management of patients exposed during a hazardous materials incident. Volumes I and II are planning guides to assist first responders and hospital emergency department personnel in planning for incidents that involve hazardous materials. Volume IIIMedical Management Guidelines for Acute Chemical Exposures-is a guide for health care professionals treating patients exposed to hazardous materials.

Fact Sheets (ToxFAQs) provide answers to frequently asked questions about toxic substances.

\section{Other Agencies and Organizations}

The National Center for Environmental Health (NCEH) focuses on preventing or controlling disease, injury, and disability related to the interactions between people and their environment outside the workplace. Contact: NCEH, Mailstop F-29, 4770 Buford Highway, NE, Atlanta, GA 30341-3724 • Phone: 770-488-7000 • FAX: 770-488-7015.

The National Institute for Occupational Safety and Health (NIOSH) conducts research on occupational diseases and injuries, responds to requests for assistance by investigating problems of health and safety in the workplace, recommends standards to the Occupational Safety and Health Administration (OSHA) and the Mine Safety and Health Administration (MSHA), and trains professionals in occupational safety and health. Contact: NIOSH, 200 Independence Avenue, SW, Washington, DC 20201 • Phone: 800-356-4674 or NIOSH Technical Information Branch, Robert A. Taft Laboratory, Mailstop C-19, 4676 Columbia Parkway, Cincinnati, OH 45226-1998 - Phone: 800-35-NIOSH.

The National Institute of Environmental Health Sciences (NIEHS) is the principal federal agency for biomedical research on the effects of chemical, physical, and biologic environmental agents on human health and well-being. Contact: NIEHS, PO Box 12233, 104 T.W. Alexander Drive, Research Triangle Park, NC 27709 • Phone: 919-541-3212.

\section{Referrals}

The Association of Occupational and Environmental Clinics (AOEC) has developed a network of clinics in the United States to provide expertise in occupational and environmental issues. Contact: AOEC, 1010 Vermont Avenue, NW, \#513, Washington, DC 20005 • Phone: 202-347-4976 •FAX: 202-347-4950•e-mail: AOEC@AOEC.ORG•Web Page: http://www.aoec.org/.

The American College of Occupational and Environmental Medicine (ACOEM) is an association of physicians and other health care providers specializing in the field of occupational and environmental medicine. Contact: ACOEM, 25 Northwest Point Boulevard, Suite 700, Elk Grove Village, IL 60007-1030 • Phone: 847-818-1800 • FAX: 847-818-9266. 


\section{CONTRIBUTORS}

\section{CHEMICAL MANAGER(S)/AUTHOR(S):}

Mike Fay, Ph.D.

John F. Risher, Ph.D.

Jewell D. Wilson, Ph.D.

ATSDR, Division of Toxicology and Environmental Medicine, Atlanta, GA

Margaret Fransen, Ph.D.

Daniel Plewak, BS.

Lisa Ingerman, Ph.D., DABT

Syracuse Research Corporation, North Syracuse, NY

THE PROFILE HAS UNDERGONE THE FOLLOWING ATSDR INTERNAL REVIEWS:

1. Health Effects Review. The Health Effects Review Committee examines the health effects chapter of each profile for consistency and accuracy in interpreting health effects and classifying end points.

2. Minimal Risk Level Review. The Minimal Risk Level Workgroup considers issues relevant to substance-specific Minimal Risk Levels (MRLs), reviews the health effects database of each profile, and makes recommendations for derivation of MRLs.

3. Data Needs Review. The Applied Toxicology Branch reviews data needs sections to assure consistency across profiles and adherence to instructions in the Guidance.

4. Green Border Review. Green Border review assures the consistency with ATSDR policy. 
This page is intentionally blank. 


\section{PEER REVIEW}

A peer review panel was assembled for xylene. The panel consisted of the following members:

1. Dr. Bruce Jarnot, Private Consultant, Prince Frederick, Maryland;

2. Dr. Kannan Krishnan, Professor of Occupational and Environmental Health, University of Montreal, Pierrefonds, Canada; and

3. Dr. Steven Lewis, President, Lewis Environmental Consulting Services, Washington, New Jersey.

These experts collectively have knowledge of xylene's physical and chemical properties, toxicokinetics, key health end points, mechanisms of action, human and animal exposure, and quantification of risk to humans. All reviewers were selected in conformity with the conditions for peer review specified in Section 104(I)(13) of the Comprehensive Environmental Response, Compensation, and Liability Act, as amended.

Scientists from the Agency for Toxic Substances and Disease Registry (ATSDR) have reviewed the peer reviewers' comments and determined which comments will be included in the profile. A listing of the peer reviewers' comments not incorporated in the profile, with a brief explanation of the rationale for their exclusion, exists as part of the administrative record for this compound.

The citation of the peer review panel should not be understood to imply its approval of the profile's final content. The responsibility for the content of this profile lies with the ATSDR. 
This page is intentionally blank. 


\section{CONTENTS}

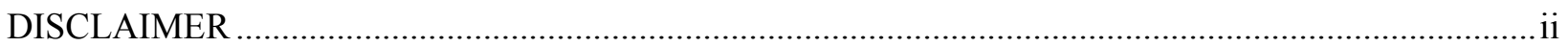

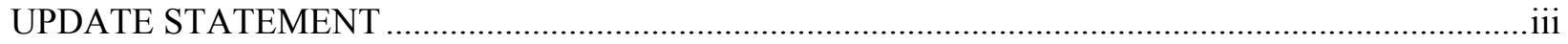

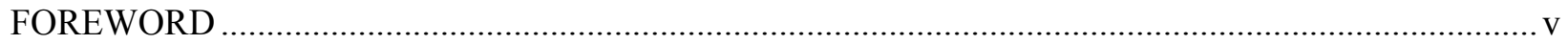

QUICK REFERENCE FOR HEALTH CARE PROVIDERS ..............................................................

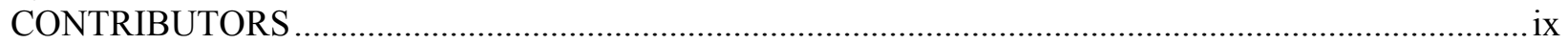

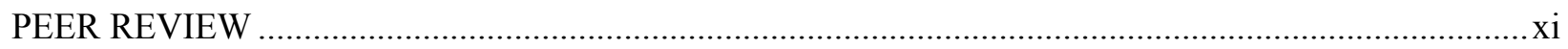

CONTENTS

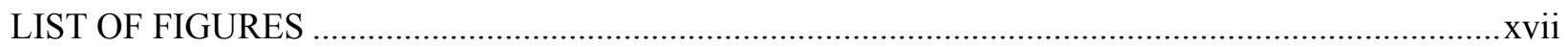

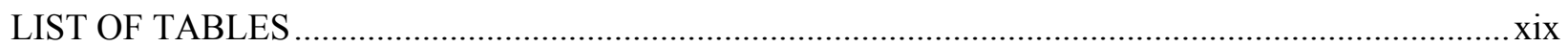

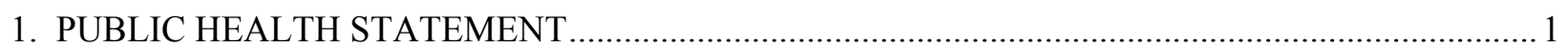

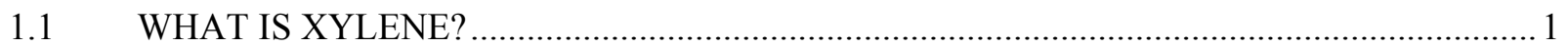

1.2 WHAT HAPPENS TO XYLENE WHEN IT ENTERS THE ENVIRONMENT?................... 2

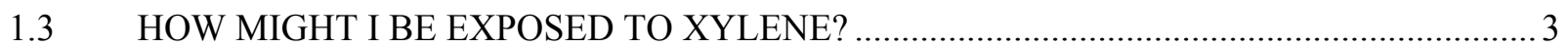

1.4 HOW CAN XYLENE ENTER AND LEAVE MY BODY? .......................................... 4

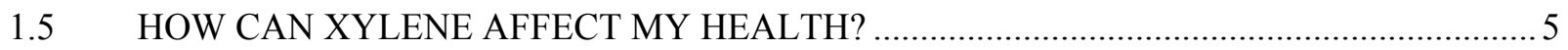

1.6 HOW CAN XYLENE AFFECT CHILDREN? ................................................................. 6

1.7 HOW CAN FAMILIES REDUCE THE RISK OF EXPOSURE TO XYLENE? ..................... 7

1.8 IS THERE A MEDICAL TEST TO DETERMINE WHETHER I HAVE BEEN

1.9 WHAT RECOMMENDATIONS HAS THE FEDERAL GOVERNMENT MADE TO

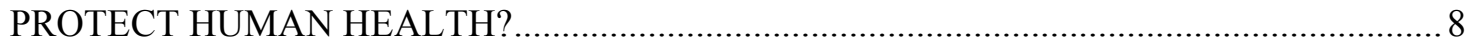

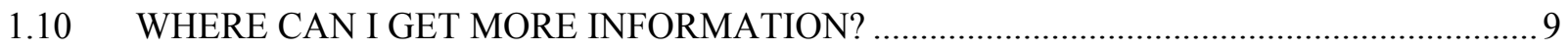

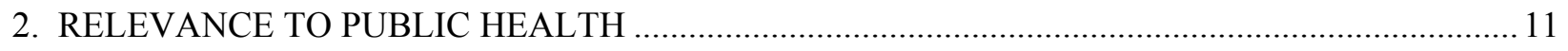

2.1 BACKGROUND AND ENVIRONMENTAL EXPOSURES TO XYLENE IN THE

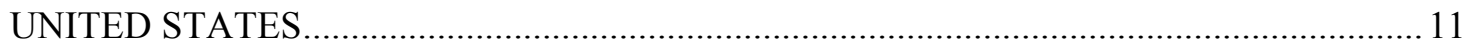

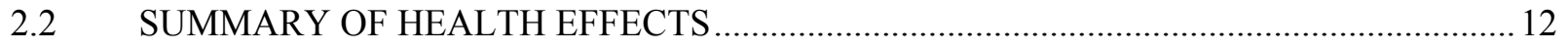

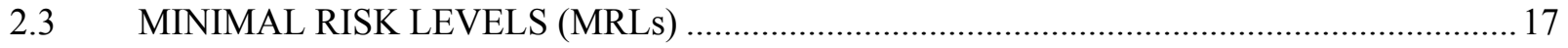

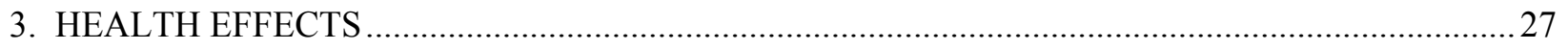

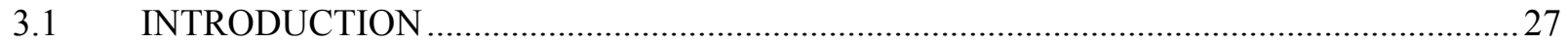

3.2 DISCUSSION OF HEALTH EFFECTS BY ROUTE OF EXPOSURE …..........................27

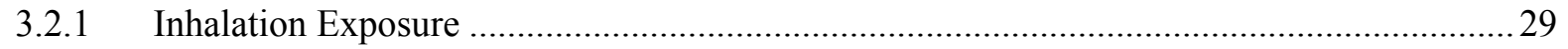

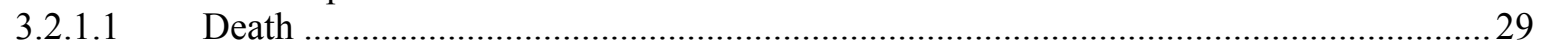

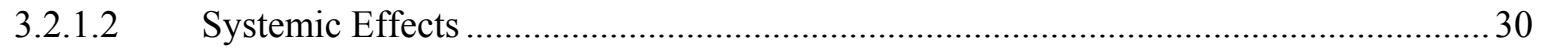

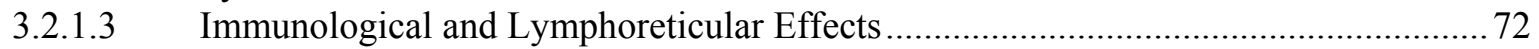

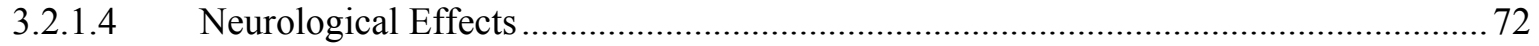

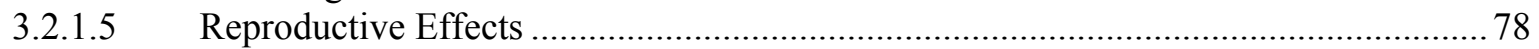

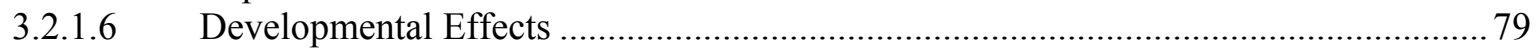

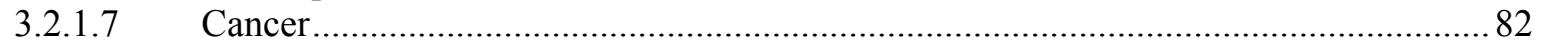

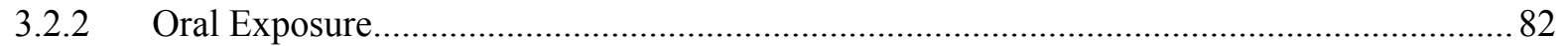

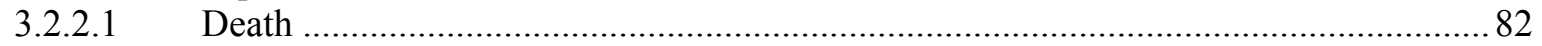

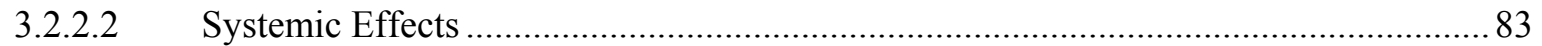

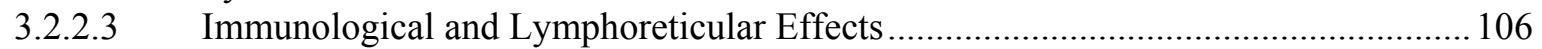

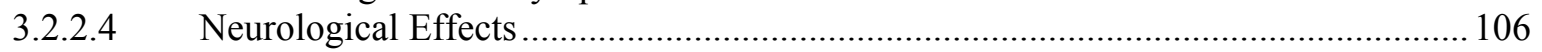

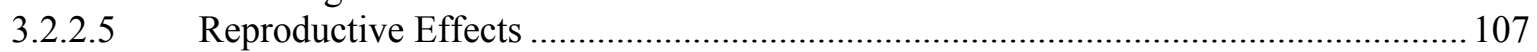

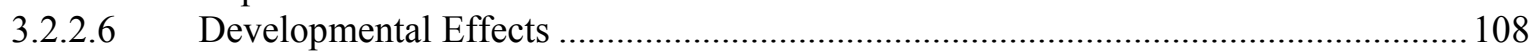

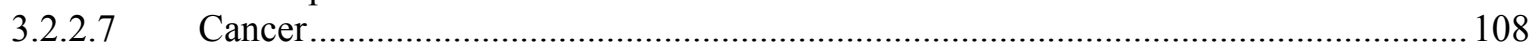




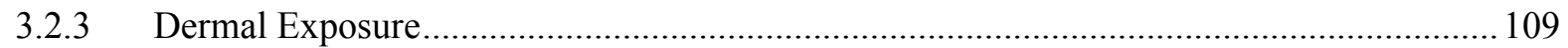

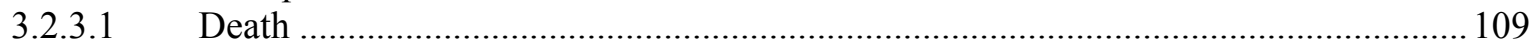

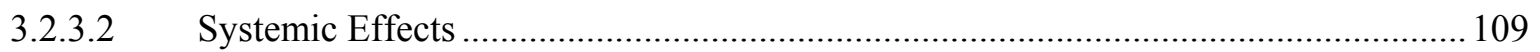

3.2.3.3 Immunological and Lymphoreticular Effects ........................................................ 115

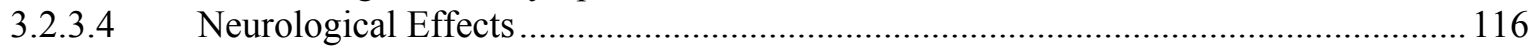

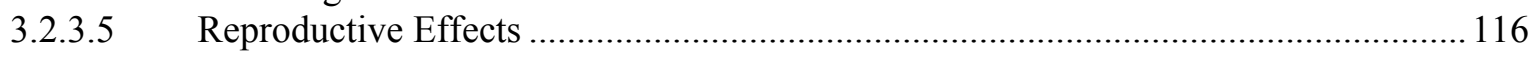

3.2.3.6 Developmental Effects ……………………………………………………....116

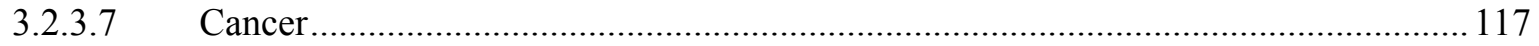

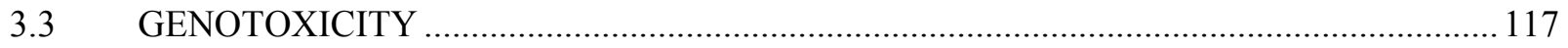

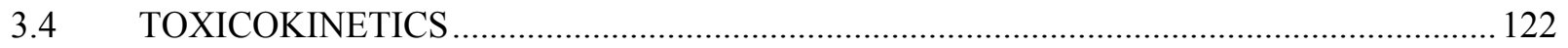

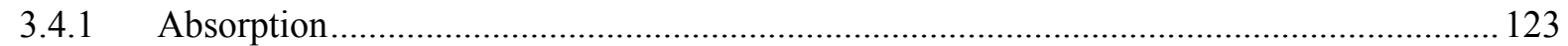

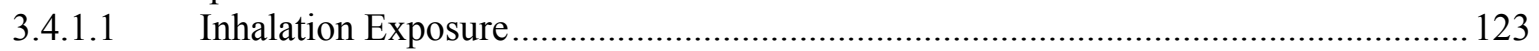

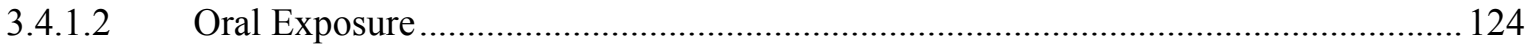

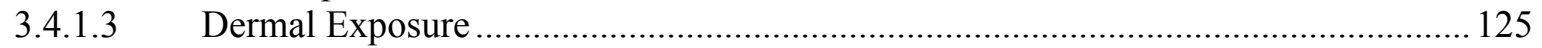

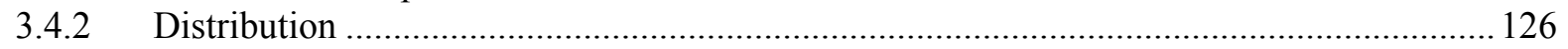

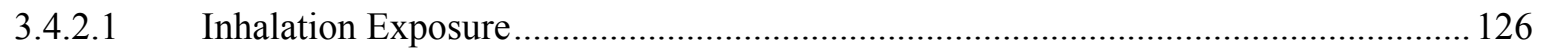

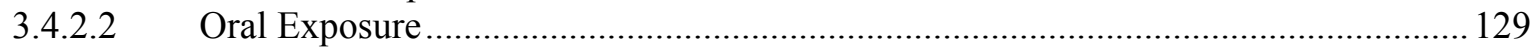

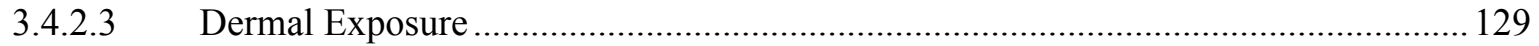

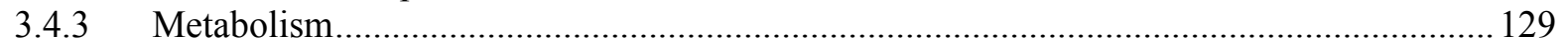

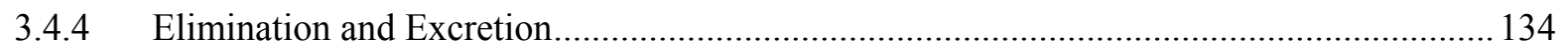

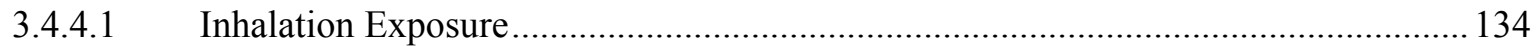

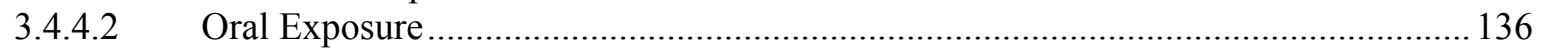

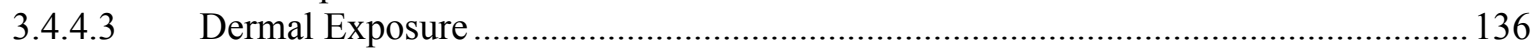

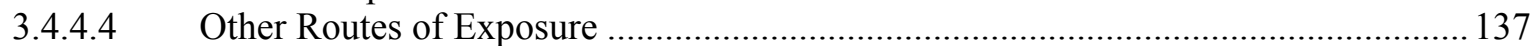

3.4.5 Physiologically Based Pharmacokinetic (PBPK)/Pharmacodynamic (PD) Models ............ 138

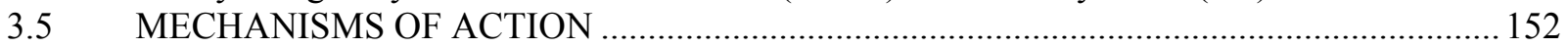

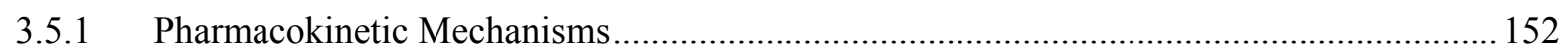

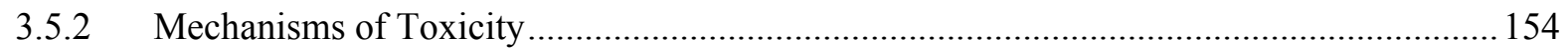

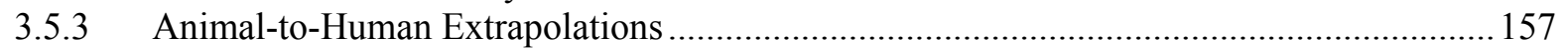

3.6 TOXICITIES MEDIATED THROUGH THE NEUROENDOCRINE AXIS ...........................157

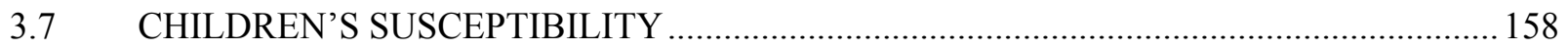

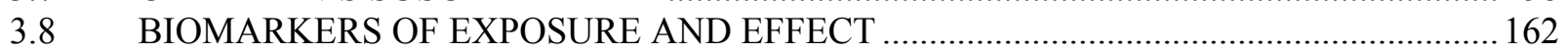

3.8.1 Biomarkers Used to Identify or Quantify Exposure to Xylene ..............................................163

3.8.2 Biomarkers Used to Characterize Effects Caused by Xylene .............................................. 164

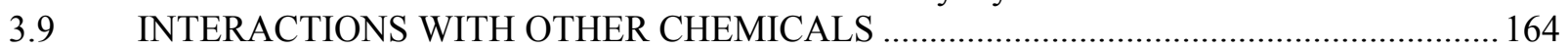

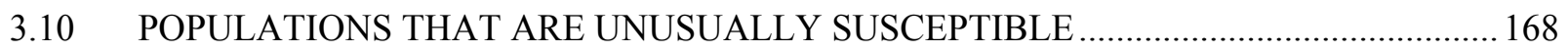

3.11 METHODS FOR REDUCING TOXIC EFFECTS ……………………………………... 170

3.11.1 Reducing Peak Absorption Following Exposure ...................................................... 170

3.11.2 Reducing Body Burden...................................................................................... 171

3.11.3 Interfering with the Mechanism of Action for Toxic Effects .......................................... 171

3.12 ADEQUACY OF THE DATABASE ........................................................................ 172

3.12.1 Existing Information on Health Effects of Xylene ………………………………......173

3.12.2 Identification of Data Needs .................................................................................. 175

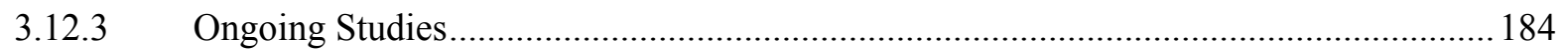

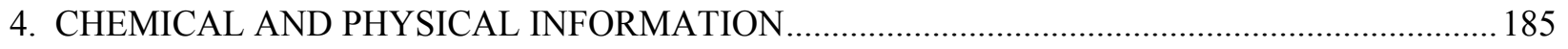

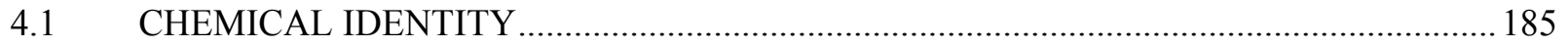

$4.2 \quad$ PHYSICAL AND CHEMICAL PROPERTIES...………………………………………... 185

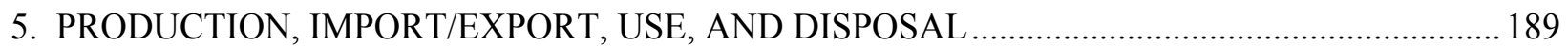

$5.1 \quad$ PRODUCTION ………………………………………………………………….... 189 


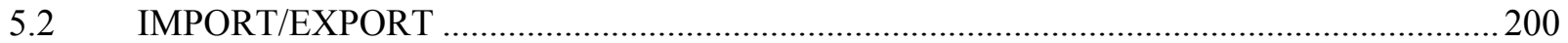

5.3 USE

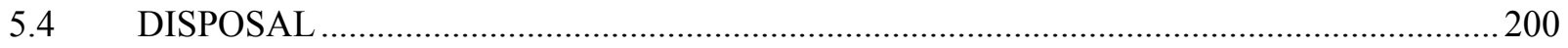

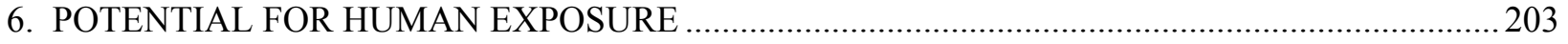

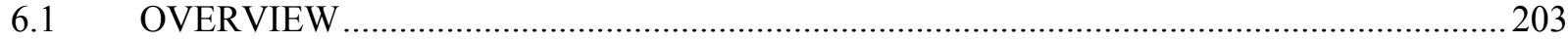

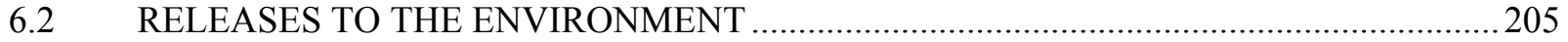

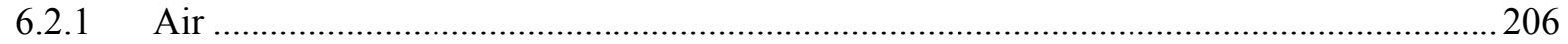

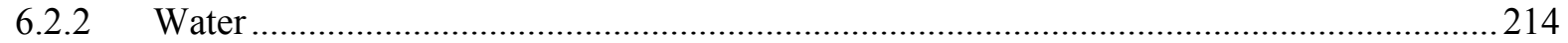

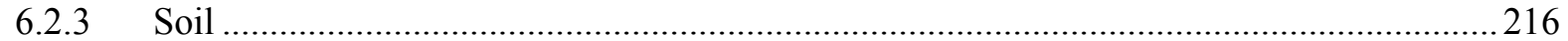

$6.3 \quad$ ENVIRONMENTAL FATE

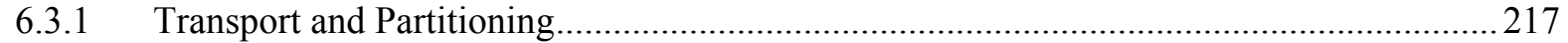

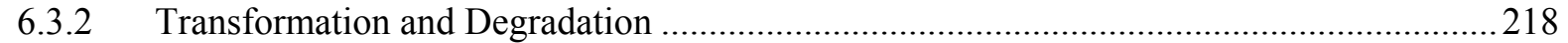

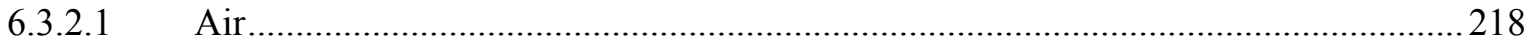

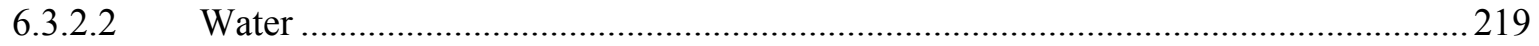

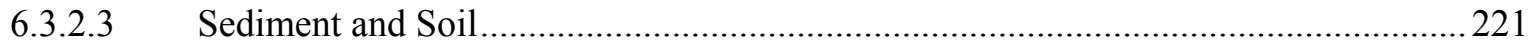

6.4 LEVELS MONITORED OR ESTIMATED IN THE ENVIRONMENT ...........................222

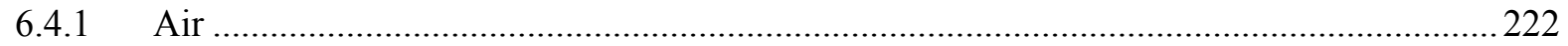

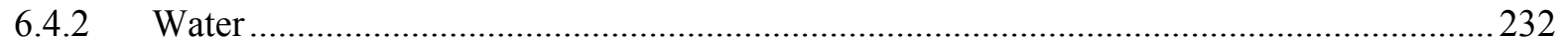

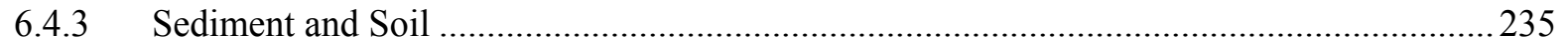

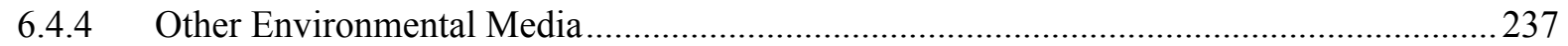

6.5 GENERAL POPULATION AND OCCUPATIONAL EXPOSURE .................................239

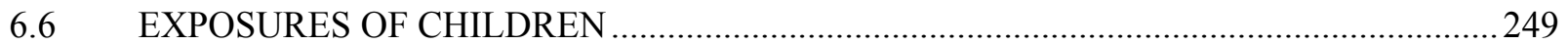

6.7 POPULATIONS WITH POTENTIALLY HIGH EXPOSURES .......................................25

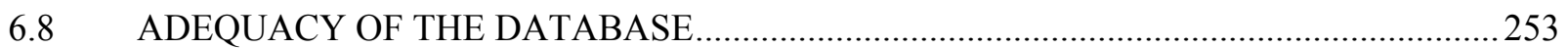

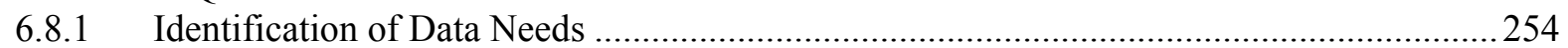

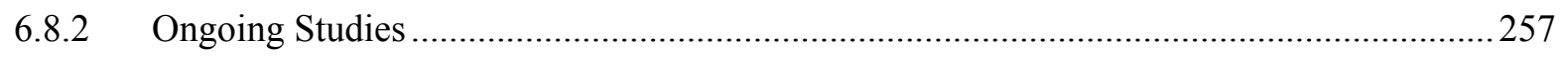

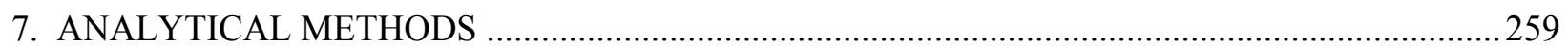

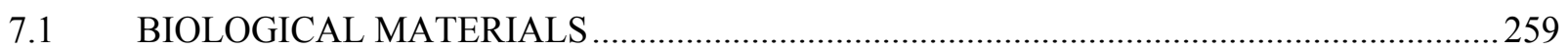

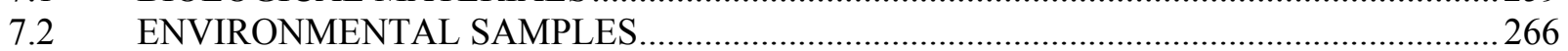

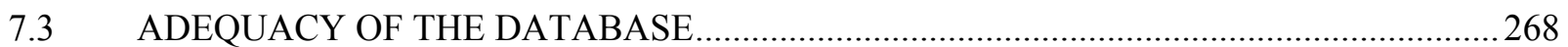

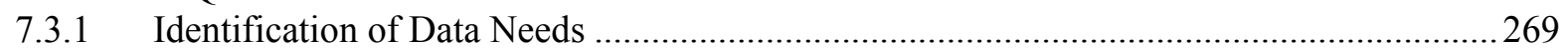

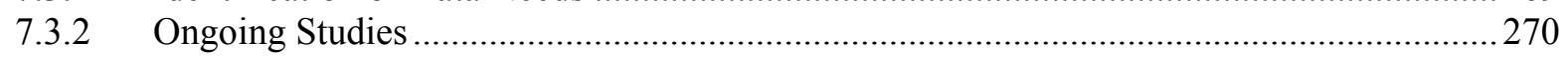

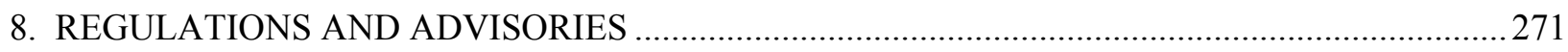

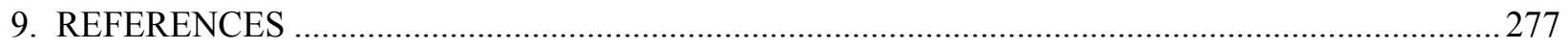

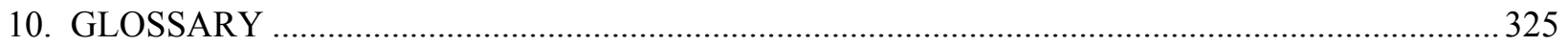

\section{APPENDICES}

A. ATSDR MINIMAL RISK LEVELS AND WORKSHEETS …......................................................A-1

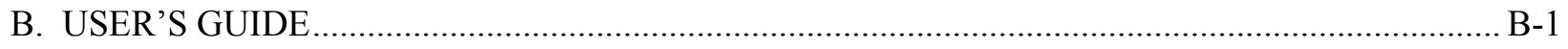

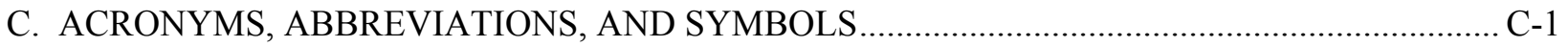

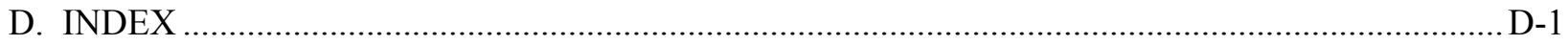


This page is intentionally blank. 


\section{LIST OF FIGURES}

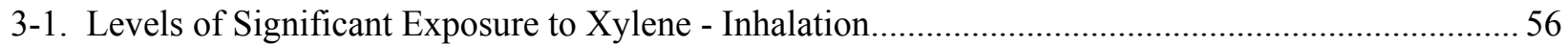

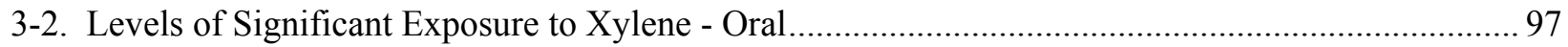

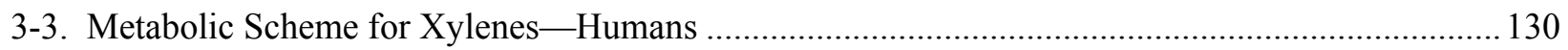

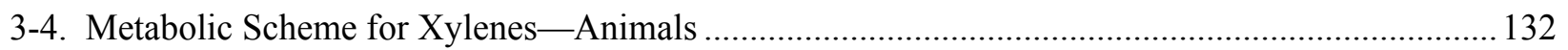

3-5. Conceptual Representation of a Physiologically Based Pharmacokinetic (PBPK) Model for a

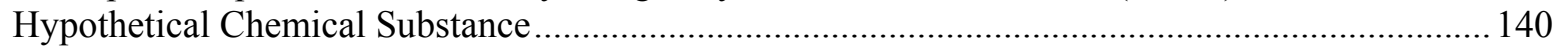

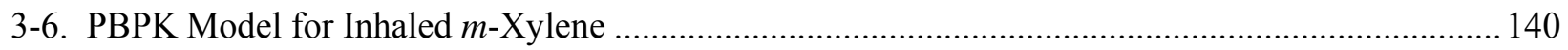

3-7. PBPK Model to Describe Dermal Absorption of $o$-Xylene ........................................................ 146

3-8. PBPK Model for Lactational Exposure to Volatile Organic Compounds (Including o-, m-, p-Xylenes) Following Maternal Occupational Inhalation Exposure ............................................. 149

3-9. Existing Information on Health Effects of Total Xylenes ....................................................... 174

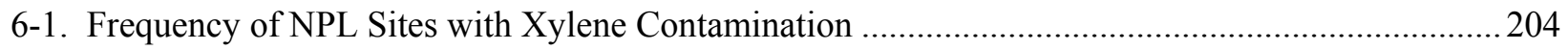


This page is intentionally blank. 


\section{LIST OF TABLES}

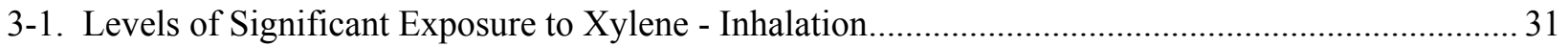

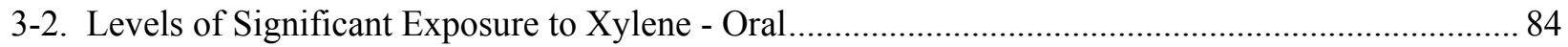

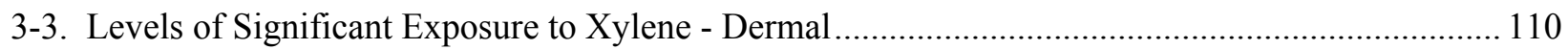

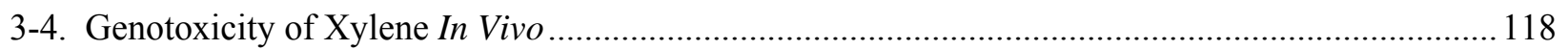

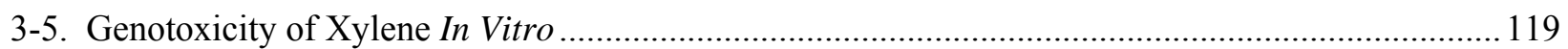

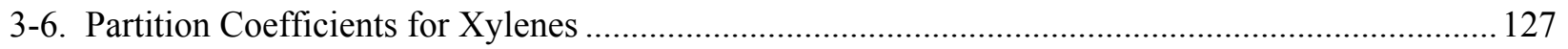

3-7. Human Parameters Used in the PBPK Inhalation Model for $m$-Xylene......................................... 142

3-8. Rat and Human Parameters Used in the PBPK Dermal Model for $o$-Xylene ................................ 145

3-9. Human Parameters Used in the PBPK Lactational Model for $o-, m-, p$-Xylene ............................ 148

4-1. Chemical Identity of $m$-Xylene, $o$-Xylene, $p$-Xylene, and Mixed Xylene .................................... 186

4-2. Physical and Chemical Properties of $m$-Xylene, $o$-Xylene, $p$-Xylene, and Mixed Xylene .............. 187

5-1. Producers of Xylene and Estimated Annual Capacities .................................................................. 190

5-2. Facilities that Produce, Process, or Use Xylene (Mixed) ............................................................ 191

5-3. Facilities that Produce, Process, or Use $m$-Xylene ........................................................................ 194

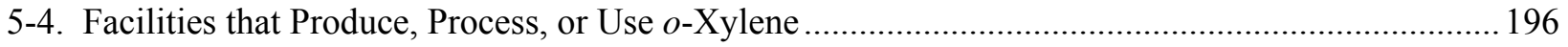

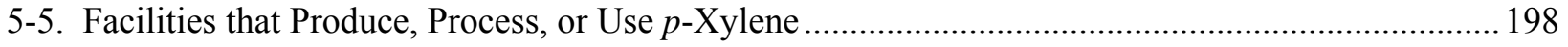

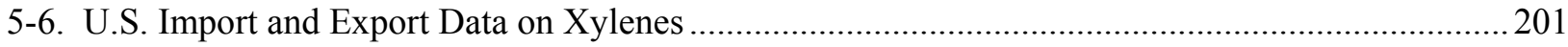

6-1. Releases to the Environment from Facilities that Produce, Process, or Use Xylene (Mixed)..........207

6-2. Releases to the Environment from Facilities that Produce, Process, or Use $m$-Xylene....................209

6-3. Releases to the Environment from Facilities that Produce, Process, or Use $o$-Xylene.....................210

6-4. Releases to the Environment from Facilities that Produce, Process, or Use $p$-Xylene.....................212

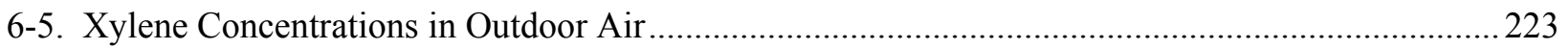

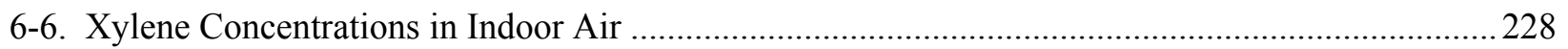

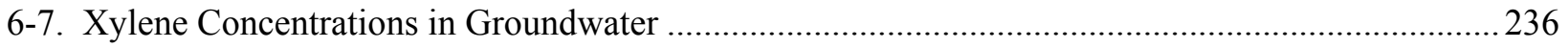


6-8. Average Personal Air, Outdoor Air, and Breath Concentrations Measured During the 1984 TEAM Study

6-9. Average Personal Air, Indoor Air, Outdoor Air, and Breath Concentrations from the 1987 TEAM Study

6-10. Concentrations of Xylene in Consumer Products.

6-11. Percentage Breakdown of NIOSH Occupational Exposure Estimates from the NOES Database

6-12. Personal, Indoor, and Outdoor Air Exposures of Inner-city Children in Minneapolis, Minnesota to Xylenes .252

7-1. Analytical Methods for Determining Xylene in Biological Materials...... 260

7-2. Analytical Methods for Determining Xylene in Environmental Samples 262

8-1. Regulations and Guidelines Applicable to Xylenes 


\section{PUBLIC HEALTH STATEMENT}

This public health statement tells you about xylene and the effects of exposure to it.

The Environmental Protection Agency (EPA) identifies the most serious hazardous waste sites in the nation. These sites are then placed on the National Priorities List (NPL) and are targeted for long-term federal clean-up activities. Xylene has been found in at least 840 of the 1,684 current or former NPL sites. Although the total number of NPL sites evaluated for this substance is not known, the possibility exists that the number of sites at which xylene is found may increase in the future as more sites are evaluated. This information is important because these sites may be sources of exposure and exposure to this substance may harm you.

When a substance is released either from a large area, such as an industrial plant, or from a container, such as a drum or bottle, it enters the environment. Such a release does not always lead to exposure. You can be exposed to a substance only when you come in contact with it. You may be exposed by breathing, eating, or drinking the substance, or by skin contact.

If you are exposed to xylene, many factors will determine whether you will be harmed. These factors include the dose (how much), the duration (how long), and how you come in contact with it. You must also consider any other chemicals you are exposed to and your age, sex, diet, family traits, lifestyle, and state of health.

\subsection{WHAT IS XYLENE?}

In this report, the terms xylene, xylenes, and total xylenes will be used interchangeably. There are three forms of xylene in which the methyl groups vary on the benzene ring: meta-xylene, ortho-xylene, and para-xylene ( $m-, o_{-}$, and $p$-xylene). These different forms are referred to as isomers. Drawings of the three different isomers are shown in Chapter 4. The term total xylenes refers to all three isomers of xylene ( $m-, o_{-}$, and $p$-xylene). Mixed xylene is a mixture of the three isomers and usually also contains $6-15 \%$ ethylbenzene. Xylene is also known as xylol or dimethylbenzene. Xylene is primarily a synthetic chemical. Chemical industries produce xylene 
from petroleum. Xylene also occurs naturally in petroleum and coal tar and is formed during forest fires, to a small extent. It is a colorless, flammable liquid with a sweet odor.

Xylene is one of the top 30 chemicals produced in the United States in terms of volume. It is primarily used as a solvent (a liquid that can dissolve other substances) in the printing, rubber, and leather industries. Along with other solvents, xylene is also widely used as a cleaning agent, a thinner for paint, and in varnishes. Xylene is used, to a lesser extent, as a material in the chemical, plastics, and synthetic fiber industries and as an ingredient in the coating of fabrics and papers. Isomers of xylene are used in the manufacture of certain polymers (chemical compounds), such as plastics. Xylene is found in small amounts in airplane fuel and gasoline.

Xylene evaporates and burns easily. Xylene does not mix well with water; however, it does mix with alcohol and many other chemicals. Most people begin to smell xylene in air at $0.08-$ 3.7 parts of xylene per million parts of air ( $\mathrm{ppm})$ and in water at $0.53-1.1 \mathrm{ppm}$. Additional information regarding chemical and physical properties and use of xylene can be found in Chapters 4 and 5.

\subsection{WHAT HAPPENS TO XYLENE WHEN IT ENTERS THE ENVIRONMENT?}

Xylene is a liquid, and it can leak into soil, surface water (creeks, streams, rivers), or groundwater. Xylene can enter the environment when it is made, packaged, shipped, or used. Most xylene that is accidentally released evaporates into the air, although some is released into rivers or lakes. Xylene can also enter soil, water, or air in large amounts after an accidental spill or as a result of an environmental leak during storage or burial at a waste site.

Since xylene evaporates easily, most xylene that gets into soil and water (if not trapped underground) is expected to go into the air where it is broken down by sunlight into other less harmful chemicals within a couple of days. For this reason, xylene is rarely found in high concentrations in topsoil or surface water (river, creeks) unless there has been a recent spill or continuing source of contamination. Any xylene that does not evaporate quickly from soil or 
water is broken down by small organisms. Only very small amounts are taken up by plants, fish, and birds.

Xylene below the soil surface may travel down through the soil and enter underground water (groundwater). Xylene may remain in groundwater for several months before it is finally broken down by small organisms. If a large amount of xylene enters soil from an accidental spill, a hazardous waste site, or a landfill, it may travel through the soil and contaminate drinking water wells. More information on what happens to xylene in the environment can be found in Chapters 5 and 6.

\subsection{HOW MIGHT I BE EXPOSED TO XYLENE?}

Xylene is primarily released from industrial sources, in automobile exhaust, and during its use as a solvent. Hazardous waste disposal sites and spills of xylene into the environment are also possible sources of exposure. You are most likely to be exposed to xylene by breathing it in contaminated air. Typical levels of xylene measured in outdoor air in the United States range from 1 to 30 parts of xylene per billion parts of air (a part per billion [ppb] is one thousandth of a part per million [ppm]; one ppm equals 1,000 ppb). Typical levels of xylene measured in indoor air range from 1 to $10 \mathrm{ppb}$.

Xylene is sometimes released into water and soil as a result of the use, storage, and transport of petroleum products. Little information exists about the amount of xylene in surface water and soil. However, levels of xylene in contaminated groundwater have been reported to be as high as $10,000 \mathrm{ppb}$.

You may be exposed to xylene by drinking or eating xylene-contaminated water or food. Xylene is not commonly found in drinking water. When it is, the levels of xylene are typically below $2 \mathrm{ppb}$. Xylene has been found in many types of foods at levels ranging from 1 to $100 \mathrm{ppb}$.

You may also come in contact with xylene from a variety of consumer products, including gasoline, paint, varnish, shellac, rust preventives, and cigarette smoke. Breathing vapors from 
these types of products can expose you to xylene. In some cases, indoor levels of xylene can be higher than outdoor levels, especially in buildings with poor ventilation. Skin contact with products containing xylene, such as solvents, lacquers, paint thinners and removers, and pesticides may also expose you to xylene.

Besides painters and paint industry workers, others who may be exposed to xylene include biomedical laboratory workers, distillers of xylene, wood processing plant workers, automobile garage workers, metal workers, and furniture refinishers. Workers who routinely come in contact with xylene-containing solvents in the workplace are the population most likely to be exposed to high levels of xylene. Additional information on the potential for human exposure can be found in Chapter 6 .

\subsection{HOW CAN XYLENE ENTER AND LEAVE MY BODY?}

Xylene is most likely to enter your body when you breathe xylene vapors. Xylene may also enter your body if eat or drink xylene-contaminated food or water. Less often, xylene enters the body through the skin following direct contact. Xylene is rapidly absorbed by your lungs after you breathe air containing it. The amount of xylene retained ranges from 50 to $75 \%$ of the amount of xylene that you inhale. Physical exercise increases the amount of xylene absorbed by the lungs. Absorption of xylene in the gut after eating food or drinking water containing it is both rapid and complete. Absorption of liquid xylene through the skin also occurs rapidly following direct contact with xylene, but absorption of xylene vapor through the skin is only about $12 \%$ of the amount absorbed by the lungs. At hazardous waste sites, the most likely ways you can be exposed are: breathing xylene vapors, drinking well water contaminated with xylene, and direct contact of the skin with xylene. Xylene passes into the blood soon after entering the body.

In people and laboratory animals, xylene is chemically changed, primarily in the liver, into a different form that is more water soluble and is rapidly removed from the body in urine. Some unchanged xylene also leaves in the breath from the lungs within a few seconds after xylene is absorbed. Small amounts of breakdown products of xylene have appeared in the urine of people 
as soon as 2 hours after breathing air containing xylene. Usually, most of the xylene that is taken in leaves the body within 18 hours after exposure ends. About $4-10 \%$ of absorbed xylene may be stored in fat, which may prolong the time needed for xylene to leave the body. Additional information on how xylene can enter and leave your body can be found in Chapter 3 .

\subsection{HOW CAN XYLENE AFFECT MY HEALTH?}

Scientists use many tests to protect the public from harmful effects of toxic chemicals and to find ways for treating persons who have been harmed.

One way to learn whether a chemical will harm people is to determine how the body absorbs, uses, and releases the chemical. For some chemicals, animal testing may be necessary. Animal testing may also help identify health effects such as cancer or birth defects. Without laboratory animals, scientists would lose a basic method for getting information needed to make wise decisions that protect public health. Scientists have the responsibility to treat research animals with care and compassion. Scientists must comply with strict animal care guidelines because laws today protect the welfare of research animals.

Scientists have found that the three forms of xylene have very similar effects on health. No health effects have been noted at the background levels that people are exposed to on a daily basis. Short-term exposure of people to high levels of xylene can cause irritation of the skin, eyes, nose, and throat; difficulty in breathing; impaired function of the lungs; delayed response to a visual stimulus; impaired memory; stomach discomfort; and possible changes in the liver and kidneys. Both short- and long-term exposure to high concentrations of xylene can also cause a number of effects on the nervous system, such as headaches, lack of muscle coordination, dizziness, confusion, and changes in one's sense of balance. Some people exposed to very high levels of xylene for a short period of time have died. Most of the information on health effects in humans exposed for long periods of time is from studies of workers employed in industries that make or use xylene. Those workers were exposed to levels of xylene in air far greater than the levels normally encountered by the general population. Many of the effects seen after their 
exposure to xylene could have been caused by exposure to other chemicals that were in the air with xylene.

Results of studies in animals indicate that large amounts of xylene can cause changes in the liver and harmful effects on the kidneys, lungs, heart, and nervous system. Short-term exposure to very high concentrations of xylene causes death in animals, as well as muscular spasms, incoordination, hearing loss, changes in behavior, changes in organ weights, and changes in enzyme activity. Animals that were exposed to xylene on their skin had irritation and inflammation of the skin. Long-term exposure of animals to low concentrations of xylene has not been well studied, but there is some information that long-term exposure of animals can cause harmful effects on the kidney (with oral exposure) or on the nervous system (with inhalation exposure).

Information from animal studies is not adequate to determine whether or not xylene causes cancer in humans. Both the International Agency for Research on Cancer (IARC) and EPA have found that there is insufficient information to determine whether or not xylene is carcinogenic and consider xylene not classifiable as to its human carcinogenicity.

Additional information regarding the health effects of xylene can be found in Chapters 2 and 3.

\subsection{HOW CAN XYLENE AFFECT CHILDREN?}

This section discusses potential health effects in humans from exposures during the period from conception to maturity at 18 years of age.

The effects of xylenes have not been studied in children, but it is likely that they would be similar to those seen in exposed adults. Although there is no direct evidence, children may be more sensitive to acute inhalation exposure than adults because their narrower airways would be more vulnerable if the surrounding tissue became swollen. (One study suggested that the narrower airway in women compared to men could be the reason women had more breathing difficulty than men after breathing xylene.) 
Although there are no conclusive studies in humans, it is possible that exposure of pregnant women to high levels of xylene may cause harmful effects to the fetus. Animal studies showed that xylene absorbed by the mother can cross the placenta and reach the fetus. The unborn animals may have reduced body weight and delayed bone mineralization if the level of xylene is high enough to be toxic to the mother. Some animal studies have shown that newborn babies that were exposed to xylene during pregnancy have problems after birth with motor coordination and the ability to orient themselves in their surroundings. In general, these developmental effects occur at exposure levels much higher than those typically seen in the background environment, levels high enough to also harm the mother.

\subsection{HOW CAN FAMILIES REDUCE THE RISK OF EXPOSURE TO XYLENE?}

If your doctor finds that you have been exposed to substantial amounts of xylene, ask whether your children might also have been exposed. Your doctor might need to ask your state health department to investigate.

Exposure to xylene as solvents (in paints or gasoline) can be reduced if the products are used with adequate ventilation and if they are stored out of the reach of small children.

Sometimes older children sniff household chemicals in attempt to get high. Talk with your children about the dangers of sniffing xylene.

If products containing xylene are spilled on the skin, then the excess should be wiped off and the area cleaned with soap and water.

\subsection{IS THERE A MEDICAL TEST TO DETERMINE WHETHER I HAVE BEEN EXPOSED TO XYLENE?}

Medical tests are available to determine if you have been exposed to xylene at higher-thannormal levels. After xylene has been absorbed, some of its breakdown products can be measured in the urine. However, a urine sample must be provided very soon (within hours) after exposure 
ends because xylene quickly leaves the body. These tests may be available in certain doctors' offices. Available tests can only indicate exposure to xylene; they cannot be used to predict which health effects, if any, will develop. More information about xylene detection can be found in Chapters 3 and 7.

\subsection{WHAT RECOMMENDATIONS HAS THE FEDERAL GOVERNMENT MADE TO PROTECT HUMAN HEALTH?}

The federal government develops regulations and recommendations to protect public health. Regulations can be enforced by law. The EPA, the Occupational Safety and Health Administration (OSHA), and the Food and Drug Administration (FDA) are some federal agencies that develop regulations for toxic substances. Recommendations provide valuable guidelines to protect public health, but cannot be enforced by law. The Agency for Toxic Substances and Disease Registry (ATSDR) and the National Institute for Occupational Safety and Health (NIOSH) are two federal organizations that develop recommendations for toxic substances.

Regulations and recommendations can be expressed as "not-to-exceed" levels, that is, levels of a toxic substance in air, water, soil, or food that do not exceed a critical value that is usually based on levels that affect animals; they are then adjusted to levels that will help protect humans. Sometimes these not-to-exceed levels differ among federal organizations because they used different exposure times (an 8-hour workday or a 24-hour day), different animal studies, or other factors.

Recommendations and regulations are also updated periodically as more information becomes available. For the most current information, check with the federal agency or organization that provides it. Some regulations and recommendations for xylene include the following:

To protect people from the potential harmful health effects of xylene, EPA regulates xylene in the environment. 
The EPA estimates that, for an adult of average weight who drinks $2 \mathrm{~L}$ of water each day for a lifetime (70 years), exposure to 7 ppm xylene in drinking water is unlikely to result in harmful noncancerous health effects.

Exposure to 40 ppm xylene in water for 1 or 10 days is unlikely to present a health risk to a small child.

The EPA has set a legally enforceable maximum level of $10 \mathrm{ppm}$ of xylene in water that is delivered to any user of a public water system.

OSHA regulates levels of xylene in the workplace. The maximum allowable amount of xylene in workroom air during an 8-hour workday in a 40-hour workweek is 100 ppm.

More information on government regulations for xylene can be found in Chapter 8.

\subsection{WHERE CAN I GET MORE INFORMATION?}

If you have any more questions or concerns, please contact your community or state health or environmental quality department, or contact ATSDR at the address and phone number below.

ATSDR can also tell you the location of occupational and environmental health clinics. These clinics specialize in recognizing, evaluating, and treating illnesses that result from exposure to hazardous substances.

Toxicological profiles are also available on-line at www.atsdr.cdc.gov and on CD-ROM. You may request a copy of the ATSDR ToxProfiles ${ }^{\mathrm{TM}} \mathrm{CD}-\mathrm{ROM}$ by calling the toll-free information 
and technical assistance number at 1-800-CDCINFO (1-800-232-4636), by e-mail at cdcinfo@cdc.gov, or by writing to:

\author{
Agency for Toxic Substances and Disease Registry \\ Division of Toxicology and Environmental Medicine \\ 1600 Clifton Road NE \\ Mailstop F-32 \\ Atlanta, GA 30333 \\ Fax: 1-770-488-4178
}

Organizations for-profit may request copies of final Toxicological Profiles from the following:

National Technical Information Service (NTIS)

5285 Port Royal Road

Springfield, VA 22161

Phone: 1-800-553-6847 or 1-703-605-6000

Web site: http://www.ntis.gov/ 


\section{RELEVANCE TO PUBLIC HEALTH}

\subsection{BACKGROUND AND ENVIRONMENTAL EXPOSURES TO XYLENE IN THE UNITED STATES}

Xylenes (mixtures of ortho-, meta-, and para-isomers) are used as industrial solvents, synthetic intermediates, and solvents in commercial products such as paints, coatings, adhesive removers, and paint thinners; they are also a component of gasoline. Xylenes are released to the atmosphere primarily as fugitive emissions from industrial sources (e.g., petroleum refineries, chemical plants), in automobile exhaust, and through volatilization from their use as solvents. Discharges into waterways and spills on land result primarily from use, storage, and transport of petroleum products and waste disposal.

When xylene is released to soil or surface water, it is expected to volatilize into the atmosphere where it is quickly degraded. The half-life for xylene in the atmosphere is 8-14 hours. Any xylene in soil or surface water that does not volatilize quickly may undergo biodegradation. Xylene may also leach into groundwater where biodegradation becomes the primary removal process. Half-lives measured for xylene in groundwater range from 25 to 287 days. Degradation rates in groundwater will vary depending on differences in conditions such as temperature, presence of oxygen, and presence of electron acceptors.

Xylene is primarily detected in air. Typical xylene concentrations range from 1 to $30 \mathrm{ppb}$ in outdoor air and from 1 to $10 \mathrm{ppb}$ in indoor air. Xylene has been detected in $<5 \%$ of samples collected during groundwater surveys conducted in the United States. Median xylene concentrations of $\leq 2 \mathrm{ppb}$ have been reported in 406 urban and 2,542 rural drinking water wells or monitoring wells in the United States. Less than $6 \%$ of drinking water samples collected during drinking water surveys contained xylene; the mean concentrations in positive samples were typically $<2 \mathrm{ppb}$. Xylene has been detected in all kinds of foods (e.g., meat, fruit, dairy, fish, vegetables, grains), with typical concentrations ranging from 1 to $100 \mathrm{ppb}$.

Because individual xylene isomers are used in large amounts in industrial settings, people who work at or live near these locations may receive a higher exposure to one xylene isomer compared to the other isomers. However, since xylenes are present as a mixture in gasoline and in the solvent components of commonly used commercial products (paint, coatings, etc.), exposure of the general population is expected to be primarily to xylenes as a mixture, and not to the separate xylene isomers.

Inhalation appears to be the major route of exposure to xylene, although exposure through the use or consumption of groundwater is also likely in areas where there is subsurface gasoline contamination. 
During exposures to xylene vapor, a small amount of dermal absorption also occurs, $\leq 2 \%$ of the amount inhaled. Humans may also be exposed to xylene through smoking, consumption of xylene-contaminated foods, and dermal contact with consumer products containing xylene. Children are expected to be exposed to xylene by the same routes as adults. Since xylene has a low affinity for adsorption onto soil and dust particles and a high volatilization rate, the risk of exposure for small children from ingesting soil or dust is likely to be low. The average daily intake of total xylene (sum of $o-, m-$, and $p$-xylene intakes) for the general population is estimated as $0.3-8.6 \mu \mathrm{g} / \mathrm{kg} /$ day from inhalation exposure and $0.06 \mu \mathrm{g} / \mathrm{kg} / \mathrm{day}$ from ingestion of drinking water assuming typical low background levels. Based on a maximal concentration of $1.5 \mathrm{mg} / \mathrm{L}$ in drinking water, the maximal daily consumption of xylenes from drinking water would be $0.04 \mathrm{mg} / \mathrm{kg} / \mathrm{day}$. These exposure levels are below the minimal risk levels (MRLs) established for xylenes (see Section 2.3 and Appendix A).

See Chapter 6 for more detailed information regarding concentrations of xylenes in environmental media.

\subsection{SUMMARY OF HEALTH EFFECTS}

Xylenes, because of their lipophilic properties, are rapidly absorbed by all routes of exposure, rapidly distributed throughout the body, and, if not metabolized, quickly eliminated in exhaled air. In humans, absorption has been estimated as $>50 \%$ through the lungs following inhalation exposure and $<50 \%$ through the gastrointestinal system. In humans exposed by inhalation, up to $2 \%$ of the absorbed dose may be absorbed through the skin. The major pathway for metabolism involves mixed function oxidases in the liver, resulting mainly in the formation of isomers of methylhippuric acid that are eliminated in the urine and are used as an index of exposure for occupational monitoring. Background urinary levels of methylhippuric acids in nonexposed workers are $<2 \mathrm{mg} / \mathrm{g}$ creatinine. Xylenes tend not to accumulate in the body, but they may be sequestered briefly in fat tissues due to their lipophilicity; elimination of xylene is slower in individuals with a greater percentage of body fat. The primary effects of xylene exposure involve the nervous system by all routes of exposure, the respiratory tract by inhalation exposure, and, at higher oral exposure levels, hepatic, renal, and body weight effects. No adverse health effects have been associated with the background levels of xylene to which the general population is typically exposed (see Section 2.1). Isomers of xylene have similar toxicokinetic properties and elicit similar toxicological effects, with no single isomer consistently exhibiting the greatest potency, depending on the end point. This issue is discussed further in the introduction to Section 2.3 and in Section 3.2. 
Information on the toxicity of xylene in humans comes from case reports, occupational studies, and studies on volunteers. At acute-duration inhalation concentrations as low as $50 \mathrm{ppm}$, xylenes produce irritant effects on the eyes, skin, and mucous membranes; impaired respiratory function; and mild central nervous system effects, including headache and dizziness. Increases in subjective reports of eye irritation, sore throat, and neurological effects (anxiety, forgetfulness, inability to concentrate, and a sensation of intoxication) were noted following chronic-duration occupational exposure at $14 \mathrm{ppm}$. Irritation of the eye may occur from contact with xylene vapor or from direct contact with xylene liquid, in which case photophobia, redness of the conjunctiva, and partial loss of the conjunctival and corneal epithelia have been reported. Slight-to-moderate eye irritation has also been observed in rabbits following direct instillation with $\geq 23 \mathrm{mg} / \mathrm{kg}$ mixed xylenes. With increasing airborne xylene concentrations of 100 $400 \mathrm{ppm}$, other neurological effects reported in acutely exposed human subjects include retardation of response times and impairments in memory and body balance. Acute exposure to an estimated 10,000 ppm xylenes elicited tremors, mental confusion, and depressant effects (narcosis) on the central nervous system that caused at least one fatality due to respiratory failure. All of these effects are related to the lipophilic properties of xylenes, which interfere with the integrity of cell membranes and alter neuronal function. In addition to neurological and respiratory effects, an increase in the reporting of nausea was noted following controlled exposure to $m$-xylene at $50 \mathrm{ppm}$. Symptoms of nausea and vomiting have also been noted in workers exposed to xylene vapors.

Other effects of xylene exposure involve the liver and kidney in humans and animals and body weight effects in laboratory animals. Hepatic effects (elevated serum transaminases and hepatocellular vacuolation) were observed in a limited number of case reports describing effects of acute exposure to an estimated 700-10,000 ppm mixed xylene, but were not observed in workers with chronic occupational exposure at $14 \mathrm{ppm}$. Hepatic effects in laboratory animals exposed orally at $\geq 750 \mathrm{mg} / \mathrm{kg} / \mathrm{day}$ or by inhalation at $\geq 300 \mathrm{ppm}$ include increases in liver weight, serum enzyme levels, and cytochrome P-450 levels, but no histopathology. However, a number of authors characterized the hepatic effects in animals as adaptive rather than adverse. Information on renal effects (distal renal tubular acidemia and abnormal clinical chemistry values) of xylene in acutely exposed humans is confounded by exposure to other compounds or uncertainties as to the duration of exposure. No alterations in renal serum biochemistry values were observed in workers exposed to $14 \mathrm{ppm}$ mixed xylene for several years. Renal effects in repeatedly exposed laboratory animals include increases in renal enzyme activity, cytochrome P-450 content, and increased kidney-to-body-weight ratios following inhalation exposure at 50-2,000 ppm or oral exposure and increased chronic nephropathy in rats exposed at $\geq 750 \mathrm{mg} / \mathrm{kg} / \mathrm{day}$. However, no renal effects were observed in rats exposed via inhalation to $810 \mathrm{ppm}$ mixed xylenes for 13 weeks, gavage 
doses of $1,000 \mathrm{mg} / \mathrm{kg} /$ day 5 days/week for 13 weeks, or gavage doses of $800 \mathrm{mg} / \mathrm{kg} /$ day to $m$ - or $p$-xylene for 90 consecutive days. It is not known whether the variability in induction of renal effects from mixed xylene is related to variations in the relative amounts of $o$-xylene or ethylbenzene or variations in the strains of rats tested. Decreased body weight gain has been observed in laboratory animals repeatedly exposed by inhalation at $\geq 700 \mathrm{ppm}$ or by oral dosing or at $\geq 700 \mathrm{mg} / \mathrm{kg} /$ day; a $5-8 \%$ reduction in body weight gain observed in male rats exposed at $500 \mathrm{mg} / \mathrm{kg}$ during the last year of a 2-year study is not considered biologically significant. Dermal exposure of humans to xylene causes skin irritation, dryness and scaling of the skin, and vasodilation. In addition, one case report demonstrated the possibility that contact urticaria can develop after several months of occupational exposure to $100 \mathrm{ppm}$ xylene vapors. Dermal effects of $m$-xylene, $o$-xylene, or mixed xylenes in laboratory animal studies included skin irritation (erythema and edema) at topical doses as low as $2.3 \mathrm{mg} / \mathrm{kg}$ and more serious effects (eschar formation in some animals and epidermal thickening) at topical doses of $\geq 114 \mathrm{mg} / \mathrm{kg}$. Rat skin that developed moderate erythema after treatment with $m$ - or $o$-xylene exhibited increases in transepidermal water loss and increases in pro-inflammatory cytokines (interleukin 1-alpha and tumor necrosis factoralpha).

Available studies of developmental or reproductive toxicity from occupational exposure to xylenes are not definitive because of the small number of subjects and/or concurrent exposure to other chemicals. In general, developmental studies in animals reported adverse fetal effects only at concentrations that caused maternal toxicity. Developmental effects in laboratory animals exposed to $\geq 350 \mathrm{ppm}$ xylenes by inhalation include delayed ossification of the skeleton at maternally toxic concentrations and reduced fetal body weight, which is also influenced by maternal body weight effects. Postnatal neurobehavioral deficits (decreased rotarod performance) have been observed in rats gestationally exposed to $m$-xylene at $500 \mathrm{ppm}$. Oral exposure to $2,060 \mathrm{mg} / \mathrm{kg} /$ day of mixed xylene has been associated with cleft plate and decreased fetal weight. Dermal exposure of rats to xylene has been associated with biochemical changes in fetal and maternal brain tissue. No reproductive effects were found in rats following inhalation of 500 ppm xylene before mating and during gestation and lactation. Histopathological examination following intermediate and chronic oral bioassays revealed no adverse effects on the reproductive organs of rats and mice dosed with mixed xylene 5 days/week at 800 and $1,000 \mathrm{mg} / \mathrm{kg} / \mathrm{day}$, respectively.

There is no definitive evidence for carcinogenic effects of xylene in humans. Epidemiological studies looking for associations with xylene exposure and specific cancers either reported no cases or a limited number of cases exposed to xylene and/or reported concurrent exposure to multiple solvents. Two-year cancer bioassays in rats and mice exposed by oral gavage provided no evidence for carcinogenicity of 
mixed xylene. The Department of Health and Human Services (DHHS) has not classified xylene as to its carcinogenicity. Both IARC and EPA have determined that xylene is not classifiable as to its carcinogenicity in humans, due to inadequate evidence for the carcinogenicity of xylenes in humans and animals.

The following sections discuss the most sensitive effects of exposure to xylenes: neurological and respiratory. These effects, as well as other adverse effects, are discussed in greater detail in Chapter 3.

Neurological Effects. The neurotoxicity of xylenes has been examined in short- and long-term inhalation studies in humans and animals and acute-duration oral studies in animals and appears to be related to the interference of unmetabolized xylene with neuronal membranes. Mild central nervous system effects (subjective symptoms of intoxication, headache, fatigue, and dizziness) have been observed following acute-duration exposure of humans to $m$-xylene at $50 \mathrm{ppm}$ and chronic-duration occupational exposure to mixed xylene at $14 \mathrm{ppm}$. Results of experimental studies with humans indicate that acute inhalation exposure to $100 \mathrm{ppm}$ mixed xylene or $200 \mathrm{ppm}$ m-xylene causes impaired short-term memory, impaired reaction time, performance decrements in numerical ability, and alterations in equilibrium and body balance. These experimental studies are supported by case reports and occupational studies that described similar neurological effects at higher exposure levels. Isolated cases of unconsciousness, amnesia, brain hemorrhage, and seizures have been associated with accidental acute inhalation exposure to unknown concentrations of xylene (estimated in one case as 10,000 ppm).

Neurological impairment has also been reported in experimental studies with laboratory animals with a similar range of concentration-related severities as in humans. The range of thresholds for specific neurological end points suggests that specific neuronal pathways or regions of the central nervous system may vary in their vulnerability to xylene exposure. Studies in animals have shown that mixed xylene and individual isomers are neurotoxic at airborne concentrations ranging from 50 to 2,000 ppm. A decreased latency of the paw-lick response was reported in rats exposed to $50 \mathrm{ppm} \mathrm{m}$-xylene for 3 months. Decreased motor performance and impaired learning have been reported in rats exposed to concentrations between 100 and 3,000 ppm. Loss of cochlear hair cells and/or hearing deficits have been observed in rats exposed by inhalation acutely to $\geq 1,450 \mathrm{ppm}$ mixed xylene and in rats exposed for 13 weeks to $900 \mathrm{ppm}$ p-xylene, but not other isomers or 1,000 ppm mixed xylenes. Changes in the levels of brain enzyme activities or neurotransmitters have been noted with acute exposure to 2,000 ppm with $m$ - or $o$-xylenes. Severe neurotoxicity was observed in rats, mice, and gerbils following acute- or intermediateduration inhalation exposure to the various xylene isomers at concentrations in excess of 1,000 ppm. 
These effects included narcosis, prostration, incoordination, tremors, muscular spasms, and labored respiration. All three xylene isomers elicited biphasic response rates in operant behavior studies in mice exposed for 30 minutes: increased responses at $\geq 1,400 \mathrm{ppm}$ and half-maximal responses at 5,179$6,176 \mathrm{ppm}$. Motor coordination was impaired at concentrations above 2,000 ppm for the para isomer and above 3,000 ppm for the meta and ortho isomers.

Animal studies have shown that oral exposure to xylenes at high concentrations (single doses of $\geq 4,000 \mathrm{mg} / \mathrm{kg}$ or repeated dosing at $2,000 \mathrm{mg} / \mathrm{kg} /$ day for 2 weeks) may result in nervous system effects such as tremors, respiratory depression, weakness, lethargy, unsteadiness, and hyperactivity. Hyperactivity was also observed in all mice after oral dosing with $1,000 \mathrm{mg} / \mathrm{kg}$ mixed xylene during weeks 4-103. Rats dosed with $p$-xylene, but not $m$ - or $o$-xylene at $900 \mathrm{mg} / \mathrm{kg} / \mathrm{day}, 5$ days/week for 2 weeks, experienced significant loss of cochlear hair cells associated with hearing at medium frequencies $(10-25 \mathrm{kHz})$.

Respiratory Effects. Numerous studies in humans identified the respiratory tract as a sensitive target of xylenes following short- or long-term inhalation exposure. Subjective symptoms of nose and throat irritation were noted following single exposures to xylene vapor at concentrations between 50 and $700 \mathrm{ppm}$, repeated intermediate-duration exposure at $100 \mathrm{ppm}$, or chronic-duration occupational exposure at $14 \mathrm{ppm}$. Mild increases in the subjective severity scores for breathing difficulty and small changes in measured pulmonary physiology parameters (reduced forced vital capacity) were observed following acute-duration exposure to $m$-xylene at $50 \mathrm{ppm}$. Labored breathing with impaired pulmonary function was reported following chronic occupational exposure to unspecified concentrations of xylene vapor. Severe lung congestion with pulmonary hemorrhages and edema was noted in a worker who died following inhalation of paint fumes containing an estimated 10,000 ppm xylene for an undetermined time $(<18$ hours) and in one case of suicide by ingestion of an undetermined amount of xylene.

Animal data from acute- and intermediate-duration inhalation studies provide supporting evidence for the respiratory effects observed in humans following inhalation exposure to xylene. Respiratory effects noted in laboratory animals exposed for a few minutes at concentrations of $\geq 690 \mathrm{ppm}$ include a $50 \%$ reduction in respiratory rate, labored breathing, irritation of the upper respiratory tract, pulmonary edema, and pulmonary inflammation. Irritation of the upper respiratory tract induced a reflex pause in the expiratory phase of respiration that resulted in an overall reduction in respiratory rate. Decreased metabolic capacity of the lungs was reported to be related to $45-80 \%$ decreases in the activities of metabolizing enzymes (cytochrome P-450) following acute exposure to mixed xylenes or individual isomers at concentrations 
between 75 and 2,000 ppm. The toxicological significance of the reduction in metabolizing enzymes is not established, but may be related to the lessened ability to reduce cellular concentrations of xylene.

\subsection{MINIMAL RISK LEVELS (MRLS)}

Estimates of exposure levels posing minimal risk to humans (MRLs) have been made for mixed xylenes. An MRL is defined as an estimate of daily human exposure to a substance that is likely to be without an appreciable risk of adverse effects (noncarcinogenic) over a specified duration of exposure. MRLs are derived when reliable and sufficient data exist to identify the target organ(s) of effect or the most sensitive health effect(s) for a specific duration within a given route of exposure. MRLs are based on noncancerous health effects only and do not consider carcinogenic effects. MRLs can be derived for acute, intermediate, and chronic duration exposures for inhalation and oral routes. Appropriate methodology does not exist to develop MRLs for dermal exposure.

Although methods have been established to derive these levels (Barnes and Dourson 1988; EPA 1990), uncertainties are associated with these techniques. Furthermore, ATSDR acknowledges additional uncertainties inherent in the application of the procedures to derive less than lifetime MRLs. As an example, acute inhalation MRLs may not be protective for health effects that are delayed in development or are acquired following repeated acute insults, such as hypersensitivity reactions, asthma, or chronic bronchitis. As these kinds of health effects data become available and methods to assess levels of significant human exposure improve, these MRLs will be revised.

Toxicological data from comparative studies demonstrate that, in some cases, the effects and effect levels of the three isomers are similar; e.g., body weight findings in the acute oral study by Condie et al. (1988) or the alveolar concentration levels associated with anaesthetic effects as described by Fang et al. (1996). Other studies have indicated different orders of relative toxicity for the isomers, but there is no consistent pattern indicating that a particular isomer is the most potent for all end points, and the differences in effect levels among the isomers may be small. For example, the ortho isomer was most potent in assays on operant behavior (Moser et al. 1985) and motor coordination in rats (Korsak et al. 1990) and in a developmental toxicity assay in rats in which mixed xylenes had the same effect levels (Saillenfait et al. 2003). The para isomer, however, was most potent in a different test for motor performance, the inverted screen test (Moser et al. 1985), and in ototoxicity assays in rats (Gagnaire et al. 2001). Given the lack of consistency among the different end points, the most sensitive effect by mixed xylenes or any isomer was chosen as the basis for MRL for mixed xylenes and all isomers for that duration and route of exposure. 


\section{Inhalation MRLs}

- An MRL of $2 \mathrm{ppm}$ has been derived for acute-duration inhalation exposure (14 days or less) to mixed xylenes

The database for acute-duration inhalation exposure to xylenes includes a number of studies in humans and laboratory animals. Studies in humans identify neurological and respiratory effects as the most sensitive end points for acute-duration inhalation exposure. Effects observed in humans at 50-690 ppm included irritation of the respiratory tract (breathing difficulty, discomfort in nose and throat, reduced forced air capacity), neurotoxicity (dizziness, headache, impaired short-term memory, increase in reaction times), and eye irritation (Carpenter et al. 1975a; Dudek et al. 1990; Gamberale et al. 1978; Nelson et al. 1943; NIOSH 1981). Other neurological effects in humans included altered visual evoked potentials following repeated exposure to $200 \mathrm{ppm} \mathrm{m}$-xylene and impaired body balance following a single 4-hour exposure at 400 ppm (Savolainen et al. 1984; Seppalainen et al. 1989).

Acute-duration neurological effects in animals were observed at concentrations of $113 \mathrm{ppm}$ and higher. These included transiently decreased operant responses in rats repeatedly exposed to $113 \mathrm{ppm}$ mixed xylene (Ghosh et al. 1987), altered responses to electric shock in rats and mice exposed once to 230 320 ppm o-xylene (Vodickova et al. 1995), and decreased axonal transport in rats repeatedly exposed to 800 ppm mixed xylene (Padilla and Lyerly 1989). Effects related to motor incoordination were observed following exposures to mixed xylenes or individual isomers in the range of 1,010-1,982 ppm (Carpenter et al. 1975a; De Ceaurriz et al. 1983; Korsak et al. 1988, 1990, 1993). Sensory-related changes (altered visual- or auditory-evoked potentials or hearing losses) occurred at exposures in the range of 1,4001,600 ppm p-xylene (Crofton et al. 1994; Dyer et al. 1988; Pryor et al. 1987; Rebert et al. 1995). Other neurological effects included disturbances in brain catecholamine or dopamine following repeated exposure to mixed xylene or individual isomers at 2,000 ppm (Andersson et al. 1981), narcosis in rats at $\geq 1,940$ ppm (Molnar et al. 1986), and ataxia and seizures in cats exposed to 9,500 ppm mixed xylene (Carpenter et al. 1975a).

The lowest effect levels for other end points affected by acute-duration inhalation exposure in animals were higher than the lowest-observed-adverse-effect level (LOAEL) for neurological effects. Rat developmental effects (reduced fetal body weight and delayed ossification) were observed at xylene exposures in the range of 350-2,000 ppm (Saillenfait et al. 2003; Ungvary et al. 1980b); maternal body weight effects were observed at $700 \mathrm{ppm}$ (Ungvary et al. 1980b). Acute-duration respiratory effects in 
animals include decreases in lung surfactant following exposure to mixed xylenes or individual isomers at 1,000 ppm or higher (Elovaara et al. 1987; Patel et al. 1978; Toftgard and Nilsen 1982) and decreased respiratory rates in mice briefly exposed to $m$ - or $o$-xylene at concentrations of 1,361-2,700 ppm (De Ceaurriz et al. 1981; Korsak et al. 1990, 1991, 1993). Increased mortality has been reported in rodents repeatedly exposed to at $700 \mathrm{ppm}$ (Ungvary et al. 1980b) or once to $\geq 2,010 \mathrm{ppm}$ (Bonnet et al. 1979; Cameron et al. 1938; Carpenter et al. 1975a; Harper et al. 1975; Hine and Zuidema 1970).

Neurological and respiratory effects are selected as co-critical effects of acute-duration inhalation exposure since they occurred at the lowest tested exposure level of $50 \mathrm{ppm}$. Ernstgard et al. (2002) was selected as the principal study for acute-duration inhalation exposure because it provides the lowest LOAEL for the co-critical effects. In this study, 28 men and 28 women were exposed to 0 or 50 ppm $m$-xylene in sessions separated by 2 weeks. There were difficulties with recruitment for the study; only $10 \% /$ sex out of the original selected group of $1,000 /$ sex agreed to participate and the drop-out rate was high $(\sim 72 \%)$ because of failure to pass the screening medical examination or a change in life circumstances. Strengths of the study include that subjects served as their own controls, exposures were controlled (with respect to solvent concentration, duration, temperature, and humidity), results were analyzed for statistical significance, and a third exposure condition (150 ppm 2-propanol) resulted in a qualitatively different pattern of adverse effects compared to $m$-xylene exposure. During exposure (3, 60, and 118 minutes from the start of exposure) and post-exposure (140 and 350 minutes from onset), the subjects rated the level of perceived discomfort using a visual analogue scale $(0-100 \mathrm{~mm})$ in a questionnaire with 10 questions. Pulmonary function measurements were conducted prior to exposure, immediately after exposure, and 3 hours post-exposure. Nasal swelling was assessed by acoustic rhinometry before, immediately after, and 3 hours after the end of exposure. Nasal lavages obtained before and 3 hours after the end of exposure were evaluated for markers of inflammation. Eye blinking was measured throughout exposure by electromyography, and color vision was assessed before, immediately after, and 3 hours post-exposure. Compared to air-exposure, exposure to $m$-xylene at $50 \mathrm{ppm}$ resulted in small statistically significant increases in the average rating of self-reported symptoms for local irritant effects (discomfort in the eyes and nose), other respiratory effects (breathing difficulty in both sexes and discomfort in the throat or airways in women), and neurological effects (feeling of intoxication in both sexes, and headache, fatigue, and dizziness in men). All self-reported symptoms were characterized as minimal. Although the subjects were not blinded with respect to the exposure conditions, there was only a weak correlation between ratings of smell and ratings of symptoms; the authors conclude that it was unlikely that the perceived exposure by itself significantly affected symptom ratings. Small statistically significant changes were observed in objective measures of respiratory 
function in exposed women, but not men, 3 hours after the end of the exposure to $m$-xylene; forced vital capacity was reduced by $2.81 \%$ (45-fold more than air exposure) and other parameters dependent on forced vital capacity were likewise altered. The authors indicated that these measured respiratory effects in women were of uncertain significance, but could be explained by possible effects of estrogen or the narrower airways in women compared to men. A minimal LOAEL of $50 \mathrm{ppm}$ is identified for neurological and respiratory effects in humans exposed to $m$-xylene. An uncertainty factor of 30 was applied to the LOAEL of $50 \mathrm{ppm}$ (3 for the use of a minimal LOAEL and 10 for human variability). The resulting MRL of $2 \mathrm{ppm}$ is considered to be protective to human health under acute-duration ( $\leq 14$ days) inhalation exposures to mixed xylenes or individual isomers.

- An MRL of 0.6 ppm has been derived for intermediate-duration inhalation exposure (15364 days) to mixed xylenes.

The database for intermediate-duration inhalation to xylenes includes one controlled-exposure study in humans and several animal bioassays. Effects in humans exposed to 100-150 ppm $p$-xylene for 17.5 hours/day, 5 days/week for 4 weeks included increased reporting of subjective symptoms of irritation of the nose and throat (NIOSH 1981). This study found no significant alterations in objective measures of neurological function (electroencephalography, tests of motor activity, and cognitive performance) and no alterations in pulmonary function in human subjects, but the study was limited in that some group sizes were small $(n=2)$. Animal toxicity bioassays that tested at concentrations below $100 \mathrm{ppm}$ reported no clinical signs of neurotoxicity or no adverse effects liver enzymes or brain weight (Hillefors-Berglund et al. 1995; Jajte et al. 2003; Jenkins et al. 1970). In a special neurobehavioral assay, a LOAEL of $50 \mathrm{ppm}$ was identified for reduced mean latency of the paw-lick response (indicative of increased sensitivity to pain) in rats exposed to $m$-xylene for 3 months (Korsak et al. 1994). Other neurobehavioral effects (impaired rotarod performance, passive avoidance learning) were observed following exposure to $100 \mathrm{ppm}$ m-xylene for 4 weeks to 6 months (Gralewicz and Wiaderna 2001; Gralewicz et al. 1995; Korsak et al. 1994); some neurological impairment persisted for 59 weeks after exposure (Gralewicz and Wiaderna 2001). Other neurological effects included distribution of astroglia cells in the brain of Mongolian gerbils exposed to $160 \mathrm{ppm}$ mixed xylene for 4 months (Rosengren et al. 1986), delayed maxillary ossification and impaired rotarod performance in rats gestationally exposed to 200 ppm technical-grade xylene for 6 hours/day (Hass and Jakobsen 1993), and impaired motor coordination and spatial orientation following gestational exposure at 500 ppm (Hass et al. 1995, 1997). Neurological effects at higher concentrations included tremors in dogs exposed to 780 ppm o-xylene for 6 hours/day, 5 days/week for 6 weeks (Jenkins et al. 1970), alterations in brain neurotransmitters following continuous exposure to $800 \mathrm{ppm}$ mixed xylenes for 
30 days (Honma et al. 1983), and auditory effects in rats (hearing loss or decreases in auditory brainstem responses) following exposure to $\geq 800 \mathrm{ppm}$ mixed xylenes or $900 \mathrm{ppm} p$-xylene for 13 weeks (Gagnaire et al. 2001, 2006; Nylen and Hagman 1994; Pryor et al. 1987).

Non-neurological effects were observed at higher concentrations. Cardiovascular effects (increased thickness of coronary microvessels) were observed in rats exposed to $230 \mathrm{ppm}$ mixed xylenes 6 hours/day, 5 days/week for 4 weeks (Morvai et al. 1987). Hepatic effects (increased liver weight) were observed at a LOAEL of $600 \mathrm{ppm}$ in rats discontinuously exposed to mixed xylenes for 4 weeks (Toftgard et al. 1981). In rats exposed gestationally to mixed xylenes or $o$-xylene, a LOAEL of $500 \mathrm{ppm}$ was identified for decreased fetal body weights in the absence of maternal toxicity (Bio/dynamics 1983). LOAELs for adult body weight effects were $1,000 \mathrm{ppm}$ or higher (Tatrai et al. 1981). Increased deaths among squirrel monkeys and rats were noted following discontinuous intermediate-duration exposure to 780 ppm o-xylene (Jenkins et al. 1970), but no systemic effects were noted in rats or dogs exposed to 810 ppm mixed xylenes (Carpenter et al. 1975a).

Neurotoxicity is selected as the critical effect of intermediate-duration inhalation exposure to xylenes, since it occurs at the lowest effect levels and may persist after exposures cease. The study by Korsak et al. (1994) on $m$-xylene was selected as the principal study because it provides the lowest LOAEL (50 ppm) for the critical effect, neurotoxicity. In this study, groups of 12-24 male Wistar rats were exposed to 0,50 , or $100 \mathrm{ppm} m$-xylene, 6 hours/day, 5 days/week for 3 months. Before the study and at the end of each month of exposure, rats were evaluated for motor coordination using the rotarod performance test. The level of analgesia was tested at termination in the paw-lick response to hot-plate test at $54{ }^{\circ} \mathrm{C}$. An increase in the failure rate on the rotarod performance test was concentration-related and statistically significant at $100 \mathrm{ppm}$, but not at $50 \mathrm{ppm}$. Mean latency of the paw-lick response was significantly reduced by $28 \%$ at $50 \mathrm{ppm}$ compared to controls; this effect is considered minimal. The rat minimal LOAEL of 50 ppm was converted to a human equivalent following the EPA (1994) dosimetric equation for an extra-respiratory effect from an inhaled category 3 gas: $\operatorname{LOAEL}_{\mathrm{HEC}}=\mathrm{LOAEL}_{\mathrm{ADJ}} \times \lambda_{\mathrm{a}} / \lambda_{\mathrm{h}}$, where $\lambda_{\mathrm{a}}$ and $\lambda_{\mathrm{h}}$ are blood:air partition coefficients for rats and humans, respectively. Reported blood:air partition coefficients for $m$-xylene are 39.9 for male Wistar rats and 26.4 for humans (Sato and Nakajima 1979). Since the rat value is higher than the human, the default value of 1 was used for $\lambda_{\mathrm{a}} / \lambda_{\mathrm{h}}$ (EPA 1994). The minimal LOAEL $\mathrm{HEC}_{\mathrm{C}}$ is therefore $50 \mathrm{ppm}$. An uncertainty factor of 90 was applied to the minimal LOAEL $_{\mathrm{HEC}}$ of $50 \mathrm{ppm}$ ( 3 for the use of a minimal LOAEL, 3 for extrapolation from animals to humans using dosimetric adjustment, and 10 for human variability). The resulting MRL of $0.6 \mathrm{ppm}$ is considered 
to be protective to human health under intermediate-duration inhalation exposures to mixed xylenes or individual isomers.

- An MRL of $0.05 \mathrm{ppm}$ has been derived for chronic-duration inhalation exposure ( $>1$ year) to mixed xylenes.

The database for chronic-duration inhalation toxicity of xylene contains two studies that provide quantitative exposure information. An occupational study reported a LOAEL of $14 \mathrm{ppm}$ for subjective respiratory and neurological effects in workers exposed to mixed xylenes for an average of 7 years (Uchida et al. 1993). This study examined 175 workers (107 men, 68 women) who were exposed to mixed xylenes (approximately 50\% $\mathrm{m}$-xylene, 30\% $\mathrm{p}$-xylene, and 15\% o-xylene) during the manufacture of boots or rubber-coated wires. Subjects were selected between 1989 and 1991 from a group of 994 solvent-exposed workers who were supplied with diffusion samplers for one 8-hour workday; exposures were corroborated by measurements of xylene metabolites in urine. Subjects were evaluated for subjective symptoms in a questionnaire and also examined for objective parameters (serum biochemistry, hematology, and urinalysis). The final group of 175 exposed workers included those showing at least $70 \%$ of their solvent exposure to be from xylene and having completed all tests. The time-weighted average (TWA) (arithmetic mean) for xylenes was $21 \mathrm{ppm}$ for an average of 7 years (geometric mean, $14 \mathrm{ppm}$ ); one man was exposed to $175 \mathrm{ppm}$. Xylenes represented at least $70 \%$ of the total solvent exposure, other chemicals being 3.4 ppm ethylbenzene (a typical component of commercial mixed xylene), $1.2 \mathrm{ppm}$ toluene and rarely, n-hexane. No subjects were exposed to benzene, and the level of toluene exposure was low enough to be insignificant. The control group included 241 non-exposed workers (116 men, 125 women) in the same factories or elsewhere. Exposed workers showed a significant increase in the reporting of subjective symptoms including increased anxiety, forgetfulness, inability to concentrate, eye and nasal irritation, and sore throats. A concentration-relationship was reported for eye irritation, sore throat, and floating sensation, the last being indicative of central nervous system effect. There were no alterations in renal or hepatic serum parameters. This study has a few limitations: the lack of precise information on the duration of exposure and the lack of information as to how the workers used xylene on the job. Reliance on subjective symptoms might be considered a limitation of the study, except that these same symptoms were noted in the principal study for acuteduration inhalation exposure (Ernstgard et al. 2002).

The only other chronic-duration study was for rats exposed to $1,096 \mathrm{ppm} o$-xylene 8 hours/day, 7 days/week for 1 year (Tatrai et al. 1989). Hepatic changes included increases in liver weight and microsomal activity and proliferating endoplasmic reticulum, but no histopathological lesions. Body 
weight was reduced by $11 \%$ in exposed rats. Calculation of a chronic-duration inhalation MRL from this rat study would not be appropriate since the study did not examine neurological effects, which are the most critical end points for inhalation exposure to xylene.

Respiratory and neurological effects are the co-critical effects of chronic-duration inhalation exposure to xylenes since they occurred at the lowest tested concentrations. The study of Uchida et al. (1993) was selected as the principal study for the chronic-duration inhalation MRL since it identified the lowest LOAEL of 14 ppm (geometric mean) for the co-critical effects. The geometric mean was used because it provides a better measure of central tendency than the arithmetic mean. The LOAEL of $14 \mathrm{ppm}$ was divided by a total uncertainty factor of 100 (10 for the use of a LOAEL and 10 for human variability) and a modifying factor of 3 to account for lack of supporting studies evaluating the chronic neurotoxicity of xylene. The resulting MRL of $0.05 \mathrm{ppm}$ is considered to be protective to human health under chronicduration ( $>1$ year) inhalation exposures to mixed xylenes or individual isomers.

\section{Oral MRLs}

- An MRL of $1 \mathrm{mg} / \mathrm{kg} / \mathrm{day}$ has been derived for acute-duration oral exposure ( $\leq 14$ days) to mixed xylenes.

Evaluation of the limited database for acute-duration oral exposure to xylenes suggests that neurotoxicity represents the most sensitive end point in laboratory animals; no quantitative human data are available. Effects observed in acute-duration oral studies in rats included altered visually evoked potentials following a single dose of $250 \mathrm{mg} / \mathrm{kg}$-xylene (Dyer et al. 1988), reduced pulmonary microsomal activity following a single dose of 1,000 mg/kg/day $p$-xylene (Patel et al. 1978), and reduced body weight gain following repeated dosing with $1,000 \mathrm{mg} / \mathrm{kg} /$ day mixed xylenes or $2,000 \mathrm{mg} / \mathrm{kg} / \mathrm{day} o$ - or $p$-xylene (Condie et al. 1988; NTP 1986). Repeated gavage dosing with $p$-xylene, but not $m$ - or $o$-xylene, at $900 \mathrm{mg} / \mathrm{kg} /$ day, 5 days/week for 2 weeks, resulted in significant loss of cochlear hair cells associated with hearing at midrange frequencies $(10-25 \mathrm{kHz})$ (Gagnaire and Langlais 2005). Repeated exposure to mixed xylenes at $2,000 \mathrm{mg} / \mathrm{kg} / \mathrm{day}$, resulted in impaired respiration (shallow and/or labored breathing) in rats and mice and increased mortality (NTP 1986). At 2,060 mg/kg/day, developmental toxicity (cleft palate) was observed in mice gestationally exposed to mixed xylenes (Marks et al. 1982). Serious neurological effects (coma, incoordination, prostration, decreased hindleg movement) were observed in rats that received single oral gavage doses of $\geq 4,000 \mathrm{mg} / \mathrm{kg}$ mixed xylenes (Muralidhara and Krishnakumari 1980; NTP 1986). 
Neurotoxicity was selected as the critical effect for acute-duration oral exposure to xylene because it was observed at the lowest exposure level and demonstrated dose-related increases in severity. The study by Dyer et al. (1988) was selected as the principal study, since it provides the lowest LOAEL for the critical effect. In this study, male Long-Evans rats with electrodes implanted for recording of brain visual potentials were dosed orally in two different experiments. In the first experiment, groups of 10-11 rats received single doses of $p$-xylene at doses of $0,500,1,000$, or $2,000 \mathrm{mg} / \mathrm{kg}$ in corn oil and were tested 75 minutes later for potentials evoked in response to a single flash in 128 trials. The latencies and amplitudes of the P1, N1, P2, N2, P3, and N3 waveforms were determined. Based on the results of this experiment, groups of 14-16 rats were dosed at 0,125 , or $250 \mathrm{mg} / \mathrm{kg}$ and tested 45 minutes later to record the N3 waveform. Forty-five minutes after administration of $250 \mathrm{mg} / \mathrm{kg}$, the amplitude of the N3 peak was decreased by $47 \%$ (statistically significant). At higher doses $(500,1,000$, and 2,000 mg/kg), the N3 peak was not observed until 75 minutes after dosing. Thus, $125 \mathrm{mg} / \mathrm{kg}$ is a NOAEL and $250 \mathrm{mg} / \mathrm{kg} /$ day is a LOAEL for suppression of visual evoked brain potentials in rats exposed once to $p$-xylene. An uncertainty factor of 100 (10 for extrapolation from animals to humans and 10 for human variability) was applied to the NOAEL. The resulting MRL of $1 \mathrm{mg} / \mathrm{kg} / \mathrm{day}$ is considered to be protective to human health under acute-duration oral exposures to mixed xylenes or individual isomers.

- An MRL of $0.4 \mathrm{mg} / \mathrm{kg} /$ day has been derived for intermediate-duration oral exposure $(\geq 14$ days to 1 year) to mixed xylenes.

The intermediate-duration oral toxicity of xylenes has been investigated in several oral gavage bioassays in rodents exposed to mixed xylene (Condie et al. 1988; NTP 1986), $m$-xylene (Elovaara et al. 1989; Wolfe 1988a), and p-xylene (Wolfe 1988b). The observed effects include increases in minimal chronic nephropathy and $17-37 \%$ increases in relative hepatic weight in rats exposed to $\geq 750 \mathrm{mg} / \mathrm{kg} / \mathrm{day}$ mixed xylene (Condie et al. 1988); 27-46.3\% increases in serum transaminase levels in rats exposed to 750 $1,500 \mathrm{mg} / \mathrm{kg} /$ day mixed or $\mathrm{m}$-xylene (Condie et al. 1988; Elovaara et al. 1989; Wolfe 1988a); reduced levels of cytochrome P-450 in the lung in rats exposed to $800 \mathrm{mg} / \mathrm{kg} \mathrm{m}$-xylene (Elovaara et al. 1989); 15$25 \%$ decreases in body weight gain in rats exposed to $800-1,000 \mathrm{mg} / \mathrm{kg} /$ day mixed, $m-$, or $p$-xylene (NTP 1986; Wolfe 1988a, 1988b); hyperactivity or increased aggressiveness subsequent to dosing with mixed xylene in rats at $1,500 \mathrm{mg} / \mathrm{kg} / \mathrm{day}$ or mice at $1,000 \mathrm{mg} / \mathrm{kg} /$ day (Condie et al. 1988; NTP 1986); and 11\% increased relative spleen weight, $16 \%$ increased relative kidney weight, and increased hematological effects (mild polycythemia and leukocytosis) in female rats dosed with 1,500 mg/kg/day mixed xylene (Condie et al. 1988). No hepatic effects were noted in F344 rats given mixed xylene at doses as high as $1,000 \mathrm{mg} / \mathrm{kg} /$ day or $\mathrm{B} \mathrm{C}_{3} \mathrm{~F}_{1}$ mice dosed at $\leq 2,000 \mathrm{mg} / \mathrm{kg} /$ day 5 days/week for 13 weeks (NTP 1986) or in Sprague-Dawley rats dosed with $p$-xylene at $800 \mathrm{mg} / \mathrm{kg} /$ day for 90 days (Wolfe 1988b). No renal effects 
were observed in rats or mice exposed to mixed xylene in studies by NTP (1986) or in rats exposed to $m$ - or $p$-xylene (Wolfe 1988a, 1988b). The lack of hepatic or renal effects in some studies may be related to strain differences, exposure differences (discontinuous vs. continuous), or isomer specificities.

A limitation of standard intermediate-duration oral bioassays for xylene is that no testing was conducted for sensitive neurological effects. The only overt neurological effect of long-term exposure to xylene was hyperactivity noted in all male and female $\mathrm{B}_{6} \mathrm{C}_{3} \mathrm{~F}_{1}$ mice (50/sex/group) immediately after oral gavage dosing 5 days/week with $1,000 \mathrm{mg} / \mathrm{kg}(710 \mathrm{mg} / \mathrm{kg} /$ day duration adjusted) mixed xylene $(9.1 \% 0-, 60.2 \%$ $m$-, and $13.6 \%$ p-xylene plus 17\% ethylbenzene) in corn oil beginning at week 4 of the 103 -week NTP (1986) bioassay; hyperactivity was not observed at $500 \mathrm{mg} / \mathrm{kg}(360 \mathrm{mg} / \mathrm{kg} /$ day, duration-adjusted). Survival was not significantly affected by treatment with xylene during the first year. Neurotoxicity (hyperactivity) observed during the first year of that study (weeks 4-51) is selected as the critical effect of intermediate-duration exposure because it was observed at the lowest LOAEL $(710 \mathrm{mg} / \mathrm{kg} / \mathrm{day}$, adjusted for intermittent exposure). Selection of neurotoxicity as the critical effect is consistent with other MRLs for xylene. The NTP (1986) study is selected as the principal study for intermediate-duration exposure to xylene because it provides the lowest adverse effect level, a LOAEL of $710 \mathrm{mg} / \mathrm{kg} / \mathrm{day}$, and a NOAEL of $500 \mathrm{mg} / \mathrm{kg}$ (360 mg/kg/day, duration-adjusted) for the critical effect. A total uncertainty factor of 100 (10 for extrapolation from animals to humans, and 10 for human variability) and a modifying factor of 10 (for the lack of testing for sensitive neurological end points and lack of developmental and multigenerational data) were applied to the duration-adjusted NOAEL. The resulting MRL of $0.4 \mathrm{mg} / \mathrm{kg} / \mathrm{day}$ is considered to be protective to human health under intermediate-duration oral exposures to mixed xylenes or individual isomers.

- An MRL of $0.2 \mathrm{mg} / \mathrm{kg} /$ day has been derived for chronic-duration oral exposure ( $\geq 1$ year) to mixed xylenes.

The available animal studies involving chronic-duration oral exposure to xylenes do not clearly identify toxic effects other than $5-8 \%$ decreases in body weight gain (not biologically significant) and unexplained reduced survival in male F344 rats at $500 \mathrm{mg} / \mathrm{kg}$ (NTP 1986) and transient hyperactivity associated with gavage administration in male and female $\mathrm{B}_{6 \mathrm{C}} 3 \mathrm{~F}_{1}$ mice at $1,000 \mathrm{mg} / \mathrm{kg} / \mathrm{day}$ (NTP 1986). These studies are standard cancer/toxicology bioassays involving administration of mixed xylenes (13.6\% p-xylene, $60.2 \% \mathrm{~m}$-xylene, $9.1 \% o$-xylene, and $17 \%$ ethylbenzene) by gavage in corn oil to groups of 50 animals/sex, 5 days/week for 103 weeks (NTP 1986). Animals were examined twice daily and clinical signs were recorded daily for the first 16 months, then once a month thereafter. Body weights were recorded weekly for the first 12 weeks and then once every 4 weeks thereafter. At termination, all 
animals were subjected to gross necropsy and histological examinations. Comprehensive histological examination of major tissues and organs revealed no exposure-related increased incidences of pathological lesions in rats or mice.

In the absence of data associating health effects in humans with chronic-duration oral exposure to xylenes, the animal bioassays provide a minimal basis for deriving an MRL for humans chronically exposed to xylenes. A NOAEL of $500 \mathrm{mg} / \mathrm{kg}$ was identified for hyperactivity in mice, but this was not selected as the basis for the chronic-duration MRL because that dose decreased survival in male rats. Therefore, the rat NOAEL of $250 \mathrm{mg} / \mathrm{kg}$ was selected as the basis of the MRL. The NOAEL was first adjusted for discontinuous exposure (5 days/7 days), resulting in a duration-adjusted NOAEL of $179 \mathrm{mg} / \mathrm{kg} /$ day. An uncertainty factor of 100 (10 for extrapolation between animals and humans and 10 for human variability) and a modifying factor of 10 were applied to the duration-adjusted NOAEL to account for the lack of testing for sensitive neurological end points (the most sensitive effects in inhalation studies and acute oral studies) and lack of developmental and multi-generational data. The resulting chronic-duration oral MRL of $0.2 \mathrm{mg} / \mathrm{kg} / \mathrm{day}$ is considered to be protective to human health under chronic-duration ( $>1$ year) oral exposures to mixed xylenes or individual isomers. 


\section{HEALTH EFFECTS}

\subsection{INTRODUCTION}

The primary purpose of this chapter is to provide public health officials, physicians, toxicologists, and other interested individuals and groups with an overall perspective on the toxicology of xylene. It contains descriptions and evaluations of toxicological studies and epidemiological investigations and provides conclusions, where possible, on the relevance of toxicity and toxicokinetic data to public health.

A glossary and list of acronyms, abbreviations, and symbols can be found at the end of this profile.

Commercial xylene is a mixture of three isomers of xylene ( $m-, o-$, and $p$-xylene) with $<20 \%$ ethylbenzene. In the following discussion of the health effects of xylene, the effects of both the mixture and the individual isomers are presented. Where possible, the effects of individual isomers will be identified and presented separately.

\subsection{DISCUSSION OF HEALTH EFFECTS BY ROUTE OF EXPOSURE}

To help public health professionals and others address the needs of persons living or working near hazardous waste sites, the information in this section is organized first by route of exposure (inhalation, oral, and dermal) and then by health effect (death, systemic, immunological, neurological, reproductive, developmental, genotoxic, and carcinogenic effects). These data are discussed in terms of three exposure periods: acute (14 days or less), intermediate (15-364 days), and chronic (365 days or more).

Levels of significant exposure for each route and duration are presented in tables and illustrated in figures. The points in the figures showing no-observed-adverse-effect levels (NOAELs) or lowestobserved-adverse-effect levels (LOAELs) reflect the actual doses (levels of exposure) used in the studies. LOAELs have been classified into "less serious" or "serious" effects. "Serious" effects are those that evoke failure in a biological system and can lead to morbidity or mortality (e.g., acute respiratory distress or death). "Less serious" effects are those that are not expected to cause significant dysfunction or death, or those whose significance to the organism is not entirely clear. ATSDR acknowledges that a considerable amount of judgment may be required in establishing whether an end point should be classified as a NOAEL, "less serious" LOAEL, or "serious" LOAEL, and that in some cases, there will be insufficient data to decide whether the effect is indicative of significant dysfunction. However, the Agency has established guidelines and policies that are used to classify these end points. ATSDR 
believes that there is sufficient merit in this approach to warrant an attempt at distinguishing between "less serious" and "serious" effects. The distinction between "less serious" effects and "serious" effects is considered to be important because it helps the users of the profiles to identify levels of exposure at which major health effects start to appear. LOAELs or NOAELs should also help in determining whether or not the effects vary with dose and/or duration, and place into perspective the possible significance of these effects to human health.

The significance of the exposure levels shown in the Levels of Significant Exposure (LSE) tables and figures may differ depending on the user's perspective. Public health officials and others concerned with appropriate actions to take at hazardous waste sites may want information on levels of exposure associated with more subtle effects in humans or animals (LOAELs) or exposure levels below which no adverse effects (NOAELs) have been observed. Estimates of levels posing minimal risk to humans (Minimal Risk Levels or MRLs) may be of interest to health professionals and citizens alike.

A change from the last edition of this profile is that a single MRL is derived for each duration (acute, intermediate, or chronic) of inhalation or oral exposure that applies equally to mixed xylenes and to the individual isomers, rather than having specific MRLs for each chemical entity. This convention is in accordance with occupational exposure levels promulgated by agencies such as ACGIH, NIOSH, and OSHA (see Table 8-1) and is supported by several lines of evidence. The isomers have similar chemical properties such as $\log \mathrm{K}_{\mathrm{ow}}$ (see Table 4-2), resulting in similar absorption, distribution, and excretion patterns (see Section 3.4 and Table 3-6). The tissue:air partition coefficients (liver, fat, and muscle) and the blood:air partition coefficients, as well as the estimated hemoglobin binding constants, for the three isomers of xylene are almost identical or comparable (Adams et al. 2005; Poulin and Krishnan 1996a, 1996b). The xylene isomers are metabolized by the same enzymes, resulting in an isomer of methylhippuric acid as the predominant metabolite in each case (see Section 3.4.3). In addition, physiologically based pharmacokinetic (PBPK) models based on the characteristics of $m$-xylene have been shown to be able to simulate the kinetics of mixed xylenes (Tardif et al. 1993a, 1995).

Toxicological data from comparative studies, as discussed by EPA (2003), demonstrate that, in some cases, the effects and effect levels of the isomers are similar; e.g., body weight findings in the acute oral study by Condie et al. (1988) or the alveolar concentration levels associated with anaesthetic effects as described by Fang et al. (1996). Other studies have indicated different orders of relative toxicity for the isomers, but there is no consistent pattern indicating that a particular isomer is the most potent for all end points, and the differences in effect levels among the isomers may be small. For example, the ortho isomer was most potent in assays on operant behavior (Moser et al. 1985) and motor coordination in rats 
(Korsak et al. 1990), and in a developmental toxicity assay in rats in which mixed xylenes had the same effect levels (Saillenfait et al. 2003). On the other hand, the para isomer was most potent in a different test for motor performance, the inverted screen test (Moser et al. 1985), and in ototoxicity assays in rats (Gagnaire and Langlais 2005; Gagnaire et al. 2001). Given the lack of consistency among the different end points, the most sensitive effect by mixed xylenes or any isomer was chosen as the basis for the MRL for mixed xylenes and all isomers for that duration and route of exposure.

A User's Guide has been provided at the end of this profile (see Appendix B). This guide should aid in the interpretation of the tables and figures for Levels of Significant Exposure and the MRLs.

\subsubsection{Inhalation Exposure}

\subsubsection{Death}

One report was located regarding death in humans following acute inhalation exposure to xylene (composition unspecified) (Morley et al. 1970). One of three men died after breathing paint fumes for several hours that contained an estimated atmospheric concentration of 10,000 ppm xylene. Xylene comprised $90 \%$ of the solvent in the paint (small amounts of toluene were also present), with the total solvent comprising $34 \%$ of the paint by weight. An autopsy of the man who died showed severe pulmonary congestion, interalveolar hemorrhage, and pulmonary edema; the brain showed hemorrhaging and evidence of anoxic damage. Clinical signs noted in the two exposed men who survived included solvent odor of the breath, cyanosis of the extremities, and neurological impairment (temporary confusion, amnesia). Both men recovered completely. The authors hypothesized that anoxia did not contribute to the effects observed in the survivors because the flow of oxygen into the area in which the men were working should have been adequate. The study was inconclusive for evaluating the toxic effects of xylene because the subjects were concurrently exposed to other chemicals in the paint. No studies were located regarding mortality in humans after intermediate or chronic inhalation exposure to mixed xylene or xylene isomers.

Acute inhalation $\mathrm{LC}_{50}$ values have been determined in animals for xylene and its isomers (Bonnet et al. 1979; Carpenter et al. 1975a; Harper et al. 1975; Hine and Zuidema 1970; Ungvary et al. 1980b). The 4-hour $\mathrm{LC}_{50}$ value for mixed xylene in rats ranged from 6,350 ppm (Hine and Zuidema 1970) to $6,700 \mathrm{ppm}$ (Carpenter et al. 1975a). The 4-hour $\mathrm{LC}_{50}$ value for $p$-xylene in rats was reported to be 4,740 ppm (Harper et al. 1975). In mice, the 6-hour $\mathrm{LC}_{50}$ values for $m-,-o-$, and $p$-xylene were determined to be $5,267,4,595$, and 3,907 ppm, respectively (Bonnet et al. 1979). These data suggest that $p$-xylene 
may be slightly more toxic than the other xylene isomers. According to the toxicity classification system of Hodge and Sterner (1949), these values indicate that mixed xylene and its isomers are slightly toxic by acute inhalation.

Mice appear to be more sensitive than rats to the lethal effects of the $m$ - and $o$-isomers of xylene (Cameron et al. 1938). While no rats died following a 24-hour exposure to 2,010 ppm m-xylene, 6 of 10 mice died as a result of a similar exposure. Similarly, a 24-hour exposure of rats to 3,062 ppm $o$-xylene resulted in a death rate of only 1 in 10 , whereas in mice, 4 of 10 died. It is unclear whether differential sensitivities exist for the $p$-isomer of xylene in mice and rats (Cameron et al. 1938).

Information regarding lethality following intermediate-duration exposures is limited to the results of a single study examining mortality in rats, guinea pigs, monkeys, and dogs following intermittent and continuous exposure to $o$-xylene (Jenkins et al. 1970). Continuous exposure to 78 ppm $o$-xylene for 90 127 days resulted in the death of only 1 of 15 rats. Intermittent exposure to $780 \mathrm{ppm} o$-xylene resulted in deaths of 3 of 15 rats; none of the 15 guinea pigs, 3 monkeys, or 2 dogs died. No data were located regarding death following chronic-duration exposure to mixed xylene or its isomers.

All $\mathrm{LC}_{50}$ values and LOAEL values from each reliable study for death in each species and duration category are recorded in Table 3-1 and plotted in Figure 3-1.

\subsubsection{Systemic Effects}

No human or animal data were available regarding dermal effects following inhalation exposure to mixed xylene or xylene isomers. The systemic effects observed after inhalation exposure to xylene are discussed below. The highest NOAEL value and all LOAEL values from each reliable study for systemic effects in each species and duration category are recorded in Table 3-1, and are plotted in Figure 3-1.

Respiratory Effects. Self-reported symptoms of respiratory irritation and impaired performance in tests of pulmonary function have been observed in studies of volunteers exposed to xylene for short periods of time under controlled conditions. In humans, nose and throat irritation has been reported following exposure to mixed xylene at $200 \mathrm{ppm}$ for 3-5 minutes (Nelson et al. 1943), to $m$-xylene at $50 \mathrm{ppm}$ for 2 hours (Ernstgard et al. 2002), and to $p$-xylene at $100 \mathrm{ppm}$ for 1-7.5 hours/day for 5 days (NIOSH 1981). However, no increase in reports of nose and throat irritation and no change in respiratory rate were seen in a study of subjects exposed to mixed xylene at a concentration of $396 \mathrm{ppm}$ for 
Table 3-1 Levels of Significant Exposure to Xylene - Inhalation

\begin{tabular}{|c|c|c|c|c|c|c|c|c|}
\hline \multirow[b]{2}{*}{$\begin{array}{l}\text { Key to } \\
\text { Figure }\end{array}$} & \multirow[b]{2}{*}{$\begin{array}{l}\text { Species } \\
\text { (Strain) }\end{array}$} & \multirow{2}{*}{$\begin{array}{l}\text { Exposurel } \\
\text { Durationl } \\
\text { Frequency } \\
\text { (Route) }\end{array}$} & & \multirow[b]{2}{*}{$\begin{array}{r}\text { NOAEL } \\
\text { (ppm) }\end{array}$} & \multicolumn{2}{|c|}{ LOAEL } & \multirow[b]{2}{*}{ Reference } & \multirow[b]{2}{*}{ Comments } \\
\hline & & & System & & $\begin{array}{c}\text { Less Serious } \\
(\mathrm{ppm})\end{array}$ & $\begin{array}{l}\text { Serious } \\
\text { (ppm) }\end{array}$ & & \\
\hline
\end{tabular}

ACUTE EXPOSURE

Death

1 Rat $24 \mathrm{hr}$

Wistar

2 Rat

$12 \mathrm{hr}$

(Wistar)

3 Rat

Harlan-

Wistar

$4 \quad$ Rat

$5 \quad$ Rat $4 \mathrm{hr}$

Long- Evans

6 Rat $7 \mathrm{~d}$

CFY $24 \mathrm{hr} / \mathrm{d}$

$7 \quad$ Mouse

SPF-Of1

$6 \mathrm{hr}$

$8 \quad$ Mouse

SPF-Of1

$6 \mathrm{hr}$

$9 \quad$ Mouse

$6 \mathrm{hr}$

SPF-Of1

\begin{tabular}{|c|c|}
\hline 3062 & (1/10 died) \\
\hline 19650 & (8/10 died) \\
\hline \multicolumn{2}{|c|}{$6700 \mathrm{M}$ (LC50) } \\
\hline $4740 \mathrm{~F}$ & (LC50) \\
\hline \multicolumn{2}{|c|}{$6350 \mathrm{M}$ (LC50) } \\
\hline $700 \mathrm{~F}$ & (4/30 died) \\
\hline $3907 \mathrm{~F}$ & (LC50) \\
\hline $5267 \mathrm{~F}$ & (LC50) \\
\hline $4595 \mathrm{~F}$ & (LC50) \\
\hline 3062 & (4/10 died) \\
\hline
\end{tabular}

Cameron et al. 1938

ortho

Cameron et al. 1938

para

Carpenter et al. 1975a

mixed

Harper et al. 1975

para

Hine and Zuidema 1970

mixed

Ungvary et al. 1980b

meta

Bonnet et al. 1979

para

Bonnet et al. 1979

meta

Bonnet et al. 1979

ortho

Cameron et al. 1938

ortho 
Table 3-1 Levels of Significant Exposure to Xylene - Inhalation

\begin{tabular}{llr}
\hline & & $\begin{array}{r}\text { Exposu } \\
\text { Duration }\end{array}$ \\
$\begin{array}{l}\text { Key to } \\
\text { Figure }\end{array}$ & $\begin{array}{r}\text { Species } \\
\text { (Strain) }\end{array}$ & $\begin{array}{r}\text { Frequen } \\
\text { (Route) }\end{array}$ \\
\hline & & \\
11 & $\begin{array}{l}\text { Mouse } \\
\text { NS }\end{array}$ & $12 \mathrm{hr}$ \\
& & \\
$12 \quad$ & Mouse \\
& NS & \\
\end{tabular}

Systemic

13 Human $0.25 \mathrm{hr}$

$\begin{array}{llll}\text { Resp } & 460 & 690 & \text { (throat irritation) } \\ \text { Ocular } & 230 & 460 & \text { (eye irritation) }\end{array}$

LOAEL

Reference

\begin{tabular}{crr} 
System & $\begin{array}{r}\text { NOAEL } \\
(\mathrm{ppm})\end{array}$ & $\begin{array}{r}\text { Less Serious } \\
(\mathrm{ppm})\end{array}$ \\
\hline
\end{tabular}

Chemical Form

13 Human 0.25 h

14 Human

$2 \mathrm{hr}$

Resp

50 (decreased forced vital

capacity; increased

severity score

for throat/airway

discomfort, breathing

difficulty, nose irritation)

19650 (9/10 died)

Cameron et al. 1938

para

$2010 \quad$ (6/10 died)

Cameron et al. 1938

meta

Carpenter et al. 1975a

mixed

Ernstgard et al. 2002

meta

Ocular $50 \quad$ (slight eye irritation)

Human 2 or $3 d$

2 or $3 \mathrm{~d}$
$70 \mathrm{~min} / \mathrm{d}$

Cardio

$299 M$

16 Human

$30 \min$

Resp

$396 \mathrm{M}$

Ocular

$396 \mathrm{M}$ 
Table 3-1 Levels of Significant Exposure to Xylene - Inhalation

\begin{tabular}{|c|c|c|c|c|}
\hline $\begin{array}{l}\text { Key to } \\
\text { Figure }\end{array}$ & $\begin{array}{l}\text { Species } \\
\text { (Strain) }\end{array}$ & $\begin{array}{l}\text { Exposurel } \\
\text { Durationl } \\
\text { Frequency } \\
\text { (Route) }\end{array}$ & System & $\begin{array}{r}\text { NOAEL } \\
(\mathrm{ppm})\end{array}$ \\
\hline \multirow[t]{3}{*}{17} & Human & $\begin{array}{l}2-6 \mathrm{~d} \\
5-5.5 \mathrm{hr} / \mathrm{d}\end{array}$ & Resp & 200 \\
\hline & & & Cardio & $200 \mathrm{M}$ \\
\hline & & & Hemato & $200 \mathrm{M}$ \\
\hline
\end{tabular}

\section{Reference}

Chemical Form

18 Human 3-5 min

Resp

200 (nose and throat irritation)

200 (eye irritation)

$100 \mathrm{~F}$ (nose and throat irritation)

NIOSH 1981

para

aine et al. 1993

meta

Nelson et al. 1943

mixed

Human

Cardio

$100 \mathrm{M}$

Ogata et al. 1970

para

Ogata et al. 1970

meta 
Table 3-1 Levels of Significant Exposure to Xylene - Inhalation

\begin{tabular}{|c|c|c|c|c|c|c|c|c|}
\hline \multirow[b]{2}{*}{$\begin{array}{l}\text { Key to } \\
\text { Figure }\end{array}$} & \multirow[b]{2}{*}{$\begin{array}{l}\text { Species } \\
\text { (Strain) }\end{array}$} & \multirow{2}{*}{$\begin{array}{l}\text { Exposurel } \\
\text { Durationl } \\
\text { Frequency } \\
\text { (Route) }\end{array}$} & \multicolumn{5}{|c|}{ LOAEL } & \multirow[b]{2}{*}{ Comments } \\
\hline & & & System & $\begin{array}{l}\text { NOAEL } \\
\text { (ppm) }\end{array}$ & $\begin{array}{c}\text { Less Serious } \\
\text { (ppm) }\end{array}$ & $\begin{array}{l}\text { Serious } \\
\text { (ppm) }\end{array}$ & $\begin{array}{l}\text { Reference } \\
\text { Chemical Form }\end{array}$ & \\
\hline
\end{tabular}

2 Human 4

$\begin{array}{lll}4 \mathrm{~d} & \text { Resp } & 200 \mathrm{M} \\ 3.67 \mathrm{hr} / \mathrm{d} & & \\ & \text { Cardio } & 200 \mathrm{M}\end{array}$

Seppalainen et al. 1989

meta

Carpenter et al. $1975 \mathrm{a}$

mixed

Elovaara 1982

meta

Elovaara et al. 1987

meta

7-ethoxycoumarin
O-deethylase activity)

$1000 \mathrm{~F}$ (decreased pulmonary microsomal activity)

Patel et al. 1978

para

Patel et al. 1978

para

Silverman and Schatz 1991

para 
Table 3-1 Levels of Significant Exposure to Xylene - Inhalation

\begin{tabular}{|c|c|c|c|c|c|c|c|c|}
\hline \multirow[b]{2}{*}{$\begin{array}{l}\text { Key to } \\
\text { Figure }\end{array}$} & \multirow[b]{2}{*}{$\begin{array}{l}\text { Species } \\
\text { (Strain) }\end{array}$} & \multirow{2}{*}{$\begin{array}{l}\text { Exposurel } \\
\text { Duration/ } \\
\text { Frequency } \\
\text { (Route) }\end{array}$} & \multicolumn{5}{|c|}{ LOAEL } & \multirow[b]{2}{*}{ Comments } \\
\hline & & & System & $\begin{array}{r}\text { NOAEL } \\
\text { (ppm) }\end{array}$ & $\begin{array}{c}\text { Less Serious } \\
\text { (ppm) }\end{array}$ & $\begin{array}{r}\text { Serious } \\
\text { (ppm) }\end{array}$ & $\begin{array}{l}\text { Reference } \\
\text { Chemical Form }\end{array}$ & \\
\hline
\end{tabular}

29 Rat 1 or $3 d$ Fischer- $3446 \mathrm{hr} / \mathrm{d}$

30 Rat Sprague- $\quad 6 \mathrm{hr} / \mathrm{d}$ Dawley

31 Rat Sprague- $\quad 6 \mathrm{hr} / \mathrm{d}$ Dawley$$
\begin{aligned}
& 3 \mathrm{~d} \\
& 6 \mathrm{hr} / \mathrm{d}
\end{aligned}
$$

Rat

$\begin{array}{ll}\text { Rat } & 3 \mathrm{~d} \\ \text { Sprague- } & 6 \mathrm{hr} / \mathrm{d} \\ \text { Dawley } & \end{array}$

Dawley

33

$\begin{array}{ll}\text { Rat } & 3 \mathrm{~d} \\ \text { Sprague- } & 6 \mathrm{hr} / \mathrm{d}\end{array}$

Dawley

$6 \mathrm{hr} / \mathrm{d}$

Resp

34

$\begin{array}{ll}\text { Rat } & 7 \mathrm{~d} \\ \text { CFY } & 24 \mathrm{hr} / \mathrm{d} \\ & \text { Gd } 7-14\end{array}$

Resp

Renal

Resp

Resp

Renal
Hepatic $\quad 1600 \mathrm{M}$

2000 M (decreased relative

kidney weight)

2000 M (decreased cytochrome

$$
\text { P-450) }
$$

Hepatic $\quad 700 \mathrm{~F}$

$\mathrm{Bd} \mathrm{Wt}$

2000 M (decreased cytochrome

P-450)

2000 M (decreased relative

kidney weight)

2000 M (decreased cytochrome

P-450)

2000 M (decreased cytochrome
P-450)

$350 \mathrm{~F}$ (ppm)

Comments

Simmons et al. 1991

para

Toftgard and Nilsen 1982

para

Toftgard and Nilsen 1982

meta

Toftgard and Nilsen 1982

ortho

Toftgard and Nilsen 1982

mixed

Ungvary et al. 1980b

meta 
Table 3-1 Levels of Significant Exposure to Xylene - Inhalation

\begin{tabular}{|c|c|c|c|c|c|c|c|c|}
\hline \multirow[b]{2}{*}{$\begin{array}{l}\text { Key to } \\
\text { Figure }\end{array}$} & \multirow[b]{2}{*}{$\begin{array}{l}\text { Species } \\
\text { (Strain) }\end{array}$} & \multirow{2}{*}{$\begin{array}{l}\text { Exposurel } \\
\text { Durationl } \\
\text { Frequency } \\
\text { (Route) }\end{array}$} & \multicolumn{5}{|c|}{ LOAEL } & \multirow[b]{2}{*}{ Comments } \\
\hline & & & System & $\begin{array}{r}\text { NOAEL } \\
\text { (ppm) }\end{array}$ & $\begin{array}{c}\text { Less Serious } \\
\text { (ppm) }\end{array}$ & $\begin{array}{l}\text { Serious } \\
\text { (ppm) }\end{array}$ & $\begin{array}{l}\text { Reference } \\
\text { Chemical Form }\end{array}$ & \\
\hline
\end{tabular}

35 Rat $7 d$

$\begin{array}{llll}\text { Rat } & 7 \mathrm{~d} & \text { Hepatic } & 700 \mathrm{~F} \\ \text { CFY } & 24 \mathrm{hr} / \mathrm{d} & & \\ & \text { Gd 7-14 } & & \\ & & & \\ & & & \end{array}$

36 Rat $\quad 7 \mathrm{~d}$ Hepatic $\quad 700 \mathrm{~F}$

$\begin{array}{llll}\text { CFY } & 24 \mathrm{hr} / \mathrm{d} & \text { Hepatic } & 700 \mathrm{~F} \\ & \text { Gd 7-14 } & & \\ & & \end{array}$

$\begin{array}{llll}37 & \text { Rat } & 9 \mathrm{~d} & \text { Hemato } \\ \text { Wistar } & 5 \mathrm{hr} / \mathrm{d} & & 2764\end{array}$

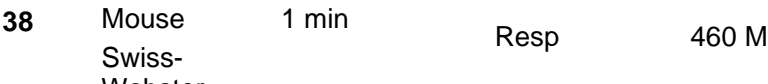

39

Mouse $5 \mathrm{~min} \quad$ Resp

Resp

$1467 \mathrm{M}$ (50\% decrease in
respiratory rate)

$2440 \mathrm{M}(50 \%$ decrease in respiratory rate)

Ungvary et al. 1980b

ortho

Ungvary et al. 1980b

para

Wronska-Nofer et al. 1991

mixed

Carpenter et al. $1975 a$

mixed

Resp

(50\% decrease

De Ceaurriz et al. 1981

ortho

Korsak et al. 1988

mixed

$41 \quad$ Mouse

6 min

Resp

$2513 \mathrm{M}(32 \%$ decrease in

respiratory rate)

Korsak et al. 1990

ortho 
Table 3-1 Levels of Significant Exposure to Xylene - Inhalation

\begin{tabular}{|c|c|c|c|c|c|c|c|}
\hline \multirow[b]{2}{*}{$\begin{array}{l}\text { Key to } \\
\text { Figure }\end{array}$} & \multirow[b]{2}{*}{$\begin{array}{l}\text { Species } \\
\text { (Strain) }\end{array}$} & \multirow{2}{*}{$\begin{array}{c}\text { Exposurel } \\
\text { Duration/ } \\
\text { Frequency } \\
\text { (Route) }\end{array}$} & \multirow[b]{2}{*}{ System } & \multirow[b]{2}{*}{$\begin{array}{r}\text { NOAEL } \\
(p p m) \\
\end{array}$} & \multicolumn{2}{|c|}{ LOAEL } & \multirow[b]{2}{*}{$\begin{array}{l}\text { Reference } \\
\text { Chemical Form }\end{array}$} \\
\hline & & & & & $\begin{array}{c}\text { Less Serious } \\
(\mathrm{ppm})\end{array}$ & $\begin{array}{r}\text { Serious } \\
(\mathrm{ppm})\end{array}$ & \\
\hline 42 & $\begin{array}{l}\text { Mouse } \\
\text { Balb/C }\end{array}$ & $6 \min$ & Resp & & $\begin{array}{c}2626 \mathrm{M} \text { (transient } 46 \% \text { decrease } \\
\text { in respiratory rate) }\end{array}$ & & $\begin{array}{l}\text { Korsak et al. } 1990 \\
\text { para }\end{array}$ \\
\hline 43 & $\begin{array}{l}\text { Mouse } \\
\text { Balb/C }\end{array}$ & $6 \min$ & Resp & & $\begin{array}{c}2700 \mathrm{M} \text { (transient } 57 \% \text { decrease } \\
\text { in respiratory rate) }\end{array}$ & & $\begin{array}{l}\text { Korsak et al. } 1990 \\
\text { meta }\end{array}$ \\
\hline 44 & $\begin{array}{l}\text { Mouse } \\
\text { Balb/c }\end{array}$ & $\begin{array}{l}\text { once } \\
6 \text { min }\end{array}$ & Resp & & & $\begin{array}{c}1361 \mathrm{M} \text { (50\% decrease in } \\
\text { respiratory rate) }\end{array}$ & $\begin{array}{l}\text { Korsak et al. } 1993 \\
\text { meta }\end{array}$ \\
\hline \multirow[t]{2}{*}{45} & $\begin{array}{l}\text { Mouse } \\
\mathrm{C} 3 \mathrm{H} / \mathrm{H} 3 \mathrm{~J}\end{array}$ & $\begin{array}{l}4 \mathrm{~d} \\
6 \mathrm{hr} / \mathrm{d}\end{array}$ & Hepatic & $1208 \mathrm{~F}$ & & & $\begin{array}{l}\text { Selgrade et al. } 1993 \\
\text { para }\end{array}$ \\
\hline & & & $\mathrm{Bd} \mathrm{Wt}$ & $1208 \mathrm{~F}$ & & & \\
\hline 46 & $\begin{array}{l}\text { Rabbit } \\
\text { New Zealand }\end{array}$ & $\begin{aligned} & 2 \mathrm{~d} \\
& \mathrm{~d} 4 \mathrm{hr} / \mathrm{d}\end{aligned}$ & Resp & & $\begin{array}{l}1000 \mathrm{M} \text { (decreased pulmonary } \\
\text { microsomal activity) }\end{array}$ & & $\begin{array}{l}\text { Patel et al. } 1978 \\
\text { para }\end{array}$ \\
\hline \multicolumn{8}{|c|}{ Neurological } \\
\hline 47 & Human & $0.25 \mathrm{hr}$ & & 460 & 690 (dizziness) & & $\begin{array}{l}\text { Carpenter et al. 1975a } \\
\text { mixed }\end{array}$ \\
\hline 48 & Human & $4 \mathrm{hr}$ & & & $100 \mathrm{M}$ (increased reaction time) & & $\begin{array}{l}\text { Dudek et al. } 1990 \\
\text { mixed }\end{array}$ \\
\hline
\end{tabular}


Table 3-1 Levels of Significant Exposure to Xylene - Inhalation

\begin{tabular}{|c|c|}
\hline $\begin{array}{l}\text { Key to } \\
\text { Figure }\end{array}$ & $\begin{array}{l}\text { Species } \\
\text { (Strain) }\end{array}$ \\
\hline 49 & Human \\
\hline
\end{tabular}

once

50 Human $2 \mathrm{~d}$

51 Human $1 d$

$70 \mathrm{~min} / \mathrm{d}$

52

Human

$30 \mathrm{~min}$

$396 \mathrm{M}$

53 Human

2-6 d

$5-5.5 \mathrm{hr} / \mathrm{d}$

$200 \mathrm{M}$

54 Human

$$
5 \mathrm{~d}
$$

$1-7.5 \mathrm{hr} / \mathrm{d}$

$100 \mathrm{~F}$ (dizziness)

$200 \mathrm{M}$

$69 \mathrm{M}$
55 Human $7 \mathrm{hr}$

56 Human
$4 \mathrm{hr}$ $50^{\mathrm{b}}$ (increased severity

scores for headache

dizziness in males:

intoxication in males and

females)

299 M (impairment in reaction

time and short-term

memory after exercising

not without exercising)

LOAEL

Serious

(ppm)

Chemical Form

Comments
Ernstgard et al. 2002

meta

\section{Gamberale et al. 1978}

mixed

Gamberale et al. 1978

mixed

Hastings et al. 1986

mixed

Laine et al. 1993

meta

NIOSH 1981

para

Ogata et al. 1970

meta

Olson et al. 1985

para 
Table 3-1 Levels of Significant Exposure to Xylene - Inhalation

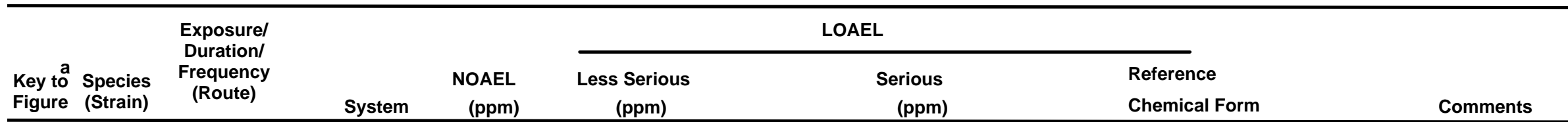

57 Human $2 \times /$ dose

$1 \mathrm{x} / \mathrm{wk}$

$281 \mathrm{M}$

58

Human $\quad 4 \mathrm{hr}$

59

$\begin{array}{ll}\text { Human } & 4 \mathrm{~d} \\ & 3.67 \mathrm{hr} / \mathrm{d}\end{array}$

60 Rat

Sprague- $\quad 6 \mathrm{hr} / \mathrm{d}$

Dawley

61 Rat

Sprague- $\quad 6 \mathrm{hr} / \mathrm{d}$

Dawley

62 Rat

$3 d$

Sprague- $\quad 6 \mathrm{hr} / \mathrm{d}$

Dawley

63 Rat

Sprague- $\quad 6 \mathrm{hr} / \mathrm{d}$

Sprague-

$\mathrm{hr} / \mathrm{d}$

Chemical Form

Savolainen 1980

meta

Savolainen et al. 1984

meta

and reaction times)

200 M (altered visual evoked potentials)

Seppalainen et al. 1989 meta

Andersson et al. 1981

para

$$
\text { catecholamine) }
$$

Andersson et al. 1981

mixed

catecholamine in brain)

Andersson et al. 1981

meta

catecholamine)

Andersson et al. 1981

ortho
2000 M (increased brain levels of catecholamine) 
Table 3-1 Levels of Significant Exposure to Xylene - Inhalation

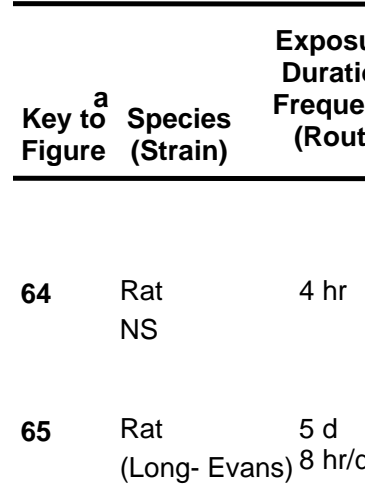

LOAEL

NOAEL

$\longrightarrow$

System

\begin{tabular}{l}
$(\mathrm{ppm})$ \\
\hline
\end{tabular}

$580 \mathrm{M}$

$800 \mathrm{M}$

$1600 \mathrm{M}$ (altered visual evoked potentials)

113 M (transiently decreased operant responding)

$99 \mathrm{M}$

114 M (transiently decreased operant responding)

2010 M
Reference

Chemical Form

Comments

Rat $\quad 5 \mathrm{hr}$

69 Rat

$3 \mathrm{~d}$

70

$4 \mathrm{hr}$

NS

(1)

(ppm)

$1300 \mathrm{M}$ (incoordination)

Carpenter et al. 1975a

mixed

$1800 \mathrm{M}$ (18-30 dB increased in

mid-range

auditory thresholds)

Crofton et al. 1994

mixed

Dyer et al. 1988

para

Ghosh et al. 1987

mixed

Ghosh et al. 1987

mixed

Ghosh et al. 1987

mixed

2870 M (impaired rotarod performance)
Korsak et al. 1988

mixed 
Table 3-1 Levels of Significant Exposure to Xylene - Inhalation

\begin{tabular}{|c|c|c|c|c|c|c|c|c|}
\hline \multirow[b]{2}{*}{$\begin{array}{l}\text { Key to } \\
\text { Figure }\end{array}$} & \multirow[b]{2}{*}{$\begin{array}{l}\text { Species } \\
\text { (Strain) }\end{array}$} & \multirow{2}{*}{$\begin{array}{l}\text { Exposurel } \\
\text { Durationl } \\
\text { Frequency } \\
\text { (Route) }\end{array}$} & \multicolumn{5}{|c|}{ LOAEL } & \multirow[b]{2}{*}{ Comments } \\
\hline & & & System & $\begin{array}{r}\text { NOAEL } \\
\text { (ppm) }\end{array}$ & $\begin{array}{c}\text { Less Serious } \\
\text { (ppm) }\end{array}$ & $\begin{array}{l}\text { Serious } \\
\text { (ppm) }\end{array}$ & $\begin{array}{l}\text { Reference } \\
\text { Chemical Form }\end{array}$ & \\
\hline
\end{tabular}

71 Rat $6 \mathrm{hr}$

72 Rat

$6 \mathrm{hr}$

NS

73 Rat

$6 \mathrm{hr}$

74 Rat

Imp:DAK

$75 \quad$ Rat

$4 \mathrm{hr}$

76

NS

$77 \quad$ Rat

$4 \mathrm{hr}$

$\begin{array}{ll}\text { Rat } & 1.5 \mathrm{wk} \\ \text { NS } & 5 \mathrm{~d} / \mathrm{wk} \\ & 6 \mathrm{hr} / \mathrm{d}\end{array}$

$800 \mathrm{M}$ (decreased axonal transport)
3000 M (impaired rotarod

performance)

3000 M (impaired rotarod performance)

3000 M (impaired rotarod performance)

1982 M (EC50 for decreased rotarod performance)

1940 M (narcosis)

2180 M (narcosis)

2100 M (narcosis)
Korsak et al. 1990

ortho

Korsak et al. 1990

para

Korsak et al. 1990

meta

Korsak et al. 1993

meta

Molnar et al. 1986

para

Molnar et al. 1986

ortho

Molnar et al. 1986

meta

Padilla and Lyerly 1989

mixed 
Table 3-1 Levels of Significant Exposure to Xylene - Inhalation

\begin{tabular}{lll}
\hline Key to & Species & $\begin{array}{c}\text { Exposurel } \\
\text { Durationl } \\
\text { Figure } \\
\text { (Strain) } \\
\text { (Route) }\end{array}$ \\
\hline & & \\
$79 \quad$ Rat & $1,3,8,13 \mathrm{~d}$ \\
& NS & $5 \mathrm{~d} / \mathrm{wk}$ \\
$6 \mathrm{hr} / \mathrm{d}$
\end{tabular}

80 Rat

$$
4 \mathrm{hr}
$$

NS

81 Rat

$8 \mathrm{hr}$

82 Rat

(Long- Evans) $8 \mathrm{hr} / \mathrm{d}$
$400 \mathrm{M}$

$1700 \mathrm{M}$

$1700 \mathrm{M}$

$102 \mathrm{M}$

$250 \mathrm{M}$
LOAEL

Less Serious Serio

(ppm)

(ppm)

Reference

Chemical Form

Comments

$\begin{array}{ll}\text { Rat } & 4 \mathrm{hr} \\ \text { (albino) } & \text { once }\end{array}$

$84 \quad$ Rat

$2 \mathrm{hr}$

F344

85 Mouse

Webster)
5 min

$800 \mathrm{M}$ (decreased axonal transport)

Padilla and Lyerly 1989

para

Pryor et al. 1987

mixed

$1450 \mathrm{M}$ (hearing loss)

2000 M (50\% decreased integrated amplitude of brainstem

auditory evoked

potentials at $16 \mathrm{kHz}$ )

$230 \mathrm{M}(18 \%$ inhibition of

electrically evoked

seizure discharge)

192 M (decreased

self-stimulation behavior)

$500 \mathrm{M}$ (decreased response

rate for

schedule-controlled
scher

operant behavior)
Pryor et al. 1987

mixed

Rebert et al. 1995

mixed

Vodickova et al. 1995

ortho

Wimolwattanapun et al. 1987

mixed

Bowen et al. 1998

meta 
Table 3-1 Levels of Significant Exposure to Xylene - Inhalation

\begin{tabular}{lll}
\hline & & $\begin{array}{r}\text { Exposu } \\
\text { Duration }\end{array}$ \\
$\begin{array}{l}\text { Key to } \\
\text { Figure }\end{array}$ & $\begin{array}{l}\text { Species } \\
\text { (Strain) }\end{array}$ & $\begin{array}{r}\text { Freque } \\
\text { (Rout }\end{array}$ \\
\hline & & \\
86 & $\begin{array}{l}\text { Mouse } \\
\text { Swiss Of1 }\end{array}$ & $4 \mathrm{hr}$ \\
& &
\end{tabular}

87

$\begin{array}{ll}\text { Mouse } & 4 \mathrm{hr} \\ \text { (albino) } & \text { once }\end{array}$

$88 \quad$ Cat

Reproductive

$89 \begin{array}{ll}\text { Rat } & 8 \mathrm{~d} \\ \text { (CFY) } & 24 \mathrm{hr} / \mathrm{d} \\ & \end{array}$

\section{Developmental}

90 Rat

$\begin{array}{ll}\text { (CFY) } & 24 \mathrm{hr} / \mathrm{d} \\ & \text { Gd } 7-14\end{array}$

91 Rat Gd 7-20

(Wistar) $6 \mathrm{hr} / \mathrm{d}$

$1010 \mathrm{M}$ (altered behavior in

swimming test)

$320 \mathrm{~F}$ (11\% decreased duration

of response

to electric shock)

LOAEL

(ppm)

Reference

Chemical Form

Comments

De Ceaurriz et al. 1983

ortho

Vodickova et al. 1995

ortho
Carpenter et al. 1975a

mixed

seizures, anesthesia)

Balogh et al. 1982

mixed

$8 \%$ decreased fertily; increased resorptions)

Balogh et al. 1982

mixed

$500 \mathrm{~F}$ (delayed air righting reflex, impaired moto coordination

on Rotarod; impaired

memory in Morris water

maze)
Hass et al. 1995

mixed

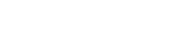


Table 3-1 Levels of Significant Exposure to Xylene - Inhalation

\begin{tabular}{lll}
\hline Key to & Species & $\begin{array}{c}\text { Exposurel } \\
\text { Duration/ } \\
\text { Figure } \\
\text { (Strain) } \\
\text { (Route) }\end{array}$ \\
\hline & & \\
92 & Rat & Gd 7-20 \\
& (Wistar) & $6 \mathrm{hr} / \mathrm{d}$
\end{tabular}

93

$10 d$

Sprague- $\quad 6 \mathrm{hr} / \mathrm{d}$

Dawley Gd 7-16

94 Rat Gd 6-20

(Sprague- $6 \mathrm{hr} / \mathrm{d}$

Dawley)

$95 \quad$ Rat

Gd 6-20

(Sprague-

Dawley)

96 Rat

Dawley)

Gd 6-20

$6 \mathrm{hr} / \mathrm{d}$

Dawley)

97 Rat

Dawley)

Gd 6-20

$6 \mathrm{hr} / \mathrm{d}$

98

$\begin{array}{ll}\text { Rat } & 9 \mathrm{~d} \\ \text { CFY } & 24 \mathrm{hr} / \mathrm{d} \\ & \text { Gd 7-15 }\end{array}$

LOAEL

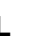

System $\quad$ NOA

(ppm)
serious

$1612 \mathrm{~F}$

500

$1000 \quad(6 \%$ decrease in fetal body weight)

100

$500 \quad(5 \%$ decrease in fetal body weight)

$500 \mathrm{~F}$ (increased latency in persisting to 28 weeks)

Reference

Chemical Form

Comments
Hass et al. 1997

mixed

Rosen et al. 1986

para

Saillenfait et al. 2003

meta

Saillenfait et al. 2003

ortho

Saillenfait et al. 2003

para

Saillenfait et al. 2003

mixed

Ungvary and Tatrai 1985

mixed 
Table 3-1 Levels of Significant Exposure to Xylene - Inhalation

(continued)

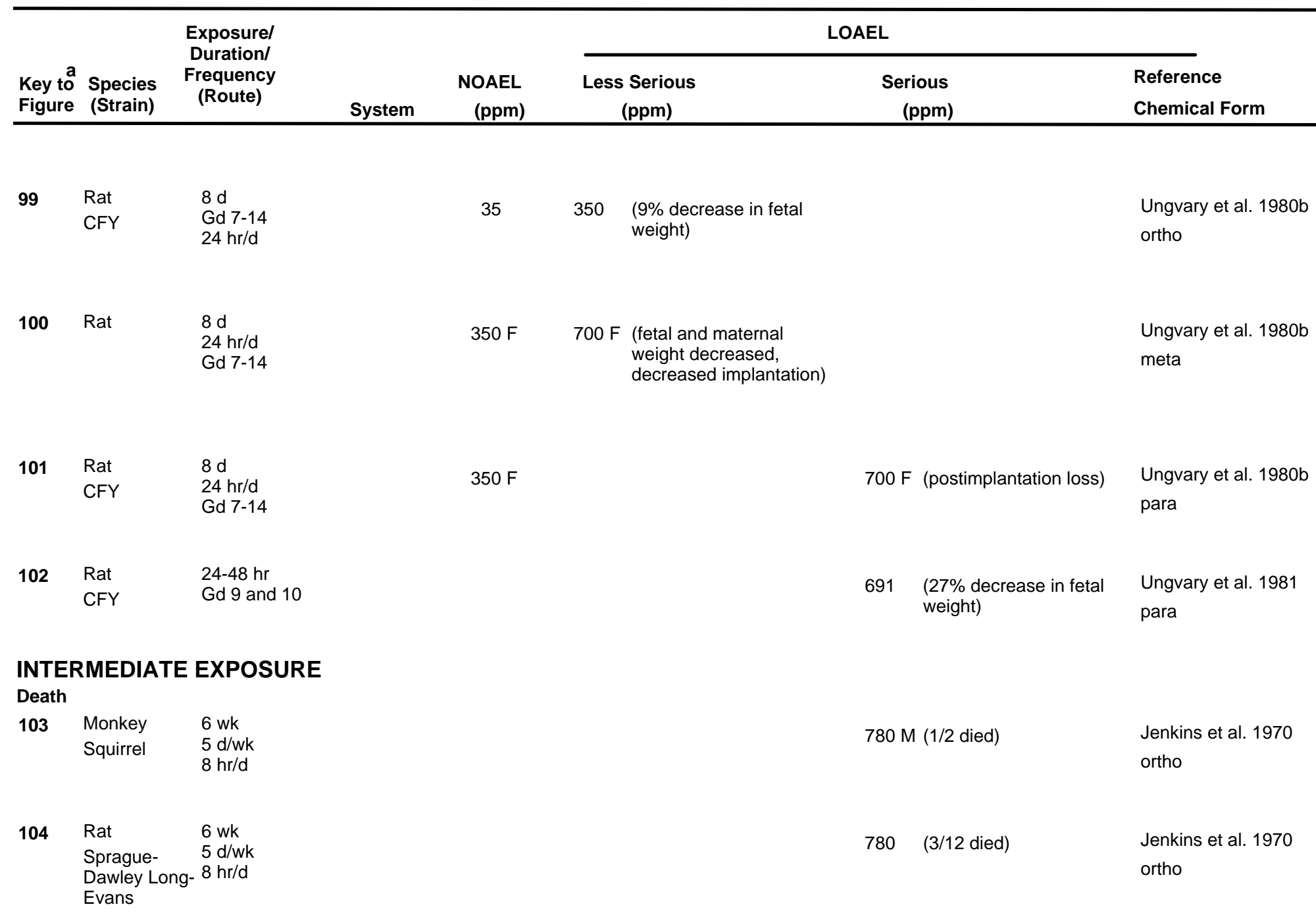


Table 3-1 Levels of Significant Exposure to Xylene - Inhalation

\begin{tabular}{|c|c|c|c|c|c|c|c|}
\hline \multirow[b]{2}{*}{$\begin{array}{l}\text { Key to } \\
\text { Figure }\end{array}$} & \multirow[b]{2}{*}{$\begin{array}{l}\text { Species } \\
\text { (Strain) }\end{array}$} & \multirow{2}{*}{$\begin{array}{l}\text { Exposurel } \\
\text { Duration/ } \\
\text { Frequency } \\
\text { (Route) }\end{array}$} & \multirow[b]{2}{*}{ System } & \multirow[b]{2}{*}{$\begin{array}{r}\text { NOAEL } \\
(\text { ppm) }\end{array}$} & \multicolumn{2}{|c|}{ LOAEL } & \multirow[b]{2}{*}{$\begin{array}{l}\text { Reference } \\
\text { Chemical Form }\end{array}$} \\
\hline & & & & & $\begin{array}{l}\text { Less Serious } \\
\text { (ppm) }\end{array}$ & $\begin{array}{l}\text { Serious } \\
(\mathrm{ppm})\end{array}$ & \\
\hline \multicolumn{8}{|c|}{ Systemic } \\
\hline 105 & Human & $\begin{array}{l}4 \mathrm{wk} \\
5 \mathrm{~d} / \mathrm{wk} \\
1-7.5 \mathrm{hr} / \mathrm{d} \\
\text { (I) }\end{array}$ & Resp & 20 & $\begin{array}{c}100 \mathrm{M} \text { (nose and throat } \\
\text { irritation) }\end{array}$ & & $\begin{array}{l}\text { NIOSH } 1981 \\
\text { para }\end{array}$ \\
\hline & & & Cardio & $150 \mathrm{M}$ & & & \\
\hline & & & Hemato & $150 \mathrm{M}$ & & & \\
\hline & & & Renal & $150 \mathrm{M}$ & & & \\
\hline & & & Ocular & $20 \mathrm{M}$ & $100 \mathrm{M}$ (eye irritation) & & \\
\hline \multirow[t]{9}{*}{106} & $\begin{array}{l}\text { Rat } \\
\text { NS }\end{array}$ & $\begin{array}{l}10 \mathrm{wk} \\
5 \mathrm{~d} / \mathrm{wk} \\
6 \mathrm{hr} / \mathrm{d}\end{array}$ & Resp & $810 \mathrm{M}$ & & & $\begin{array}{l}\text { Carpenter et al. 1975a } \\
\text { mixed }\end{array}$ \\
\hline & & & Cardio & $810 \mathrm{M}$ & & & \\
\hline & & & Gastro & $810 \mathrm{M}$ & & & \\
\hline & & & Hemato & $810 \mathrm{M}$ & & & \\
\hline & & & Musc/skel & $810 \mathrm{M}$ & & & \\
\hline & & & Hepatic & $810 \mathrm{M}$ & & & \\
\hline & & & Renal & $810 \mathrm{M}$ & & & \\
\hline & & & Endocr & $810 \mathrm{M}$ & & & \\
\hline & & & $\mathrm{Bd} \mathrm{Wt}$ & $810 \mathrm{M}$ & & & \\
\hline 107 & $\begin{array}{l}\text { Rat } \\
\text { NS }\end{array}$ & $\begin{array}{l}5,9,14 \text {, or } \\
18 \mathrm{wk} \\
5 \mathrm{~d} / \mathrm{wk} \\
6 \mathrm{hr} / \mathrm{d}\end{array}$ & Hepatic & $300 \mathrm{M}$ & & & $\begin{array}{l}\text { Elovaara et al. } 1980 \\
\text { mixed }\end{array}$ \\
\hline
\end{tabular}


Table 3-1 Levels of Significant Exposure to Xylene - Inhalation

\begin{tabular}{|c|c|c|c|c|c|c|c|}
\hline \multirow[b]{2}{*}{$\begin{array}{l}\text { Key to } \\
\text { Figure }\end{array}$} & \multirow[b]{2}{*}{$\begin{array}{l}\text { Species } \\
\text { (Strain) }\end{array}$} & \multirow{2}{*}{$\begin{array}{l}\text { Exposurel } \\
\text { Durationl } \\
\text { Frequency } \\
\text { (Route) }\end{array}$} & \multirow[b]{2}{*}{ System } & \multirow[b]{2}{*}{$\begin{array}{r}\text { NOAEL } \\
\text { (ppm) }\end{array}$} & \multicolumn{2}{|c|}{ LOAEL } & \multirow[b]{2}{*}{$\begin{array}{l}\text { Reference } \\
\text { Chemical Form }\end{array}$} \\
\hline & & & & & $\begin{array}{c}\text { Less Serious } \\
(\mathrm{ppm})\end{array}$ & $\begin{array}{r}\text { Serious } \\
(\mathrm{ppm})\end{array}$ & \\
\hline 108 & $\begin{array}{l}\text { Rat } \\
\text { (Sprague- } \\
\text { Dawley) }\end{array}$ & $\begin{array}{l}13 \mathrm{wk} \\
6 \mathrm{~d} / \mathrm{wk} \\
6 \mathrm{hr} / \mathrm{d}\end{array}$ & $\mathrm{Bd} \mathrm{Wt}$ & $1800 \mathrm{M}$ & & & $\begin{array}{l}\text { Gagnaire et al. } 2001 \\
\text { meta }\end{array}$ \\
\hline 109 & $\begin{array}{l}\text { Rat } \\
\text { (Sprague- } \\
\text { Dawley) }\end{array}$ & $\begin{array}{l}13 \mathrm{wk} \\
6 \mathrm{~d} / \mathrm{wk} \\
6 \mathrm{hr} / \mathrm{d}\end{array}$ & $\mathrm{Bd} \mathrm{Wt}$ & $1800 \mathrm{M}$ & & & $\begin{array}{l}\text { Gagnaire et al. } 2001 \\
\text { ortho }\end{array}$ \\
\hline 110 & $\begin{array}{l}\text { Rat } \\
\text { (Sprague- } \\
\text { Dawley) }\end{array}$ & $\begin{array}{l}13 \mathrm{wk} \\
6 \mathrm{~d} / \mathrm{wk} \\
6 \mathrm{hr} / \mathrm{d}\end{array}$ & $\mathrm{Bd} \mathrm{Wt}$ & $1800 \mathrm{M}$ & & & $\begin{array}{l}\text { Gagnaire et al. } 2001 \\
\text { para }\end{array}$ \\
\hline 111 & $\begin{array}{l}\text { Rat } \\
\text { (Wistar) }\end{array}$ & $\begin{array}{l}3 \mathrm{mo} \\
5 \mathrm{~d} / \mathrm{wk} \\
6 \mathrm{hr} / \mathrm{d}\end{array}$ & $\mathrm{Bd} \mathrm{Wt}$ & $1000 \mathrm{M}$ & & & $\begin{array}{l}\text { Gralewicz et al. } 1995 \\
\text { meta }\end{array}$ \\
\hline \multirow[t]{2}{*}{112} & $\begin{array}{l}\text { Rat } \\
\text { (Wistar) }\end{array}$ & $\begin{array}{l}5 \mathrm{mo} \\
5 \mathrm{~d} / \mathrm{wk} \\
5 \mathrm{hr} / \mathrm{d}\end{array}$ & Hepatic & $92 \mathrm{M}$ & & & $\begin{array}{l}\text { Jajte et al. } 2003 \\
\text { meta }\end{array}$ \\
\hline & & & $\mathrm{Bd} \mathrm{Wt}$ & $92 \mathrm{M}$ & & & \\
\hline \multirow[t]{5}{*}{113} & $\begin{array}{l}\text { Rat } \\
\text { Sprague- } \\
\text { Dawley Long- } \\
\text { Evans }\end{array}$ & $\begin{array}{l}90-127 \mathrm{~d} \\
24 \mathrm{hr} / \mathrm{d} \\
g-\end{array}$ & Resp & 78 & & & $\begin{array}{l}\text { Jenkins et al. } 1970 \\
\text { ortho }\end{array}$ \\
\hline & & & Cardio & 78 & & & \\
\hline & & & Hemato & 78 & & & \\
\hline & & & Hepatic & 78 & & & \\
\hline & & & Renal & 78 & & & \\
\hline
\end{tabular}


Table 3-1 Levels of Significant Exposure to Xylene - Inhalation

\begin{tabular}{|c|c|c|c|c|}
\hline $\begin{array}{l}\text { Key to } \\
\text { Figure }\end{array}$ & $\begin{array}{l}\text { Species } \\
\text { (Strain) }\end{array}$ & $\begin{array}{l}\text { Exposurel } \\
\text { Durationl } \\
\text { Frequency } \\
\text { (Route) }\end{array}$ & System & $\begin{array}{l}\text { NOAE } \\
\text { (ppn }\end{array}$ \\
\hline \multirow[t]{5}{*}{114} & $\begin{array}{l}\text { Rat } \\
\text { Sprague- } \\
\text { Dawley Long } \\
\text { Evans }\end{array}$ & $\begin{array}{c}6 \mathrm{wk} \\
5 \mathrm{~d} / \mathrm{wk} \\
\text { g- } 8 \mathrm{hr} / \mathrm{d}\end{array}$ & Resp & 780 \\
\hline & & & Cardio & 780 \\
\hline & & & Hemato & 780 \\
\hline & & & Hepatic & 780 \\
\hline & & & Renal & 780 \\
\hline
\end{tabular}

115 Rat $3 \mathrm{mo}$

Wistar $\quad 5 \mathrm{~d} / \mathrm{wk}$

$5 \mathrm{~d} / \mathrm{wk}$
$6 \mathrm{hr} / \mathrm{d}$

Hemato

$1000 \mathrm{M}$

$\mathrm{Bd} W \mathrm{t}$

$116 \quad$ Rat $3 \mathrm{mo}$

(Wistar) $\quad 5 \mathrm{~d} / \mathrm{wk}$

$6 \mathrm{hr} / \mathrm{d}$

Hemato

$50 \mathrm{M}$

100 M (19\% decreased

erythrocytes;

$35 \%$ increase

Korsak et al. 1992

meta

Korsak et al. 1994

meta

230 M (increased wall thickness Morvai et al. 1987

in coronary

micro-vessels)

mixed

Rydzynski et al. 1992

meta

ortho 
Table 3-1 Levels of Significant Exposure to Xylene - Inhalation

\begin{tabular}{|c|c|c|c|c|c|c|c|c|}
\hline \multirow[b]{2}{*}{$\begin{array}{l}\text { Key to } \\
\text { Figure }\end{array}$} & \multirow[b]{2}{*}{$\begin{array}{l}\text { Species } \\
\text { (Strain) }\end{array}$} & \multirow{2}{*}{$\begin{array}{l}\text { Exposurel } \\
\text { Duration/ } \\
\text { Frequency } \\
\text { (Route) }\end{array}$} & & \multicolumn{4}{|c|}{ LOAEL } & \multirow[b]{2}{*}{ Comments } \\
\hline & & & System & $\begin{array}{l}\text { NOAEL } \\
\text { (ppm) }\end{array}$ & $\begin{array}{c}\text { Less Serious } \\
\text { (ppm) }\end{array}$ & $\begin{array}{l}\text { Serious } \\
\text { (ppm) }\end{array}$ & $\begin{array}{l}\text { Reference } \\
\text { Chemical Form }\end{array}$ & \\
\hline
\end{tabular}

\begin{tabular}{|c|c|c|c|c|c|}
\hline 119 & $\begin{array}{l}\text { Rat } \\
\text { Wistar }\end{array}$ & $\begin{array}{l}3 \mathrm{mo} \\
5 \mathrm{~d} / \mathrm{wk} \\
6 \mathrm{hr} / \mathrm{d}\end{array}$ & Hepatic & $1000 \mathrm{M}$ & $\begin{array}{l}\text { Rydzynski et al. } 1992 \\
\text { meta }\end{array}$ \\
\hline \multirow[t]{2}{*}{120} & $\begin{array}{l}\text { Rat } \\
\text { CFY }\end{array}$ & $\begin{array}{l}6 \mathrm{mo} \\
7 \mathrm{~d} / \mathrm{wk} \\
8 \mathrm{hr} / \mathrm{d}\end{array}$ & Hepatic & $1096 \mathrm{M}$ & $\begin{array}{l}\text { Tatrai et al. } 1981 \\
\text { ortho }\end{array}$ \\
\hline & & & $\mathrm{Bd} \mathrm{Wt}$ & $\begin{array}{c}1096 \mathrm{M} \text { ( } 12 \% \text { decrease in body } \\
\text { weight) }\end{array}$ & \\
\hline 121 & $\begin{array}{l}\text { Rat } \\
\text { Sprague- } \\
\text { Dawley }\end{array}$ & $\begin{array}{l}4 \mathrm{wk} \\
5 \mathrm{~d} / \mathrm{wk} \\
6 \mathrm{hr} / \mathrm{d}\end{array}$ & Hepatic & $\begin{array}{l}600 \mathrm{M}(11 \% \text { increase in relative } \\
\text { liver weight) }\end{array}$ & $\begin{array}{l}\text { Toftgard et al. } 1981 \\
\text { mixed }\end{array}$ \\
\hline \multirow[t]{8}{*}{122} & Dog & $\begin{array}{l}13 \mathrm{wk} \\
5 \mathrm{~d} / \mathrm{wk} \\
6 \mathrm{hr} / \mathrm{d}\end{array}$ & Resp & $810 \mathrm{M}$ & $\begin{array}{l}\text { Carpenter et al. 1975a } \\
\text { mixed }\end{array}$ \\
\hline & & & Cardio & $810 \mathrm{M}$ & \\
\hline & & & Gastro & $810 \mathrm{M}$ & \\
\hline & & & Hemato & $810 \mathrm{M}$ & \\
\hline & & & Musc/skel & $810 \mathrm{M}$ & \\
\hline & & & Hepatic & $810 \mathrm{M}$ & \\
\hline & & & Renal & $810 \mathrm{M}$ & \\
\hline & & & Endocr & $810 \mathrm{M}$ & \\
\hline \multicolumn{6}{|c|}{ Neurological } \\
\hline 123 & Human & $\begin{array}{l}4 \mathrm{wk} \\
5 \mathrm{~d} / \mathrm{wk} \\
1-7.5 \mathrm{hr} / \mathrm{d}\end{array}$ & & $150 \mathrm{M}$ & $\begin{array}{l}\text { NIOSH } 1981 \\
\text { para }\end{array}$ \\
\hline
\end{tabular}


Table 3-1 Levels of Significant Exposure to Xylene - Inhalation

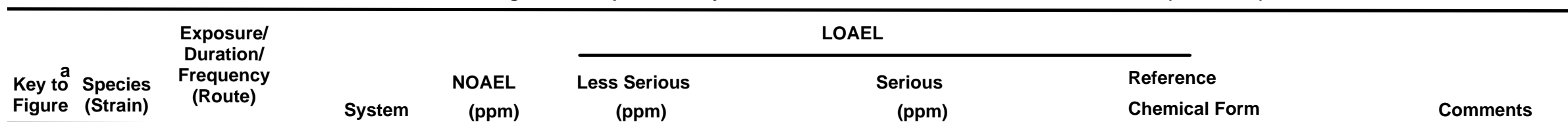

124 Monkey 90-127

$78 \mathrm{M}$

Jenkins et al. 1970

Squirrel

125 Monkey $6 \mathrm{wk}$

Squirrel $\quad 5 \mathrm{~d} / \mathrm{wk}$

$8 \mathrm{hr} / \mathrm{d}$

$1800 \mathrm{M}$

$13 \mathrm{wk}$

(Sprague- $\quad 6 \mathrm{~d} / \mathrm{wk}$

Dawley) $\quad 6 \mathrm{hr} / \mathrm{d}$

$1800 \mathrm{M}$

ortho

Jenkins et al. 1970

ortho

Gagnaire et al. 2001

meta

$128 \quad$ Rat 13 wk

(Sprague- $\quad 6 \mathrm{~d} / \mathrm{wk}$

Dawley) $6 \mathrm{hr} / \mathrm{d}$

$450 \mathrm{M}$

$900 \mathrm{M}$ (loss of cochlear hair cells without functiona hearing loss)

1800 M (extensive cochlear hair Gagnaire et al. 2001

cell loss; altered auditory para

evoked potentials;

persistent 35-42 dB

hearing loss)

Gagnaire et al. 2001

ortho

129 Rat 13 wk

(Sprague- $6 \mathrm{~d} / \mathrm{wk}$

Dawley) $\quad 6 \mathrm{hr} / \mathrm{d}$

$500 \mathrm{M}$

1000 M (13-19 dB hearing losses Gagnaire et al. 2006

in 2-16 kHz frequencies; mixed

in all rats, significant loss

of outer hair cells of

cochlea)
$20 \%$ o-xylene, $20 \%$

p-xylene, $40 \%$

m-xylene, $20 \%$

ethylbenzene. 
Table 3-1 Levels of Significant Exposure to Xylene - Inhalation

\begin{tabular}{|c|c|c|c|c|c|c|c|c|}
\hline \multirow[b]{2}{*}{$\begin{array}{l}\text { Key to } \\
\text { Figure }\end{array}$} & \multirow[b]{2}{*}{$\begin{array}{l}\text { Species } \\
\text { (Strain) }\end{array}$} & \multirow{2}{*}{$\begin{array}{l}\text { Exposurel } \\
\text { Durationl } \\
\text { Frequency } \\
\text { (Route) }\end{array}$} & \multirow[b]{2}{*}{ System } & \multirow[b]{2}{*}{$\begin{array}{r}\text { NOAEL } \\
\text { (ppm) } \\
\end{array}$} & \multicolumn{2}{|c|}{ LOAEL } & \multirow[b]{2}{*}{$\begin{array}{l}\text { Reference } \\
\text { Chemical Form }\end{array}$} & \multirow[b]{2}{*}{ Comments } \\
\hline & & & & & $\begin{array}{c}\text { Less Serious } \\
(\mathrm{ppm}) \\
\end{array}$ & $\begin{array}{r}\text { Serious } \\
(\mathrm{ppm})\end{array}$ & & \\
\hline 130 & $\begin{array}{l}\text { Rat } \\
\text { (Sprague- } \\
\text { Dawley) }\end{array}$ & $\begin{array}{l}13 \mathrm{wk} \\
6 \mathrm{~d} / \mathrm{wk} \\
6 \mathrm{hr} / \mathrm{d}\end{array}$ & & $500 \mathrm{M}$ & & $\begin{array}{c}1000 \mathrm{M} \text { (in all rats, significant } \\
\text { loss of hair cells in outer } \\
\text { rows of organ of Corti) }\end{array}$ & $\begin{array}{l}\text { Gagnaire et al. } 2006 \\
\text { mixed }\end{array}$ & $\begin{array}{l}30 \% \text { o-xylene, } 10 \% \\
\text { p-xylene, } 50 \% \\
\text { m-xylene, } 10 \% \\
\text { ethylbenzene. }\end{array}$ \\
\hline 131 & $\begin{array}{l}\text { Rat } \\
\text { (Wistar) }\end{array}$ & $\begin{array}{l}4 \mathrm{wk} \\
5 \mathrm{~d} / \mathrm{wk} \\
6 \mathrm{hr} / \mathrm{d}\end{array}$ & & & $\begin{array}{l}100 \mathrm{M} \text { (impaired passive and } \\
\text { active avoidance } \\
\text { learning) }\end{array}$ & & $\begin{array}{l}\text { Gralewicz and Wiaderna } 2001 \\
\text { meta }\end{array}$ & \\
\hline 132 & $\begin{array}{l}\text { Rat } \\
\text { (Wistar) }\end{array}$ & $\begin{array}{l}3 \mathrm{mo} \\
5 \mathrm{~d} / \mathrm{wk} \\
6 \mathrm{hr} / \mathrm{d}\end{array}$ & & & $\begin{array}{c}100 \mathrm{M} \text { (learning deficit in radial } \\
\text { arm maze test) }\end{array}$ & & $\begin{array}{l}\text { Gralewicz et al. } 1995 \\
\text { meta }\end{array}$ & \\
\hline 133 & $\begin{array}{l}\text { Rat } \\
\text { (Sprague- } \\
\text { Dawley) }\end{array}$ & $\begin{array}{l}4 \mathrm{wk} \\
5 \mathrm{~d} / \mathrm{wk} \\
6 \mathrm{hr} / \mathrm{d}\end{array}$ & & $80 \mathrm{M}$ & & & $\begin{array}{l}\text { Hillefors-Berglund et al. } 1995 \\
\text { mixed }\end{array}$ & \\
\hline 134 & $\begin{array}{l}\text { Rat } \\
\text { Albino }\end{array}$ & $\begin{array}{l}30 \mathrm{~d} \\
24 \mathrm{hr} / \mathrm{d}\end{array}$ & & & $\begin{array}{l}800 \mathrm{M} \text { (decreased acetylcholine } \\
\text { in striatum, increased } \\
\text { glutamine in midbrain, } \\
\text { and norepinephrine in } \\
\text { hypothalmus) }\end{array}$ & & $\begin{array}{l}\text { Honma et al. } 1983 \\
\text { mixed }\end{array}$ & \\
\hline 135 & $\begin{array}{l}\text { Rat } \\
\text { Wistar }\end{array}$ & $\begin{array}{l}3 \mathrm{mo} \\
5 \mathrm{~d} / \mathrm{wk} \\
6 \mathrm{hr} / \mathrm{d}\end{array}$ & & & $\begin{array}{l}1000 \mathrm{M} \text { (decreased rotarod } \\
\text { performance and } \\
\text { spontaneous motor } \\
\text { activity) }\end{array}$ & & $\begin{array}{l}\text { Korsak et al. } 1992 \\
\text { meta }\end{array}$ & \\
\hline
\end{tabular}


Table 3-1 Levels of Significant Exposure to Xylene - Inhalation

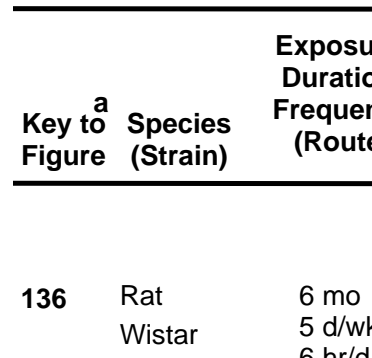

$$
6 \mathrm{hr} / \mathrm{d}
$$

137 Rat $3 \mathrm{mo}$

(Wistar) $\quad 5 \mathrm{~d} / \mathrm{wk}$

138 Rat $61 \mathrm{~d}$

Sprague- $\quad 7 \mathrm{~d} / \mathrm{wk}$

Dawley $\quad 8 \mathrm{hr} / \mathrm{d}$

139 Rat $6 \mathrm{wk}$

Fischer- $3447 \mathrm{~d} / \mathrm{wk}$

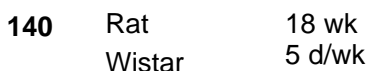

$\begin{array}{ll}\text { Wistar } & 5 \mathrm{~d} / \mathrm{wk} \\ & 6 \mathrm{hr} / \mathrm{d}\end{array}$

141 Rat 18 wk

$\begin{array}{ll}\text { Wistar } & 5 \mathrm{~d} / \mathrm{wk} \\ & 6 \mathrm{hr} / \mathrm{d}\end{array}$

142 Mouse $7 \mathrm{wk}$ NMRI- BOM $5 \mathrm{~d} / \mathrm{wk}$
$100 \mathrm{M}$ (decreased rotarod

performance and

spontaneous motor

activity)

$50^{\mathrm{C}} \mathrm{M}$ (decreased latency of

paw-lick response)

$1009 \mathrm{M}$ (reversible decrease in auditory brainstem response)

$800 \mathrm{M}$ (hearing loss)

$300 \mathrm{M}$ (decreased membrane lipids in axon membranes)

$300 \mathrm{M}$ (transient decreases in preening behavior)

$1600 \mathrm{~F}$ (decreased alpha-adrenergic binding in brain)
Reference

Chemical Form

Comments
Korsak et al. 1992

meta

Korsak et al. 1994

meta

Nylen and Hagman 1994

mixed

Pryor et al. 1987

mixed

Savolainen and Seppalainen

1979

mixed

Savolainen et al. 1979a

mixed

Rank 1985

meta 
Table 3-1 Levels of Significant Exposure to Xylene - Inhalation

(continued)

\begin{tabular}{lll}
\hline $\begin{array}{l}\text { Key to } \\
\text { Figure }\end{array}$ & $\begin{array}{r}\text { Species } \\
\text { (Strain) }\end{array}$ & $\begin{array}{r}\text { Exposure } \\
\text { Duration } \\
\text { Frequency } \\
\text { (Route) }\end{array}$ \\
\hline & & \\
143 & Dog & $90-127$ \\
& Beagle & $24 \mathrm{hr} / \mathrm{d}$ \\
& & \\
144 & Dog & $6 \mathrm{wk}$ \\
& Beagle & $5 \mathrm{~d} / \mathrm{wk}$ \\
& & $8 \mathrm{hr} / \mathrm{d}$ \\
& & \\
145 & Gerbil & $3 \mathrm{mo}$ \\
& Mongolian & $30 \mathrm{~d} / \mathrm{mo}$ \\
& & $24 \mathrm{hr} / \mathrm{d}$
\end{tabular}

LOAEL

Reference

Chemical Form

Comments

System $\quad \begin{array}{r}\text { NOAEL } \\ (\mathrm{ppm})\end{array}$

(ppm)

(ppm)

Jenkins et al. 1970

ortho

$780 \mathrm{M}$ (tremor)

Jenkins et al. 1970

ortho

160 (regional increases in

DNA and astro-glial

proteins)

Rosengren et al. 1986

mixed

Nylen et al. 1989

mixed

Bio/dynamics 1983

mixed

Developmental

147 Rat

$166 \mathrm{~d}$

$6 \mathrm{hr} / \mathrm{d}$ 
Table 3-1 Levels of Significant Exposure to Xylene - Inhalation

(continued)

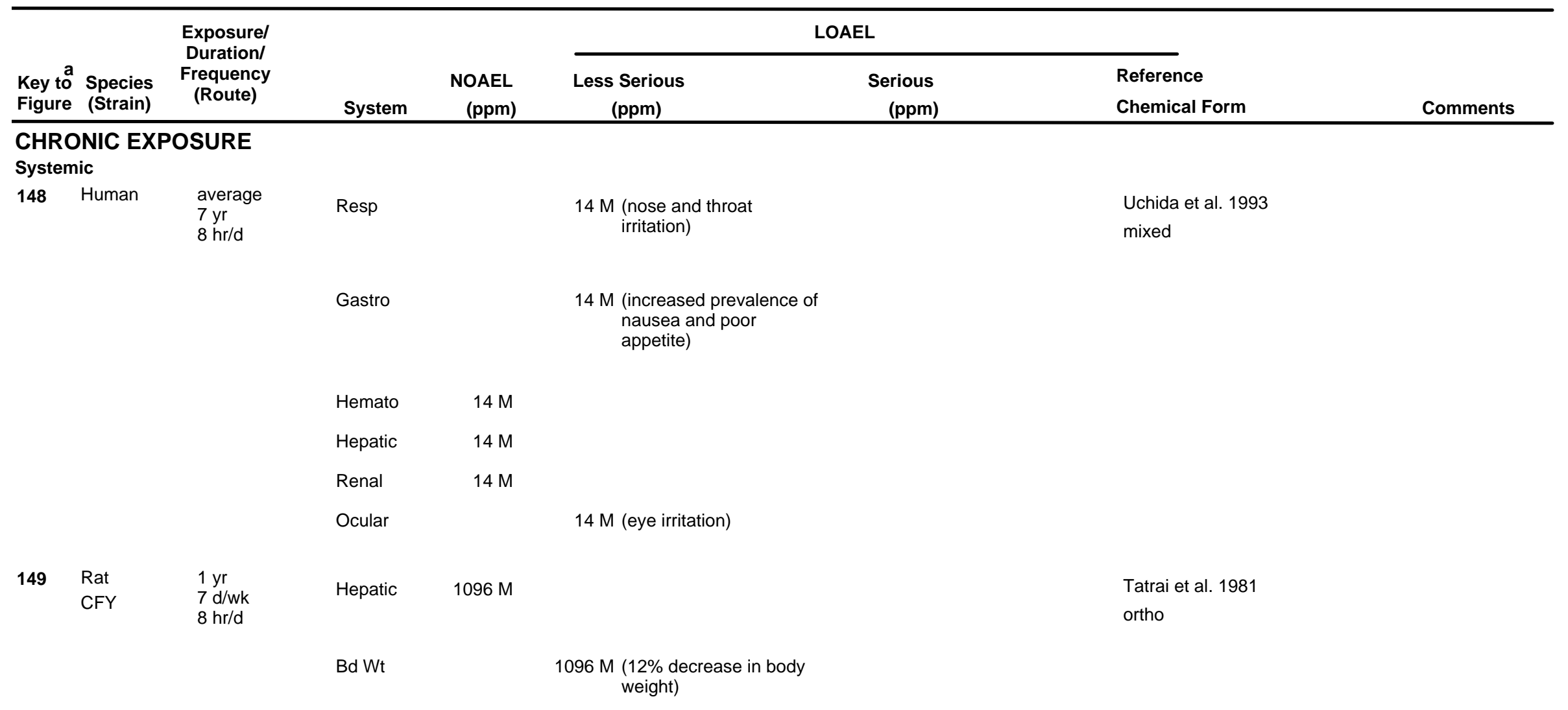


Table 3-1 Levels of Significant Exposure to Xylene - Inhalation

(continued)

\begin{tabular}{|c|c|c|c|c|c|c|c|}
\hline \multirow[b]{2}{*}{$\begin{array}{l}\text { Key to } \\
\text { Figure }\end{array}$} & \multirow[b]{2}{*}{$\begin{array}{l}\text { Species } \\
\text { (Strain) }\end{array}$} & \multirow{2}{*}{$\begin{array}{l}\text { Exposurel } \\
\text { Durationl } \\
\text { Frequency } \\
\text { (Route) }\end{array}$} & \multirow[b]{2}{*}{ System } & \multirow[b]{2}{*}{$\begin{array}{r}\text { NOAEL } \\
(p p m) \\
\end{array}$} & \multicolumn{2}{|c|}{ LOAEL } & \multirow[b]{2}{*}{$\begin{array}{l}\text { Reference } \\
\text { Chemical Form }\end{array}$} \\
\hline & & & & & $\begin{array}{c}\text { Less Serious } \\
(\mathrm{ppm})\end{array}$ & $\begin{array}{r}\text { Serious } \\
\text { (ppm) }\end{array}$ & \\
\hline \multicolumn{8}{|c|}{ Neurological } \\
\hline 150 & Human & $\begin{array}{l}\text { average } \\
7 \mathrm{yr} \\
8 \mathrm{hr} / \mathrm{d}\end{array}$ & \multicolumn{4}{|c|}{$\begin{array}{l}\text { (increased prevalence of } \\
\text { anxiety, forgetfulness, } \\
\text { inability to concentrate } \\
\text { and other subjective } \\
\text { symptoms) }\end{array}$} & $\begin{array}{l}\text { Uchida et al. } 1993 \\
\text { mixed }\end{array}$ \\
\hline
\end{tabular}

a The number corresponds to the entries in Figure 3-1.

b Used to derive an acute-duration minimal risk level (MRL) for mixed xylenes based on a minimal LOAEL of 50 ppm for m-xylene in humans; concentration divided by an uncertainty factor of 30 ( 3 for use of a minimal LOAEL and 10 for human variability).

c Used to derive an intermediate-duration minimal risk level (MRL) for mixed xylenes based on a minimal LOAEL of 50 ppm for m-xylene in rats; this LOAEL was converted to a human equivalent concentration using a dosimetric adjustment (EPA 1994). The human equivalent LOAEL of 50 ppm was divided by an uncertainty factor of 90 (3 for use of a minimal LOAEL, 3 for extrapolation from animals to humans with dosimetric adjustment, and 10 for human variability).

d Used to derive a chronic-duration minimal risk level (MRL) for mixed xylenes based on a LOAEL of 14 ppm (geometric mean) for mixed xylenes in humans; concentration divided by an uncertainty factor of 100 (10 for use of a LOAEL and 10 for human variability) and a modifying factor of 3 to account for the lack of supporting studies evaluating the chronic neurotoxicity of xylene.

$\mathrm{Bd} \mathrm{Wt}=$ body weight; Cardio = cardiovascular; $\mathrm{d}=$ day $(\mathrm{s}) ; \mathrm{dB}=$ decibel; $\mathrm{EC} 50=$ effective concentration; Endocr = endocrine; $\mathrm{F}=\mathrm{Female} ; \mathrm{Gastro}=$ gastrointestinal; $\mathrm{Gd}=$ gestational day, $50 \%$; hemato = hematological; $\mathrm{hr}=$ hour(s); $\mathrm{KHz}=$ kilohertz; LC50 = lethal concentration, $50 \% ; \mathrm{LOAEL}=$ lowest-observed-adverse-effect level; $\mathrm{M}=$ male; min = minute(s); $\mathrm{mo}=$ month(s); Musc/skel = musculoskeletal; NOAEL = no-observed-adverse-effect level; Resp = respiratory; $x=\operatorname{time}(s) ; w k=$ week(s); $y r=y e a r(s)$ 
Figure 3-1 Levels of Significant Exposure to Xylene - Inhalation

Acute ( $\leq 14$ days)

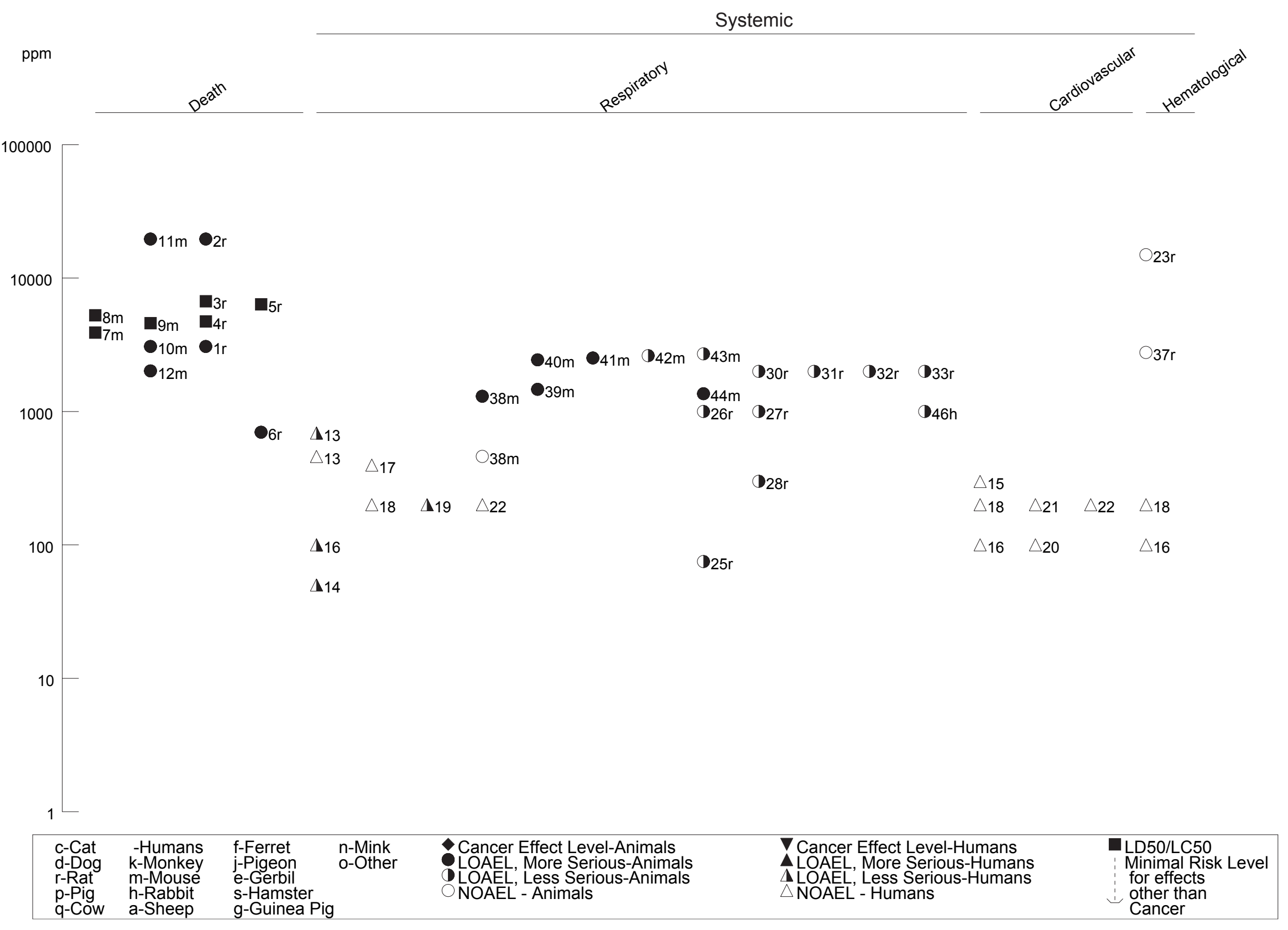


Figure 3-1 Levels of Significant Exposure to Xylene - Inhalation (Continued)

Acute ( $\leq 14$ days)

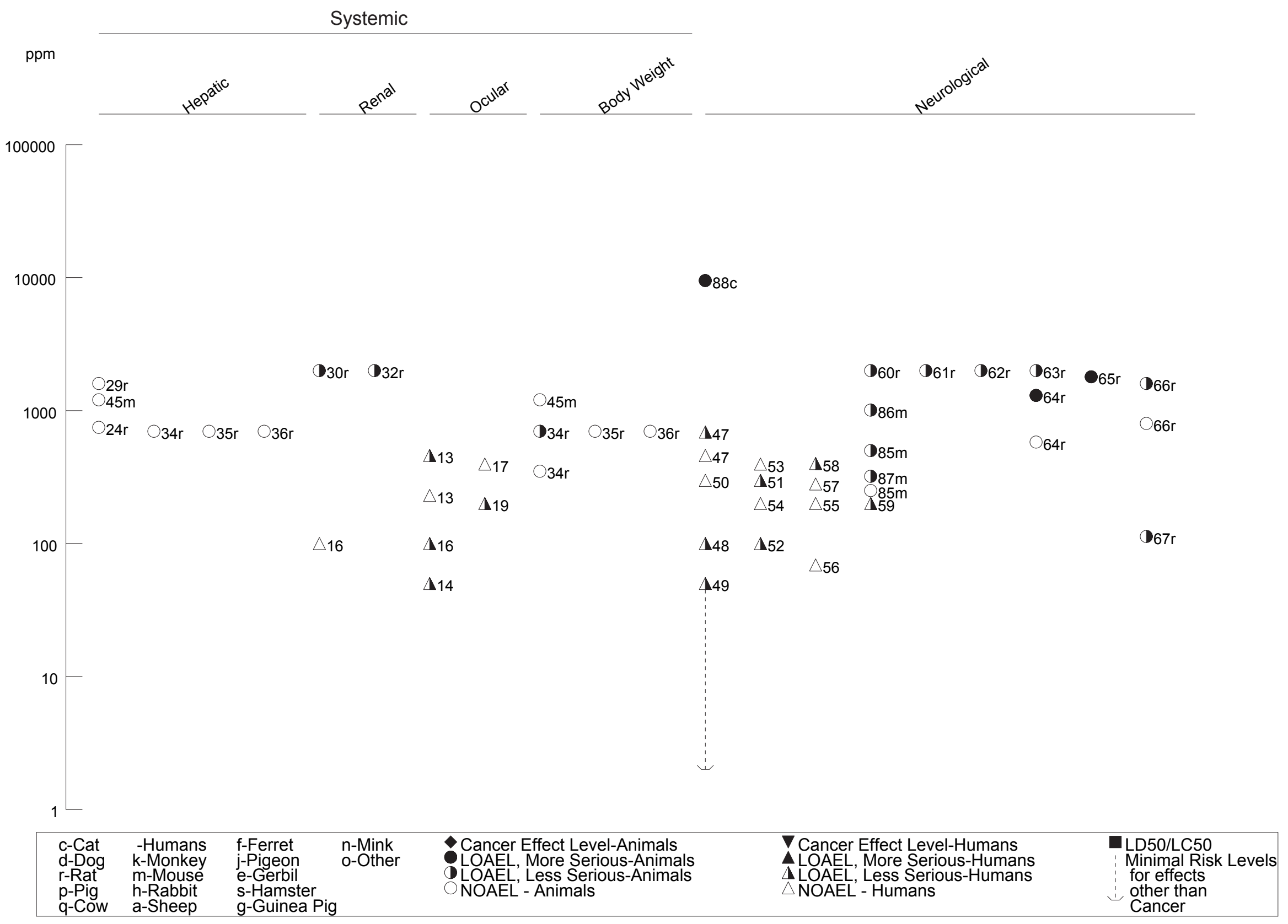


Figure 3-1 Levels of Significant Exposure to Xylene - Inhalation (Continued)

Acute ( $\leq 14$ days)

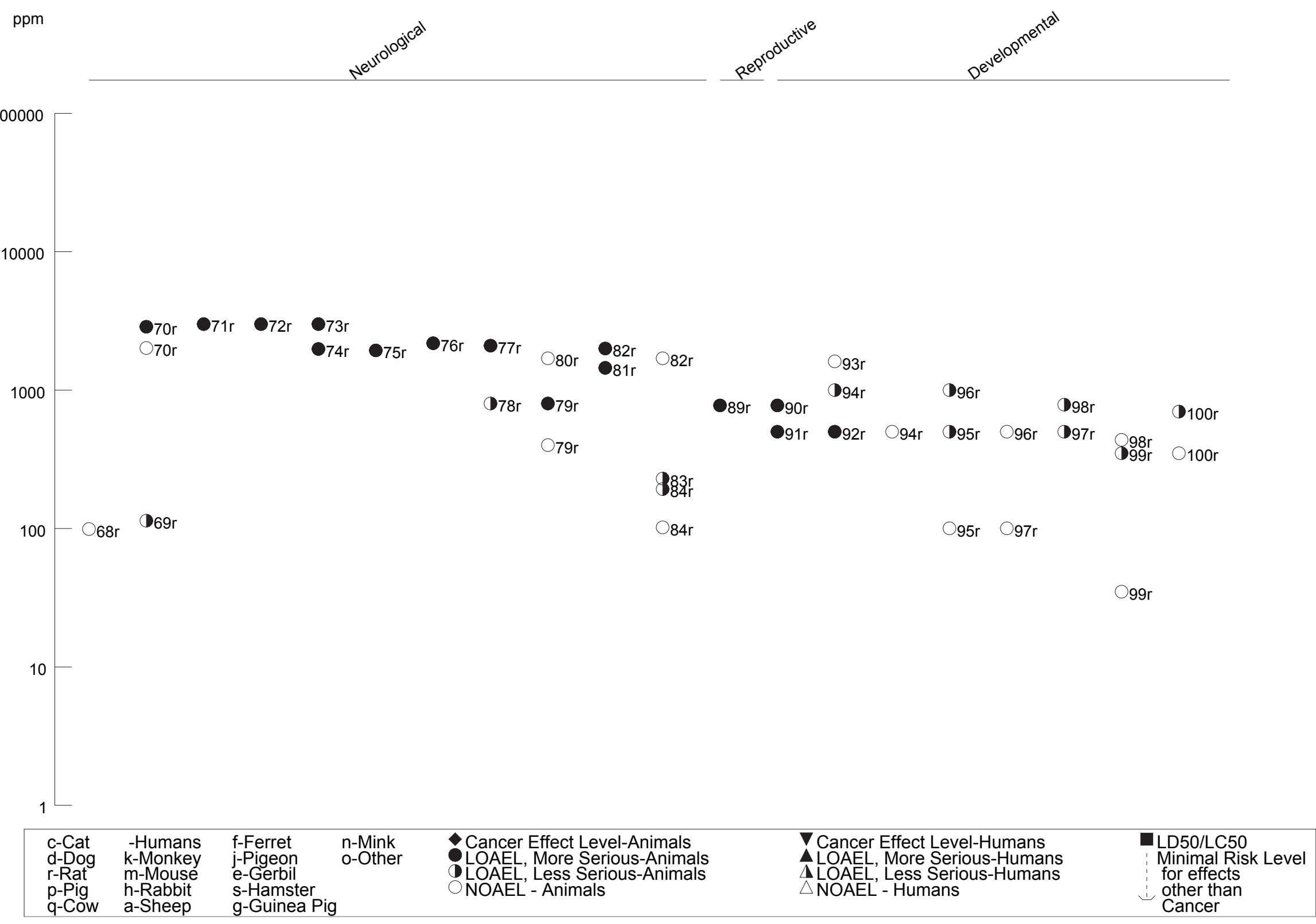


Figure 3-1 Levels of Significant Exposure to Xylene - Inhalation (Continued)

Acute $(\leq 14$ days $)$
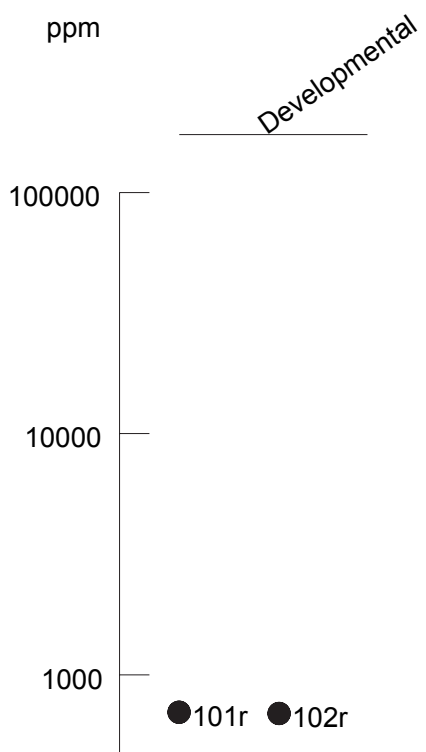

O101r

100

$$
\text { - }
$$$$
10-
$$$$
\leftarrow
$$

\begin{tabular}{|c|c|c|c|c|c|c|}
\hline $\begin{array}{l}\text { c-Cat } \\
\text { d-Dog } \\
\text { r-Rat } \\
\text { p-Pig } \\
\text { q-Cow }\end{array}$ & $\begin{array}{l}\text {-Humans } \\
\text { k-Monkey } \\
\text { m-Mouse } \\
\text { h-Rabbit } \\
\text { a-Sheep }\end{array}$ & $\begin{array}{l}\text { f-Ferret } \\
\text { j-Pigeon } \\
\text { e-Gerbil } \\
\text { s-Hamster } \\
\text { g-Guinea Pi }\end{array}$ & $\begin{array}{l}\text { n-Mink } \\
\text { o-Other }\end{array}$ & $\begin{array}{l}\text { Cancer Effect Level-Animals } \\
\text { LOAEL, More Serious-Animals } \\
\text { LOAEL, Less Serious-Animals } \\
\text { NOAEL - Animals }\end{array}$ & $\begin{array}{l}\nabla \text { Cancer Effect Level-Humans } \\
\Delta \text { LOAEL, More Serious-Humans } \\
\Delta \text { LOAEL, Less Serious-Humans } \\
\triangle \text { NOAEL - Humans }\end{array}$ & $\begin{array}{l}\text { LD50/LC50 } \\
\text { Minimal Risk Level } \\
\text { for effects } \\
\text { other than } \\
\text { Cancer }\end{array}$ \\
\hline
\end{tabular}


Figure 3-1 Levels of Significant Exposure to Xylene - Inhalation (Continued)

Intermediate (15-364 days)

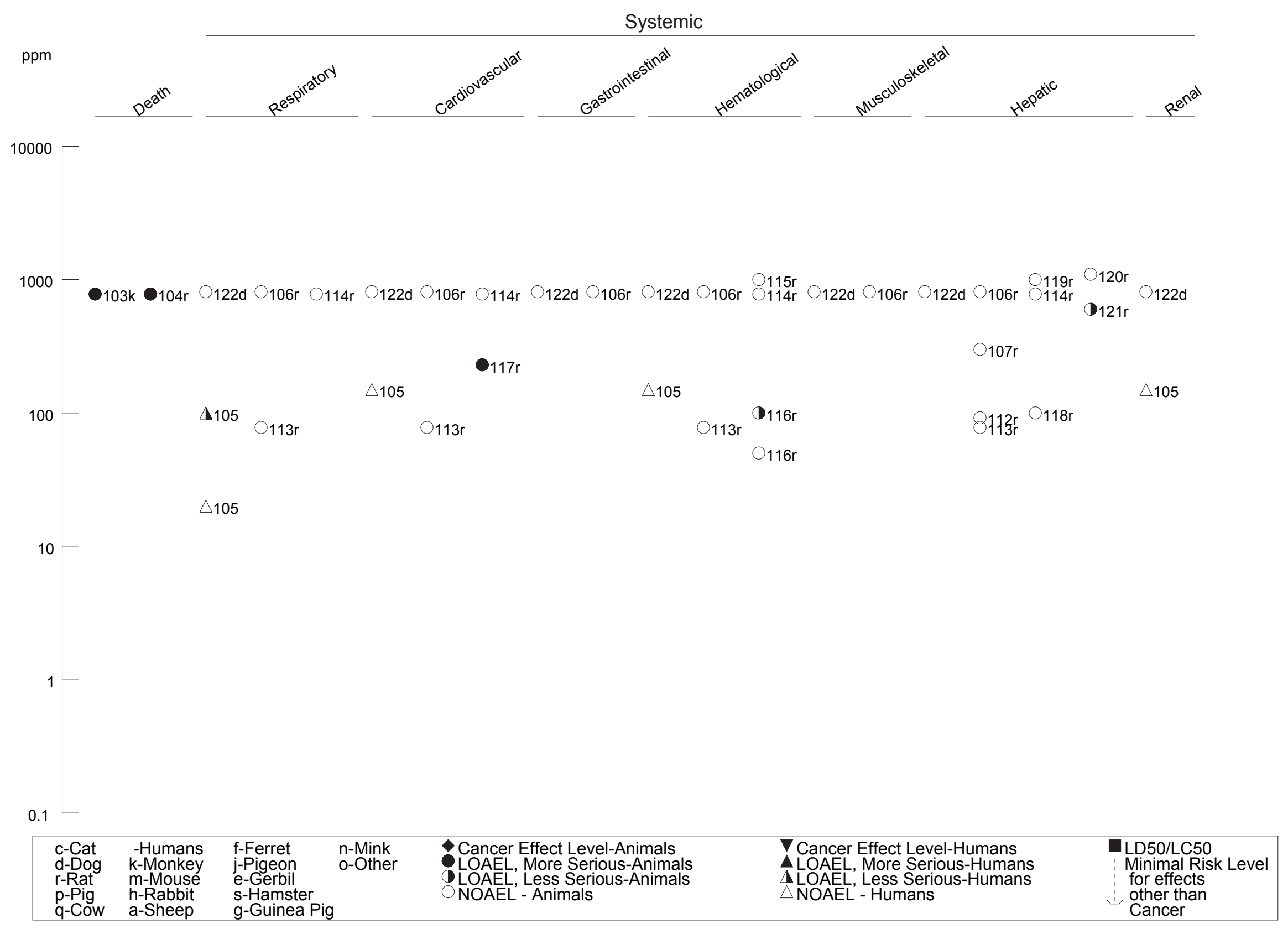


Figure 3-1 Levels of Significant Exposure to Xylene - Inhalation (Continued)

Intermediate (15-364 days)

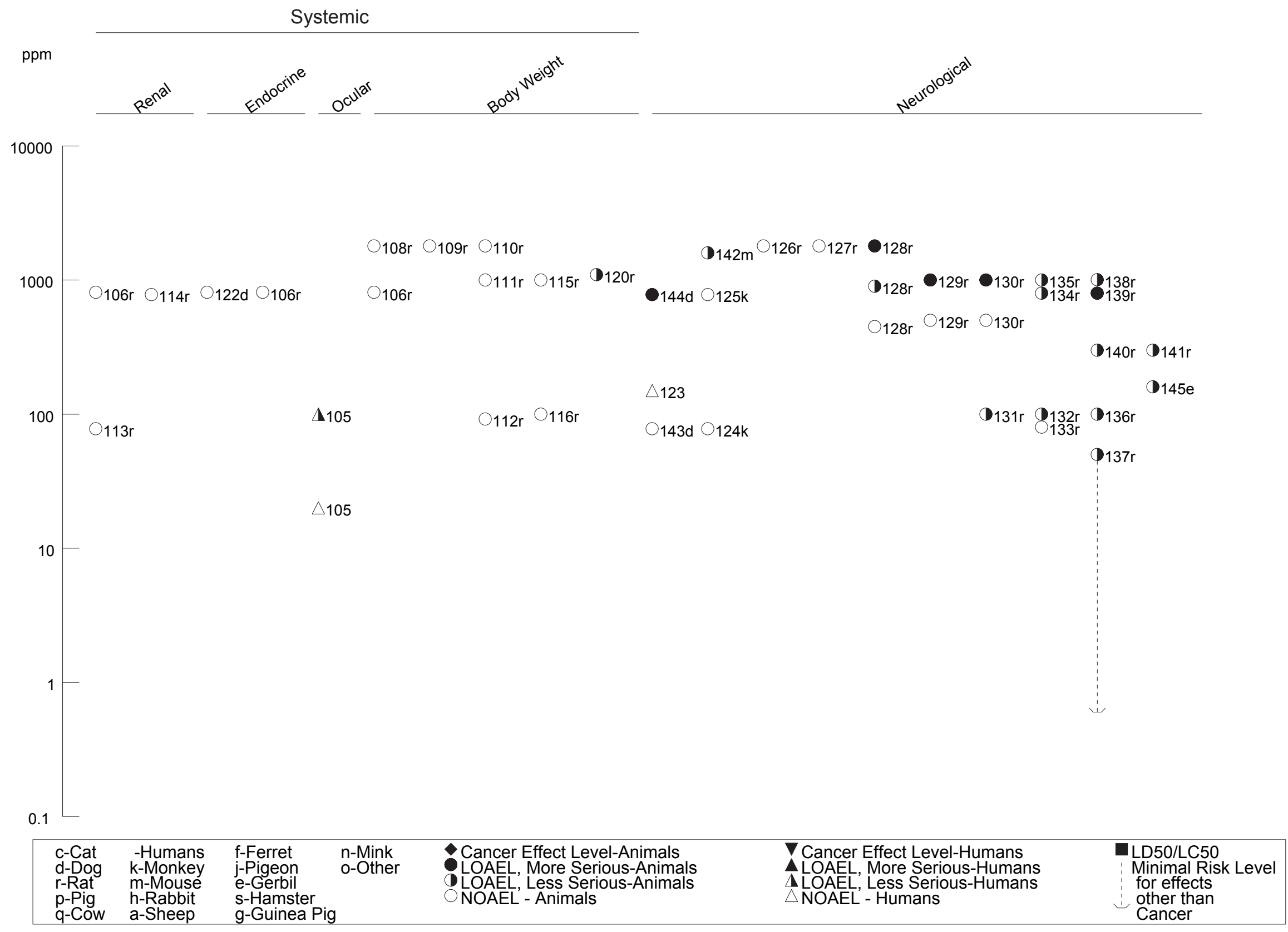


Figure 3-1 Levels of Significant Exposure to Xylene - Inhalation (Continued)

Intermediate (15-364 days)

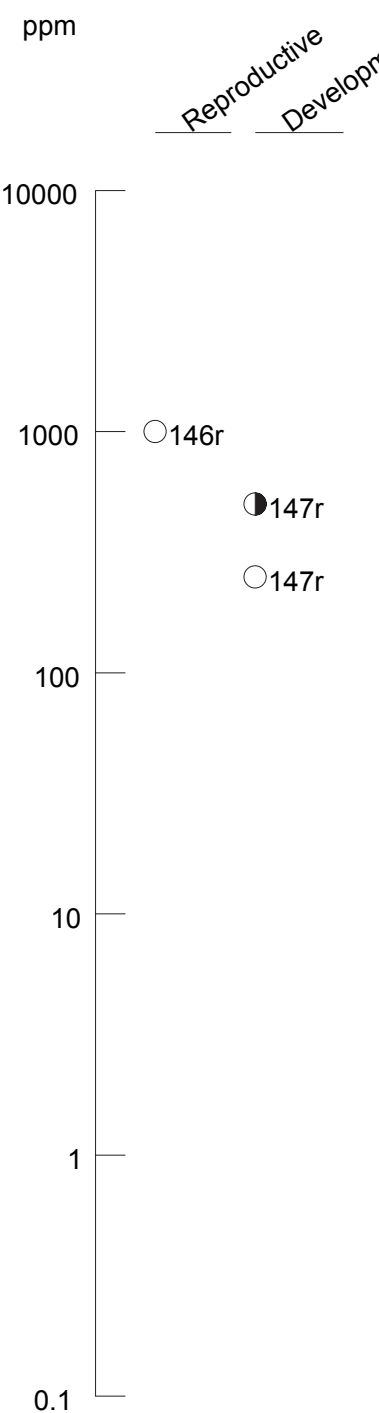

\begin{tabular}{|c|c|c|c|c|c|c|}
\hline $\begin{array}{l}\text { c-Cat } \\
\text { d-Dog } \\
\text { r-Rat } \\
\text { p-Pig } \\
\text { q-Cow }\end{array}$ & $\begin{array}{l}\text {-Humans } \\
\text { k-Monkey } \\
\text { m-Mouse } \\
\text { h-Rabbit } \\
\text { a-Sheep }\end{array}$ & $\begin{array}{l}\text { f-Ferret } \\
\text { j-Pigeon } \\
\text { e-Gerbil } \\
\text { s-Hamster } \\
\text { g-Guinea Pig }\end{array}$ & $\begin{array}{l}\text { n-Mink } \\
\text { o-Other }\end{array}$ & $\begin{array}{l}\text { Cancer Effect Level-Animals } \\
\text { LOAEL, More Serious-Animals } \\
\text { LOAEL, Less Serious-Animals } \\
\text { NOAEL - Animals }\end{array}$ & $\begin{array}{l}\nabla \text { Cancer Effect Level-Humans } \\
\Delta \text { LOAEL, More Serious-Humans } \\
\Delta \text { LOAEL, Less Serious-Humans } \\
\triangle \text { NOAEL - Humans }\end{array}$ & $\begin{array}{l}\text { LD50/LC50 } \\
\text { Minimal Risk Level } \\
\text { for effects } \\
\text { other than } \\
\text { Cancer }\end{array}$ \\
\hline
\end{tabular}


Figure 3-1 Levels of Significant Exposure to Xylene - Inhalation (Continued) Chronic ( $\geq 365$ days)

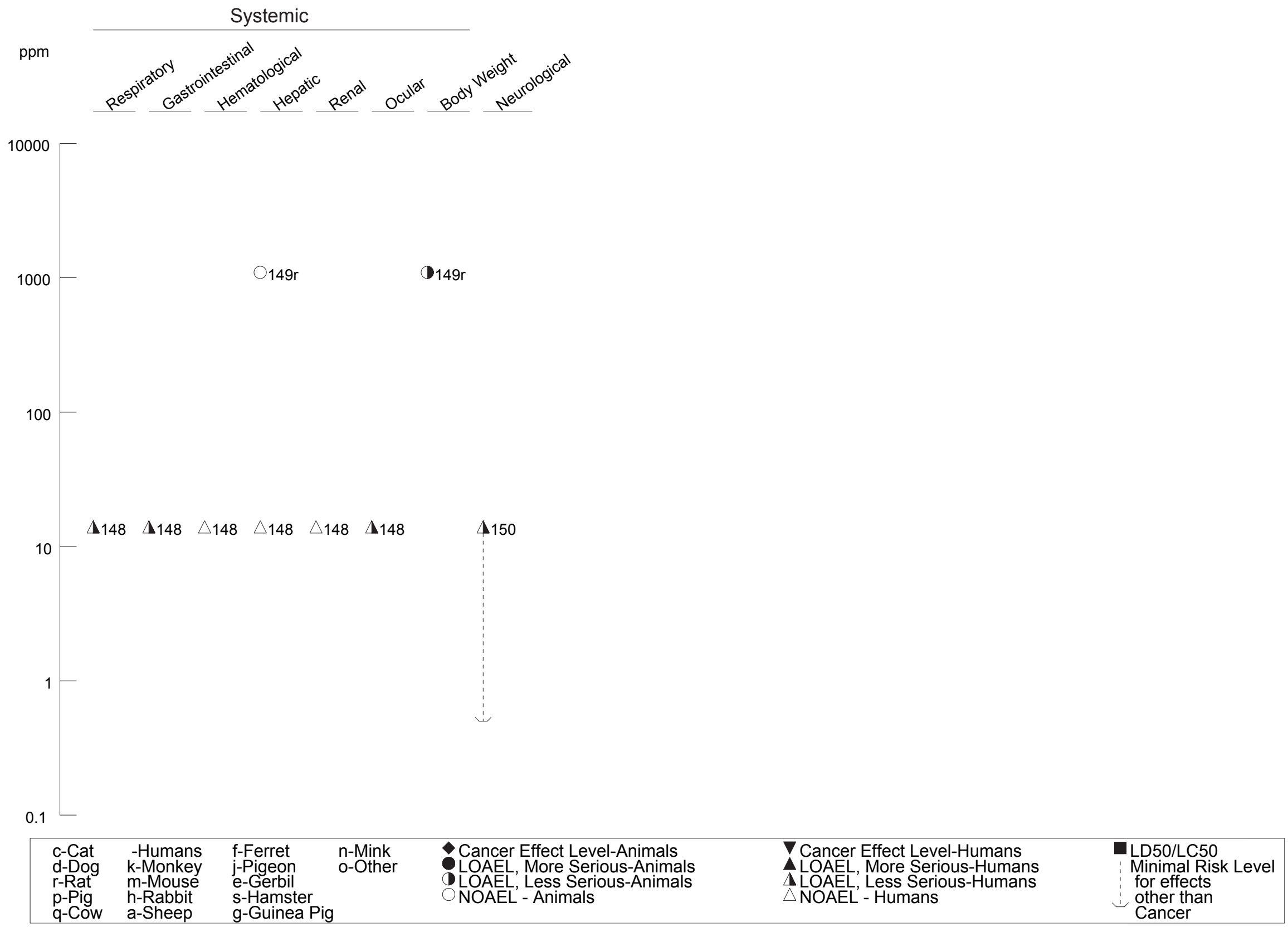


30 minutes (Hastings et al. 1986). Slight, but statistically significant increases in the average rating for subjective symptoms of respiratory effects were observed following exposure to $m$-xylene at $50 \mathrm{ppm}$ (Ernstgard et al. 2002) for discomfort in the nose in both sexes after 60 and 118 minutes, in discomfort in the throat or airways in women after 60 minutes, and in breathing difficulty in men at 118 minutes and women at both timepoints. Small but statistically significant changes in objective tests of pulmonary function were reported in women, but not men, measured 3 hours after the end of the 2-hour exposure: decreased forced vital capacity $(\mathrm{FVC})$, increased forced expiratory flow at $75 \% \mathrm{FVC}\left(\mathrm{FEF}_{75}\right)$, and increased ratio of forced expiratory volume in 1 minute $\left(\mathrm{FEV}_{1}\right)$ to forced vital capacity $\left(\mathrm{FEV}_{1} / \mathrm{FVC}\right)$. This study is the basis for the acute-duration inhalation MRL for which respiratory and neurologic toxicity are the critical effects. Chest x-rays obtained from volunteers exposed to a time-weightedaverage (TWA) concentration of $200 \mathrm{ppm} m$-xylene for 3.67 hours/day for 4 days showed no adverse effects on the lungs (Seppalainen et al. 1989). Also, no effects on pulmonary ventilation volume were observed in volunteers exposed to $150 \mathrm{ppm} p$-xylene for 5 days/week in a 4-week trial (NIOSH 1981).

At much higher concentrations, however, the lung may be adversely affected. An autopsy revealed that exposure to an estimated 10,000 ppm of xylene produced severe lung congestion with focal intra-alveolar hemorrhage and pulmonary edema in one worker who died following exposure to xylene fumes for several hours while painting (Morley et al. 1970). Another worker exposed in the same incident exhibited patchy diffuse opacities in radiograms and moist rales in both lungs; a third exposed worker showed no evidence of lung effects. Case reports indicate that acute-duration inhalation exposure to mixed xylene and $p$-xylene has been associated with irritation of the nose and throat (Carpenter et al. 1975a; Klaucke et al. 1982; Nelson et al. 1943; Nersesian et al. 1985; NIOSH 1981). A worker at a chemical company who was exposed to heated xylene from a pressurized hose experienced throat pain and dyspnea (Narvaez and Song 2003).

Chronic occupational exposure of workers to an unspecified concentration of vapors of mixed xylene has also been associated with labored breathing and impaired pulmonary function (Hipolito 1980; Roberts et al. 1988). A significant $(\mathrm{p}<0.01)$ increase in the prevalence of nose and throat irritation was reported by workers chronically exposed to mixed xylene vapors at a geometric mean TWA concentration of $14 \mathrm{ppm}$ (Uchida et al. 1993). This study is the basis for the chronic-duration inhalation MRL for which respiratory and neurological toxicity are the critical effects.

Adverse respiratory effects noted in rats, mice, and guinea pigs following acute and intermediate inhalation exposure to xylene are similar to those observed in humans. They include decreased 
respiration, labored breathing, irritation of the respiratory tract, pulmonary edema, pulmonary hemorrhage, and pulmonary inflammation (Carpenter et al. 1975a; De Ceaurriz et al. 1981; Furnas and Hine 1958; Korsak et al. 1990). Exposure to concentrations of 2,440 ppm mixed xylene for 6 minutes (Korsak et al. 1988) to 1,467 ppm o-xylene for 5 minutes (De Ceaurriz et al. 1981), or to 1,361 ppm $m$-xylene for 6 minutes (Korsak et al. 1993) produced a 50\% decrease in respiratory rate in mice. Comparison of the individual xylene isomers showed that the irritant effects of $m$ - and $o$-xylene as quantified by measurements of respiratory rate in mice are more pronounced than those of $p$-xylene, with $o$-xylene having the most prolonged effect (Korsak et al. 1990). In rats that died as a result of exposure to 9,900 ppm mixed xylene for 4 hours, atelectasis, hemorrhage, and edema of the lungs were observed (Carpenter et al. 1975a). Biochemical changes detected in the lungs after acute-duration intermittent exposure include transiently decreased lung surfactant levels at $300 \mathrm{ppm} p$-xylene (Silverman and Schatz 1991) and decreased pulmonary microsomal enzyme activities at 2,000 ppm mixed xylene, 75-2,000 ppm $m$-xylene, 2,000 ppm o-xylene, or 1,000 or 3,400 ppm p-xylene (Day et al. 1992; Elovaara et al. 1980, 1987; Patel et al. 1978; Silverman and Schatz 1991; Toftgard and Nilsen 1982). The LOAEL of 75 ppm for $m$-xylene was based on decreased P-450 and 7-ethoxycoumarin $O$-deethylase activities noted in the lungs of rats exposed for 24 hours (Elovaara et al. 1987). The decrease in pulmonary microsomal activity by selective inactivation of enzymes can result from damage to lung tissue caused by the toxic metabolite of xylene, a methylbenzaldehyde (Carlone and Fouts 1974; Patel et al. 1978; Smith et al. 1982); the selective inactivation of enzymes may also result in anoxia.

No effect on absolute or relative lung weights was observed in male rats intermittently exposed to $m$-xylene at concentrations as high as 100 ppm for 13 weeks (Korsak et al. 1994). No histopathological changes in the lungs were evident in rats, dogs, guinea pigs, or monkeys following intermediate exposure for 90-127 days to concentrations of 78 ppm o-xylene on a continuous basis (Jenkins et al. 1970) or 13 weeks to $810 \mathrm{ppm}$ mixed or 6 weeks to $780 \mathrm{ppm} o$-xylene, 5 weeks to $300 \mathrm{ppm} m$-xylene, or for 5 days to $300 \mathrm{ppm}$ p-xylene on an intermittent basis (Carpenter et al. 1975a; Elovaara et al. 1987; Jenkins et al. 1970; Silverman and Schatz 1991).

No animal studies were located that evaluated the respiratory effects of mixed xylene or single xylene isomers following chronic inhalation exposure.

An acute-duration inhalation MRL of 2 ppm was calculated for mixed xylenes based on a LOAEL for neurological and respiratory effects in human subjects exposed to $50 \mathrm{ppm} \mathrm{m}$-xylene for 2 hours (Ernstgard et al. 2002; see footnote in Table 3-1). A chronic-duration inhalation MRL of 0.05 ppm was 
calculated for mixed xylenes based on a LOAEL of $14 \mathrm{ppm}$ for subjective neurological and respiratory symptoms in workers exposed to mixed xylene 8 hours/day, 5 days/week for an average of 7 years (Uchida et al. 1993; see footnote in Table 3-1).

Cardiovascular Effects. Limited human data are available regarding the cardiovascular effects of xylene following inhalation exposure. Although tachycardia was reported by one of nine persons exposed to unidentified levels of xylene as a result of its use in a sealant in a heating duct, no effects on heart rate, blood pressure, or cardiac function were noted in humans exposed to $\leq 299 \mathrm{ppm}$ mixed xylene for an acute duration (70 minutes to 7 hours) (Gamberale et al. 1978), 200 ppm m-xylene (Ogata et al. 1970; Seppalainen et al. 1989), or 150 ppm p-xylene (NIOSH 1981; Ogata et al. 1970). Furthermore, two survivors exposed to an estimated 10,000 ppm xylene in an industrial accident had normal pulse, blood pressure, and heart sounds upon hospitalization. Chronic occupational exposure to xylene along with other chemical agents has resulted in complaints of heart palpitations, chest pain, and an abnormal electrocardiogram (ECG) (Hipolito 1980; Kilburn et al. 1985). However, the contribution of other chemical exposures to these effects cannot be eliminated.

Data regarding cardiovascular effects in animals are limited. Morphological changes in coronary microvessels (increased wall thickness) were noted in rats exposed to $230 \mathrm{ppm}$ xylene (unspecified composition) for 4 weeks (Morvai et al. 1987). Other effects seen in rats inhaling unspecified (lethal) concentrations of xylene of unknown composition included ventricular repolarization disturbances and occasional arrhythmias; the toxicity of unknown components was not reported (Morvai et al. 1976). However, no adverse effects on the heart were observed upon histopathological examination of rats and dogs exposed intermittently for 10-13 weeks to mixed xylene at concentrations as high as $810 \mathrm{ppm}$ (Carpenter et al. 1975a) or rats, guinea pigs, dogs, or monkeys exposed to $o$-xylene at $78 \mathrm{ppm}$ on a continuous basis for 90-127 days or 780 ppm on an intermittent basis for 6 weeks (Jenkins et al. 1970). No effect on absolute or relative heart weights was observed in male rats intermittently exposed to $m$-xylene at concentrations as high as 100 ppm for 13 weeks (Korsak et al. 1994). No information was located regarding cardiovascular effects in animals after chronic exposure to mixed xylene or its individual isomers.

Gastrointestinal Effects. Symptoms of nausea, vomiting, and gastric discomfort have been noted in workers exposed to xylene vapors (concentration unspecified) (Goldie 1960; Hipolito 1980; Klaucke et al. 1982; Nersesian et al. 1985; Uchida et al. 1993). These symptoms subsided after cessation of the xylene exposure. Anorexia and vomiting were also observed in a patient admitted to the hospital after sniffing 
paint containing xylene and other unknown substances over a 2-week period in an effort to become intoxicated (Martinez et al. 1989) and nausea was more frequently reported in males acutely exposed to m-xylene for 2 hours, as compared to controls (Ernstgard et al. 2002).

Limited data were located regarding gastrointestinal effects in animals. No lesions were observed in the gastrointestinal tract of rats and dogs exposed to concentrations as high as $810 \mathrm{ppm}$ mixed xylene for 13 weeks (Carpenter et al. 1975a). No studies were located regarding gastrointestinal effects in animals after acute or chronic inhalation exposure to mixed xylene or the isomers of xylene.

Hematological Effects. Human data are limited regarding the effects of xylene on the blood. Female volunteers had normal blood counts after exposure to $100 \mathrm{ppm} p$-xylene for $1-7.5$ hours/day for 5 days (NIOSH 1981). Hemoglobin content of the blood was unaffected in two workers exposed to an estimated 10,000 ppm of mixed xylene in an industrial accident (Morley et al. 1970). Decreased white blood cell counts were observed in two women with chronic occupational exposure to xylene (Hipolito 1980; Moszczynski and Lisiewicz 1983, 1984a), but exposure to other chemicals cannot be ruled out as an alternative explanation for the effects observed.

Previously, chronic occupational exposure to xylene by inhalation was thought to be associated with a variety of hematological effects (NIOSH 1975). However, exposure in all cases was to solvent mixtures known or suspected to contain benzene as well. Because benzene is an agent known to cause leukemia and other blood dyscrasias in humans (Agency for Toxic Substances and Disease Registry 2005), these effects cannot be solely attributed to xylene.

An occupational study in which no benzene exposure was involved (Uchida et al. 1993) found no hematological effects (red blood cell, white blood cell and platelet counts, and hemoglobin concentrations were unchanged). Workers (175) were exposed to a geometric mean TWA of 14 ppm xylene for an average of 7 years, and mixed xylene exposure accounted for $70 \%$ or more of the total exposure (Uchida et al. 1993). This study suggests that occupational exposure to relatively low concentrations of xylenes does not cause hematological effects.

No effect on erythrocyte fragility was observed in rats exposed to $15,000 \mathrm{ppm}$ mixed xylene for 45 minutes (Carpenter et al. 1975a). No adverse hematological effects have been observed in rats exposed to 2,764 ppm mixed xylene for 5 hours/day for 9 days (Wronska-Nofer et al. 1991). In rats intermittently exposed to $100 \mathrm{ppm} m$-xylene for 90 days, erythrocyte counts were reduced by $18.5 \%$ and 
leukocyte counts were increased by $35 \%$ (Korsak et al. 1994). Increases in leukocyte count were reported in rats and dogs exposed intermittently to 780 ppm o-xylene for 6 weeks (Jenkins et al. 1970), but it is unknown whether these increases were statistically significant. However, no effects on hematological parameters were observed in rats or dogs following intermediate-duration intermittent exposure to concentrations as high as $810 \mathrm{ppm}$ of mixed xylene (Carpenter et al. 1975a) or in guinea pigs exposed to 78 ppm o-xylene continuously or 780 ppm o-xylene intermittently (Jenkins et al. 1970) for an intermediate duration.

Musculoskeletal Effects. A 1993 occupational study indicates that workers exposed to xylenes (geometric mean TWA $14 \mathrm{ppm}$ ) reported reduced grasping power and reduced muscle power in the extremities more frequently than the unexposed controls (Uchida et al. 1993). This effect was a neurological effect rather than a direct effect on the muscles. No additional data were available regarding musculoskeletal effects in humans following inhalation exposure to mixed xylene or its individual isomers. Animal data regarding musculoskeletal effects following xylene inhalation are limited but provide no indication that xylene produces musculoskeletal effects. No lesions were observed in the skeletal muscle of rats and dogs exposed for an intermediate exposure to concentrations as high as 810 ppm mixed xylene (Carpenter et al. 1975a).

Hepatic Effects. Human data regarding hepatic effects following inhalation of xylene are limited to several case and occupational studies (Klaucke et al. 1982; Morley et al. 1970; Uchida et al. 1993); other occupational studies involve exposure to other compounds such as toluene (Dolara et al. 1982; Kurppa and Husman 1982). Two of these studies suggest that acute-duration exposure to high levels of xylene may result in hepatic toxicity. Two painters who survived exposure to an estimated 10,000 ppm of xylene and several workers who were exposed to an estimated $700 \mathrm{ppm}$ of xylene had transiently elevated serum transaminase levels (Klaucke et al. 1982; Morley et al. 1970). The one painter who died had hepatocellular vacuolation following exposure to xylene for 18.5 hours. An occupational study in which workers were exposed an average of 7 years to $>70 \%$ mixed xylenes (geometric mean TWA 14 ppm) found no changes in serum biochemistry values that reflect liver function (total bilirubin, aspartate aminotransferase, alanine aminotransferase, gamma glutamyl transpeptidase, alkaline phosphatase, and leucine aminopeptidase) (Uchida et al. 1993). This study suggests that low-level occupational exposure to xylenes does not result in hepatic effects.

Animal studies using rats indicate that mixed xylene, $m$-xylene, $o$-xylene, or $p$-xylene generally induce a wide variety of hepatic enzymes, as well as increased hepatic cytochrome P-450 content in rats (Elovaara 
1982; Elovaara et al. 1980; Patel et al. 1979; Savolainen et al. 1978; Selgrade et al. 1993; Toftgard and Nilsen 1981, 1982; Toftgard et al. 1981; Ungvary et al. 1980a). Following acute exposures to mixed xylene (Savolainen et al. 1978; Ungvary 1990; Wisniewska-Knypl et al. 1989), m-xylene (Elovaara 1982; Ungvary et al. 1980b), o-xylene (Tatrai and Ungvary 1980; Ungvary et al. 1980a), or $p$-xylene (Patel et al. 1979; Simmons et al. 1991; Ungvary et al. 1980b), effects have been observed including increased relative liver weight (Simmons et al. 1991; Tatrai and Ungvary 1980; Ungvary et al. 1980a, 1980b), cytochrome P-450 content (Simmons et al. 1991; Ungvary 1990; Ungvary et al. 1980a; WisniewskaKnypl et al. 1989), microsomal protein (Elovaara 1982), microsomal enzyme activity (Elovaara 1982; Savolainen et al. 1978; Ungvary 1990; Ungvary et al. 1980a; Wisniewska-Knypl et al. 1989), proliferation of the endoplasmic reticulum (Ungvary 1990; Wisniewska-Knypl et al. 1989), and decreased hexobarbital sleep time (Ungvary 1990; Ungvary et al. 1980a). Similar changes were observed in rabbits and mice (Ungvary 1990). Although histopathological examination of livers in most studies showed no adverse effects (Elovaara 1982; Simmons et al. 1991; Ungvary et al. 1980b), minor histopathological changes suggesting mild hepatic toxicity included decreased glycogen content, dilation of the cisterns of the rough endoplasmic reticulum, separation of ribosomes from the membranes, variously shaped mitochondria, and increased autophagous bodies (Tatrai and Ungvary 1980; Ungvary 1990). Also, increased serum transaminases were observed following a 4-hour exposure of rats to 1,000 ppm $p$-xylene (Patel et al. 1979).

Many similar hepatic effects appear after intermediate-duration exposure to mixed xylene or $O$-xylene. They include increased absolute and/or relative hepatic weight in rats (Kyrklund et al. 1987; Tatrai and Ungvary 1980; Tatrai et al. 1981; Toftgard et al. 1981; Ungvary 1990; Ungvary et al. 1980a), increased cytochrome P-450 (Tatrai et al. 1981; Ungvary 1990; Ungvary et al. 1980a); increased microsomal enzyme activity (Elovaara et al. 1980, 1987; Tatrai et al. 1981; Toftgard et al. 1981; Ungvary 1990; Ungvary et al. 1980a), proliferation of the smooth and rough endoplasmic reticulum (Rydzynski et al. 1992; Tatrai and Ungvary 1980; Tatrai et al. 1981; Ungvary 1990) and decreased hexobarbital sleeping time because of enhanced metabolism of the drug (Tatrai et al. 1981; Ungvary 1990; Ungvary et al. 1980a). Similar effects were observed in rabbits and mice (Ungvary 1990). As in the acute studies, several intermediate-duration studies in rats, guinea pigs, monkeys, or dogs, reported no effect on serum transaminases (Carpenter et al. 1975a; Tatrai et al. 1981) or hepatic morphology (Carpenter et al. 1975a; Jenkins et al. 1970). Ultrastructural examination of livers showed only minor changes: decreased hepatic glycogen in rats (Tatrai and Ungvary 1980; Ungvary 1990; Ungvary et al. 1980b), ultrastructural changes in hepatic rough endoplasmic reticulum and mitochondria in rats (Tatrai and Ungvary 1980; Ungvary 1990), increased autophagous bodies (Tatrai et al. 1981; Ungvary 1990), and changes in the distribution 
of hepatocellular nuclei in rats (Tatrai and Ungvary 1980). Some authors have characterized the hepatic changes as adaptive rather than adverse (Tatrai and Ungvary 1980; Ungvary 1990). No effects on hepatic microsomal proteins, cytochrome P-450, lipid peroxidation (as indicated by levels of malondialdehyde), triglycerides, serum enzymes (AST, ALT, SDH), or absolute or relative liver weights were observed in rats exposed to $m$-xylene at concentrations as high as $100 \mathrm{ppm}$ for 13 weeks (Korsak et al. 1994). No changes in the levels of lipid peroxidation (malondialdehyde levels), glutathione, or glutathione-Stransferase activity were observed in rats intermittently exposed to $92 \mathrm{ppm} m$-xylene for 5 months (Jajte et al. 2003).

Increased liver weight and microsomal enzyme activity were reported in a study in which rats were exposed to 1,096 ppm o-xylene for 1 year (Tatrai et al. 1981). Electron microscopic examination of liver revealed a proliferation of the endoplasmic reticulum and only very minor effects on mitochondria as exemplified by increased numbers of peroxisomes.

Renal Effects. Although urinalyses (using a dip-stick technique) of volunteers exposed to $p$-xylene at $100 \mathrm{ppm}$ for 5 days or up to $150 \mathrm{ppm}$ in a multi-week exposure paradigm showed no adverse effects on the kidneys (NIOSH 1981), limited data from case reports and occupational studies suggest that inhalation exposure to solvent mixtures containing xylene may be associated with adverse renal effects in humans (Martinez et al. 1989; Morley et al. 1970). These effects included increased blood urea (Morley et al. 1970), distal renal tubular acidemia (Martinez et al. 1989), and decreased urinary clearance of endogenous creatinine (Morley et al. 1970). Other studies that reported increased urinary levels of $\beta$-glucuronidase (Franchini et al. 1983), or increased urinary excretion of albumin, erythrocytes, and leukocytes (Askergren 1981, 1982) are confounded by concurrent exposure to substantial amounts of toluene, a known renal toxicant.

In an occupational study in which the exposure was predominantly to mixed xylenes (geometric mean TWA $14 \mathrm{ppm}$ ) for an average of 7 years (Uchida et al. 1993), no effects on measures of kidney function (serum creatinine or urinalysis for urobilinogen, sugar, protein, and occult bleeding) were noted. This study suggests that low-level occupational exposure to xylenes does not result in kidney effects.

The renal effects of mixed xylene and $o$-xylene following inhalation exposure have been evaluated in acute and intermediate studies with rats, guinea pigs, dogs, and monkeys (Carpenter et al. 1975a; Elovaara 1982; Jenkins et al. 1970; Toftgard and Nilsen 1982). Effects noted in these studies at xylene concentrations of 50-2,000 ppm have included increased renal enzyme activity, increased renal 
cytochrome P-450 content, and increased kidney-to-body weight ratios (o-xylene-exposed rats) (Elovaara 1982; Toftgard and Nilsen 1982). However, histopathologic examination of rats, guinea pigs, dogs, and monkeys did not reveal any renal lesions after inhalation of $810 \mathrm{ppm}$ mixed xylene or $78 \mathrm{ppm} o$-xylene for an intermediate period of 13 weeks and 90-127 days, respectively (Carpenter et al. 1975a; Jenkins et al. 1970). No effect on absolute or relative kidney weights was observed in male rats intermittently exposed to $m$-xylene at concentrations as high as 100 ppm for 13 weeks (Korsak et al. 1994).

No studies were located regarding renal effects following chronic inhalation exposure to mixed xylene or its isomers.

Endocrine Effects. No human data were available regarding endocrine effects following inhalation exposure to mixed xylene or xylene isomers. Inhalation exposure to $810 \mathrm{ppm}$ mixed xylene for 13 weeks produced no adverse adrenal, thyroid, or parathyroid effects in the dog (Carpenter et al. 1975a). No effect on absolute or relative adrenal weights was observed in male rats intermittently exposed to $m$-xylene at concentrations as high as 100 ppm for 13 weeks (Korsak et al. 1994).

Ocular Effects. Human data indicate that acute inhalation exposures to $460 \mathrm{ppm}$ mixed xylene and $100 \mathrm{ppm}$ p-xylene vapors produce mild and transient eye irritation (Carpenter et al. 1975a; Hastings et al. 1986; Klaucke et al. 1982; Nelson et al. 1943; Nersesian et al. 1985; NIOSH 1981). This effect is probably the result of direct contact of the xylene vapor with the eye and as such is described under Ocular Effects in Section 3.2.3.2.

No animal data were available regarding ocular effects following inhalation exposure to mixed xylenes or xylene isomers.

Body Weight Effects. No studies were located regarding body weight effects in humans following inhalation exposure to mixed xylenes or xylene isomers.

A number of intermediate-duration intermittent inhalation studies of xylene have examined body weight effects in animals (Carpenter et al. 1975a; Gagnaire et al. 2001, 2006; Gralewicz and Wiaderna 2001; Jajte et al. 2003; Korsak et al. 1992, 1994; Rosengren et al. 1986; Tatrai et al. 1981). Except for the study by Tatrai et al. (1981) in which a $12 \%$ decrease in body weight was observed in rats exposed to 1,096 ppm o-xylene for 6 months, no significant adverse effects on body weight were noted. 
Metabolic Effects. Metabolic acidosis was reported in a man who sniffed paint containing xylenes, but other solvents in the paint may have contributed to the effect (Martinez et al. 1989). No data were located concerning metabolic effects in animals following inhalation exposure to xylenes.

\subsubsection{Immunological and Lymphoreticular Effects}

Limited data were available regarding immunological and lymphoreticular effects of xylene in humans. Decreased lymphocytes (Moszczynski and Lisiewicz 1983, 1984a) and decreased serum complement (Smolik et al. 1973) have been observed in workers exposed to xylene. However, no determination can be made regarding the association between inhalation of xylene and immunological effects from the available human studies, because workers were concurrently exposed to other chemical agents.

Acute exposure (4 days, 4 hours/day) of mice to 1,208 ppm p-xylene had no effect on natural killer cell activity, although mortality from murine cytomegalovirus was increased (Selgrade et al. 1993). The investigators (Selgrade et al. 1993) attributed the enhanced virus susceptibility to increased liver toxicity rather than to an effect on the immune system. Intermittent exposure of rats and dogs to mixed xylenes for 10 or 13 weeks at concentrations as high as $810 \mathrm{ppm}$ resulted in no effect on spleen weight (Carpenter et al. 1975a).

\subsubsection{Neurological Effects}

The neurological effects of xylene in humans following inhalation exposure have been evaluated in a number of experimental studies, case reports, and occupational studies. Results of experimental studies with humans indicate that acute inhalation exposure to mixed xylene or $m$-xylene causes impaired shortterm memory, impaired reaction time, performance decrements in numerical ability, and alterations in equilibrium and body balance (Carpenter et al. 1975a; Dudek et al. 1990; Gamberale et al. 1978; Riihimaki and Savolainen 1980; Savolainen and Linnavuo 1979; Savolainen and Riihimaki 1981a; Savolainen et al. 1979b, 1984, 1985a).

Dizziness was reported by the majority of subjects exposed to $690 \mathrm{ppm}$ mixed xylene for 15 minutes, but in only one of six persons exposed at $460 \mathrm{ppm}$ (Carpenter et al. 1975a). In objective measures of neurological function, exposure to $100 \mathrm{ppm}$ mixed xylene for 4 hours resulted in prolonged reaction time (Dudek et al. 1990) and exposure to $299 \mathrm{ppm}$ mixed xylene for 70 minutes during exercise resulted in impaired short-term memory and reaction time (Gamberale et al. 1978). No impairment in performance tests was observed in sedentary subjects exposed at 299 ppm for 70 minutes (15 men) (Gamberale et al. 
1978) or at $396 \mathrm{ppm}$ for 30 minutes (10 men) (Hastings et al. 1986). The difference between the effects in the absence and presence of exercise may be due to increased xylene respiratory uptake during exercise.

Slight, but statistically significant, increases in the average rating for subjective symptoms of neurological effects were observed following exposure to $50 \mathrm{ppm} \mathrm{m}$-xylene vapor compared to controls (Ernstgard et al. 2002). After 60 and 118 minutes of exposure, severity ratings for feelings of intoxication were elevated in men and women, and ratings for headache were elevated in men. The ratings for dizziness were increased in exposed men after 118 minutes of exposure. (This study was chosen as the basis for the acute-duration inhalation MRL, for these subjective neurological effects and changes in objective and subjective measures of respiratory function.) Electroencephalograms obtained from nine men exposed to $m$-xylene at 200 ppm (TWA) for 4 hours showed only minor changes (Seppalainen et al. 1991). These changes were characterized as a slight increase in alpha-wave frequency and percentage early in the exposure period and a decrease in exercise-induced increases in theta and delta waves indicating central nervous system effects. Studies using the $m$-isomer of xylene have also indicated that some tolerance may occur during acute exposures. While exposure to stable concentrations of $m$-xylene for 7 hours or 4 hours, twice a week in the range of up to approximately $280 \mathrm{ppm}$ had no effect on body sway, coordination, or reaction time (Ogata et al. 1970; Savolainen 1980; Savolainen et al. 1980b), exposure for 6 hours or 6-9 days to levels fluctuating between 64 and 400 ppm produced impairment in human body balance and/or reaction time (Savolainen and Linnavuo 1979; Savolainen and Riihimaki 1981a; Savolainen et al. 1979b, 1980a, 1984, 1985a). A 3-hour exposure of nine male volunteers to $m$-xylene at $200 \mathrm{ppm}$ during exercise resulted in a slight but significant $(\mathrm{p}<0.05)$ change in the N135 component of a pattern visual evoked potential (Seppalainen et al. 1989). Laine et al. (1993) saw no clear effects on visual reaction times or auditive choice reaction times in nine male volunteers exposed to levels of $m$-xylene fluctuating between 135 and 400 ppm (TWA $200 \mathrm{ppm}$ ) with or without exercise. Levels of $m$-xylene fluctuating between 135 and 400 ppm produced a slight decrease in the latency of visual evoked potentials (Seppalainen et al. 1989), but no clear effects on visual reaction times or auditory choice reaction times (Laine et al. 1993).

Objective measures of neurological function (electroencephalography, tests of motor activity and cognitive performance) in humans are not affected by acute or intermediate, intermittent or continuous inhalation exposure to $p$-xylene for 4 hours or up to 7 hours for 5 days at concentrations ranging from 69 to 150 ppm (NIOSH 1981; Olson et al. 1985). Differences in such factors as the xylene isomer, the neurological parameter, exposure conditions and concentrations, rapid development of tolerance, and total 
xylene uptake may account for the variability in results. However, some sex difference in subjective reports of central nervous system effects was observed (NIOSH 1981). Three women exposed to p-xylene at $100 \mathrm{ppm}$ for 1-7.5 hours/day, for 5 days, showed no effects on electroencephalograms, evoked potentials, or cognitive performance, but frequently reported headache and dizziness as a result of exposure (NIOSH 1981). In contrast, four men exposed at concentrations of up to $150 \mathrm{ppm} p$-xylene under the same exposure conditions reported no increase in headaches or dizziness.

Available case reports and occupational studies together provide suggestive evidence that acute and chronic inhalation exposure to xylene or solvent mixtures containing xylene may be associated with neurological effects; however, most studies are difficult to evaluate because the exposure conditions either have not been well characterized or the subjects may have been exposed to other chemicals in addition to xylene. The neurological symptoms observed in these studies include headache, nausea, dizziness, difficulty concentrating, impaired memory, slurred speech, ataxia, fatigue, agitation, confusion, tremors, labored breathing, and sensitivity to noise (Arthur and Curnock 1982; Goldie 1960; Gupta et al. 1990; Hipolito 1980; Klaucke et al. 1982; Martinez et al. 1989; Morley et al. 1970; Nersesian et al. 1985; Roberts et al. 1988). In several case reports, isolated instances of unconsciousness, amnesia, brain hemorrhage, and epileptic seizure have been associated with acute inhalation exposure to solvent mixtures containing xylene (Arthur and Curnock 1982; Goldie 1960; Martinez et al. 1989; Morley et al. 1970). Long-term occupational exposure ( $\geq 10$ years) to mixed solvents among spray painters was associated with an increase in depression and "loss of interest," but no significant effects on psychological performance tests or CAT-scan measures of brain atrophy (Triebig et al. 1992a, 1992b). Workers exposed to mixed solvents for $<10->30$ years exhibited significantly reduced conduction velocities in the radial and tibial nerves, as well as duration-related increases in symptoms of numbness, cramps, and weakness (Jovanovic et al. 2004). Because other chemicals were present with xylenes in many of these studies, the effects observed cannot be conclusively attributed to xylene exposure.

Another occupational study in which xylene exposure was most well defined and represented $70 \%$ of the solvent exposure (Uchida et al. 1993) reported an increase in subjective symptoms including an increased prevalence of anxiety, forgetfulness, inability to concentrate, and dizziness among workers exposed to an average TWA concentration of $21 \mathrm{ppm}$ (14 ppm geometric mean) of mixed xylenes for an average of 7 years. No objective measures of neurological impairment were tested in this study. Subjective symptoms of neurological and respiratory toxicity in this study were selected as co-critical effects for the chronic-duration inhalation MRL. 
Results of experimental studies with animals also provide evidence that mixed xylene and its isomers are neurotoxic following inhalation exposure. Signs of neurotoxicity observed in rats, mice, dogs, cats, and gerbils following acute and intermediate inhalation exposure to the various xylene isomers include narcosis, prostration, incoordination, tremors, muscular spasms, labored breathing, behavioral changes, hyperreactivity to stimuli, altered visual evoked potentials, elevated auditory thresholds, hearing loss, and decreased acetylcholine in midbrain and norepinephrine in hypothalamus (suggestive of effect on motor control, sleep, and memory maintenance) (Andersson et al. 1981; Bushnell 1989; Carpenter et al. 1975a; De Ceaurriz et al. 1983; Furnas and Hine 1958; Ghosh et al. 1987; Honma et al. 1983; Korsak et al. 1988, 1990; Kyrklund et al. 1987; Molnar et al. 1986; Pryor et al. 1987; Rank 1985; Rosengren et al. 1986; Savolainen and Seppalainen 1979; Savolainen et al. 1978, 1979b; Wimolwattanapun et al. 1987).

Exposure levels associated with neurological effects in animals are well defined. A comparative study determined that the minimal alveolar concentrations needed to induce anesthesia in rats were similar for all three isomers $\left(0.00118,0.00139\right.$, and $0.00151 \mathrm{~atm}$, respectively, for $o^{-}, \mathrm{m}-$, and $p$-xylene $)$, but only $p$-xylene also induced excitation (strong tremors) (Fang et al. 1996). Acute exposure to unspecified levels of mixed xylene resulted in respiratory paralysis (Morvai et al. 1976), 1,600 ppm $p$-xylene produced hyperactivity (Bushnell 1989), and 1,300 ppm mixed xylene produced incoordination in rats, which did not persist after exposure ended; no overt signs of toxicity were noted at $580 \mathrm{ppm}$ (Carpenter et al. 1975a). All three xylene isomers produced narcosis in rats after 1-4 hours of exposure to concentrations of approximately 2,000 ppm (Molnar et al. 1986). No behavioral signs of xylene intoxication were observed in dogs or monkeys exposed continuously to $78 \mathrm{ppm} o$-xylene for up to 127 days, but dogs exposed to $780 \mathrm{ppm} o$-xylene intermittently for 6 weeks exhibited tremors during exposure (Jenkins et al. 1970).

The neurotoxicity of xylenes has been evaluated in neurobehavioral tests on animals exposed by inhalation. Mice exposed for 30 minutes by inhalation to any of the isomers exhibited impaired operant performance at the same minimal effective concentration, $1,400 \mathrm{ppm}$, but the median effective concentrations varied to a limited degree- $5,179 \mathrm{ppm}$ for $o$-xylene, 5,611 ppm for $p$-xylene, and $6,176 \mathrm{ppm}$ for $m$-xylene (Moser et al. 1985). The order of potency was different for impairment of motor coordination in mice undergoing the inverted screen test, with a minimal effective concentration of 2,000 ppm for $p$-xylene and 3,000 ppm for the two other isomers (Moser et al. 1985); the median effective concentrations were 2,676,3,640, and 3,790 ppm, respectively, for $p$-, $o-$, and $m$-xylene. Acute exposures to concentrations inducing behavioral changes in rats and mice ranged from $114 \mathrm{ppm}$ for effects of mixed xylene on operant conditioning or self-stimulation behavior (Ghosh et al. 1987; 
Wimolwattanapun et al. 1987), $500 \mathrm{ppm}$ for reduced response rate in schedule-controlled operant behavior in mice exposed to $m$-xylene (Bowen et al. 1998), to 1,010 ppm for $o$-xylene-induced immobility in a "behavioral despair swimming test" (De Ceaurriz et al. 1983). Exposure of male rats to $230 \mathrm{ppm}$ o-xylene for 4 hours shortened the duration of response (extension of hindlimbs) to an applied electrical shock by $18.8 \%$ (Vodickova et al. 1995); doubling the exposure concentration correspondingly doubled the magnitude of the response. In the same report, exposure of female mice to $320 \mathrm{ppm} o$-xylene for 2 hours shortened the duration of response (velocity of tonic extension, i.e., the reciprocal of the latency) was reduced by $11 \%$; unlike rats, exposure at twice the concentration, increased the magnitude of the response by a factor of 3.7. Impaired rotarod performance was observed in rats acutely exposed to mixed xylene and the individual xylene isomers at concentrations of $\geq 3,000 \mathrm{ppm}$ (Korsak et al. 1990). In intermediate-duration inhalation studies with rats, exposure to $100 \mathrm{ppm} m$-xylene intermittently for 3 or 6 months or to $1,000 \mathrm{ppm}$ for 3 months showed decreased rotarod performance and decreased spontaneous activity (Korsak et al. 1992, 1994). The effect was greater following the 3-month exposure at $1,000 \mathrm{ppm}$ than the 6-month exposure at $100 \mathrm{ppm}$ suggesting that for effects on motor activity, concentration is more important than duration of exposure. The persistence of neurological effects of xylene was examined in some studies that evaluated tested animals some weeks after the last exposure. Rats intermittently exposed to $100 \mathrm{ppm} m$-xylene for 4 weeks exhibited impaired passive avoidance learning (tested 5 weeks after exposure) and impaired acquisition, but not retention, of the two-way active avoidance response (tested 9 weeks after exposure) (Gralewicz and Wiaderna 2001). Exposure had no lasting effect in tests for short-term memory, responsiveness to a thermal stimulus (paw-lick latency), or spontaneous activity (open-field test) or the retention of a learned active avoidance response.

Sensory deficits resulting from xylene exposure have been observed under controlled testing conditions. Acute exposure to 1,600 ppm, but not $800 \mathrm{ppm} p$-xylene depressed the amplitude of visual evoked potentials (Dyer et al. 1988). Hearing deficits have been reported in animals exposed to xylene for acute or intermediate durations. Hearing loss occurred in rats exposed to 1,450 ppm mixed xylene for 8 hours, whereas exposure to 1,700 ppm for 4 hours produced no effects on hearing (Pryor et al. 1987) indicating that the duration of exposure is important for the observation of ototoxic effects in conditioned avoidance tests. In rats acutely exposed to 1,800 ppm mixed xylenes, 18-30 dB hearing losses were observed at mid-range frequencies (Crofton et al. 1994). The amplitude of brainstem auditory evoked potentials was reduced by $50 \%$ in rats acutely exposed at $2,000 \mathrm{ppm}$, but not at 1,700 ppm (Rebert et al. 1995). Hearing loss was also evident after exposure for 6 weeks to $800 \mathrm{ppm}$ mixed xylene (Pryor et al. 1987). In rats exposed intermittently to $900 \mathrm{ppm} p$-xylene for 13 weeks, there was some hair cell loss in the organ of Corti, but no effect on auditory neurophysiology (Gagnaire et al. 2001). At 1,800 ppm, extensive 
ototoxicity (altered brainstem auditory evoked potentials, measurable hearing loss [deficits of 35-42 dB] and significant hair cell loss in the organ of Corti) was observed as early as the fourth week and did not improve during an 8-week recovery period; neither $o$-xylene nor $m$-xylene were ototoxic in this study. All rats exposed for 13 weeks to 1,000 or 2,000 ppm mixed xylenes containing ethylbenzene exhibited significant irreversible ototoxicity including hearing deficits and loss of hair cells (Gagnaire et al. 2006). Exposures to these mixtures at 250 or $500 \mathrm{ppm}$ resulted in a small loss of hair cells in one of eight rats per group, but no effect on auditory thresholds. According to the authors, ethylbenzene contributes significantly to the ototoxicity of technical xylene.

Changes in neuronal cells and brain biochemistry have been noted in animals following exposure to xylenes. Acute exposure to $p$-xylene caused decreased axonal transport at concentrations as low as 800 ppm (Padilla and Lyerly 1989); however, no such decrease was apparent 3 days after exposures had ceased. At 1,600 ppm, however, the decrease in axonal transport persisted for 13 days after exposure. Acute inhalation of 2,000 ppm mixed xylene produced increased dopamine and/or noradrenaline levels in the hypothalamus of rats; no behavioral changes were assessed (Andersson et al. 1981). Levels of these catecholamines in the hypothalamus of rats were also increased following inhalation of $2,000 \mathrm{ppm} \mathrm{m-,} \mathrm{O}^{-}$, or $p$-xylene (Andersson et al. 1981). Brain concentrations of deoxyribonucleic acid (DNA) and/or astroglial proteins increased in rats (at 300-320 ppm) and gerbils (at $160 \mathrm{ppm}$ ) after intermediate continuous exposure of 3-4.5 months to xylene (Rosengren et al. 1986; Savolainen and Seppalainen 1979). In addition, increased levels of brain enzymes, changes in axon membranes, and behavioral changes occurred in rats after exposure to $300 \mathrm{ppm}$ of mixed xylene for 18 weeks (Savolainen and Seppalainen 1979; Savolainen et al. 1979a). Alterations in neurotransmitter levels were observed in some brain areas at 800 ppm mixed xylene for 30 days (Honma et al. 1983). However, no significant long-term alterations in fatty acid levels were noted in the brains of rats after intermediate-duration exposure of 30 or 90 days to $320 \mathrm{ppm}$ mixed xylene (Kyrklund et al. 1987). At 1,600 ppm m-xylene for 7 weeks, decreased $\alpha$-adrenergic binding compared to the controls was observed in the hypothalamus of exposed mice (Rank 1985). No persistent changes in brain weight or weights of the caudate-putamen or subcortical limbic areas, or in agonist binding to the dopamine $\mathrm{D}_{2}$ receptor in those regions occurred in rats exposed to $80 \mathrm{ppm} p$-xylene for 4 weeks and examined 5 weeks after the last exposure (HilleforsBerglund et al. 1995).

No animal studies were located regarding neurological effects following chronic inhalation exposure to mixed xylene or its isomers. 
The highest NOAEL values and all LOAEL values for each reliable study for neurological effects in each species and duration category are recorded in Table 3-1 and plotted in Figure 3-1. An acute-duration inhalation MRL of 2 ppm was calculated for mixed xylenes based on a LOAEL for subjective neurological effects and measured and subjective respiratory effects in human subjects exposed to $50 \mathrm{ppm}$ m-xylene for 2 hours (Ernstgard et al. 2002; see footnote in Table 3-1). An intermediate-duration inhalation MRL of $0.6 \mathrm{ppm}$ was calculated for mixed xylenes based on a minimal LOAEL of $50 \mathrm{ppm}$ for decreased mean latency of the paw-lick response in rats exposed to $m$-xylene for 6 hours/day, 5 days/week for 3 months (Korsak et al. 1994; see footnote in Table 3-1). A chronic-duration inhalation MRL of $0.05 \mathrm{ppm}$ was calculated for mixed xylenes based on a LOAEL of $14 \mathrm{ppm}$ for subjective neurological and respiratory symptoms in workers exposed to mixed xylene 8 hours/day, 5 days/week for an average of 7 years (Uchida et al. 1993; see footnote in Table 3-1).

\subsubsection{Reproductive Effects}

A few occupational studies evaluated reproductive effects in workers exposed to xylenes. A case-control study of spontaneous abortions among Finnish workers for whom there was biomonitoring data for exposure to organic solvents found no statistically significant increase in the odds ratio associated with exposure to xylene (Lindbohm et al. 1990). Spontaneous abortions in early pregnancy were significantly increased among 37 women exposed to xylene and formalin in pathology or histology laboratories (Taskinen et al. 1994). Although the increased odds ratio $(\mathrm{OR}=3.1 ; 95 \%$ confidence interval=1.3-7.5) for spontaneous abortion was statistically significant for women exposed to xylene at least 3-4 days/week, the analysis cannot be considered definitive because of simultaneous exposure to other solvents and chemicals. A cross-sectional study among 1,408 petrochemical workers in China reported an increased prevalence of oligomenorrhea among workers exposed to organic solvents, but the contribution of xylene cannot be definitively determined as all workers who were exposed to xylene were also exposed to other solvents such as benzene, toluene, and styrene (Cho et al. 2001).

Continuous exposure of CFY rats for 8 days on days 7-14 during pregnancy to $775 \mathrm{ppm}$ mixed xylene produced an increased number of resorptions without any maternal toxicity; reduced fertility was also observed (Balogh et al. 1982). However, no adverse reproductive effects were noted following inhalation exposure of male and female $\mathrm{CD}$ rats to mixed xylene at concentrations as high as $500 \mathrm{ppm}$ during premating, mating, pregnancy, and lactation (Bio/dynamics 1983). No effect on absolute or relative testicular weights was observed in rats intermittently exposed to $m$-xylene at concentrations as high as 100 ppm for 13 weeks (Korsak et al. 1994). Inhalation exposure of male Sprague-Dawley rats to 
1,000 ppm mixed xylene for 61 days produced no alterations in testes, accessory glands, or circulating male hormone levels (Nylen et al. 1989). Strain differences may account for the differential response to mixed xylene in these studies. The highest NOAEL and LOAEL values for each reliable study for reproductive effects in rat for each duration category are recorded in Table 3-1 and plotted in Figure 3-1.

\subsubsection{Developmental Effects}

Although the human data regarding the developmental effects of xylene suggest a possible relationship between solvent (unspecified) exposure and developmental toxicity, these data are limited for assessing the relationship between inhalation of xylene and developmental effects because the available studies involved concurrent exposure to other solvents in addition to xylene in the workplace (Holmberg and Nurminen 1980; Kucera 1968; Taskinen et al. 1989; Windham et al. 1991), and because of the small number of subjects ranging from 9 to 61 (Taskinen et al. 1989; Windham et al. 1991).

Both mixed xylene and the individual isomers produce fetotoxic effects in laboratory animals. Effects of mixed xylene observed in rats, mice, and rabbits included increased incidences of skeletal variations in fetuses, delayed ossification, fetal resorptions, hemorrhages in fetal organs, and decreased fetal body weight (Balogh et al. 1982; Bio/dynamics 1983; Hass and Jakobsen 1993; Hudak and Ungvary 1978; Litton Bionetics 1978a; Mirkova et al. 1983; Ungvary 1985; Ungvary and Tatrai 1985). Balogh et al. (1982) reported delayed ossification in all exposure levels (53-775 ppm), but as the incidences were inversely related to dose, this end point is not included in LSE Table 3-1. The levels at which these effects were observed depended upon the composition and concentration of mixed xylene, the time of exposure, and the choice of strain and test species used. In addition, animals in a number of studies were exposed 24 hours/day (Balogh et al. 1982; Hudak and Ungvary 1978; Ungvary 1985; Ungvary and Tatrai 1985), whereas animals in other studies (Bio/dynamics 1983; Hass and Jakobsen 1993; Litton Bionetics 1978a; Mirkova et al. 1983) were exposed 6 hours/day. The study conducted by Litton Bionetics (1978a) used a formulation of mixed xylene with a comparatively high percentage (36\%) of ethylbenzene. Developmental effects were reported following maternal exposure to concentrations as low as $12 \mathrm{ppm}$ mixed xylene in rats (Mirkova et al. 1983), but the health of the test animals may have been compromised due to poor animal husbandry. This is suggested by the relatively low conception rates and the high incidence of fetal hemorrhages seen in the controls. Maternal toxicity was observed at $775 \mathrm{ppm}$ in the study by Balogh et al. (1982) and at 138 ppm in the study by Ungvary (1985); however, no maternal toxicity occurred at exposure levels of 100-400 ppm in the studies by Bio/dynamics (1983), Hass and Jacobsen (1993), Hudak and Ungvary (1978), and Litton Bionetics (1978a). Insufficient evidence was 
presented to determine whether maternal toxicity occurred in the studies by Mirkova et al. (1983) and Ungvary and Tatrai (1985). Many of the studies (Balogh et al. 1982; Bio/dynamics 1983; Hudak and Ungvary 1978; Mirkova et al. 1983; Ungvary 1985; Ungvary and Tatrai 1985) had limitations that made them difficult to assess (e.g., unknown composition of xylene and insufficient number of doses to form a dose-response relationship; lack of detail with regard to both methods and data obtained). Furthermore, several of these studies (Balogh et al. 1982; Ungvary and Tatrai 1985; Ungvary et al. 1980b) reported the incidence of fetal effects, such as delayed skeletal ossification and extra ribs, on the basis of total fetuses per exposure group, but not on a per-litter basis. Without litter-specific results, it is not possible to adjust for possible covariance with litter size, as reported by Litton Bionetics (1978a). Given the uncertainty of the significance of these effects, increases in the incidences of delayed ossification or skeletal variants were not used to establish NOAELs or LOAELs unless litter-specific data were provided. Based on the reliable data in these reports (fetal weight, fetal death, implantation losses), developmental toxicity in rats occurred at concentrations of $\geq 350 \mathrm{ppm}$; these effects are noted in Table 3-1.

A number of studies reported neurobehavioral deficits in rats resulting from exposure to xylene during gestation. ATSDR considers these effects to be serious. Hass and Jakobsen (1993) reported decreased rotarod performance in 1- and 2-day-old rat pups exposed to $200 \mathrm{ppm}$ mixed xylenes 6 hours/day on gestation days 4-20. Female offspring of rats exposed to $500 \mathrm{ppm}$ mixed xylenes for 6 hours/day on gestation days 7-20 showed significant reductions in absolute brain weight after weaning and neurobehavioral deficits (delayed air righting reflex, nonsignificant trends for reduced motor coordination in the Rotarod test, and increased latencies in the Morris water maze test) (Hass et al. 1995). Hass et al. (1995) suggested that the delayed air righting reflex and impaired spatial navigation in the water maze (locating a re-situated platform) may have been related to ototoxicity, since both tasks involve the vestibular system and cochlear hair cells. The latency in locating a moved platform was significantly increased in the first learning trial by exposed offspring tested at week 28 , but was not significantly different from controls at 55 weeks (Hass et al. 1997).

Inhalation of $o$ - or $p$-xylene at concentrations similar to those at which mixed xylene caused fetal toxicity, produced decreased fetal weight, skeletal retardation, and post-implantation loss in rats, mice, and rabbits following maternal acute exposure during gestation days 7-14/15 (Ungvary and Tatrai 1985; Ungvary et al. 1980b, 1981); no maternal toxicity was observed. A NOAEL value of 1,612 ppm $p$-xylene for developmental effects was determined from one study with rats (Rosen et al. 1986). The large variation in concentrations of xylene producing developmental effects and those producing no developmental effects may be influenced by a number of factors (e.g., strain and species of animal, purity of xylene, 
method of exposure, exposure pattern and duration, etc.). For example, Rosen et al. (1986) exposed animals for 6 hours/day, whereas animals were exposed 24 hours/day in studies by Ungvary and Tatrai (1985) and Ungvary et al. (1980b, 1981). No information on maternal toxicity was available for the studies by Ungvary and Tatrai (1985) or Ungvary et al. (1981); however, in the studies by Rosen et al. (1986) and Ungvary et al. (1980b), signs of maternal toxicity in rats following inhalation of the isomers included decreased weight gain, decreased food consumption, and increased liver-to-body weight ratios. $m$-Xylene was the only isomer that resulted in lasting maternal growth inhibition or maternal mortality (Ungvary et al. 1980b). Thus, it is difficult to determine from these studies whether mixed xylenes are selectively toxic to the fetus or the observed developmental toxicity was secondary to maternal toxicity. As mentioned above, retardation in skeletal growth or variations, in the absence of litter-specific data, were not used to determine NOAELs or LOAELs for exposure to specific isomers of xylene (Ungvary et al. 1980b).

Results of a well-documented comparative standard developmental toxicity assay in rats exposed for 6 hours/day on gestational days 6-20, suggest that developmental toxicities of $m$ - and $p$-xylene are secondary to maternal toxicity (Saillenfait et al. 2003). Statistically significant maternal effects (reduced 'corrected weight gain' - body weight gain exclusive of gravid uterus weight) and fetal toxicity (reduced fetal weight) occurred at exposures of $\geq 1,000 \mathrm{ppm}$, but not $500 \mathrm{ppm}$ for these two isomers. Both $m$ - and p-xylene at 2,000 ppm induced significant increases in delayed ossification, a skeletal variation related to retarded growth, although the affected bones were different for the two isomers. Dams exposed at $\geq 1,000 \mathrm{ppm}$ to $o$-xylene or mixed xylenes showed similar effects, although the reduced corrected weight gain for mixed xylenes was only significant for gestational days 6-13. However, both $o$-xylene and mixed xylene caused significantly reduced fetal body weights at $500 \mathrm{ppm}$, the maternal NOAEL. Exposure to mixed xylenes did not elicit any developmental structural anomalies at concentrations as high as 2,000 ppm, whereas $o$-xylene elicited increases in the percent of fetuses per litter with skeletal variations (incomplete ossification) at 2,000 ppm and in the percent of fetuses with any variation at $1,000 \mathrm{ppm}$. This assay did not include testing for postnatal neurobehavioral effects.

The highest NOAEL value and all LOAEL values from each reliable study for developmental effects in each species and duration category are recorded in Table 3-1 and plotted in Figure 3-1. 


\subsubsection{Cancer}

Human data regarding cancer are limited to three occupational studies and one population-based study. Two studies examined the cancer and leukemia risks among solvent-exposed workers and suggested a possible relationship between coal-based xylene exposure and leukemia (Arp et al. 1983; Wilcosky et al. 1984). Both contain limitations (e.g., small number of subjects ranging from 9 to 85 male workers, no exposure concentrations, unknown composition of xylene) that preclude a definitive conclusion regarding inhalation of xylene and cancer. A retrospective cohort mortality study evaluated 14,457 workers at an aircraft maintenance facility, 108 of whom had been exposed to xylene (Spirtas et al. 1991; Stewart et al. 1991). In this study, evaluations of deaths from multiple myeloma or non-Hodgkin's lymphoma revealed no cases among xylene-exposed workers, representing 1,837 and 444 person-years of exposure, for men and women, respectively (Spirtas et al. 1991; Stewart et al. 1991). A population-based case-control study that estimated job-related exposure to xylene reported limited evidence of increased risks for cancers of the colon and rectum following high exposure to xylene (Gerin et al. 1998). The authors indicated that the marginally significant excesses in rectal cancer in xylene-exposed workers may have been due to the confounding effect of exposure to styrene or another material associated with styrene exposure. Limitations of this study included a lack of information on exposure concentrations and the composition of xylene, a small number of cases, and the fact that most of the xylene-exposed workers were also exposed to toluene and benzene.

No studies were located regarding cancer in animals exposed via inhalation to mixed xylene or xylene isomers.

\subsubsection{Oral Exposure}

\subsubsection{Death}

Death in humans following accidental or intentional ingestion of xylene was reported by Abu Al Ragheb et al. (1986). Levels of xylene found in blood and gastric and duodenal contents were 110, 8,800, and $33,000 \mathrm{mg} / \mathrm{L}$, respectively, indicating ingestion of a large, but undetermined, quantity of xylene. Death was attributed to respiratory failure secondary to depression of the respiratory center in the brain.

Mortality was observed in laboratory animals following the ingestion of mixed xylene or isomers of xylene. Reported acute oral $\mathrm{LD}_{50}$ values in rats for mixed xylene range from 3,523 $\mathrm{mg} / \mathrm{kg}$ when administered in corn oil (NTP 1986) to $8,600 \mathrm{mg} / \mathrm{kg}$ when administered undiluted (Hine and Zuidema 
1970). It appears that the absorption of xylene was enhanced by corn oil due to its greater lipophilicity. The acute oral $\mathrm{LD}_{50}$ values for mixed xylene administered in corn oil to male and female mice were 5,627 and 5,251 mg/kg, respectively (NTP 1986). The acute oral $\mathrm{LD}_{50}$ for $m$-xylene (neat) in rats was $6,661 \mathrm{mg} / \mathrm{kg}$ (Smyth et al. 1962). According to a study by Gerarde (1959), $m$-xylene may be slightly less toxic than the other two isomers, since a single oral dose of $4,320 \mathrm{mg} / \mathrm{kg}$ of $\mathrm{m}$-xylene resulted in death in $3 / 10$ rats, whereas $4,400 \mathrm{mg} / \mathrm{kg}$ of $o$-xylene or $4,305 \mathrm{mg} / \mathrm{kg}$ of $p$-xylene produced death in $7 / 10$ and $6 / 10$ rats, respectively. In acute-duration repeated-dose gavage studies, mortality was observed in 8 of 10 rats given $2,000 \mathrm{mg} / \mathrm{kg}$ mixed xylene and 10 of 10 mice given $4,000 \mathrm{mg} / \mathrm{kg}$ mixed xylene in corn oil for 14 days (NTP 1986) and in 2 females from a group of 10 male and 10 female rats that received $2,000 \mathrm{mg} / \mathrm{kg} / \mathrm{day} p$-xylene for 10 days (Condie et al. 1988).

Survival was significantly lowered in male rats exposed to mixed xylene 5 days/week at chronic oral doses of $500 \mathrm{mg} / \mathrm{kg}$ but not at $250 \mathrm{mg} / \mathrm{kg}$ (NTP 1986); females were not affected. Although mortality appeared to be dose related in the treated rats, many of the early deaths were related to an error in gavage methodology; non-accidental deaths occurred with an incidence of $22 \%$ in controls, $32 \%$ at $250 \mathrm{mg} / \mathrm{kg} / \mathrm{day}$, and $38 \%$ at $500 \mathrm{mg} / \mathrm{kg} /$ day.

All $\mathrm{LD}_{50}$ values and LOAEL values from each reliable study for death in each species and duration category are recorded in Table 3-2 and plotted in Figure 3-2.

\subsubsection{Systemic Effects}

The systemic effects observed after oral exposure to xylene are discussed below. The highest NOAEL value and all LOAEL values from each reliable study for systemic effects in each species and duration category are recorded in Table 3-2 and plotted in Figure 3-2.

Respiratory Effects. Limited information was located regarding respiratory effects in humans following oral exposure to mixed xylene or xylene isomers. Postmortem examination of a man who committed suicide by ingesting xylene showed pulmonary congestion and edema (Abu Al Ragheb et al. 1986). Death resulted from centrally mediated respiratory depression.

A single oral dose of $4,000 \mathrm{mg} / \mathrm{kg}$ in mice or daily oral dosing of rats and mice by gavage with mixed xylene for 14 days at $2,000 \mathrm{mg} / \mathrm{kg} /$ day resulted in shallow and labored breathing immediately after dosing, but no compound-related effects were observed in the lungs at necropsy (NTP 1986). Mice given 
Table 3-2 Levels of Significant Exposure to Xylene - Oral

\begin{tabular}{|c|c|c|c|c|c|c|c|c|}
\hline \multirow[b]{2}{*}{$\begin{array}{l}\text { Key to } \\
\text { Figure }\end{array}$} & \multirow[b]{2}{*}{$\begin{array}{c}\text { Species } \\
\text { (Strain) }\end{array}$} & \multirow{2}{*}{$\begin{array}{l}\text { Exposurel } \\
\text { Durationl } \\
\text { Frequency } \\
\text { (Route) }\end{array}$} & \multirow[b]{2}{*}{ System } & \multirow[b]{2}{*}{$\begin{array}{l}\text { NOAEL } \\
\text { (mg/kg/day) }\end{array}$} & \multicolumn{2}{|c|}{ LOAEL } & \multirow[b]{2}{*}{$\begin{array}{l}\text { Reference } \\
\text { Chemical Form }\end{array}$} & \multirow[b]{2}{*}{ Comments } \\
\hline & & & & & $\begin{array}{r}\text { Less Serious } \\
\text { (mg/kg/day) }\end{array}$ & $\begin{array}{l}\text { Serious } \\
\text { (mg/kg/day) }\end{array}$ & & \\
\hline
\end{tabular}

ACUTE EXPOSURE

Death

1 Rat $10 \mathrm{~d}$

Sprague- $\quad 1 \times / d$

Dawley (GO)

2 Rat once

NS (GO)

3 Rat once

NS (GO)

4 Rat once

NS (GO)

5 Rat once

Long- Evans (G)

6 Rat once

Albino-Wistar (GO)

CFT

7 Rat $\quad 14 \mathrm{~d}$

$\begin{array}{ll}\text { F344/N } & 1 \times / d \\ & (G O)\end{array}$

8 Rat once

F344/N (GO)

9 Rat once

Carworth- (G)

Wistar
$2000 \mathrm{~F}$ (2/20 died)

4305

(6/10 rats died)

4400

(7/10 died)

432

(3/10 died)

8640 M (LD50)

$5950 \mathrm{~F}$ (4/6 died)

$2000 \quad$ (8/10 died)

3523 M (LD50)

6661 M (LD50)
Condie et al. 1988

para

Gerarde 1959

para

Gerarde 1959

ortho

Gerarde 1959

meta

Hine and Zuidema 1970

mixed

Muralidhara and Krishnakumari

1980

mixed

NTP 1986

mixed

NTP 1986

mixed

Smyth et al. 1962

meta 
Table 3-2 Levels of Significant Exposure to Xylene - Oral

(continued)

\begin{tabular}{|c|c|c|c|c|c|c|c|}
\hline \multirow[b]{2}{*}{$\begin{array}{l}\text { Key to } \\
\text { Figure }\end{array}$} & \multirow[b]{2}{*}{$\begin{array}{l}\text { Species } \\
\text { (Strain) }\end{array}$} & \multirow{2}{*}{$\begin{array}{l}\text { Exposurel } \\
\text { Duration/ } \\
\text { Frequency } \\
\text { (Route) }\end{array}$} & \multirow[b]{2}{*}{ System } & \multirow[b]{2}{*}{$\begin{array}{l}\text { NOAEL } \\
\text { (mg/kg/day) }\end{array}$} & \multicolumn{3}{|c|}{ LOAEL } \\
\hline & & & & & $\begin{array}{r}\text { Less Serious } \\
\text { (mg/kg/day) }\end{array}$ & $\begin{array}{l}\text { Serious } \\
\text { (mg/kg/day) }\end{array}$ & $\begin{array}{l}\text { Reference } \\
\text { Chemical }\end{array}$ \\
\hline 10 & $\begin{array}{l}\text { Mouse } \\
\text { B6C3F1 }\end{array}$ & $\begin{array}{l}\text { once } \\
\text { (GO) }\end{array}$ & & & & $\begin{array}{l}5627 \mathrm{M}(\mathrm{LD} 50) \\
\mathrm{b} \\
5251 \mathrm{~F}(\mathrm{LD} 50)\end{array}$ & $\begin{array}{l}\text { NTP } 1986 \\
\text { mixed }\end{array}$ \\
\hline 11 & $\begin{array}{l}\text { Mouse } \\
\text { B6C3F1 }\end{array}$ & $\begin{array}{l}14 \mathrm{~d} \\
1 \times / d \\
(\mathrm{GO})\end{array}$ & & & & (10/10 died) & $\begin{array}{l}\text { NTP } 1986 \\
\text { mixed }\end{array}$ \\
\hline
\end{tabular}

Systemic

$\begin{array}{llll}\begin{array}{l}\text { Rat } \\ \begin{array}{l}\text { Sprague- } \\ \text { Dawley }\end{array}\end{array} & \begin{array}{l}10 \mathrm{~d} \\ 1 \mathrm{x} / \mathrm{d}\end{array} & \text { Hemato } & 2000 \\ & (\mathrm{GO}) & & \\ & & \text { Renal } & 2000 \\ & & \text { Bd Wt } & 2000\end{array}$

$13 \begin{array}{lll}\text { Rat } & 10 & \text { Hemato } \\ \text { Sprague- } & 1 \mathrm{x} / \mathrm{d} & \end{array}$

$\begin{array}{lll}\text { Sprague- } & 1 \times / d & \text { Hemato } \\ \text { Dawley } & (\mathrm{GO}) & \end{array}$

Renal 2000

Bd Wt 1000

$2000 \mathrm{M}(13 \%$ decrease in body weight)

14 Rat

$\begin{array}{ll} & 10 \mathrm{~d} \\ \text { Sprague- } & 1 \mathrm{x} / \mathrm{d}\end{array}$

Hemato 2000

Dawley (GO)

Renal $\quad 2000$

Bd Wt $\quad 1000 \quad 2000$ M (14\% decrease in body weight)

Condie et al. 1988 meta
Condie et al. 1988 ortho 
Table 3-2 Levels of Significant Exposure to Xylene - Oral

\begin{tabular}{|c|c|c|c|c|c|c|c|c|}
\hline \multirow[b]{2}{*}{$\begin{array}{l}\text { Key to } \\
\text { Figure }\end{array}$} & \multirow[b]{2}{*}{$\begin{array}{l}\text { Species } \\
\text { (Strain) }\end{array}$} & \multirow{2}{*}{$\begin{array}{l}\text { Exposurel } \\
\text { Duration/ } \\
\text { Frequency } \\
\text { (Route) }\end{array}$} & \multicolumn{5}{|c|}{ LOAEL } & \multirow[b]{2}{*}{ Comments } \\
\hline & & & System & $\begin{array}{l}\text { NOAEL } \\
\text { (mg/kg/day) }\end{array}$ & $\begin{array}{r}\text { Less Serious } \\
\text { (mg/kg/day) }\end{array}$ & $\begin{array}{l}\text { Serious } \\
\text { (mg/kg/day) }\end{array}$ & $\begin{array}{l}\text { Reference } \\
\text { Chemical Form }\end{array}$ & \\
\hline
\end{tabular}

15 Rat once Long- Evans (GO)

16 Rat $\begin{array}{ll}\text { F344/N } & 14 \mathrm{~d} \\ & 1 \mathrm{x} / \mathrm{d} \\ & (\mathrm{GO})\end{array}$

17 Rat SpragueDawley

\section{once}

(GO)
Other

$1000 \mathrm{M}$

2000 M (mild hypothermia)

Resp $\quad 1000$

500

$1000 \mathrm{M}(18 \%$ decrease in body

Resp

\section{$14 d$}

$14 \mathrm{~d}$

(GO)

Resp

$\mathrm{Bd} \mathrm{Wt}$

2000

Immunol Lymphoret

19 Rat $10 \mathrm{~d}$

Sprague- $1 \mathrm{x} / \mathrm{d}$

Dawley

(GO)

Neurological

20 Rat

once

Long- Evans (GO)

(mg/kg/day)

Comments

weight gain in males)

Dyer et al. 1988

para

$2000 \quad \begin{aligned} & \text { (shallow and labored } \\ & \text { breathing) }\end{aligned}$

mixed

Patel et al. 1978

para

microsomal activity)

2000 (shallow breathing)

NTP 1986

mixed

Condie et al. 1988

para

Dyer et al. 1988

para 
Table 3-2 Levels of Significant Exposure to Xylene - Oral

(continued)

\begin{tabular}{|c|c|c|c|c|c|c|c|c|}
\hline \multirow[b]{2}{*}{$\begin{array}{l}\text { Key to } \\
\text { Figure }\end{array}$} & \multirow[b]{2}{*}{$\begin{array}{c}\text { Species } \\
\text { (Strain) }\end{array}$} & \multirow{2}{*}{$\begin{array}{l}\text { Exposurel } \\
\text { Duration/ } \\
\text { Frequency } \\
\text { (Route) }\end{array}$} & \multirow[b]{2}{*}{ System } & \multirow[b]{2}{*}{$\begin{array}{l}\text { NOAEL } \\
\text { (mg/kg/day) }\end{array}$} & \multicolumn{2}{|c|}{ LOAEL } & \multirow[b]{2}{*}{$\begin{array}{l}\text { Reference } \\
\text { Chemical Form }\end{array}$} & \multirow[b]{2}{*}{ Comments } \\
\hline & & & & & $\begin{array}{r}\text { Less Serious } \\
(\mathrm{mg} / \mathrm{kg} / \mathrm{day})\end{array}$ & $\begin{array}{l}\text { Serious } \\
\text { (mg/kg/day) }\end{array}$ & & \\
\hline
\end{tabular}

21 Rat $2 \mathrm{wk}$

(Sprague- $\quad 5 \mathrm{~d} / \mathrm{w}$

Dawley) $1 \mathrm{x} / \mathrm{d}$

22 Rat $2 w k$

$\begin{array}{ll}\text { (Sprague- } & 5 \mathrm{~d} / \mathrm{wk} \\ & 1 \mathrm{x} / \mathrm{d}\end{array}$

$\begin{array}{ll}\text { Dawley) } & 1 \mathrm{x} / \mathrm{d} \\ & (\mathrm{GO})\end{array}$

23 Rat

$\begin{array}{ll}\text { Rat } & 2 \mathrm{wk} \\ \text { (Sprague- } & 5 \mathrm{~d} / \mathrm{wk}\end{array}$

Dawley) $1 \mathrm{x} / \mathrm{d}$

(GO)

24 Rat

Albino- Wistar (GO)

$25 \quad$ Rat

F344/N (GO)

$26 \begin{array}{ll}\text { Mouse } & 1 \mathrm{x} / \mathrm{d} \\ \text { B6C3F1 } & 5 \mathrm{~d} / \mathrm{wk}\end{array}$

$\begin{array}{ll}\text { B6C3F1 } & 5 \mathrm{~d} / \mathrm{wk} \\ & 13 \mathrm{wk}\end{array}$

(GO)
$900 \mathrm{M}$

$900 \mathrm{M}$ (partial loss of outer hair

$$
\text { cells in cochlea }
$$

responsive to medium

frequencies: $10-25 \mathrm{kHz}$ )

$900 \mathrm{M}$

5950 F (coma)

4000 (decreased hindleg

movement,

incoordination,

prostration)

2000 (weakness, lethargy

unsteadiness, tremors,

and partial paralysis)
Gagnaire and Langlais 2005

meta

Gagnaire and Langlais 2005

para

Gagnaire and Langlais 2005

ortho

Muralidhara and Krishnakumar

1980

mixed

NTP 1986

mixed 
Table 3-2 Levels of Significant Exposure to Xylene - Oral

(continued)

\begin{tabular}{|c|c|c|c|c|c|c|c|}
\hline \multirow[b]{2}{*}{$\begin{array}{l}\text { Key to } \\
\text { Figure }\end{array}$} & \multirow[b]{2}{*}{$\begin{array}{l}\text { Species } \\
\text { (Strain) }\end{array}$} & \multirow{2}{*}{$\begin{array}{l}\text { Exposurel } \\
\text { Durationl } \\
\text { Frequency } \\
\text { (Route) }\end{array}$} & \multirow[b]{2}{*}{ System } & \multirow[b]{2}{*}{$\begin{array}{l}\text { NOAEL } \\
\text { (mg/kg/day) }\end{array}$} & \multicolumn{2}{|c|}{ LOAEL } & \multirow[b]{2}{*}{$\begin{array}{l}\text { Reference } \\
\text { Chemical Form }\end{array}$} \\
\hline & & & & & $\begin{array}{r}\text { Less Serious } \\
\text { (mg/kg/day) }\end{array}$ & $\begin{array}{l}\text { Serious } \\
\text { (mg/kg/day) }\end{array}$ & \\
\hline \multicolumn{8}{|c|}{ Developmental } \\
\hline 27 & $\begin{array}{l}\text { Mouse } \\
\text { CD-1 }\end{array}$ & $\begin{array}{l}10 d \\
\text { Gd 6-15 } \\
3 \times / d\end{array}$ & & $1030 \mathrm{~F}$ & & $2060 \mathrm{~F}$ (cleft palate) & $\begin{array}{l}\text { Marks et al. } 1982 \\
\text { mixed }\end{array}$ \\
\hline & & (GO) & & & & & \\
\hline 28 & $\begin{array}{l}\text { Mouse } \\
\text { ICR/SIM }\end{array}$ & $\begin{array}{l}5 d \\
1 x / d \\
\text { Gd 8-12 }\end{array}$ & & $2000 \mathrm{~F}$ & & & $\begin{array}{l}\text { Seidenberg et al. } 1986 \\
\text { meta }\end{array}$ \\
\hline
\end{tabular}

\section{INTERMEDIATE EXPOSURE}

Systemic

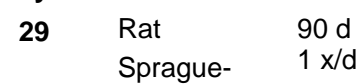

Sprague- $\quad 1 \times / d$

Hemato

$750 \mathrm{~F}$

$1500 \mathrm{~F}$ (mild polycythemia and leukocytosis; increased

spleen weight)

Condie et al. 1988

mixed

$\begin{array}{lll}\text { Hepatic } \quad 150 \quad 750 & \begin{array}{l}\text { (14\% increase in serum } \\ \text { alanine aminotransferase } \\ \text { in females, } 14-17 \% \\ \text { increase in relative liver } \\ \text { weight in both sexes) }\end{array} \\ \text { Renal } \quad 150 \mathrm{~F} \quad 750 \mathrm{~F} & \begin{array}{l}\text { (minimal chronic } \\ \text { nephropathy in } 6 / 10 \\ \text { females) }\end{array} \\ \text { Bd Wt } \quad 1500 & \end{array}$


Table 3-2 Levels of Significant Exposure to Xylene - Oral

(continued)

\begin{tabular}{|c|c|c|c|c|c|}
\hline $\begin{array}{l}\text { Key to } \\
\text { Figure }\end{array}$ & $\begin{array}{l}\text { Species } \\
\text { (Strain) }\end{array}$ & $\begin{array}{l}\text { Exposurel } \\
\text { Durationl } \\
\text { Frequency } \\
\text { (Route) }\end{array}$ & System & $\begin{array}{l}\text { NOAEL } \\
\text { (mg/kg/day) }\end{array}$ & $\begin{array}{r}\text { Less Serious } \\
(\mathrm{mg} / \mathrm{kg} / \mathrm{day})\end{array}$ \\
\hline \multirow[t]{2}{*}{30} & $\begin{array}{l}\text { Rat } \\
\text { NS }\end{array}$ & $\begin{array}{l}3.5 \mathrm{wk} \\
5 \mathrm{~d} / \mathrm{wk} \\
1 \mathrm{x} / \mathrm{d}\end{array}$ & Resp & & $\begin{array}{c}800 \mathrm{M} \text { (decreased cytochrome } \\
\text { P-450) }\end{array}$ \\
\hline & & & Hepatic & & $\begin{array}{l}800 \mathrm{M} \text { (increased plasma SGP } \\
\text { and relative } \\
\text { liver weight) }\end{array}$ \\
\hline
\end{tabular}

31 Rat $13 w k$

$13 \mathrm{wk}$
$5 \mathrm{~d} / \mathrm{wk}$
$1 \mathrm{x} / \mathrm{d}$

Resp $\quad 1000$

LOAEL

F344/N $\quad 1 \mathrm{x} / \mathrm{d}$

(GO)

Serious

Reference

Chemical Form

Comments

\begin{tabular}{|c|c|c|}
\hline Cardio & 1000 & \\
\hline Gastro & 1000 & \\
\hline Hemato & 1000 & \\
\hline Musc/skel & 1000 & \\
\hline Hepatic & 1000 & \\
\hline Renal & 1000 & \\
\hline Ocular & 1000 & \\
\hline $\mathrm{Bd} \mathrm{Wt}$ & $500 \mathrm{M}$ & $\begin{array}{c}1000 \text { M ( } 15 \% \text { decrease in body } \\
\text { weight gain) }\end{array}$ \\
\hline
\end{tabular}

NTP 1986

mixed
Elovaara et al. 1989

meta 
Table 3-2 Levels of Significant Exposure to Xylene - Oral

(continued)

\begin{tabular}{|c|c|c|c|c|c|c|c|c|}
\hline \multirow[b]{2}{*}{$\begin{array}{l}\text { Key to } \\
\text { Figure }\end{array}$} & \multirow[b]{2}{*}{$\begin{array}{c}\text { Species } \\
\text { (Strain) }\end{array}$} & \multirow{2}{*}{$\begin{array}{l}\text { Exposurel } \\
\text { Duration/ } \\
\text { Frequency } \\
\text { (Route) }\end{array}$} & \multirow[b]{2}{*}{ System } & \multirow[b]{2}{*}{$\begin{array}{l}\text { NOAEL } \\
\text { (mg/kg/day) }\end{array}$} & \multicolumn{2}{|c|}{ LOAEL } & \multirow[b]{2}{*}{$\begin{array}{l}\text { Reference } \\
\text { Chemical Form }\end{array}$} & \multirow[b]{2}{*}{ Comments } \\
\hline & & & & & $\begin{array}{r}\text { Less Serious } \\
\text { (mg/kg/day) }\end{array}$ & $\begin{array}{l}\text { Serious } \\
\text { (mg/kg/day) }\end{array}$ & & \\
\hline
\end{tabular}

$32 \quad$ Rat 13 wk

Sprague- $\quad 7 \mathrm{~d} / \mathrm{wk}$

Resp

800

Wolfe 1988a

$\begin{array}{ll}\text { Dawley } & 1 \mathrm{x} / \mathrm{d} \\ & (\mathrm{GO})\end{array}$

meta

\begin{tabular}{|c|c|c|}
\hline Cardio & 800 & \\
\hline Gastro & 800 & \\
\hline Hemato & 800 & \\
\hline Musc/skel & 800 & \\
\hline Hepatic & $800 \mathrm{~F}$ & $\begin{array}{c}800 \mathrm{M}(37 \% \text { increased serum } \\
\text { alanine } \\
\text { aminotransferase })\end{array}$ \\
\hline Renal & 800 & \\
\hline Dermal & 800 & \\
\hline Ocular & 800 & \\
\hline $\mathrm{Bd} \mathrm{Wt}$ & $200 \mathrm{M}$ & $\begin{array}{l}800 \mathrm{M} \text { (body weight gain } \\
\text { decreased by } 15 \%)\end{array}$ \\
\hline
\end{tabular}


Table 3-2 Levels of Significant Exposure to Xylene - Oral

(continued)

\begin{tabular}{|c|c|c|c|c|c|c|c|c|}
\hline \multirow[b]{2}{*}{$\begin{array}{l}\text { Key to } \\
\text { Figure }\end{array}$} & \multirow[b]{2}{*}{$\begin{array}{c}\text { Species } \\
\text { (Strain) }\end{array}$} & \multirow{2}{*}{$\begin{array}{l}\text { Exposurel } \\
\text { Duration/ } \\
\text { Frequency } \\
\text { (Route) }\end{array}$} & \multirow[b]{2}{*}{ System } & \multirow[b]{2}{*}{$\begin{array}{l}\text { NOAEL } \\
\text { (mg/kg/day) }\end{array}$} & \multicolumn{2}{|c|}{ LOAEL } & \multirow[b]{2}{*}{$\begin{array}{l}\text { Reference } \\
\text { Chemical Form }\end{array}$} & \multirow[b]{2}{*}{ Comments } \\
\hline & & & & & $\begin{array}{r}\text { Less Serious } \\
\text { (mg/kg/day) }\end{array}$ & $\begin{array}{l}\text { Serious } \\
\text { (mg/kg/day) }\end{array}$ & & \\
\hline
\end{tabular}

33 Rat

$\begin{array}{ll}\text { Rat } & 13 \mathrm{wk} \\ \text { Sprague- } & 7 \mathrm{~d} / \mathrm{wk}\end{array}$

Dawley $\quad 1 \mathrm{x} / \mathrm{d}$

(GO)

Resp

800

$\begin{array}{ll}\text { Cardio } & 800 \\ \text { Gastro } & 800 \\ \text { Hemato } & 800 \\ \text { Musc/skel } & 800 \\ \text { Hepatic } & 800 \\ \text { Renal } & 800 \\ \text { Dermal } & 800 \\ \text { Ocular } & 800 \\ \text { Bd Wt } & 800\end{array}$

34

\begin{tabular}{|c|c|c|c|}
\hline \multirow[t]{8}{*}{$\begin{array}{l}\text { Mouse } \\
\text { B6C3F1 }\end{array}$} & $\begin{array}{l}13 \mathrm{wk} \\
5 \mathrm{~d} / \mathrm{wk} \\
1 \mathrm{x} / \mathrm{d} \\
(\mathrm{GO})\end{array}$ & Cardio & 2000 \\
\hline & & Gastro & 2000 \\
\hline & & Hemato & 2000 \\
\hline & & Musc/skel & 2000 \\
\hline & & Hepatic & 2000 \\
\hline & & Renal & 2000 \\
\hline & & Ocular & 2000 \\
\hline & & $\mathrm{Bd} W \mathrm{t}$ & 2000 \\
\hline
\end{tabular}

Wolfe $1988 b$

para
NTP 1986

mixed 
Table 3-2 Levels of Significant Exposure to Xylene - Oral

(continued)

\begin{tabular}{|c|c|c|c|c|c|c|c|c|}
\hline \multirow[b]{2}{*}{$\begin{array}{l}\text { Key to } \\
\text { Figure }\end{array}$} & \multirow[b]{2}{*}{$\begin{array}{l}\text { Species } \\
\text { (Strain) }\end{array}$} & \multirow{2}{*}{$\begin{array}{l}\text { Exposurel } \\
\text { Duration/ } \\
\text { Frequency } \\
\text { (Route) }\end{array}$} & \multicolumn{5}{|c|}{ LOAEL } & \multirow[b]{2}{*}{ Comments } \\
\hline & & & System & $\begin{array}{l}\text { NOAEL } \\
\text { (mg/kg/day) }\end{array}$ & $\begin{array}{r}\text { Less Serious } \\
\text { (mg/kg/day) }\end{array}$ & $\begin{array}{l}\text { Serious } \\
\text { (mg/kg/day) }\end{array}$ & $\begin{array}{l}\text { Reference } \\
\text { Chemical Form }\end{array}$ & \\
\hline
\end{tabular}

Neurological

35

$\begin{array}{ll}\text { Rat } & 90 \mathrm{~d} \\ \text { Sprague- } & 1 \mathrm{x} / \mathrm{d}\end{array}$

Dawley

(GO)

$750 \mathrm{M}$

$1500 \mathrm{M}$ (increased

aggressiveness)

Condie et al. 1988

mixed

36 Rat

$\begin{array}{ll}\text { Rat } & 13 \mathrm{wk} \\ \text { F344/N } & 5 \mathrm{~d} / \mathrm{wk} \\ & 1 \mathrm{x} / \mathrm{d}\end{array}$

1000

NTP 1986

$1 \mathrm{x} / \mathrm{d}$

(GO)

$37 \quad$ Rat $13 w k$

Sprague- $\quad 7 \mathrm{~d} / \mathrm{wk}$

$\begin{array}{ll}\text { Dawley } & 1 \mathrm{x} / \mathrm{d} \\ & (\mathrm{GO})\end{array}$

800

Wolfe 1988a

meta

Wolfe 1988b

para

Sprague- $\quad 7 \mathrm{~d} / \mathrm{wk}$

$\begin{array}{ll}\text { Dawley } & 1 \mathrm{x} / \mathrm{d} \\ & (\mathrm{GO})\end{array}$

800

$39 \begin{array}{lll}\text { Mouse } & 103 \mathrm{wk} \\ \text { B6C3F1 } & 5 \mathrm{~d} / \mathrm{wk}\end{array}$

(GO)

$500^{d}$

1000 (hyperactivity weeks

4-52)

NTP 1986

mixed

Reproductive

40 Rat $13 \mathrm{wk}$

F344/N $\quad \begin{array}{ll}5 \mathrm{~d} / \mathrm{wk} \\ 1 \mathrm{x} / \mathrm{d}\end{array}$

$1 \mathrm{x} / \mathrm{d}$

1000

NTP 1986

mixed

$41 \quad$ Rat $\quad 13 w k$

$\begin{array}{ll}\text { Sprague- } & 7 \mathrm{~d} / \mathrm{wk} \\ \text { Dawley } & 1 \mathrm{x} / \mathrm{d}\end{array}$

800

Wolfe $1988 \mathrm{a}$

meta 
Table 3-2 Levels of Significant Exposure to Xylene - Oral

\begin{tabular}{cc}
\hline & $\begin{array}{c}\text { Exposurel } \\
\text { Durationl }\end{array}$ \\
Key to Species & $\begin{array}{c}\text { Frequency } \\
\text { (Route) }\end{array}$
\end{tabular}

Exposurel
Durationl

Figure (Strain) (Route)

42 Rat

$\begin{array}{ll}\text { Rat } & 13 \mathrm{wk} \\ \text { Sprague- } & 7 \mathrm{~d} / \mathrm{wk}\end{array}$

Dawley $\quad 1 \mathrm{x} / \mathrm{d}$

(GO)

43 Mouse

$13 \mathrm{wk}$
$5 \mathrm{~d} / \mathrm{wk}$

$1 \mathrm{x} / \mathrm{d}$

(GO)

\section{CHRONIC EXPOSURE}

\section{Death}

44 Rat

$\begin{array}{ll}\text { Rat } & 103 \mathrm{wk} \\ \text { F344/N } & 5 \mathrm{~d} / \mathrm{wk} \\ & 1 \mathrm{x} / \mathrm{d} \\ & (\mathrm{GO})\end{array}$

LOAEL

NOAEL Less Serious

(mg/kg/day)

800

Wolfe $1988 b$

para

NTP 1986

mixed

$500 \mathrm{M}$ (survival decreased by

16\%)

NTP 1986

mixed 
Table 3-2 Levels of Significant Exposure to Xylene - Oral

(continued)

\begin{tabular}{|c|c|c|c|c|c|c|c|c|}
\hline \multirow[b]{2}{*}{$\begin{array}{l}\text { Key to } \\
\text { Figure }\end{array}$} & \multirow[b]{2}{*}{$\begin{array}{c}\text { Species } \\
\text { (Strain) }\end{array}$} & \multirow{2}{*}{$\begin{array}{l}\text { Exposurel } \\
\text { Durationl } \\
\text { Frequency } \\
\text { (Route) }\end{array}$} & \multirow[b]{2}{*}{ System } & \multirow[b]{2}{*}{$\begin{array}{l}\text { NOAEL } \\
\text { (mg/kg/day) }\end{array}$} & \multicolumn{2}{|c|}{ LOAEL } & \multirow[b]{2}{*}{$\begin{array}{l}\text { Reference } \\
\text { Chemical Form }\end{array}$} & \multirow[b]{2}{*}{ Comments } \\
\hline & & & & & $\begin{array}{r}\text { Less Serious } \\
\text { (mg/kg/day) }\end{array}$ & $\begin{array}{l}\text { Serious } \\
\text { (mg/kg/day) }\end{array}$ & & \\
\hline
\end{tabular}

Systemic

$\begin{array}{llll}\text { Rat } & \begin{array}{l}103 \mathrm{wk} \\ 5 \mathrm{~d} / \mathrm{wk} \\ 1 \mathrm{x} / \mathrm{d} \\ (\mathrm{GO})\end{array} & \text { Resp } & 500 \\ & & \\ & \text { Cardio } & 500 \\ & \text { Gastro } & 500 \\ & \text { Hemato } & 500 & \\ & \text { Musc/skel } & 500 & \\ & \text { Hepatic } & 500 & \\ & \text { Renal } & 500 & \\ & \text { Dermal } & 500 & 500 \text { M (body weight decreased } \\ & \text { Ocular } & 500 & \text { 5-8\% after week 59) }\end{array}$

NTP 1986

mixed 
Table 3-2 Levels of Significant Exposure to Xylene - Oral

(continued)

\begin{tabular}{|c|c|c|c|c|c|c|c|c|}
\hline \multirow[b]{2}{*}{$\begin{array}{l}\text { Key to } \\
\text { Figure }\end{array}$} & \multirow[b]{2}{*}{$\begin{array}{c}\text { Species } \\
\text { (Strain) }\end{array}$} & \multirow{2}{*}{$\begin{array}{l}\text { Exposurel } \\
\text { Duration/ } \\
\text { Frequency } \\
\text { (Route) }\end{array}$} & \multirow[b]{2}{*}{ System } & \multirow[b]{2}{*}{$\begin{array}{l}\text { NOAEL } \\
\text { (mg/kg/day) }\end{array}$} & \multicolumn{2}{|c|}{ LOAEL } & \multirow[b]{2}{*}{$\begin{array}{l}\text { Reference } \\
\text { Chemical Form }\end{array}$} & \multirow[b]{2}{*}{ Comments } \\
\hline & & & & & $\begin{array}{r}\text { Less Serious } \\
(\mathrm{mg} / \mathrm{kg} / \mathrm{day})\end{array}$ & $\begin{array}{l}\text { Serious } \\
\text { (mg/kg/day) }\end{array}$ & & \\
\hline
\end{tabular}

46

$\begin{array}{llll}\text { Mouse } & \begin{array}{l}103 \mathrm{wk} \\ 5 \mathrm{~d} / \mathrm{wk} \\ 1 \mathrm{x} / \mathrm{d} \\ (\mathrm{GO})\end{array} & \text { Resp } & 1000 \\ & & & \\ & & & \\ & & & \\ & & \text { Gardio } & 1000 \\ & & \text { Hemato } & 1000 \\ & & \text { Musc/skel } & 1000 \\ & \text { Hepatic } & 1000 \\ & & \text { Renal } & 1000 \\ & & \text { Dermal } & 1000 \\ & & \text { Ocular } & 1000 \\ & & \text { Bd Wt } & 1000\end{array}$

NTP 1986

mixed

Neurological

47 Rat

$103 \mathrm{wk}$

$5 \mathrm{~d} / \mathrm{wk}$

$500^{e}$

NTP 1986

$1 \mathrm{x} / \mathrm{d}$

(GO)

Reproductive

48 Rat

$\begin{array}{ll}\text { Rat } & 103 \mathrm{wk} \\ \text { F344/N } & 5 \mathrm{~d} / \mathrm{wk} \\ & 1 \mathrm{x} / \mathrm{d} \\ & \text { (GO) }\end{array}$

$103 w k$

$5 \mathrm{~d} / \mathrm{wk}$

500

NTP 1986

mixed

(GO) 
Table 3-2 Levels of Significant Exposure to Xylene - Oral

(continued)

\begin{tabular}{|c|c|c|c|c|c|c|c|c|}
\hline \multirow[b]{2}{*}{$\begin{array}{l}\text { Key to } \\
\text { Figure }\end{array}$} & \multirow[b]{2}{*}{$\begin{array}{c}\text { Species } \\
\text { (Strain) }\end{array}$} & \multirow{2}{*}{$\begin{array}{l}\text { Exposurel } \\
\text { Duration/ } \\
\text { Frequency } \\
\text { (Route) }\end{array}$} & \multirow[b]{2}{*}{ System } & \multirow[b]{2}{*}{$\begin{array}{l}\text { NOAEL } \\
\text { (mg/kg/day) }\end{array}$} & \multicolumn{2}{|c|}{ LOAEL } & \multirow[b]{2}{*}{$\begin{array}{l}\text { Reference } \\
\text { Chemical Form }\end{array}$} & \multirow[b]{2}{*}{ Comments } \\
\hline & & & & & $\begin{array}{r}\text { Less Serious } \\
(\mathrm{mg} / \mathrm{kg} / \mathrm{day})\end{array}$ & $\begin{array}{l}\text { Serious } \\
\text { (mg/kg/day) }\end{array}$ & & \\
\hline
\end{tabular}

$49 \quad$ Mouse

$$
103 w k
$$

$\begin{array}{ll}\text { B6C3F1 } & 5 \mathrm{~d} / \mathrm{wk} \\ & 1 \mathrm{x} / \mathrm{d}\end{array}$

NTP 1986

(GO)

mixed

a The number corresponds to the entries in Figure 3-2.

b Differences in levels of health effects and cancer effects between male and females are not indicated in Figure 3-2. Where such differences exist, only the levels of effect for the most sensitive gender are presented.

c Used to derive an acute-duration minimal risk level (MRL) for mixed xylenes based on a NOAEL of $125 \mathrm{mg} / \mathrm{kg} / \mathrm{day} \mathrm{p}$-xylene. The NOAEL was divided by an uncertainty factor of 100 (10 for extrapolation from animals to humans and 10 for human variability)

d Used to derive an intermediate-duration minimal risk level (MRL) for mixed xylenes based on a NOAEL of $500 \mathrm{mg} / \mathrm{kg}$ and a LOAEL of $1000 \mathrm{mg} / \mathrm{kg}$ for mice exposed to mixed xylenes 5 days/week. The NOAEL was adjusted for intermittent exposure ( 5 days/7 days) and divided by an uncertainty factor of 100 (10 for extrapolation from animals to humans and 10 for human variability) and a modifying factor of 10 to account for the lack of testing for sensitive neurological endpoints and lack of developmental and multi-generational data.

e Used to derive a chronic-duration minimal risk level (MRL) for mixed xylenes based on a NOAEL of $250 \mathrm{mg} / \mathrm{kg}$ in rats exposed to mixed xylenes 5 days/week. At $500 \mathrm{mg} / \mathrm{kg}$, a decrease in survival was observed, thus the next lowest dose was selected as the basis of the MRL. The NOAEL of $250 \mathrm{mg} / \mathrm{kg} / \mathrm{was}$ adjusted for intermittent exposure (5 days/7 days) and divided by an uncertainty factor of 100 (10 for extrapolation from animals to humans and 10 for human variability) and a modifying factor of 10 to account for the lack of testing for sensitive neurological endpoints and lack of developmental and multi-generational data.

Bd Wt = body weight; Cardio = cardiovascular; d = day(s); F = Female; Gastro = gastrointestinal; Gd = gestational day; hemato = hematological; LD50 = lethal dose, 50\% kill; LOAEL = lowest-observed-adverse-effect level; $\mathrm{M}$ = male; Musc/skel = musculoskeletal; NOAEL = no-observed-adverse-effect level; Resp = respiratory; SGPT = serum glutamic pyruvic transaminase; $\mathrm{x}=$ time(s); wk = week(s) 
Figure 3-2 Levels of Significant Exposure to Xylene - Oral

Acute ( $\leq 14$ days)

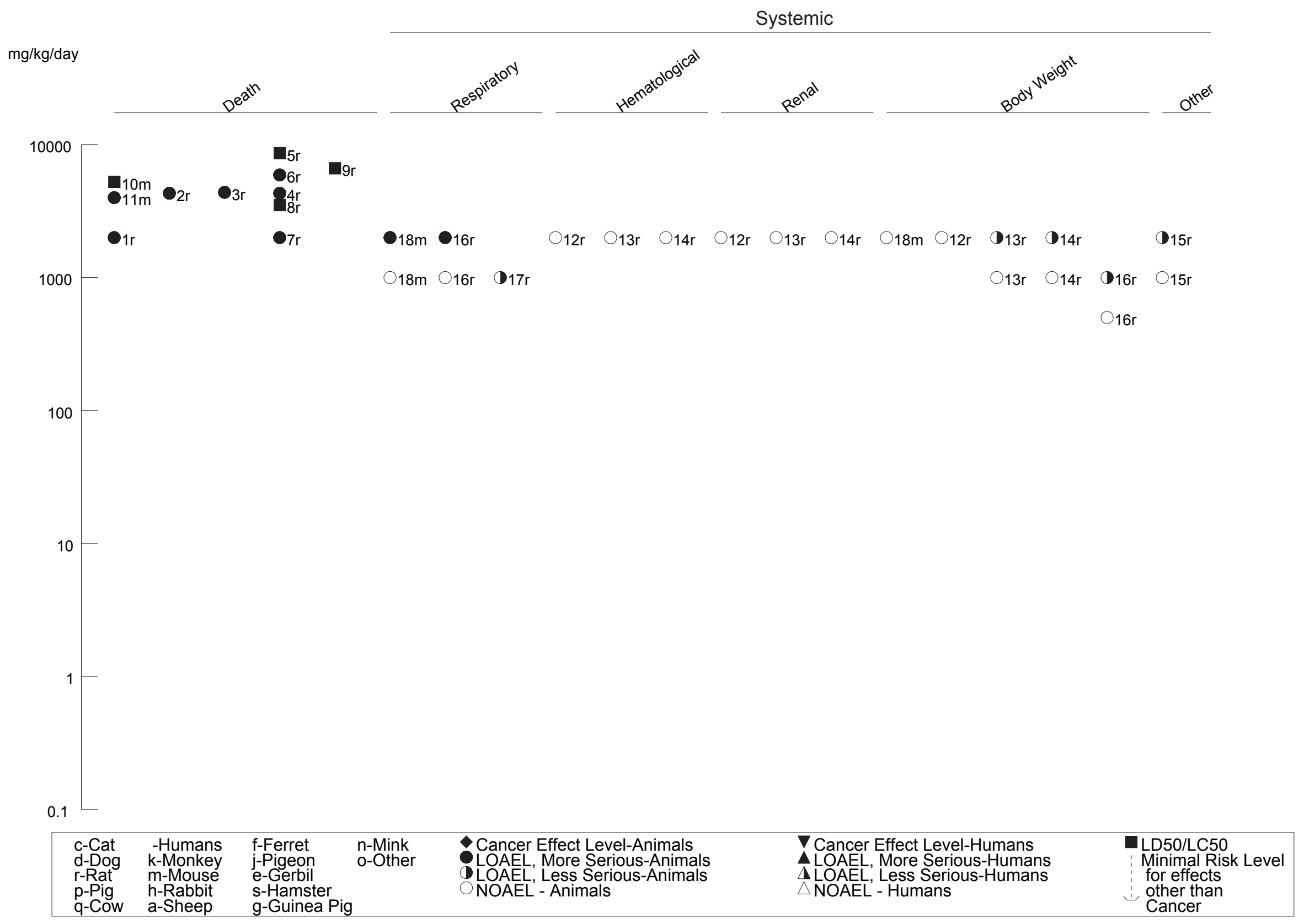


Figure 3-2 Levels of Significant Exposure to Xylene - Oral (Continued)

Acute ( $\leq 14$ days)

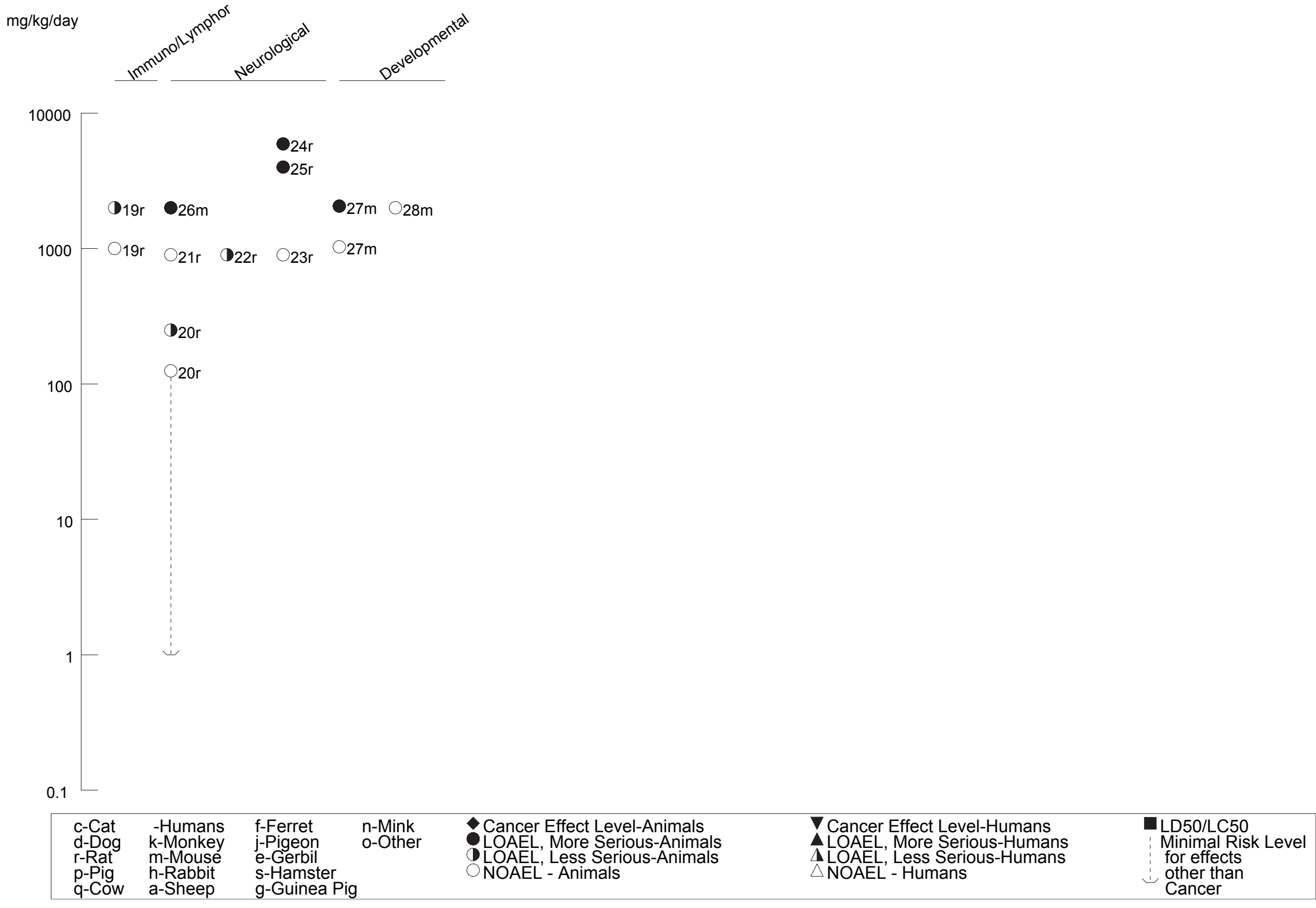


Figure 3-2 Levels of Significant Exposure to Xylene - Oral (Continued)

Intermediate (15-364 days)

Systemic

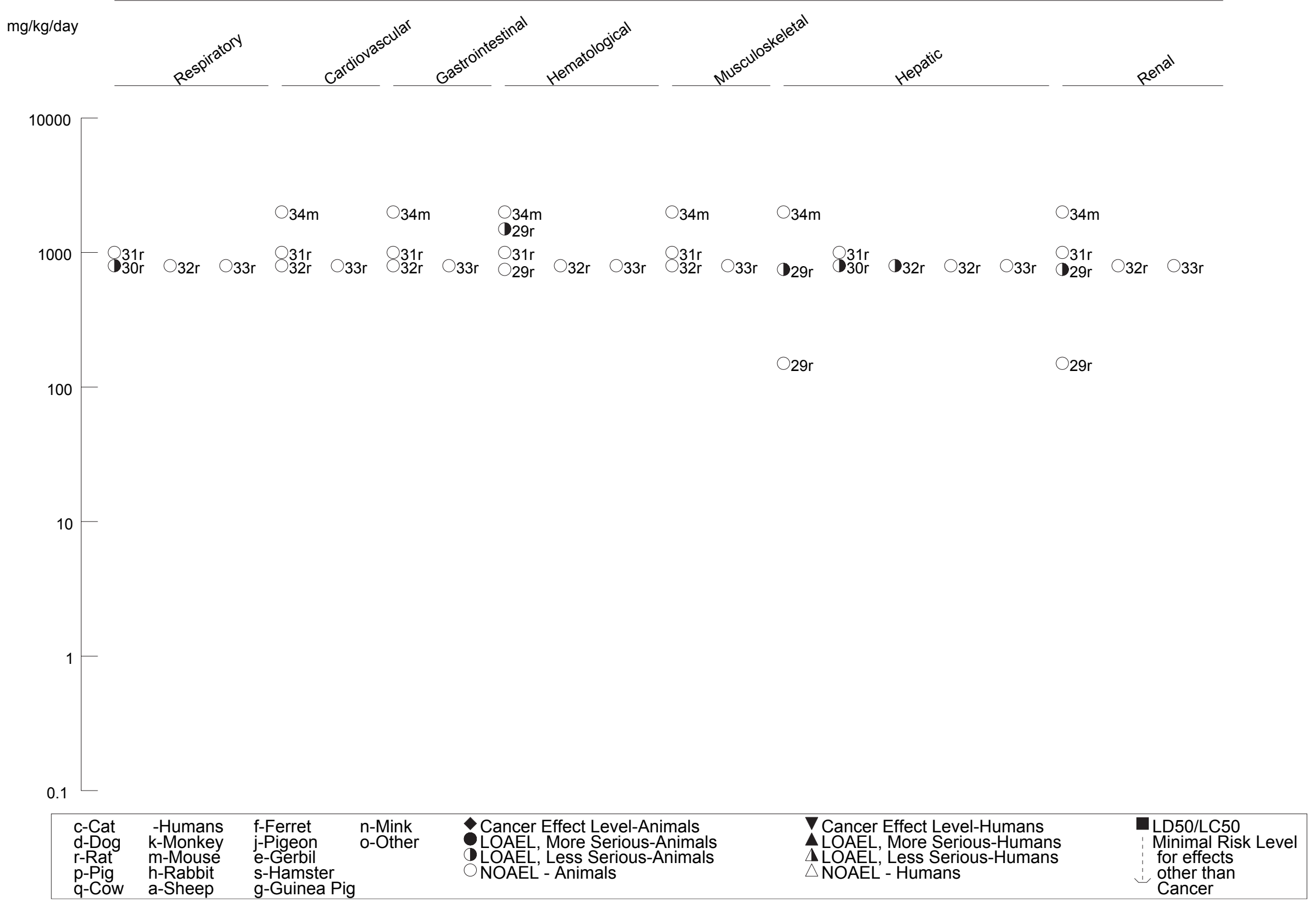


Figure 3-2 Levels of Significant Exposure to Xylene - Oral (Continued)

Intermediate (15-364 days)

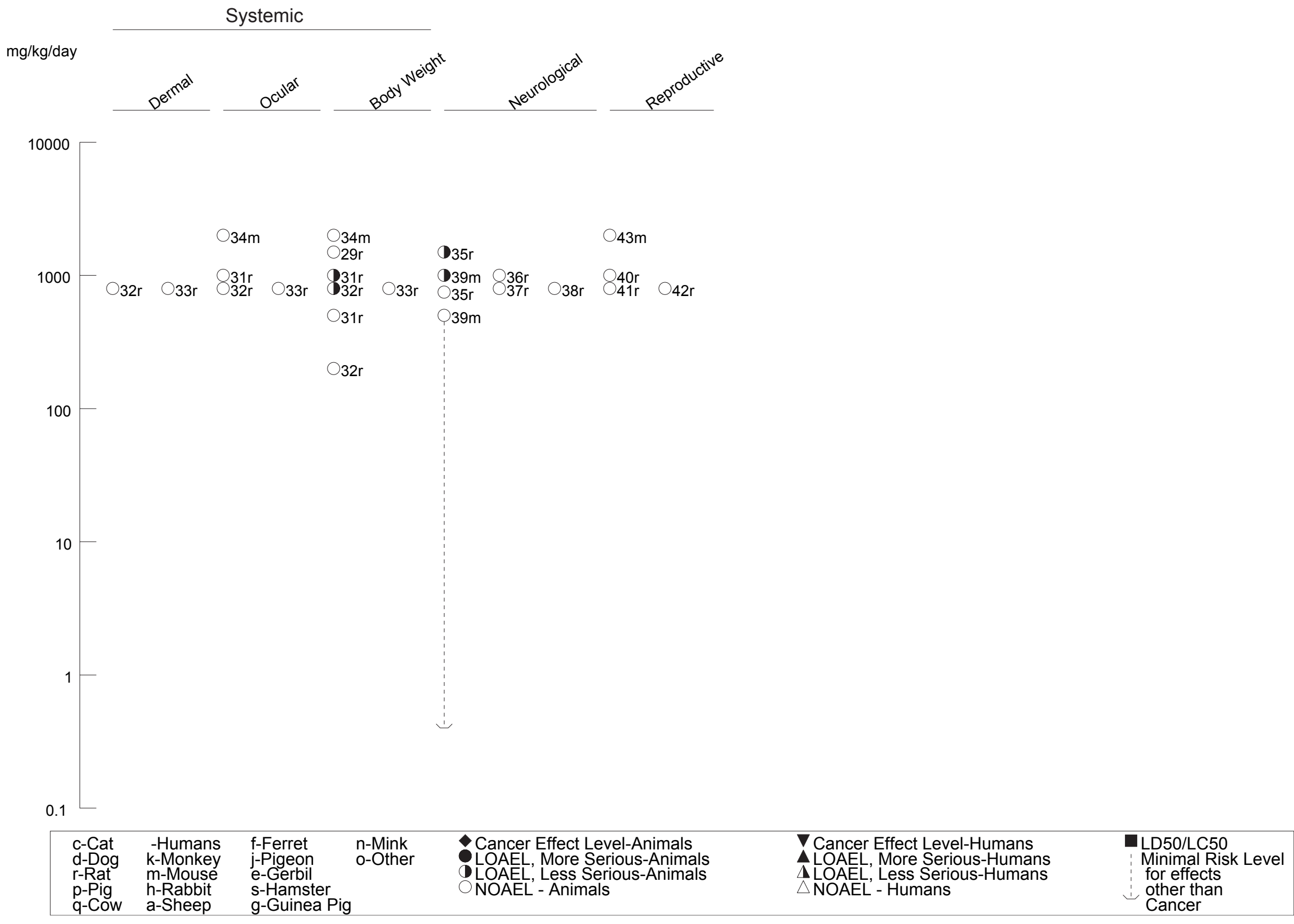


Figure 3-2 Levels of Significant Exposure to Xylene - Oral (Continued)

Chronic ( $\geq 365$ days)

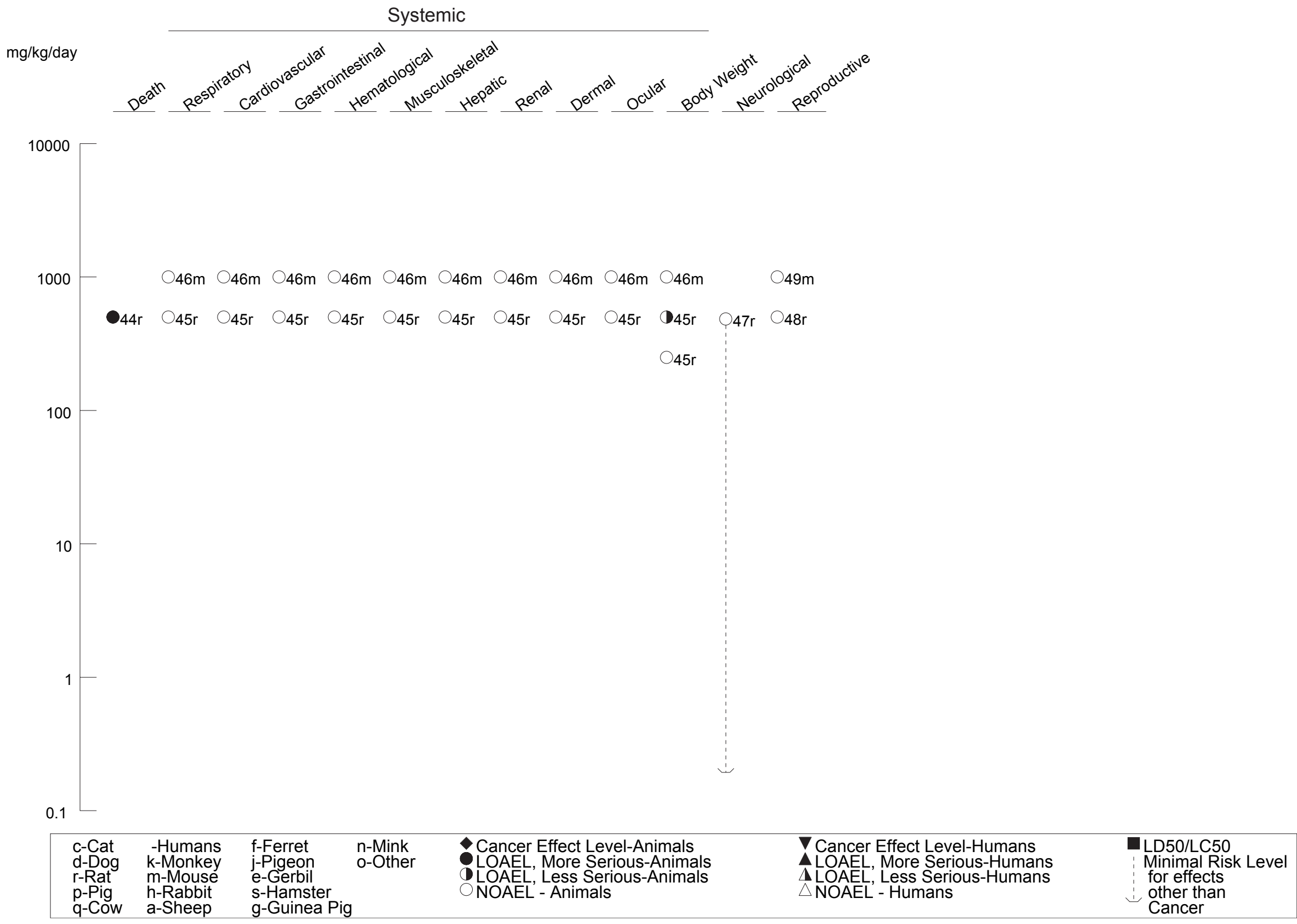


daily oral doses of 2,000 mg/kg/day, 5 days/week, for 13 weeks exhibited similar effects 15-60 minutes after dosing (NTP 1986). Histopathological examination of the lungs and mainstem bronchi of rats and mice administered mixed xylene 5 days/week at doses as high as $1,000 \mathrm{mg} / \mathrm{kg}$ in rats and 2,000 $\mathrm{mg} / \mathrm{kg}$ in mice for 13 weeks or $500 \mathrm{mg} / \mathrm{kg}$ in rats and $1,000 \mathrm{mg} / \mathrm{kg}$ in mice for up to 2 years revealed no adverse effects (NTP 1986). Gross and histopathological examination of rats administered $m$ - or $p$-xylene for 13 weeks at doses as high as $800 \mathrm{mg} / \mathrm{kg} /$ day revealed no treatment-related effects (Wolfe 1988a, 1988b).

Decreased pulmonary microsomal enzyme activity was observed in rats after a single oral dose of $1,000 \mathrm{mg} / \mathrm{kg}$ of $p$-xylene (Patel et al. 1978) and decreased pulmonary cytochrome P-450 content were observed in rats after gavage dosing with $800 \mathrm{mg} / \mathrm{kg}$ /day, 5 days/week, for 3 weeks (Elovaara et al. 1989), suggesting some direct toxicity of xylene in the lungs. Selective inactivation of enzymes can result in damage to tissue caused by the toxic metabolite of xylene, a methylbenzaldehyde (Carlone and Fouts 1974; Patel et al. 1978; Smith et al. 1982). The formation of the methylbenzaldehydes has not been confirmed in humans.

Cardiovascular Effects. Limited information was located regarding cardiovascular effects in humans following oral exposure to mixed xylene or its isomers. Postmortem examination showed no adverse effects on the heart or coronary arteries of a man who committed suicide by ingesting a large but unknown quantity of xylene (Abu Al Ragheb et al. 1986). No adverse cardiovascular effects were noted following histopathological examination of the heart in rats and mice exposed 5 days/week to mixed xylene at $\approx 63-2,000 \mathrm{mg} / \mathrm{kg}$ for 13 or 103 weeks (NTP 1986). No treatment-related effects were noted upon gross or histopathological examination of the heart in rats administered $m$ - or $p$-xylene at doses as high as $800 \mathrm{mg} / \mathrm{kg} /$ day for 13 weeks (Wolfe 1988a, 1988b).

Gastrointestinal Effects. No superficial erosions, deep ulcerations, or other lesions were observed during postmortem examination of the gastric mucosa of a person who died following ingestion of a "large quantity" of xylene (Abu Al Ragheb et al. 1986). Histopathological examination of rats administered doses 5 days/week as high as $1,000 \mathrm{mg} / \mathrm{kg}$ of mixed xylene and mice administered doses as high as $2,000 \mathrm{mg} / \mathrm{kg}$ of mixed xylene for 13 weeks or in rats and mice administered doses as high as 500 and $1,000 \mathrm{mg} / \mathrm{kg}$, respectively, for 2 years revealed no adverse effects on the stomach, small intestine, or colon (NTP 1986). Administration of $p$-xylene up to $800 \mathrm{mg} / \mathrm{kg} / \mathrm{day}$ for 13 weeks also had no significant effect on gastrointestinal organs of rats (Wolfe 1988a, 1988b). 
Hematological Effects. No studies were located regarding hematological effects in humans following oral exposure to mixed xylene or xylene isomers. Exposure of rats to 2,000 mg/kg/day p-xylene for 10 days resulted in no effects detectable in routine hematological analysis (Condie et al. 1988). Exposure to $o$ - and $m$-xylene at $2,000 \mathrm{mg} / \mathrm{kg} /$ day for 10 days produced a decrease in the spleen weight of male rats (Condie et al. 1988); however, hematological analyses in these rats were normal. Mild polycythemia and leukocytosis in both male and female rats and an increase in spleen weight in females were observed in rats exposed to $1,500 \mathrm{mg} / \mathrm{kg} / \mathrm{day}$ mixed xylene for 90 days (Condie et al. 1988). No effects were observed upon histopathological examination of the bone marrow following exposure to $800 \mathrm{mg} / \mathrm{kg} /$ day of $p$-xylene in rats and mice (Wolfe 1988a), or on administration 5 days/week of $1,000 \mathrm{mg} / \mathrm{kg}$ mixed xylene in rats for 13 weeks (NTP 1986), 2,000 mg/kg mixed xylene in mice for 13 weeks (NTP 1986), or $500 \mathrm{mg} / \mathrm{kg}$ mixed xylene in rats and $1,000 \mathrm{mg} / \mathrm{kg}$ in mice for 2 years (NTP 1986).

Musculoskeletal Effects. No studies were located regarding musculoskeletal effects in humans following oral exposure to mixed xylene or xylene isomers. In two animal bioassays, no musculoskeletal effects were observed in rats and mice upon histopathological examination of the femur, sternebrae, or vertebrae following intermediate or chronic exposure 5 days/week to mixed xylene up to $2,000 \mathrm{mg} / \mathrm{kg}$ for mice and $1,000 \mathrm{mg} / \mathrm{kg}$ for rats for 13 weeks and $1,000 \mathrm{mg} / \mathrm{kg}$ for mice or $500 \mathrm{mg} / \mathrm{kg}$ for rats for 103 weeks (NTP 1986). No adverse effects were observed in the sternum (with marrow), thigh musculature, or femur upon histopathological examination of rats administered $m$ - or $p$-xylene at doses up to $800 \mathrm{mg} / \mathrm{kg} /$ day for 13 weeks (Wolfe $1988 \mathrm{a}, 1988 \mathrm{~b}$ ).

Hepatic Effects. No studies were located regarding hepatic effects in humans following oral exposure to mixed xylene or xylene isomers. In general, studies in animals have shown mild changes in the liver in response to oral exposure to mixed xylene. These changes included increased activity of liver enzymes and ultrastructural changes indicative of increased metabolic activity, but no evidence of histopathological changes in the liver tissue (Ungvary 1990). In acute and intermediate studies with rats, oral exposure to mixed xylene (Condie et al. 1988; Ungvary 1990) and its isomers (Condie et al. 1988; Elovaara et al. 1989; Pyykko 1980) has been associated with hepatic enzyme induction and increased hepatic weight. In the study by Condie et al. (1988), acute exposure to $p$-xylene at $250 \mathrm{mg} / \mathrm{kg} / \mathrm{day}$ and $\mathrm{m}$ - and $o$-xylene at $1,000 \mathrm{mg} / \mathrm{kg} / \mathrm{day}$ for 10 days caused increases in liver weight. Administration of doses of $1,060 \mathrm{mg} / \mathrm{kg} /$ day of all three xylene isomers for 3 days also produced increased cytochrome $b_{5}$ content and increased activities of liver enzymes in rats (Pyykko 1980), with the different isomers showing different enzyme induction potencies. Increased liver weight was observed with $m$ - and 
$o$-xylene, but not $p$-xylene, and increased cytochrome P-450 was observed only with $m$-xylene. Administration of mixed xylene to rats for 90 days caused $13.8-27 \%$ increases in relative liver weight ratios at doses between 750 and 1,500 mg/kg/day (Condie et al. 1988); a statistically significant 5.7\% increase in relative liver weight in male rats at $150 \mathrm{mg} / \mathrm{kg} /$ day is not biologically significant. No treatment-related histopathological changes were observed in the liver, but mild increases in serum transaminases were observed at $750 \mathrm{mg} / \mathrm{kg} /$ day. Similar increases in serum alanine aminotransferase were observed following ingestion of $800 \mathrm{mg} / \mathrm{kg} /$ day of $m$-xylene for 3 or 13 weeks (Elovaara et al. 1989; Wolfe 1988a). No effects were noted upon histopathological examination of the liver of rats and mice that were administered mixed xylene 5 days/week for a chronic or intermediate period of time with doses as high as 2,000 mg/kg for mice; $1,000 \mathrm{mg} / \mathrm{kg}$ for rats for 13 weeks; and 1,000 mg/kg for mice and $500 \mathrm{mg} / \mathrm{kg}$ for rats for 103 weeks (NTP 1986). Administration of doses as high as $800 \mathrm{mg} / \mathrm{kg} / \mathrm{day}$ of p-xylene in rats for 13 weeks produced no adverse hepatic effects (Wolfe 1988b).

Renal Effects. No studies were located regarding renal effects in humans following oral exposure to mixed xylene or xylene isomers. Data are available for renal effects in laboratory animals following acute-duration exposure to individual isomers, but not to mixed xylene (Condie et al. 1988; Pyykko 1980). At $1,060 \mathrm{mg} / \mathrm{kg} /$ day for 3 days, increases in kidney weight were observed with $\mathrm{m}$-xylene and increases in microsomal enzyme content and activity were observed with all three isomers (Pyykko 1980). No effects on urine parameters were noted after a 10-day exposure to $2,000 \mathrm{mg} / \mathrm{kg} /$ day of any of the isomers (Condie et al. 1988). The majority of studies using mixed xylene or its isomers for intermediate or chronic durations also showed no adverse effects on the kidneys. The only toxic change observed was increased hyaline droplet change in males (not relevant to humans) and increased early chronic nephropathy in females at $150-1,500 \mathrm{mg} / \mathrm{kg} /$ day mixed xylene for 90 days; the incidence in females at $150 \mathrm{mg} / \mathrm{kg} /$ day was not statistically significant. Although urine from these rats were normal (Condie et al. 1988), continued hyaline droplet accumulation can result in cell damage. Increased relative kidney weight was also observed in male rats given mixed xylene at $750 \mathrm{mg} / \mathrm{kg} / \mathrm{day}$ and in female rats at $1,500 \mathrm{mg} / \mathrm{kg} /$ day for 90 days (Condie et al. 1988). Similarly, increased kidney weight and microsomal enzyme activity were observed in rats exposed to $800 \mathrm{mg} / \mathrm{kg} /$ day of $m$-xylene, 5 days/week, for 3 weeks (Elovaara et al. 1989). Increased relative kidney weight was also observed in male rats administered $800 \mathrm{mg} \mathrm{m}$-xylene/kg/day for 13 weeks (Wolfe 1988a, 1988b). Histopathology of the kidneys and urinary bladder were normal. Also, no adverse effects were noted upon histopathological examination of the kidneys of rats and mice following intermediate or chronic exposure 5 days/week to doses of mixed xylene as high as 2,000 mg/kg (for 13 weeks in mice) and $1,000 \mathrm{mg} / \mathrm{kg}$ (for 103 weeks in mice) (NTP 1986). 
Endocrine Effects. No studies were located regarding endocrine effects in humans or animals following oral exposure to mixed xylene or xylene isomers.

Dermal Effects. No studies were located regarding dermal effects in humans following oral exposure to mixed xylene or xylene isomers. Limited information was located regarding dermal effects in animals. No adverse effects were noted during microscopic examination of the skin of rats and mice administered mixed xylene 5 days/week at doses as high as 2,000 mg/kg in mice and 1,000 mg/kg for rats for an intermediate (13 weeks) period of time or as high as $1,000 \mathrm{mg} / \mathrm{kg}$ for mice and $500 \mathrm{mg} / \mathrm{kg}$ for rats for a chronic (103 weeks) period of time (NTP 1986). The skin of rats administered doses as high as $800 \mathrm{mg} / \mathrm{kg} /$ day of $\mathrm{m}$ - or $p$-xylene for 13 weeks appeared normal upon histopathological examination (Wolfe 1988a, 1988b).

Ocular Effects. No studies were located regarding ocular effects in humans following oral exposure to mixed xylenes or xylene isomers. Histopathological examination of the eyes of rats and mice orally exposed to mixed xylenes (NTP 1986) or to $m$ - or $p$-xylene (Wolfe 1988a, 1988b) for 13 or 103 weeks showed no effects. No additional data regarding ocular effects in animals following oral exposure to xylenes were available.

Body Weight Effects. Effects on body weight were observed in several acute studies of the effects of mixed xylene and its isomers (Condie et al. 1988; NTP 1986; Pyykko 1980). Exposure to $m-$, $o-$, and p-xylene for 3 days resulted in weight losses of between 2.5 and 3 times that observed in control rats (Pyykko 1980). A 14-day exposure of rats and mice to mixed xylene resulted in an $18 \%$ decrease in body weight gain in male rats at $1,000 \mathrm{mg} / \mathrm{kg} /$ day and an $89 \%$ decrease in bodyweight gain in male mice at $2,000 \mathrm{mg} / \mathrm{kg} / \mathrm{day}$ (NTP 1986). Body weights of male rats given $2,000 \mathrm{mg} / \mathrm{kg} / \mathrm{day} o$ - or $p$-xylene, but not $m$-xylene, for 10 days showed $14 \%$ and $13 \%$ decreases, respectively, relative to controls (Condie et al. 1988). No significant body weight changes were observed in male or female rats dosed daily with $\leq 1,500 \mathrm{mg} / \mathrm{kg}$ /day mixed xylenes for 13 weeks (Condie et al. 1988), but a $16 \%$ decrease was noted in female mice dosed 5 days/week for 13 weeks at 2,000 mg/kg (NTP 1986). Body weights were decreased by $25 \%$ in males (with reduced food consumption) and by $15.5 \%$ in females (with normal food consumption) treated by daily oral gavage with $800 \mathrm{mg} m$-xylene/kg/day for 13 weeks (Wolfe 1988a). In a parallel study with $p$-xylene, body weights were reduced by $21 \%$ in males and by $11 \%$ in females treated at $800 \mathrm{mg} / \mathrm{kg} /$ day, although food consumption was significantly higher than controls (Wolfe 1988b). Body weight in male rats at $1,000 \mathrm{mg} / \mathrm{kg}$ and in female mice at 2,000 mg/kg mixed xylene were 
decreased 15\% and 16\%, respectively, in a 13-week study (NTP 1986). After week 59 in a 2-year study, mean body weights were reduced by $5-8 \%$ in male rats exposed by oral gavage 5 days/week to $500 \mathrm{mg} / \mathrm{kg}$ mixed xylenes, but this change is not biologically significant (NTP 1986). No body weight effects were observed in female rats exposed at doses as high as $500 \mathrm{mg} / \mathrm{kg}$ or in mice exposed at doses as high as $1,000 \mathrm{mg} / \mathrm{kg} /$ day (NTP 1986).

\subsubsection{Immunological and Lymphoreticular Effects}

No studies were located regarding immunological or lymphoreticular effects in humans after oral exposure to mixed xylene or xylene isomers. The only information suggesting a possible toxic effect of mixed xylene or its isomers on the immune system was a decrease in spleen and thymus weight observed in rats exposed for 10 days to 2,000 mg/kg/day p-xylene (Condie et al. 1988). Organ weight changes were not accompanied by histopathological changes.

The NOAEL and LOAEL values for immunological effects of $p$-xylene in rats are recorded in Table 3-2 and plotted in Figure 3-2.

\subsubsection{Neurological Effects}

Information concerning possible neurological effects associated with the ingestion of xylene is limited. Xylene produced a coma that persisted for more than 26 hours in a person who accidentally ingested an unknown amount (Recchia et al. 1985). The composition of the xylene was also unknown.

Clinical signs consistent with central nervous system toxicity have been observed in rats and mice following oral exposure to mixed xylene. A single oral dose of $4,000 \mathrm{mg} / \mathrm{kg}$ caused incoordination, prostration, decreased hindleg movement, and hunched posture in rats and tremors, prostration, and/or slowed breathing in mice (NTP 1986). In 13-week gavage assays in rats, neurotoxic signs included hyperactivity, convulsions, salivation, and epistaxis following exposure to $800 \mathrm{mg} / \mathrm{kg} / \mathrm{day} m$ - or $p$-xylene (Wolfe 1988a, 1988b) and increased aggression following exposure to $1,500 \mathrm{mg} / \mathrm{kg} /$ day mixed xylenes (Condie et al. 1988). Clinical signs observed in mice in the hour after gavage dosing with mixed xylenes included weakness, lethargy, unsteadiness, tremors, and partial paralysis of hindlimbs at $2,000 \mathrm{mg} / \mathrm{kg}$ in a 13-week assay and hyperactivity at 1,000 mg/kg beginning week 4 in 13-week and 2-year assays (NTP 1986). Mild sedation at $2,000 \mathrm{mg} / \mathrm{kg}$ and increases in latency of several peaks in flash-evoked potentials at doses of $250 \mathrm{mg} / \mathrm{kg}$ and higher were observed following single doses of $p$-xylene; no effect was seen at 
$125 \mathrm{mg} / \mathrm{kg} /$ day (Dyer et al. 1988). This NOAEL was used to derive an MRL of $1 \mathrm{mg} / \mathrm{kg} / \mathrm{day}$ for acute oral exposure to mixed xylenes or individual xylene isomers.

Histological damage to the outer hair cells of the organ of Corti provided evidence of ototoxicity in rats exposed by oral gavage to $p$-xylene, but not $m$ - or $o$-xylene, at a dose of $900 \mathrm{mg} / \mathrm{kg} / \mathrm{day}, 5$ days/week for 2 weeks (Gagnaire and Langlais 2005). The losses of hair cells occurred in the area of the cochlea responsive to medium frequencies $(10-25 \mathrm{kHz})$. No histopathology of the brain or spinal cord was observed in rats or mice administered mixed xylenes 5 days/week at doses as high as $1,000 \mathrm{mg} / \mathrm{kg}$ (rats) or $2,000 \mathrm{mg} / \mathrm{kg} / \mathrm{day}$ (mice) for 13 weeks or $1,000 \mathrm{mg} / \mathrm{kg}$ for 2 years (NTP 1986). Similarly, no neurohistopathology was observed in rats administered doses of $m$ - or $p$-xylene as high as $800 \mathrm{mg} / \mathrm{kg} / \mathrm{day}$ for 13 weeks, although the brain-to-body weight ratio was increased in males dosed with $800 \mathrm{mg} / \mathrm{kg} / \mathrm{day}$ of $m$-xylene (Wolfe 1988a, 1988b).

An acute-duration oral MRL of $1 \mathrm{mg} / \mathrm{kg} / \mathrm{day}$ for mixed xylenes or individual isomers was calculated based on a NOAEL of $125 \mathrm{mg} / \mathrm{kg}$ and a LOAEL of $250 \mathrm{mg} / \mathrm{kg}$ for altered visual evoked brain potentials in male Long-Evans rats given single gavage doses of $p$-xylene (Dyer et al. 1988; NTP 1986) as described in the footnote in Table 3-2. An intermediate-duration oral MRL of $0.4 \mathrm{mg} / \mathrm{kg} /$ day for mixed xylenes or individual isomers was calculated based on a NOAEL of $500 \mathrm{mg} / \mathrm{kg}$ and a LOAEL of $1,000 \mathrm{mg} / \mathrm{kg}$ for hyperactivity in male and female $\mathrm{B} 6 \mathrm{C} 3 \mathrm{~F}_{1}$ mice dosed with mixed xylenes by gavage 5 days/week for 4 51 weeks (NTP 1986) as described in the footnote in Table 3-2.

The highest NOAEL value and all LOAEL values from each reliable study for neurological effects in rats and mice and for each exposure duration are recorded in Table 3-2 and plotted in Figure 3-2.

\subsubsection{Reproductive Effects}

No studies were located regarding reproductive effects in humans following oral exposure to mixed xylene or individual isomers.

No studies in animals directly examining reproductive function following oral administration of mixed xylene or its isomers were located; however, histological examination of rats and mice administered mixed xylene 5 days $/$ week at doses as high as $1,000 \mathrm{mg} / \mathrm{kg}$ in rats and 2,000 mg/kg in mice for 13 weeks revealed no adverse effects on the prostate/testes (male), ovaries/uterus, or mammary glands (female) (NTP 1986). The reproductive system organs of rats administered doses of $m$ - or $p$-xylene as high as 
$800 \mathrm{mg} / \mathrm{kg} /$ day appeared comparable to controls after 13 weeks of treatment (Wolfe 1988a, 1988b). In chronic studies, no adverse histopathological changes were observed in the reproductive organs in rats at doses as high as $500 \mathrm{mg} / \mathrm{kg}$ and in mice at doses as high as $1,000 \mathrm{mg} / \mathrm{kg}$ administered 5 days/week for 103 weeks (NTP 1986). The highest NOAEL value from each reliable study for reproductive effects are recorded in Table 3-2 and plotted in Figure 3-2.

\subsubsection{Developmental Effects}

No studies were located regarding developmental effects in humans following oral exposure to mixed xylene or xylene isomers.

Significantly increased incidences of cleft palate and decreased fetal body weight were reported following maternal oral exposure during gestation days $6-15$ to doses of $2,060 \mathrm{mg} / \mathrm{kg} /$ day mixed xylene in mice (Marks et al. 1982). Mixed xylene was also toxic to the dams, producing $31.5 \%$ mortality at $3,100 \mathrm{mg} / \mathrm{kg} / \mathrm{day}$. It is unclear whether the observation of cleft palate in this study is associated with maternal toxicity or a predisposition of mice under stress to give birth to offspring with this birth defect. In a teratology screening study, $2,000 \mathrm{mg} / \mathrm{kg} /$ day of $\mathrm{m}$-xylene produced no evidence of fetal toxicity in mice (Seidenberg et al. 1986). Given the limited amount of animal data, no conclusion can be made regarding the relationship between oral exposure of xylene and adverse developmental effects. The highest NOAEL value and all LOAEL values from each reliable study for developmental effects are recorded in Table 3-2 and plotted in Figure 3-2.

\subsubsection{Cancer}

No data were located regarding cancer in humans following oral exposure to mixed xylene or xylene isomers.

The carcinogenicity of mixed xylene following oral exposure has been evaluated in chronic studies with rats and mice; however, no animal studies were available on the carcinogenic effects of $m-, o_{-}$, or $p$-xylene following oral exposure. Results of the chronic oral studies with mixed xylene have been negative (NTP 1986) or equivocal (Maltoni et al. 1983, 1985). In a chronic bioassay, rats and mice of both sexes received mixed xylene by gavage at 0,250 , or $500 \mathrm{mg} / \mathrm{kg}$ and 0,500 , or $1,000 \mathrm{mg} / \mathrm{kg}$, respectively, 5 days/week for 103 weeks. The interpretation of the results of the NTP bioassay was compromised by the large number of gavage-related deaths early in the study in the high-dose male rats. In the other chronic study (Maltoni et al. 1983, 1985), male and female rats were fed xylene (unspecified) 
by gavage at 0 or $500 \mathrm{mg} / \mathrm{kg}, 4-5$ days/week for 104 weeks. The Maltoni studies were weakened because of methodological flaws such as failure to report site-specific neoplasia, insufficient toxicity data, and absence of statistical analyses. Therefore, given the limited data, no definitive conclusion can be made regarding the carcinogenicity of mixed xylene in animals following oral exposure.

\subsubsection{Dermal Exposure}

In addition to studies that have directly examined the health effects of dermal exposure to xylene, a number of reports of health effects resulting from occupational exposure to xylene have been included in this section. Dermal contact with xylene is likely in many occupational situations, and absorption of xylene has been demonstrated in humans (Engstrom et al. 1977; Riihimaki 1979b; Riihimaki and Pfaffli 1978). The results of the occupational studies must be interpreted with caution, however, because of coexposure to other compounds.

\subsubsection{Death}

No reports of death in humans following dermal exposure to xylene were located. Limited animal data suggest that mixed xylene and $m$-xylene can cause death when applied dermally (Hine and Zuidema 1970; Smyth et al. 1962). The acute dermal $\mathrm{LD}_{50}$ in rabbits has been determined to be $3,228 \mathrm{mg} / \mathrm{kg} / \mathrm{day}$ for $m$-xylene and $>114 \mathrm{mg} / \mathrm{kg} /$ day for mixed xylene for 4 hours or more (Hine and Zuidema 1970; Smyth et al. 1962).

The $\mathrm{LD}_{50}$ value for death in rabbits as a result of acute-duration exposure to $m$-xylene is recorded in Table 3-3.

\subsubsection{Systemic Effects}

No studies were located regarding musculoskeletal, endocrine, or body weight effects in humans or animals following dermal exposure to mixed xylenes or xylene isomers. The systemic effects that were observed after dermal exposure to xylene are discussed below. All LOAEL values from each reliable study for systemic effects in each species and duration category are recorded in Table 3-3.

Respiratory Effects. Case reports of dryness of the throat (Goldie 1960) in painters and decreased pulmonary function and dyspnea in histology technicians with chronic exposure to xylene (Hipolito 1980) have been published. It is likely that these effects represent direct effects of xylene or other solvents on 
Table 3-3 Levels of Significant Exposure to Xylene - Dermal

\begin{tabular}{|c|c|c|c|c|c|c|c|c|c|}
\hline \multirow[b]{2}{*}{$\begin{array}{l}\text { Species } \\
\text { (Strain) }\end{array}$} & \multirow{2}{*}{$\begin{array}{l}\text { Exposurel } \\
\text { Durationl } \\
\text { Frequency } \\
\text { (Route) }\end{array}$} & \multicolumn{7}{|c|}{ LOAEL } & \multirow[b]{2}{*}{ Comments } \\
\hline & & System & NOAEL & \multicolumn{3}{|c|}{ Less Serious } & Serious & $\begin{array}{l}\text { Reference } \\
\text { Chemical Form }\end{array}$ & \\
\hline \multirow{2}{*}{\multicolumn{10}{|c|}{$\begin{array}{l}\text { ACUTE EXPOSURE } \\
\text { Death }\end{array}$}} \\
\hline & & & & & & & & & \\
\hline $\begin{array}{l}\text { Mouse } \\
\text { Hall }\end{array}$ & once & & & & & $\begin{array}{c}57 \\
\mathrm{mg} / \mathrm{kg}\end{array}$ & (8/120 died) & $\begin{array}{l}\text { Pound and Withers } 1963 \\
\text { mixed }\end{array}$ & \\
\hline $\begin{array}{l}\text { Rabbit } \\
\text { Albino New } \\
\text { Zealand }\end{array}$ & $24 \mathrm{hr}$ & & & & & $\begin{array}{l}3228 \mathrm{M} \\
\mathrm{mg} / \mathrm{kg}\end{array}$ & (LD50) & $\begin{array}{l}\text { Smyth et al. } 1962 \\
\text { meta }\end{array}$ & \\
\hline \multicolumn{10}{|l|}{ Systemic } \\
\hline $\begin{array}{l}\text { Rat } \\
\text { (hairless) }\end{array}$ & $\begin{array}{l}4 d \\
5 \times / d\end{array}$ & Dermal & & $\begin{array}{c}66 \\
\mathrm{mg} / \mathrm{day}\end{array}$ & $\begin{array}{l}\text { (2-fold increase in } \\
\text { transepidermal water } \\
\text { loss, moderate erythema } \\
5 \text {-fold increase in } \\
\text { pro-inflammatory } \\
\text { cytokine TNF-alpha) }\end{array}$ & & & $\begin{array}{l}\text { Ahaghotu et al. 2005; Singh } \\
2006\end{array}$ & $\begin{array}{l}66 \mathrm{mg} / \text { day delivered in } \\
5 \text { fractions of } 13.2 \mathrm{mg} .\end{array}$ \\
\hline $\begin{array}{l}\text { Rat } \\
\text { (hairless) }\end{array}$ & $1 \mathrm{hr}$ & Dermal & & $\begin{array}{l}199 \mathrm{M} \\
\mathrm{mg}\end{array}$ & $\begin{array}{l}\text { (2-fold higher } \\
\text { transepidermal water } \\
\text { loss, 30\% reduced } \\
\text { moisture content, } \\
\text { moderate erythema, } \\
\text { 2.5-fold increase in } \\
\text { pro-inflammatory } \\
\text { TNF-alpha) }\end{array}$ & ortho & & $\begin{array}{l}\text { Chatterjee et al. } 2005 \\
\text { meta }\end{array}$ & Occlusive exposure. \\
\hline
\end{tabular}


Table 3-3 Levels of Significant Exposure to Xylene - Dermal

(continued)

\begin{tabular}{|c|c|c|c|c|c|c|c|c|}
\hline \multirow[b]{2}{*}{$\begin{array}{l}\text { Species } \\
\text { (Strain) }\end{array}$} & \multirow{2}{*}{$\begin{array}{l}\text { Exposurel } \\
\text { Durationl } \\
\text { Frequency } \\
\text { (Route) }\end{array}$} & \multicolumn{6}{|c|}{ LOAEL } & \multirow[b]{2}{*}{ Comments } \\
\hline & & System & NOAEL & Less Seri & ous & Serious & $\begin{array}{l}\text { Reference } \\
\text { Chemical Form }\end{array}$ & \\
\hline $\begin{array}{l}\text { Rat } \\
\text { (hairless) }\end{array}$ & $\begin{array}{l}4 d \\
5 \times / d\end{array}$ & Dermal & & $\begin{array}{l}64.8 \mathrm{M} \\
\mathrm{mg} / \text { day }\end{array}$ & $\begin{array}{l}\text { (2-fold increase in } \\
\text { transepidermal water } \\
\text { loss, } 22 \% \text { reduced wate } \\
\text { content, moderate } \\
\text { erythema, 5.7-fold } \\
\text { increase in } \\
\text { pro-inflammatory } \\
\text { TNF-alpha) }\end{array}$ & & $\begin{array}{l}\text { Chatterjee et al. } 2005 \\
\text { meta }\end{array}$ & $\begin{array}{l}\text { Unocclusive dermal } \\
\text { exposure. } 64.8 \mathrm{mg} / \text { day } \\
\text { delivered in } 5 \text { fractions } \\
\text { of } 12.96 \mathrm{mg} \text {. }\end{array}$ \\
\hline $\begin{array}{l}\text { Rat } \\
\text { (Fischer- 344) }\end{array}$ & $\begin{array}{l}1 \mathrm{hr} \\
\text { once }\end{array}$ & Dermal & & $\begin{array}{l}1200 \mathrm{M} \\
\mathrm{mg} / \mathrm{kg}\end{array}$ & $\begin{array}{l}\text { (separation of epidermis } \\
\text { and dermis) }\end{array}$ & & $\begin{array}{l}\text { Gunasekar et al. } 2003 \\
\text { meta }\end{array}$ & \\
\hline $\begin{array}{l}\text { Mouse } \\
\text { (BALB/c) }\end{array}$ & once & Dermal & & $\begin{array}{l}3136 \mathrm{~F} \\
\mu \mathrm{g} / \mathrm{kg}\end{array}$ & (skin edema) & & $\begin{array}{l}\text { Iyadomi et al. } 2000 \\
\text { meta }\end{array}$ & \\
\hline $\begin{array}{l}\text { Mouse } \\
\text { Hall }\end{array}$ & once & Dermal & & $\begin{array}{c}57 \\
\mathrm{mg} / \mathrm{kg}\end{array}$ & $\begin{array}{l}\text { (edema, irritation, } \\
\text { scaliness of skin) }\end{array}$ & & $\begin{array}{l}\text { Pound and Withers } 1963 \\
\text { mixed }\end{array}$ & \\
\hline $\begin{array}{l}\text { Gn Pig } \\
\text { Dunkin } \\
\text { Hartley }\end{array}$ & $\begin{array}{l}3 d \\
3 \times / d\end{array}$ & Dermal & & $\begin{array}{c}2.3 \mathrm{~F} \\
\mathrm{mg} / \mathrm{kg} / \mathrm{day}\end{array}$ & (skin irritation) & & $\begin{array}{l}\text { Anderson et al. } 1986 \\
\text { mixed }\end{array}$ & \\
\hline $\begin{array}{l}\text { Rabbit } \\
\text { NS }\end{array}$ & once & Ocular & & $\begin{array}{r}87 \\
\mathrm{mg}\end{array}$ & (mild irritation of eyes) & & $\begin{array}{l}\text { Consumer Product Testing } \\
1976\end{array}$ & $0.1 \mathrm{~mL} / \mathrm{eye}$. \\
\hline
\end{tabular}


Table 3-3 Levels of Significant Exposure to Xylene - Dermal

(continued)

\begin{tabular}{|c|c|c|c|c|c|c|c|c|c|}
\hline \multirow[b]{2}{*}{$\begin{array}{l}\text { Species } \\
\text { (Strain) }\end{array}$} & \multirow{2}{*}{$\begin{array}{c}\text { Exposurel } \\
\text { Duration/ } \\
\text { Frequency } \\
\text { (Route) }\end{array}$} & \multirow[b]{2}{*}{ System } & & \multicolumn{4}{|c|}{ LOAEL } & \multirow[b]{2}{*}{$\begin{array}{l}\text { Reference } \\
\text { Chemical Form }\end{array}$} & \multirow[b]{2}{*}{ Comments } \\
\hline & & & NOAEL & Less Ser & ous & & Serious & & \\
\hline \multirow[t]{2}{*}{$\begin{array}{l}\text { Rabbit } \\
\text { New Zealand } \\
\text { white }\end{array}$} & once & Dermal & & & & $\begin{array}{c}114 \mathrm{M} \\
\mathrm{mg} / \mathrm{kg}\end{array}$ & $\begin{array}{l}\text { (moderate to severe skir } \\
\text { irritation) }\end{array}$ & $\begin{array}{l}\text { Hine and Zuidema } 1970 \\
\text { mixed }\end{array}$ & $\begin{array}{l}0.1 \mathrm{~mL} / \mathrm{eye} ; 0.5 \mathrm{~mL} \text { to } \\
\text { skin. }\end{array}$ \\
\hline & & Ocular & & $\begin{array}{l}87 \mathrm{M} \\
\mathrm{mg}\end{array}$ & $\begin{array}{l}\text { (moderately irritating to } \\
\text { the conjunctiva) }\end{array}$ & & & & \\
\hline $\begin{array}{l}\text { Rabbit } \\
\text { New Zealand } \\
\text { white }\end{array}$ & once & Ocular & & $\begin{array}{l}87 \mathrm{M} \\
\mathrm{mg}\end{array}$ & (eye irritation) & & & $\begin{array}{l}\text { Hine and Zuidema } 1970 \\
\text { mixed }\end{array}$ & $0.1 \mathrm{~mL} / \mathrm{eye}$. \\
\hline $\begin{array}{l}\text { Rabbit } \\
\text { (New } \\
\text { Zealand) }\end{array}$ & once & Ocular & & $\begin{array}{r}87 \\
\mathrm{mg}\end{array}$ & (minimal eye irritation) & & & $\begin{array}{l}\text { Kennah et al. } 1989 \\
\text { mixed }\end{array}$ & $0.1 \mathrm{~mL} / \mathrm{eye}$. \\
\hline $\begin{array}{l}\text { Rabbit } \\
\text { New Zealand } \\
\text { albino }\end{array}$ & once & Ocular & & $\begin{array}{l}432 \\
\mathrm{mg}\end{array}$ & (eye irritation) & & & $\begin{array}{l}\text { Smyth et al. } 1962 \\
\text { meta }\end{array}$ & $0.5 \mathrm{~mL} / \mathrm{eye}$. \\
\hline $\begin{array}{l}\text { Rabbit } \\
\text { Albino }\end{array}$ & once & Dermal & & $\begin{array}{c}2.3 \\
\mathrm{mg} / \mathrm{kg}\end{array}$ & (skin irritation) & & & $\begin{array}{l}\text { Smyth et al. } 1962 \\
\text { meta }\end{array}$ & \\
\hline
\end{tabular}

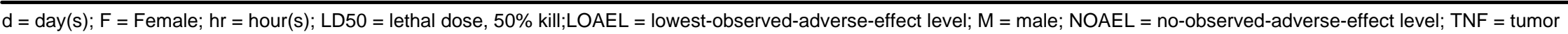
necrosis factor; $x=$ time(s) 
the respiratory tissues and they are discussed in more detail in Section 3.2.1.2. No studies were located regarding respiratory effects in animals following dermal exposure to mixed xylene or xylene isomers, although some of the inhalation studies also involved exposure via dermal route as well.

Cardiovascular Effects. Cases of flushing, chest pains, and palpitations in histology technicians have been reported (Hipolito 1980). These studies also involved exposure via inhalation route. It is unclear whether these effects are directly attributable to xylene exposure because of possible exposure to other chemicals. No studies were located regarding cardiovascular effects in animals after dermal exposure to mixed xylene or xylene isomers.

Gastrointestinal Effects. Gastric discomfort in painters (Goldie 1960) and nausea in histology technicians (1980) have been reported; these studies also involved exposure via inhalation route. However, other chemicals in the workplace may have contributed to these effects. No studies were located regarding gastrointestinal effects in animals following dermal exposure to mixed xylene or xylene isomers.

Hematological Effects. Decreased white blood cell count has been observed in histology technicians (Hipolito 1980) and in workers with occupational exposure to benzene, toluene, and xylene (Moszczynski and Lisiewicz 1983, 1984a); these studies also involved exposure via inhalation route. However, chemicals other than xylene may have caused these decreases. No studies were located regarding hematological effects in animals following dermal exposure to mixed xylene or xylene isomers.

Hepatic Effects. When compared with unexposed controls, workers with occupational exposure via dermal and inhalation routes to toluene, xylene, and pigments had significantly increased urinary D-glucaric acid content in the urine indicating hepatic microsomal enzyme induction (Dolara et al. 1982). Serum antipyrine half-life was increased, suggesting possible hepatotoxicity. No effect on serum aminotransferases was observed in workers exposed to a mixture of solvents (Kurppa and Husman 1982). These studies also involved exposure via inhalation route. These studies are limited in that multiple chemical exposures occurred and the effects observed cannot be directly attributed to xylene. No studies were located regarding hepatic effects in animals following dermal exposure to mixed xylene or xylene isomers.

Renal Effects. Occupational exposure to a mixture of mainly xylene and toluene resulted in elevated albumin, erythrocytes, and leukocytes in the urine (Askergren 1981, 1982). In addition, increased 
$\beta$-glucuronidase was observed in the urine of painters (Franchini et al. 1983). However, these studies are limited in that the effects observed may be attributable to exposure to toluene, which is a known renal toxicant, and that inhalation as well as dermal exposure could have occurred. No studies were located regarding renal effects in animals following dermal exposure to mixed xylene or xylene isomers.

Dermal Effects. Acute dermal exposure of human subjects to undiluted $m$-xylene in hand immersion studies has been associated with transient skin erythema (irritation), vasodilation of the skin, and dryness and scaling of the skin (Engstrom et al. 1977; Riihimaki 1979b). Urticaria was reported in a female cytology worker exposed predominantly to xylene vapors (Palmer and Rycroft 1993). Because this response probably had an immunological component, it is discussed further in the Immunological and Lymphoreticular Effects section.

Mild-to-severe skin irritation was noted in rabbits, guinea pigs, and mice treated topically with mixed xylene (2.3-114 mg/kg/day), $m$-xylene (65-199 mg/day) or $o$-xylene (66 mg/day) in acute studies (Ahaghotu et al. 2005, isomer identified in personal communication by Singh 2006; Anderson et al. 1986; Chatterjee et al. 2005; Consumer Products Testing 1976; Food and Drug Research Labs 1976a; Hine and Zuidema 1970; Pound and Withers 1963; Smyth et al. 1962). The extent of the irritation appeared to increase with duration of exposure; the most severe dermal irritation ratings were obtained in the longest exposures of 10-days (Hine and Zuidema 1970). Application of $0.250 \mathrm{~mL}(1,200 \mathrm{mg} / \mathrm{kg}$ ) of $\mathrm{m}$-xylene to the skin of rats for 1 hour resulted in a significant increase in oxidative species and DNA fragmentation (increased low-molecular-weight DNA) within 2 hours (Gunasekar et al. 2003; Rogers et al. 2001). Treatment with $m$ - or $o$-xylene increased levels of inducible nitric oxide synthetase and tumor necrosis factor-alpha (TNF-alpha), a pro-inflammatory cytokine, in skin, as well as plasma levels of interleukin-1alpha (IL-1alpha) (Ahaghotu et al. 2005; Chatterjee et al. 2005; Gunasekar et al. 2003). Dermal swelling following application of $69 \mathrm{mg} \mathrm{m}$-xylene to the ear of mice resulted in significant increases in the thickness of the ear as measured 0.5-25 hours after application (Iyadomi et al. 2000); peak thickness was observed 6 hours after treatment. Histopathological effects of exposure to $m$ - or $o$-xylene included swelling and disruption of the stratum corneum, granulocyte infiltration of the epidermis, and separation of the epidermis and dermis, with evidence of local inflammation (accumulation of mast cells, plasma cells) (Ahaghotu et al. 2005; Chatterjee et al. 2005; Gunasekar et al. 2003). Impairment of skin function was evident in the increase in transepidermal water loss in hairless rats following occlusive exposure (199 $\mathrm{mg}$ for 1 hour) or unocclusive exposure $(64.8 \mathrm{mg} /$ day in 5 divided daily fractions over 4 days $)$ to $m$-xylene or unocclusive exposure (66 mg/day in 5 divided doses, for 4 days) to $o$-xylene and the reduction in skin moisture content observed in rats treated unocclusively as above with $m$-xylene 
(Ahaghotu et al. 2005; Chatterjee et al. 2005). Moderate-to-marked irritation and moderate necrosis were observed in rabbits with a 2-4-week dermal exposure to undiluted xylene (Wolf et al. 1956). No chronic animal studies evaluating the dermal effects of xylene were located.

Ocular Effects. There are a few case reports in humans describing the effects of direct contact of the eye with heated xylene from a pressurized hose in one case or with paint containing xylene as the solvent in two cases (Ansari 1997; Narvaez and Song 2003). Both kinds of exposures resulted in photophobia, redness of the conjunctiva, and partial loss of the conjunctival and corneal epithelia. The effects in the two men exposed to paint were characterized as equivalent to grade II chemical burns and, with medical therapy, largely reversed after about a week (Ansari 1997). The other case also experienced subconjunctival hemorrhage, more extensive loss of the corneal epithelium, and xylene keratopathy (melting of the corneal stroma), the latter of which persisted to 4 weeks following the initial injury (Narvaez and Song 2003). It is possible combined thermal, physical, and chemical injury increased the severity of ocular effects in this case.

Several studies in humans have reported on ocular effects of exposure to xylene vapor. In the controlled exposure situations, eye irritation was reported at concentrations of mixed xylene as low as $200 \mathrm{ppm}$ for 3-5 minutes (Nelson et al. 1943) and of $p$-xylene as low as $100 \mathrm{ppm}$ for 1-7.5 hours/day for 5 days (NIOSH 1981). Case reports have also demonstrated transient eye irritation in humans exposed to vapors of mixed xylene or p-xylene (Carpenter et al. 1975a; Hastings et al. 1986; Klaucke et al. 1982; Nelson et al. 1943; Nersesian et al. 1985; NIOSH 1981). Eye irritation was more frequently reported by workers exposed to mixed xylene (geometric mean TWA 14 ppm) than by the controls (Uchida et al. 1993).

Instillation of $0.1 \mathrm{~mL}(87 \mathrm{mg})$ of mixed xylene or $0.5 \mathrm{~mL}(432 \mathrm{mg})$ of $m$-xylene into the eyes of rabbits resulted in slight-to-moderate eye irritation (Consumer Products Testing 1976; Hine and Zuidema 1970; Kennah et al. 1989; Smyth et al. 1962). No corneal effects were observed in these studies.

\subsubsection{Immunological and Lymphoreticular Effects}

Limited data were located regarding immunological and lymphoreticular effects in humans following dermal exposure to xylene. Occupational exposure to benzene, toluene, and xylene resulted in decreased serum complement (Smolik et al. 1973) and in decreased lymphocytes, but there was no effect on lymphocyte reactions when stimulated with phytohemagglutinin (Moszczynski and Lisiewicz 1983, 1984a). Interpretation of these studies is limited in that chemicals other than xylene may have accounted 
for the effects observed. Exposures via inhalation and dermal routes also may have occurred. Contact urticaria was reported in a female cytology worker exposed for several months predominantly to $<100$ ppm xylene vapors (Palmer and Rycroft 1993). A closed patch test resulting in severe erythema and wealing provides evidence that the effect was a result of direct contact of xylene vapor with the skin and suggests that the reaction was immunological.

No studies were located regarding immunological or lymphoreticular effects in animals after dermal exposure to mixed xylene or xylene isomers.

\subsubsection{Neurological Effects}

Occupational exposure to xylene has been reported to result in headache, dizziness, malaise, a feeling of drunkenness, irritability, fine tremor, dysphasia, hyperreflexia, and/or impaired concentration and memory (Goldie 1960; Hipolito 1980; Kilburn et al. 1985; Roberts et al. 1988). These studies are limited, however, because other chemical exposures in the workplace may have been responsible for the effects observed and that exposures via inhalation and dermal routes may have occurred.

As described in a brief report, pregnant rats dermally exposed to xylene (form not specified) at $2,000 \mathrm{mg} / \mathrm{kg} /$ day throughout gestation showed a statistically significant $27 \%$ reduction, compared to controls, in motor activity in an open field test, suggesting a neurotoxic effect of xylene (Mirkova et al. 1979). In this study, dosing at 200 or $2,000 \mathrm{mg} / \mathrm{kg}$ /day reduced brain cholinesterase activities in dams by $35-38 \%$ compared to controls; fetal brain cholinesterase was also reported to be 'inhibited' at those doses. The report did not discuss whether any measures were taken to prevent ingestion of the test material.

\subsubsection{Reproductive Effects}

No studies were located regarding reproductive effects in humans or animals after dermal exposure to mixed xylene or xylene isomers.

\subsubsection{Developmental Effects}

The human data regarding the developmental effects of xylene suggest a possible relationship between occupational solvent exposure and developmental toxicity (Holmberg and Nurminen 1980; Kucera 1968; Taskinen et al. 1989; Windham et al. 1991). However, these data are limited for assessing the relationship between dermal exposure to xylene and developmental effects because the available studies 
involved concurrent exposure to other chemical agents in addition to xylene in the workplace (Holmberg and Nurminen 1980; Kucera 1968; Taskinen et al. 1989; Windham et al. 1991), because few subjects were tested (Taskinen et al. 1989; Windham et al. 1991), and because it is extremely difficult to have a pure dermal exposure since such exposure in the absence of respiratory protection is accompanied by inhalation exposure.

As described in a brief report, dermal exposure of pregnant rats to doses as low as $200 \mathrm{mg} / \mathrm{kg} / \mathrm{day}$ of xylene (unspecified concentration and isomer) throughout gestation produced decreases in enzyme activity (cholinesterase, cytochrome oxidase) in fetal and maternal brain tissue (Mirkova et al. 1979).

\subsubsection{Cancer}

Studies of workers occupationally exposed to solvents have examined the cancer and leukemia risks and suggest a possible relationship between coal-based xylene exposure and leukemia (Arp et al. 1983; Wilcosky et al. 1984). Both contain limitations (e.g., small number of subjects, no exposure concentrations, unknown composition of xylene and possible exposure to benzene and other chemicals) that preclude a definitive conclusion regarding dermal exposure to xylene and cancer; development of these studies probably also involved exposure via inhalation.

Limited information was located regarding the carcinogenicity of dermal exposure to xylene in animals (Berenblum 1941; Pound 1970; Pound and Withers 1963). Application of xylene (concentration, purity, and amount unspecified) to the skin for 25 weeks resulted in no increase in skin tumors, and did not potentiate the number of skin tumors produced by benz[a]pyrene (Berenblum 1941). However, two studies showed that a single xylene pretreatment slightly enhanced the number of tumors produced by a combination of ultraviolet light irradiation (initiation) and croton oil (promotion) (Pound 1970) or urethane (initiation) and croton oil (promotion) (Pound and Withers 1963). These findings suggest that xylene may be a promoter for skin cancer and might also act as initiator or cocarcinogen. These studies are limited in that tumors other than skin tumors were not assessed and untreated controls were not used. Furthermore, it is not known whether the xylene was analyzed for the presence of other aromatic hydrocarbons such as benzene that are known to be tumorigenic.

\subsection{GENOTOXICITY}

The preponderance of data from testing in vivo (Table 3-4) and in vitro (Table 3-5) indicates that xylenes are not mutagenic and do not induce chromosomal anomalies. A few studies indicate that DNA 
Table 3-4. Genotoxicity of Xylene In Vivo

\begin{tabular}{|c|c|c|c|c|c|}
\hline $\begin{array}{l}\text { Species (test } \\
\text { system) }\end{array}$ & End point & $\begin{array}{l}\text { Exposure route; } \\
\text { dose }\end{array}$ & Results & Reference & Isomer \\
\hline \multicolumn{6}{|l|}{ Mammalian cells: } \\
\hline $\begin{array}{l}\text { Human peripheral } \\
\text { lymphocytes }\end{array}$ & $\begin{array}{l}\text { Sister chromatid } \\
\text { exchange and } \\
\text { chromosomal } \\
\text { abberations }\end{array}$ & $\begin{array}{l}\text { Inhalation } \\
\text { (occupational } \\
\text { exposure) }\end{array}$ & - & $\begin{array}{l}\text { Haglund et al. } \\
1980\end{array}$ & Not reported \\
\hline $\begin{array}{l}\text { Human peripheral } \\
\text { lymphocytes }\end{array}$ & $\begin{array}{l}\text { Sister chromatid } \\
\text { exchange }\end{array}$ & $\begin{array}{l}\text { Inhalation } \\
\text { (occupational } \\
\text { exposure) }\end{array}$ & - & $\begin{array}{l}\text { Pap and Varga } \\
1987\end{array}$ & Mixed xylene \\
\hline $\begin{array}{l}\text { Human peripheral } \\
\text { lymphocytes }\end{array}$ & $\begin{array}{l}\text { Sister chromatid } \\
\text { exchange }\end{array}$ & $\begin{array}{l}\text { Inhalation (three } \\
\text { exposures; } \\
40 \text { ppm, } \\
7 \text { hours/day) }\end{array}$ & - & $\begin{array}{l}\text { Richer et al. } \\
1993\end{array}$ & Mixed xylene \\
\hline Mouse reticulocytes & $\begin{array}{l}\text { Chromosomal } \\
\text { aberrations or } \\
\text { Micronuclei } \\
\text { formation }\end{array}$ & $\begin{array}{l}\text { Oral (single } \\
\text { exposure); } \\
\leq 1,000 \mathrm{mg} / \mathrm{kg}\end{array}$ & - & Feldt 1986 & Mixed xylene \\
\hline $\begin{array}{l}\text { Rat (Fischer 344) } \\
\text { skin }\end{array}$ & DNA damage & $\begin{array}{l}\text { Dermal (250 } \mu \mathrm{L} ; \\
1 \text { hour) }\end{array}$ & + & $\begin{array}{l}\text { Rogers et al. } \\
2001\end{array}$ & $m$-Xylene \\
\hline Rat bone marrow & $\begin{array}{l}\text { Chromosomal } \\
\text { aberrations }\end{array}$ & $\begin{array}{l}\text { Intraperitoneal } \\
\text { (single exposure) }\end{array}$ & - & $\begin{array}{l}\text { Litton Bionetics } \\
1978 b\end{array}$ & $\begin{array}{l}\text { Mixed xylene } \\
(11.4 \% \\
o \text {-xylene, } 0.3 \% \\
p \text {-xylene, } 36.1 \% \\
\text { ethylbenzene) }\end{array}$ \\
\hline Rat bone marrow & $\begin{array}{l}\text { Chromosomal } \\
\text { aberrations }\end{array}$ & $\begin{array}{l}\text { Intraperitoneal } \\
\text { (five exposures) }\end{array}$ & - & $\begin{array}{l}\text { Litton Bionetics } \\
\text { 1978b }\end{array}$ & $\begin{array}{l}\text { Mixed xylene } \\
(0.3 \% \text { p-xylene, } \\
36.1 \% \text { ethyl- } \\
\text { benzene })\end{array}$ \\
\hline $\begin{array}{l}\text { Mouse bone marrow } \\
\text { polychromatic- } \\
\text { erythrocyte assay } \\
\text { (micro-nucleus test) }\end{array}$ & $\begin{array}{l}\text { Micronuclei } \\
\text { formation }\end{array}$ & $\begin{array}{l}\text { Intraperitoneal } \\
\text { (two exposures } \\
\text { at } \leq 650 \mathrm{mg} / \mathrm{kg} \text { ) }\end{array}$ & - & $\begin{array}{l}\text { Mohtashami- } \\
\text { pur et al. } 1985\end{array}$ & $\begin{array}{l}\text { m-Xylene, } \\
\text { o-xylene, } \\
\text { p-xylene }\end{array}$ \\
\hline $\begin{array}{l}\text { Rat sperm-head } \\
\text { morphology assay }\end{array}$ & $\begin{array}{l}\text { Sperm-head } \\
\text { abnormalities }\end{array}$ & $\begin{array}{l}\text { Intraperitoneal } \\
\text { (two exposures } \\
\text { at } 435 \mathrm{mg} / \mathrm{kg} \text { ) }\end{array}$ & - & $\begin{array}{l}\text { Washington et } \\
\text { al. } 1983\end{array}$ & o-Xylene \\
\hline
\end{tabular}

$-=$ negative result

$+=$ positive result 
Table 3-5. Genotoxicity of Xylene In Vitro

\begin{tabular}{|c|c|c|c|c|c|}
\hline \multirow[b]{2}{*}{ Species (test system) } & \multirow[b]{2}{*}{ End point } & \multicolumn{2}{|c|}{ Results } & \multirow[b]{2}{*}{ Reference } & \multirow[b]{2}{*}{ Isomer } \\
\hline & & $\begin{array}{l}\text { With } \\
\text { activation }\end{array}$ & $\begin{array}{l}\text { Without } \\
\text { activation }\end{array}$ & & \\
\hline \multicolumn{6}{|l|}{ Prokaryotic organisms: } \\
\hline $\begin{array}{l}\text { Salmonella typhimurium } \\
\text { TA97, TA98, TA100, TA1535/ } \\
\text { plate incorporation assay }\end{array}$ & $\begin{array}{l}\text { Reverse } \\
\text { Mutation }\end{array}$ & - & - & NTP 1986 & $\begin{array}{l}\text { Mixed } \\
\text { xylene }\end{array}$ \\
\hline $\begin{array}{l}\text { S. typhimurium TA98, TA100, } \\
\text { TA1535, TA1537/plate } \\
\text { incorporation assay }\end{array}$ & $\begin{array}{l}\text { Reverse } \\
\text { Mutation }\end{array}$ & - & - & $\begin{array}{l}\text { Haworth et al. } \\
1983\end{array}$ & $\begin{array}{l}m \text {-Xylene; } \\
\text { o-xylene; } \\
\text { p-xylene }\end{array}$ \\
\hline $\begin{array}{l}\text { S. typhimurium TA98, TA100, } \\
\text { UTH8414, UTH8413/plate } \\
\text { incorporation assay }\end{array}$ & $\begin{array}{l}\text { Reverse } \\
\text { Mutation }\end{array}$ & - & - & $\begin{array}{l}\text { Connor et al. } \\
1985\end{array}$ & $\begin{array}{l}m \text {-Xylene; } \\
\text { o-xylene; } \\
\text { p-xylene }\end{array}$ \\
\hline $\begin{array}{l}\text { S. typhimurium TA98, TA100, } \\
\text { TA1535, TA1537, TA1538/ } \\
\text { plate incorporation assay }\end{array}$ & $\begin{array}{l}\text { Reverse } \\
\text { Mutation }\end{array}$ & - & - & Bos et al. 1981 & $\begin{array}{l}m \text {-Xylene; } \\
\text { o-xylene; } \\
\text { p-xylene }\end{array}$ \\
\hline $\begin{array}{l}\text { S. typhimurium TA98, TA100, } \\
\text { TA1535, TA1537/spot and } \\
\text { plate incorporation assays }\end{array}$ & $\begin{array}{l}\text { Reverse } \\
\text { Mutation }\end{array}$ & - & - & Florin et al. 1980 & $\begin{array}{l}m \text {-Xylene; } \\
p \text {-xylene }\end{array}$ \\
\hline $\begin{array}{l}\text { S. typhimurium TA98, TA100, } \\
\text { TA1535, TA1537, TA1538/ } \\
\text { suspension and plate } \\
\text { incorporation assays }\end{array}$ & $\begin{array}{l}\text { Reverse } \\
\text { Mutation }\end{array}$ & - & - & $\begin{array}{l}\text { Litton Bionetics } \\
1978 \mathrm{~b}\end{array}$ & $\begin{array}{l}\text { Mixed } \\
\text { xylene }\end{array}$ \\
\hline $\begin{array}{l}\text { S. typhimurium TA98, TA100, } \\
\text { TA1535, TA1537, TA1538/ } \\
\text { plate incorporation assay }\end{array}$ & $\begin{array}{l}\text { Reverse } \\
\text { Mutation }\end{array}$ & - & - & $\begin{array}{l}\text { Shimizu et al. } \\
1985\end{array}$ & $p$-Xylene \\
\hline $\begin{array}{l}\text { S. typhimurium TA1535/ } \\
\text { pSK1002 (umuC-lacZ gene } \\
\text { expression) }\end{array}$ & SOS induction & - & - & $\begin{array}{l}\text { Nakamura et al. } \\
1987\end{array}$ & $\begin{array}{l}\text { Mixed } \\
\text { xylene }\end{array}$ \\
\hline $\begin{array}{l}\text { Escherichia coli WP2uvrA/ } \\
\text { plate incorporation assay }\end{array}$ & Mutation & - & - & $\begin{array}{l}\text { Shimizu et al. } \\
1985\end{array}$ & $p$-Xylene \\
\hline $\begin{array}{l}\text { E. coli WP2 }(\lambda) \text { (Ionii, sulA1, } \\
\text { trpE65, uvrA155, lamB+), } \\
\text { microscreen prophage- } \\
\text { induction assay }\end{array}$ & Mutation & - & - & $\begin{array}{l}\text { DeMarini et al. } \\
1991\end{array}$ & $\begin{array}{l}\text { Mixed } \\
\text { xylene }\end{array}$ \\
\hline $\begin{array}{l}\text { E. coli WP2, WP2uvrA, WP67, } \\
\text { CM611, WP100, } \\
\text { W3110polA+, p3478pola-I } \\
\text { DNA repair microsuspension } \\
\text { assay }\end{array}$ & DNA damage & - & - & $\begin{array}{l}\text { McCarroll et al. } \\
\text { 1981b }\end{array}$ & $\begin{array}{l}\text { Not reported } \\
\text { (technical } \\
\text { grade) }\end{array}$ \\
\hline $\begin{array}{l}\text { Bacillus subtilis } \mathrm{H} 17 \\
\text { M45/modified rec assay }\end{array}$ & DNA damage & - & - & $\begin{array}{l}\text { McCarroll et al. } \\
1981 a\end{array}$ & $\begin{array}{l}\text { Not reported } \\
\text { (technical } \\
\text { grade) }\end{array}$ \\
\hline \multicolumn{6}{|l|}{ Eukaryotic organisms: } \\
\hline $\begin{array}{l}\text { Saccharomyces cerevisiae } \\
\text { D4/suspension and plate } \\
\text { incorporation assays }\end{array}$ & $\begin{array}{l}\text { Mitotic gene } \\
\text { conversion }\end{array}$ & - & - & $\begin{array}{l}\text { Litton Bionetics } \\
1978 \mathrm{~b}\end{array}$ & $\begin{array}{l}\text { Mixed } \\
\text { xylene }\end{array}$ \\
\hline
\end{tabular}


Table 3-5. Genotoxicity of Xylene In Vitro

\begin{tabular}{|c|c|c|c|c|c|}
\hline \multirow[b]{2}{*}{ Species (test system) } & \multirow[b]{2}{*}{ End point } & \multicolumn{2}{|c|}{ Results } & \multirow[b]{2}{*}{ Reference } & \multirow[b]{2}{*}{ Isomer } \\
\hline & & $\begin{array}{l}\text { With } \\
\text { activation }\end{array}$ & $\begin{array}{l}\text { Without } \\
\text { activation }\end{array}$ & & \\
\hline \multicolumn{6}{|l|}{ Mammalian cells: } \\
\hline $\begin{array}{l}\text { Cultured mouse lymphoma } \\
\text { cells (L5178Y, TK+/-) }\end{array}$ & $\begin{array}{l}\text { Forward } \\
\text { mutation }\end{array}$ & - & - & $\begin{array}{l}\text { Litton Bionetics } \\
1978 \mathrm{~b}\end{array}$ & $\begin{array}{l}\text { Mixed } \\
\text { xylene }\end{array}$ \\
\hline Cultured human lymphocytes & $\begin{array}{l}\text { Sister chromatid } \\
\text { exchange and } \\
\text { chromosomal } \\
\text { aberrations }\end{array}$ & Not tested & - & $\begin{array}{l}\text { Gerner-Smidt } \\
\text { and Friedrich } \\
1978\end{array}$ & Not reported \\
\hline Cultured human lymphocytes & $\begin{array}{l}\text { Sister chromatid } \\
\text { exchange }\end{array}$ & Not tested & - & $\begin{array}{l}\text { Richer et al. } \\
1993\end{array}$ & $\begin{array}{l}\text { Mixed } \\
\text { xylene }\end{array}$ \\
\hline Cultured human lymphocytes & $\begin{array}{l}\text { DNA-damage } \\
\text { (comet assay) }\end{array}$ & Not tested & $+\mathrm{T}$ & $\begin{array}{l}\text { Morrozzi et al. } \\
1999\end{array}$ & $\begin{array}{l}\text { Mixed } \\
\text { xylene }\end{array}$ \\
\hline $\begin{array}{l}\text { Cultured Chinese hamster } \\
\text { ovary cells }\end{array}$ & $\begin{array}{l}\text { Sister chromatid } \\
\text { exchange and } \\
\text { chromosomal } \\
\text { aberrations }\end{array}$ & - & - & $\begin{array}{l}\text { Anderson et al. } \\
1990\end{array}$ & $\begin{array}{l}\text { Mixed } \\
\text { xylene }\end{array}$ \\
\hline $\begin{array}{l}\text { Cultured Syrian hamster } \\
\text { embryo cells-Simian } \\
\text { adenovirus SA7 }\end{array}$ & $\begin{array}{l}\text { Enhanced viral } \\
\text { transformation }\end{array}$ & & - & Casto 1981 & $\begin{array}{l}\text { Mixed } \\
\text { xylene }\end{array}$ \\
\hline
\end{tabular}

$-=$ negative result; $+\mathrm{T}=$ damage associated with cytotoxcity ( $14 \%$ viability) 
fragmentation may occur if cells are exposed to levels that cause cytotoxicity, presumably related to the activity of nucleases within dying cells.

Limited human data are available regarding the genotoxic effects of mixed xylene following inhalation exposure. No inhalation studies were located regarding the genotoxicity of $m-, o-$, or $p$-xylene in humans and animals. Results of studies by Pap and Varga (1987) and Richer et al. (1993) suggest that inhalation exposure of humans to mixed xylene is not associated with the induction of sister chromatid exchanges or chromosomal aberrations in peripheral lymphocytes. Results of other investigations were also negative for chromosomal aberrations in humans or rats exposed by inhalation to xylene; however, as the isomeric composition of the xylene in these studies was not reported (Haglund et al. 1980; Zhong et al. 1980), it is difficult to assess the contribution of the individual isomers of xylene. The rat study was limited by the lack of details regarding exposure concentrations and duration of exposure.

No studies were located regarding genotoxic effects in humans after oral exposure to mixed xylene or xylene isomers. No chromosomal aberrations or change in the incidence of micronuclei were observed in reticulocytes isolated from mice receiving oral doses of xylenes as high as $1,000 \mathrm{mg} / \mathrm{kg}$ within a 24-hour period (Feldt 1986).

No studies were located regarding genotoxic effects in humans after dermal exposure to mixed xylene or xylene isomers. A significant increase in lower molecular weight genomic DNA was detected in the skin of rats that received a dermal application of $250 \mu \mathrm{L}(1,200 \mathrm{mg} / \mathrm{kg}) m$-xylene for 1 hour (Rogers et al. 2001). Electrophoretic patterns indicative of DNA fragmentation appeared 2 hours after exposure ended and coincided with the significant increase in oxidative species in skin cells. Although the level of oxidative species subsequently was reduced - the authors speculate as a result of endogenous antioxidant activity-lower molecular weight DNA levels remained significantly elevated at 4 and 6 hours after exposure. DNA fragmentation coincided with histopathological evidence of skin irritation, inflammation, and separation of the epidermis and dermis (Gunasekar et al. 2003).

The absence of genotoxic effects following parenteral in vivo exposure to xylene has been reported in the bone marrow chromosomal aberration test with rats (Litton Bionetics 1978b), the bone marrow micronucleus test with mice (Mohtashamipur et al. 1985), and the sperm morphology test with rats (Washington et al. 1983). 
Mixed xylene, and the individual xylene isomers, have been tested for genotoxicity in a variety of shortterm in vitro assays. Results of standard mutagenicity assays indicate that mixed xylene and xylene isomers are not mutagenic in bacteria, yeast, or mammalian cells (Bos et al. 1981; Connor et al. 1985; DeMarini et al. 1991; Florin et al. 1980; Haworth et al. 1983; Litton Bionetics 1978b; McCarroll et al. 1981a, 1981b; NTP 1986; Shimizu et al. 1985). Mixed xylenes did not induce umu gene expression, part of the SOS response to DNA damage, in Salmonella typhimurium TA1535/pSK1002 (Nakamura et al. 1987). Mixed xylene did not induce chromosomal anomalies (chromosomal aberrations or sister chromatid exchanges) in cultured mammalian cells (Anderson et al. 1990; Gerner-Smidt and Friedrich 1978; Richer et al. 1993). Increased DNA damage in human lymphocytes exposed to a single concentration of mixed xylene $(5 \mu \mathrm{M})$ was associated with reduced cell viability (14\% of controls) and likely caused by nuclease activity in the dying cells (Morozzi et al. 1999). Mixed xylenes did not enhance the adenovirus transformation of hamster embryo cells (Casto 1981).

No mutagenic activity was demonstrated for any of the various metabolites of xylene in bacterial test systems. S. typhimurium strains TA98, TA100, TA1535, TA1537, and TA1538, with and without S9 metabolic activation, have been used to test the mutagenic activity of p-xylenol (Epler et al. 1979; Florin et al. 1980; Hejtmankova et al. 1979; Pool and Lin 1982), m-xylenol (Epler et al. 1979; Florin et al. 1980), and o-methylbenzyl alcohol (Bos et al. 1981). 2,4-Dimethylphenol has been evaluated in a gene reversion assay with Escherichia coli strain Sd-4-73 (Szybalski 1958).

In summary, genotoxicity studies on mixed xylene and the individual isomers of xylene have provided consistently negative results in a variety of in vitro and in vivo assays and test systems (bacteria, yeast, insects, cultured mammalian cells, mice, rats, and humans). Thus, there is sufficient evidence to conclude that mixed xylene, $m$-xylene, $o$-xylene, and $p$-xylene are nonmutagenic. Xylenes may cause DNA fragmentation at cytotoxic concentrations because of nucleases released from lysosomes in moribund cells. There is also limited evidence from bacterial test systems that suggests that xylene metabolites, specifically $m$-xylenol, $p$-xylenol, 2,4-dimethylphenol, and o-methylbenzyl alcohol, are also nonmutagenic.

\subsection{TOXICOKINETICS}

Studies in humans and animals have shown that xylenes are well absorbed by the inhalation and oral routes. Approximately $60 \%$ of inspired xylene is retained and approximately $90 \%$ of ingested xylene is absorbed. Absorption of xylene also occurs by the dermal route, but to a much lesser extent than by the 
inhalation and oral routes especially following exposure to xylene vapor. Following absorption, xylene is rapidly distributed throughout the body by way of the systemic circulation. In the blood, xylene is primarily bound to serum proteins. Xylene accumulates primarily in adipose tissue. All three isomers of xylene are primarily metabolized by oxidation of a methyl group and conjugation with glycine to yield the methylhippuric acid. In humans exposed to xylene, $>90 \%$ of the absorbed xylene is excreted in the urine as the methylhippuric acid. Aromatic hydroxylation of xylene to xylenol occurs to only a limited extent in humans. Less than $2 \%$ of an absorbed dose is excreted in the urine as xylenol. Other minor metabolites found in urine include methylbenzyl alcohol and glucuronic acid conjugates of the oxidized xylene. Metabolism in animals is qualitatively similar, but glucuronide conjugates make up a larger proportion of the urinary excretion products (see Figures 3-3 and 3-4). In addition, methylbenzaldehyde (the product of the action of alcohol dehydrogenase on methylbenzyl alcohol) has been detected in animals, where it may exert toxic effects, but its presence has not been confirmed in humans. In humans, about $95 \%$ of the absorbed xylene is excreted in the urine, with about $5 \%$ excreted unchanged in the exhaled air. Elimination from most tissue compartments is rapid, with slower elimination from muscle and adipose tissue.

\subsubsection{Absorption}

\subsubsection{Inhalation Exposure}

Xylenes are very soluble in blood and are therefore absorbed easily into the systemic circulation during inhalation exposure (Astrand 1982). Evidence for absorption of xylene by humans following inhalation exposure is provided by the observation that urine metabolites increase in proportion to exposure (Inoue et al. 1993; Jonai and Sato 1988; Kawai et al. 1991; Ogata et al. 1970; Riihimaki and Pfaffli 1978; Riihimaki et al. 1979b; Sedivec and Flek 1976b; Senczuk and Orlowski 1978; Wallen et al. 1985) and in proportion to increased ventilatory rates during exercise (Astrand 1982; Astrand et al. 1978; Bergert and Nestler 1991; Engstrom and Bjurstrom 1978; Riihimaki and Savolainen 1980; Riihimaki et al. 1979b). Absorption of the retained isomers appears to be similar, regardless of exposure duration or dose. The alveolar air concentrations of xylenes in male volunteers exposed at rest to $100 \mathrm{ppm}$ mixed xylenes for 70 minutes reached equilibrium within 10 minutes (Gamberale et al. 1978).

Many authors have measured the retention of xylene in the lungs following inhalation exposure. It is this retained xylene that is available for absorption into the systemic circulation. In experimental studies with human subjects, retention of the various isomers was similar following inhalation of $m-, o-$, or $p$-xylene, and averaged 63.6\% (Sedivec and Flek 1976b). Other authors have estimated that between 49.8 and 
72.8\% of inhaled xylene is retained (David et al. 1979; Ogata et al. 1970; Riihimaki and Pfaffli 1978; Riihimaki and Savolainen 1980; Wallen et al. 1985). Pulmonary retention does not appear to differ on the basis of sex (Senczuk and Orlowski 1978). However, one study reported that uptake of $m$-xylene during a 2-hour exposure at $200 \mathrm{mg} / \mathrm{m}^{3}$ was slightly higher in women than in men (Ernstgard et al. 2003). The enhanced pulmonary ventilation and cardiac output associated with physical exertion can increase the amount of xylene retained and subsequently absorbed into the body (Astrand et al. 1978; Riihimaki et al. 1979b). The study by Astrand et al. (1978) suggests that retention efficiency decreases as exposure duration increases.

In pregnant mice, approximately $30 \%$ of an administered inhalation dose of $600 \mathrm{ppm} p$-xylene was absorbed following a 10-minute exposure period (Ghantous and Danielsson 1986). Absorption was not quantified in other animal studies, but absorption can be inferred from the observed effects on pulmonary microsomal enzymes and the appearance of methylhippuric acid in the urine following inhalation of xylene (Carlsson 1981; David et al. 1979; Elovaara 1982; Elovaara et al. 1987; Patel et al. 1978).

\subsubsection{Oral Exposure}

Limited information is available on the absorption of xylene in humans and animals following ingestion. Excretion of urinary metabolites indicated that absorption had occurred following oral doses of either 40 or $80 \mathrm{mg} / \mathrm{kg}$ of $o$-xylene or $\mathrm{m}$-xylene in humans (Ogata et al. 1979). Although absorption was not fully quantified, recovery of specific metabolites demonstrated that at least $34 \%$ of $o$-xylene and $53 \%$ of $m$-xylene had been absorbed from the administered doses of $40 \mathrm{mg} / \mathrm{kg}$.

Measurement of urinary metabolites in rats over 24 hours demonstrated almost complete absorption (8792\%) following oral gavage dosing with $1.8 \mathrm{~g} \mathrm{~m}$-xylene, or $1.74 \mathrm{~g} \mathrm{o}$ - or $p$-xylene (Bray et al. 1949). Experiments in which $0.15 \mathrm{~mL}$ of radiolabelled $m$-xylene $(0.27 \mathrm{mg} / \mathrm{kg})$ was administered by oral gavage in 5\% aqueous gum acacia to male and female rats indicated that absorption was rapid (Turkall et al. 1992); peak blood levels of radioactivity were observed within 20 minutes. The half-life of absorption of $m$-xylene was twice as fast in females than in males, although the total amount absorbed over 24 hoursthe area under the curve (AUC) for plasma radioactivity — was the same in both sexes (about $0.2 \%$ of the initial dose/mL/hour). When $m$-xylene was first adsorbed to $0.5 \mathrm{~g}$ of a sandy soil before mixing with the vehicle and gavage administration, the absorption half-life was twice as long in female rats but unaffected in males (Turkall et al. 1992); adsorption to clay soil did not produce a biologically significant difference 
from $m$-xylene administered alone. Adsorption to sandy soil doubled the peak blood levels of radioactivity and increased the plasma AUC by $75 \%$ in female rats, but had no such effects in males.

\subsubsection{Dermal Exposure}

Results of experimental studies with humans indicate that $m$-xylene is absorbed following dermal exposure; however, the extent of penetration and absorption of $m$-xylene through skin is not nearly as great as that resulting from inhalation (Engstrom et al. 1977; Riihimaki 1979b; Riihimaki and Pfaffli 1978). Dermal absorption may occur via exposure to $m$-xylene vapors, as well as through direct dermal contact with the solvent (Dutkiewicz and Tyras 1968; Engstrom et al. 1977; Kezic et al. 2000; Loizou et al. 1999; Riihimaki 1979b; Riihimaki and Pfaffli 1978). Absorption of $m$-xylene through the skin was measured at a maximal permeation rate, occurring between 15 and 25 minutes at a flux of $46 \mathrm{nmol} / \mathrm{cm}^{2} /$ minute (Kezic et al. 2001). In humans, the estimated absorption rate following immersion of both hands in $m$-xylene for 15 minutes was approximately $2 \mu \mathrm{g} / \mathrm{cm}^{2} /$ minute (Engstrom et al. 1977). Another study measured a rate of absorption of $75-160 \mu \mathrm{g} / \mathrm{cm}^{2} /$ minute when xylene was applied to forearm skin (Dutkiewicz and Tyras 1968). Absorption of $m$-xylene vapor through the skin has been estimated at between 0.1 and $2 \%$ of the inhaled dose (Kezic et al. 2000; Loizou et al. 1999; Riihimaki and Pfaffli 1978). Dermal permeability constants averaging $0.25 \mathrm{~cm} /$ hour were calculated from exposure of subjects to 600 ppm m-xylene for 3.5 hours (McDougal et al. 1990; Riihimaki and Pfaffli 1978). In human subjects exposed to $6770 \mathrm{ppm} m$-xylene vapor on the hand and forearm the average flux through the skin was $0.091 \mathrm{mg} / \mathrm{L} /$ hour for a 20 -minute exposure, falling to $0.061 \mathrm{mg} / \mathrm{L} /$ hour for a 180 -minute exposure (Kezic et al. 2004). Simultaneously, the maximal flux into the blood was $0.034 \mathrm{mg} / \mathrm{L} / \mathrm{hour}$ after 20 minutes, rising to $0.063 \mathrm{mg} / \mathrm{L} /$ hour after a 180-minute exposure. Maximum permeation rates of $m$-xylene vapor were achieved within 90 minutes (Kezic et al. 2004). Dermal absorption of $m$-xylene following topical administration or exposure to vapor was 3 times greater in subjects with atopic dermatitis compared to normal subjects (Engstrom et al. 1977; Riihimaki and Pfaffli 1978).

Limited information is available regarding the absorption of xylene following dermal exposure in animals. Permeability of rat skin to $m$-xylene was estimated from blood levels obtained during dermal exposure to liquid m-xylene (Morgan et al. 1991; Skowronski et al. 1990) or $m$-xylene vapors (McDougal et al. 1990). Peak blood levels of $m$-xylene were reached within 2 hours of topical application from sealed dermal enclosures in rats and slowly declined during the 24-hour observation period (Morgan et al. 1991); an average of $562 \mathrm{mg}(0.65 \mathrm{~mL})$ was absorbed across a $3.1 \mathrm{~cm}^{2}$ area of skin $(1 \%$ of total surface area) from the initial volume of $2 \mathrm{~mL}$ over 24 hours. The total amount absorbed was reduced when 
$m$-xylene was administered in aqueous solution (Morgan et al. 1991). Dermal permeability constants calculated for rats exposed to 5,000 ppm $m$-xylene averaged $0.723 \mathrm{~cm} /$ hour, about 3 times greater than those calculated for humans (McDougal et al. 1990); the flux was calculated as $0.0151 \mathrm{mg} / \mathrm{cm}^{2} / \mathrm{hour}$. Skin:air partition values for a series of solvents including $m$-xylene correlated well with permeability constants (McDougal et al. 1990), but not with octanol-water partition coefficients. $m$-Xylene adsorbed on sandy soil or clay soils showed lower peak absorption than for $m$-xylene alone and clay soil significantly prolonged the absorption half-life, but the total amount absorbed over an unspecified period was unchanged (Abdel-Rahman et al. 1993; Skowronski et al. 1990).

Dermal penetration rates of xylene have been estimated using excised skin of rats exposed to liquid or vapor. The absorption of $o$-xylene by excised abdominal skin from rats increased with the time of contact (Tsuruta 1982); the penetration rate was estimated to be $0.006 \mathrm{mg} / \mathrm{cm}^{2} /$ hour. The flux of $o$-xylene through the dorsal skin of hairless rats was measured as $0.22 \mathrm{mg} / \mathrm{cm}^{2} /$ hour (Ahaghotu et al. 2005). The skin:air partition coefficient for $m$-xylene was found to be $50.4 \pm 1.7$ using rat skin in vitro exposed to 203 ppm $m$-xylene vapor (Mattie et al. 1994); the concentration of $m$-xylene in the skin reached equilibrium in 2 hours. Dermal absorption studies using excised skin are not directly comparable to in vivo studies because of the lack of an intact blood supply, but diffusion through the skin appears to be the limiting factor rather than removal from the skin by the blood (McDougal et al. 1990).

\subsubsection{Distribution}

Results of experiments to derive tissue:air partition coefficients for xylene indicate that the isomers are expected to have similar distributions in the body (Table 3-6). Factors influencing the distribution of xylene in blood are discussed in Section 3.5.1.

\subsubsection{Inhalation Exposure}

In human subjects exposed by inhalation to $\leq 44 \mathrm{ppm}$ deuterium-labeled xylene isomers for 2 hours, peak blood xylene concentrations were highest exactly at the end of exposure and then declined (Adams et al. 2005). Absorbed xylene in venous blood was distributed in cells (12\%) and serum (88\%), with about $9 \%$ of the total amount in blood associated with protein-free serum (Riihimaki et al. 1979b). Following systemic circulation, xylene is distributed primarily to adipose tissue. Estimates of the amount of xylene accumulated in human adipose tissue range from 4 to 10\% of the absorbed dose (Astrand 1982; Engstrom and Bjurstrom 1978; Riihimaki et al. 1979b). It has been suggested that following prolonged 
Table 3-6. Partition Coefficients for Xylenes

\begin{tabular}{|c|c|c|c|c|}
\hline \multirow[b]{2}{*}{ Reference } & \multirow[b]{2}{*}{ Species } & \multicolumn{3}{|c|}{ Partition coefficient $^{a}$} \\
\hline & & $m$-Xylene & o-Xylene & $p$-Xylene \\
\hline & & \multicolumn{3}{|c|}{ Blood:Air } \\
\hline Gargas et al. 1989 & Rat (male F-344) & $46 \pm 1.5$ & $44.3 \pm 2.0$ & $41.3 \pm 3.5$ \\
\hline Kaneko et al. 1991a & Rat (male Wistar) & $39.9 \pm 7.18^{b}$ & NR & NR \\
\hline $\begin{array}{l}\text { Kumarathasan et al. } \\
1998\end{array}$ & $\begin{array}{l}\text { Rat (male } \\
\text { Sprague-Dawley) }\end{array}$ & $40.3 \pm 1.4$ & NR & $37.0 \pm 0.4$ \\
\hline Thrall et al. $2002^{\mathrm{C}}$ & Rat & & 37.8 & \\
\hline $\begin{array}{l}\text { Sato and Nakajima } \\
1979\end{array}$ & Human & $26.4 \pm 0.9^{b}$ & $31.1 \pm 2.3^{b}$ & $37.6 \pm 3.5^{b}$ \\
\hline Pierce et al. 1996 & Human & $31.9 \pm 0.45$ & $35.2 \pm 0.45$ & $39.0 \pm 0.70$ \\
\hline \multirow[t]{2}{*}{ Thrall et al. $2002^{c}$} & Human & & 34.0 & \\
\hline & & \multicolumn{3}{|c|}{ Fat:Air } \\
\hline Gargas et al. 1989 & Rat (male F-344) & $1,859 \pm 93$ & $1,877 \pm 132$ & $1,748 \pm 65$ \\
\hline Kaneko et al. 1991a & Rat (male Wistar) & $2,050 \pm 459^{b}$ & NR & NR \\
\hline Pierce et al. 1996 & Rat (male F-344) & $2,325 \pm 194$ & $2,930 \pm 260$ & NR \\
\hline $\begin{array}{l}\text { Kumarathasan et al. } \\
1998\end{array}$ & $\begin{array}{l}\text { Rat (male } \\
\text { Sprague-Dawley) }\end{array}$ & $1,970 \pm 34$ & NR & $1,863 \pm 37$ \\
\hline \multirow[t]{2}{*}{ Pierce et al. 1996} & Human & $1,919 \pm 53$ & $2,460 \pm 63$ & $2,019 \pm 102$ \\
\hline & & \multicolumn{3}{|c|}{ Liver:Air } \\
\hline Gargas et al. 1989 & Rat (male F-344) & $90.9 \pm 4.4$ & $108 \pm 7$ & $90.0 \pm 4.3$ \\
\hline Kaneko et al. 1991a & Rat (male Wistar) & $79.9 \pm 9.42^{b}$ & NR & NR \\
\hline \multirow[t]{2}{*}{$\begin{array}{l}\text { Kumarathasan et al. } \\
1998\end{array}$} & $\begin{array}{l}\text { Rat (male } \\
\text { Sprague-Dawley) }\end{array}$ & $66.8 \pm 6.6$ & NR & $62.1 \pm 5.0$ \\
\hline & & \multicolumn{3}{|c|}{ Muscle:Air } \\
\hline Gargas et al. 1989 & Rat (male F-344) & $41.9 \pm 5.7$ & $51.5 \pm 6.7$ & $38.4 \pm 4.1$ \\
\hline Kaneko et al. 1991a & Rat (male Wistar) & $79.7 \pm 20.2^{b}$ & NR & NR \\
\hline \multirow[t]{2}{*}{$\begin{array}{l}\text { Kumarathasan et al. } \\
1998\end{array}$} & $\begin{array}{l}\text { Rat (male } \\
\text { Sprague-Dawley) }\end{array}$ & $41.3 \pm 0.1$ & NR & $43.21 \pm 3.4$ \\
\hline & & \multicolumn{3}{|c|}{ Milk:Air } \\
\hline Fisher et al. 1997 & Human & \multicolumn{3}{|c|}{$133 \pm 54.58$ for $o-, m-, p$-xylenes } \\
\hline
\end{tabular}


occupational exposure to xylene, significant amounts of the solvent could accumulate in adipose tissue (Astrand 1982; Engstrom and Bjurstrom 1978).

Studies in mice (Bergman 1983; Ghantous and Danielsson 1986) and rats (Carlsson 1981; Ito et al. 2002) indicate that the distribution of $m$ - or $p$-xylene and their metabolites is characterized by high uptake in lipid-rich tissues, such as brain and fat. High uptake also occurs in well-perfused organs, such as the liver and kidney. Using low-temperature (cryogenic) whole-body autoradiography of male mice exposed by inhalation to ${ }^{14} \mathrm{C}$-labeled $m$-xylene for 10 minutes, Bergman (1983) observed a high level of unmetabolized $m$-xylene (volatile radioactivity) in body fat, bone marrow, white matter of the brain, spinal cord, spinal nerves, liver, and kidney immediately after exposure. High levels of metabolites (nonvolatile radioactivity) were present in the blood, liver, lung, kidney, and adrenal medulla. Unmetabolized $m$-xylene persisted in the central nervous system and spinal nerves for up to 1 hour postexposure, whereas a considerable amount persisted in body fat at 4-8 hours after exposure. Metabolites were detectable post-exposure in the adrenal medulla for 0.5 hours, in the liver for 2 hours, in the kidney for up to 8 hours, in bile for 2-8 hours, in the intestinal lumen for up to 24 hours, and in the nasal mucosa and bronchi for 2-24 hours. No radioactivity was detectable by 48 hours. In male Sprague-Dawley rats exposed to 2,000 ppm $m$-xylene vapor 4 hours/day for 5 consecutive days, $m$-xylene concentrations were measured in tissues by gas chromatography (Ito et al. 2002). The highest concentrations of $m$-xylene were observed in peri-intestinal fat and subcutaneous fat, whereas concentrations more than 40 times lower were detected in lung, spleen, testis, heart, kidney, liver, and psoas muscle. In the brain subdivided into four parts, the concentration of $m$-xylene was 3-6 times lower than in subcutaneous fat. The uneven distribution of inhaled $m$-xylene in the brain was associated with changes in $\mathrm{GABA}_{\mathrm{A}}$ receptor binding (discussed in Section 3.5.2).

According to an intermediate-duration animal study, the level of xylene stored in body fat may decrease as exposure continues due to an increase in metabolic rate possibly by inducing its own metabolism (Savolainen et al. 1979a). Levels of $m$-xylene in perirenal fat of rats exposed to $300 \mathrm{ppm}$ technical xylene decreased from 67.6 to $36.6 \mu \mathrm{g} / \mathrm{g}$ tissue as exposure duration increased from 5 to 18 weeks (Savolainen et al. 1979a).

$p$-Xylene and $o$-xylene have been shown to readily cross the placenta and were distributed in amniotic fluid and embryonic and fetal tissues (Ghantous and Danielsson 1986; Ungvary et al. 1980b). The level detected in fetal tissues (brain, liver, lung, and kidney), which are low in lipids, was only $2 \%$ of that 
detected in the maternal brain tissue, which contains large amounts of lipids (Ghantous and Danielsson 1986). Also, higher levels were detected in fetal tissues than in amniotic fluid (Ungvary et al. 1980b).

\subsubsection{Oral Exposure}

No studies were located regarding distribution in humans following oral exposure to mixed xylene or xylene isomers. In rats administered $m$-xylene by gavage, fat contained the highest tissue concentration of radioactivity; approximately $0.3 \%$ of the administered dose was found per gram of fat in females and $0.1 \%$ per gram of fat in males (Turkall et al. 1992).

\subsubsection{Dermal Exposure}

No studies were located regarding distribution of xylene in humans following dermal exposure to mixed xylene or individual isomers. Extremely limited information was located regarding distribution in animals following dermal absorption. After topical administration, $m$-xylene was rapidly detectable in plasma, reaching peak concentrations in about 2 hours (Skowronski et al. 1990). About $0.003 \%$ of the initial dose was detected per gram of subcutaneous fat 48 hours after exposure.

\subsubsection{Metabolism}

The biotransformation of xylene in humans proceeds primarily by the oxidation of a side-chain methyl group by microsomal enzymes (mixed function oxidases) in the liver to yield toluic acids (methylbenzoic acids). These toluic acids conjugate with glycine to form toluic acids (methylhippuric acids) that are excreted into the urine (Astrand et al. 1978; Norstrom et al. 1989; Ogata et al. 1970, 1979; Riihimaki et al. 1979a; Sedivec and Flek 1976b; Senczuk and Orlowski 1978). This metabolic pathway accounts for almost all of the absorbed dose of xylene, regardless of the isomer, route of administration, administered dose, or duration of exposure. Minor metabolic pathways that account for $<10 \%$ of the absorbed dose include the elimination of unchanged compound in the exhaled breath and in the urine, and the urinary elimination of methylbenzyl alcohols, $o$-toluylglucuronides (o-toluic acid glucuronide), xylene mercapturic acid (Norstrom et al. 1988), and xylenols (dimethylphenols). Dimethylphenylmercapturic acid (DPMA) was detected in only 9 out of 27 samples of exposed workers and occurred at a ratio of only $0.0003 \%$ compared to the main metabolite, methylhippuric acid (Gonzalez-Reche et al. 2003). The metabolism of the various xylene isomers in humans is presented in Figure 3-3. 


\section{Figure 3-3. Metabolic Scheme for Xylenes-Humans}

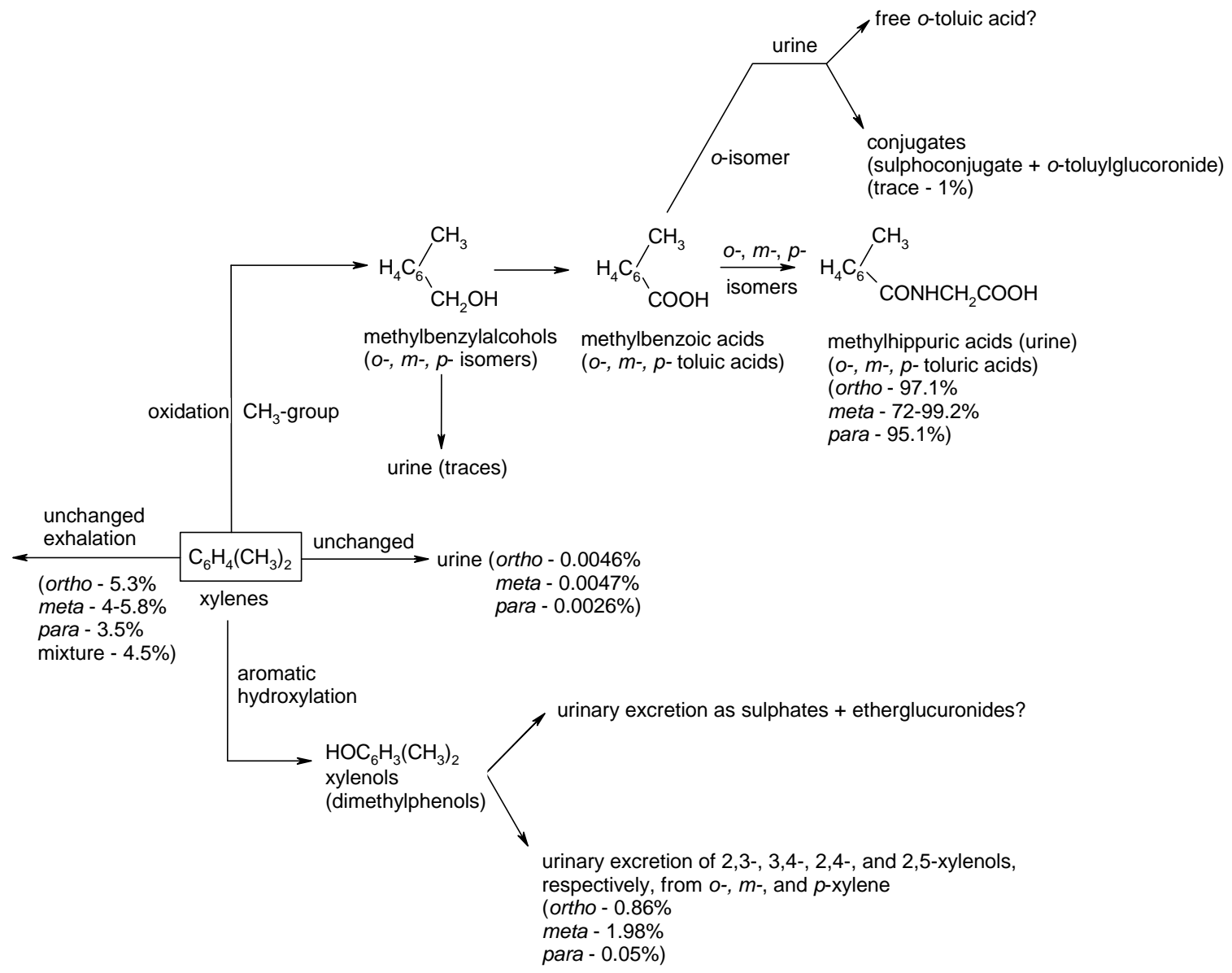

Sources: derived from Astrand et al. 1978; Ogata et al. 1980; Riihimaki et al. 1979a, 1979b; Sedivec and Flek 1976b; Senczuk and Orlowski 1978; Toftgard and Gustafsson 1980 
In physiologically based pharmacokinetic (PBPK) modeling of blood concentration data from male subjects exposed by inhalation to 0-44 ppm of deuterium-labeled xylene isomers for 2 hours, the rate of hepatic metabolism was held constant scaled to body weight (Adams et al. 2005). Simulations revealed that apparent systemic clearance of isomers sometimes exceeded that predicted by the hepatic blood flow parameter, suggesting that extrahepatic tissues could play a significant role in xylene metabolism in certain individuals.

The metabolism of xylene in animals is qualitatively similar to that of humans, though quantitative differences do exist (Bakke and Scheline 1970; Bray et al. 1949; Ogata et al. 1979; Sugihara and Ogata 1978; van Doorn et al. 1980). The metabolism of the various isomers in animals is presented in Figure 3-4. The major quantitative difference between animals and humans occurs in the metabolism of the metabolic intermediate methylbenzoic acid (toluic acid). In rats given $m-, o-$, or $p$-xylene by intraperitoneal injection, $10-56.6 \%$ of the administered dose of $o$-xylene was excreted in the urine as $o$-toluylglucuronide, whereas approximately $1 \%$ of the administered doses of $m$ - and $p$-xylene was metabolized to the appropriate toluylglucuronide (Ogata et al. 1979; van Doorn et al. 1980). The amounts of $m$-methylhippuric acid and $p$-methylhippuric acid excreted in the urine accounted for 49-63 and 64$75 \%$ of the administered dose, respectively (Ogata et al. 1979; Sugihara and Ogata 1978). Similar results were seen in rats administered $m$-xylene by gavage (Turkall et al. 1992). In studies with rabbits, $60 \%$ of an administered $o$-xylene dose, $81 \%$ of an $m$-xylene dose, and $88 \%$ of a $p$-xylene dose were excreted in the urine as methylhippuric acids (Bray et al. 1949). Minor quantities of methylbenzyl alcohols and xylenols have also been detected in the urine of experimental animals administered xylene isomers (Bakke and Scheline 1970; Ogata et al. 1979; Turkall et al. 1992; van Doorn et al. 1980). In a study of metabolites formed by aromatic hydroxylation, rats administered $100 \mathrm{mg} / \mathrm{kg}$ doses of xylene isomers eliminated $0.1 \%$ of a dose of $o$-xylene in the urine as 3,4-xylenol and $0.03 \%$ as 2,3 -xylenol, $0.9 \%$ of a dose of $m$-xylene as 2,4-xylenol, and 1\% of a dose of $p$-xylene as 2,5-xylenol (Bakke and Scheline 1970); A trace of the methylbenzyl alcohol was also detected in the urine of rats given $o$ - and $m$-xylene, but not in rats given $p$-xylene. In rats administered $m$-xylene by the dermal route, the major metabolite in the urine over a 24-hour period was identified as methylhippuric acid (82.3\%), with xylenol comprising 7.2\% and unchanged $m$-xylene comprising 3.8\% of the urinary products (Skowronski et al. 1990). In rats given $m$-xylene adsorbed onto sandy soil, the proportion of xylenol present in the urine over the first 12 hours of excretion was significantly increased.

Studies in animals have also shown that the metabolism of xylene may be influenced by prior exposures to xylene (Elovaara et al. 1989). Pretreatment of rats with $m$-xylene increased the percentage of 


\section{Figure 3-4. Metabolic Scheme for Xylenes-Animals}

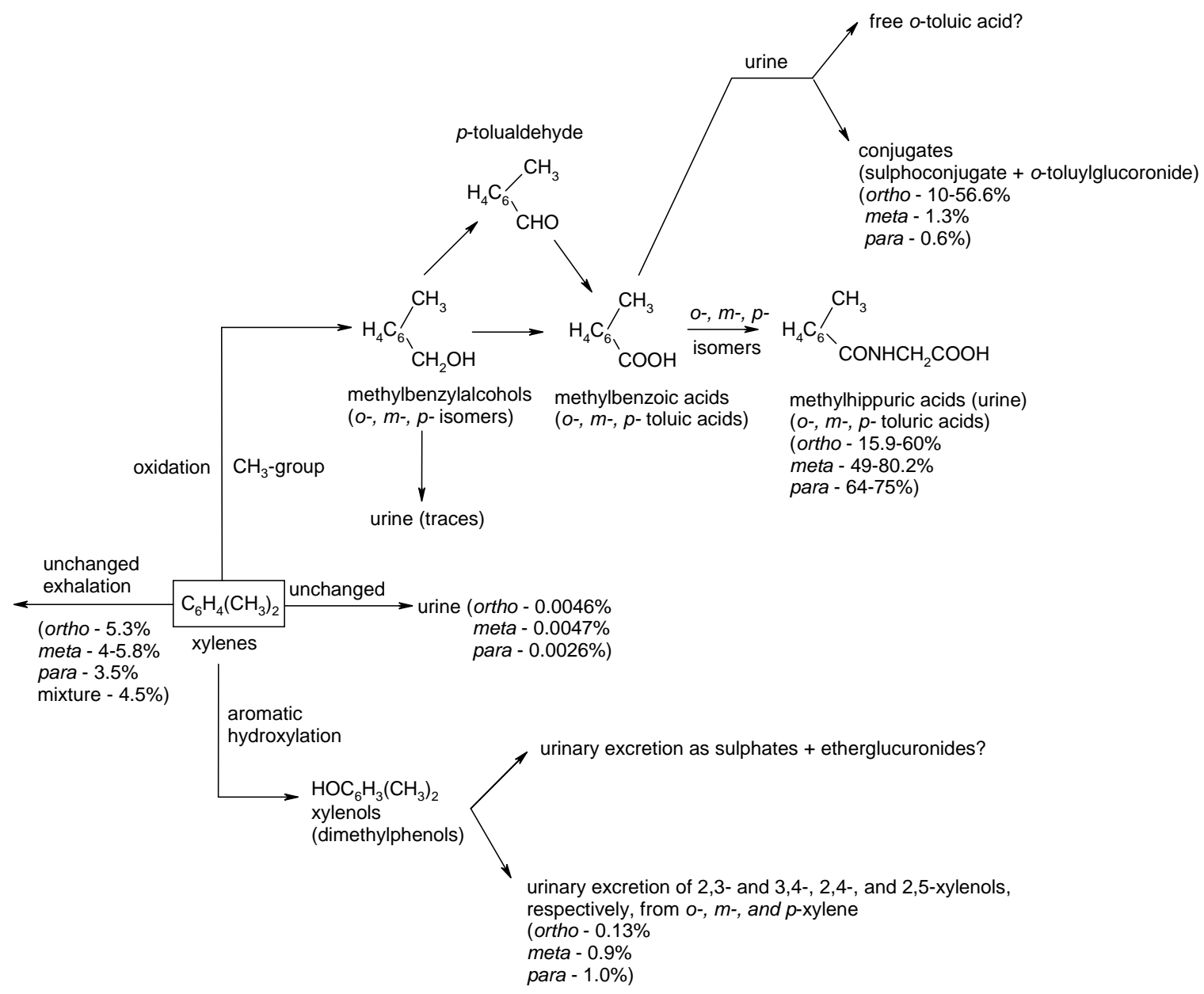

Sources: derived from Bakke and Scheline 1970; Bray et al. 1949; Ogata et al. 1980; Sugihara and Ogata 1978; Toftgard and Gustafsson 1980; van Doorn et al. 1980 
methylhippuric acid and thioethers in the urine by approximately 10\%. A toxic metabolite of xylene in rats and rabbits appears to be methylbenzaldehyde (tolualdehyde) (Carlone and Fouts 1974; Patel et al. 1978; Smith et al. 1982). It is formed by the action of alcohol dehydrogenase on methylbenzyl alcohol in lung and liver tissues (Elovaara et al. 1987); the presence of methylbenzaldehyde has not been confirmed in humans. Lung tissue can be damaged by this intermediate because of its selective inactivation of enzymes involved in microsomal electron transport (mixed function oxidases, cytochrome P-450) (Elovaara et al. 1987).

The differences in xylene metabolism observed between humans and animals may, in part, be explained by differences in the size of the doses given to humans and animals in experimental studies (David et al. 1979; Ogata et al. 1979; van Doorn et al. 1980). The formation of glucuronic acid derivatives may be an emergency mechanism that is activated when the organism can no longer conjugate all acids with glycine (Ogata et al. 1979; Sedivec and Flek 1976b; van Doorn et al. 1980). Humans dosed with $19 \mathrm{mg} / \mathrm{kg}$ xylene excreted only methylhippuric acids in the urine, whereas rabbits exposed to $600 \mathrm{mg} / \mathrm{kg}$ excreted both methylhippuric acids and derivatives of glucuronic acid (Sedivec and Flek 1976b). The second-phase conjugation of the main oxidized intermediate (methylbenzoic acid with glycine to form methylhippuric acid) may be the rate-limiting step in humans. The maximum rate of glycine mobilization limits the rate of conjugation to a level below $200 \mu \mathrm{mol} /$ minute (Riihimaki et al. 1979a, 1979b). If this limit is approached, other elimination pathways may be activated, such as conjugation with glucuronic acid or aromatic hydroxylation to form xylenols. The capacity of the first-phase oxidation reaction, encompassing both side-chain and aromatic oxidation, is not known. Aromatic oxidation of xylene could possibly produce toxic intermediates and phenolic end-metabolites (Riihimaki et al. 1979b); however, this is a minor metabolic pathway.

Using a low-temperature (cryogenic), whole-body autoradiography technique for mice exposed by inhalation to radiolabeled $m$-xylene for 10 minutes, Bergman (1983) observed high levels of metabolites (non-volatile radioactivity) in the blood, liver, lung, kidney, and adrenal medulla immediately after exposure. Metabolites were detectable post-exposure in the adrenal medulla for 0.5 hours, in the liver for 2 hours, in the kidney for up to 8 hours, in bile for 2-8 hours, in the intestinal lumen for up to 24 hours, and in the nasal mucosa and bronchi for 2-24 hours. No radioactivity was detectable 48 hours after exposure, indicating that there was no significant binding of xylene metabolites to tissue macromolecules. 


\subsubsection{Elimination and Excretion}

\subsubsection{Inhalation Exposure}

In humans, about $95 \%$ of absorbed xylene is biotransformed and excreted as urinary metabolites, almost exclusively as methylhippuric acids; the remaining 5\% is eliminated unchanged in the exhaled breath (Astrand et al. 1978; Ogata et al. 1979; Pellizzari et al. 1992; Riihimaki et al. 1979b; Sedivec and Flek 1976b; Senczuk and Orlowski 1978). Less than $0.005 \%$ of the absorbed dose of xylene isomers is eliminated unchanged in the urine, and $<2 \%$ is eliminated as xylenols (Sedivec and Flek 1976b). The excretion of methylhippuric acids is rapid and a significant amount is detected in the urine within 2 hours of exposure. The amount of methylhippuric acid increases with time. Differences in the amount of the metabolites excreted depend on the interpersonal differences in lung ventilation and retention, not on the isomer of xylene (Sedivec and Flek 1976b).

There appear to be at least two distinct phases of elimination, a relatively rapid one (half-life, 1 hour) and a slower one (half-life, 20 hours). These phases of elimination are consistent with the distribution of xylene into three main tissue compartments; the rapid and slower elimination phases correspond to elimination from the muscles and the adipose tissue, respectively, whereas the elimination of xylene from the parenchymal organs is so rapid that the available studies could not monitor it (Ogata et al. 1970; Riihimaki et al. 1979a, 1979b). It is also possible that the renal excretion of the most common xylene metabolite, methylhippuric acid, takes place via the tubular active secretion mechanism of organic acids. Renal excretion is not a rate-limiting step in the elimination of absorbed xylene under normal physiological conditions (Riihimaki et al. 1979b). Physiologically based pharmacokinetic modeling suggests that the urinary excretion of $m$-methylhippuric acid following $m$-xylene exposure of humans is linear at concentrations up to $500 \mathrm{ppm}$, and that elimination of $m$-methylhippuric acid is slower in individuals with a greater percentage of body fat (e.g., women) (Kaneko et al. 1991a, 1991b). Systemic clearance rates in male subjects following inhalation of $\leq 44 \mathrm{ppm}$ deuterium-labeled xylene isomers for 2 hours ranged between 116 and $129 \mathrm{~L} /$ hour (Adams et al. 2005). The terminal half-life averaged 34 hours for all three isomers among all subjects, but varied widely among individuals from 12 to 108 hours.

Volunteers acutely exposed by inhalation to 100 or $200 \mathrm{ppm} m$-xylene for 7 hours excreted 54 and $61 \%$, respectively, of the administered dose by 18 hours after exposure ended (Ogata et al. 1970). Following intermittent acute exposure of men and women to 23, 69, or $138 \mathrm{ppm} \mathrm{m-xylene,} \mathrm{excretion} \mathrm{of}$ $m$-methylhippuric acid peaked 6-8 hours after exposure began. It decreased rapidly, regardless of 
exposure level or sex, after exposure had ended. Almost no xylene or $m$-methylhippuric was detected 24 hours later (Senczuk and Orlowski 1978). Following exposure to $200 \mathrm{mg} / \mathrm{m}^{3} \mathrm{~m}$-xylene for 2 hours, small differences in elimination were noted between genders (Ernstgard et al. 2003). Women excreted $31 \%$ more (AUC) unmetabolized $m$-xylene in exhaled air than men, whereas men excreted $37 \%$ more (AUC) $m$-methylhippuric acid in urine than women.

Exercise increased the amount of xylene absorbed and thus increased the amount of $m$-methylhippuric acid and 2,4-xylenol eliminated in the urine of men exposed to $m$-xylene (Riihimaki et al. 1979b). No evidence of saturation of metabolism of xylene was observed at exposures as high as $200 \mathrm{ppm}$ (Riihimaki et al. 1979b). The excretion of $m$-methylhippuric acid appeared to correspond very closely to the estimated xylene uptake and expired xylene represented about $4-5 \%$ of the absorbed xylene in all exposure groups (Riihimaki et al. 1979b). Urinary excretion of methylhippuric acid correlated well with exposure (Kawai et al. 1992; Lapare et al. 1993; Skender et al. 1993), and based on a study of workers occupationally exposed to mixed xylenes (geometric mean TWA 14 ppm), Inoue et al. (1993) estimated a slope of $13 \mathrm{mg}$ methylhippuric acid/L/ppm (11.1 mg/g creatinine/ppm) for all three isomers. A sexrelated difference in the urinary excretion of methylhippuric acids was not observed (Inoue et al. 1993).

A few studies have evaluated the kinetics of elimination of the metabolites of xylene following inhalation exposure of experimental animals. The primary urinary metabolite is methylhippuric acid (David et al. 1979). A dose-relationship in the urinary elimination of $m$-methylhippuric acid in rats was observed immediately after exposure to 50 or $500 \mathrm{ppm} m$-xylene for 6 hours (Kaneko et al. 2000). Another study found the increase of urinary elimination of methylhippuric acid metabolites to be linear in rats exposed for 5 hours to mixed xylene between 75 and $150 \mathrm{ppm}$, but non-linear between exposures at 150 and 225 ppm (Tardif et al. 1992). Concomitantly, there were non-linear increases in the amount of xylene excreted unchanged in exhaled air, indicating possible saturation of metabolism at $225 \mathrm{ppm}$.

Using a low-temperature (cryogenic), whole-body autoradiography technique for mice exposed by inhalation to radiolabeled $m$-xylene for 10 minutes, Bergman (1983) observed elimination of metabolites (non-volatile radioactivity) in bile and feces. Clearance of metabolites was completed in the adrenal medulla by 0.5 hours, in the liver after a little over 2 hours, in the kidney after 8 hours, in bile after 8 hours, in the intestinal lumen after 24 hours, and in the nasal mucosa and bronchi after 24 hours. No radioactivity was detectable 48 hours after exposure. 


\subsubsection{Oral Exposure}

Limited information is available on the elimination of the metabolites of xylene following ingestion in humans. In an unspecified number of male volunteers given oral doses of $40 \mathrm{mg} / \mathrm{kg} / \mathrm{day}$ of $o$-xylene or $m$-xylene, the molar (mol) excretion ratios (total excretion [mol] in urine during appropriate interval/dose administered [mol] x 100[\%]) for o-methylhippuric acid and m-methylhippuric acid were 33.1 and 53.1, respectively (Ogata et al. 1979). More of the $m$-xylene is eliminated as methylhippuric acid than the ortho derivative for $o$-xylene. The molar excretion ratio for $o$-toluic acid glucuronide (o-toluylglucuronide) was 1.0 in men given $o$-xylene as an oral dose of $40 \mathrm{mg} / \mathrm{kg} /$ day. The amounts of $o$-methylhippuric acid (o-toluic acid) and of $o$-toluic acid glucuronide excreted in the urine attained maximal levels in 3-6 hours of exposure, while that of $m$-methylhippuric acid attained peak levels in 13 hours (Ogata et al. 1979). These results indicate that the major elimination pathway of $o$-xylene is the formation of $o$-methylhippuric acid in humans. The formation of $o$-toluic acid glucuronide is a minor pathway for the elimination of $o$-toluic acid, but would be available in the event of saturation of the major pathway.

Excretion of radioactivity by rats following an oral dose of $m$-xylene showed most excretion occurred in the urine during the first 12 hours after dosing ( $50-59 \%$ of the dose), with a total of $96.2 \%$ eliminated in urine in males and 73.7\% in urine in females over 48 hours (Turkall et al. 1992). Approximately 8 and $22 \%$ of the total dose was excreted in exhaled air by males and females, respectively, during the first 12 hours. $m$-Methylhippuric acid comprised $67-75 \%$ of the urinary radioactivity, with xylenol comprising $2-18 \%$, and unchanged xylene comprising approximately $1 \%$. When $m$-xylene adsorbed onto sandy soil matrix was administered, the excretion of radioactivity in urine and exhaled air in female rats was significantly decreased compared to $m$-xylene during the first 12 hours (Turkall et al. 1992). In males given xylene in a sandy or clay soil matrix, the excretion of radioactivity was increased in exhaled air during the first 12 hours. In a study of aromatic hydroxylation products of xylene metabolism, rats administered $100 \mathrm{mg} / \mathrm{kg}$ doses of xylene isomers eliminated $1 \%$ or less of the administered dose as xylenols in the urine, along with trace amounts of methylbenzyl alcohols for $o$ - and $m$-xylene only (Bakke and Scheline 1970).

\subsubsection{Dermal Exposure}

The elimination of $m$-xylene has been studied in human subjects exposed dermally either to liquid or to vapor. The elimination of liquid $m$-xylene absorbed dermally in humans following a 15-minute exposure was through the exhaled breath and urine (Engstrom et al. 1977; Riihimaki and Pfaffli 1978). Elimination 
in the exhaled breath followed a two-phase elimination curve with a rapid half-life of 1 hour and a longer half-life of 10 hours. In human subjects who immersed both hands in $m$-xylene for 15 minutes, the rate of excretion of $m$-methylhippuric acid was approximately $50 \mu \mathrm{mol} /$ hour at 2 hours and $2 \mu \mathrm{mol} /$ hour 3 hours later (Riihimaki 1979b). In male subjects whose hand and forearm were exposed dermally to 6,770 ppm $m$-xylene vapor for up to 3 hours, elimination of xylene in the exhaled breath was detected within 10 minutes and continued up to several hours after the exposure ended (Kezic et al. 2004).

In rats receiving a dermal application of $15 \mathrm{mg}$ of radiolabeled $m$-xylene per $\mathrm{cm}^{2}$, elimination over 48 hours was primarily in unmetabolized expired air ( $61.9 \%$ of the initial dose), with $42.7 \%$ excreted as metabolites in the urine and $0.1 \%$ in feces (Skowronski et al. 1990). The majority of the excretion in expired air occurred within the first 12 hours, with excretion in the urine occurring primarily during the first 24 hours; fecal excretion was detected the second day. If $m$-xylene was applied to the skin in the form of a sandy soil matrix, the excretion was similar to that seen with $m$-xylene alone, but if the $m$-xylene was applied adsorbed onto clay soil matrix, approximately equal amounts were excreted in exhaled air and in the urine (46 and 53\%, respectively).

\subsubsection{Other Routes of Exposure}

Limited information was available on the elimination of xylene metabolites in rats following intraperitoneal injection (Ogata et al. 1979; Sugihara and Ogata 1978; van Doorn et al. 1980). The urinary metabolites of xylene are similar regardless of route of exposure; however, the amounts of the various metabolites differ. The elimination profile of xylene isomers is related more to absorption than it is with dose or duration of exposure. In rats, $49-62.6 \%$ of various doses of $m$-xylene or $64-75 \%$ of various doses of $p$-xylene were excreted in the urine as $m$-methylhippuric acid or $p$-methylhippuric acid, respectively (Sugihara and Ogata 1978). Urinary excretion of $o$-toluic acid glucuronide and o-methylhippuric acid accounted for 57 and 16\%, respectively, of single intraperitoneal dose of 1,240 mg $o$-xylene $/ \mathrm{kg}$ give to rats (Ogata et al. 1979). The amount of $o$-toluic acid glucuronide and o-methylhippuric acid excreted reached a maximum 8-24 hours after dosing. Mercapturic acid derivatives were present in the urine of rats following an intraperitoneal dose of $m-, o-$, or $p$-xylene (Tanaka et al. 1990; van Doorn et al. 1980). The percentages ranged from $0.6 \%$ (p-xylene) to 10-29\% (o-xylene). 


\subsubsection{Physiologically Based Pharmacokinetic (PBPK)/Pharmacodynamic (PD) Models}

Physiologically based pharmacokinetic (PBPK) models use mathematical descriptions of the uptake and disposition of chemical substances to quantitatively describe the relationships among critical biological processes (Krishnan et al. 1994). PBPK models are also called biologically based tissue dosimetry models. PBPK models are increasingly used in risk assessments, primarily to predict the concentration of potentially toxic moieties of a chemical that will be delivered to any given target tissue following various combinations of route, dose level, and test species (Clewell and Andersen 1985). Physiologically based pharmacodynamic (PBPD) models use mathematical descriptions of the dose-response function to quantitatively describe the relationship between target tissue dose and toxic end points.

PBPK/PD models refine our understanding of complex quantitative dose behaviors by helping to delineate and characterize the relationships between: (1) the external/exposure concentration and target tissue dose of the toxic moiety, and (2) the target tissue dose and observed responses (Andersen and Krishnan 1994; Andersen et al. 1987). These models are biologically and mechanistically based and can be used to extrapolate the pharmacokinetic behavior of chemical substances from high to low dose, from route to route, between species, and between subpopulations within a species. The biological basis of PBPK models results in more meaningful extrapolations than those generated with the more conventional use of uncertainty factors.

The PBPK model for a chemical substance is developed in four interconnected steps: (1) model representation, (2) model parameterization, (3) model simulation, and (4) model validation (Krishnan and Andersen 1994). In the early 1990s, validated PBPK models were developed for a number of toxicologically important chemical substances, both volatile and nonvolatile (Krishnan and Andersen 1994; Leung 1993). PBPK models for a particular substance require estimates of the chemical substancespecific physicochemical parameters, and species-specific physiological and biological parameters. The numerical estimates of these model parameters are incorporated within a set of differential and algebraic equations that describe the pharmacokinetic processes. Solving these differential and algebraic equations provides the predictions of tissue dose. Computers then provide process simulations based on these solutions.

The structure and mathematical expressions used in PBPK models significantly simplify the true complexities of biological systems. If the uptake and disposition of the chemical substance(s) are adequately described, however, this simplification is desirable because data are often unavailable for 
many biological processes. A simplified scheme reduces the magnitude of cumulative uncertainty. The adequacy of the model is, therefore, of great importance, and model validation is essential to the use of PBPK models in risk assessment.

PBPK models improve the pharmacokinetic extrapolations used in risk assessments that identify the maximal (i.e., the safe) levels for human exposure to chemical substances (Andersen and Krishnan 1994). PBPK models provide a scientifically sound means to predict the target tissue dose of chemicals in humans who are exposed to environmental levels (for example, levels that might occur at hazardous waste sites) based on the results of studies where doses were higher or were administered in different species. Figure 3-5 shows a conceptualized representation of a PBPK model.

If PBPK models for xylene exist, the overall results and individual models are discussed in this section in terms of their use in risk assessment, tissue dosimetry, and dose, route, and species extrapolations.

\section{Kaneko et al. (1991a) Inhalation Exposure Model}

Kaneko et al. (1991a) developed a PBPK model for inhalation of $m$-xylene by rats and humans, based on the trichloroethylene model of Endoh et al. (1989). The model simulates concentrations of $m$-xylene in seven modeled tissue compartments and the amount of metabolites formed (Figure 3-6 and Table 3-7).

Description of the Model. The model consists of compartments for lung (lung arterial blood and one-third of the tidal volume), liver, fat (adipose tissues and yellow bone marrow), slowly-perfused tissues (red bone marrow), richly-perfused tissues (brain, heart, kidneys and glandular tissues), muscle (muscle and skin), and gastrointestinal tract (portal system excluding the liver), plus an arteriovenous shunt. Model parameters included tissue volumes and blood flows, calculated for each compartment based on values by Davis and Mapleson (1981), and several values that were determined experimentally for adult male Wistar rats: partition coefficients, metabolic constants $\left(\mathrm{V}_{\max }\right.$ and $\left.\mathrm{K}_{\mathrm{m}}\right)$, and the rate constant for urinary excretion of $m$-methyl hippuric acid. The concentration flowing out of a given compartment (in venous blood) was set as equilibrated with the concentration in that compartment. All metabolism of $m$-xylene was assumed to occur in the liver and was expressed as a Michaelis-Menten process. The metabolism of $m$-xylene considered only the main pathway, (i.e., the conversion to $m$-toluic acid, which is conjugated to glycine and excreted in the urine as $m$-methylhippuric acid); the pathway from $m$-xylene to coefficients for rat blood, lung, brain, heart, kidney, testis, intestine, spleen, liver, fat, and muscle were determined by vial equilibration, and tissue/blood values were calculated by dividing the tissue/air 


\section{Figure 3-5. Conceptual Representation of a Physiologically Based Pharmacokinetic (PBPK) Model for a Hypothetical Chemical Substance}

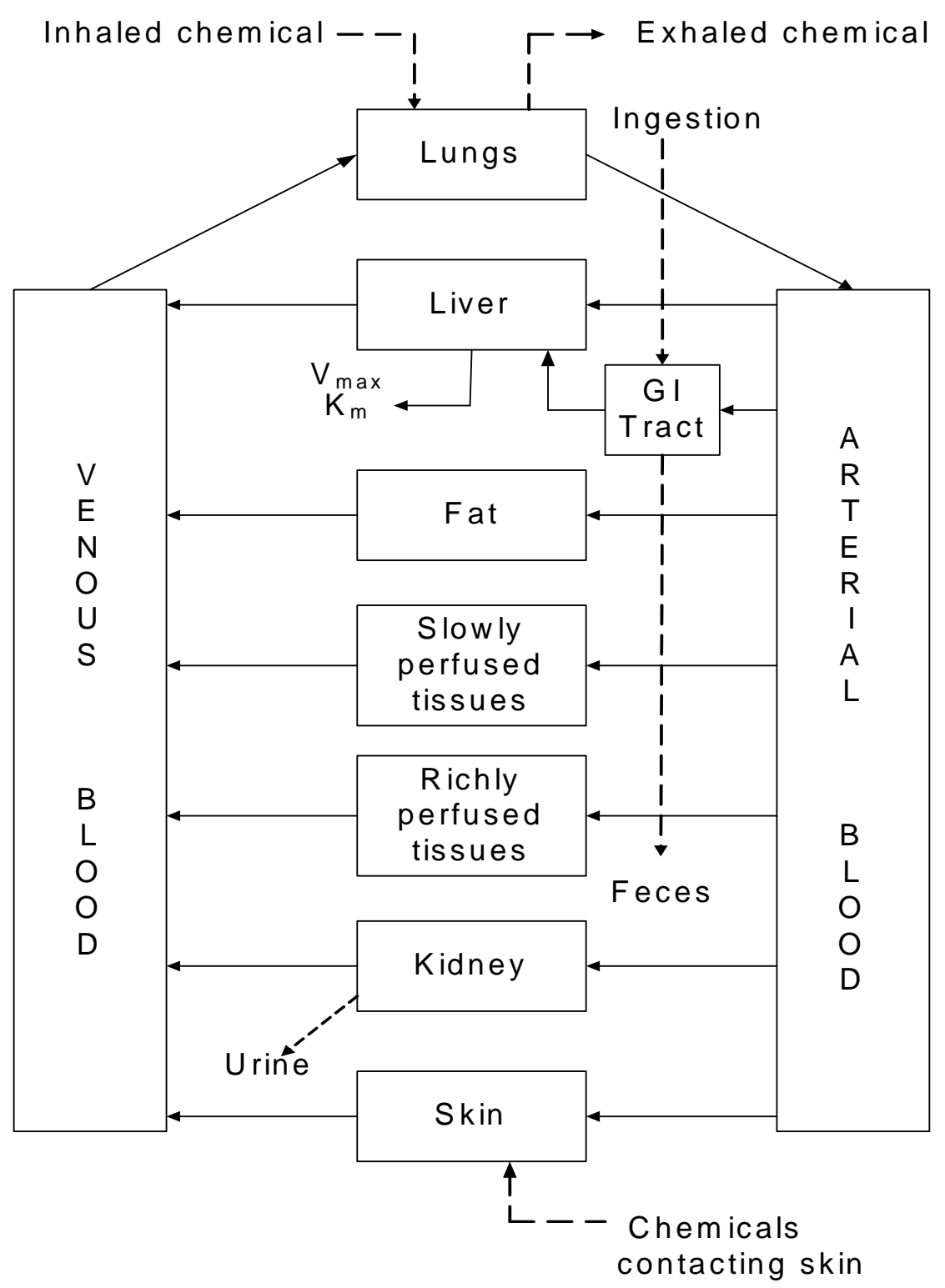

Note: This is a conceptual representation of a physiologically based pharmacokinetic (PBPK) model for a hypothetical chemical substance. The chemical substance is shown to be absorbed via the skin, by inhalation, or by ingestion, metabolized in the liver, and excreted in the urine or by exhalation. 
Figure 3-6. PBPK Model for Inhaled $m$-Xylene

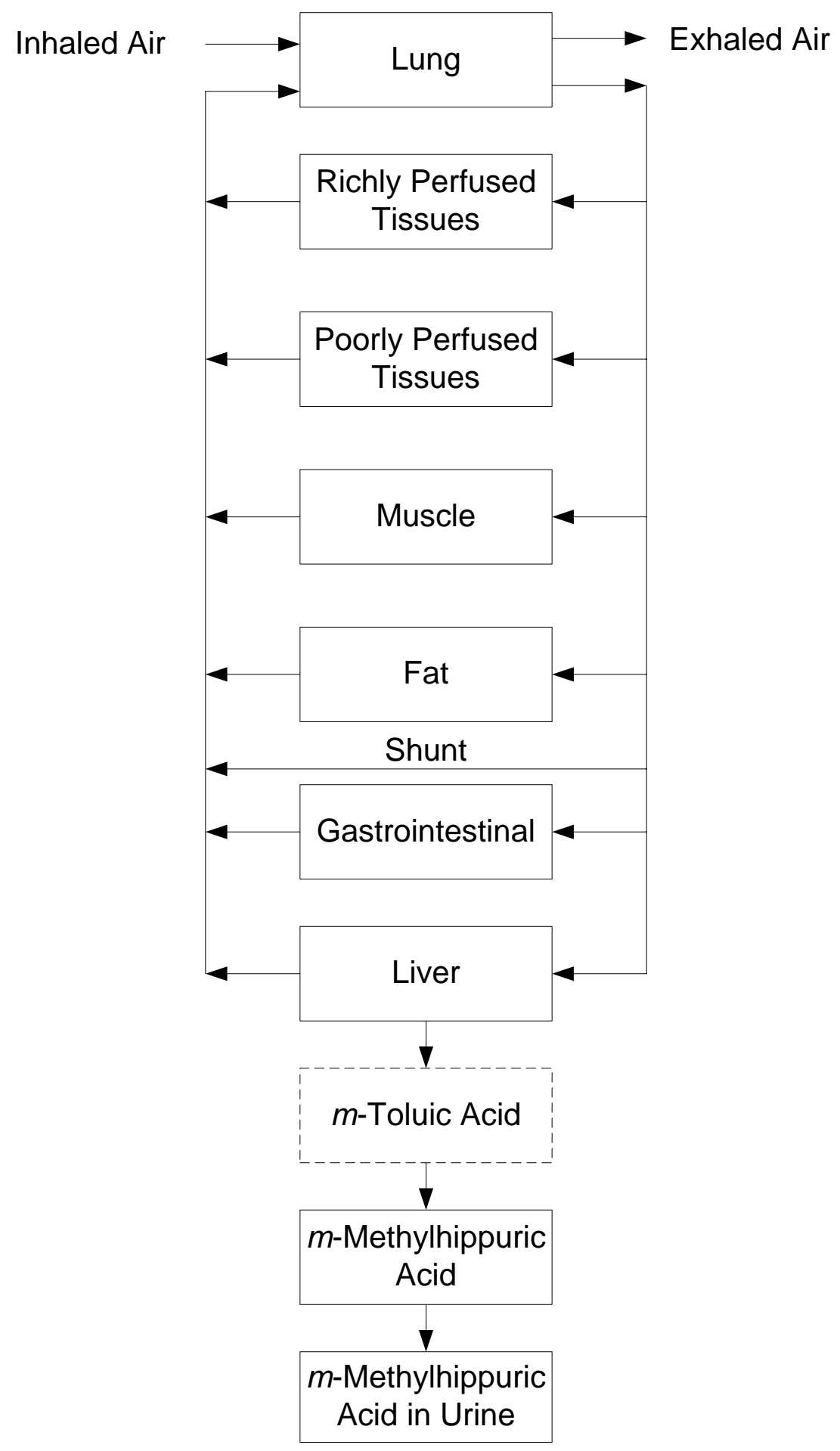




\section{Table 3-7. Human Parameters Used in the PBPK Inhalation Model for $\boldsymbol{m}$-Xylene}

\begin{tabular}{|c|c|c|}
\hline \multicolumn{3}{|c|}{ Tissue volume (percent body weight) } \\
\hline Lung & \multicolumn{2}{|l|}{ calculated $^{\mathrm{a}}$} \\
\hline Richly perfused & \multicolumn{2}{|l|}{3} \\
\hline Slowly perfused & \multicolumn{2}{|l|}{8.5} \\
\hline Muscle & \multicolumn{2}{|l|}{41.5} \\
\hline Fat & \multicolumn{2}{|l|}{21.1} \\
\hline Gastrointestinal tract & \multicolumn{2}{|l|}{1.9} \\
\hline Liver & \multicolumn{2}{|l|}{2.3} \\
\hline Cardiac output (L/minute) & \multicolumn{2}{|l|}{$0.296(\mathrm{bw})^{0.7}$} \\
\hline \multicolumn{3}{|c|}{ Blood flow (percent cardiac output) } \\
\hline Lung & \multicolumn{2}{|l|}{100} \\
\hline Richly perfused & \multicolumn{2}{|l|}{37.9} \\
\hline Slowly perfused & \multicolumn{2}{|l|}{6.3} \\
\hline Muscle & \multicolumn{2}{|l|}{11.4} \\
\hline Fat & \multicolumn{2}{|l|}{5.3} \\
\hline Gastrointestinal tract & \multicolumn{2}{|l|}{17.1} \\
\hline Liver & \multicolumn{2}{|l|}{6.9} \\
\hline Shunt & \multicolumn{2}{|l|}{15.1} \\
\hline \multicolumn{3}{|l|}{ Air flow } \\
\hline Alveolar ventilation (L/hour) ${ }^{\mathrm{b}}$ & \multicolumn{2}{|l|}{$0.296(\mathrm{bw})^{0.7}$} \\
\hline \multicolumn{3}{|l|}{ Partition coefficients } \\
\hline Lung:blood & \multicolumn{2}{|l|}{4.09} \\
\hline Richly perfused:blood & \multicolumn{2}{|l|}{4.42} \\
\hline Slowly perfused:blood & \multicolumn{2}{|l|}{2.01} \\
\hline Muscle:blood & \multicolumn{2}{|l|}{3.01} \\
\hline Fat:blood & \multicolumn{2}{|l|}{77.8} \\
\hline Gastrointestinal tract:blood & \multicolumn{2}{|l|}{4.67} \\
\hline Liver:blood & \multicolumn{2}{|l|}{3.02} \\
\hline Blood:air & \multicolumn{2}{|l|}{26.4} \\
\hline \multicolumn{3}{|l|}{ Metabolic constants } \\
\hline $\mathrm{V}_{\max }{ }^{\mathrm{c}}(\mathrm{mmol} / \mathrm{minute})$ & $\mathrm{V}_{\max 1}=1.394 \times 10^{-3}(\mathrm{bw})^{0.7}$ & $\mathrm{~V}_{\max 2}=1.115 \times 10^{-2}(\mathrm{bw})^{0.7}$ \\
\hline $\mathrm{K}_{\mathrm{m}}^{\mathrm{d}}(\mathrm{mmol} / \mathrm{L})$ & $\mathrm{K}_{\mathrm{m} 1}=0.033$ & $\mathrm{~K}_{\mathrm{m} 2}=0.330$ \\
\hline $\mathrm{K}_{\mathrm{ex}}^{\mathrm{d}}$ (per minute) & 0.012 & \\
\hline
\end{tabular}

Source: Adapted from Kaneko et al. (1991a)

${ }^{\mathrm{a}}$ Lung volume $=$ functional residual capacity + one third tidal volume $+($ volume arterial blood $\mathrm{x}$ blood:air partition coefficient + volume of lung tissue x lung:air partition coefficient.

${ }^{\mathrm{b}}$ Set equal to cardiac output.

${ }^{\mathrm{C}} \mathrm{Rat} \mathrm{V}_{\max }$ and $\mathrm{K}_{\mathrm{m}}$ were determined experimentally for two different exposure concentrations. Rat $\mathrm{V}_{\max 1}$ $\left(0.6 \times 10^{-3} \mathrm{mmol} /\right.$ minute $)$ and $\mathrm{V}_{\max 2}\left(4.8 \times 10^{-3} \mathrm{mmol} /\right.$ minute $)$ values were corrected for body surface area (body weight) ${ }^{0.7}$.

${ }^{\mathrm{d}}$ Set equal to experimentally determined rat value.

bw = body weight 
coefficient by the blood/air coefficient. The richly perfused coefficient was set equal to that of the liver. Human coefficients were calculated as the rat tissue/air partition coefficients divided by the human blood/air coefficient reported by Sato and Nakajima (1979). Metabolic rate constants were experimentally determined for high and low concentrations. For the human model, cardiac output and metabolic rate constants were estimated from rat values using a (body weight) ${ }^{0.70}$ scalar, but the rat $\mathrm{K}_{\mathrm{m}}$ was used directly. Simulations were performed for a 70-kg man inhaling $100 \mathrm{ppm} \mathrm{m}$-xylene for 6 hours as reported by Riihimaki et al. (1979b). The blood concentration was set equivalent to the concentration flowing out of the richly-perfused compartment.

Validation of the Model. The model parameters were optimized against the human data of Riihimaki et al. (1979b). Comparisons of model simulations against data from studies other than those used in model development were not presented.

Additional Predictions. Kaneko et al. (1991b) used the model to evaluate the relationship of external and internal doses of $m$-xylene and determine the effects of body weight, body fat content, sex, and physical activity on blood concentrations and urinary excretion of metabolite. Several different exposure scenarios were simulated: continuous exposure of a 70-kg male to $50 \mathrm{ppm}$ for 8 hours, intermittent exposure of a 70-kg male to 100 ppm for 1 hour 4 times at 1-hour intervals, continuous exposure of a $70-\mathrm{kg}$ male for 8 hours to various concentrations between 0 and 4,000 ppm. In the simulation, larger bodies absorbed more $m$-xylene, and excreted more metabolite, but there was no significant change in blood concentration. Increased physical activity increased both the blood concentration of $m$-xylene and the rate of urinary excretion of metabolite. The concentration of $m$-xylene in the blood during exposure was lower in women than in men, but was higher in women 10 hours after exposure. The rate of urinary excretion of metabolite was lower in women both during and after exposure.

\section{Adams et al. (2005) Inhalation Exposure Model}

Adams et al. (2005) developed a PBPK model for inhalation of deuterium-labeled xylene isomers in humans, using biomonitoring data (isomer levels in blood and exhaled air) to quantify the kinetics of each isomer in adult Caucasian men. The model simulates concentrations in six modeled tissue compartments and assumes systematic clearance of xylenes by metabolism primarily in the liver, but also in the lung.

Description of the Model. The model consists of compartments for lung blood, adipose tissue, slowly perfused tissue, rapidly perfused tissue, and liver. The model used subject-specific values for 
body weight, adipose tissue fraction, and exposure concentration. Cardiac output and all blood flows were scaled to (body weight) ${ }^{0.74}$. Published values were used for fractional tissue compartment volumes, fractional blood flows, tissue/blood partition coefficients, and hepatic metabolic constants. Alveolar ventilation, blood flows, and blood/air partition coefficients were assigned as Bayesian parameters to be optimized during model fitting. As xylene was assumed to diffuse rapidly into tissues, the transfer of xylene from arterial blood to tissues was assumed to be flow-limited. Changes in the amount of xylenes in nonmetabolizing tissues, in the metabolizing hepatic compartment, and the mass balance of xylene in the lung (including a metabolic component varying from 0 to $25 \%$ of hepatic metabolism) were defined by a series of simultaneous differential equations. Extrahepatic metabolism was indicated when the apparent systematic clearance of xylenes exceeded hepatic blood flow.

Validation of the Model. The model parameters were optimized against human data collected by the investigators (Adams et al. 2005). Twenty-seven male Caucasian volunteers were exposed at rest to atmospheres containing 0-40 ppm each of ${ }^{2} \mathrm{H}_{10}$-meta-, ${ }^{2} \mathrm{H}_{10}$-ortho-, and ${ }^{2} \mathrm{H}_{10}$-para-xylene for 2 hours through a gated mouthpiece. Eight subjects experienced replicate exposures (at a minimal 2-week interval), bringing the total number of exposures to 37. Inhaled and exhaled breath concentrations of deuterium-labeled isomers were measured continuously during exposure. Doses were estimated for all subjects as the product of exposure concentration, exposure duration, and model-fitted subject-specific alveolar ventilation rates. Blood concentrations of isomers were measured in 1 venous blood sample collected before exposure and in 15-20 samples collected at the end of exposure for 4 days; collection frequency was every 15 minutes for the first several hours to every 12 hours after day 1 . Model-fitted values for systemic clearance, terminal half-lives, and blood:air partition coefficients were similar to values reported by other investigators (Pierce et al. 1996; Sato 1988; Wallen et al. 1985). Subjects exposed during multiple sessions showed significant intra-individual variability in respiratory and blood physiology parameters as well as metabolism rates. The study authors suggested that differences in diet, alcohol consumption, and stress could affect variation in these parameters.

\section{Thrall and Woodstock (2003) Dermal Exposure Model}

Thrall and Woodstock (2003) adapted a PBPK model for binary inhalation exposures to toluene and $m$-xylene by Tardif et al. (1993a) to construct a PBPK model for dermal exposures to $o$-xylene in rats and humans (Table 3-8 and Figure 3-7). 
Table 3-8. Rat and Human Parameters Used in the PBPK Dermal Model for o-Xylene

\begin{tabular}{|c|c|c|}
\hline & Rat & Human \\
\hline Body weight (kg) & $0.2-0.25$ & $83.9,48.5,80.7$ \\
\hline \multicolumn{3}{|c|}{ Tissue volume (percent body weight) } \\
\hline Liver & 4 & 4 \\
\hline Fat & 8 & $30.7,22.3,26.4$ \\
\hline Richly perfused & 5 & 5 \\
\hline Slowly perfused & 64 & Variable \\
\hline Total skin & 10 & 10 \\
\hline Cardiac output (L/hour) & 5.4 & 348 \\
\hline \multicolumn{3}{|c|}{ Blood flow (percent cardiac output) } \\
\hline Liver & 25 & 25 \\
\hline Fat & 5 & 6 \\
\hline Richly perfused & 51 & 49 \\
\hline Slowly perfused & 15 & 15 \\
\hline Total skin & 5 & 5 \\
\hline \multicolumn{3}{|l|}{ Air flow } \\
\hline Alveolar ventilation (L/hour) & 5.4 & 348 \\
\hline \multicolumn{3}{|l|}{ Partition coefficients } \\
\hline Saline:air & 2.6 & 2.6 \\
\hline Blood:air & 37.8 & 34.0 \\
\hline Liver:blood & 3.4 & 3.8 \\
\hline Fat:blood & 55.7 & 61.9 \\
\hline Muscle:blood & 2.0 & 2.2 \\
\hline Skin:air & 65.4 & 65.4 \\
\hline \multicolumn{3}{|l|}{ Metabolic constants } \\
\hline $\mathrm{V}_{\max }(\mathrm{mg} / \mathrm{kg} / \mathrm{hour})$ & 8.4 & 8.4 \\
\hline $\mathrm{K}_{\mathrm{m}}(\mathrm{mg} / \mathrm{L})$ & 0.2 & 0.2 \\
\hline
\end{tabular}

Source: Adapted from Thrall and Woodstock (2003) 
Figure 3-7. PBPK Model to Describe Dermal Absorption of o-Xylene

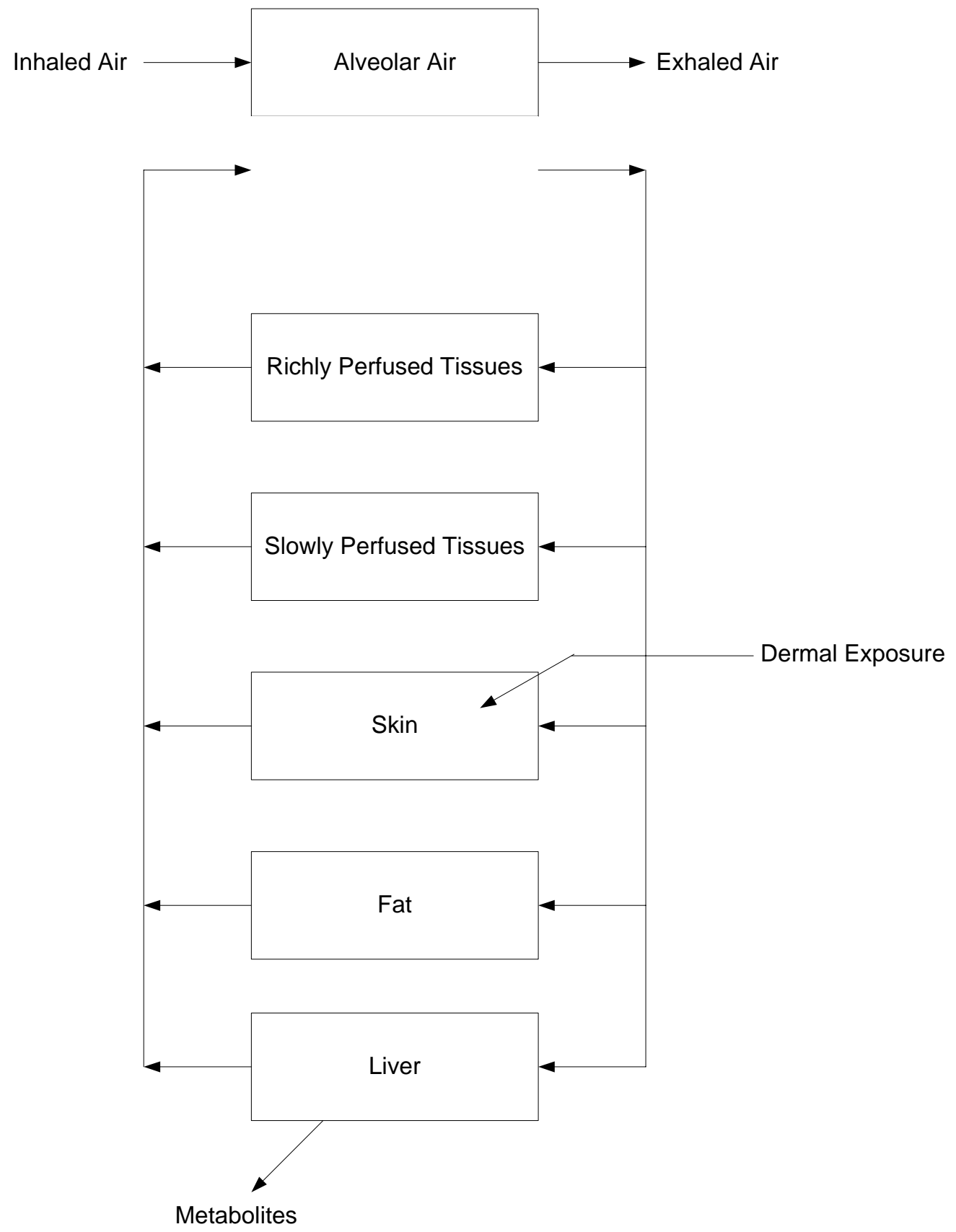

Source: adapted from Thrall and Woodstock (2003) 
Description of the Model. The model consists of compartments for lung, blood, richly perfused tissues, slowly perfused tissues, skin, fat, and liver. The skin compartment represents exposed skin, whereas nonexposed skin is incorporated into the slowly perfused compartment. Total skin volume (10\% of body weight) was assumed to receive $5 \%$ of the total cardiac output. The volume and blood flow for exposed skin were calculated according to the methods of Jepson and McDougal (1997). Model parameters were as presented in Table 3-8. Metabolism was assumed to occur in the liver. Dermal exposure was simulated by relating the rate of change in the concentration of xylene in the skin compartment to the rate of penetration through the skin (the flux) and the rate of delivery because of blood flow and the arterial concentration (the perfusion) (Thrall et al. 2000). The model also considered the amount of xylene lost through evaporation from nonoccluded skin. The kinetics of dermal absorption was determined empirically in experiments in rats and humans based on the appearance of xylene in exhaled air. A total of $400 \mu \mathrm{g}$ of $o$-xylene in aqueous solution $(0.02 \%)$ was applied to the skin of rats under an occlusive covering for 4 hours. Human subjects submerged their lower legs into a hydrotherapy tub containing $500 \mu \mathrm{g} / \mathrm{L} o$-xylene (a concentration 400 times lower than for rats) for 10-30 minutes and were monitored unexposed for an additional 30 minutes. An algorithm was used to vary the skin permeability coefficient to achieve an optimal fit to the time course data for each subject and then averaged. The calculated skin permeability rate constants of $0.058 \pm 0.009 \mathrm{~cm} /$ hour for rat and $0.005 \pm 0.001 \mathrm{~cm} /$ hour for human adequately described the respective individual dermal exposure data sets. Both species rapidly absorbed aqueous xylene through the skin as shown by the chemical's appearance in exhaled air within a few seconds of exposure.

Validation of the Model. The dermal PBPK model was used to simulate the exhaled breath data from experiments in humans dermally exposed for 3 minutes to undiluted $m$-xylene (Kezic et al. 2001). The calculated skin permeability coefficient was identical to the average value of $0.005 \mathrm{~cm} /$ hour derived by Thrall and Woodstock (2003). Simulation of blood concentration data from F344 rats dermally exposed to saturated $m$-xylene for 24 hours (Morgan et al. 1991) resulted in a calculated skin permeability coefficient of $0.0727 \mathrm{~cm} /$ hour, which was in the range of values determined by Thrall and Woodstock (2003).

\section{Fisher et al. (1997) Lactational Model for Inhalation Exposure}

Fisher et al. (1997) adapted the PBPK model of Ramsey and Andersen (1984) to create a lactational model for maternal occupational exposure to volatile organic solvents, including $o^{-}, \mathrm{m}-$, and $p$-xylenes (Table 3-9 and Figure 3-8). 


\section{Table 3-9. Human Parameters Used in the PBPK Lactational Model for} $o-, m$-, $p$-Xylene

\begin{tabular}{|c|c|}
\hline & Human \\
\hline Body weight (kg) & 60 \\
\hline \multicolumn{2}{|l|}{ Tissue volume (percent body weight) } \\
\hline Liver & 1.5 \\
\hline Fat & 25 \\
\hline Richly perfused & 10 \\
\hline Slowly perfused & 54 \\
\hline Milk (mL) & $10-125$ \\
\hline Cardiac output (L/hour) & $15 \times$ body weight ${ }^{0.74}$ \\
\hline \multicolumn{2}{|l|}{ Blood flow (percent cardiac output) } \\
\hline Liver & 29 \\
\hline Fat & 10 \\
\hline Richly perfused & 35 \\
\hline Slowly perfused & 19 \\
\hline Milk & 7 \\
\hline \multicolumn{2}{|l|}{ Air flow } \\
\hline Alveolar ventilation (L/hour) & $24 \times$ body weight ${ }^{0.74}$ \\
\hline \multicolumn{2}{|l|}{ Milk compartment } \\
\hline Rate of ingestion of breast milk by nursing infant (per hour) & 20 \\
\hline Rate of breast milk production at 1.5-3.0 months lactation (L/hour) & 0.06 \\
\hline \multicolumn{2}{|l|}{ Partition coefficients } \\
\hline Liver:blood & 2.14 \\
\hline Fat:blood & 41.28 \\
\hline Milk:blood & 2.98 \\
\hline Richly perfused:blood & 2.14 \\
\hline Slowly perfused:blood & 0.97 \\
\hline \multicolumn{2}{|l|}{ Metabolic constants } \\
\hline $\mathrm{V}_{\max }^{\mathrm{a}}$ & Not reported \\
\hline $\mathrm{K}_{\mathrm{m}}{ }^{\mathrm{a}}$ & Not reported \\
\hline
\end{tabular}

Source: Adapted from Fisher et al. (1997) 
Figure 3-8. PBPK Model for Lactational Exposure to Volatile Organic Compounds (Including $0-, m-, p$-Xylenes) Following Maternal Occupational Inhalation Exposure

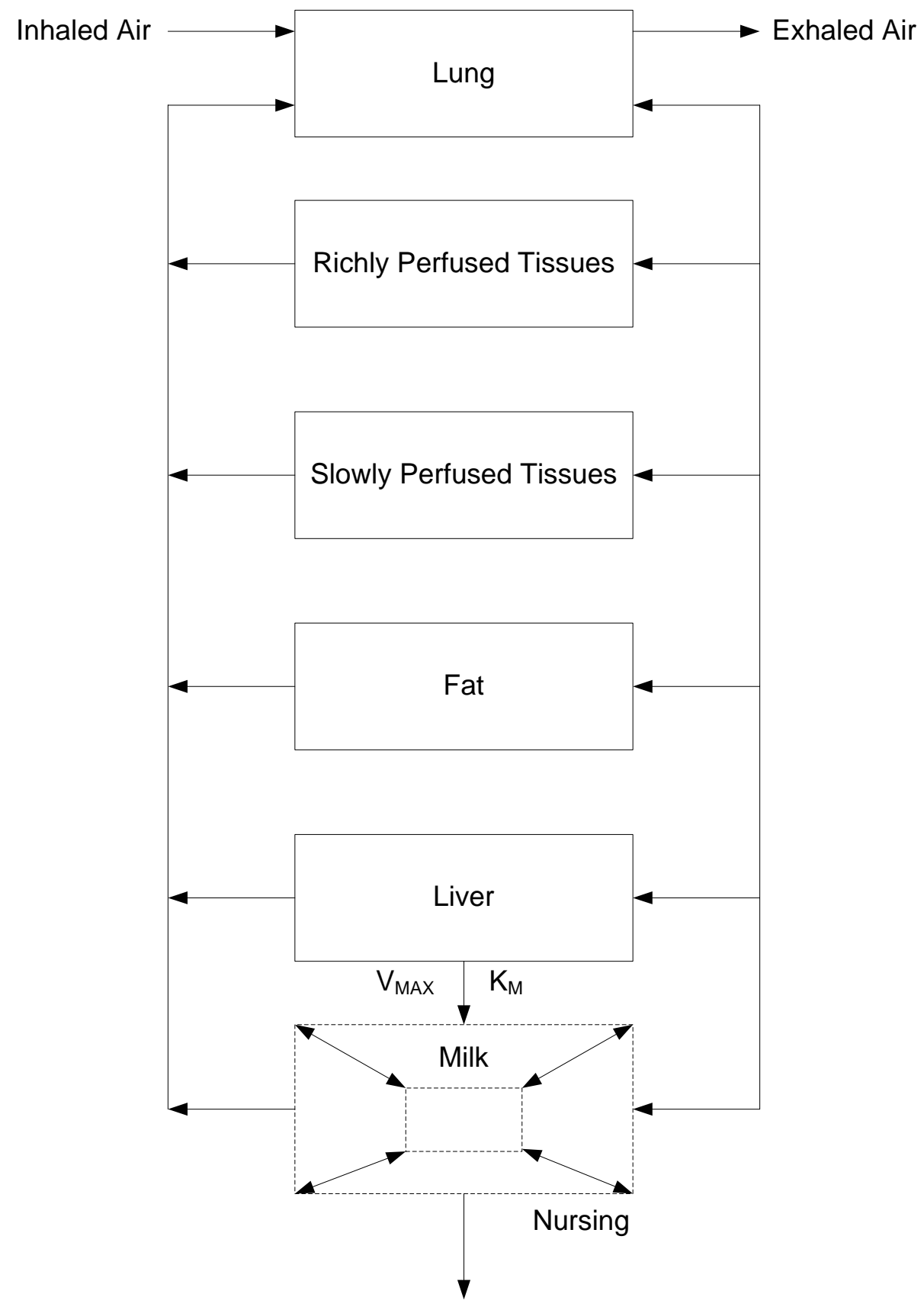

Infant Chemical Exposure 
Description of the Model. In addition to the standard compartments for lung, richly- and slowlyperfused tissues, fat, and lung (the site of metabolism), the model contained a milk compartment. Milk production was described as a zero-order process at $0.06 \mathrm{~L} /$ hour. Milk letdown from nursing (infant nursing rate) was described as a first order process (20/hour). The milk volume in mammary tissue was $0.125 \mathrm{~L}$ before nursing and $0.010 \mathrm{~L}$ at the end of nursing. The equation describing the rate of change in the amount of chemical ingested by the nursing infant included terms for the chemical concentration in milk, the amount of milk in the mammary tissue lumen, the infant nursing rate, and a switch to turn on or off nursing over 24 hours. Integration of this equation provided the cumulative amount of chemical ingested by a nursing infant in 24 hours. Partition coefficients for blood:air and milk:air were experimentally derived to calculate the milk:blood partition coefficient (Table 3-9). The human tissue:blood coefficient for xylenes was calculated by dividing the rat tissue:air coefficients of Gargas et al. (1989) by the human blood:air coefficient. The published paper did not report the constants ( $\mathrm{V}_{\max }$ and $\mathrm{K}_{\mathrm{m}}$ ) used to describe the rate of metabolism of xylenes in humans, so it appears that the authors may have assumed zero metabolism for xylenes.

The lactational model was used to simulate lactational transfer of xylenes following maternal exposure for 8 hours at the threshold limit value (TLV) of $100 \mathrm{ppm}$. The model predicted that approximately $1-2 \%$ of inhaled xylenes would be transferred to milk and that the daily intake for a $10 \mathrm{~kg}$ infant would be $6.59 \mathrm{mg}$.

\section{Interaction Models}

In addition to those described above, a number of PBPK models have been developed for exposures to xylene in the presence of other chemicals. Tardif et al. (1993a, 1995) first developed a PBPK model for inhalation exposures to binary mixtures of $m$-xylene and toluene in the rat and later adapted the model for humans. The models based on parameters for $m$-xylene were found to adequately simulate the kinetics of mixed xylenes as well. A later model was developed for inhalation of a ternary mixture of alkyl benzenes (m-xylene, toluene, and ethylbenzene) in rats and humans (Tardif et al. 1997). Haddad et al. (2000) validated a PBPK model for inhalation of mixtures of benzene, toluene, ethyl benzene, and $m$-xylene (BTEX), with the addition of a model for dichloromethane. Kaneko et al. (1995) modeled the effect of phenobarbital pretreatment with exposure to $m$-xylene orally or intraperitoneally at the same doses or by inhalation. The interactions between xylene and the other chemicals are presented in Section 3.9. 
MacDonald et al. (2002) used PBPK modeling to estimate central nervous system effects following exposure to a time-weighted-average $100 \mathrm{ppm}$ of $m$-xylene for 8 hours. The model incorporated the inhalation PBPK model for $m$-xylene by Kaneko et al. (1991a) and a model for ingested ethanol by Derr (1993). Other components included a competitive enxyme (CYP2E1) inhibition model for the two chemicals, as well as a population PBPK simulation model derived from statistical distributions of the PBPK model parameters with Monte Carlo sampling. Finally, since central nervous system effects were assumed to be dependent on blood levels of $m$-xylene, a pharmacodynamic (PD) threshold model was included for peak venous concentrations of $m$-xylene. The deterministic PBPK model for $m$-xylene was validated against available human data (Loizou et al. 1999; Riihimaki et al. 1982b). The population PBPK-PD model for $m$-xylene was run to establish the population distribution for peak venous blood $m$-xylene concentrations $\left(\mathrm{C}_{\mathrm{V}, \max }\right)$ following exposure at the U.K. occupational exposure standard of $100 \mathrm{ppm}$ over 8 hours and for a 4-hour exposure at $200 \mathrm{ppm}$ that elicited central nervous system disturbances in healthy volunteers (Savolainen et al. 1985a). The corresponding $80^{\text {th }}$ percentile on the $\mathrm{C}_{\mathrm{V}, \max }$ cumulative frequency distribution, $20.35 \mu \mathrm{mol} / \mathrm{L}$, was selected as the pharmacokinetic/ pharmcodynamic threshold for central nervous system depression elicited by exposure to $m$-xylene. Simulations were also reported for blood levels of ethanol alone, combined $m$-xylene and ethanol, and population PBPK-PD modeling for the combined exposure scenario. In the model simulations, increasing exposure to ethanol increased the probability of central nervous system effects from exposure to $m$-xylene.

PBPK modeling has been employed to assess possible toxicological interactions between chemicals, such as inhalation exposure to $m$-xylene and methylchloroform (Tardif and Charest-Tardif 1999).

Haddad et al. (2001) developed a PBPK modeling-based risk assessment approach for mixtures of volatile chemicals that would incorporate pharmacokinetic interactions, such as changes in hepatic metabolism, among components of the mixture.

\section{Model Applications}

In deriving a chronic-duration inhalation reference concentration (RfC) for xylenes, the EPA (2003) examined the application of the inhalation PBPK models for $m$-xylene developed by Tardif and colleagues (Haddad et al. 1999; Tardif et al. 1991, 1992, 1993a, 1993b, 1995) to extrapolate rat exposure levels from the intermediate-duration inhalation study by Korsak et al. (1994) to human equivalent exposure levels. Using a time-weighted-average steady-state concentration of $m$-xylene in blood as the 
internal dose surrogate, it was found that model-predicted human equivalent concentrations $\left(46.5 \mathrm{mg} / \mathrm{m}^{3}\right)$ were similar, although not identical, to human equivalent concentrations $\left(39 \mathrm{mg} / \mathrm{m}^{3}\right)$ calculated from rat experimental exposure levels using the 1994 EPA default cross-species dosimetric equations (EPA 2003). EPA (2003) noted that, as neurobehavioral effects were the targets in the study by Korsak et al. (1994), the brain concentration of xylene would have been a more optimal dose surrogate, but the model did not include such a compartment.

Pelekis et al. (2001) used the PBPK models of Tardif et al. (1993a, 1995) to examine possible differences between children and adults with respect to estimated blood and tissue doses following inhalation exposure to xylene. The model simulations incorporated published physiological parameters for a $10-\mathrm{kg}$ 1 -year-old child and high and low values for adults. The simulations revealed that the parameters having a significant effect on within-species variability of tissue and blood doses following inhalation exposures were the body weight, ventilation rate, fraction of cardiac output flowing to the liver, blood:air partition coefficient, and hepatic extraction ratio.

Jang and Droz (1997) used PBPK modeling to evaluate possible ethnic differences in metabolism in individuals exposed by inhalation to $100 \mathrm{ppm} \mathrm{m}$-xylene for 6 hours (Jang et al. 1997). No significant differences were noted between six Caucasians and six Asians in metabolic clearance of methylhippuric acid.

\subsection{MECHANISMS OF ACTION}

\subsubsection{Pharmacokinetic Mechanisms}

Absorption. The isomers of xylene are expected to have similar absorption behavior because of their relatively small molecular size and similar chemical properties. Penetration of xylenes is rapid by all routes of exposure because of the lipophilic nature of the chemicals, as indicated by the $\log \mathrm{K}_{\mathrm{ow}}$ values (see Table 4-2). The similar blood-air partition coefficients for the xylene isomers also reflect the expected similarities in absorption from inhaled air through the lung (Table 3-6). PBPK modeling of human data revealed little inter-subject variability with respect to blood:air partition coefficients (Adams et al. 2005).

Distribution. Distribution of xylenes is rapid throughout the body although their water solubility is low (Table 4-2). Xylenes, because of their lipophilic properties, are readily distributed to fat and tissues rich in cell membranes, such as the brain. The lipophilic nature of xylenes is generally responsible for 
their penetration across the placenta to fetal tissues and for distribution into milk (Fisher et al. 1997). Distribution of xylenes is a simple diffusion process. As shown by PBPK modeling, the factors affecting tissue and blood distributions of inhaled xylenes include the body weight (since several physiological parameters are scaled to body weight), ventilation rate, fraction of cardiac output flowing to the liver, blood:air partition coefficient, and hepatic extraction ratio (Pelekis et al. 2001). In addition, hepatic blood flow can be significantly affected by changes in posture, exercise, and thermal stress (Adams et al. 2005). All of these factors may contribute to intra- and inter-individual variations in tissue distributions of xylenes (Adams et al. 2005; Pelekis et al. 2001).

In developing a tissue composition-based algorithm for predicting tissue:air partition coefficients for volatile organic compounds, including isomers of xylene, Poulin and Krishnan (1996a, 1996b) uncovered an anomaly regarding blood compared to other tissues. The algorithm was based on tissue composition with respect to water and total lipid content by weight, and phospholipids and neutral lipids as fractions of total lipids. For liver, muscle, and adipose tissue, the algorithm, which used the solubilities of chemicals in water, neutral lipid, and phospholipid, predicted tissue:air partition coefficients that were similar to values in the published literature (ratios of predicted vs experimental between 0.93 and 1.57 for xylene isomers on average). For blood, however, the algorithm seriously underpredicted the partition coefficients of hydrophobic organic compounds, for xylenes by a factor of 5, indicating that solubility characteristics alone could not account for partitioning. Poulin and Krishnan (1996b) suggested that retention of hydrophobic organic compounds in blood was partly the result of reversible binding to hydrophobic holes in hemoglobin molecules. Association constants $\left(\mathrm{K}_{\mathrm{a}}\right)$ of 1,512, 1,853, and 1,609 per mole were calculated for reversible binding to hemoglobin by $o-, m$-, and $p$-xylene, respectively (Poulin and Krishnan 1996b).

In PBPK modeling of data from humans exposed by inhalation, fitted values for partitioning between blood and slowly perfused tissues exhibited significant inter-individual variability, ranging from 0.68 to 13.78 for $m$-xylene (mean 3.05 \pm 3.77 ), $0.65-17.44$ for $o$-xylene (mean $2.88 \pm 3.37$ ), and $0.55-12.27$ for p-xylene (2.25 \pm 2.58$)$ (Adams et al. 2005). The basis for this variability is not understood.

Metabolism. Phase I metabolism of xylenes is primarily by the oxidation of side-chain methyl groups by mixed function oxidases in the liver, forming methylbenzoic (toluic) acids. Phase II metabolism is primarily through conjugation with glycine, which is rate limiting in humans (Riihimaki et al. 1979a, 1979b). In the absence of glycine, minor pathways are employed, such as conjugation with glucuronic acid or aromatic hydroxylation to form xylenols. In humans, hepatic microsomal CYP2E1 is the primary 
enzyme involved with the metabolism of xylenes to methylbenzylalcohols, the dominant pathway leading to the formation of methylhippuric acid isomers (Tassaneeyakul et al. 1996); human CYP1A2 causes the ring hydroxylation of $m$-xylene to form 2,4-dimethylphenol.

Inter-individual variations in rates of metabolism of xylenes may be affected by lifestyle differences. Treatment with ethanol prior to xylene exposure increases rates of xylene metabolism by the liver, presumably by inducing hepatic microsomal enzymes (Tardif et al. 1994; Wisniewska-Knypl et al. 1989).

Excretion. The lipophilic properties of unmetabolized xylene are responsible for the diffusion out of the lungs into exhaled breath. The water solubility of conjugated metabolites of xylene is responsible for their transported out of the body in urine. As estimated by PBPK modeling of human data, systemic clearance of $m$-xylene (fitted average of $131 \mathrm{~L} /$ hour) proceeds more rapidly than for $o$-xylene (116 L/hour) or p-xylene (119 L/hour) (Adams et al. 2005).

\subsubsection{Mechanisms of Toxicity}

The lipophilic effects of xylenes, which dissolve lipid membranes, is responsible for the irritant effects on eyes, mucous membranes and skin (Riihimaki 1979b). In addition, the lipophilicity of xylenes is responsible for their narcotic and anaesthetic properties, which are similar for the three isomers (Fang et al. 1996). The para isomer differs in also eliciting excitation reactions in rats (Fang et al. 1996). The mechanism of anesthetics in general is not well understood, but probably relates to intercalation of the chemical into neuronal cell membranes, changing membrane properties that affect transmission of nerve impulses (Desi et al. 1967; EPA 1985a; Gerarde 1959; Savolainen and Pfaffli 1980; Tahti 1992). The mechanism could be either by a disruption of the lipid environment in which membrane proteins function or by direct interaction with the hydrophobic/hydrophilic conformation of proteins in the neuronal membrane. A statistically significant $28 \%$ increase in binding ( $\mathrm{fmol} / \mathrm{mg}$ tissue) of radiolabeled $t$-butylbicyclophosphorothionate, a ligand to the picrotoxin/convulsant binding stite of $\mathrm{GABA}_{\mathrm{A}}$ receptors, was observed in the molecular layer of the cerebellum of rats exposed by inhalation to $m$-xylene at a high concentration (Ito et al. 2002). The results of this study suggest that the high concentration of $m$-xylene in the cerebellum (see Section 3.2.2.1) increased GABA release and/or enhanced $\mathrm{GABA}_{\mathrm{A}}$ receptor function, consequently increasing $\mathrm{GABA}_{\mathrm{A}}$ receptor binding activity. The increased inhibitory effect of GABAergic neurotransmission in the cerebellum is consistent with the adverse effect of $m$-xylene on motor coordination (Ito et al. 2002). 
Changes in levels of various neurotransmitters and lipid composition have been observed in several brain areas following acute- and intermediate-duration exposure to xylene (Andersson et al. 1981; Honma et al. 1983; Savolainen and Seppalainen 1979). It is unclear whether these represent direct effects of xylene or are secondary changes resulting from nonspecific central nervous system depression. Some authors have also suggested that metabolic intermediates, such as arene oxides or methylbenzaldehyde, may be responsible for the toxic effects of xylene (Savolainen and Pfaffli 1980). Oxidation of xylene to these intermediates by microsomal enzyme systems may occur within brain cells (Savolainen and Pfaffli 1980). Acute-duration oral or intermediate-duration inhalation exposures to high concentrations of $p$-xylene, but not the other isomers, results in death of cochlear hair cells and hearing loss in rats (Gagnaire and Langlais 2005; Gagnaire et al. 2001). The reason for the isomer-specificity of the effect is not known, but Gagnaire and Langlais (2005) have indicated that ototoxicity from exposure to aromatic solvents is generally associated with structural constraints: the presence of a single short side chain on the benzene ring. They imply that the meta- and ortho- placements of a second short side chain in xylene interfere with ototoxic potency, whereas the para- position does not. Direct effects of xylene on the brain may be responsible for degraded performance of rats in tests of motor coordination, but the precise brain areas affected have not been identified (Gralewicz and Wiaderna 2001; Gralewicz et al. 1995; Korsak et al. 1990, 1992, 1993, 1994).

Inhibition of pulmonary microsomal enzymes has been observed by several investigators (Elovaara et al. 1987; Foy and Schatz 2004; Foy et al. 1996; Park et al. 1994; Patel et al. 1978; Silverman and Schatz 1991; Smith et al. 1982; Stickney et al. 1989). The exact mechanism of the enzyme inhibition is unknown but has been attributed to the formation of a toxic reactive metabolite (such as methylbenzaldehyde) that binds directly to microsomal protein and inactivates the microsomal enzymes (Patel et al. 1978; Smith et al. 1982). Direct effects on microsomal membrane fluidity and/or lipid content do not appear to be involved (Stickney et al. 1989). Inhalation exposure to $m$-xylene for 6 hours caused a condensation-dependent reduction in cytochromes CYP 2B1, 2E1, and 4B1 in the lung and 2B1 and 2E1 in the nasal mucosa (Vaidyanathan et al. 2003).

The cytotoxicity of $m$-xylene has been associated with reductions in cellular antioxidant status (Coleman et al. 2003). In rat dermal fibroblasts cultured in a collagen matrix (dermal equivalents), exposure to $m$-xylene caused duration- and concentration-related reductions in viability, endogenous catalase activity, and endogenous thiol levels (thiols detected with 5,5-dithiobis[2-nitrobenzoic acid], DTNB). Supplementation with the antioxidant $N$-acetylcysteine, a hydroxyl radical scavenger as well as a 
precursor to glutathione, increased survival of cultured fibroblasts exposed to $m$-xylene. These results suggest that hydroxyl radicals may play a role in xylene-induced cytotoxicity.

The mechanism for xylene's toxic effects on the kidneys is also unknown, but may be related to formation of reactive metabolites and subsequent irritation or direct membrane fluidization (EPA 1985a). Support for the former idea is provided in several experiments using porcine proximal tubular cells (LLC-PK1, an established cell line) cultured in the presence of $p$-xylene (Al-Ghamdi et al. 2003a, 2003b, 2004). Treatment for up to 48 hours with $5 \mathrm{mM}$ p-xylene reduced cell viability, increased the activity of CYP2E1, and increased the release of malondialdehyde (indicative of lipid peroxidation), but did not cause an increase in DNA fragmentation (Al-Ghamdi et al. 2003a). Disulfuram, an inhibitor of CYP2E1, reduced the activity of this enzyme, as well as the release of malondialdehyde occurring from treatment with $p$-xylene. Experiments using a free radical scavenger (catalase) also counteracted the effect of $p$-xylene. These experiments suggest that renal toxicity of $p$-xylene may be related to its metabolism by CYP2E1, which generates the production of oxidative intermediates and subsequent necrosis; the authors suggest that this mechanism would be relevant to acute high-level exposures to xylene. Results of additional experiments with LLC-PK1 cells treated for 96 hours with $1 \mathrm{mM} p$-xylene suggested that nephrotoxicity from xylene may involve induction of apoptosis through the activation of mitochondrial caspase-9 and caspase-3 (Al-Ghamdi et al. 2003b, 2004); typical features of apoptosis, DNA fragmentation and upregulation of Bax protein compared to Bcl-2 protein (increasing the ratio of Bax/Bcl-2), were observed with xylene treatment. In humans exposed to solvent mixtures containing xylene and a substantial proportion of toluene, a number of effects were noted (lysozymuria and increased urinary excretion of beta-glucuronidase, albumen, erythrocytes and leukocytes) that are attributable to renal injury (Askergren, 1982; Franchini et al. 1983). However, these effects are likely caused by toluene, which is a known renal toxicant.

Developmental toxicity of $m$ - and $p$-xylenes in standard animal bioassays is largely related to maternal toxicity (Saillenfait et al. 2003). Effects on fetal body weight and delay of skeletal ossification are both growth related and impaired by compound-induced reductions in maternal body weight. The reason that fetal body weight effects occur at doses lower than the maternal LOAEL for $o$-xylene and mixed xylenes is not known. The fact that mixed xylenes did not elicit the skeletal variants or delayed ossification seen with the individual isomers at the same concentration suggests that the effects of the isomers are not additive. Thus, it is possible that there may be isomer-specific targets relating to skeletal development, as indicated by the slightly different pattern of effects; for example, the incidence of incomplete ossification 
in thoracic vertebral centra was significantly elevated in fetuses exposed to $m$-xylene, but not the other isomers.

The mechanism of neurobehavioral deficits observed in rats exposed gestationally following maternal inhalation to $m$-xylene is not known (Hass and Jakobsen 1993; Hass et al. 1995, 1997). Toxicity to vestibular structures involved with balance or hippocampal neurons involved with sensory processing have been suggested as targets associated with impaired motor coordination and spatial navigation following xylene exposure (Hass et al. 1997).

Xylenes have not been shown to be mutagenic in short-term bioassays, but DNA degradation was reported in the skin of rats exposed dermally to $m$-xylene (Rogers et al. 2001). Penetration of liquid xylene may impair the integrity of cell membranes, resulting in release of nucleases from membranebound lysosomal stores and concomitant fragmentation of DNA. This process appears to be associated with cell death.

\subsubsection{Animal-to-Human Extrapolations}

In general, toxic effects of xylenes observed in humans, such as depression of the central nervous system and irritant effects, are also observed in animals. These similarities are to be expected given that they are a consequence of the chemical properties of unmetabolized xylenes and are not likely to be affected by minor species differences in cell type. Toxicokinetic processes, including metabolic pathways for xylenes, are similar in animals and humans, but would be limited by physiological differences in respiration. EPA (1994) has provided guidance for extrapolating from inhalation exposure in animals to human equivalent exposure levels in humans. PBPK models (see Section 3.4.5) have been employed to estimate human equivalent exposures for toxicity in exposed rats.

\subsection{TOXICITIES MEDIATED THROUGH THE NEUROENDOCRINE AXIS}

Recently, attention has focused on the potential hazardous effects of certain chemicals on the endocrine system because of the ability of these chemicals to mimic or block endogenous hormones. Chemicals with this type of activity are most commonly referred to as endocrine disruptors. However, appropriate terminology to describe such effects remains controversial. The terminology endocrine disruptors, initially used by Thomas and Colborn (1992), was also used in 1996 when Congress mandated the EPA to develop a screening program for “...certain substances [which] may have an effect produced by a naturally occurring estrogen, or other such endocrine effect[s]...”. To meet this mandate, EPA convened a 
panel called the Endocrine Disruptors Screening and Testing Advisory Committee (EDSTAC), and in 1998, the EDSTAC completed its deliberations and made recommendations to EPA concerning endocrine disruptors. In 1999, the National Academy of Sciences released a report that referred to these same types of chemicals as hormonally active agents. The terminology endocrine modulators has also been used to convey the fact that effects caused by such chemicals may not necessarily be adverse. Many scientists agree that chemicals with the ability to disrupt or modulate the endocrine system are a potential threat to the health of humans, aquatic animals, and wildlife. However, others think that endocrine-active chemicals do not pose a significant health risk, particularly in view of the fact that hormone mimics exist in the natural environment. Examples of natural hormone mimics are the isoflavinoid phytoestrogens (Adlercreutz 1995; Livingston 1978; Mayr et al. 1992). These chemicals are derived from plants and are similar in structure and action to endogenous estrogen. Although the public health significance and descriptive terminology of substances capable of affecting the endocrine system remains controversial, scientists agree that these chemicals may affect the synthesis, secretion, transport, binding, action, or elimination of natural hormones in the body responsible for maintaining homeostasis, reproduction, development, and/or behavior (EPA 1997b). Stated differently, such compounds may cause toxicities that are mediated through the neuroendocrine axis. As a result, these chemicals may play a role in altering, for example, metabolic, sexual, immune, and neurobehavioral function. Such chemicals are also thought to be involved in inducing breast, testicular, and prostate cancers, as well as endometriosis (Berger 1994; Giwercman et al. 1993; Hoel et al. 1992).

No in vivo or in vitro studies were located regarding endocrine disruption in human and/or animals after exposure to mixed xylenes or individual isomers of xylene. Evidence for endocrine effects has not been seen in studies on reproductive, developmental, or chronic toxicity of xylenes.

\subsection{CHILDREN'S SUSCEPTIBILITY}

This section discusses potential health effects from exposures during the period from conception to maturity at 18 years of age in humans, when most biological systems will have fully developed. Potential effects on offspring resulting from exposures of parental germ cells are considered, as well as any indirect effects on the fetus and neonate resulting from maternal exposure during gestation and lactation. Relevant animal and in vitro models are also discussed. 
Children are not small adults. They differ from adults in their exposures and may differ in their susceptibility to hazardous chemicals. Children's unique physiology and behavior can influence the extent of their exposure. Exposures of children are discussed in Section 6.6, Exposures of Children.

Children sometimes differ from adults in their susceptibility to hazardous chemicals, but whether there is a difference depends on the chemical (Guzelian et al. 1992; NRC 1993). Children may be more or less susceptible than adults to health effects, and the relationship may change with developmental age (Guzelian et al. 1992; NRC 1993). Vulnerability often depends on developmental stage. There are critical periods of structural and functional development during both prenatal and postnatal life, and a particular structure or function will be most sensitive to disruption during its critical period(s). Damage may not be evident until a later stage of development. There are often differences in pharmacokinetics and metabolism between children and adults. For example, absorption may be different in neonates because of the immaturity of their gastrointestinal tract and their larger skin surface area in proportion to body weight (Morselli et al. 1980; NRC 1993); the gastrointestinal absorption of lead is greatest in infants and young children (Ziegler et al. 1978). Distribution of xenobiotics may be different; for example, infants have a larger proportion of their bodies as extracellular water, and their brains and livers are proportionately larger (Altman and Dittmer 1974; Fomon 1966; Fomon et al. 1982; Owen and Brozek 1966; Widdowson and Dickerson 1964). The infant also has an immature blood-brain barrier (Adinolfi 1985; Johanson 1980) and probably an immature blood-testis barrier (Setchell and Waites 1975). Many xenobiotic metabolizing enzymes have distinctive developmental patterns. At various stages of growth and development, levels of particular enzymes may be higher or lower than those of adults, and sometimes unique enzymes may exist at particular developmental stages (Komori et al. 1990; Leeder and Kearns 1997; NRC 1993; Vieira et al. 1996). Whether differences in xenobiotic metabolism make the child more or less susceptible also depends on whether the relevant enzymes are involved in activation of the parent compound to its toxic form or in detoxification. There may also be differences in excretion, particularly in newborns who all have a low glomerular filtration rate and have not developed efficient tubular secretion and resorption capacities (Altman and Dittmer 1974; NRC 1993; West et al. 1948). Children and adults may differ in their capacity to repair damage from chemical insults. Children also have a longer remaining lifetime in which to express damage from chemicals; this potential is particularly relevant to cancer.

Certain characteristics of the developing human may increase exposure or susceptibility, whereas others may decrease susceptibility to the same chemical. For example, although infants breathe more air per kilogram of body weight than adults breathe, this difference might be somewhat counterbalanced by their 
alveoli being less developed, which results in a disproportionately smaller surface area for alveolar absorption (NRC 1993).

No data are available regarding the effects of exposure to xylenes in children, but it is expected that children would experience the same effects as exposed adults. In controlled acute inhalation experiments on $m$-xylene, one reason offered for the greater impairment in respiratory function (reduced forced vital capacity) in exposed women to compared to exposed men was that the airways in women are narrower and therefore more sensitive to swelling (Ernstgard et al. 2002). On this basis, it is likely that children exposed to xylenes by inhalation would be more sensitive to respiratory impairment than exposed adults.

A few epidemiological studies on laboratory workers suggested that maternal exposure to xylenes might increase the frequency of spontaneous abortion, but the validity of the association is confounded by simultaneous exposure to other chemicals (Lindbohm et al. 1990; Taskinen et al. 1994).

The lipophilic properties of xylenes suggest that the absorption and distribution in children are likely to be similar to those of adults. PBPK modeling estimated that about $1-2 \%$ of xylene absorbed from maternal occupational inhalation exposure at $100 \mathrm{ppm}$ would be transferred to breast milk (Fisher et al. 1997). The daily intake of xylene by a 10-kg nursing infant under this scenario was estimated to be $6.59 \mathrm{mg}$. Studies in mice have demonstrated that xylene absorbed by pregnant females is transferred to the fetus, but that uptake by fetal tissues was lower than in maternal tissues (Ghantous and Danielsson 1986). The most reliable developmental toxicity assay in rats exposed by inhalation indicated that fetotoxicity (reduced fetal weight and delayed ossification, a skeletal variation that is affected by impaired growth) from maternal exposure to $m$ - and $p$-xylene only occurs at maternally toxic exposure levels (Saillenfait et al. 2003); exposure to $o$-xylene or mixed xylenes resulted in fetal body weight effects at concentrations below the LOAEL for maternal effects. The pattern of skeletal variations (i.e., the bones affected) was slightly different for each isomer and no serious malformations were observed at concentrations as high as $2,000 \mathrm{ppm}$. No significant increase in skeletal variations was noted for gestational exposure to mixed xylenes, suggesting that the effect of the isomers was not additive (Saillenfait et al. 2003). Other developmental inhalation studies reported skeletal variations and delayed ossification at lower concentrations, but the results were not considered for determining NOAEL or LOAEL values because fetal effects were not reported on a per-litter basis and little information on maternal toxicity was provided (see Section 3.2.1.6). Fetal toxicity in oral rodent studies occurred at relatively high doses near the maternal effect level (Marks et al. 1982). A potentially serious developmental effect of xylene is impaired neurobehavioral function, which has been observed in rats 
gestationally exposed at a level (500 ppm) that was not toxic to dams (Hass and Jakobson 1995; Hass et al. 1997). In these studies, motor coordination was impaired and deficiencies in a water maze test for spatial navigation persisted to postnatal week 28 in the offspring of exposed dams. These results suggest that in humans, excess maternal exposure to xylene might result in postnatal neurobehavioral deficits even if no overt structural anomalies occurred.

The main phase I metabolizing enzyme for xylene, CYP2E1, is present in the fetus and in children. The presence of CYP2E1 facilitates the elimination of xylene from the body. Total fetal liver CYP content is a relatively constant $30 \%$ of the adult level from the end of the first trimester of gestation up to 1 year of age (EPA 2001b). mRNA for CYP2E1 has been detected in human first-trimester placentas (Hakkola and Saarinen 1996). Low levels of CYP2E1 protein have been detected in human fetal brain as early as gestational day 46, substantially increasing around day 50 (Boutelet-Bochan et al. 1997; Brzezinski et al. 1999). In the fetal liver, CYP2E1 protein was not detectable at 10 weeks of gestation, but was present at 16 weeks (Carpenter et al. 1996). Additionally, there is some evidence that maternal alcohol consumption induces placental CYP2E1 in humans (Rasheed et al. 1997). Hepatic levels of CYP2E1 mRNA increase significantly during the first 24 hours after birth, largely resulting from demethylation that allows transcription to proceed (Vieira et al. 1996). Major accumulations of CYP2E1 occur between 1 and 3 months of age and values comparable to those of adults are achieved sometime between 1 and 10 years of age (EPA 2001b; Vieira et al. 1996). Thus, elimination of xylene metabolites in exposed children would be expected to be similar to exposed adults.

Aside from the model described above (and in Section 3.4.5) for lactational transfer to nursing infants by Fisher et al. (1997), no PBPK models have been developed for children or for fetuses or pregnant women exposed to xylenes.

Ginsberg et al. (2004) considered developmental and pharmacokinetic differences between adults and children that could impact risk assessments of children exposed to toxicants. The higher inhalation rate per respiratory surface area in children compared to adults might result in an apparent higher dose to the lungs in children for the first several years compared to adults exposed to xylene at the same atmospheric concentration. As the stratum corneum of the epidermis is well-developed in newborn infants, dermal absorption of xylene by newborns would be generally expected to be similar to adults. The one exception is for the infants born prematurely, in which the stratum corneum attains a normal thickness only by the second postnatal week. The relatively larger ratio of liver weight to body weight in children, especially during the first 2 years postnatally, and the lower body lipid content for the first 3 months postnatally, 
compared to adults, would suggest that retention of absorbed xylene is lower and systemic clearance of absorbed xylene is faster in young children than in adults. These factors are countered by the possibly lower rates of metabolism of xylenes during the first two postnatal months (see above). The larger brain weight to body weight ratios in children, especially during the first two postnatal years, suggests that proportionally more xylene may be delivered to the brain of children, rendering them more vulnerable to the neurotoxic effects of xylene than similarly exposed adults. No compound-specific experimental data are available to confirm these suppositions regarding the exposure of children to xylenes.

\subsection{BIOMARKERS OF EXPOSURE AND EFFECT}

Biomarkers are broadly defined as indicators signaling events in biologic systems or samples. They have been classified as markers of exposure, markers of effect, and markers of susceptibility (NAS/NRC 1989).

Due to a nascent understanding of the use and interpretation of biomarkers, implementation of biomarkers as tools of exposure in the general population is very limited. A biomarker of exposure is a xenobiotic substance or its metabolite(s) or the product of an interaction between a xenobiotic agent and some target molecule(s) or cell(s) that is measured within a compartment of an organism (NAS/NRC 1989). The preferred biomarkers of exposure are generally the substance itself, substance-specific metabolites in readily obtainable body fluid(s), or excreta. However, several factors can confound the use and interpretation of biomarkers of exposure. The body burden of a substance may be the result of exposures from more than one source. The substance being measured may be a metabolite of another xenobiotic substance (e.g., high urinary levels of phenol can result from exposure to several different aromatic compounds). Depending on the properties of the substance (e.g., biologic half-life) and environmental conditions (e.g., duration and route of exposure), the substance and all of its metabolites may have left the body by the time samples can be taken. It may be difficult to identify individuals exposed to hazardous substances that are commonly found in body tissues and fluids (e.g., essential mineral nutrients such as copper, zinc, and selenium). Biomarkers of exposure to xylene are discussed in Section 3.8.1.

Biomarkers of effect are defined as any measurable biochemical, physiologic, or other alteration within an organism that, depending on magnitude, can be recognized as an established or potential health impairment or disease (NAS/NRC 1989). This definition encompasses biochemical or cellular signals of tissue dysfunction (e.g., increased liver enzyme activity or pathologic changes in female genital epithelial cells), as well as physiologic signs of dysfunction such as increased blood pressure or decreased lung 
capacity. Note that these markers are not often substance specific. They also may not be directly adverse, but can indicate potential health impairment (e.g., DNA adducts). Biomarkers of effects caused by xylene are discussed in Section 3.8.2.

A biomarker of susceptibility is an indicator of an inherent or acquired limitation of an organism's ability to respond to the challenge of exposure to a specific xenobiotic substance. It can be an intrinsic genetic or other characteristic or a preexisting disease that results in an increase in absorbed dose, a decrease in the biologically effective dose, or a target tissue response. If biomarkers of susceptibility exist, they are discussed in Section 3.10, Populations That Are Unusually Susceptible.

\subsubsection{Biomarkers Used to Identify or Quantify Exposure to Xylene}

Xylene levels in the blood and levels of its metabolite, methylhippuric acid, in the urine are the primary markers used to detect exposure to xylene. Xylene is very soluble in the blood and is readily absorbed into the circulation during exposure (Astrand 1982). Measurement of blood levels of xylene is limited by the rapid metabolism of xylene. Moreover, there are no data on background concentrations of xylene in blood or urine. Xylene and xylene isomers have been detected at concentrations of up to $400 \mathrm{nM}$ in urine of workers exposed to xylene at geometric mean concentration of $1.9 \mathrm{ppm}$ with a maximum concentration of $27.3 \mathrm{ppm}$ (Takeuchi et al. 2002). Xylenes are metabolized almost exclusively to methylhippuric acids in humans. Detection of methylhippuric acid in the urine is the most widely used indicator of xylene exposure (ACGIH 1986). A strong association has been shown between urinary methylhippuric acid concentrations and exposure to xylene (Daniell et al. 1992; Jonai and Sato 1988; Kawai et al. 1991); during an 8-hour workshift, a concentration of $57.8 \mathrm{mg} / \mathrm{L}$ of methylhippuric acid isomers (i.e., all isomers combined) was found to correlate with exposure to $3.8 \mathrm{ppm}$ (geometric mean concentration) of total xylenes (Kawai et al. 1991). In a study of Chinese men and women occupationally exposed to mixed xylenes, Inoue et al. (1993) estimated that $13 \mathrm{mg}$ of methylhippuric acid would be excreted in a liter of urine for each ppm of xylene exposure (or $11.1 \mathrm{mg} / \mathrm{g}$ creatinine/ppm). This relationship was true for both men and women as well as for mixed and individual isomers. Within 2 hours of an inhalation exposure, methylhippuric acid may be detected in the urine (Sedivec and Flek 1976b). The excretion of methylhippuric acid is complete within 1 or 2 days of exposure to xylene, limiting the utility of this biomarker to the detection of only very recent exposures. With chronic exposure to xylene, the metabolism is enhanced, further limiting the time following exposure that xylene levels may be measured in the blood (Savolainen et al. 1979a). Since the methylhippuric acid background levels in persons not exposed to xylenes are very low, methylhippuric acids are specific markers for xylenes, except for 
exposure to alkyl toluenes in which the number of carbon atoms in the alkyl group is odd. A minor metabolite of xylene, $N$-acetyl-S-xylyl cysteine (a trioether), may also be detected in the urine (Tanaka et al. 1990; van Doorn et al. 1980); however, it is at such low levels in the urine during experimental exposures that it is ineffective as a biomarker (Norstrom et al. 1988). For additional information on the kinetics of xylene absorption, distribution, metabolism, or excretion, see Section 3-4.

\subsubsection{Biomarkers Used to Characterize Effects Caused by Xylene}

The following changes are potential biomarkers of effect for xylenes; however, none of the changes are unique to xylene exposure. Xylenes have been observed to enhance the activity of a variety of microsomal enzymes and increase hepatic cytochrome P-450 content (Elovaara 1982; Elovaara et al. 1980; Patel et al. 1979; Savolainen et al. 1978; Tatrai et al. 1981; Toftgard and Nilsen 1981, 1982; Toftgard et al. 1981). Scores consistent with memory impairment and decreased reaction time have been observed using standard intelligence tests and measures of reaction time (Gamberale et al. 1978; Riihimaki and Savolainen 1980; Savolainen and Riihimaki 1981a; Savolainen et al. 1979b, 1984, 1985a). These effects are likely to be elicited by any solvent that has anaesthetic properties. Erythema of the skin is a biomarker of dermal exposure to xylene, but this is a common reaction to compounds with irritant properties (Ahaghotu et al. 2005; Anderson et al. 1986; Chatterjee et al. 2005; Hine and Zuidema 1970; Pound and Withers 1963; Smyth et al. 1962). Increases in blood levels of interleukin 1-alpha, as demonstrated in rats, may represent a biomarker of inflammatory processes in skin following dermal exposure to xylene, but might apply to any chemical that elicits dermal irritation (Ahaghotu et al. 2005; Chatterjee et al. 2005).

\subsection{INTERACTIONS WITH OTHER CHEMICALS}

The interaction of xylene with alcohol, drugs (aspirin, phenobarbitol), and various solvents (1,1,1-trichlorethane, benzene, toluene, ethylbenzene, methyl ethyl ketone) has been evaluated in experimental studies with humans and animals. Xylene has a high potential to interact with numerous substances because the isomers induce microsomal enzymes in the liver (Blanchard and Morris 1994; Liira et al. 1991), while microsomal enzymes in the lungs are inhibited by xylene exposure (Blanchard and Morris 1994; Elovaara et al. 1987; Patel et al. 1978; Silverman and Schatz 1991; Toftgard and Nilsen 1982). Which enzymes will be affected is isomer dependent. For example, $m$-xylene is a more potent inducer of P-450 2B enzymes than p-xylene (Backes et al. 1993). The isomer differences, as well as organ differences in effects on xenobiotic metabolizing enzymes, make it difficult to predict the interaction of xylene with other substances. 
The effects from combined exposure to xylene and ethanol have been studied most extensively because of the reasonable expectancy that some workers will consume alcoholic beverages and subsequently might be exposed to xylene occupationally by inhalation. Results of studies with humans and animals indicate that metabolic interaction between xylene and ethanol occurs. Co-administered ethanol appears to inhibit the metabolism of xylene, probably by competition for mixed function oxidases, resulting in elevated blood levels of xylene and decreased excretion of methylhippuric acid (Elovaara et al. 1980; Riihimaki et al. 1982a, 1982b; Romer et al. 1986; Savolainen 1980; Savolainen et al. 1978, 1979b, 1980b). A kinetic study in rats (Kaneko et al. 1993) suggests that ethanol inhibition of xylene metabolism occurs only at high airborne concentrations $(500 \mathrm{ppm})$. Paradoxically, ethanol pretreatment has a synergistic effect on metabolism of xylene as a result of the induction of microsomal enzymes in the liver (Wisniewska-Knypl et al. 1989). Ethanol pretreatment (137 g taken orally) enhanced the metabolism of $m$-xylene in human subjects exposed at $400 \mathrm{ppm}$, but had no effect at $100 \mathrm{ppm}$ (Tardif et al. 1994). This would enhance the metabolic capacity of the liver and modify biological effects of other chemicals that are either detoxified or converted to toxic metabolites by the microsomal enzymes. In summary, it cannot be stated with certainty whether alcohol and xylene would interact to produce synergistic or antagonistic effects in humans and animals because there are reasons why both would occur. MacDonald et al. (2002) used PBPK modeling to evaluate the likelihood of central nervous system effects in people exposed to a timeweighted-average concentration of $100 \mathrm{ppm} m$-xylene for 8 hours and also exposed to ethanol. In the model simulations, increasing exposure to ethanol increased the probability of central nervous system effects from exposure to $m$-xylene. Combined exposure to $m$-xylene and ethanol increased lipid peroxidation in the rat liver (Jajte et al. 2003).

Kaneko et al. (1995) modeled the effect of phenobarbital pretreatment ( $80 \mathrm{mg} / \mathrm{kg} / \mathrm{day}$ for 3 days) with exposure to $m$-xylene orally or intraperitoneally at 8.6 or $86.4 \mathrm{mg} / \mathrm{kg}$ or by inhalation for 6 hours at 40 or $400 \mathrm{ppm}$. Phenobarbital treatment increased the metabolism of inhaled $m$-xylene at $400 \mathrm{ppm}$. This was related to a decrease in xylene blood concentrations and increased urinary excretion. Phenobarbital had no significant effect on metabolism of ingested xylene. The metabolism of $m$-xylene was not affected by coexposure to $n$-butyl alcohol in rats exposed by inhalation for 7 hours to $100 \mathrm{ppm}$ of each chemical (Swiercz et al. 1995).

Combined exposure to ethanol and xylene results in macrocytosis and decreased erythrocyte membrane fluidity (Wronska-Nofer et al. 1991). These effects were not observed when either chemical was 
administered alone. It is unclear whether this interaction is pharmacological or pharmacokinetic in nature.

Acute inhalation exposure to a mixture of toluene and xylene resulted in more-than-additive respiratory and central nervous system toxicity (Korsak et al. 1988, 1992). Elevated blood levels of xylene and toluene and decreased excretion of the major metabolites of xylene and toluene in the urine (Tardif et al. 1992) suggest mutual metabolic inhibition. However, simultaneous exposures in humans indicate that a threshold exists for this interaction (Tardif et al. 1991). No increase in blood levels of these substances was observed during combined exposures to $50 \mathrm{ppm}$ toluene and $40 \mathrm{ppm}$ xylene over 3 consecutive days, whereas increases in blood levels and levels in exhaled air were observed during a combined 4-hour exposure to $95 \mathrm{ppm}$ toluene and $80 \mathrm{ppm}$ xylene. Thus, combined exposures at below threshold level are unlikely to produce greater than additive toxicity (Tardif et al. 1991). Physiologically based toxicokinetic modeling studies using rat data suggests that the interaction between toluene and xylene is competitive, with toluene a more potent inhibitor of xylene metabolism than xylene is of toluene metabolism (Tardif et al. 1993a, 1993b, 1995). PBPK modeling studies of ternary mixtures predicted that humans exposed to permissible levels for the mixture (33 ppm $m$-xylene, $17 \mathrm{ppm}$ toluene, and $33 \mathrm{ppm}$ ethylbenzene), would not exhibit biologically significant metabolic inhibition of the components (Tardif et al. 1997). In these ternary simulations, after $0.5-8$ hours of exposure, the concentration of xylene in alveolar air was slightly, but significantly higher than expected compared to exposure of xylene alone; the blood concentration of xylene following exposure to the ternary mixture was slightly elevated in venous blood after 5.5-8 hours of exposure. Studies in rats exposed to binary or ternary mixtures $(m$-xylene and toluene with or without ethylbenzene) at component concentrations of 100 or $200 \mathrm{ppm}$ demonstrated that the kinetics of metabolism was less affected by metabolic interactions when metabolism was near saturation (Tardif et al. 1996).

Exposure to xylene combined with benzene or ethylbenzene may also produce mutual inhibition of the metabolism of both solvents (Engstrom et al. 1984; Nakajima and Sato 1979b). Ethylbenzene is found in commercial xylene. In contrast, ethyl acetate exposure in combination with exposure to $m$-xylene caused a reduction in blood xylene levels (Freundt et al. 1989).

Combined exposures to $m$-xylene and methyl ethyl ketone (2-butanone) produced a synergistic induction of microsomal enzymes in rats (Liira et al. 1991), but in humans, inhibited the metabolism of $m$-xylene to methylhippuric acid, resulting in corresponding increases in levels of xylene in blood and fat (Liira et al. 1988, 1991). While the side-chain oxidation of xylene to methylhippuric acid was inhibited, an increase 
in ring oxidation (xylenol production) was observed (Liira et al. 1991), indicating that the inhibition was specific to a particular oxidation reaction. Thus, it is not known as to whether 2-butanone and $m$-xylene would interact to produce additive or antagonist effects in humans and animals.

Inhalation of $m$-xylene following pretreatment with phenobarbital was associated with both increased pulmonary retention of $m$-xylene and increased urinary excretion of $m$-methylbenzoic acid (David et al. 1979). Combined inhalation of 1,1,1-trichloroethane (methylchloroform) and $m$-xylene had no effect on the metabolism of $m$-xylene, as indicated by blood concentrations of the parent compound and urinary concentrations of $m$-methylhippuric acid (Tardif and Charest-Tardif 1999). Surprisingly, inhalation of $m$-xylene and 1,1,1-trichloroethane has been associated with slight improvements in certain psychophysiological parameters, including reaction time and equilibrium in humans as compared with pre-exposure measurements (Savolainen et al. 1982a, 1982b), and impairment in others such as visual evoked potentials and equilibrium (Savolainen et al. 1982a; Seppalainen et al. 1983). Also, a protective effect of xylene on $n$-hexane-induced testicular atrophy and peripheral nerve effects were observed when rats were exposed to $n$-hexane and xylene simultaneously (Nylen and Hagman 1994; Nylen et al. 1989), although combined exposure to xylene and $n$-hexane increased loss of auditory sensitivity (Nylen and Hagman 1994; Nylen 1996). Bromobenzene, which requires metabolic activation, showed greater toxicity to the liver in $p$-xylene exposed rats, while lung toxicity was not affected (Day et al. 1992).

Following concurrent exposure to $100 \mathrm{ppm} m$-xylene and 1,500 $\mathrm{mg}$ aspirin orally in human subjects, the urinary excretion of metabolites conjugated to glycine ( $m$-methylhippuric acid and salicyluric acid, respectively) was reduced by $50 \%$ for both compounds (Campbell et al. 1988). The authors speculate that the cause was competition for the enzymes involved in conjugation with glycine (acyl-CoA synthetase and glycine $\mathrm{N}$-acylase), resulting in saturation of the conjugation pathway for both chemicals (Campbell et al. 1988). Administration of aspirin to pregnant rats during inhalation exposure to xylene caused greater-than-additive potentiation of maternal and fetal toxic effects (Ungvary 1985). This was postulated to be due to the interference with metabolism of aspirin by xylene and vice versa.

Oral exposure to a relatively high dose of sodium benzoate, a food additive, 3 hours before intraperitoneal injection of $o$-xylene altered the metabolism of $o$-xylene in rats (Moriwaki et al. 2005). Pre-treatment with sodium benzoate (more than twice the amount of $o$-xylene on a molar basis) reduced the excretion of $o$-methylhippuric acid but increased the excretion of $o$-toluic acid conjugates and unmodified $o$-xylene in urine. These changes were attributed to competition for the enzymes involved in glycine conjugation (benzoyl-Coenzyme A synthetase and benzoyl-CoA:glycine N-acyltransferase). 
Exposure to xylene has been shown to inhibit several microsomal enzymes in the lung (Blanchard and Morris 1994; Elovaara et al. 1987; Patel et al. 1978; Silverman and Schatz 1991; Toftgard and Nilsen 1982). $m$-Xylene administered intraperitoneally or exposure to $o$-xylene by inhalation altered the pulmonary microsomal metabolism of benzo[a]pyrene in rats, resulting in inhibition of its detoxification and increased production of toxic, mutagenic metabolites (bay region diols) (Park and Schatz 1999; Stickney et al. 1991). One study suggested that xylene acts as a promotor or cocarcinogen for the induction of skin tumors in mice, but the purity of the tested material was not reported (Pound 1970). If verified, these findings could be relevant in combined human exposures to xylene and polyaromatic hydrocarbons present in cigarette smoke and combustion emissions and especially to petrochemical workers who could be exposed to xylene, crude oils (promotor), and ultraviolet light (initiator).

Dilution in polyethylene glycol 600 (PEG 600) increased the severity of eye irritation elicited by xylene and other aromatic compounds in rabbits compared to the undiluted compounds (Kennah et al. 1989). Undiluted xylene (not specified but presumed to be mixed isomers) elicited only minimal eye irritation whereas dilutions in PEG 600 resulted in slight or moderate irritation (3-5-fold increases in Draize scores); similar increases were observed with toluene and styrene. Because other diluents (cellosolve acetate or methyl isobutyl ketone) did not have the same effect, the study authors proposed that the aromatic compounds interacted with PEG 600 to form a more potent ocular irritant.

In addition to interacting with other chemicals, exposure to xylene at high concentrations has also been shown to increase the effects of a virus. Acute exposure of mice to 1,208 ppm (but not $595 \mathrm{ppm}$ ) $p$-xylene (4 days, 4 hours/day) increased the mortality resulting from the murine cytomegalovirus (Selgrade et al. 1993). This effect was a result of potentiation of the liver damage cause by the virus rather than an immunological effect.

\subsection{POPULATIONS THAT ARE UNUSUALLY SUSCEPTIBLE}

A susceptible population will exhibit a different or enhanced response to xylene than will most persons exposed to the same level of xylene in the environment. Reasons may include genetic makeup, age, health and nutritional status, and exposure to other toxic substances (e.g., cigarette smoke). These parameters result in reduced detoxification or excretion of xylene, or compromised function of organs affected by xylene. Populations who are at greater risk due to their unusually high exposure to xylene are discussed in Section 6.7, Populations with Potentially High Exposures. 
Available data indicate that subsets of the human population may be unusually susceptible to the toxic effects of xylene. Pregnant women, fetuses, and very young children may be at greater risk of adverse health effects from xylene exposure than the population in general (Barlow and Sullivan 1982; Holmberg and Nurminen 1980; Hudak and Ungvary 1978; Kucera 1968; Marks et al. 1982; Mirkova et al. 1983; Ungvary et al. 1980b, 1981). Although no human studies were located indicating maternal or fetal toxicity following mixed xylene exposure, animal studies that involved exposure to $m$-xylene and aspirin or xylene alone suggest there may be a relationship between exposure to the agents and developmental effects (Hudak and Ungvary 1978; Marks et al. 1982; Ungvary 1985; Ungvary et al. 1980b, 1981). In summary, although it is not clear how toxic xylene might be to fetuses and infants, for safety's sake caution is urged. The ability of fetuses and very young children to metabolize certain xenobiotics, including possibly xylene, is reduced because of their immature enzyme detoxification systems (Calabrese 1978). Thus, for pregnant women exposed to xylene, ingestion of aspirin is likely to potentiate adverse effects of xylene in both the mother and the offspring.

People with subclinical and clinical epilepsy are at increased risk of seizures if exposed to xylene because of its excitatory central nervous system effects (Arthur and Curnock 1982; Goldie 1960; Riihimaki and Hanninen 1987). It has also been demonstrated in human (Goldie 1960; Riihimaki et al. 1982a; Savolainen 1980; Savolainen et al. 1978, 1980b) and animal studies (Elovaara et al. 1980; Savolainen et al. 1979b) that alcohol consumption potentiates xylene toxicity. Some people appear particularly susceptible to the interaction and may develop dizziness, nausea, and dermal flush (Riihimaki et al. 1982b; Savolainen et al. 1980b).

People with clinical or subclinical renal, hepatic, or cardiac disease may be more susceptible to the effects of xylene. Evidence from occupational and case studies indicates that exposure to high levels of xylene might cause renal impairment and some hepatic effects, as well as cardiac manifestations, including tachycardia and ECG abnormalities (Goldie 1960; Hipolito 1980; Morley et al. 1970; NIOSH 1975; Von Burg 1982). However, exposure to xylene in these studies was confounded with exposure to other chemical agents.

Limited human data suggest that people with respiratory diseases, such as asthma, could potentially be at risk with regard to the adverse effects of xylene following inhalation exposure (Hipolito 1980; Morley et al. 1970). 


\subsection{METHODS FOR REDUCING TOXIC EFFECTS}

This section will describe clinical practice and research concerning methods for reducing toxic effects of exposure to xylene. However, because some of the treatments discussed may be experimental and unproven, this section should not be used as a guide for treatment of exposures to xylene. When specific exposures have occurred, poison control centers and medical toxicologists should be consulted for medical advice. The following texts provide specific information about treatment following exposures to xylene:

Ellenhorn MJ, Barceloux DG, eds. 1988. Medical toxicology: Diagnosis and treatment of human poisoning. New York, NY: Elsevier, 1422-1426, 1730-1731.

IPCS. 1992. Xylene. Poisons information. Monograph 565. International Programme on Chemical Safety. http://www.inchem.org/documents/pims/chemical/xylene.htm. April 7, 2005.

\subsubsection{Reducing Peak Absorption Following Exposure}

General recommendations reported for reducing absorption following acute high-dose exposure to xylene include removal of the patient from the source of exposure to fresh air and decontamination of the skin with mild soap and water (Bronstein and Currance 1988; Ellenhorn and Barceloux 1988; Goldfrank et al. 1990; Stutz and Janusz 1988). When the eyes have been involved, copious rinsing with tepid water or normal saline has been used for decontamination (Bronstein and Currance 1988; Stutz and Janusz 1988).

Strategies for reducing xylene absorption following ingestion are limited because light petroleum products such as xylene cause severe aspiration pneumonitis (Ellenhorn and Barceloux 1988; IPCS 1992). Gastric lavage is the preferred method recommended for emptying the stomach contents, as long as a cuffed endotracheal tube is used to limit the possibility of aspiration (Goldfrank et al. 1990). Induction of emesis (vomiting) with syrup of ipecac is generally not recommended, especially in pediatric cases (Goldfrank et al. 1990); emesis is contraindicated if unprovoked emesis has already occurred or if the patient is not alert or has an impaired gag reflex (Ellenhorn and Barceloux 1988; Goldfrank et al. 1990). The only circumstances in which emesis is recommended are when very large quantities have been ingested, such as in a suicide attempt (Ellenhorn and Barceloux 1988; Goldfrank et al. 1990), or another highly toxic substance has been ingested together with xylene (Goldfrank et al. 1990). In summary, gastric lavage is recommended to reduce xylene absorption from the gastrointestinal tract only when one is certain that aspiration is not likely to occur. 
Although the use of activated charcoal and/or cathartics to limit intestinal absorption is recommended in some treatment protocols (Stutz and Janusz 1988), their use has been reported to be equivocal (Ellenhorn and Barceloux 1988). No studies have shown that activated charcoal is effective in adsorbing petroleum distillates or that cathartics are effective in speeding excretion (Goldfrank et al. 1990). Furthermore, because of low viscosity, oil-based cathartics may increase aspiration pneumonitis and absorption (Goldfrank et al. 1990).

\subsubsection{Reducing Body Burden}

In acute exposure situations, most xylene absorbed by the body is excreted in the urine or exhaled air within a day after exposure (see Section 3.4.4). However, charcoal hemoperfusion has been used to speed the removal of xylene from the body and to reverse its acute toxicity (Recchia et al. 1985). Sevcik et al. (1992) also used hemoperfusion and hemodialysis in an attempt to speed removal of xylene. Whether the relative gain from these treatment methods is worth the potential risks of invasive treatment remains to be established. A small percentage of absorbed xylene is retained in body fat. It has been suggested that over a prolonged period of exposure, significant amounts of xylene could accumulate in adipose tissue (Astrand 1982; Engstrom and Bjurstrom 1978). However, xylene has been shown to induce its own metabolism with the result that greater amounts of metabolites are excreted and less is available for storage (Elovaara et al. 1989; Savolainen et al. 1979a). No information was located regarding methods for reducing adipose stores of xylene; removal of xylene from adipose tissue is slow, limited by the lipophilic nature of the chemical and the poor vascular perfusion of adipose tissue (Riihimaki et al. 1979b). Use of agents known to induce microsomal enzyme activity is a possible experimental method for enhancing excretion of xylene released from adipose stores by increasing the gradient of diffusion.

\subsubsection{Interfering with the Mechanism of Action for Toxic Effects}

No information was located on established therapies designed to interfere with the mechanism of action of xylene. However, some speculation is possible regarding areas for future research in this regard. For example, the central nervous system toxicity of xylene is believed to be similar to that produced by other nonspecific central nervous system depressants (Desi et al. 1967; EPA 1985a; Gerarde 1959; Savolainen and Pfaffli 1980; Tahti 1992). If circulating xylene levels could be reduced, then the central nervous system toxicity may likewise be reduced (see Section 3.11.2).

Since the exact metabolite responsible for the pulmonary toxicity of xylene has not been identified, it is difficult to speculate on steps to avert its synthesis or speed its excretion. In animals, selective 
inactivation of enzymes can result in damage to tissue caused by the toxic metabolite of xylene, methylbenzaldehyde. This effect has not been confirmed in humans. Decreased pulmonary microsomal enzyme activity was seen in rats administered a single dose or repeated doses of $p$-xylene for 3 weeks (Elovaara et al. 1989; Patel et al. 1978). However, the inhibition of pulmonary microsomal enzymes decreases to some extent with continued exposure to xylene (Silverman and Schatz 1991); this may indicate that xylene-induced activation of metabolizing enzymes and thereby acceleration of its own metabolism (Elovaara et al. 1989) may be limiting the production of the toxic metabolite.

At least one in vitro study indicated that cytotoxicity caused by $m$-xylene is associated with reductions in cellular antioxidants and that supplementation with $\mathrm{N}$-acetylcysteine partially ameliorates these effects (Coleman et al. 2003). $\mathrm{N}$-acetylcysteine has been employed as a therapeutic antagonist for acetaminophen poisoning, but its effectiveness for reducing toxicity of xylenes in vivo has not been assessed.

The available information on the mechanisms of renal and fetotoxicity is insufficient to allow speculation on potential means for blocking these effects.

\subsection{ADEQUACY OF THE DATABASE}

Section 104(I)(5) of CERCLA, as amended, directs the Administrator of ATSDR (in consultation with the Administrator of EPA and agencies and programs of the Public Health Service) to assess whether adequate information on the health effects of xylene is available. Where adequate information is not available, ATSDR, in conjunction with the National Toxicology Program (NTP), is required to assure the initiation of a program of research designed to determine the health effects (and techniques for developing methods to determine such health effects) of xylene.

The following categories of possible data needs have been identified by a joint team of scientists from ATSDR, NTP, and EPA. They are defined as substance-specific informational needs that if met would reduce the uncertainties of human health assessment. This definition should not be interpreted to mean that all data needs discussed in this section must be filled. In the future, the identified data needs will be evaluated and prioritized, and a substance-specific research agenda will be proposed. 


\subsubsection{Existing Information on Health Effects of Xylene}

The existing data on health effects of inhalation, oral, and dermal exposure of humans and animals to xylene are summarized in Figure 3-9. The purpose of this figure is to illustrate the existing information concerning the health effects of xylene. Each dot in the figure indicates that one or more studies provide information associated with that particular effect. The dot does not necessarily imply anything about the quality of the study or studies, nor should missing information in this figure be interpreted as a "data need". A data need, as defined in ATSDR's Decision Guide for Identifying Substance-Specific Data Needs Related to Toxicological Profiles (Agency for Toxic Substances and Disease Registry 1989), is substance-specific information necessary to conduct comprehensive public health assessments. Generally, ATSDR defines a data gap more broadly as any substance-specific information missing from the scientific literature.

People may be exposed to xylene at hazardous waste sites by inhalation of contaminated air, drinking contaminated water, or dermal contact with contaminated water or subsurface soils and sediments. Volatilization of xylene from surface water and soil occurs rapidly; therefore, inhalation is the most likely route of exposure to xylene at these sites. The human health effects of xylene by inhalation exposure have been studied to the greatest extent. There is little information available regarding health effects in humans following oral or dermal exposure to xylene. The bulk of the information on health effects in humans associated with dermal exposure comes from reports of occupational exposures, which are likely to be combined inhalation and dermal exposures. As noted above, ingestion of xylene may be of concern because of the potential for xylene to contaminate sources of drinking water (groundwater) and certain soils. Dermal exposure to xylene is of concern not only because of potential workplace exposures, but also because members of the general public are potentially exposed to xylene contained in paints, glues, and other household products. As noted above, dermal exposure to soils and water contaminated with xylene at waste sites could also occur.

Human fatalities following both inhalation and ingestion of xylene have been reported in the literature. Acute inhalation exposure of humans to xylene has resulted in hepatic and cardiovascular effects as well as neurologic effects. Very limited data regarding the systemic health effects of intermediate-duration human exposure to xylene were located in the literature. Also, very limited human carcinogenicity data were reported in the literature. Very little information is available on the chronic systemic, immunologic, developmental, reproductive, and genotoxic health effects of xylene exposure in humans. Interpretation of the large number of human studies examining the health effects of inhaled xylene vapor is difficult 
Figure 3-9. Existing Information on Health Effects of Total Xylenes
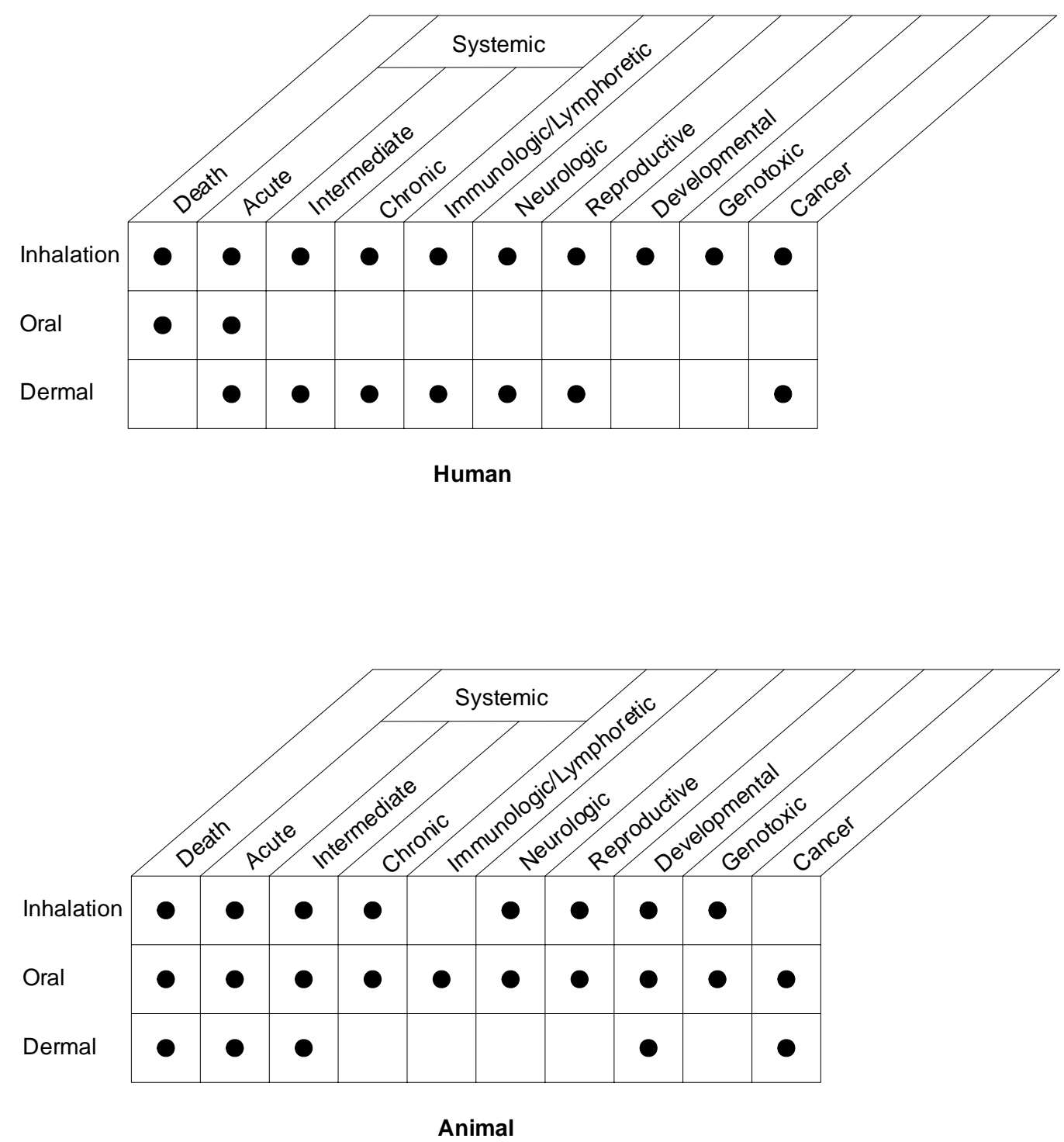

- Existing Studies 
because of study design limitations, such as inadequate characterization of exposure and concurrent exposure to other solvents such as toluene and benzene.

Studies conducted on experimental animals have been fairly extensive (Figure 3-9) and have focused on the adverse health effects following inhalation and oral exposure to xylene. Data are comprehensive on neurological and systemic effects. There are several developmental studies in animals, although most have limitations. Limited information exists on the carcinogenicity of mixed xylenes. A large number of studies on the genotoxicity of xylene are available, with the majority reporting negative results.

\subsubsection{Identification of Data Needs}

Acute-Duration Exposure. There are acute exposure data in humans and/or animals that indicate that the central nervous system (Andersson et al. 1981; Arthur and Curnock 1982; Bushnell 1989; Carpenter et al. 1975a; De Ceaurriz et al. 1983; Dudek et al. 1990; Dyer et al. 1988; Ernstgard et al. 2002; Furnas and Hine 1958; Gamberale et al. 1978; Ghosh et al. 1987; Klaucke et al. 1982; Korsak et al. 1988; Martinez et al. 1989; Molnar et al. 1986; Morley et al. 1970; Muralidhara and Krishnakumari 1980; Nersesian et al. 1985; NIOSH 1981; NTP 1986; Padilla and Lyerly 1989; Pryor et al. 1987; Savolainen and Linnavuo 1979; Savolainen et al. 1978, 1979b, 1984, 1985a; Seppalainen et al. 1989; Wimolwattanapun et al. 1987) and possibly the developing fetus (Balogh et al. 1982; Hudak and Ungvary 1978; Marks et al. 1982; Ungvary 1985; Ungvary and Tatrai 1985; Ungvary et al. 1980b, 1981) are the major targets of acute xylene toxicity by the inhalation and oral routes. Limited information is available on the nervous system effects of dermal exposure to xylenes (Goldie 1960; Hipolito 1980; Kilburn et al. 1985; Roberts et al. 1988). Death has been observed to occur as a result of exposure by inhalation, oral, and dermal exposure, and lethal and nonlethal levels of total xylenes have been determined (Abu $\mathrm{Al}$ Ragheb et al. 1986; Bonnet et al. 1979; Cameron et al. 1938; Carpenter et al. 1975a; Condie et al. 1988; Dyer et al. 1988; Furnas and Hine 1958; Gerarde 1959; Harper et al. 1975; Hine and Zuidema 1970; Morley et al. 1970; Muralidhara and Krishnakumari 1980; NTP 1986; Pound and Withers 1963; Smyth et al. 1962; Ungvary et al. 1980b; Wolf et al. 1956). Acute studies have demonstrated that xylene is irritating to the skin and eyes (Anderson et al. 1986; Carpenter et al. 1975a; Consumer Products Testing 1976; De Ceaurriz et al. 1981; Engstrom et al. 1977; Ernstgard et al. 2002; Food and Drug Research Labs 1976a; Hine and Zuidema 1970; Klaucke et al. 1982; Nelson et al. 1943; Nersesian et al. 1985; NIOSH 1981; Pound and Withers 1963; Riihimaki 1979b; Smyth et al. 1962; Wolf et al. 1956). Inhalation of xylenes has also been shown to cause irritation of the respiratory tract and dyspnea (Carpenter et al. 
1975a; De Ceaurriz et al. 1981; Ernstgard et al. 2002; Furnas and Hine 1958; Klaucke et al. 1982; Korsak et al. 1988; Morvai et al. 1976; Nelson et al. 1943; Nersesian et al. 1985; NIOSH 1981).

Data were sufficient to determine an acute-duration inhalation MRL for mixed xylenes based on increased respiratory effects (slight irritation and impaired lung function) and subjective neurological symptoms (headache, dizziness, feeling of intoxication) in humans exposed to $m$-xylene (Ernstgard et al. 2002). The acute oral MRL for mixed xylenes is based on a NOAEL for neurological effects in rats exposed to p-xylene (Dyer et al. 1988). Additional information on the effects observed after acute dermal exposure is needed due to the likelihood that acute duration skin contact with xylenes could occur in the home, workplace, and possibly at hazardous waste sites. Pharmacokinetic data and toxicity data indicate that xylene is absorbed through the skin (Dutkiewicz and Tyras 1968; Engstrom et al. 1977; Kezic et al. 2001; McDougal et al. 1990; Morgan et al. 1991; Riihimaki 1979b; Riihimaki and Pfaffli 1978; Skowronski et al. 1990), although the relative absorption by this route is difficult to ascertain because of the rapid evaporation of xylenes from the skin. A PBPK model has been developed for dermal exposure (Thrall and Woodstock 2003). Additional acute-duration inhalation and oral studies clarifying which nervous system effects are the most sensitive are needed to provide critical, reliable guidance values for acute exposure.

Intermediate-Duration Exposure. Intermediate-duration inhalation, oral, and dermal studies have identified the central nervous system (Condie et al. 1988; Goldie 1960; Honma et al. 1983; Jenkins et al. 1970; NTP 1986; Pryor et al. 1987; Rank 1985; Savolainen and Seppalainen 1979; Savolainen et al. 1979a), liver (Condie et al. 1988; Elovaara et al. 1989; Ungvary 1990), kidneys (Condie et al. 1988), and possibly the developing fetus (Bio/dynamics 1983; Mirkova et al. 1979, 1983; Taskinen et al. 1989) as the primary targets of intermediate-duration xylene exposure. Very few studies were located that examined the effects associated with intermediate-duration dermal exposure to xylenes (Mirkova et al. 1979; Wolf et al. 1956). Pharmacokinetic data indicate that absorption of xylenes occurs through the skin; however, it is difficult to determine whether similar end points would be expected after repeated dermal exposure to xylenes. Human skin may be repeatedly exposed to xylene as a result of occupational and home use. Repeated exposure of the skin to contaminated media at hazardous waste sites may also occur. Therefore, a well-designed and well-conducted intermediate-duration dermal study may help to better characterize the potential health hazards associated with repeated dermal exposure to xylenes.

An intermediate-duration inhalation MRL was derived based on a NOAEL for decreased rotarod performance in rats discontinuously exposed to $m$-xylene for 3 months (Korsak et al. 1994). Data were 
sufficient to determine an intermediate-duration oral MRL for mixed xylenes based on a LOAEL for hyperactivity in animals (Condie et al. 1988). Deficiencies of the intermediate-duration oral toxicity database include the lack of testing for neurobehavioral effects, which appear to be the most sensitive end points in acute-duration oral toxicity studies, and lack of developmental and multi-generational data. Additional intermediate-duration oral studies that identify a NOAEL and LOAEL for sensitive neurobehavioral effects are needed to provide reliable guidance values for intermediate-duration exposure.

Chronic-Duration Exposure and Cancer. Few human (Arp et al. 1983; Askergren 1981, 1982; Askergren et al. 1981b, 1981c; Brasington and Thorpe-Swenson 1991; Dolara et al. 1982; Franchini et al. 1983; Gupta et al. 1990; Hipolito 1980; Holmberg and Nurminen 1980; Kilburn et al. 1985; Kucera 1968; Kurppa and Husman 1982; Moszczynski and Lisiewicz 1983, 1984a; Roberts et al. 1988; Smolik et al. 1973; Triebig et al. 1992a, 1992b; Uchida et al. 1993; Wilcosky et al. 1984) or animal studies (Tatrai et al. 1981; Maltoni et al. 1983, 1985; NTP 1986) were available regarding the health effects associated with chronic exposure to xylenes. The central nervous system (Gupta et al. 1990; Hipolito 1980; NTP 1986; Roberts et al. 1988) and the kidney (Askergren 1981, 1982; Askergren et al. 1981b, 1981c; Franchini et al. 1983) appear to be the primary targets of chronic xylene exposure. However, the study by Uchida et al. (1993) suggests that in healthy individuals, kidney effects are unlikely to occur at concentrations below those that cause neurological effects and eye and respiratory tract irritation. A chronic-duration inhalation MRL was derived based on the subjective neurological and respiratory effects noted in the Uchida et al. (1993) study. It is not clear if the effects noted in this study were a result of exposure at the TWA (14 ppm) or a result of short-term exposure at higher concentrations. Studies that focus on neurological effects with different exposure scenarios resulting in the same TWA may help to distinguish between effects caused by transient exposure to higher concentrations and those caused by stable lowlevel exposure. A chronic-duration oral MRL was calculated based on a NOAEL for decreased body weight at a dose that reduced survival in rats exposed to mixed xylenes for 2 years (NTP 1986). No specific target organ was identified in this study and there is uncertainty as to the NOAEL because there is no information on sensitive neurobehavioral effects. No chronic dermal studies of xylenes were identified. Since the inhalation and oral routes of exposure are the most important for individuals living near hazardous waste sites or in occupational settings, additional inhalation and oral studies are needed to provide critical, reliable guidance values for chronic exposure to xylenes.

Few epidemiological studies were available regarding the development of cancer in humans following inhalation, oral, or dermal exposure to mixed xylene or xylene isomers (Arp et al. 1983; Wilcosky et al. 
1984). Several oral carcinogenicity bioassays involving lifetime exposure have been conducted with mixed xylene in rats and mice (Maltoni et al. 1983, 1985; NTP 1986); however, all of these bioassays contained limitations that preclude a definitive conclusion regarding the carcinogenicity of xylene. Several dermal studies are available in which xylene (unspecified isomeric content) was evaluated for its ability to enhance tumor induction by tumor-initiating and tumor-promoting agents (Berenblum 1941; Pound 1970; Pound and Withers 1963); however, these studies are less than lifetime and have often involved exposures to more than one chemical agent. No animal cancer bioassays involving inhalation exposure to mixed xylene or isomers of xylene have been conducted. Because the issue of the potential carcinogenicity of xylenes has not been resolved, additional bioassays are needed. Chronic inhalation exposure to low levels is needed in these studies because chronic exposure by this route may be encountered in the workplace, home, or in the vicinity of hazardous waste sites.

Genotoxicity. Limited data are available regarding the genotoxicity of inhalation of xylenes in humans (Haglund et al. 1980; Pap and Varga 1987; Richer et al. 1993). No data are available regarding the potential genotoxicity of xylenes in humans following oral or dermal exposure. Animal studies examining the genotoxicity of inhalation (Zhong et al. 1980) or oral (Feldt 1986) exposure to xylenes have been almost uniformly negative. The only positive assay was for DNA fragmentation in skin of rats exposed dermally (Rogers et al. 2001); this effect appears to be related to cell death. Also, a variety of in vitro assays (Anderson et al. 1990; Bos et al. 1981; Connor et al. 1985; DeMarini et al. 1991; Epler et al. 1979; Florin et al. 1980; Gerner-Smidt and Friedrich 1978; Haworth et al. 1983; Hejtmankova et al. 1979; Litton Bionetics 1978b; McCarroll et al. 1981a, 1981b; NTP 1986; Pool and Lin 1982; Richer et al. 1993; Shimizu et al. 1985) produced negative results. Because of the large number of negative studies that exist, additional in vivo or in vitro assays of the genotoxicity potential of xylenes are not needed.

Reproductive Toxicity. One epidemiological study suggested that paternal exposure to xylenes in the workplace may increase the likelihood of abortions; however, this study was limited by the size of the sample population (Taskinen et al. 1989). Only one animal inhalation study has been conducted to test the potential reproductive toxicity of mixed xylene (Bio/dynamics 1983). No studies of reproductive function have been conducted on either mixed xylene or the individual xylene isomers in animals following exposure via oral or dermal routes. Histopathological examination of reproductive organs of rats and mice following intermediate (NTP 1986; Wolfe 1988a, 1988b) and chronic (NTP 1986) oral bioassays revealed no adverse effects; however, given the high potential for human exposure to xylene and its isomers and their ability to cross the placenta (Ghantous and Danielsson 1986; Ungvary et al. 1980b), additional studies in animals and epidemiological studies in humans are needed to assess more 
fully the reproductive toxicity of xylene and its isomers. A multi-generation reproductive toxicity study in animals is needed, possibly combined with a study examining developmental neurotoxicity end points.

Developmental Toxicity. Congenital defects of the central nervous system in children whose mothers were exposed occupationally to mixed xylene vapors were reported in two case studies (Holmberg and Nurminen 1980; Kucera 1968). However, the studies have many limitations, and no conclusion can be made. Animal inhalation, oral, and dermal studies have provided some information on the developmental effects of xylene and its isomers (Balogh et al. 1982; Bio/dynamics 1983; Hudak and Ungvary 1978; Litton Bionetics 1978a; Marks et al. 1982; Mirkova et al. 1979, 1983; Rosen et al. 1986; Seidenberg et al. 1986; Ungvary 1985; Ungvary and Tatrai 1985; Ungvary et al. 1980b, 1981); however, the quality of many of these studies precludes drawing conclusions. An inhalation developmental study in rats evaluated all three isomers and mixed xylenes using standard contemporary protocols, and found all tested xylenes could produce developmental toxicity (Saillenfait et al. 2003). Ingestion of aspirin by pregnant rats exposed to xylene have been shown to potentiate adverse maternal and fetal effects (Ungvary 1985). The most critical developmental studies in animals relate to neurobehavioral deficits observed in offspring following inhalation exposure of dams to xylenes, since these effects persist for months (Hass and Jakobsen 1993, 1995; Hass et al. 1997). No developmental neurobehavioral studies of xylenes have been conducted in animals exposed orally. Such studies are needed to better assess the potential of developmental toxicity and dose-response relationships associated with oral exposure to xylene. More information is needed on the mechanism of xylene-induced developmental toxicity, particularly of the developing nervous system, because this appears to be a sensitive target of xylene exposure. Such a study could be combined with a multi-generational reproductive toxicity study.

Immunotoxicity. Several occupational studies have been conducted to evaluate the immunological effects of xylene (Moszczynski and Lisiewicz 1983, 1984a; Smolik et al. 1973); however, workers in these studies were exposed to other chemical agents in addition to xylene. No animal studies involving exposure by any route have been conducted to examine directly the immunotoxicity of mixed xylene or the xylene isomers, although a decrease in thymus weight was observed in one oral study (Condie et al. 1988). Inhalation exposure studies in animals employing only xylene or its isomers may remove uncertainties about the immunotoxicity potential of xylene. One case report indicates that dermal sensitization to xylene is possible (Palmer and Rycroft 1993). Dermal sensitization tests would provide additional information on whether an allergic response to xylene is likely, since the potential for skin contact by humans occurs in occupational settings and in soil and water at hazardous waste sites. 
Neurotoxicity. Human and animal studies regarding neurologic effects have been conducted following inhalation, oral, and dermal exposures to xylene (Andersson et al. 1981; Carpenter et al. 1975a; Condie et al. 1988; Dyer et al. 1988; Ernstgard et al. 2002; Gagnaire and Langlais 2005; Gagnaire et al. 2001, 2006; Gamberale et al. 1978; Gralewicz and Wiaderna 2001; Gralewicz et al. 1995; Klaucke et al. 1982; Korsak et al. 1990, 1991, 1992, 1993, 1994; Morley et al. 1970; NIOSH 1981; NTP 1986; Nylen and Hagman 1994; Ogata et al. 1970; Savolainen et al. 1984, 1985a; Wolfe 1988a, 1988b) (see Sections 3.2.1.4 and 3.2.2.4 for additional data). Data from such studies indicate that xylene adversely affects the nervous system; all inhalation MRLs and the acute oral MRL are based, at least in part, on neurological effects of xylene. The majority of studies in humans and animals concentrated on the neurobehavioral effects of xylene. Further studies attempting to elucidate the mechanism of action of xylenes on the nervous system are needed to increase understanding of the neurotoxic effects produced by high concentrations of xylenes. An occupational study of workers exposed to low concentrations of mixed solvents including xylenes for 10-44 years found no significant effects on CAT-scan measures of brain atrophy (Triebig et al. 1992a), but specific information on xylene is not available. Additional wellconducted studies in animals on the histopathologic changes of the central nervous system following intermediate or chronic exposure are needed to provide useful information on permanent structural alterations induced by xylene. More epidemiological and/or animal data are needed to better assess sensitive neurological end points (subjective effects, behavior, performance) for intermediate and chronic exposures by both inhalation and oral routes. The priorities in descending order of need are chronic oral $>$ intermediate oral $=$ chronic inhalation $>$ intermediate inhalation .

Epidemiological and Human Dosimetry Studies. Limited epidemiological studies (Arp et al. 1983; Askergren 1981, 1982; Askergren et al. 1981b, 1981c; Dolara et al. 1982; Franchini et al. 1983; Gupta et al. 1990; Holmberg and Nurminen 1980; Kilburn et al. 1985; Kucera 1968; Kurppa and Husman 1982; Moszczynski and Lisiewicz 1983, 1984a; Smolik et al. 1973; Taskinen et al. 1989; Uchida et al. 1993; Wilcosky et al. 1984) and no human dosimetry studies on any of the xylenes have been conducted. Much of the available information on the effects of xylene in humans comes from case reports (Abu Al Ragheb et al. 1986; Arthur and Curnock 1982; Brasington and Thorpe-Swenson 1991; Goldie 1960; Hipolito 1980; Klaucke et al. 1982; Martinez et al. 1989; Morley et al. 1970; Nersesian et al. 1985; Roberts et al. 1988) and occupational studies in which subjects were exposed to other chemical agents in addition to xylene (Arp et al. 1983; Askergren 1981, 1982; Askergren et al. 1981b, 1981c; Dolara et al. 1982; Franchini et al. 1983; Gupta et al. 1990; Holmberg and Nurminen 1980; Jovanovic et al. 2004; Kilburn et al. 1985; Kucera 1968; Kurppa and Husman 1982; Moszczynski and Lisiewicz 1983, 1984a; Smolik et al. 1973; Taskinen et al. 1989; Uchida et al. 1993; Wilcosky et al. 1984). The best 
characterization of exposure, verified with urinary metabolite measurements, was conducted in the occupational study by Uchida et al. (1993); subjects were exposed to 70\% xylene with little involvement of other chemicals, besides ethylbenzene, a component of mixed xylene. Many of the case reports and occupational studies were also limited because exposure conditions were not well characterized.

Additional well-designed and well-controlled epidemiological studies of people living near waste sites or industries using xylene, or occupational studies in which xylene exposure conditions are better characterized, may be useful to better describe potential neurotoxic effects and their dose-response relationships. Epidemiological studies examining the nervous system, reproductive outcome, and renal effects associated with xylene exposure would be particularly useful since these have been shown to be sensitive end points.

Human Sensory Studies on Oral Exposure. A sensory study is needed using human subjects on the detection of taste and smell thresholds for drinking water containing mixed xylenes. This data need is prompted by the observation that the guidance values for xylene $(0.6-1 \mathrm{mg} / \mathrm{kg} /$ day $)$ translated to typical daily water consumption ( 2 L/day) are only slightly below the maximum solubility of xylene in water $(\sim 145 \mathrm{mg} / \mathrm{L})$. Information on the detectability of xylene at maximum solubility would be a useful rule of thumb for public health communications. This information might allow individuals to rule out water contamination at levels of concern in many situations.

\section{Biomarkers of Exposure and Effect.}

Exposure. Methods are available for determining xylene and its metabolite, methylhippuric acid, in biological tissues and fluids (Daniell et al. 1992; Jonai and Sato 1988; Kawai et al. 1991; Sedivec and Flek 1976b). These biomarkers of exposure are specific for xylene exposure and are sufficient for determining recent exposure to xylenes but are incapable of distinguishing short-term from intermediateand chronic-duration exposures. It would be useful to determine if a biomarker of longer-term exposure could be derived, although it is not known whether one could be found.

Effect. No specific biomarkers of effects have been identified for xylenes. Xylenes have been demonstrated to cause a number of adverse health effects including central nervous system depression (Gamberale et al. 1978; Riihimaki and Savolainen 1980; Savolainen and Linnavuo 1979; Savolainen and Riihimaki 1981b; Savolainen et al. 1979b, 1984, 1985a). A number of neurological and cognitive function tests exist and have been used to identify central nervous system changes produced by xylenes. However, until the mechanism for nervous system disruption is identified, it is unlikely that a specific test 
could predict xylene-specific intoxication. Similarly, xylene treatment of the skin elicits increases in proinflammatory cytokines in blood (interleukin 1-alpha) and skin (tumor necrosis factor-alpha) (Ahaghotu et al. 2005; Chatterjee et al. 2005; Gunasekar et al. 2003), but these responses are not specific to xylene exposure. Assessment of hepatic enzyme induction is difficult without obtaining liver tissue. Demonstration of enhanced metabolism of substances by the microsomal enzyme system could be interpreted as microsomal induction; however, a large number of substances other than xylenes also induce enhanced enzyme activity. Renal impairment also has been associated with high levels of xylene exposure. Increased excretion of albumin, leukocytes, and erythrocytes demonstrates kidney damage of the type ascribed to xylene exposure, but these effects are not specific for xylenes. However, limited data are available associating levels of xylene in human tissues and fluids with adverse health effects. Available human studies have focused on the blood concentrations of $m$-xylene associated with central nervous system effects. Additional animal studies evaluating the association between xylene (or xylene metabolite) levels in other human tissues or fluids and adverse health effects are needed.

Absorption, Distribution, Metabolism, and Excretion. The absorption, metabolism, and excretion of xylenes following inhalation, oral, and dermal exposures in humans and/or animals have been well characterized (Astrand 1982; Engstrom et al. 1977; Inoue et al. 1993; Jonai and Sato 1988; Kawai et al. 1991; Kezic et al. 2001; Ogata et al. 1970, 1979; Riihimaki 1979b; Riihimaki and Pfaffli 1978; Riihimaki et al. 1979a, 1979b; Skowronski et al. 1990). The distribution of xylene has been well characterized in animals and identified to a small extent in humans. The database for absorption, distribution, and excretion of xylene isomers in humans and/or animals after inhalation exposure is most extensive. The database for oral and dermal exposures is not as extensive but has been well described. Differences in the rate of metabolism of xylenes after short-term or chronic exposure have been identified. Differences in the toxicokinetics of xylene seen when exposure occurs with xylene adsorbed to sandy or clay soil have also been examined. Dermal penetration and resulting doses of xylene could be better characterized.

Although a number of route-specific PBPK models are available, a model is needed that would enable route-to-route extrapolation from intermediate- or chronic-duration inhalation data for oral exposure. Such a model would help address uncertainties in existing oral exposure data resulting from experimental bolus delivery to animals. Since environmental oral exposures involve consumption of contaminated drinking water during the course of a day, toxicity values based on bolus dosing would tend to overestimate risk. 
Comparative Toxicokinetics. The target organs and adverse health effects of xylenes are similar across species. Toxicokinetic studies have been performed in humans, rats, mice, rabbits, and monkeys (Adams et al. 2005; Astrand et al. 1978; Bakke and Scheline 1970; Bray et al. 1949; Ogata et al. 1979; Patel et al. 1978; Smith et al. 1982; Sugihara and Ogata 1978; van Doorn et al. 1980). There is reasonable correlation between the end points examined in these studies. The metabolism of $m$ - and $p$-xylenes is similar in rats and humans. However, a difference in the metabolism of $o$-xylene in rats and in humans exists. Whereas $o$-xylene is almost exclusively metabolized to $o$-methylhippuric acid in humans, $10-56 \%$ of $o$-xylene is also conjugated by glucuronide and glutathione in rats. Toxic metabolic intermediates of xylene such as benzaldehyde found in rats have not been found in humans. Additional studies are needed to determine whether other differences exist in the metabolism of xylenes among species. Although Inoue et al. (1993) did not observe a sex-related difference in excretion in men and women occupationally exposed to xylenes, sex-related differences in the toxicokinetics of xylene have been identified in animals. Additional studies concerning sex/genetic factors controlling xylene metabolism in humans might by useful.

Methods for Reducing Toxic Effects. Current methods used for reducing toxic effects of xylenes after acute exposures concentrate on decreasing absorption (HSDB 2007). Additional research on speeding excretion of xylene and reducing its concentration at its target organs are needed. As future research identifies the mechanisms underlying the toxic effects of xylenes, additional methods may be developed for combating the effects of xylene at the molecular level.

Children's Susceptibility. Data needs relating to both prenatal and childhood exposures, and developmental effects expressed either prenatally or during childhood, are discussed in detail in the Developmental Toxicity subsection above. There are no studies examining health effects in children exposed to xylenes, but the effects are likely to be the same as those observed in exposed adults. As mentioned in the Developmental Toxicity and Neurotoxicity subsections, there is a need for more data on neurological (neurobehavioral) effects of xylenes in laboratory animals. Studies in rats indicated that inhalation exposure to xylenes during gestation can affect neurobehavioral performance of offspring (Hass and Jakobson 1995; Hass et al. 1997). Studies in laboratory animals exposed by the oral route could identify dose-response relationships for neurological effects that would help in the assessment of health risks for children exposed to drinking water containing xylene.

There is currently insufficient age-specific information relevant to the absorption, distribution, metabolism, and elimination of xylenes in children. Although the absorption and distribution of xylenes 
are driven by their lipophilic properties and diffusion, and the main metabolizing enzyme (CYP2E1) is available at adult levels in children by year 1 (Vieira et al. 1996), not enough information is available for children $<1$ year old. No data are available relative to the possible contribution of other microsomal enzymes to xylene metabolism in young animals. The elimination of xylene and its metabolites from the body is relatively rapid and expected to be similar in children and adults. However, the balance between factors tending to facilitate xylene clearance in children (lower body fat content, larger liver-to-body weight ratio) and factors tending to slow clearance (immaturity of enzyme systems) is not well understood. Data are especially needed to compare pulmonary metabolism of xylenes to hepatic metabolism, since the contribution of pulmonary metabolism may be significant for inhalation exposures (Adams et al. 2005). These suggestions are consistent with the recommendations of the peer consultation meeting of the Voluntary Children's Chemical Evaluation Program (VCCEP 2006). PBPK modeling studies indicate that xylenes can be excreted into breast milk (Fisher et al. 1997), but no PBPK models have been developed for children, fetuses, or pregnant women exposed to xylenes. Placental transfer of xylenes has been demonstrated in mice, but uptake by fetal tissues was lower than in maternal tissues (Ghantous and Danielsson 1986). Elimination of xylene and its metabolites is so rapid that there does not seem to be a need for pediatric-specific methods to reduce peak absorption.

Child health data needs relating to exposure are discussed in Section 6.8.1, Identification of Data Needs: Exposures of Children.

\subsubsection{Ongoing Studies}

The Federal Research in Progress database (FEDRIP 2006) listed research by Dr. Karla Thrall on the assessment of human exposure to volatile organic chemicals, including xylene, at Superfund sites. The study, which is supported by the National Institute of Environmental Health Sciences, is intended to extend existing PBPK models to describe uptake kinetics and brain dosimetry following exposure. The results of this research program are intended to improve the extrapolation from animal studies to relevant human exposure situations and to develop biomarkers of susceptibility and response in potentially exposed populations. Dr. Steven Dewey is developing methods for quantifying exposure of fetal macaques after administration of positron-labeled inhalants such as ${ }^{\mathrm{C} 11}$ toluene to the macaque mother. This research, sponsored by the National Institute on Drug Abuse, could be useful in the future for evaluating fetal and postnatal behavioral deficits resulting from maternal exposure to such inhalants. 


\section{CHEMICAL AND PHYSICAL INFORMATION}

\subsection{CHEMICAL IDENTITY}

Information regarding the chemical identity of $m-, o-$, and $p$-xylene and mixed xylene is located in

Table 4-1. Commercial or mixed xylene generally contains about $40-65 \% \mathrm{~m}$-xylene and up to $20 \%$ each of o-xylene, $p$-xylene, and ethylbenzene (Fishbein 1985).

\subsection{PHYSICAL AND CHEMICAL PROPERTIES}

Information regarding the physical and chemical properties of $m-, o-$, and $p$-xylene and mixed xylene is located in Table 4-2. 


\section{Table 4-1. Chemical Identity of $m$-Xylene, $o$-Xylene, $p$-Xylene, and Mixed Xylene ${ }^{a}$}

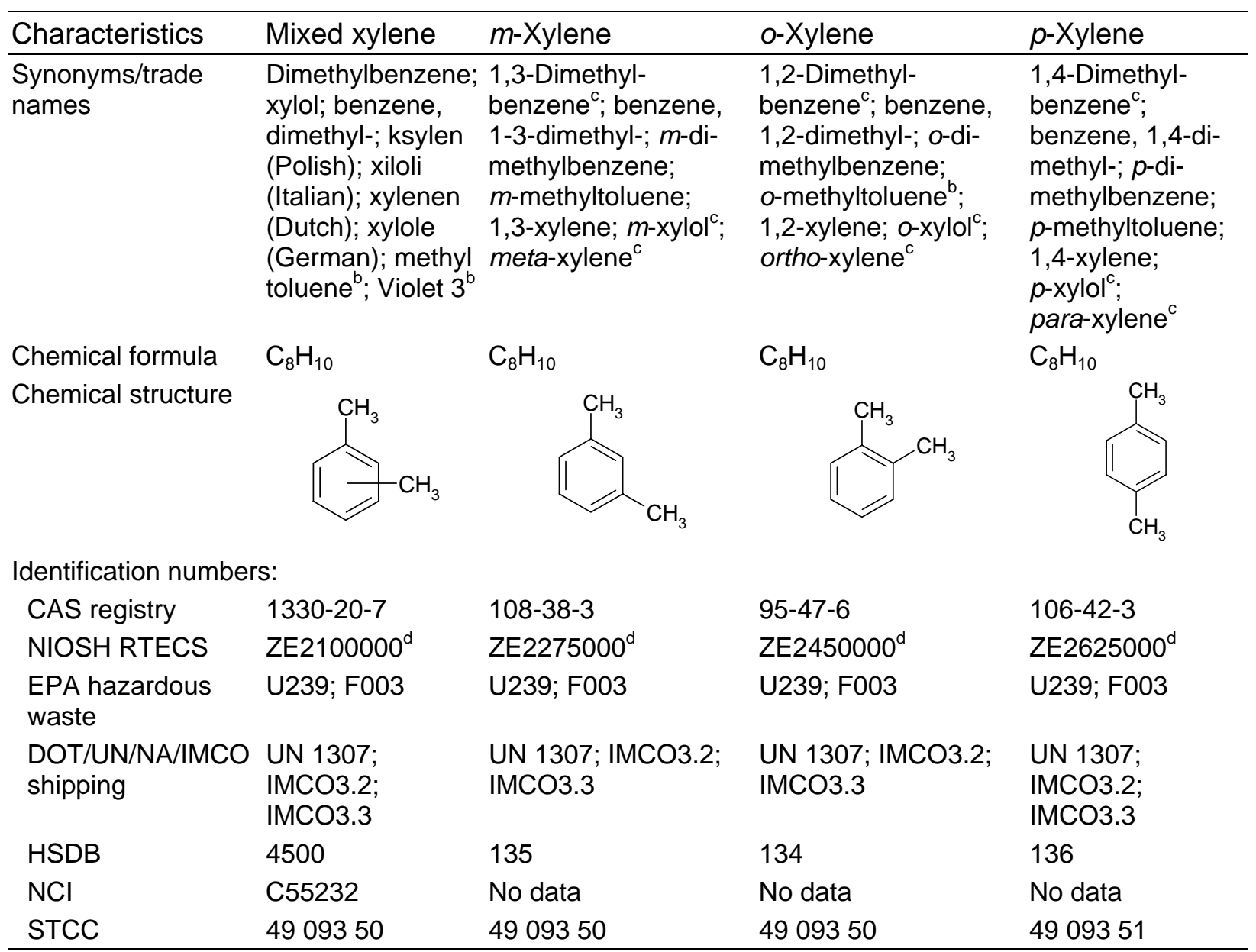

${ }^{a}$ All information obtained from HSDB 2007 except where noted.

${ }^{\mathrm{b}}$ Lewis 2000

${ }^{\mathrm{C}} \mathrm{NIOSH} 2005$

${ }^{\mathrm{d}}$ RTECS 2007

CAS = Chemical Abstracts Services; DOT/UN/NA/IMCO = Department of Transportation/United Nations/North America/Intergovernmental Maritime Dangerous Goods Code; EPA = Environmental Protection Agency; HSDB = Hazardous Substances Data Bank; NCI = National Cancer Institute; NIOSH = National Institute for Occupational Safety and Health; RTECS = Registry of Toxic Effects of Chemical Substances; STCC = Standard Transport Commodity Code 
Table 4-2. Physical and Chemical Properties of $m$-Xylene, $o$-Xylene, $p$-Xylene, and Mixed Xylene ${ }^{\mathrm{a}}$

\begin{tabular}{|c|c|c|c|c|}
\hline Property & Mixed xylene & $m$-Xylene & o-Xylene & $p$-Xylene \\
\hline Molecular weight & $106.16^{\mathrm{b}}$ & $106.16^{\mathrm{b}}$ & $106.16^{\mathrm{b}}$ & $106.16^{\mathrm{b}}$ \\
\hline Color & Clear $^{c}$ & Colorless $^{c}$ & Colorless $^{c}$ & Colorless $^{c}$ \\
\hline Physical state & Liquid $^{c}$ & Liquid $^{c}$ & Liquid $^{c}$ & Liquid $^{c}$ \\
\hline Melting point & No data & $-47.8{ }^{\circ} C^{d}$ & $-25.2{ }^{\circ} C^{d}$ & $13.2^{\circ} \mathrm{C}^{\mathrm{d}}$ \\
\hline Boiling point & $137-140^{\circ} \mathrm{C}^{\mathrm{b}}$ & $139.1{ }^{\circ} \mathrm{C}^{d}$ & $144.5^{\circ} \mathrm{C}^{\mathrm{d}}$ & $138.4^{\circ} \mathrm{C}^{\mathrm{d}}$ \\
\hline Density at $20^{\circ} \mathrm{C} / 4^{\circ} \mathrm{C}$ & $0.864 \mathrm{~g} / \mathrm{cm}^{3 \mathrm{e}}$ & $0.864 \mathrm{~g} / \mathrm{cm}^{3 \mathrm{e}}$ & $0.880 \mathrm{~g} / \mathrm{cm}^{3 e}$ & $0.8611 \mathrm{~g} / \mathrm{cm}^{3 \mathrm{e}}$ \\
\hline Odor & Sweet & Sweet & Sweet & Sweet \\
\hline \multicolumn{5}{|l|}{ Odor threshold: } \\
\hline Water & No data & No data & No data & No data \\
\hline Air & $\begin{array}{l}0.0045 \mathrm{mg} / \mathrm{L} \\
(1.0 \mathrm{ppm})^{\mathrm{g}}\end{array}$ & $0.05 \mathrm{ppm}^{f}$ & $0.05 \mathrm{ppm}^{f}$ & $0.05 \mathrm{ppm}^{f}$ \\
\hline \multicolumn{5}{|l|}{ Solubility: } \\
\hline Water at $25^{\circ} \mathrm{C}$ & $106 \mathrm{mg} / \mathrm{L}^{\mathrm{i}}$ & $161 \mathrm{mg} / \mathrm{L}^{\mathrm{j}}$ & $178 \mathrm{mg} / \mathrm{L}^{\mathrm{j}}$ & $162 \mathrm{mg} / \mathrm{L}^{\mathrm{j}}$ \\
\hline Organic solvent(s) & $\begin{array}{l}\text { Miscible with alcohol } \\
\text { and ether }\end{array}$ & $\begin{array}{l}\text { Miscible with } \\
\text { alcohol, ether, and } \\
\text { other solvents }^{\mathrm{b}}\end{array}$ & $\begin{array}{l}\text { Miscible with } \\
\text { alcohol and } \\
\text { ether }^{b}\end{array}$ & $\begin{array}{l}\text { Soluble in alcohol, } \\
\text { ether, and other } \\
\text { organic solvents }^{b}\end{array}$ \\
\hline \multicolumn{5}{|l|}{ Partition coefficients: } \\
\hline $\log K_{o w}$ & No data & $3.2^{k}$ & $3.12^{k}$ & $3.15^{k}$ \\
\hline $\log K_{o c}$ & No data & $2.22^{1}$ & $2.11^{\prime}$ & $2.31^{\prime}$ \\
\hline Vapor pressure & $6.72 \mathrm{mmHg}$ at $21^{\circ} \mathrm{C}^{\mathrm{e}}$ & $\begin{array}{l}8.29 \mathrm{mmHg} \text { at } \\
25^{\circ} \mathrm{C}^{\mathrm{m}}\end{array}$ & $\begin{array}{l}6.61 \mathrm{mmHg} \text { at } \\
25^{\circ} \mathrm{C}^{\mathrm{n}}\end{array}$ & $8.84 \mathrm{~mm} \mathrm{Hg}$ at $25^{\circ} \mathrm{C}^{\mathrm{m}}$ \\
\hline Henry's law constant & No data & $\begin{array}{l}7.18 \times 10^{-3} \mathrm{~atm}- \\
\mathrm{m}^{3} / \mathrm{mol}^{j}\end{array}$ & $\begin{array}{l}5.18 \times 10^{-3} \mathrm{~atm}- \\
\mathrm{m}^{3} / \mathrm{mol}^{j}\end{array}$ & $6.90 \times 10^{-3} \mathrm{~atm}-\mathrm{m}^{3} / \mathrm{mol}^{0}$ \\
\hline $\begin{array}{l}\text { Autoignition } \\
\text { temperature }\end{array}$ & $464^{\circ} \mathrm{C}\left(867^{\circ} \mathrm{F}\right)$ & $527^{\circ} \mathrm{C}$ & $463^{\circ} \mathrm{C}$ & $528^{\circ} \mathrm{C}$ \\
\hline Flashpoint & $29^{\circ} \mathrm{C}(\mathrm{CC})^{\mathrm{b}}$ & $27^{\circ} \mathrm{C}(\mathrm{CC})^{\mathrm{b}}$ & $32{ }^{\circ} \mathrm{C}(\mathrm{CC})^{\mathrm{b}}$ & $27^{\circ} \mathrm{C}(\mathrm{CC})^{\mathrm{b}}$ \\
\hline Flammability limits & No data & $1.1-7.0 \%$ & $1.0-7.0 \%$ & $1.1-7.0 \%$ \\
\hline $\begin{array}{l}\text { NFPA Flammability } \\
\text { Classification }\end{array}$ & $3^{p}$ & $3^{p}$ & $3^{p}$ & $3^{p}$ \\
\hline $\begin{array}{l}\text { NFPA Reactivity } \\
\text { Classification }\end{array}$ & $0^{9}$ & $0^{9}$ & $0^{9}$ & $0^{9}$ \\
\hline
\end{tabular}


Table 4-2. Physical and Chemical Properties of $m$-Xylene, $o$-Xylene, $p$-Xylene, and Mixed Xylene ${ }^{\mathrm{a}}$

\begin{tabular}{|c|c|c|c|c|}
\hline Property & Mixed xylene & $m$-Xylene & o-Xylene & $p$-Xylene \\
\hline Air conversion factors ${ }^{r}$ & $1 \mathrm{ppm}=4.34 \mathrm{mg} / \mathrm{m}^{3 /}$ & $\begin{array}{l}1 \mathrm{ppm}= \\
4.34 \mathrm{mg} / \mathrm{m}^{3 \mathrm{~s}} \\
1 \mathrm{ppm} \mathrm{=} \\
4.41 \mathrm{mg} / \mathrm{m}^{3 \mathrm{t}} \\
1 \mathrm{mg} / \mathrm{m}^{3}= \\
0.23 \mathrm{ppm}\end{array}$ & $\begin{array}{l}1 \mathrm{ppm}= \\
4.34 \mathrm{mg} / \mathrm{m}^{31} \\
1 \mathrm{ppm}= \\
4.41 \mathrm{mg} / \mathrm{m}^{3 \mathrm{t}} \\
1 \mathrm{mg} / \mathrm{m}^{3}= \\
0.23 \mathrm{ppm}\end{array}$ & $\begin{array}{l}1 \mathrm{ppm}=4.34 \mathrm{mg} / \mathrm{m}^{3 !} ; \\
1 \mathrm{ppm}=4.41 \mathrm{mg} / \mathrm{m}^{3 \mathrm{t}} \\
1 \mathrm{mg} / \mathrm{m}^{3}=0.23 \mathrm{ppm}\end{array}$ \\
\hline
\end{tabular}

${ }^{a}$ All information obtained from HSDB 2007 unless otherwise noted.

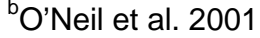

'Lewis 1997

dide 2005

e Lewis 2001

${ }^{\mathrm{f}}$ CHRIS 1999

${ }^{9}$ Carpenter et al. 1975 a

${ }^{\mathrm{h}}$ Gerarde 1959

'Yalkowsky and He 1992

'Sanemasa et al. 1982

kHansch et al. 1995

'Abdul et al. 1987

${ }^{\mathrm{m}}$ Chao et al. 1983

${ }^{\mathrm{n}} \mathrm{AIChE} 1996$

${ }^{\circ}$ Foster et al. 1994

${ }^{\mathrm{P}}$ Classified by the NFPA as a liquid that can be ignited under almost all normal temperature conditions.

${ }^{\mathrm{q}}$ Classified by the NFPA as a liquid that is normally stable even under fire exposure conditions and that is not reactive with water.

${ }^{r}$ In air, ppb is a volume-to-volume ratio for vapor-phase compounds like xylenes. When air monitoring data are reported in terms of ppb or ppm, it is understood that this is equivalent to ppbv or ppmv (parts per billion or parts per million volume-to-volume ratio).

sSandmeyer 1981

tVerschueren 2001

$\mathrm{CC}=$ closed cup $;$ NFPA $=$ National Fire Protection Association $; \mathrm{TOC}=$ tag open cup 


\section{PRODUCTION, IMPORT/EXPORT, USE, AND DISPOSAL}

\subsection{PRODUCTION}

U.S. manufacturers had an estimated annual production capacity of 18 billion pounds of mixed xylene in 2006 (SRI 2006). This figure is based on maximum plant production volumes. Table 5-1 lists producers of mixed xylene and their estimated annual capacities. In 1990 and 1991, U.S. production of xylene totaled 6.2 and 6.1 billion pounds, respectively (Reisch 1992). These figures represent the total amount of mixed xylene actually produced by U.S. manufacturers based on data from trade associations and industry sources. The production of mixed xylene during the year 1995 was reported to be 9.5 billion pounds (Cannella 1998). According to data collected under the Toxic Substances Control Act Inventory Update Rule, the total production volume of mixed xylene reported by U.S. manufacturers has remained above 1 billion pounds during each reporting year (1986, 1990, 1994, 1998, and 2002) (EPA 2002a).

Table 5-2 summarizes the number of facilities in each state that manufactured or processed mixed xylene in 2004, the ranges of maximum amounts on site, if reported, and the activities and uses as reported in the Toxics Release Inventory (TRI) (TRI04 2006). The data listed in this table should be used with caution since only certain types of facilities are required to report. This is not an exhaustive list.

The 2006 estimated U.S. annual production capacities of $o$ - and $p$-xylene were 1 and 11 billion pounds, respectively (SRI 2006). These figures are based on maximum plant production volumes. Table 5-1 lists producers of $o$ - and $p$-xylene and their estimated annual capacities. Production capacity data for $m$-xylene were not reported.

According to estimates, over 943 million and 5.2 billion pounds of $o$ - and $p$-xylene, respectively, were produced in the United States in 1990 (Reisch 1992; USITC 1991). The production volumes of $o$-xylene reported by United States manufacturers during 1986, 1990, 1994, 1998, and 2002 were within the ranges of $>500$ million pounds -1 billion pounds, $>1$ billion pounds, $>500$ million pounds -1 billion pounds, $>1$ billion pounds, and $>500$ million pounds-1 billion pounds, respectively (EPA 2002a). The ranges of production volumes reported during these years have remained unchanged for both $m$-xylene $(>100-$ 500 million pounds) and $p$-xylene ( $>1$ billion pounds).

It is not clear whether there is any relationship between the amounts of xylene isomers produced at an individual facility and the total amount of mixed xylenes produced at the same facility. Sources reporting production and capacity data do not specify whether companies that report amounts of both mixed 
Table 5-1. Producers of Xylene and Estimated Annual Capacities ${ }^{a}$

\begin{tabular}{|c|c|c|c|c|c|}
\hline \multirow[b]{2}{*}{ Producers } & \multirow[b]{2}{*}{ Location(s) } & \multicolumn{4}{|c|}{ Annual capacity (millions of pounds) } \\
\hline & & Mixed xylene & $m$-Xylene & o-Xylene & $p$-Xylene \\
\hline \multirow[t]{3}{*}{ BP America, Inc. } & Texas City, Texas & 2,215 & NR & - & 2,693 \\
\hline & Whiting, Indiana & 1,735 & - & - & - \\
\hline & Decatur, Alabama & - & - & - & 2,420 \\
\hline $\begin{array}{l}\text { Chevron Phillips } \\
\text { Chemical Company LP } \\
\text { Olefins and Polyolefins } \\
\text { Business Unit }\end{array}$ & Pascagoula, Mississippi & 1,025 & - & - & 1,001 \\
\hline $\begin{array}{l}\text { Chevron Phillips } \\
\text { Chemical Puerto Rico } \\
\text { Core Inc. }\end{array}$ & Guayama, Puerto Rico & 639 & - & 150 & 727 \\
\hline $\begin{array}{l}\text { CITGO Petroleum } \\
\text { Corporation }\end{array}$ & Corpus Christi, Texas & 538 & - & - & - \\
\hline \multirow{2}{*}{$\begin{array}{l}\text { ConocoPhillips } \\
\text { Refining, Marketing \& } \\
\text { Transportation Division }\end{array}$} & Alliance, Louisiana & 432 & - & - & - \\
\hline & Sweeny, Texas & 1,049 & - & - & - \\
\hline \multirow{3}{*}{$\begin{array}{l}\text { ExxonMobil Chemical } \\
\text { Company }\end{array}$} & Baytown, Texas & 1,710 & - & 280 & 1,316 \\
\hline & Beaumont, Texas & 705 & - & - & 606 \\
\hline & Chalmette, Louisiana & 505 & - & 150 & 419 \\
\hline \multirow{2}{*}{$\begin{array}{l}\text { Flint Hills Resources } \\
\text { L.P. }\end{array}$} & Corpus Christi, Texas & 397 & NR & 397 & 1,322 \\
\hline & Corpus Christi, Texas & 1,677 & - & - & - \\
\hline HOVENSA, L.L.C. & St. Croix, Virgin Islands & 833 & - & - & - \\
\hline $\begin{array}{l}\text { Lyondell-Citgo Refining, } \\
\text { LP }\end{array}$ & Houston, Texas & 765 & - & - & 399 \\
\hline \multirow{2}{*}{$\begin{array}{l}\text { Marathon Petroleum } \\
\text { LLC }\end{array}$} & Catlettsburg, Kentucky & 183 & - & - & - \\
\hline & Texas City, Texas & 73 & - & - & - \\
\hline $\begin{array}{l}\text { Shell Chemical } \\
\text { Company }\end{array}$ & Deer Park, Texas & 355 & - & - & - \\
\hline \multirow[t]{3}{*}{ Sunoco, Inc. } & Marcus Hook, Pennsylvania & 187 & - & - & - \\
\hline & Toledo, Ohio & 377 & - & - & - \\
\hline & Westville, New Jersey & 441 & - & - & \\
\hline $\begin{array}{l}\text { Total Petrochemicals } \\
\text { USA, Inc. }\end{array}$ & Port Arthur, Texas & 855 & - & - & - \\
\hline \multirow{3}{*}{$\begin{array}{l}\text { Valero Energy } \\
\text { Corporation }\end{array}$} & Corpus Christi, Texas & 500 & - & - & - \\
\hline & Corpus Christi, Texas & 652 & - & - & - \\
\hline & Three Rivers, Texas & 361 & - & - & - \\
\hline Totals & & 18,209 & NR & 976 & 10,903 \\
\hline
\end{tabular}

${ }^{a}$ Derived from SRI 2006

$-=$ not produced; $N R=$ the $x y l e n e$ was produced, but the amount was not reported 


\section{Table 5-2. Facilities that Produce, Process, or Use Xylene (Mixed)}

\begin{tabular}{|c|c|c|c|c|}
\hline State $^{a}$ & $\begin{array}{l}\text { Number of } \\
\text { facilities }\end{array}$ & $\begin{array}{l}\text { Minimum } \\
\text { amount on site } \\
\text { in pounds }\end{array}$ & $\begin{array}{l}\text { Maximum } \\
\text { amount on site } \\
\text { in pounds }{ }^{b}\end{array}$ & 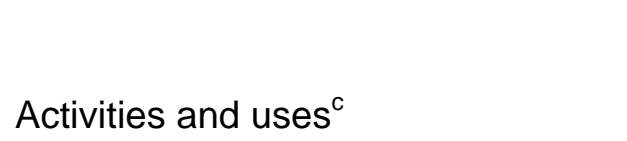 \\
\hline$\overline{A K}$ & 26 & 10,000 & $499,999,999$ & $1,2,3,4,5,7,8,9,10,11,12$ \\
\hline AL & 181 & 0 & $499,999,999$ & $1,2,3,4,5,6,7,8,9,10,11,12,13,14$ \\
\hline AR & 97 & 0 & 9,999,999 & $1,2,3,4,5,6,7,8,9,10,11,12,13$ \\
\hline AS & 2 & 1,000 & $9,999,999$ & 9,11 \\
\hline$A Z$ & 64 & 0 & $9,999,999$ & $1,2,3,4,5,7,8,9,10,11,12,13$ \\
\hline CA & 307 & 0 & $999,999,999$ & $1,2,3,4,5,6,7,8,9,10,11,12,13,14$ \\
\hline $\mathrm{CO}$ & 65 & 100 & 49,999,999 & $1,2,3,4,5,6,7,8,9,10,11,12,13$ \\
\hline CT & 73 & 0 & $49,999,999$ & $1,2,3,4,6,7,8,9,10,11,12,13$ \\
\hline DE & 29 & 0 & $99,999,999$ & $1,2,3,5,6,7,8,9,10,11,12,13$ \\
\hline $\mathrm{FL}$ & 99 & 0 & $999,999,999$ & $1,2,3,4,5,6,7,8,9,10,11,12$ \\
\hline GA & 140 & 0 & $499,999,999$ & $1,2,3,4,5,6,7,8,9,10,11,12,13,14$ \\
\hline GU & 4 & $1,000,000$ & $9,999,999$ & $2,3,4,7,9,12,13$ \\
\hline $\mathrm{HI}$ & 20 & 100 & $49,999,999$ & $1,2,3,4,5,6,7,8,9,10,12,13,14$ \\
\hline IA & 121 & 0 & $49,999,999$ & $1,2,3,4,5,6,7,8,9,10,11,12,13,14$ \\
\hline ID & 21 & 100 & 9,999,999 & $1,2,3,4,5,7,8,9,10,11,12$ \\
\hline IL & 255 & 0 & $10,000,000,000$ & $1,2,3,4,5,6,7,8,9,10,11,12,13,14$ \\
\hline IN & 234 & 0 & $499,999,999$ & $1,2,3,4,5,6,7,8,9,10,11,12,13,14$ \\
\hline KS & 158 & 0 & $999,999,999$ & $1,2,3,4,5,6,7,8,9,10,11,12,13,14$ \\
\hline KY & 135 & 0 & $499,999,999$ & $1,2,3,4,5,6,7,8,9,10,11,12,13,14$ \\
\hline LA & 253 & 0 & $999,999,999$ & $1,2,3,4,5,6,7,8,9,10,11,12,13,14$ \\
\hline MA & 95 & 0 & 49,999,999 & $2,3,4,5,6,7,8,9,10,11,12,13$ \\
\hline MD & 69 & 0 & $49,999,999$ & $1,2,3,4,5,6,7,8,9,10,11,12,13$ \\
\hline ME & 30 & 100 & $49,999,999$ & $2,3,4,6,7,8,9,10,11,12$ \\
\hline MI & 289 & 0 & $499,999,999$ & $1,2,3,4,5,6,7,8,9,10,11,12,13,14$ \\
\hline MN & 117 & 0 & 99,999,999 & $1,2,3,4,5,6,7,8,9,10,11,12,13,14$ \\
\hline MO & 147 & 0 & $499,999,999$ & $1,2,3,4,5,6,7,8,9,10,11,12$ \\
\hline MP & 6 & 100 & 999,999 & $2,3,4,7,9$ \\
\hline MS & 135 & 0 & $49,999,999$ & $1,2,3,4,5,6,7,8,9,10,11,12,13,14$ \\
\hline MT & 32 & 100 & $499,999,999$ & $1,2,3,4,5,6,7,8,9,10,11,12,13,14$ \\
\hline NC & 140 & 0 & $499,999,999$ & $1,2,3,4,5,6,7,8,9,10,11,12,13,14$ \\
\hline ND & 26 & 100 & $49,999,999$ & $1,2,3,4,6,7,8,9,10,11,12$ \\
\hline NE & 67 & 0 & 9,999,999 & $1,2,3,4,5,6,7,8,9,10,11,12,13$ \\
\hline $\mathrm{NH}$ & 41 & 0 & 999,999 & $2,3,6,7,8,9,10,11,12,13$ \\
\hline NJ & 199 & 0 & $499,999,999$ & $1,2,3,4,5,6,7,8,9,10,11,12,13,14$ \\
\hline NM & 35 & 100 & 9,999,999 & $1,2,3,4,5,6,7,8,9,10,11,12,13$ \\
\hline NV & 27 & 100 & $9,999,999$ & $1,2,3,4,5,6,7,8,9,10,11,12$ \\
\hline NY & 161 & 0 & $499,999,999$ & $1,2,3,4,5,6,7,8,9,10,11,12,13$ \\
\hline $\mathrm{OH}$ & 300 & 0 & $499,999,999$ & $1,2,3,4,5,6,7,8,9,10,11,12,13,14$ \\
\hline
\end{tabular}




\section{Table 5-2. Facilities that Produce, Process, or Use Xylene (Mixed)}

\begin{tabular}{|c|c|c|c|c|}
\hline State $^{a}$ & $\begin{array}{l}\text { Number of } \\
\text { facilities }\end{array}$ & $\begin{array}{l}\text { Minimum } \\
\text { amount on site } \\
\text { in pounds }^{b}\end{array}$ & $\begin{array}{l}\text { Maximum } \\
\text { amount on site } \\
\text { in pounds }{ }^{b}\end{array}$ & 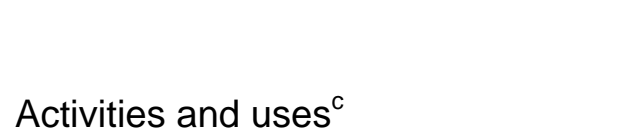 \\
\hline $\mathrm{OK}$ & 107 & 0 & $499,999,999$ & $1,2,3,4,5,6,7,8,9,10,11,12,13,14$ \\
\hline OR & 73 & 0 & 49,999,999 & $1,2,3,4,5,7,8,9,10,11,12,13$ \\
\hline PA & 224 & 0 & $499,999,999$ & $1,2,3,4,5,6,7,8,9,10,11,12,13,14$ \\
\hline PR & 89 & 0 & $10,000,000,000$ & $1,2,3,4,5,6,7,8,9,10,11,12,13$ \\
\hline $\mathrm{RI}$ & 48 & 0 & $49,999,999$ & $1,2,3,4,6,7,8,9,10,11,12$ \\
\hline SC & 100 & 0 & $499,999,999$ & $1,2,3,4,5,6,7,8,9,10,11,12,13$ \\
\hline SD & 35 & 0 & $99,999,999$ & $2,3,7,8,10,11,12$ \\
\hline $\mathrm{TN}$ & 166 & 0 & $99,999,999$ & $1,2,3,4,5,6,7,8,9,10,11,12,13,14$ \\
\hline TX & 535 & 0 & $10,000,000,000$ & $1,2,3,4,5,6,7,8,9,10,11,12,13,14$ \\
\hline UT & 87 & 0 & $49,999,999$ & $1,2,3,4,5,6,7,8,9,10,11,12,13$ \\
\hline VA & 125 & 0 & $99,999,999$ & $1,2,3,4,5,6,7,8,9,10,11,12,13$ \\
\hline VI & 11 & 100,000 & $10,000,000,000$ & $1,2,3,4,5,6,7,8,9,12$ \\
\hline VT & 12 & 100 & 999,999 & $5,7,10,11,12$ \\
\hline WA & 121 & 0 & $10,000,000,000$ & $1,2,3,4,5,6,7,8,9,10,11,12,13,14$ \\
\hline WI & 170 & 0 & $99,999,999$ & $1,2,3,4,5,6,7,8,9,10,11,12,13,14$ \\
\hline WV & 85 & 0 & $499,999,999$ & $1,2,3,4,5,6,7,8,9,10,11,12,13$ \\
\hline WY & 42 & 100 & $49,999,999$ & $1,2,3,4,5,6,7,8,9,10,11,12,13,14$ \\
\hline
\end{tabular}

${ }^{a}$ Post office state abbreviations used

${ }^{\mathrm{b}}$ Amounts on site reported by facilities in each state

${ }^{\mathrm{c} A c t i v i t i e s / \text { Uses: }}$
1. Produce
6. Impurity
2. Import
3. Onsite use/processing
7. Reactant
11. Chemical Processing Aid
4. Sale/Distribution
8. Formulation Component
12. Manufacturing Aid
5. Byproduct
9. Article Component
13. Ancillary/Other Uses
14. Process Impurity

Source: TRI04 2006 (Data are from 2004) 
xylenes and the individual isomers have separated the amounts of the isomers from the mixed xylenes (in which case, they would be counting these amounts twice) or whether they are reporting total amounts of mixed xylenes and xylene isomers separately that are finished and ready for use.

Tables 5-3, 5-4, and 5-5 list the U.S. facilities that manufacture or process $m-, o_{-}^{-}$, and $p$-xylene, respectively, with their corresponding location, range of amounts on site, and activities and uses. This information is based on the release data reported to the TRI for 2004 (TRI04 2006). The data listed in these tables should be used with caution since only certain types of facilities are required to report and the actual figures may be higher.

Mixed xylene consists of a mixture of ethylbenzene and the $m-, o-$, and $p$-isomers of xylene; $m$-xylene predominates. In addition to ethylbenzene, mixed xylene may contain nonxylene hydrocarbons, such as benzene, toluene, trimethylbenzene, phenol, thiophene, and pyridine; the combined volume of these nonxylene hydrocarbons is only a fraction of a percentage point of the composition of mixed xylene (Gerarde 1960; Riihimaki and Hanninen 1987; Sandmeyer 1981). Current formulations of mixed xylene are relatively free $(<0.001 \%)$ of benzene contamination (Gosselin et al. 1984; Riihimaki and Hanninen 1987). The exact composition of mixed xylene depends on the manufacturing method used. Currently, nearly all mixed xylene is produced as a catalytic reformate of petroleum and consists of approximately 44\% m-xylene, 20\% o-xylene, 20\% p-xylene, and 15\% ethylbenzene (HSDB 2007; NIOSH 1975). Mixed xylene may also be manufactured from coal tar, yielding a mixture of approximately $45-70 \%$ $m$-xylene, $23 \%$ p-xylene, 10-15\% o-xylene, and 6-10\% ethylbenzene (HSDB 2007). Other production processes include gasoline pyrolysis and disproportionation of toluene, both of which produce a mixture free of ethylbenzene, and recovery from coke-oven light oil (HSDB 2007; NIOSH 1975; Ransley 1984).

The xylene isomers are produced from mixed xylene. $m$-Xylene is obtained from mixed xylene via crystallization to remove $p$-xylene and fractionation to remove $o$-xylene and ethylbenzene, or via complexing with hydrofluoric acid and boron trifluoride (HSDB 2007). $o$-Xylene is isolated from mixed xylene via distillation but can also be produced by the isomerization of $m$-xylene (HSDB 2007). p-Xylene is derived from mixed xylene by crystallization, solvent extraction, or adsorption (HSDB 2007; Lewis 2001). 
Table 5-3. Facilities that Produce, Process, or Use $\boldsymbol{m}$-Xylene

\begin{tabular}{|c|c|c|c|c|}
\hline State $^{a}$ & $\begin{array}{l}\text { Number of } \\
\text { facilities }\end{array}$ & $\begin{array}{l}\text { Minimum } \\
\text { amount on site } \\
\text { in pounds }\end{array}$ & $\begin{array}{l}\text { Maximum } \\
\text { amount on site } \\
\text { in pounds }{ }^{b}\end{array}$ & Activities and uses $^{c}$ \\
\hline$\overline{\mathrm{AL}}$ & 16 & 1,000 & $499,999,999$ & $1,2,3,4,5,6,7,10,11,12,13$ \\
\hline AR & 10 & 100 & $9,999,999$ & $7,8,9,11,12$ \\
\hline$A Z$ & 2 & 10,000 & 99,999 & 10,12 \\
\hline $\mathrm{CA}$ & 35 & 0 & $99,999,999$ & $1,2,3,4,5,6,7,8,9,10,11,12,13$ \\
\hline $\mathrm{CO}$ & 1 & $1,000,000$ & $9,999,999$ & 1,4 \\
\hline $\mathrm{CT}$ & 2 & 10,000 & 99,999 & 10 \\
\hline $\mathrm{DE}$ & 1 & 10,000 & 99,999 & 7,12 \\
\hline FL & 7 & 1,000 & $9,999,999$ & $2,3,10,11,12$ \\
\hline GA & 7 & 100 & 999,999 & $1,4,7,10,11,13$ \\
\hline GU & 2 & 0 & 9,999 & 9 \\
\hline $\mathrm{HI}$ & 7 & $1,000,000$ & $9,999,999$ & $1,2,5,7,10,12,13,14$ \\
\hline IA & 8 & 100 & 99,999 & $8,9,10,11,12$ \\
\hline IL & 21 & 100 & $49,999,999$ & $1,2,3,5,6,7,9,11,12$ \\
\hline IN & 7 & 100 & 9,999,999 & $6,7,8,11,12$ \\
\hline KS & 10 & 100 & 999,999 & $5,6,7,9,10,12$ \\
\hline KY & 9 & 1,000 & 99,999 & $1,4,5,10,11,12$ \\
\hline LA & 17 & 1,000 & $49,999,999$ & $1,2,3,4,5,6,7,9,12,13,14$ \\
\hline MA & 2 & 0 & 99,999 & $2,3,7,10$ \\
\hline MD & 2 & 10,000 & 99,999 & $3,4,5,11$ \\
\hline MI & 5 & 100 & 99,999 & $7,8,10,11,12$ \\
\hline MN & 3 & 1,000 & 99,999 & 7,10 \\
\hline MO & 10 & 1,000 & 999,999 & $2,7,9,10,11,12$ \\
\hline MS & 21 & 1,000 & $99,999,999$ & $1,2,3,4,5,6,7,10,11,12,13,14$ \\
\hline MT & 1 & 10,000 & 99,999 & 2,4 \\
\hline NC & 9 & 0 & 999,999 & $1,3,5,6,7,8,10,12$ \\
\hline NE & 9 & 100 & 999,999 & $2,3,7,11,12$ \\
\hline $\mathrm{NH}$ & 3 & 0 & 99,999 & $7,11,12$ \\
\hline NJ & 10 & 1,000 & $49,999,999$ & $2,3,6,7,10,11,12$ \\
\hline NM & 6 & 100,000 & 9,999,999 & $1,2,3,4,7,9,12$ \\
\hline NV & 1 & 100,000 & 999,999 & 9 \\
\hline NY & 8 & 0 & 99,999 & $2,3,6,7,9,10,11$ \\
\hline $\mathrm{OH}$ & 7 & 1,000 & 999,999 & $6,7,8,9,10,11,12$ \\
\hline OK & 3 & 1,000 & $9,999,999$ & $4,7,9,12$ \\
\hline OR & 3 & 1,000 & 99,999 & $7,10,12$ \\
\hline PA & 20 & 100 & $49,999,999$ & $1,3,4,5,7,8,9,10,11,12,13$ \\
\hline PR & 18 & 1,000 & $10,000,000,000$ & $1,2,3,4,5,6,7,9,10,12,13$ \\
\hline SC & 9 & 1,000 & 999,999 & $6,7,10,11,12$ \\
\hline TN & 20 & 100 & $49,999,999$ & $1,2,3,4,5,6,7,8,9,10,11,12,13$ \\
\hline TX & 71 & 0 & $499,999,999$ & $1,2,3,4,5,6,7,8,9,10,11,12,13,14$ \\
\hline
\end{tabular}


Table 5-3. Facilities that Produce, Process, or Use $\boldsymbol{m}$-Xylene

\begin{tabular}{|c|c|c|c|c|}
\hline State $^{a}$ & $\begin{array}{l}\text { Number of } \\
\text { facilities }\end{array}$ & $\begin{array}{l}\text { Minimum } \\
\text { amount on site } \\
\text { in pounds }\end{array}$ & $\begin{array}{l}\text { Maximum } \\
\text { amount on site } \\
\text { in pounds }\end{array}$ & Activities and uses $^{c}$ \\
\hline UT & 5 & 1,000 & $9,999,999$ & $1,4,9,10$ \\
\hline VA & 6 & 1,000 & $9,999,999$ & $1,2,4,9,10,11,12$ \\
\hline WA & 1 & 100 & 999 & 12 \\
\hline WI & 6 & 0 & 99,999 & $6,7,8,10,11,12,13$ \\
\hline WV & 3 & 10,000 & $9,999,999$ & $1,4,6,7,8$ \\
\hline
\end{tabular}

${ }^{\text {a }}$ Post office state abbreviations used

${ }^{\mathrm{b}}$ Amounts on site reported by facilities in each state ${ }^{\mathrm{c}}$ Activities/Uses:
1. Produce
6. Impurity
2. Import
7. Reactant
11. Chemical Processing Aid
3. Onsite use/processing
8. Formulation Component
12. Manufacturing Aid
4. Sale/Distribution
9. Article Component
13. Ancillary/Other Uses
5. Byproduct
10. Repackaging
14. Process Impurity

Source: TRI04 2006 (Data are from 2004) 
Table 5-4. Facilities that Produce, Process, or Use o-Xylene

\begin{tabular}{|c|c|c|c|c|}
\hline State $^{a}$ & $\begin{array}{l}\text { Number of } \\
\text { facilities }\end{array}$ & $\begin{array}{l}\text { Minimum } \\
\text { amount on site } \\
\text { in pounds }\end{array}$ & $\begin{array}{l}\text { Maximum } \\
\text { amount on site } \\
\text { in pounds }{ }^{b}\end{array}$ & 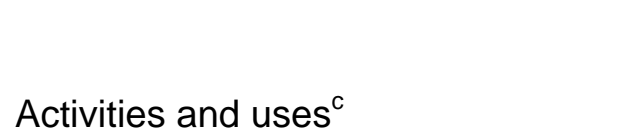 \\
\hline$\overline{A L}$ & 25 & 0 & $99,999,999$ & $1,2,3,4,5,6,7,10,11,12,13$ \\
\hline AR & 9 & 0 & 9,999,999 & $2,3,7,9,10,12$ \\
\hline$A Z$ & 3 & 1,000 & 99,999 & $3,7,10,12$ \\
\hline CA & 46 & 0 & $99,999,999$ & $1,2,3,4,5,6,7,8,9,10,12,13$ \\
\hline $\mathrm{CO}$ & 2 & 10,000 & $9,999,999$ & $1,4,12$ \\
\hline CT & 4 & 0 & 999,999 & $7,10,11,12$ \\
\hline DE & 2 & 100,000 & 999,999 & $7,10,11$ \\
\hline $\mathrm{FL}$ & 2 & 1,000 & $9,999,999$ & 7,12 \\
\hline GA & 15 & 0 & 999,999 & $7,10,11,12$ \\
\hline GU & 2 & 0 & 9,999 & 9 \\
\hline $\mathrm{HI}$ & 7 & $1,000,000$ & $9,999,999$ & $1,2,5,7,10,12,13,14$ \\
\hline IA & 7 & 0 & 99,999 & $8,10,11,12$ \\
\hline IL & 19 & 0 & 49,999,999 & $2,3,6,7,10,11,12$ \\
\hline IN & 5 & 100 & 99,999 & $7,10,11,12$ \\
\hline KS & 11 & 1,000 & $9,999,999$ & $1,2,3,4,6,7,9,10,12$ \\
\hline KY & 1 & 10,000 & 99,999 & 10 \\
\hline LA & 24 & 0 & $10,000,000,000$ & $1,2,3,4,5,6,7,9,10,11,12,13$ \\
\hline MA & 6 & 0 & 99,999 & $2,3,7,9,10,12$ \\
\hline MD & 3 & 1,000 & 99,999 & $2,3,4,5,6,10,11$ \\
\hline MI & 7 & 0 & 99,999 & $1,5,6,7,9,10,11,12,13$ \\
\hline MN & 1 & 0 & 99 & $2,3,12$ \\
\hline MO & 14 & 0 & 49,999,999 & $2,3,6,7,9,10,12$ \\
\hline MS & 17 & 1,000 & $99,999,999$ & $1,2,3,4,5,6,7,10,12,13,14$ \\
\hline MT & 1 & 10,000 & 99,999 & 2,4 \\
\hline NC & 12 & 0 & 999,999 & $1,2,3,5,7,10,11,12,13$ \\
\hline NE & 8 & 100 & 99,999 & $2,3,7,11,12$ \\
\hline $\mathrm{NH}$ & 2 & 0 & 99 & 11,12 \\
\hline NJ & 24 & 0 & $99,999,999$ & $2,3,4,6,7,9,10,11,12$ \\
\hline NM & 2 & 100,000 & 9,999,999 & $1,2,3,4,7,12$ \\
\hline NY & 6 & 0 & 99,999 & $2,3,9,10,11,12$ \\
\hline $\mathrm{OH}$ & 11 & 0 & 99,999 & $2,3,7,8,9,10,11,12$ \\
\hline OK & 1 & 0 & 0 & $7,10,12$ \\
\hline PA & 26 & 0 & $9,999,999$ & $1,2,3,4,5,6,7,8,9,10,11,12,13$ \\
\hline PR & 25 & 1,000 & $10,000,000,000$ & $1,2,3,4,5,6,7,10,11,12,13$ \\
\hline RI & 4 & 10,000 & 999,999 & $7,10,12$ \\
\hline SC & 10 & 1,000 & $9,999,999$ & $6,10,11,12,14$ \\
\hline TN & 22 & 0 & $49,999,999$ & $1,2,3,4,5,7,8,9,10,11,12,13$ \\
\hline TX & 76 & 0 & $499,999,999$ & $1,2,3,4,5,6,7,8,9,10,11,12,13,14$ \\
\hline UT & 5 & 10,000 & $9,999,999$ & $1,3,4,9$ \\
\hline
\end{tabular}


5. PRODUCTION, IMPORT/EXPORT, USE, AND DISPOSAL

Table 5-4. Facilities that Produce, Process, or Use o-Xylene

\begin{tabular}{|c|c|c|c|c|}
\hline State $^{a}$ & $\begin{array}{l}\text { Number of } \\
\text { facilities }\end{array}$ & $\begin{array}{l}\text { Minimum } \\
\text { amount on site } \\
\text { in pounds }\end{array}$ & $\begin{array}{l}\text { Maximum } \\
\text { amount on site } \\
\text { in pounds }\end{array}$ & Activities and uses $^{c}$ \\
\hline VA & 2 & 10,000 & $9,999,999$ & $1,2,4,9,12$ \\
\hline WA & 4 & 100 & $9,999,999$ & $6,7,8,12,13$ \\
\hline WI & 6 & 1,000 & 999,999 & 10,12 \\
\hline WV & 7 & 100 & $9,999,999$ & $1,2,3,4,6,7,8,10$ \\
\hline
\end{tabular}

${ }^{\mathrm{a}}$ Post office state abbreviations used

${ }^{\mathrm{b}}$ Amounts on site reported by facilities in each state ${ }^{\mathrm{c} A c t i v i t i e s / \text { Uses: }}$
1. Produce
6. Impurity
11. Chemical Processing Aid
2. Import
7. Reactant
12. Manufacturing Aid
3. Onsite use/processing
8. Formulation Component
13. Ancillary/Other Uses
4. Sale/Distribution
9. Article Component
14. Process Impurity
5. Byproduct
10. Repackaging

Source: TRI04 2006 (Data are from 2004) 
Table 5-5. Facilities that Produce, Process, or Use $p$-Xylene

\begin{tabular}{|c|c|c|c|c|}
\hline State $^{a}$ & $\begin{array}{l}\text { Number of } \\
\text { facilities }\end{array}$ & $\begin{array}{l}\text { Minimum } \\
\text { amount on site } \\
\text { in pounds }{ }^{b}\end{array}$ & $\begin{array}{l}\text { Maximum } \\
\text { amount on site } \\
\text { in pounds }{ }^{b}\end{array}$ & 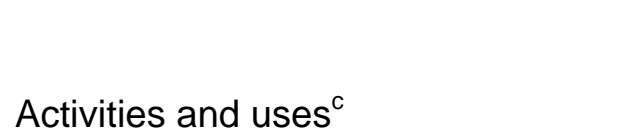 \\
\hline$\overline{A L}$ & 14 & 1,000 & $499,999,999$ & $1,2,3,4,5,6,7,11,12,13$ \\
\hline AR & 6 & 1,000 & 9,999,999 & $7,9,10$ \\
\hline$A Z$ & 1 & 10,000 & 99,999 & 12 \\
\hline $\mathrm{CA}$ & 33 & 0 & $49,999,999$ & $1,2,3,4,5,6,7,8,9,10,12,13$ \\
\hline $\mathrm{CO}$ & 2 & 100,000 & $9,999,999$ & 1,4 \\
\hline DE & 1 & 10,000 & 99,999 & 7,12 \\
\hline FL & 1 & 1,000 & 9,999 & 10 \\
\hline GA & 6 & 100 & 99,999 & $2,3,7,10,11,12$ \\
\hline $\mathrm{HI}$ & 7 & $1,000,000$ & $9,999,999$ & $1,2,5,7,10,12,13,14$ \\
\hline IA & 6 & 0 & 99,999 & $8,10,11$ \\
\hline IL & 7 & 0 & 999,999 & $1,6,7,10,11,13$ \\
\hline IN & 1 & 10,000 & 99,999 & 7 \\
\hline KS & 4 & 1,000 & 99,999 & $7,10,12$ \\
\hline KY & 1 & 1,000 & 9,999 & 12 \\
\hline LA & 11 & 0 & 49,999,999 & $1,3,4,6,7,13$ \\
\hline MA & 3 & 1,000 & 99,999 & $7,9,10$ \\
\hline MD & 1 & 10,000 & 99,999 & $3,4,5$ \\
\hline MI & 2 & 1,000 & 99,999 & 7,9 \\
\hline MN & 1 & 1,000 & 9,999 & 11 \\
\hline MO & 2 & 1,000 & 9,999 & 10,12 \\
\hline MS & 9 & 1,000 & $99,999,999$ & $1,2,3,4,5,7,12,13,14$ \\
\hline MT & 1 & 10,000 & 99,999 & 2,4 \\
\hline NC & 12 & 0 & $499,999,999$ & $1,2,3,5,6,9,10$ \\
\hline NE & 9 & 100 & 99,999 & $2,3,7,11,12$ \\
\hline $\mathrm{NH}$ & 1 & 0 & 99 & 12 \\
\hline $\mathrm{NJ}$ & 6 & 100 & $9,999,999$ & $3,6,7,9,10,11,12$ \\
\hline NM & 2 & 100,000 & 999,999 & $1,2,3,4,7,12$ \\
\hline NY & 7 & 100 & 999,999 & $6,7,9,10,11$ \\
\hline $\mathrm{OH}$ & 2 & 1,000 & 99,999 & $8,11,12$ \\
\hline PA & 17 & 100 & $49,999,999$ & $1,3,4,5,7,8,9,12,13$ \\
\hline PR & 10 & 10,000 & $10,000,000,000$ & $1,2,3,4,6,10$ \\
\hline SC & 6 & 10,000 & 499,999,999 & $6,12,14$ \\
\hline TN & 20 & 0 & $49,999,999$ & $1,2,3,4,5,6,7,8,9,10,11,12,13$ \\
\hline $\mathrm{TX}$ & 61 & 0 & $499,999,999$ & $1,2,3,4,5,6,7,8,9,10,11,12,13,14$ \\
\hline UT & 5 & 10,000 & 9,999,999 & $1,3,4,9,10$ \\
\hline VA & 1 & $1,000,000$ & $9,999,999$ & $1,4,12$ \\
\hline VI & 6 & 100,000 & $10,000,000,000$ & $1,2,3,4,6,7$ \\
\hline WA & 1 & 100 & 999 & 12 \\
\hline
\end{tabular}


5. PRODUCTION, IMPORT/EXPORT, USE, AND DISPOSAL

Table 5-5. Facilities that Produce, Process, or Use p-Xylene

\begin{tabular}{lccrl}
\hline & $\begin{array}{l}\text { Mumber of } \\
\text { amount on site } \\
\text { facilities }\end{array}$ & $\begin{array}{l}\text { Maximum } \\
\text { in pounds }\end{array}$ & $\begin{array}{l}\text { Mamount on site } \\
\text { in pounds }\end{array}$ & ${\text { Activities and } \text { uses }^{\mathrm{c}}}^{\text {in }}$ \\
\hline WI & 4 & 1,000 & 99,999 & $7,11,12$ \\
WV & 5 & 1,000 & $9,999,999$ & $1,4,6,7,8,10$ \\
\hline
\end{tabular}

${ }^{\mathrm{a}}$ Post office state abbreviations used

${ }^{\mathrm{b}}$ Amounts on site reported by facilities in each state

${ }^{\mathrm{c} A c t i v i t i e s / \text { Uses: }}$
1. Produce
6. Impurity
2. Import
7. Reactant
3. Onsite use/processing
8. Formulation Component
11. Chemical Processing Aid
4. Sale/Distribution
9. Article Component
5. Byproduct
10. Repackaging
12. Manufacturing Aid
13. Ancillary/Other Uses
14. Process Impurity

Source: TRI04 2006 (Data are from 2004) 


\subsection{IMPORT/EXPORT}

Available import and export data for mixed, $o-, m-$, and $p$-xylene are shown in Table 5-6. Amounts of U.S. imports and exports reported for mixed xylenes appear to be independent of the amounts reported for the individual xylene isomers. U.S. imports and exports of total xylenes during 2005 were approximately 1.3 billion liters and 280 million liters, respectively (USDOC 2005).

\subsection{USE}

Approximately $70 \%$ of mixed xylene is used in the production of ethylbenzene and the $\mathrm{m}-, \mathrm{o}-$, and $p$-isomers. The remaining mixed xylene is used as a solvent, in products such as paints and coatings, or blended into gasoline (Fishbein 1988; HSDB 2007; NCI 1985; Riihimaki and Hanninen 1987).

The xylene isomers are used primarily for internal industrial operations as solvents and intermediates in synthetic reactions. $m$-Xylene is a chemical intermediate in the production of isophthalic acid and isophthalonitrile; isophthalic acid, in turn, is used in the manufacture of polyesters. $o$-Xylene is a chemical intermediate in the synthesis of phthalic anhydride (for plasticizers), phthalonitrile, 4,4-(trifluoro-1-(trifluoromethyl) ethylidene) diphthalic anhydride (for polyimide polymers), $o$-toluic acid, vitamins, and pharmaceuticals. $p$-Xylene is a chemical intermediate for the synthesis of dimethyl terephthalate, terephthalic acid (for polyesters), dimethyl tetrachloroterephthalate, vitamins, and pharmaceuticals. Both $o$-xylene and $p$-xylene are used as components of insecticides (HSDB 2007; Lewis 2001).

\subsection{DISPOSAL}

Various methods of incineration are used in the disposal of xylene isomers, such as fluidized bed rotary kiln and liquid injection incinerator methods (EPA 1981a; HSDB 2007). The addition of a more flammable solvent has been suggested to make the process easier (HSDB 2007).

Criteria for the disposal of xylenes are currently subject to significant revision. Under the Resource Conservation and Recovery Act, waste product, off-specification batches, and spill residues of xylenes $>1,000$ pounds are subject to handling, reporting, and recordkeeping requirements. This applies also to spent xylene solvents and still bottoms from the refining of these solvents (EPA 1980b, 1981b). 
Table 5-6. U.S. Import and Export Data on Xylenes ${ }^{a}$

\begin{tabular}{crrrrrrrrl}
\hline & \multicolumn{9}{c}{ Import } \\
\cline { 2 - 8 } Year & Mixed & o-Xylene & $m$-Xylene & $p$-Xylene Mixed & o-Xylene & $m$-Xylene & $p$-Xylene Reference \\
\hline 1986 & 284 & 3 & NA & 141 & NA & NA & NA & NA & HSDB 2007 \\
1995 & 713 & 47 & 6 & 343 & 713 & 90 & 37 & 713 & Canella 1998 \\
2000 & $1,302^{\mathrm{b}}$ & 3 & 51 & 189 & $141^{\mathrm{c}}$ & 50 & NA & 152 & USDOC 2005 \\
2005 & $1,303^{\mathrm{b}}$ & 0 & 11 & 0 & $263^{\mathrm{c}}$ & 10 & NA & 12 & USDOC 2005 \\
\hline
\end{tabular}

${ }^{a}$ Values reported in millions of liters

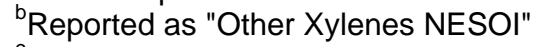

${ }^{\mathrm{C}}$ Reported as "Other Xylenes NESOI (except $o$ - or $p$-Xylene)"

$\mathrm{NA}=$ data not available 
5. PRODUCTION, IMPORT/EXPORT, USE, AND DISPOSAL

This page is intentionally blank. 


\section{POTENTIAL FOR HUMAN EXPOSURE}

\subsection{OVERVIEW}

Xylene has been identified in at least 840 of the 1,684 hazardous waste sites that have been proposed for inclusion on the EPA National Priorities List (NPL) (HazDat 2006). However, the number of sites evaluated for xylene is not known. The frequency of these sites can be seen in Figure 6-1. Of these sites, 835 are located within the United States, 2 are located in the Commonwealth of Puerto Rico (not shown), 1 is located in Guam (not shown), and 2 are located on the Virgin Islands (not shown).

Xylenes are released to the atmosphere primarily as fugitive emissions from industrial sources (e.g., petroleum refineries, chemical plants), in automobile exhaust, and through volatilization from their use as solvents. Discharges into waterways and spills on land result primarily from use, storage, and transport of petroleum products and waste disposal. When xylenes are released to soil or surface water, they are expected to volatilize into the atmosphere. However, based on the mobility of these substances in soil, xylenes may also leach into groundwater, where they may persist for several months.

Xylene is rapidly transformed in the troposphere where photooxidation by hydroxyl radicals is the dominant process. Xylene is stable to hydrolysis and oxidation in the aquatic environment, but is expected to undergo biodegradation. However, based on the volatility of xylene, biotransformation of this substance in surface waters is not expected to compete with its evaporation into the air. Xylene is also expected to volatilize from soil surfaces. Biodegradation is an important process in subsurface soils and groundwater where volatilization is hindered. Xylene is not expected to adsorb strongly to soil; however, soil adsorption increases as organic matter content increases. Xylene has been found to bioaccumulate to very modest levels (e.g., bioconcentration factors of $<100$ ), and food-chain biomagnification has not been observed.

As is expected based on the partitioning and environmental fate of xylenes, these substances are primarily detected in air. Typical xylene concentrations range from 1 to $30 \mathrm{ppb}$ in outdoor air and from 1 to $10 \mathrm{ppb}$ in indoor air (see note at the end of Table 4-2). According to groundwater surveys conducted in the United States, xylenes are generally detected in $<5 \%$ of groundwater samples. However, xylene concentrations in contaminated groundwater have been reported as high as $10,000 \mathrm{ppb}$. Little data are available reporting the concentrations of xylene in soil or surface water. Less than $6 \%$ of drinking water samples collected during U.S. drinking water surveys contained xylenes. Mean concentrations in positive 
Figure 6-1. Frequency of NPL Sites with Xylene Contamination
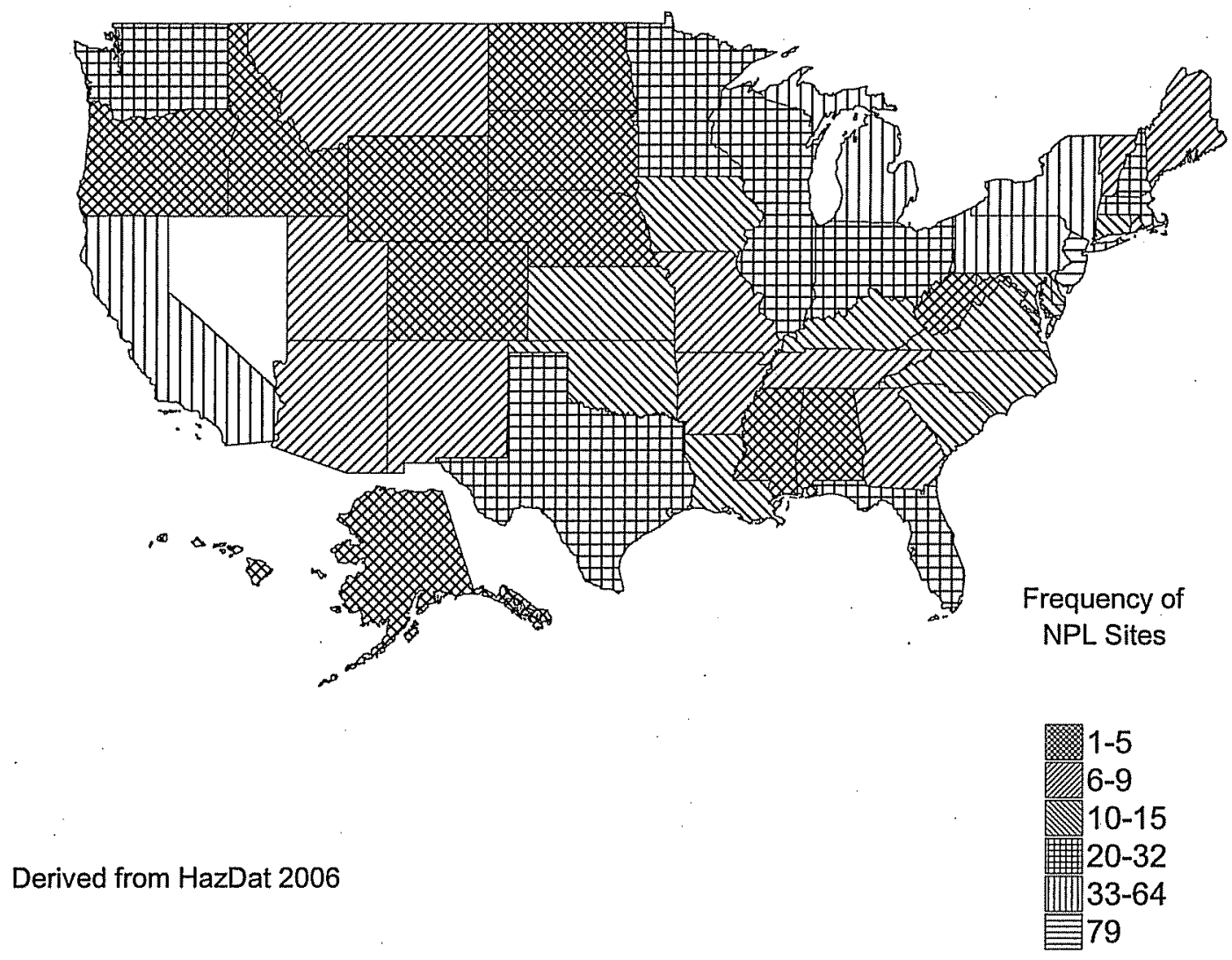
samples were typically $<2 \mathrm{ppb}$. Xylene has been detected in a variety of foods with typical concentrations ranging from 1 to $100 \mathrm{ppb}$.

Because individual xylene isomers are used in large amounts in industrial settings, people who work at or live near these locations may receive a higher exposure to one xylene isomer compared to the other isomers. However, since xylenes are present as a mixture in gasoline and in the solvent components of commonly used commercial products (paint, etc.), exposure of the general population is expected to be primarily to xylenes as a mixture, and not to the separate xylene isomers.

Xylene or its metabolites have been detected in human urine, blood, and expired air samples among members of the general population. Human exposure to xylene is believed to occur via inhalation of indoor and workplace air, inhalation of automobile exhaust, ingestion of contaminated drinking water, smoking, and inhalation and dermal absorption of solvents containing xylene.

When analyzing biological and environmental samples for xylene, it is difficult to separate the $m$ - and $p$-isomers. Therefore, the concentrations of these two isomers are typically reported together as a sum (m-/p-xylene).

\subsection{RELEASES TO THE ENVIRONMENT}

The Toxics Release Inventory (TRI) data should be used with caution because only certain types of facilities are required to report (EPA 2005g). This is not an exhaustive list. Manufacturing and processing facilities are required to report information to the TRI only if they employ 10 or more full-time employees; if their facility is included in Standard Industrial Classification (SIC) Codes 10 (except 1011, 1081, and 1094), 12 (except 1241), 20-39, 4911 (limited to facilities that combust coal and/or oil for the purpose of generating electricity for distribution in commerce), 4931 (limited to facilities that combust coal and/or oil for the purpose of generating electricity for distribution in commerce), 4939 (limited to facilities that combust coal and/or oil for the purpose of generating electricity for distribution in commerce), 4953 (limited to facilities regulated under RCRA Subtitle C, 42 U.S.C. section 6921 et seq.), 5169, 5171, and 7389 (limited S.C. section 6921 et seq.), 5169, 5171, and 7389 (limited to facilities primarily engaged in solvents recovery services on a contract or fee basis); and if their facility produces, imports, or processes $\geq 25,000$ pounds of any TRI chemical or otherwise uses $>10,000$ pounds of a TRI chemical in a calendar year (EPA 2005g). 


\subsubsection{Air}

Estimated releases of mixed xylenes, $m$-xylene, $o$-xylene, and $p$-xylene to the atmosphere were 34.8 million pounds ( 16,000 metric tons), 0.5 million pounds ( 240 metric tons), 0.6 million pounds (290 metric tons), and 1.4 million pounds (640 metric tons), respectively, to the atmosphere from 2,794, 58,67 , and 39 domestic manufacturing and processing facilities, respectively, in 2004. These releases accounted for about $91,93,95$, and $98 \%$ of the estimated total environmental releases of mixed xylenes, $m$-xylene, $o$-xylene, and $p$-xylene, respectively, from facilities required to report to the TRI (TRI04 2006). These releases are summarized in Tables 6 1, 6-2, 6-3, and 6-4, respectively.

Xylene has been identified in air samples collected at 143 of the 840 NPL hazardous waste sites where it was detected in some environmental media (HazDat 2006).

Most annual releases of xylene are refinery losses into the atmosphere during the production, transportation, and processing of petroleum. Other significant sources of xylene emissions are from the use of solvents, as a component of automobile exhaust gases, evaporation of gasoline into the air during its transportation and distribution, and releases from the chemical industry (Merian and Zander 1982). Based on vapor pressures of 6-8 mm Hg (AIChE 1996; Chao et al. 1983; Lewis 2000), volatilization from soil or surface water is also expected to be an important pathway for xylenes to enter the atmosphere.

Releases of xylenes are associated with outgassing from landfills where disposal of industrial, hazardous, and mixed municipal wastes occur. In Finland during 1989-1990, all three xylene isomers were detected in gases released from closed municipal landfills at average concentrations of $0.86,3.6$, and $1.2 \mathrm{mg} / \mathrm{m}^{3}$ $(0.20,0.83$, and $0.28 \mathrm{ppm})$ for the $o-, m-$, and $p$-isomers, respectively (Assmuth and Kalevi 1992). Concentrations of all of the xylene isomers in off-gases from an active landfill were 30-35 times higher. In 1987-1988, air emissions of mixed xylene from facilities in the Houston, Texas, ship channel industrial area were 551 tons per year (LaGrone 1991). The emissions resulted in an ambient concentration in this area of 1.6 parts per billion volume (ppbv). The mean concentrations of $o$-xylene and $m$-/ $p$-xylene in landfill gas from the Fresh Kills Municipal Solid-waste Landfill in New York City (Staten Island) were 2.17 and 5.97 ppmv, respectively (Eklund et al. 1998).

Eitzer (1995) measured volatile organic compound (VOC) emissions at eight municipal solid waste compositing facilities. Respective mean $p$-xylene and $m$ - $/ o$-xylene concentrations were 1 and $2 \mu \mathrm{g} / \mathrm{m}^{3}$ 
Table 6-1. Releases to the Environment from Facilities that Produce, Process, or Use Xylene (Mixed) ${ }^{a}$

\begin{tabular}{|c|c|c|c|c|c|c|c|c|c|}
\hline \multirow[b]{3}{*}{ State $^{c}$} & \multirow[b]{3}{*}{$\mathrm{RF}^{\mathrm{d}}$} & \multicolumn{8}{|c|}{ Reported amounts released in pounds per year ${ }^{b}$} \\
\hline & & \multirow[b]{2}{*}{ Air $^{\mathrm{e}}$} & \multirow{2}{*}{\multicolumn{2}{|c|}{ Water ${ }^{f} \mathrm{UI}^{\mathrm{g}}$}} & \multirow[b]{2}{*}{ Land $^{\mathrm{h}}$} & \multirow[b]{2}{*}{ Other ${ }^{i}$} & \multicolumn{3}{|c|}{ Total release } \\
\hline & & & & & & & On-site & Off-site ${ }^{k}$ & $\begin{array}{l}\text { On- and } \\
\text { off-site }\end{array}$ \\
\hline$\overline{\mathrm{AK}}$ & 10 & 22,415 & 41 & 0 & 820 & 0 & 23,268 & 7 & 23,275 \\
\hline$A L$ & 76 & $2,051,596$ & 190 & 50 & 27,628 & 12,182 & $2,078,986$ & 12,660 & $2,091,646$ \\
\hline AR & 35 & 571,748 & 46 & 0 & 74 & 0 & 571,794 & 74 & 571,868 \\
\hline$A Z$ & 27 & 87,092 & 0 & 0 & 5,901 & 768 & 87,097 & 6,664 & 93,761 \\
\hline CA & 155 & 504,919 & 266 & 204 & 28,895 & 8,789 & 529,014 & 14,059 & 543,074 \\
\hline $\mathrm{CO}$ & 19 & 70,584 & 0 & 0 & 10 & 479 & 70,584 & 489 & 71,073 \\
\hline CT & 20 & 18,978 & 3 & 0 & 3 & 6,759 & 18,981 & 6,762 & 25,743 \\
\hline $\mathrm{DE}$ & 9 & 93,557 & 0 & 0 & 51 & 0 & 93,557 & 51 & 93,608 \\
\hline $\mathrm{FL}$ & 67 & 457,196 & 29 & 0 & 1 & 549 & 457,225 & 550 & 457,775 \\
\hline GA & 67 & $1,070,406$ & 252 & 0 & 589 & 755 & $1,071,198$ & 805 & $1,072,003$ \\
\hline GU & 3 & 4,407 & 1 & 0 & 0 & 1,417 & 4,408 & 1,417 & 5,825 \\
\hline $\mathrm{HI}$ & 11 & 25,708 & 43 & 0 & 1,710 & 2,795 & 25,758 & 4,498 & 30,256 \\
\hline IA & 62 & 883,083 & 0 & 0 & 0 & 0 & 883,083 & 0 & 883,083 \\
\hline ID & 4 & 39,534 & No data & 0 & 0 & 0 & 39,534 & 0 & 39,534 \\
\hline IL & 134 & $1,287,226$ & 296 & 0 & 6,483 & 6,504 & $1,288,763$ & 11,746 & $1,300,509$ \\
\hline IN & 165 & $2,964,117$ & 469 & 0 & 11,642 & 2,469 & $2,973,971$ & 4,726 & $2,978,698$ \\
\hline KS & 52 & 773,525 & 146 & 293 & 1,033 & 50 & 774,006 & 1,041 & 775,047 \\
\hline KY & 82 & $1,159,382$ & 484 & 0 & 138,771 & 25,662 & $1,160,156$ & 164,143 & $1,324,299$ \\
\hline LA & 80 & $1,096,942$ & 710 & 27,957 & 13,236 & 7,374 & $1,127,021$ & 19,199 & $1,146,219$ \\
\hline MA & 43 & 68,132 & 152 & 0 & 139 & 24,187 & 68,284 & 24,326 & 92,610 \\
\hline MD & 23 & 102,410 & 266 & 0 & 139 & 250 & 102,677 & 388 & 103,065 \\
\hline ME & 8 & 14,187 & 80 & 0 & 0 & 1,820 & 14,266 & 1,820 & 16,086 \\
\hline MI & 137 & $2,768,110$ & 27 & 0 & 49,160 & 155,142 & $2,777,445$ & 194,994 & $2,972,439$ \\
\hline MN & 45 & 655,287 & 30 & 0 & 597 & 70 & 655,354 & 630 & 655,984 \\
\hline $\mathrm{MO}$ & 77 & $1,797,940$ & 0 & 0 & 510 & 1,748 & $1,797,940$ & 2,258 & $1,800,198$ \\
\hline MP & 2 & 587 & 0 & 0 & 0 & 10 & 587 & 10 & 597 \\
\hline MS & 47 & $1,056,657$ & 50 & 0 & 1,826 & 0 & $1,058,407$ & 126 & $1,058,533$ \\
\hline MT & 8 & 41,341 & 5 & 0 & 75 & 5,600 & 41,357 & 5,664 & 47,021 \\
\hline NC & 62 & 609,558 & 0 & 0 & 558 & 4,296 & 609,558 & 4,854 & 614,412 \\
\hline ND & 5 & 60,533 & 1 & 4 & 13 & 0 & 60,534 & 17 & 60,551 \\
\hline NE & 19 & 231,469 & No data & 0 & 26,437 & 510 & 231,469 & 26,947 & 258,416 \\
\hline $\mathrm{NH}$ & 6 & 32,052 & No data & 0 & 0 & 500 & 32,052 & 500 & 32,552 \\
\hline $\mathrm{NJ}$ & 77 & 262,906 & 1,454 & 0 & 20,425 & 6,810 & 270,720 & 20,876 & 291,596 \\
\hline NM & 13 & 71,564 & 5 & 25 & 230 & 5,468 & 71,824 & 5,468 & 77,292 \\
\hline NV & 9 & 3,149 & No data & 0 & 902,695 & 0 & 905,844 & 0 & 905,844 \\
\hline NY & 76 & 278,303 & 366 & 0 & 1,492 & 7,499 & 278,675 & 8,985 & 287,660 \\
\hline
\end{tabular}




\section{Table 6-1. Releases to the Environment from Facilities that Produce, Process, or Use Xylene (Mixed) ${ }^{a}$}

\begin{tabular}{|c|c|c|c|c|c|c|c|c|c|}
\hline \multirow[b]{3}{*}{ State $^{c}$} & \multirow[b]{3}{*}{$\mathrm{RF}^{\mathrm{d}}$} & \multicolumn{8}{|c|}{ Reported amounts released in pounds per year ${ }^{b}$} \\
\hline & & \multirow[b]{2}{*}{ Air ${ }^{e}$} & \multirow{2}{*}{\multicolumn{2}{|c|}{ Water ${ }^{f} \mathrm{UI}^{\mathrm{g}}$}} & \multirow[b]{2}{*}{ Land $^{\mathrm{h}}$} & \multirow[b]{2}{*}{ Other ${ }^{i}$} & \multicolumn{3}{|c|}{ Total release } \\
\hline & & & & & & & On-site & Off-site $^{k}$ & $\begin{array}{l}\text { On- and } \\
\text { off-site }\end{array}$ \\
\hline $\mathrm{OH}$ & 184 & $2,367,513$ & 90 & 2,114 & 226,194 & 7,205 & $2,370,589$ & 232,528 & $2,603,117$ \\
\hline OK & 37 & 471,134 & 47 & 109 & 560 & 50 & 471,566 & 333 & 471,899 \\
\hline OR & 25 & 252,626 & 1,001 & 0 & 1,038 & 20,770 & 254,416 & 21,019 & 275,435 \\
\hline PA & 147 & $1,080,111$ & 725 & 0 & 11,167 & 18,372 & $1,080,857$ & 29,517 & $1,110,375$ \\
\hline PR & 16 & 45,826 & 2 & 0 & 0 & 6 & 45,828 & 6 & 45,834 \\
\hline RI & 9 & 8,395 & 6 & 0 & 0 & 7,305 & 8,401 & 7,305 & 15,706 \\
\hline SC & 40 & $1,028,025$ & 627 & 0 & 3,172 & 14,124 & $1,029,472$ & 16,476 & $1,045,948$ \\
\hline SD & 16 & 399,993 & No data & 0 & 0 & 0 & 399,993 & 0 & 399,993 \\
\hline TN & 86 & $1,918,718$ & 80 & 0 & 1,137 & 9,962 & $1,918,799$ & 11,098 & $1,929,897$ \\
\hline TX & 271 & $3,517,712$ & 18,881 & $1,098,590$ & 27,896 & 75,766 & $4,517,614$ & 221,231 & $4,738,845$ \\
\hline UT & 23 & 135,919 & 1,000 & 0 & 1,051 & 2,141 & 137,169 & 2,942 & 140,111 \\
\hline VA & 58 & 886,351 & 255 & 0 & 575 & 5 & 886,856 & 330 & 887,185 \\
\hline VI & 3 & 49,114 & 0 & 0 & 1,089 & 0 & 49,114 & 1,089 & 50,203 \\
\hline VT & 1 & 1,880 & No data & 0 & 0 & 0 & 1,880 & 0 & 1,880 \\
\hline WA & 30 & 175,968 & 4 & 0 & 596 & 137 & 176,299 & 405 & 176,705 \\
\hline WI & 80 & 830,359 & 5 & 0 & 25,478 & 1,665 & 855,764 & 1,743 & 857,507 \\
\hline WV & 25 & 396,903 & 418 & 0 & 4,524 & 65 & 397,321 & 4,589 & 401,910 \\
\hline WY & 8 & 25,986 & 0 & 0 & 69 & 0 & 26,055 & 0 & 26,055 \\
\hline Total & 2,794 & $34,829,134$ & 28,554 & $1,129,345$ & $1,545,689$ & 448,034 & $36,883,391$ & $1,097,365$ & $37,980,757$ \\
\hline
\end{tabular}

${ }^{a}$ The TRI data should be used with caution since only certain types of facilities are required to report. This is not an exhaustive list. Data are rounded to nearest whole number.

${ }^{\mathrm{b}}$ Data in TRI are maximum amounts released by each facility.

${ }^{\mathrm{c}}$ Post office state abbreviations are used.

${ }^{\mathrm{d}}$ Number of reporting facilities.

${ }^{\mathrm{e}}$ The sum of fugitive and point source releases are included in releases to air by a given facility.

'Surface water discharges, waste water treatment-(metals only), and publicly owned treatment works (POTWs) (metal and metal compounds).

${ }^{9}$ Class I wells, Class II-V wells, and underground injection.

${ }^{\mathrm{h}}$ Resource Conservation and Recovery Act (RCRA) subtitle C landfills; other on-site landfills, land treatment, surface impoundments, other land disposal, other landfills.

iStorage only, solidification/stabilization (metals only), other off-site management, transfers to waste broker for disposal, unknown

j'The sum of all releases of the chemical to air, land, water, and underground injection wells.

${ }^{\mathrm{k}}$ Total amount of chemical transferred off-site, including to POTWs.

$\mathrm{RF}=$ reporting facilities; $\mathrm{UI}=$ underground injection

Source: TRI04 2006 (Data are from 2004) 
Table 6-2. Releases to the Environment from Facilities that Produce, Process, or Use $m$-Xylene ${ }^{a}$

\begin{tabular}{|c|c|c|c|c|c|c|c|c|c|}
\hline \multirow[b]{3}{*}{ State $^{c}$} & \multirow[b]{3}{*}{$\mathrm{RF}^{\mathrm{d}}$} & \multicolumn{8}{|c|}{ Reported amounts released in pounds per year ${ }^{b}$} \\
\hline & & \multirow[b]{2}{*}{ Air ${ }^{\mathrm{e}}$} & \multirow[b]{2}{*}{ Water $^{f}$} & \multirow[b]{2}{*}{$\mathrm{UI}^{\mathrm{g}}$} & \multirow[b]{2}{*}{ Land $^{\mathrm{h}}$} & \multirow[b]{2}{*}{ Other ${ }^{i}$} & \multicolumn{3}{|c|}{ Total release } \\
\hline & & & & & & & On-site $^{j}$ & Off-site $^{k}$ & On- and off-site \\
\hline$\overline{\mathrm{AL}}$ & 1 & 201,000 & 22,000 & 0 & 250 & 0 & 223,250 & 0 & 223,250 \\
\hline AR & 1 & 1,137 & No data & 0 & 5 & 0 & 1,137 & 5 & 1,142 \\
\hline $\mathrm{CA}$ & 1 & 0 & No data & No data & 0 & No data & No data & 0 & 0 \\
\hline $\mathrm{FL}$ & 1 & 4,505 & No data & 0 & 0 & 0 & 4,505 & 0 & 4,505 \\
\hline GA & 4 & 38,421 & No data & 0 & 0 & 0 & 38,421 & 0 & 38,421 \\
\hline IA & 3 & 14,891 & No data & 0 & 0 & 0 & 14,891 & 0 & 14,891 \\
\hline IL & 2 & 6,271 & 4 & 0 & 173 & 0 & 6,276 & 173 & 6,449 \\
\hline IN & 4 & 2,835 & No data & 0 & 0 & 0 & 2,835 & 0 & 2,835 \\
\hline KS & 1 & 39,870 & No data & 0 & 0 & 0 & 39,870 & 0 & 39,870 \\
\hline KY & 2 & 2,535 & No data & 0 & 0 & 0 & 2,535 & 0 & 2,535 \\
\hline LA & 2 & 1,463 & 0 & 0 & 213 & 0 & 1,463 & 213 & 1,676 \\
\hline MO & 1 & 933 & 0 & 0 & 0 & 0 & 933 & 0 & 933 \\
\hline MS & 1 & 30,100 & 25 & 0 & 1,006 & 0 & 30,126 & 1,005 & 31,131 \\
\hline NE & 2 & 11,265 & No data & 0 & 0 & 0 & 11,265 & 0 & 11,265 \\
\hline $\mathrm{NJ}$ & 1 & 0 & No data & No data & 0 & No data & No data & 0 & 0 \\
\hline NM & 2 & 190 & No data & 0 & 0 & 0 & 190 & 0 & 190 \\
\hline NY & 1 & 920 & No data & 0 & 0 & 0 & 920 & 0 & 920 \\
\hline OK & 1 & 500 & 1 & 0 & 0 & 0 & 501 & 0 & 501 \\
\hline PA & 1 & 0 & No data & No data & 0 & No data & No data & 0 & 0 \\
\hline PR & 1 & 11,530 & 0 & 0 & 0 & 0 & 11,530 & 0 & 11,530 \\
\hline SC & 2 & 33,508 & No data & 0 & 0 & 0 & 33,508 & 0 & 33,508 \\
\hline TN & 1 & 500 & No data & 0 & 0 & 0 & 500 & 0 & 500 \\
\hline TX & 21 & 118,647 & 2,149 & 10,190 & 2,569 & 1,397 & 132,711 & 2,242 & 134,953 \\
\hline UT & 1 & 0 & No data & No data & 0 & No data & No data & 0 & 0 \\
\hline Total & 58 & 521,021 & 24,180 & 10,190 & 4,216 & 1,397 & 557,366 & 3,638 & 561,004 \\
\hline
\end{tabular}

${ }^{a}$ The TRI data should be used with caution since only certain types of facilities are required to report. This is not an exhaustive list. Data are rounded to nearest whole number.

${ }^{\mathrm{b}}$ Data in TRI are maximum amounts released by each facility.

${ }^{c}$ Post office state abbreviations are used.

${ }^{\mathrm{d}}$ Number of reporting facilities.

${ }^{\mathrm{e}}$ The sum of fugitive and point source releases are included in releases to air by a given facility.

${ }^{f}$ Surface water discharges, waste water treatment-(metals only), and publicly owned treatment works (POTWs) (metal and metal compounds).

${ }^{\mathrm{g}}$ Class I wells, Class II-V wells, and underground injection.

${ }^{h}$ Resource Conservation and Recovery Act (RCRA) subtitle C landfills; other on-site landfills, land treatment, surface impoundments, other land disposal, other landfills.

iStorage only, solidification/stabilization (metals only), other off-site management, transfers to waste broker for disposal, unknown

${ }^{\mathrm{j}}$ The sum of all releases of the chemical to air, land, water, and underground injection wells.

${ }^{\mathrm{k}}$ Total amount of chemical transferred off-site, including to POTWs.

$\mathrm{RF}=$ reporting facilities; $\mathrm{UI}=$ underground injection

Source: TRI04 2006 (Data are from 2004) 
Table 6-3. Releases to the Environment from Facilities that Produce, Process, or Use o-Xylene ${ }^{a}$

\begin{tabular}{|c|c|c|c|c|c|c|c|c|c|}
\hline \multirow[b]{3}{*}{ State $^{c}$} & \multirow[b]{3}{*}{$\mathrm{RF}^{\mathrm{d}}$} & \multicolumn{8}{|c|}{ Reported amounts released in pounds per year ${ }^{b}$} \\
\hline & & \multirow[b]{2}{*}{ Air ${ }^{\mathrm{e}}$} & \multirow[b]{2}{*}{ Water ${ }^{f}$} & \multirow[b]{2}{*}{$U^{g}$} & \multirow[b]{2}{*}{ Land $^{\text {h }}$} & \multirow[b]{2}{*}{ Other ${ }^{i}$} & \multicolumn{3}{|c|}{ Total release } \\
\hline & & & & & & & On-site & Off-site ${ }^{k}$ & $\begin{array}{l}\text { On- and } \\
\text { off-site }\end{array}$ \\
\hline$\overline{\mathrm{AL}}$ & 4 & 224,186 & 9,500 & 0 & 252 & 0 & 233,938 & 0 & 233,938 \\
\hline AR & 2 & 29,007 & 0 & 82 & 5 & 0 & 29,089 & 5 & 29,094 \\
\hline CA & 2 & 114 & 0 & 0 & 0 & 0 & 114 & 0 & 114 \\
\hline $\mathrm{FL}$ & 1 & 0 & No data & 0 & 0 & No data & No data & 0 & 0 \\
\hline GA & 4 & 598 & No data & 0 & 3 & 0 & 598 & 3 & 601 \\
\hline IA & 2 & 2,247 & No data & 0 & 0 & 0 & 2,247 & 0 & 2,247 \\
\hline IL & 3 & 110,203 & 4 & 0 & 0 & 0 & 110,207 & 0 & 110,207 \\
\hline IN & 1 & 424 & No data & 0 & 0 & 0 & 424 & 0 & 424 \\
\hline KS & 2 & 17,966 & No data & 0 & 0 & 0 & 17,966 & 0 & 17,966 \\
\hline KY & 1 & 0 & No data & 0 & 0 & No data & No data & 0 & 0 \\
\hline LA & 2 & 2,739 & 0 & 0 & 2 & 0 & 2,739 & 2 & 2,741 \\
\hline MD & 1 & 0 & No data & 0 & 0 & 0 & 0 & 0 & 0 \\
\hline MO & 2 & 5,161 & 5 & 0 & 5 & 0 & 5,171 & 0 & 5,171 \\
\hline MS & 1 & 16,400 & 25 & 0 & 503 & 0 & 16,425 & 503 & 16,928 \\
\hline NC & 2 & 3,383 & 18 & 0 & 0 & 0 & 3,401 & 0 & 3,401 \\
\hline ND & 1 & 0 & No data & 0 & 0 & No data & No data & 0 & 0 \\
\hline NE & 2 & 1,144 & No data & 0 & 0 & 0 & 1,144 & 0 & 1,144 \\
\hline $\mathrm{NJ}$ & 3 & 509 & No data & 0 & 0 & 0 & 509 & 0 & 509 \\
\hline NY & 2 & 45,231 & No data & 0 & 0 & 0 & 45,231 & 0 & 45,231 \\
\hline PA & 2 & 0 & No data & 0 & 0 & No data & No data & 0 & 0 \\
\hline PR & 2 & 4,658 & 0 & 0 & 0 & 0 & 4,658 & 0 & 4,658 \\
\hline SC & 1 & 12 & No data & 0 & 0 & 0 & 12 & 0 & 12 \\
\hline $\mathrm{TN}$ & 2 & 16,509 & No data & 0 & 0 & 0 & 16,509 & 0 & 16,509 \\
\hline TX & 20 & 162,940 & 1 & 25,174 & 1,096 & 614 & 189,107 & 718 & 189,825 \\
\hline UT & 1 & 0 & No data & 0 & 0 & No data & No data & 0 & 0 \\
\hline
\end{tabular}




\section{Table 6-3. Releases to the Environment from Facilities that Produce, Process, or Use o-Xylene ${ }^{\mathrm{a}}$}

\begin{tabular}{|c|c|c|c|c|c|c|c|c|c|}
\hline \multirow[b]{3}{*}{ State $^{c}$} & \multirow[b]{3}{*}{$R^{d}$} & \multicolumn{8}{|c|}{ Reported amounts released in pounds per year ${ }^{\mathrm{b}}$} \\
\hline & & \multirow[b]{2}{*}{ Air ${ }^{\mathrm{e}}$} & \multirow[b]{2}{*}{ Water ${ }^{\mathrm{f}}$} & \multirow[b]{2}{*}{$U^{g}$} & \multirow[b]{2}{*}{ Land $^{\mathrm{h}}$} & \multirow[b]{2}{*}{ Other ${ }^{i}$} & \multicolumn{3}{|c|}{ Total release } \\
\hline & & & & & & & On-site & Off-site ${ }^{k}$ & $\begin{array}{l}\text { On- and } \\
\text { off-site }\end{array}$ \\
\hline$\overline{\mathrm{WI}}$ & 1 & 1,452 & 0 & 0 & 0 & 0 & 1,452 & 0 & 1,452 \\
\hline Total & 67 & 644,883 & 9,553 & 25,256 & 1,866 & 614 & 680,941 & 1,231 & 682,172 \\
\hline
\end{tabular}

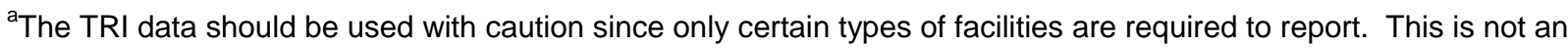
exhaustive list. Data are rounded to nearest whole number.

${ }^{b}$ Data in TRI are maximum amounts released by each facility.

${ }^{\mathrm{C}}$ Post office state abbreviations are used.

${ }^{\mathrm{d}}$ Number of reporting facilities.

${ }^{\mathrm{e}}$ The sum of fugitive and point source releases are included in releases to air by a given facility.

f Surface water discharges, waste water treatment-(metals only), and publicly owned treatment works (POTWs) (metal and metal compounds).

${ }^{\mathrm{g}}$ Class I wells, Class II-V wells, and underground injection.

${ }^{\mathrm{h}}$ Resource Conservation and Recovery Act (RCRA) subtitle C landfills; other on-site landfills, land treatment, surface impoundments, other land disposal, other landfills.

'Storage only, solidification/stabilization (metals only), other off-site management, transfers to waste broker for disposal, unknown

${ }^{\mathrm{j}}$ The sum of all releases of the chemical to air, land, water, and underground injection wells.

${ }^{\mathrm{k}}$ Total amount of chemical transferred off-site, including to POTWs.

$\mathrm{RF}=$ reporting facilities; $\mathrm{UI}=$ underground injection

Source: TRI04 2006 (Data are from 2004) 
Table 6-4. Releases to the Environment from Facilities that Produce, Process, or Use $p$-Xylene ${ }^{a}$

\begin{tabular}{|c|c|c|c|c|c|c|c|c|}
\hline \multirow[b]{3}{*}{ State $^{c}$} & \multirow[b]{3}{*}{$\mathrm{RF}^{\mathrm{d}}$} & \multicolumn{7}{|c|}{ Reported amounts released in pounds per yearb } \\
\hline & & \multirow[b]{2}{*}{ Air $^{\mathrm{e}}$} & \multirow[b]{2}{*}{ Water $^{f}$} & \multirow[b]{2}{*}{$\mathrm{UI}^{\mathrm{g}}$} & \multirow[b]{2}{*}{ Land $^{\mathrm{h}}$} & \multicolumn{3}{|c|}{ Total release } \\
\hline & & & & & & On-site & Off-site ${ }^{k}$ & On- and off-site \\
\hline$\overline{\mathrm{AL}}$ & 1 & 380,000 & 360 & 0 & 4,150 & 380,610 & 3,900 & 384,510 \\
\hline AR & 2 & 354 & 0 & 0 & 5 & 354 & 5 & 359 \\
\hline $\mathrm{CA}$ & 1 & 12,862 & No data & 0 & 0 & 12,862 & 0 & 12,862 \\
\hline GA & 1 & 11,410 & No data & 0 & 0 & 11,410 & 0 & 11,410 \\
\hline IA & 2 & 487 & No data & 0 & 0 & 487 & 0 & 487 \\
\hline IN & 1 & 2,496 & No data & 0 & 0 & 2,496 & 0 & 2,496 \\
\hline KS & 1 & 8,284 & No data & 0 & 0 & 8,284 & 0 & 8,284 \\
\hline KY & 1 & 0 & No data & No data & 0 & No data & 0 & 0 \\
\hline LA & 1 & 0 & No data & 0 & 0 & 0 & 0 & 0 \\
\hline MS & 1 & 123,000 & 25 & 0 & 503 & 123,025 & 503 & 123,528 \\
\hline NC & 3 & 1,032 & No data & 0 & 0 & 1,032 & 0 & 1,032 \\
\hline NE & 1 & 423,442 & 268 & 0 & 2,550 & 423,710 & 2,550 & 426,260 \\
\hline PA & 1 & 0 & No data & No data & 0 & No data & 0 & 0 \\
\hline PR & 1 & 18,338 & 0 & 0 & 0 & 18,338 & 0 & 18,338 \\
\hline SC & 3 & 276,970 & 0 & 0 & 0 & 276,970 & 0 & 276,970 \\
\hline TN & 2 & 24,482 & No data & 0 & 0 & 24,482 & 0 & 24,482 \\
\hline $\mathrm{TX}$ & 15 & 136,545 & 2,144 & 10,539 & 1,724 & 150,479 & 473 & 150,952 \\
\hline UT & 1 & 0 & No data & No data & 0 & No data & 0 & 0 \\
\hline Total & 39 & $1,419,702$ & 2,797 & 10,539 & 8,932 & $1,434,539$ & 7,431 & $1,441,970$ \\
\hline
\end{tabular}

${ }^{a}$ The TRI data should be used with caution since only certain types of facilities are required to report. This is not an exhaustive list. Data are rounded to nearest whole number.

${ }^{\mathrm{b}}$ Data in TRI are maximum amounts released by each facility.

${ }^{\mathrm{C}}$ Post office state abbreviations are used.

${ }^{\mathrm{d}}$ Number of reporting facilities.

${ }^{\mathrm{e}}$ The sum of fugitive and point source releases are included in releases to air by a given facility.

'Surface water discharges, waste water treatment-(metals only), and publicly owned treatment works (POTWs) (metal and metal compounds).

${ }^{\mathrm{g}}$ Class I wells, Class II-V wells, and underground injection.

${ }^{\mathrm{h}}$ Resource Conservation and Recovery Act (RCRA) subtitle C landfills; other on-site landfills, land treatment, surface impoundments, other land disposal, other landfills.

'Storage only, solidification/stabilization (metals only), other off-site management, transfers to waste broker for disposal, unknown

${ }^{\mathrm{j}}$ The sum of all releases of the chemical to air, land, water, and underground injection wells.

${ }^{\mathrm{k}}$ Total amount of chemical transferred off-site, including to POTWs.

$\mathrm{RF}=$ reporting facilities; $\mathrm{UI}=$ underground injection

Source: TRI04 2006 (Data are from 2004) 
(0.23 and $0.46 \mathrm{ppb}$ ) in background air; 910 and 2,600 $\mu \mathrm{g} / \mathrm{m}^{3}$ (209 and $600 \mathrm{ppb)} \mathrm{in} \mathrm{tipping} \mathrm{air;} \mathrm{1,600} \mathrm{and}$ $3,700 \mu \mathrm{g} / \mathrm{m}^{3}$ (370 and $850 \mathrm{ppb}$ ) in shredder air; 110 and $250 \mu \mathrm{g} / \mathrm{m}^{3}$ (25 and $58 \mathrm{ppb)} \mathrm{in} \mathrm{indoor} \mathrm{air;} 250$ and $420 \mu \mathrm{g} / \mathrm{m}^{3}$ (56 and $97 \mathrm{ppb)}$ in digester air; 250 and $520 \mu \mathrm{g} / \mathrm{m}^{3}$ (58 and $120 \mathrm{ppb)} \mathrm{in} \mathrm{fresh} \mathrm{compost} \mathrm{air;}$ 76 and $86 \mu \mathrm{g} / \mathrm{m}^{3}$ (18 and $\left.20 \mathrm{ppb}\right)$ in mid-aged compost air; 31 and $55 \mu \mathrm{g} / \mathrm{m}^{3}(7.1$ and $13 \mathrm{ppb})$ in old compost air; and 4 and $6 \mu \mathrm{g} / \mathrm{m}^{3}$ (0.92 and $\left.1.4 \mathrm{ppb}\right)$ in curing compost air.

The mean concentration of xylene in the post combustion region of a German incineration plant (prior to any emission reduction) was $7.881 \mu \mathrm{g} / \mathrm{m}^{3}$ (1.816 ppb) (Zimmermann et al. 2001). Jay and Stieglitz (1995) reported xylene concentrations of 7.77 and $1.79 \mu \mathrm{g} / \mathrm{m}^{3}(1.79$ and $0.412 \mathrm{ppb})$ in the emissions of a municipal waste incineration plant at an unspecified location in Germany. Schauer et al. (2001) reported emissions of $o$-xylene and $\mathrm{m}$-/ $p$-xylene during pine wood burning of 18.1 and $60.0 \mathrm{mg}$ per $\mathrm{kg}$ of wood burned, respectively.

$o$-Xylene was identified in the volatile emissions of a waste water treatment plant in Broward County, Florida with an emission rate of approximately $16 \mathrm{mg} / \mathrm{s}$ (1,400 g/day) (Tansel and Eyma 1999). The volatile emissions of xylenes measured in five municipal waste water treatment plants in Ontario, Canada were 770, 129, 213, 408, and $0.16 \mathrm{~g} /$ day (Quigley and Corsi 1995). The volatile emissions of total xylenes from four publicly owned treatment works servicing the Los Angeles, California area were 3,683, 215, 294, and $72 \mathrm{~kg} /$ year (Mayer et al. 1994)

Xylenes have been detected among other VOCs in the emissions of newly built homes (Hodgson et al. 2000; Zabiegala et al. 1999). The geometric mean (range) concentrations of $m$-/ $p$-xylene in the indoor air of four manufactured houses and seven site built houses in the eastern and southeastern United States were $1.0 \mathrm{ppb}(0.5-2.7 \mathrm{ppb})$ and $3.9 \mathrm{ppb}(1.4-11.5 \mathrm{ppb})$, respectively (Hodgson et al. 2000). The geometric mean (range) emission rates of $m$ - $/ p$-xylene from these houses were $3.2 \mu \mathrm{g} / \mathrm{m}^{2}$-hour $(<1.5-$ $9.1 \mu \mathrm{g} / \mathrm{m}^{2}$-hour) and $9.5 \mu \mathrm{g} / \mathrm{m}^{2}$-hour $\left(<4.1-24.2 \mu \mathrm{g} / \mathrm{m}^{2}\right.$-hour $)$, respectively. Brown et al. (1994) reported weighted average geometric mean and 98 th percentile $m$ - $/ p$-xylene concentrations of 280 and $2,700 \mu \mathrm{g} / \mathrm{m}^{3}$ (65 and $620 \mathrm{ppb}$ ), respectively, in seven new dwellings, 25 and $240 \mu \mathrm{g} / \mathrm{m}^{3}$ (5.8 and $55 \mathrm{ppb}$ ), respectively, in four new office buildings, and 9 and $85 \mu \mathrm{g} / \mathrm{m}^{3}$ (2 and $20 \mathrm{ppb}$ ), respectively, in one new school building. The concentration of $m$ - $/ p$-xylene in volatiles released from a tufted textile floor covering with styrenebutadiene rubber backing ranged from 85.1 to $155 \mathrm{ng} / \mathrm{L}$ (19.6-35.7 ppt) (Sollinger et al. 1994).

$o$-Xylene and $m-/ p$-xylene emission rates from diesel-powered medium duty trucks have been measured as 830 and 2,330 $\mu \mathrm{g} / \mathrm{km}(0.83$ and $2.33 \mathrm{mg} / \mathrm{km})$, respectively (Schauer et al. 1999). Schauer et al. (2002) 
reported tailpipe $o$-xylene and $m$-/p-xylene emission rates of 5,410 and $14,300 \mu \mathrm{g} / \mathrm{km} \mathrm{(5.41} \mathrm{and}$ $14.3 \mathrm{mg} / \mathrm{km}$ ), respectively, for catalyst-equipped gasoline-powered vehicles and 562,000 and $1,720,000 \mu \mathrm{g} / \mathrm{km}(562$ and 1,720 mg/km), respectively, for noncatalyst-equipped gasoline-powered vehicles. Mean $o$-xylene and $m$ - $/ p$-xylene tail pipe emission rates from cars that use a catalyst were $0.0042-0.0110$ and $0.0054-0.0270 \mathrm{~g} / \mathrm{km}(4.2-11$ and $5.4-27 \mathrm{mg} / \mathrm{km})$, respectively, compared to mean emission rates of 0.0330 and $0.0868 \mathrm{~g} / \mathrm{km}(33$ and $86.8 \mathrm{mg} / \mathrm{km})$, respectively, from a car that did not use a catalyst (Chan et al. 1995). These authors reported mean $o$-xylene and tail pipe emission rates of approximately $0.010 \mathrm{~g} / \mathrm{km}(10 \mathrm{mg} / \mathrm{km})$ from noncatalyst motorcycle engines and $0.016 \mathrm{~g} / \mathrm{km}(37 \mathrm{mg} / \mathrm{km})$ from a catalyst motorcycle engine. Mean $m-/ p$-xylene tail pipe emission rates were approximately $0.028 \mathrm{~g} / \mathrm{km}(28 \mathrm{mg} / \mathrm{km})$ from the noncatalyst motorcycle engines and $0.037 \mathrm{~g} / \mathrm{km}(37 \mathrm{mg} / \mathrm{km})$ from the catalyst motorcycle engine. Gabele (1997) reported that $o$-xylene and $m$-/ $p$-xylene made up 1.58 and $4.55 \%$, respectively, of total organic emissions in the exhaust of 10 four-stroke lawnmower engines.

$o$-Xylene and $m$-/ $/ p$-xylene emission values measured in the Craeybeckx tunnel in Antwerp, Belgium were 0.148 and $0.307 \mathrm{~g} / \mathrm{kg}$ carbon, respectively, under highway conditions and 0.216 and $0.442 \mathrm{~g} / \mathrm{kg}$ carbon, respectively, under congested conditions (De Fré et al. 1994). Average concentrations in the tunnel air were $17 \mu \mathrm{g} / \mathrm{m}^{3}$ (3.9 ppb) for $o$-xylene and $34 \mu \mathrm{g} / \mathrm{m}^{3}$ (7.8 ppb) for $m$ - $/ p$-xylene, compared to background concentrations of $1.0 \mu \mathrm{g} / \mathrm{m}^{3}(0.23 \mathrm{ppb})$ for $o$-xylene and $2.4 \mu \mathrm{g} / \mathrm{m}^{3}(0.55 \mathrm{ppb})$ for $m-/ p$-xylene. The authors estimated total yearly vehicle emissions in Belgium of 824 metric tons/year (1.82 million pounds/year) for $o$-xylene and 1,690 metric tons/year (3.72 million pounds/year) for $m$-/ $p$-xylene based on the congested traffic emissions data. Vehicle emission rates measured in the Los Angeles roadway tunnel in 1993 were $200 \mathrm{mg} / \mathrm{L}$ of consumed fuel for $o$-xylene and $557 \mathrm{mg} / \mathrm{L}$ of consumed fuel for $m$ - $/ p$-xylene (Fraser et al. 1998b). Lawryk and Weisel (1996) measured mean $o$-xylene and $m$-/ $p$-xylene in vehicle concentrations of 20.7 and $52.9 \mu \mathrm{g} / \mathrm{m}^{3}$ (4.77 and $12.2 \mathrm{ppb}$ ), respectively, in the Lincoln Tunnel and 12.5 and $31.4 \mu \mathrm{g} / \mathrm{m}^{3}$ (2.88 and $\left.7.47 \mathrm{ppb}\right)$, respectively, on the New Jersey Turnpike.

\subsubsection{Water}

Estimated releases of mixed xylenes, $m$-xylene, $o$-xylene, and $p$-xylene to surface water were 29,000 pounds (13 metric tons), 24,000 pounds (11 metric tons), 10,000 pounds (4.3 metric tons), and 3,000 pounds (1.3 metric tons), respectively, to the atmosphere from 2,794, 58, 67, and 39 domestic manufacturing and processing facilities, respectively, in 2004. These releases accounted for $<0.1,4.3$, 1.4 , and $<0.1 \%$ of the estimated total environmental releases of mixed xylenes, $m$-xylene, $o$-xylene, and 
p-xylene, respectively, from facilities required to report to the TRI (TRI04 2006). These releases are summarized in Tables 6-1, 6-2, 6-3, and 6-4.

Xylene has been identified in groundwater and surface water samples collected at 556 and 150 of the 840 NPL hazardous waste sites, respectively, where it was detected (HazDat 2006).

Xylenes may be introduced into groundwater by fuel oil, gasoline, or solvent spills, infiltration of polluted surface waters, leaking underground petroleum storage tanks, or leaching from disposed wastes (Giger and Schaffner 1981).

The mean concentrations of xylenes in three groundwater monitoring wells at a Shell Oil service station site in San Diego, California were 0.39, 1.23, and $19.35 \mathrm{mg} / \mathrm{L}$ (ppm) (Odermatt 1994). Groundwater extract from an oil spill site in northern Virginia contained xylene at a concentration of $2.8 \mathrm{ppm}$ (Mushrush et al. 1994).

A total xylene concentration (concentration includes ethylbenzene) of $1.2 \mathrm{ppb}$ was detected in effluent from containment ponds in the containment area of an oil spill that accumulated along the banks of the Atigun River, Alaska (Lysyj et al. 1980). Treated effluents from offshore oil drilling platforms in the Gulf of Mexico contained an average concentration of $0.3 \mathrm{mg} / \mathrm{L}(\mathrm{ppm})$ (concentration includes ethylbenzene) (Lysyj et al. 1980).

The mean influent concentrations of $o$-xylene and $m-/ p$-xylene upstream of the Metropolitan Wastewater Treatment Plant of St. Paul, Minnesota were 4.09 and $9.03 \mu \mathrm{g} / \mathrm{L}(\mathrm{ppb})$, respectively, in grab samples and 4.04 and $2.64 \mu \mathrm{g} / \mathrm{L}$ (ppb), respectively, in composite samples (Balogh et al. 1998). Final effluent from a Los Angeles County waste-water treatment plant, sampled between November 1980 and August 1981, contained $o$-xylene and $p$-xylene at concentrations of 40 and $30 \mu \mathrm{g} / \mathrm{L}(\mathrm{ppb})$, respectively (Gossett et al. 1983).

All three xylenes have been detected in the leachate from landfills at concentrations ranging from 10 to 4,400 $\mu \mathrm{g} / \mathrm{L}(\mathrm{ppb}$ ) for hazardous waste landfills and from 3.7 to $38 \mu \mathrm{g} / \mathrm{L}$ (ppb) for domestic landfills (Först et al. 1989a, 1989b). Roy (1994) reported a concentration range of $2.5-320 \mu \mathrm{g} / \mathrm{L}$ (ppb) for xylenes in municipal landfill leachate. The concentration of xylenes detected in 6 of 13 landfills sampled in Minnesota ranged from 1.3 to $950 \mu \mathrm{g} / \mathrm{L}$ (ppb) (Roy 1994). The concentration of $o$-xylene and $m-/ p$-xylene in groundwater samples collected at the Orange County Landfill in central Florida range 
from 0.04 to $38.78 \mu \mathrm{g} / \mathrm{L}$ (ppb) and from 0.10 to $3.18 \mu \mathrm{g} / \mathrm{L}$ (ppb), respectively (Chen and Zolteck 1995). The concentration of $o$-xylene and $m$-/ $p$-xylene in surface water samples at this site ranged from 0.07 to $0.09 \mu \mathrm{g} / \mathrm{L}(\mathrm{ppb})$ and from 0.07 to $0.10 \mu \mathrm{g} / \mathrm{L}(\mathrm{ppb})$, respectively.

Xylenes are also released to waterways through the use of gasoline powered outboard boat engines (Gabele 2000; Juttner 1994). The rates of release of $o$-xylene and $m$-/ $p$-xylene to water by an unleaded gasoline powered four-stroke outboard motor were 3.7 and $7.1 \mathrm{mg} /$ minute, respectively (Juttner 1994). The aqueous emission rates of xylenes from a two-stroke outboard engine and a four-stroke outboard engine range from 0.27 to $0.86 \mathrm{~g} / \mathrm{kW}$-hour $(0.075-0.24 \mathrm{~kg} / \mathrm{kJ})$ and from 0.07 to $0.09 \mathrm{~g} / \mathrm{kW}$-hour $(0.019$ $0.025 \mathrm{~kg} / \mathrm{kJ}$ ), respectively (Gabele 2000).

\subsubsection{Soil}

Estimated releases of mixed xylenes, $m$-xylene, $o$-xylene, and $p$-xylene to soil were 1.5 million pounds (700 metric tons), 4,200 pounds (1.9 metric tons), 2,900 pounds (1.3 metric tons), and 8,900 pounds (4.0 metric tons), respectively, to the atmosphere from 2,794, 58, 67, and 39 domestic manufacturing and processing facilities, respectively in 2004 . These releases accounted for $4,0.8,0.4$, and $0.6 \%$ of the estimated total environmental releases of mixed xylenes, $m$-xylene, $o$-xylene, and $p$-xylene, respectively, from facilities required to report to the TRI (TRI04 2006).

An additional 1.1 million pounds (510 metric tons) of mixed xylenes, 10,000 pounds (4.6 metric tons) of $m$-xylene, 25,000 pounds (11.4 metric tons) of $o$-xylene, and 11,000 (4.8 metric tons) of $p$-xylene, constituting about $3,1.8,3.7$, and $0.7 \%$, respectively of the total environmental emissions, were released via underground injection (TRI04 2006). These releases are summarized in Tables 6-1, 6-2, 6-3, and 6-4.

Xylene has been identified in soil and sediment samples collected at 469 and 139 of the 840 NPL hazardous waste sites, respectively, where it was detected (HazDat 2006).

No quantitative information was available in the literature regarding total releases of xylene to soil. Atmospheric xylene may reach soils either by wet deposition by precipitation or through dry deposition of material adsorbed to particulate matter in air. Xylene may also reach soils from the introduction of manmade wastes (e.g., landfills) or as a result of accidental releases (e.g., spills). 
Xylene was detected in only 4 out of 30 sediment samples collected adjacent to combined sewer overflow outfalls along the lower Passaic River in New Jersey at concentrations of $15,7,6$, and $5 \mu \mathrm{g} / \mathrm{kg}$ (ppb) (Iannuzzi et al. 1997).

\subsection{ENVIRONMENTAL FATE}

The environmental fate (transport, partitioning, transformation, and degradation) is expected to be similar for each of the xylene isomers based on the similarities of their physical and chemical properties.

\subsubsection{Transport and Partitioning}

Based on Henry's law constants of 0.005-0.007 atm- $\mathrm{m}^{3} / \mathrm{mol}$ (Foster et al. 1994; Sanemasa et al. 1982), volatilization is expected to be the dominant transport mechanism for xylenes in surface water. The halflife associated with the volatilization of $o$-xylene from surface waters at a depth of 1 meter is reported to be 5.6 hours (Mackay and Leinonen 1975). This value will vary in accordance with turbulence and water depth. Once xylenes enter the atmosphere, they undergo rapid photooxidation such that washout and long-range atmospheric transport are not expected to be important processes.

Soil organic carbon sorption coefficient $\left(\mathrm{K}_{\mathrm{oc}}\right)$ values ranging from 25.4 to 540 indicate that xylenes are mobile in soil and will not adsorb strongly to organic matter, although adsorption does increase with increasing organic matter (Green et al. 1981; Kango and Quinn 1989; Nathwani and Phillips 1977; Seip et al. 1986; Swann et al. 1983). Based on the Henry's law constants and vapor pressures (6.6-8.8 $\mathrm{mm} \mathrm{Hg}$ ) (AIChE 1996; Chao et al. 1983; Lewis 2000), xylenes that are released to soil are expected to volatilize if near the surface. However, the mobility of xylenes in soil indicates that these substances may also leach into groundwater, especially when volatilization is hindered as is the case with underground releases from gasoline storage tanks.

When xylene was spilled at an application depth of $7.2 \mathrm{~cm}$ (2.9 inches) or less on loam-textured soil at moisture contents ranging from 0.15 to $0.26 \mathrm{~kg} / \mathrm{kg}, 1-4 \%$ volatilized, $0.5-35 \%$ leached, $50-85 \%$ degraded, and 6-12\% remained after about 80 days in the soil (Aurelius and Brown 1987). Most of the observed volatilization occurred immediately after application. A greater percentage of xylene initially evaporated when it was applied to the wet soil compared to the dry soil. However, after the initial retention in the dry soil, there was less moisture to entrap the subsurface soil and prevent it from later volatilizing or degrading. The xylene that remained in the wet soil after the initial evaporation was then blocked by the moisture and was less able to volatilize or degrade. Therefore, xylene is expected to 
evaporate more rapidly when spilled onto wet soil than when spilled onto dry soil; however, removal is expected to be more complete in the dry soil after the initial retention period.

Xylene moves through unsaturated (drier) soil faster than water and other polar solvents (Amoozegar et al. 1986; Barbee and Brown 1986). For example, diffusion coefficients of approximately 0.02 and $0.005 \mathrm{~cm}^{2} /$ second were measured in dry and wet (12-15\%) cores taken from the first natural barriers at the Los Alamos National Laboratory chemical waste site (Fuentes et al. 1991). Additional field data suggesting that concentrated organics may leach 10-1,000 times faster than water in unsaturated soil were provided by Griffin et al. (1984). This increased conductivity is probably due to cracks in the soil through which the organics move rapidly (Aurelius and Brown 1987).

Measured $\log$ octanol-water partition coefficient $\left(\log \mathrm{K}_{\mathrm{ow}}\right)$ values are 2.77, 3.15, and 3.20 for $o-, p$-, and $m$-xylene, respectively (Chiou et al. 1982; Gherini et al. 1989). The rapid oxidation of xylene isomers to their corresponding polar metabolites seems to preclude bioconcentration in higher animal systems and, therefore, bioaccumulation up the food chain is unlikely (NRC 1980). Bioconcentration factors (BCFs) for $o-, m-$, and $p$-xylenes have been estimated to be 45,105 , and 95, respectively (EPA 1985a). Bioconcentration of xylene has been observed in shrimp (Pandalus platyceros) (Sanborn and Malins 1980), manila clams (Tapes semidecussata) (Nunes and Benville 1979), and eels (Anguilla japonica) (Ogata and Miyake 1978). A bioconcentration factor of 6 has been reported for tissue uptake in clams throughout an 8-day exposure to $o$-, $m$-, and $p$-xylenes (Nunes and Benville 1979), and bioconcentration factors of 21.4, 23.6, and 23.6 have been reported for eels exposed to $50 \mathrm{ppm}$ of $o_{-}^{-}, \mathrm{m}$-, and $p$-xylenes, respectively (Ogata and Miyake 1978). Tissue levels reached a steady state after 10 days. The green alga Selenastrum capricornutum has bioconcentration factors of 257, 251, and 218 for $p$-, $m$-, and $o$-xylenes, respectively (Herman et al. 1991). Bioconcentration has been predicted for all isomers of xylene because of their tendency to partition into the octanol phase of the octanol-water system (EPA 1978). Scientists generally regard BCFs below 20 as indicative of little or no bioconcentration, 20-100 as equivocal, and $<1,000$ as indicative of modest bioconcentration.

\subsubsection{Transformation and Degradation}

\subsubsection{Air}

Xylene is transformed in the atmosphere by photooxidation. Based on experimental reaction rate constants of $13.7 \times 10^{-12}-23.6 \times 10^{-12} \mathrm{~cm}^{3} /$ molecule-sec (Atkinson 1989; Kwok and Atkinson 1995), the calculated half-life of xylenes in air is $8-14$ hours using a hydroxyl radical concentration of 
$5.00 \times 10^{5}$ molecules $/ \mathrm{cm}^{3}$ and a 12-hour day (Atkinson 1990). The transformation of xylene by reaction with hydroxyl radicals prevails over that of reaction with ozone (half-life 3-78 years) and nitrate radicals (half-life 80-220 days) (Atkinson and Carter 1984; Atkinson et al. 1982, 1984, 1991; ECETOC 1986; Fox et al. 1984; Mill 1980; Roberts et al. 1984). Direct photolysis is not expected because xylene does not significantly absorb light at wavelengths $>290 \mathrm{~nm}$ (Jori et al. 1986). Major photodegradation products formed by the cleavage of the aromatic ring in the presence of nitric oxide are: $o$-tolualdehyde, methylglyoxal, 4-nitro-o-xylene, and 2,3-dimethylphenol for o-xylene; 2,6-dimethylphenol, 2,4-dimethylphenol, methylglyoxal, and $m$-tolualdehyde for $m$-xylene; and $p$-tolualdehyde and 2,5-dimethylphenol for $p$-xylene (Atkinson et al. 1991; Gery et al. 1987). Glyoxal and methylglyoxal may form approximately $30-50 \%$ of the photooxidation products (Tuazon et al. 1986). o-Xylene also forms formaldehyde, acetaldehyde, biacetyl nitrate, and peroxyacetylnitrate (Bandow and Washida 1985; Darnall et al. 1979; Shepson et al. 1984; Takagi et al. 1980). The products of photoreaction with hydroxyl radicals are ultimately degraded to carbon dioxide and water after absorption in the hydrosphere (Guisti et al. 1974).

\subsubsection{Water}

The primary removal process for xylene in surface water is expected to be volatilization. Xylenes are not expected to undergo hydrolysis, and biodegradation is not fast enough to be a competing process (Rathbun 1998). However, biodegradation is expected to an important removal process for xylenes in groundwater since volatilization is hindered under these conditions (Rathbun 1998).

Data describing the degradation of xylenes in surface waters are limited. The first-order degradation-rate coefficients for $o$-xylene and $m$-/ $p$-xylene in a strip-pit pond formed as a result of coal-mining operations were 3.6 and 6.5 day $^{-1}$, respectively, after amendment with nutrients (Bouwer 1989; Rathbun 1998). These rate coefficients correspond to half-lives of approximately 4.6 and 2.6 hours, respectively. Clapp et al. (1994) observed the biodegradation of priority pollutants in waste water treated in both an activated sludge reactor and a biological aerated filter reactor. Influent concentrations were $1.929 \mu \mathrm{g} / \mathrm{L}(\mathrm{ppb}$ ) for $o$-xylene and $5.53 \mu \mathrm{g} / \mathrm{L}(\mathrm{ppb})$ for $m$-/ $p$-xylene, while effluent and percent removal concentrations were $<1.2 \mu \mathrm{g} / \mathrm{L}(\mathrm{ppb})$ and $>38 \%$, respectively, for $o$-xylene and $<1.2 \mu \mathrm{g} / \mathrm{L}(\mathrm{ppb})$ and $>78 \%$, respectively, for $m-/ p$-xylene in both types of reactors.

The majority of xylene biodegradation studies have examined the fate of this substance in groundwater. During a field experiment conducted at Base Borden in Ontario, Canada, xylenes were degraded to 50\% 
of initial concentrations $(1,080-1,290 \mu \mathrm{g} / \mathrm{L}(\mathrm{ppb}))$ in a shallow sand aquifer in approximately $20-40$ days (Barker 1987). The authors reported similar results in aerobic laboratory biodegradation tests. Biodegradation of xylenes is expected to be slower under anaerobic conditions. The aerobic biodegradation half-lives of $o_{-}, m-$, and $p$-xylene in an unconfined, uncontaminated aquifer after injection with 100\% PS6 gasoline were 116, 70, and 100 days, respectively (API 1994). The gasoline was injected above a landfill leachate zone. Although biodegradation of petroleum hydrocarbons (including xylene) spilled in northern Alaska was observed, the authors concluded that this process is likely to be limited by a short annual thaw season (Braddock and McCarthy 1996).

The half-life of xylenes measured in groundwater from an anaerobic aquifer in Seal Beach, California was approximately 25 days (Beller et al. 1995). Xylene degradation metabolites observed by these authors included the methylated homologs of benzylsuccinic acid, benzylfumaric acid, and E-phenylitaconate (Beller 2000; Beller et al. 1995). o-Xylene was anaerobically degraded by approximately $75-100 \%$ 30 days in groundwater after it was injected into contaminated aquifers (Reusser et al. 2002). Detection of methylbenzylsuccinic acid in the same wells following injection was additional evidence of xylene biodegradation. Between 92 and $95 \%$ of xylene released from a gasoline spill at an underground storage tank site was degraded over a distance of $80 \mathrm{~m}$ from the spill location (Kao and Wang 2001). The major removal process was biodegradation under iron-reducing conditions. Thierrin et al. (1995) measured a half-life of 125-170 days for xylenes under anoxic groundwater conditions. The first-order anaerobic biodegradation rate constants for $o-, m$-, and $p$-xylene were $0.028-0.11,0.026-0.10$, and $0.017-$ 0.067 week $^{-1}$ (corrected for dilution), respectively, in an underground gasoline storage tank spill plume at the Sleeping Bear Dunes National Lakeshore in Michigan. These rate constants correspond to half-lives of 7-25 weeks for $o$-xylene, 7-27 weeks for $m$-xylene, and 10-41 weeks for $p$-xylene (Wilson et al. 1994). Hunt et al. (1997) did not observe anaerobic biodegradation of xylenes at Sleeping Bear Dunes National Lakeshore and reported that this process may be hindered by the presence of acetate and other non-BTEX organic carbon. These authors did observe anaerobic biodegradation of xylenes in groundwater at a site near Rocky Point, North Carolina. Weidemeier et al. (1995a) concluded that BTEX was biodegraded both aerobically and anaerobically at two U.S. air force bases (AFB) (Hill AFB and Patrick AFB) with sulfite reduction being the primary removal mechanism at Hill AFB and methanogenesis being the primary removal mechanism at Patrick AFB. Greater than $99 \%$ of xylene at the Hill AFB site was degraded after traveling $305 \mathrm{~m}$ downgradient from the contamination source. Only $38 \%$ of xylene ( $34 \%$ o-xylene, $60 \%$ m-xylene, and $21 \% p$-xylene) at the Patrick AFB site was degraded after traveling $98 \mathrm{~m}$ downgradient from the contamination source. Differences in the degradation rates 
and half-lives reported in these studies are most likely due to differences in study conditions such as temperature, presence of oxygen, and presence of electron acceptors.

\subsubsection{Sediment and Soil}

If xylene is released to soil, the dominant removal process is expected to be volatilization. However, in situations where volatilization is hindered, biodegradation is expected to be an important fate process.

$o$-Xylene in vials containing unsaturated soil was biodegraded within 5 days after a lag period of 10 days (English and Loehr 1991). However, subsequent additions of $o$-xylene to the soil were degraded without a lag period. The half-life after the sixth addition to the soil was less than 3 hours. $o$ - and $p$-Xylene applied to a soil microcosm at $3 \mu \mathrm{L} / \mathrm{g}(2.6 \mathrm{mg} / \mathrm{g})$ were aerobically degraded in less than 25 days (Tsao et al. 1998). $\mathrm{CO}_{2}$ production measured during the experiment ranged from approximately $35-65 \%$. p-Xylene, at an initial concentration of $45 \mathrm{ppm}$, was completely degraded in natural potting soil microcosm in less than 27 days at $20^{\circ} \mathrm{C}$ (Eriksson et al. 1999). It was not degraded at $6{ }^{\circ} \mathrm{C}$.

Elshahed et al. (2001) reported that xylenes were degraded anaerobically in an aquifer microflora laboratory test under sulfate-reducing conditions, but not under methanogenic conditions. $m$-Xylene was completely degraded in less than 25 days under sulfate-reducing conditions. The authors proposed that the successive metabolites involved in the ultimate anaerobic degradation of xylenes are methylbenzylsuccinic acid, toluic acid, phthalic acid, and benzoic acid (finally degraded to carbon dioxide).

The first-order biodegradation rate constants for xylene calculated from data collected at a gasoline spill site in Beaufort, South Carolina ranged from 0.10 to $0.31 \mathrm{~d}^{-1}$ near the water table (Lahvis et al. 1999). These rates correspond to half-lives of 2-7 days. Sovik et al. (2002) calculated first-order biodegradation rate constants of $0.1-0.11 \mathrm{~d}^{-1}$ for xylene applied to a lysimeter trench at the Gardermoen delta north of Oslo, Norway. These rates correspond to half-lives of 6-7 days.

Total VOCs (including xylene) added to sewage sludge-amended fields were reduced to a concentration similar to that in control fields within 36 days in a plowed plot and within 8 days in a pasture plot (Wilson and Jones 1996; Wilson et al. 1997). The half-lives calculated for $o$-xylene and $m$-/ $p$-xylene were 7.6 and 5.8 days, respectively, in the plowed plot and 0.7 and 0.3 days, respectively, in the pasture plot. During a laboratory soil study conducted by these authors, $92.6 \%$ of the total VOC concentration was lost after 65 days from an unsterilized treatment compared to $88.1 \%$ loss from a sterilized treatment. This indicates 
that abiotic factors, such as volatilization, are primarily responsible for this degradation, with biodegradation accounting for approximately $5 \%$ of the loss.

\subsection{LEVELS MONITORED OR ESTIMATED IN THE ENVIRONMENT}

Reliable evaluation of the potential for human exposure to xylene depends in part on the reliability of supporting analytical data from environmental samples and biological specimens. Concentrations of xylene in unpolluted atmospheres and in pristine surface waters are often so low as to be near the limits of current analytical methods. In reviewing data on xylene levels monitored or estimated in the environment, it should also be noted that the amount of chemical identified analytically is not necessarily equivalent to the amount that is bioavailable. The analytical methods available for monitoring xylene in a variety of environmental media are detailed in Chapter 7.

\subsubsection{Air}

Since one of the largest sources of xylene release into the atmosphere is auto emissions, atmospheric concentrations are related to urbanization. Ambient air concentrations of xylene in industrial and urban areas of the United States have been reported to range from 0.003 to $0.38 \mathrm{mg} / \mathrm{m}^{3}$ (1-88 ppb) (Merian and Zander 1982). Median $o$-xylene concentrations calculated from a compilation of atmospheric data on organic chemicals were $0.41 \mu \mathrm{g} / \mathrm{m}^{3}$ (0.094 ppb) in rural/remote areas (114 observations), $5.2 \mu \mathrm{g} / \mathrm{m}^{3}$ (1.2 ppb) in urban/suburban areas (1,885 observations), and $3.5 \mu \mathrm{g} / \mathrm{m}^{3}(0.81 \mathrm{ppb})$ in source-dominated areas (183 observations) (EPA 1983). The median concentrations for the combined $m$ - and $p$-isomers were $0.38 \mu \mathrm{g} / \mathrm{m}^{3}(0.088 \mathrm{ppb})$ in rural $/$ remote areas (115 observations), $12 \mu \mathrm{g} / \mathrm{m}^{3}$ (2.8 ppb) in urban/suburban areas (1,911 observations), and $7.4 \mu \mathrm{g} / \mathrm{m}^{3}$ (1.7 ppb) in source-dominated areas (186 observations) (EPA 1983). Air samples in highly industrialized areas of Illinois (Chicago and East St. Louis) between 1986 and 1990 had mean concentrations of 3.9-16 $\mu \mathrm{g} / \mathrm{m}^{3}$ (0.90-3.7 ppb) (for $m$ - and $p$-xylene combined) and 2.9-3.3 $\mu \mathrm{g} / \mathrm{m}^{3}(0.67-0.76 \mathrm{ppb})$ (for $o$-xylene and styrene combined). Levels in rural areas of Illinois were $1.2 \mu \mathrm{g} / \mathrm{m}^{3}(0.28 \mathrm{ppb})$ (for $m$ - and $p$-xylene combined) and $1.1 \mu \mathrm{g} / \mathrm{m}^{3}(0.25 \mathrm{ppb})$ (for $o$-xylene and styrene combined). Paints (including automobile paint), chemical plants, and vehicle exhaust were the major sources of these chemicals (Sweet and Vermette 1992). Concentrations of xylenes measured in outdoor air at various locations are listed in Table 6-5. Based on these data, typical xylene concentrations in outdoor air range from 1 to $30 \mathrm{ppb}$.

Air samples collected at 12 cities around the United States between 1979 and 1984 contained average concentrations of 1.0-10.2 ppb for $m-/ p$-xylene and 0.3-4.2 ppb for $o$-xylene (Singh et al. 1985). In 
Table 6-5. Xylene Concentrations in Outdoor Air

\begin{tabular}{|c|c|c|c|c|c|}
\hline \multirow[b]{2}{*}{ Location } & \multirow{2}{*}{$\begin{array}{l}\text { Type of } \\
\text { value }\end{array}$} & \multicolumn{3}{|c|}{ Concentration (ppb) } & \multirow[b]{2}{*}{ Reference } \\
\hline & & o-Xylene & $m-/ p$-Xylene & Total xylenes $^{a}$ & \\
\hline \multicolumn{6}{|l|}{ Urban } \\
\hline $\begin{array}{l}\text { Urban/suburban } \\
\text { United States }\end{array}$ & Median & 1.2 & 2.8 & 4 & EPA 1983 \\
\hline $\begin{array}{l}\text { Industrial/Urban } \\
\text { United States }\end{array}$ & Range & - & - & $1-88^{b}$ & $\begin{array}{l}\text { Merian and Zander } \\
1982\end{array}$ \\
\hline $\begin{array}{l}\text { Urban } 12 \text { U.S. } \\
\text { cities }\end{array}$ & Mean & $0.3-4.2$ & $1.0-10.2$ & - & Singh et al. 1985 \\
\hline 39 U.S. cities & Range & $0.9-79$ & $1.3-338$ & $7.2^{\mathrm{C}}$ & EPA 1989 \\
\hline 39 U.S. cities & Median & 8.0 & 20.0 & 28.0 & Mohamed et al. 2002 \\
\hline 9 major U.S. cities & Median & $0.3-3.1$ & $0.7-8.6$ & - & Mohamed et al. 2002 \\
\hline $\begin{array}{l}\text { Chicago, East St. } \\
\text { Louis }\end{array}$ & Mean & $0.67-0.76^{d}$ & $0.9-3.7$ & - & $\begin{array}{l}\text { Sweet and Vermette } \\
1992\end{array}$ \\
\hline Los Angeles & Range & $4-13$ & $11-45$ & - & $\begin{array}{l}\text { Grosjean and Fung } \\
1984\end{array}$ \\
\hline Las Vegas & Range & $0-1.1$ & $0-0.46$ & - & Tran et al. 2000 \\
\hline Phoenix & Mean & 1.07 & 2.92 & 3.99 & Zielinska et al. 1998 \\
\hline Tucson & Mean & 0.46 & 1.21 & 1.67 & Zielinska et al. 1998 \\
\hline Columbus, Ohio & Mean & 0.31 & 0.82 & 1.1 & Spicer et al. 1996 \\
\hline \multicolumn{6}{|l|}{ Rural } \\
\hline $\begin{array}{l}\text { Rural } \\
\text { United States }\end{array}$ & Median & 0.094 & 0.088 & 0.18 & EPA 1983 \\
\hline Rural Illinois & Mean & $0.25^{d}$ & 0.28 & $0.53^{d}$ & $\begin{array}{l}\text { Sweet and Vermette } \\
1992\end{array}$ \\
\hline Rural Arizona & Mean & $0.30,0.43$ & $0.82,1.13$ & $1.12,1.56$ & Zielinska et al. 1998 \\
\hline \multicolumn{6}{|l|}{ Mixed Locations } \\
\hline $\begin{array}{l}25 \text { sites in } \\
\text { Minnesota }\end{array}$ & Mean & 0.18 & $1.18^{\mathrm{e}}$ & 1.36 & Pratt et al. 2000 \\
\hline $\begin{array}{l}\text { Southern } \\
\text { California }\end{array}$ & Mean & 1.7 & 4.5 & 6.2 & Fraser et al. 2000 \\
\hline $\begin{array}{l}\text { Approximately } \\
100 \text { U.S. locations }\end{array}$ & Median & 0.51 & $1.96^{\mathrm{e}}$ & 0.51 & $\begin{array}{l}\text { EPA 1993; Kelly et al. } \\
1994\end{array}$ \\
\hline \multicolumn{6}{|l|}{ Remote } \\
\hline Remote Arizona & Mean & 0.02 & 0.03 & 0.05 & Zielinska et al. 1998 \\
\hline North Atlantic & Mean & 0.003 & $0.0068^{\mathrm{e}}$ & 0.0098 & Eichmann et al. 1979 \\
\hline Indian Ocean & Mean & 0.00021 & $0.00028^{\mathrm{e}}$ & 0.00049 & Eichmann et al. 1980 \\
\hline Pacific Ocean & Range & $0.01-0.77$ & $<0.05-0.31$ & - & $\begin{array}{l}\text { Greenberg and } \\
\text { Zimmerman } 1984\end{array}$ \\
\hline
\end{tabular}


Table 6-5. Xylene Concentrations in Outdoor Air

\begin{tabular}{llllll}
\hline & Type of & \multicolumn{3}{c}{ Concentration $(\mathrm{ppb})$} \\
\cline { 3 - 5 } Location & value & $o-$ Xylene & $m-/ p-$ Xylene & Total xylenes ${ }^{\mathrm{a}}$ & Reference \\
\hline Brazilian & Range & - & - & $<0.05-0.12^{\mathrm{b}}$ & Greenberg and \\
Rainforest & & & & & Zimmerman 1984 \\
\hline
\end{tabular}

a Unless otherwise indicated, total xylene concentrations listed here are the sum of the $o$-xylene and $m$ - $/ p$-xylene concentrations.

${ }^{\mathrm{b}}$ Range of total xylenes reported in source

cMedian total xylenes concentration reported in source

dIncludes styrene

${ }^{\text {e }}$ Sum of $m$ - and $p$-xylene concentrations (reported separately in source) 
1981, atmospheric concentrations of $m$-/ $p$-xylene and $o$-xylene, measured at a downtown Los Angeles location, were 11-45 and 4-13 ppbv, respectively (Grosjean and Fung 1984). Between 1984 and 1986, ambient air monitoring was conducted in 39 cities nationwide. All three isomers of xylene were detected in all cities at a median concentration of 7.2 ppb (range, 1.3-338 ppb for $m-/ p$-xylene and 0.9-79 ppb for $o$-xylene) (EPA 1989). In 1990, o-xylene was measured at $4.2-6.9 \mu \mathrm{g} / \mathrm{m}^{3}(0.97-1.6 \mathrm{ppb})$ in ambient outdoor air in Atlanta, Georgia (EPA 1991a).

$o$-Xylene and $m$-/ $p$-xylene were detected in ambient air at Las Vegas, Nevada at concentrations of 0 4.6 and $0-2.0 \mu \mathrm{g} / \mathrm{m}^{3}(0-1.1$ and $0-0.46 \mathrm{ppb})$, respectively (Tran et al. 2000). Trost et al. (1997) reported urban air concentrations of $o$-xylene and $m-/ p$-xylene of 0.5 and $1.2 \mathrm{ppbv}$, respectively, in Boston, Massachusetts, 0.5 and 0.9 ppbv, respectively, in Chicago, Illinois, 1.1 and 2.9 ppbv, respectively, in Los Angeles, California, and 18 and 38 ppbv, respectively, in Houston, Texas. Mean concentrations of $o$-xylene and $m$-/p-xylene were 1.07 and $2.92 \mathrm{ppbv}$, respectively, in urban air from Phoenix, Arizona, 0.46 and $1.21 \mathrm{ppbv}$, respectively, in urban air from Tucson, Arizona, 0.30 and $0.82 \mathrm{ppbv}$, respectively, in rural air from Payson, Arizona, 0.43 and 1.13 ppbv, respectively, in rural air from Casa Grande, Arizona, and 0.02 and $0.03 \mathrm{ppbv}$, respectively in remote mountain top air near Hillside, Arizona (Zielinska et al. 1998). The mean (maximum) concentrations of $o$-xylene and $m$-/ $p$-xylene in 142 air samples collected in the Columbus, Ohio area were $0.31 \mathrm{ppb}(1.22 \mathrm{ppb})$ and $0.82 \mathrm{ppb}(3.88 \mathrm{ppb})$, respectively (Spicer et al. 1996). Concentrations of xylenes in snow samples collected in Denver, Colorado during February, 1995 were $<10,86,47,32$, and $38 \mathrm{ng} / \mathrm{L}$ (Bruce and McMahon 1996). Mohamed et al. (2002) reported median $o$-xylene and $m$-/p-xylene concentrations of 8.0 and $20.0 \mathrm{ppb}$, respectively, measured in the air of 39 U.S. cities. Median $o$-xylene and $m$ - $/ p$-xylene concentrations reported for nine major U.S. cities were $0.3-$ 3.1 and $0.7-8.6 \mathrm{ppb}$, respectively.

The mean and median concentrations of xylenes in air samples collected at 25 sites across Minnesota were 0.79 and $0.5 \mu \mathrm{g} / \mathrm{m}^{3}$ (0.18 and $\left.0.12 \mathrm{ppb}\right)$, respectively, for $o$-xylene, 2.94 and $1.87 \mu \mathrm{g} / \mathrm{m}^{3}$ (0.686 and $\left.0.43 \mathrm{ppb}\right)$, respectively, for $m$-xylene, and 2.11 and $1.36 \mu \mathrm{g} / \mathrm{m}^{3}(0.49$ and $0.31 \mathrm{ppb})$, respectively, for $p$-xylene (Pratt et al. 2000). The observed ambient mean concentrations of $o$-xylene and $m-/ p$-xylene in Southern California based on data from Los Angeles, Azusa, and Claremont, California were 7.3 and $19.6 \mu \mathrm{g} / \mathrm{m}^{3}(1.7$ and $4.5 \mathrm{ppb}$ ), respectively (Fraser et al. 2000). EPA (1993) and Kelly et al. (1994) reported the ambient air concentrations of $o^{-}, \mathrm{m}$-, and $p$-xylene that were measured in 104, 98, and 102 U.S. locations, respectively. Median concentrations were $2.2 \mu \mathrm{g} / \mathrm{m}^{3}(0.51 \mathrm{ppb})$ in 8,542 samples for $o$-xylene, $4.2 \mu \mathrm{g} / \mathrm{m}^{3}(0.97 \mathrm{ppb})$ in 8,431 samples for $m$-xylene, and $4.3 \mu \mathrm{g} / \mathrm{m}^{3}(0.99 \mathrm{ppb})$ in 3,597 samples for $p$-xylene. 
Mean concentrations of $o$-xylene and $m$-/ $p$-xylene measured in vehicle tunnels were 61.79 and $148.04 \mathrm{ppb}$ carbon, respectively, in the Caldecott tunnel of San Francisco, California, 17 and $34 \mu \mathrm{g} / \mathrm{m}^{3}$ (3.9 and $7.8 \mathrm{ppb})$, respectively, in the Craeybeckx tunnel in Antwerp, Belgium, 39.6 and $69.8 \mu \mathrm{g} / \mathrm{m}^{3}(9.12$ and $16.1 \mathrm{ppb})$, respectively, in the Zefun tunnel of Taipei, Taiwan, and 74.4 and $104.0 \mu \mathrm{g} / \mathrm{m}^{3}(17.1$ and $24.0 \mathrm{ppb}$ ) respectively, in the Lishin motorcycle tunnel of Taipei, Taiwan (De Fré et al. 1994; Hsieh et al. 1999; Zielinska and Fung 1994).

Mean in-vehicle concentrations of $o$-xylene and $m$-/ $p$-xylene reported by Leung and Harrison (1999) for several locations in the United States were 2.5 and $2.7 \mathrm{ppb}$, respectively, in Birmingham, Alabama, 1.7 and 4.8 ppb, respectively, during the winter in Boston, Massachusetts, 2.6 and $7.1 \mathrm{ppb}$, respectively, during the summer in Raleigh, North Carolina, and 2.1 and $5.5 \mathrm{ppb}$, respectively, during the winter in New Jersey. The mean and range of xylene concentrations measured in the cabins of automobiles with gasoline engines in Germany were 6.2 and $1.4-22.6 \mu \mathrm{g} / \mathrm{m}^{3}$ (1.4 and 0.3-5.2 ppb), respectively, for $o$-xylene and 19.1 and $3.9-66.3 \mu \mathrm{g} / \mathrm{m}^{3}$ (4.4 and 0.9-15 ppb), respectively, for $m$-/ $p$-xylene (Ilgen et al. 2001c). The mean and range of xylene concentrations in the cabins of automobiles with diesel engines were 2.7 and $1.0-5.9 \mu \mathrm{g} / \mathrm{m}^{3}$ (0.6 and $0.2-1.4 \mathrm{ppb}$ ), respectively, for 0 -xylene and 7.9 and $3.2-15.2 \mu \mathrm{g} / \mathrm{m}^{3}$ (1.8 and 0.7-3.5 ppb), respectively, for $m-/ p$-xylene. Mean in-vehicle concentrations during suburban New Jersey-New York City commutes were $10.1 \mu \mathrm{g} / \mathrm{m}^{3}(2.33 \mathrm{ppb})$ for $o$-xylene and $22.5 \mu \mathrm{g} / \mathrm{m}^{3}$ (5.18 ppb) for $m$ - $/ p$-xylene with high ventilation and $14.5 \mu \mathrm{g} / \mathrm{m}^{3}$ (3.34 ppb) for $o$-xylene and $34.6 \mu \mathrm{g} / \mathrm{m}^{3}$ $(7.97 \mathrm{ppb})$ for $m-/ p$-xylene with low ventilation. The mean (range) xylene concentration measured in the air inside patrol cars was $4.5 \mathrm{ppb}(1.4-12.1 \mathrm{ppb})$ compared to a concentration of 1.0 ppb (0.04-2.4 ppb) in air at an ambient site and 1.0 ppb (0.4-1.9 ppb) in roadside air (Riediker et al. 2003).

Chan et al. (1994) reported the concentrations of VOCs that commuters were exposed to in Taipei, Taiwan during the spring of 1992. The mean, median, and maximum concentrations of $o$-xylene were 95, 90 , and $265 \mu \mathrm{g} / \mathrm{m}^{3}(22,21$, and $61 \mathrm{ppb})$, respectively, in the bus, 161, 140, and $405 \mu \mathrm{g} / \mathrm{m}^{3}$ (37, 32, and $93 \mathrm{ppb})$, respectively, in the car, and 244, 225, and $467 \mu \mathrm{g} / \mathrm{m}^{3}(56,52$, and $108 \mathrm{ppb})$, respectively, on a motorcycle. The mean, median, and maximum concentrations of $m-/ p$-xylene were 149,141 , and $480 \mu \mathrm{g} / \mathrm{m}^{3}$ (34, 32, and $111 \mathrm{ppb}$ ), respectively, in the bus, 240, 212, and $479 \mu \mathrm{g} / \mathrm{m}^{3}$ (55, 49, and $110 \mathrm{ppb}$ ), respectively, in the car, and 362, 350, and $698 \mu \mathrm{g} / \mathrm{m}^{3}$ (83, 81, and $\left.161 \mathrm{ppb}\right)$, respectively, on a motorcycle.

Jo and Choi (1996) reported higher concentrations of xylenes in urban vehicles compared to suburban vehicles during commutes in Korea. Mean, median, and maximum concentrations of $o$-xylene were 22.8, 17.7 , and $90.1 \mu \mathrm{g} / \mathrm{m}^{3}(5.2,4.1$, and $21 \mathrm{ppb})$, respectively, in urban automobiles and 16.6, 12.4 and 
$65.6 \mu \mathrm{g} / \mathrm{m}^{3}(3.8,2.9$, and $15 \mathrm{ppb})$, respectively, in urban buses. Mean, median, and maximum concentrations of $m-/ p$-xylene were $28.9,23.2$, and $82.7 \mu \mathrm{g} / \mathrm{m}^{3}(6.7,5.3$, and $19 \mathrm{ppb})$, respectively, in urban automobiles and 23.1, 16.3, and $64.3 \mu \mathrm{g} / \mathrm{m}^{3}(5.3,3.8$, and $15 \mathrm{ppb})$, respectively, in urban buses. Mean, median, and maximum concentrations of $o$-xylene were $13.1,11.0$, and $33.9 \mu \mathrm{g} / \mathrm{m}^{3}(3.0,2.5$, and $7.8 \mathrm{ppb})$, respectively, in suburban automobiles and $9.5,7.5$, and $36.4 \mu \mathrm{g} / \mathrm{m}^{3}(2.2,1.7$, and $8.4 \mathrm{ppb}$ ), respectively, in suburban buses. Mean, median, and maximum concentrations of $m-/ p$-xylene were 18.6, 14.9 , and $47.0 \mu \mathrm{g} / \mathrm{m}^{3}(4.3,3.4$, and $11 \mathrm{ppb})$, respectively, in suburban automobiles and $12.5,9.8$, and $34.2 \mu \mathrm{g} / \mathrm{m}^{3}(2.9,2.3$, and $7.9 \mathrm{ppb})$, respectively, in urban buses.

Xylene has also been detected in remote locations. Average background levels of $o-, m-$, and $p$-xylene, measured over the North Atlantic in 1977, were 13, 21, and $9.2 \mathrm{ng} / \mathrm{m}^{3}(0.003,0.0048$, and $0.002 \mathrm{ppb})$, respectively (Eichmann et al. 1979). Similar measurements over the Indian Ocean gave average concentrations of $0.9,1.1$, and $0.15 \mathrm{ng} / \mathrm{m}^{3}(0.21,0.25$, and $0.03 \mathrm{ppt})$, respectively (Eichmann et al. 1980), while over the Pacific Ocean, concentrations ranged from below the detection limit ( $0.05 \mathrm{ppbv})$ to $0.31 \mathrm{ppbv}$ for $m$ - and $p$-xylene and from 0.01 to $0.77 \mathrm{ppbv}$ for $o$-xylene (Greenberg and Zimmerman 1984). All three xylene isomers were detected in the air of the Brazilian rainforest in 1979-1980, at concentrations ranging from below the detection limit $(0.05 \mathrm{ppbv})$ to $0.12 \mathrm{ppbv}$ (Greenberg and Zimmerman 1984). The concentrations of $o$-xylene and the combination of $m$ - and $p$-xylene measured in Antarctic snow collected near the coast of the Ross Sea ranged from 1 to $80 \mathrm{ng} / \mathrm{L}$ and from 8 to $198 \mathrm{ng} / \mathrm{L}$, respectively (Desideri et al. 1994, 1998). It is unlikely that xylenes were carried to these locations by long-range atmospheric transport since the half-life of these substances in air is $8-14$ hours. One explanation given for the presence of xylenes in the remote oceanic locations was that they were being released in marine aerosols from contaminated ocean water (Desideri et al. 1994, 1998; Eichmann et al. 1979, 1980). However, sources of this marine contamination were not identified.

Recent studies have indicated that xylene is also a common but low contaminant of indoor air both at home and in the workplace. Xylene concentrations measured in indoor air are listed in Table 6-6. Based on these data, the concentrations of xylene in indoor air typically range from 1 to $10 \mathrm{ppb}$.

Concentrations of $m$ - and $p$-xylene measured in homes at 15 locations in the United States ranged from 10 to $47 \mu \mathrm{g} / \mathrm{m}^{3}$ (2.3-10.8 ppb) (Seifert and Abraham 1982). Similar results were reported during a 1981 study of the correlation between breath concentration and personal and outdoor air concentrations of 350 New Jersey residents (Wallace et al. 1986). The weighted median indoor air concentrations of $o$-xylene and the combined $m$ - and $p$-xylene isomers were 4.9 and $14 \mu \mathrm{g} / \mathrm{m}^{3}(1.1$ and $3.2 \mathrm{ppb})$, 
Table 6-6. Xylene Concentrations in Indoor Air

\begin{tabular}{|c|c|c|c|c|c|}
\hline \multirow[b]{2}{*}{ Location } & \multirow[b]{2}{*}{$\begin{array}{l}\text { Type of } \\
\text { value }\end{array}$} & \multicolumn{3}{|c|}{ Concentration (ppb) } & \multirow[b]{2}{*}{ Reference } \\
\hline & & o-Xylene & $\begin{array}{l}m-/ p- \\
\text { Xylene }\end{array}$ & $\begin{array}{l}\text { Total } \\
\text { xylenes }^{a}\end{array}$ & \\
\hline \multicolumn{6}{|l|}{ U.S. Locations } \\
\hline 15 homes & Range & - & $2.3-10.8$ & - & $\begin{array}{l}\text { Seifert and Abraham } \\
1982\end{array}$ \\
\hline $\begin{array}{l}\text { Homes of } 350 \text { New Jersey } \\
\text { residents }\end{array}$ & Median & 1.1 & 3.2 & 4.3 & Wallace et al. 1986 \\
\hline $\begin{array}{l}\text { Homes of } 36 \text { students in } \\
\text { New York City }\end{array}$ & & & & & Kinney et al. 2002 \\
\hline Summer & Mean & 0.52 & 1.48 & 2.0 & \\
\hline Winter (30 students) & Mean & 0.77 & 2.40 & 3.2 & \\
\hline $\begin{array}{l}\text { Library of Congress in } \\
\text { Washington, DC }\end{array}$ & Mean & 0.7 & $1.7^{\mathrm{b}}$ & $2.4^{\mathrm{b}}$ & NIOSH 1990a \\
\hline 50 "normal" houses & Median & 0.41 & $1.3^{\mathrm{b}}$ & $1.71^{\mathrm{b}}$ & Kostiainen 1995 \\
\hline 7 "sick" houses & Median & 0.77 & $2.2^{b}$ & $2.97^{b}$ & Kostiainen 1995 \\
\hline "Sick" building & Range & $0.99-1.6$ & $2.8-5.1$ & - & Weschler et al. 1990 \\
\hline $\begin{array}{l}\text { Office buildings in United } \\
\text { States }\end{array}$ & & & & & Shields et al. 1996 \\
\hline $\begin{array}{l}50 \text { telecommunications } \\
\text { offices }\end{array}$ & $\begin{array}{l}\text { Geometric } \\
\text { mean }\end{array}$ & $0.65^{\mathrm{C}}$ & 0.99 & $1.64^{\mathrm{C}}$ & \\
\hline 9 data centers & $\begin{array}{l}\text { Geometric } \\
\text { mean }\end{array}$ & $1.1^{\mathrm{c}}$ & 1.5 & $2.6^{\mathrm{c}}$ & \\
\hline $\begin{array}{l}11 \text { administrative } \\
\text { offices }\end{array}$ & $\begin{array}{l}\text { Geometric } \\
\text { mean }\end{array}$ & $0.81^{\mathrm{c}}$ & 1.11 & $1.92^{\mathrm{C}}$ & \\
\hline Canadian homes & & & & & Fellin and Otson 1994 \\
\hline Winter (185 homes) & Mean & 0.94 & $3.72^{d}$ & 4.66 & \\
\hline Spring (178 homes) & Mean & 1.59 & $4.73^{d}$ & 6.32 & \\
\hline Summer (197 homes) & Mean & 0.70 & $2.43^{d}$ & 3.13 & \\
\hline Fall (194 homes) & Mean & 1.89 & $8.10^{d}$ & 9.99 & \\
\hline U.K. Indoor locations & & & & & Kim et al. 2001 \\
\hline 64 homes & Mean & 0.44 & $1.64^{d}$ & 2.08 & \\
\hline 12 offices & Mean & 0.41 & $1.08^{d}$ & 1.49 & \\
\hline 6 restaurants, 6 pubs & Mean & $1.4,1.6$ & $5.1,5.7^{d}$ & $6.5,7.3$ & \\
\hline $\begin{array}{l}8 \text { department stores, } \\
6 \text { cinemas, } 3 \text { perfume } \\
\text { shops }\end{array}$ & Mean & $\begin{array}{l}0.81,1.4 \\
0.55\end{array}$ & $\begin{array}{l}2.81,4.9 \\
1.91^{\mathrm{d}}\end{array}$ & $\begin{array}{l}3.62,6.3 \\
2.46\end{array}$ & \\
\hline 6 libraries & Mean & 0.76 & $2.81^{d}$ & 3.57 & \\
\hline 8 labs & Mean & 0.18 & $0.58^{d}$ & 0.76 & \\
\hline
\end{tabular}


Table 6-6. Xylene Concentrations in Indoor Air

\begin{tabular}{|c|c|c|c|c|c|}
\hline \multirow[b]{2}{*}{ Location } & \multirow[b]{2}{*}{$\begin{array}{l}\text { Type of } \\
\text { value }\end{array}$} & \multicolumn{3}{|c|}{ Concentration (ppb) } & \multirow[b]{2}{*}{ Reference } \\
\hline & & o-Xylene & $\begin{array}{l}m-/ p- \\
\text { Xylene }\end{array}$ & $\begin{array}{l}\text { Total } \\
\text { xylenes }^{a}\end{array}$ & \\
\hline German homes & & & & & Ilgen et al. 2001 \\
\hline Rural homes (summer) & Mean & 0.16 & 0.744 & 0.904 & \\
\hline Rural homes (winter) & Mean & 0.36 & 1.1 & 1.46 & \\
\hline Urban homes (summer) & Mean & 0.36 & 1.09 & 1.45 & \\
\hline Urban homes (winter) & Mean & 0.64 & 1.91 & 2.55 & \\
\hline
\end{tabular}

${ }^{a}$ Unless otherwise indicated, total xylene concentrations listed here are the sum of the $o$-xylene and $m-/ p$-xylene concentrations.

${ }^{\mathrm{b}}$ Does not include $m$-xylene concentration because it was not reported in the source.

"Includes styrene

${ }^{\mathrm{d}}$ Sum of $m$ - and $p$-xylene concentrations (reported separately in source) 
respectively. Breath concentrations showed significant correlation with personal air concentrations but only weak correlation with outdoor air concentrations. Concentrations in indoor air were usually higher than in outdoor air, indicating that the source of the xylene was building materials or household products (e.g., cleaning agents) (Wallace et al. 1986, 1987b). However, in areas where heavy automotive traffic has increased outdoor xylene concentrations, ventilating homes by leaving windows open may actually result in additional increases in indoor xylene levels (Hung and Liao 1991). That indoor sources of xylene contribute significantly to the levels of these chemicals in interior spaces was also demonstrated in an indoor air monitoring study conducted in February and March 1989 on the premises of the Library of Congress in Washington, DC. $p$-Xylene and $o$-xylene were present at mean levels of $7.2 \mu \mathrm{g} / \mathrm{m}^{3}(1.7 \mathrm{ppb})$ and $3.2 \mu \mathrm{g} / \mathrm{m}^{3}(0.7 \mathrm{ppb})$ in indoor air and $3.2 \mu \mathrm{g} / \mathrm{m}^{3}(0.7 \mathrm{ppb})$ and $1.2 \mu \mathrm{g} / \mathrm{m}^{3}(0.3 \mathrm{ppb})$ in outdoor air, respectively (NIOSH 1990a).

Analysis of a building with "sick building syndrome" in the summer of 1987 showed significant differences in the concentrations of xylenes in indoor and outdoor air. Combined $m$ - and $p$-xylene concentrations were $12-22 \mu \mathrm{g} / \mathrm{m}^{3}(2.8-5.1 \mathrm{ppb})$ and $3.9 \mu \mathrm{g} / \mathrm{m}^{3}(0.9 \mathrm{ppb})$ for indoor and outdoor air, respectively, while $o$-xylene concentrations were $4.3-6.8 \mu \mathrm{g} / \mathrm{m}^{3}(0.99-1.6 \mathrm{ppb})$ for indoor air and $2.8 \mu \mathrm{g} / \mathrm{m}^{3}$ (0.64 ppb) for outdoor air. Higher levels of the xylenes were detected during autumn for indoor but not outdoor samples. Potential sources were tobacco smoke and other consumer products including carpet adhesive (Weschler et al. 1990). Kostianen (1995) measured the concentrations of VOCs in the indoor air of 50 "normal" houses and 38 "sick" houses. Median concentrations of $o$-xylene and $p$-xylene in the "normal" houses were 1.8 and $5.77 \mu \mathrm{g} / \mathrm{m}^{3}$ (0.41 and $\left.1.3 \mathrm{ppb}\right)$, respectively. Concentrations were only provided for 7 of the 38 "sick" houses. o-Xylene concentrations in these houses ranged from 1.74 to $1,100 \mu \mathrm{g} / \mathrm{m}^{3}(0.40-253 \mathrm{ppb})$ with median and geometric mean values of 3.35 and $8.74 \mu \mathrm{g} / \mathrm{m}^{3}(0.77$ and $2.01 \mathrm{ppb})$, respectively. $p$-Xylene concentrations ranged from 5.46 to $2,240 \mu \mathrm{g} / \mathrm{m}^{3}$ (1.26-516 ppb) with median and geometric mean values of 9.53 and $23.9 \mu \mathrm{g} / \mathrm{m}^{3}$ (2.20 and $\left.5.51 \mathrm{ppb}\right)$, respectively.

Ilgen et al. (2001b) measured geometric mean $o$-xylene and $m-/ p$-xylene concentrations of 0.68 and $3.23 \mu \mathrm{g} / \mathrm{m}^{3}$ (0.16 and $\left.0.744 \mathrm{ppb}\right)$, respectively, in German rural homes and 1.56 and $4.74 \mu \mathrm{g} / \mathrm{m}^{3}(0.36$ and $1.09 \mathrm{ppb})$, respectively, in German urban homes during the summer. Winter $o$-xylene and $m$ - $/ p$-xylene concentrations were 1.57 and $4.97 \mu \mathrm{g} / \mathrm{m}^{3}$ (0.36 and $\left.1.1 \mathrm{ppb}\right)$, respectively, in the rural homes, and 2.78 and $8.27 \mu \mathrm{g} / \mathrm{m}^{3}$ (0.64 and $\left.1.91 \mathrm{ppb}\right)$, respectively, in the urban homes.

Fellin and Otson (1994) measured seasonal indoor air concentrations of VOCs in Canadian homes during 1991. The mean concentrations of $o-, m-$, and $p$-xylene were $4.09,11.21$, and $4.96 \mu \mathrm{g} / \mathrm{m}^{3}(0.94,2.58$, and 
$1.14 \mathrm{ppb})$, respectively, in 185 homes during the winter, $6.89,13.17$, and $7.38 \mu \mathrm{g} / \mathrm{m}^{3}(1.59,3.03$, and $1.70 \mathrm{ppb})$, respectively, in 178 homes during the spring, 3.03, 6.81 , and $3.73 \mu \mathrm{g} / \mathrm{m}^{3}(0.698,1.57$, and $0.86 \mathrm{ppb})$, respectively, in 197 homes during the summer, and 8.19, 26.03, and $9.12 \mu \mathrm{g} / \mathrm{m}^{3}(1.89,6.00$, and $2.10 \mathrm{ppb}$ ), respectively, in 194 homes during the fall.

Graham et al. (2004) studied the effect of vehicle emission infiltration from an attached garage on the concentrations of VOCs in house air. The median levels of xylenes measured in 15 Canadian homes before and after running a warm vehicle in the garage during the afternoon were 18 and $34.5 \mathrm{ng} / \mathrm{L}$, respectively. Median levels in these houses before and after running the vehicles following a cold start in the morning were 22.1 and $47.8 \mathrm{ng} / \mathrm{L}$, respectively. Median concentrations of xylenes measured in the garage were $274 \mathrm{ng} / \mathrm{L}$ after running the vehicles in the afternoon and 1,001 $\mathrm{ng} / \mathrm{L}$ after running the vehicles in the morning.

The mean concentrations of $o$-xylene and $m-/ p$-xylene were 1.52 and $4.46 \mu \mathrm{g} / \mathrm{m}^{3}(0.350$ and $1.03 \mathrm{ppb})$, respectively, in the home outdoor air and 3.36 and $10.4 \mu \mathrm{g} / \mathrm{m}^{3}(0.774$ and $2.40 \mathrm{ppb})$, respectively, in the home indoor air of 36 students from west central Harlem in New York City during the winter (Kinney et al. 2002). $o$-Xylene and $m$-/p-xylene concentrations during the summer were 2.00 and $5.77 \mu \mathrm{g} / \mathrm{m}^{3}$ (0.461 and $1.33 \mathrm{ppb}$ ) respectively, in the home outdoor air and 2.27 and $6.44 \mu \mathrm{g} / \mathrm{m}^{3}(0.523$ and $1.48 \mathrm{ppb})$, respectively, in the home indoor air of 30 students from this area. Mean $o$-xylene and $m$ - $/ p$-xylene concentrations measured in the personal air of the students were 2.24 and $6.71 \mu \mathrm{g} / \mathrm{m}^{3}(0.516$ and $1.55 \mathrm{ppb})$, respectively, during the winter and 3.93 and $10.9 \mu \mathrm{g} / \mathrm{m}^{3}(0.906$ and $2.51 \mathrm{ppb})$, respectively, during the summer.

Jo et al. (2003) did not observe a large difference in xylene indoor air concentrations at high apartment floors compared to the air at low floors. In contrast, the concentrations of other chemicals such as methyl tertiary butyl ether, toluene, and benzene were higher in the air at the lower floors. Median $o$-xylene and $m$-/ $p$-xylene concentrations were 4.6 and $13.1 \mu \mathrm{g} / \mathrm{m}^{3}$ (1.1 and $\left.3.02 \mathrm{ppb}\right)$, respectively, in air from low floors compared to 4.8 and $14.2 \mu \mathrm{g} / \mathrm{m}^{3}$ (1.1 and $\left.3.27 \mathrm{ppb}\right)$, respectively, in air from high floors. The type of ventilation used in the apartment buildings was not indicated.

Ilgen et al. (2001b) reported that xylene concentrations in the indoor air of homes dramatically increased after redecoration, painting, and varnishing. Elevated $o$-xylene and $m-/ p$-xylene levels attributed to these activities ranged from 11.2 to $103.8 \mu \mathrm{g} / \mathrm{m}^{3}$ (2.6-23.9 ppb) and from 28.5 to $352.4 \mu \mathrm{g} / \mathrm{m}^{3}$ (6.6-81 ppb), respectively, compared to normal levels ranging from 0.7 to $1.4 \mu \mathrm{g} / \mathrm{m}^{3}(0.16-0.32 \mathrm{ppb})$ and from 2.6 to 
$5.9 \mu \mathrm{g} / \mathrm{m}^{3}$ (0.60-1.4 ppb), respectively. Leung and Harrison (1998) reported xylene concentrations measured in various microenvironments. Values were highest in the painted room and traffic tunnel microenvironments with mean $o$-xylene concentrations of 9.0 and $5.1 \mathrm{ppb}$, respectively, and mean $m-/ p$-xylene concentrations of 24.6 and $22.7 \mathrm{ppb}$, respectively.

In the United Kingdom, Kim et al. (2001) measured xylene concentrations in the air of 64 homes, 12 offices, 6 restaurants, 6 pubs, 8 department stores, 6 cinemas, 3 perfume shops, 6 libraries, and 8 labs. Mean concentrations at these locations were $0.8-6.9 \mu \mathrm{g} / \mathrm{m}^{3}(0.18-1.6 \mathrm{ppb})$ for $o$-xylene, $1.9-18.3 \mu \mathrm{g} / \mathrm{m}^{3}$ (0.44-4.2 ppb) for $m$-xylene, and $0.6-6.5 \mu \mathrm{g} / \mathrm{m}^{3}(0.14-1.5 \mathrm{ppb})$ for $p$-xylene. Mean concentrations reported for each location are listed in Table 6-6. These authors also measured xylene in the air of 12 train stations, 12 coach stations, 12 trafficked roads, 18 trains, 18 busses, and 35 cars. Xylene levels were highest in the cars, with mean concentrations of $54.2,127.2$, and $52.5 \mu \mathrm{g} / \mathrm{m}^{3}(12.4,29.3$, and $12.1 \mathrm{ppb}$ ) for $o_{-}, \mathrm{m}-$, and $p$-xylene, respectively. Mean concentrations at the other locations were 3.5$13.2 \mu \mathrm{g} / \mathrm{m}^{3}$ (0.81-3 ppb) for $o$-xylene, $10.1-32.3 \mu \mathrm{g} / \mathrm{m}^{3}$ (2.3-7.4 ppb) for $m$-xylene, and 3.5-11.6 $\mu \mathrm{g} / \mathrm{m}^{3}$ (0.81-2.7 ppb) for $p$-xylene.

Shields et al. (1996) measured the VOC concentrations in the air of 70 outdoor locations, 50 telecommunications offices, 11 administrative offices, and 9 data centers throughout the United States. The geometric mean concentrations of $m$ - $/ p$-xylene at these locations were $2.7,4.3,4.6$, and $6.7 \mu \mathrm{g} / \mathrm{m}^{3}(0.62$, $0.99,1.1$, and $1.5 \mathrm{ppb}$ ), respectively. The geometric mean concentrations of $o$-xylene (reported in combination with styrene) at these locations were $1.4,2.8,3.5$, and $4.8 \mu \mathrm{g} / \mathrm{m}^{3}(0.32,0.65,0.81$, and $1.1 \mathrm{ppb}$ ) respectively. The concentration of $o$-xylene and $m-/ p$-xylene measured in the air of three photocopy centers ranged from 0.5 to $16.8 \mathrm{ppb}$ and from 0.7 to $9.4 \mathrm{ppb}$, respectively (Stefaniak et al. 2000). The concentrations of xylene in 22 air samples collected from nonproduction departments of 6 pulp, paper, and paper product mills ranged from $<1$ to $82 \mathrm{ppm}$ with mean and median concentrations of 23 and 17 ppm, respectively (Teschke et al. 1999).

\subsubsection{Water}

Limited monitoring data are available on ambient concentrations of xylenes in surface waters. In view of the rapid volatilization of xylenes, their presence in surface waters is unlikely to be significant. Surface waters generally contain average xylene concentrations of $<1 \mathrm{ppb}$ total xylenes except in areas where there are fuel processing activities, such as petroleum refining (ECETOC 1986; Otson et al. 1982b; Sauer 
et al. 1978). Typical surface water concentrations range from not detected to $2 \mu \mathrm{g} / \mathrm{L}$ (ppb) (Otson et al. 1982b; Sauer et al. 1978).

According to 1999-2005 nationwide U.S monitoring data from the STORET database, mixed xylenes were detected in 1,252 out of 9,189 water samples with a median (range) concentration of $5.5 \mu \mathrm{g} / \mathrm{L}(\mathrm{ppb}$ ) $(0.05-75,000 \mu \mathrm{g} / \mathrm{L}[\mathrm{ppb}])($ EPA 2005h). o-Xylene was detected in 414 out of 3,623 STORET water samples with a median (range) concentration of $0.32 \mu \mathrm{g} / \mathrm{L}$ (ppb) $(0.001-2,400 \mu \mathrm{g} / \mathrm{L}$ [ppb]). m-Xylene was detected in 76 out of 532 STORET water samples with a median (range) concentration of $0.5 \mu \mathrm{g} / \mathrm{L}$ (ppb) $(0.02-2.18 \mu \mathrm{g} / \mathrm{L}[\mathrm{ppb}]) . p$-Xylene was detected in 73 out of 526 STORET water samples with a median (range) concentration of $0.5 \mu \mathrm{g} / \mathrm{L}$ (ppb) $(0.01-2 \mu \mathrm{g} / \mathrm{L}$ [ppb]). $m-/ p$-Xylene was detected in 358 out of 3,147 STORET water samples with a median (range) concentration of $0.53 \mu \mathrm{g} / \mathrm{L}(\mathrm{ppb})(0.004$ $4,300 \mu \mathrm{g} / \mathrm{L}[\mathrm{ppb}])$.

The concentrations of $o$-xylene measured in estuarine water from Humber, Tyne, Wear, and Tweed, United Kingdom were all $<10 \mathrm{ng} / \mathrm{L}$ (ppt); however, $o$-xylene was detected at Tees, United Kingdom at concentrations up to 1,340 ng/L (ppt) (Dewulf and Van Langenhove 1997). This high concentration of $O$-xylene was attributed to the use of this isomer as an intermediate in the production of phthalic anhydride. Concentrations of $o$-xylene and the combination of $m$ - and $p$-xylene in estuarine water from Sheldt, Netherlands/Belgium were 21.0 and $23.0 \mathrm{ng} / \mathrm{L}$ (ppt), respectively. Mixed xylenes have been detected in estuarine water from Brazos, United States at concentrations ranging from 1 to $56 \mathrm{ng} / \mathrm{L}$ (ppt). Xylenes have been detected in beach, bay, shelf sea, and coastal waters in locations around the world. $o$-Xylene concentrations were 1.8-42 ng/L (ppt) at Vineyard Sound, United States, $210 \mathrm{ng} / \mathrm{L}$ (ppt) at La Pineda, Spain, 0.3-10.1 ng/L (ppt) at the Gulf of Mexico, $4.0 \mathrm{ng} / \mathrm{L}$ (ppt) at Vilanova/Sitges, Spain, $6.1 \mathrm{ng} / \mathrm{L}$ (ppt) at Barcelona, Spain, $<1-13 \mathrm{ng} / \mathrm{L}$ (ppt) at the Campeche Shelf, $<10-35 \mathrm{ng} / \mathrm{L}$ (ppt) at the North Sea, and 9.8-11.9 ng/L (ppt) at the Belgian Continental Shelf. The concentrations of $m$-/ $p$-xylene at these locations were $2.5-66,34,27-24.4,72,15,22-59,<10-50$, and $11.0-15.1 \mathrm{ng} / \mathrm{L}$ (ppt), respectively. The concentration of $o$-xylene was $<10 \mathrm{ng} / \mathrm{L}$ (ppt) in water samples from five coastal locations in the United Kingdom.

Xylenes were detected in stormwater samples collected from basins around the state of North Carolina at mean, median, and maximum concentrations of $0.15,0.08$, and $2.37 \mu \mathrm{g} / \mathrm{L}(\mathrm{ppb})$, respectively, for $o$-xylene and 0.14, 0.09, and 1.62 $\mu \mathrm{g} / \mathrm{L}$ (ppb), respectively, for $m-/ p$-xylene (Borden et al. 2002). Concentrations of detected xylene in 592 stormwater samples from 16 cities and metropolitan areas across 
the United States ranged from 0.2 to $15 \mu \mathrm{g} / \mathrm{L}(\mathrm{ppb})$ with a median concentration of $0.4 \mu \mathrm{g} / \mathrm{L}(\mathrm{ppb})$ (percent detected not reported) (Delzer et al. 1997).

Data on the occurrence of xylene in public drinking water supplies are available from several federal, regional, and state surveys (EPA 1985a). In most cases, $<6 \%$ of the groundwater and surface water systems sampled contained detectable levels of xylenes (EPA 1985a). Typical xylene concentrations (all isomers) ranged from 0.2 to $9.9 \mu \mathrm{g} / \mathrm{L}$ (ppb) with mean concentrations of $<2 \mu \mathrm{g} / \mathrm{L}$ (ppb) (EPA 1985a; Keith et al. 1976; Williams et al. 1982). The Unregulated Contaminant Information System (URCIS) database round 1 contains public water system contaminant data for a 24-state cross-section (EPA 2001a). $o$-Xylene was detected in $2.41 \%$ of 1,450 surface water public water systems and $1.69 \%$ of 12,638 groundwater public water systems. m-Xylene was detected in $2.12 \%$ of 1,276 surface water public water systems and $1.47 \%$ of 10,145 groundwater public water systems. $p$-Xylene was detected in $2.36 \%$ of 1,230 surface water public water systems and $1.49 \%$ of 8,956 groundwater public water systems. Total xylenes were detected in $10.75 \%$ of 670 surface water public water systems and $2.51 \%$ of 8,841 groundwater public water systems. The $99^{\text {th }}$ percentile concentrations of $o-, m-, p-$, and total xylenes for the round 1 data were $<5.0,<4.0,<5.0$, and $0.6 \mu \mathrm{g} / \mathrm{L}(\mathrm{ppb})$, respectively. The California EPA Office of Drinking Water quality-monitoring database contains drinking water contaminant data for both surface water and groundwater sources throughout the state of California (Storm 1994). According to these data, xylene has been detected in 23 out of 11,589 California public drinking water sources $(0.2 \%)$ with mean and median detected concentrations of 22.91 and $3.80 \mu \mathrm{g} / \mathrm{L}(\mathrm{ppb})$, respectively.

The migration of petroleum products from leaking underground storage tanks and pipelines poses a groundwater contamination problem. Gasoline-contaminated groundwater in Los Angeles contained levels of xylenes as high as $153 \mu \mathrm{g} / \mathrm{L}$ (ppb) (Karlson and Frankenberger 1989). Contaminated groundwater from a New Jersey industrial site contained xylenes at a concentration of $3.68 \mathrm{mg} / \mathrm{L}$ (ppm) (Adey et al. 1996).

The concentration of $o$-xylene in groundwater collected from a sampling well at a crude oil spill site in north central Minnesota was $269 \mu \mathrm{g} / \mathrm{L}$ (ppb) (Eganhouse et al. 1996). The concentrations of total xylenes detected in the leachate plume of a closed landfill in Norman, Oklahoma were $0-1 \mu \mathrm{g} / \mathrm{L}$ (ppb) (Eganhouse et al. 2001). The concentration of xylenes measured in groundwater from a hydrocarboncontaminated site in Southern California was 1,010 $\mathrm{g} / \mathrm{L}$ (ppb) (Kent and Spycher 1994). The concentrations of $m$-xylene and the combination of $o$ - and $p$-xylene measured at the Biscayne Aquifer Superfund site study area were 20 and $23 \mu \mathrm{g} / \mathrm{L}$ (ppb), respectively (Canter and Sabatini 1994). 
Seventeen out of 338 water samples collected from four sites in Iowa using drain tiles, observations wells, or lysimeters contained xylene with a mean concentration of $3.0 \mu \mathrm{g} / \mathrm{L}(\mathrm{ppb})$ and a maximum concentration of $5 \mu \mathrm{g} / \mathrm{L}(\mathrm{ppb}$ ) (Wang et al. 1995). Xylenes were detected above a minimum detection limit of $0.2 \mu \mathrm{g} / \mathrm{L}(\mathrm{ppb})$ in approximately $15 \%$ of groundwater samples collected from 30 wells located throughout the Denver, Colorado area with a maximum concentration of $33 \mu \mathrm{g} / \mathrm{L}$ (ppb) (Bruce and McMahon 1996). During the National Water Quality Assessment Program of the U.S. Geological Survey, mixed xylenes were detected above a reporting level of $0.2 \mu \mathrm{g} / \mathrm{L}(\mathrm{ppb})$ in $2.2 \%$ (9 of 406$)$ of urban wells with median and maximum detected concentrations of approximately 2 and $50 \mu \mathrm{g} / \mathrm{L}(\mathrm{ppb})$, respectively (Kolpin et al. 1997; Squillace et al. 1999). Mixed xylenes were detected above $0.2 \mu \mathrm{g} / \mathrm{L}$ (ppb) in $0.9 \%$ (23 of 2,542) of rural wells with median and maximum detected concentrations of approximately 0.5 and $200 \mu \mathrm{g} / \mathrm{L}$ (ppb), respectively. Concentrations of xylenes measured in groundwater are listed in Table 6-7.

Concentrations of $o$-xylene and the combination of $m$ - and $p$-xylene in Antarctic pack ice located at the coast of the Ross Sea range from 10 to $20 \mathrm{ng} / \mathrm{L}$ (ppt) and from 29 to $54 \mathrm{ng} / \mathrm{L}$ (ppt), respectively (Desideri et al. 1998).

\subsubsection{Sediment and Soil}

Although several investigators (Aurelius and Brown 1987; Barbee and Brown 1986; Griffin et al. 1984) refer to leaching of xylene from waste disposal sites as a source of xylene levels in groundwater samples, little data are available on actual measurements of xylene in soil. The rapid volatilization of this chemical makes its presence in surface soils unlikely. According to 1999-2005 nationwide U.S. monitoring data from the STORET database, mixed xylene was detected in 90 out of 528 soil samples with a median (range) concentration of $0.038 \mathrm{mg} / \mathrm{kg}(0.001-190 \mathrm{mg} / \mathrm{kg})($ EPA $2005 \mathrm{~h})$.

The concentrations of $o$-xylene and $m$-/p-xylene ranged from $<0.05$ to $3.2 \mathrm{ng} / \mathrm{g}$ (ppb) (mean $0.89 \mathrm{ng} / \mathrm{g}$ [ppb]) and from 0.08 to $5.2 \mathrm{ng} / \mathrm{g}$ (ppb) (mean $1.4 \mathrm{ng} / \mathrm{g}$ [ppb]) in sediment samples collected from seven rivers and a port located in the Niigata Perfecture, Japan (Kawata et al. 1997). The concentration of xylenes measured in an aquifer matrix from a hydrocarbon-contaminated site in Southern California was 5,300 $\mu \mathrm{g} / \mathrm{kg}$ (ppb), respectively (Kent and Spycher 1994). According to 1999-2005 nationwide U.S. monitoring data from the STORET database, mixed xylene was detected in 14 out of 165 sediment samples with a median (range) concentration of $0.087 \mathrm{mg} / \mathrm{kg}(0.001-41 \mathrm{mg} / \mathrm{kg})(\mathrm{EPA} 2005 \mathrm{~h})$. 
Table 6-7. Xylene Concentrations in Groundwater ${ }^{\mathrm{a}}$

\begin{tabular}{|c|c|c|c|c|c|}
\hline \multirow[b]{2}{*}{ Location } & \multirow{2}{*}{$\begin{array}{l}\text { Percent } \\
\text { detected }\end{array}$} & \multicolumn{3}{|c|}{ Positive concentration (ppb) } & \multirow[b]{2}{*}{ Reference } \\
\hline & & Mean & Median & Maximum & \\
\hline \multicolumn{6}{|l|}{ General surveys } \\
\hline 4 sites in lowa & 5 & 3.0 & - & 10,000 & Wang et al. 1995 \\
\hline $\begin{array}{l}30 \text { wells around } \\
\text { Denver, Colorado }\end{array}$ & 15 & - & - & 33 & Bruce and McMahon 1996 \\
\hline 406 urban U.S. wells & 2.2 & - & 2.0 & 50 & Squllace et al. 1999 \\
\hline 2,542 rural U.S. wells & 0.9 & - & 0.5 & 200 & Squllace et al. 1999 \\
\hline \multicolumn{6}{|l|}{$\begin{array}{l}\text { Individual contaminated } \\
\text { sites }\end{array}$} \\
\hline $\begin{array}{l}\text { Los Angeles site } \\
\text { (gasoline } \\
\text { contamination) }\end{array}$ & - & - & - & 153 & $\begin{array}{l}\text { Karlson and } \\
\text { Frankenberger } 1989\end{array}$ \\
\hline $\begin{array}{l}\text { New Jersey industrial } \\
\text { site }\end{array}$ & - & $3,680^{b}$ & - & - & Adey et al. 1996 \\
\hline $\begin{array}{l}\text { Minnesota crude oil } \\
\text { spill site }\end{array}$ & - & $269^{c}$ & - & - & Eganhouse et al. 1996 \\
\hline $\begin{array}{l}\text { Leachate from closed } \\
\text { Oklahoma landfill }\end{array}$ & - & - & - & 1 & Eganhouse et al. 2001 \\
\hline $\begin{array}{l}\text { Southern California } \\
\text { site }\end{array}$ & - & $1,010^{b}$ & - & - & Kent and Spycher 1994 \\
\hline $\begin{array}{l}\text { Biscayne Aquifer } \\
\text { Superfund site }\end{array}$ & - & $43^{d}$ & - & - & Canter and Sabatini 1994 \\
\hline
\end{tabular}

${ }^{\mathrm{a}}$ Reported in sources as concentrations of xylene unless otherwise specified

${ }^{b}$ Value reported by source as single concentration of xylenes at that site

'Value reported by source as single concentration of $o$-xylene at that site $(m$ - and $p$-xylene not reported)

${ }^{d}$ Value is the sum of reported single $o$ - and $p$-xylene concentrations at that site ( $m$-xylene not reported) 


\subsubsection{Other Environmental Media}

Xylene has been detected in cigarette smoke, consumer products, and some foods. The gas phase delivery of $p$-xylene in ultra-low tar delivery cigarette smoke ranges from $<0.01$ to $8 \mu \mathrm{g} /$ cigarette, while the ranges for $m$ - and $o$-xylene are $<0.01-20$ and $<0.005-10 \mu \mathrm{g} /$ cigarette, respectively (Higgins et al. 1983). Data were not located for regular strength or unfiltered cigarettes. The 1,095 household products surveyed by the Consumer Product Safety Commission (Fishbein 1985) contained an average of 9.5\% mixed xylene. The largest number of products containing mixed xylene were household aerosols and paints, varnishes, shellac, and rust preventatives. The concentrations of $o$-xylene measured in interior alkyd primer and three interior alkyd enamel paints were 1.39, 4.91, 5.48, and $6.92 \mathrm{mg} / \mathrm{g}(1,390,4,910$, 5,480 , and $6,920 \mathrm{ppm}$ ) (Fortmann et al. 1998). The concentrations of $p$-xylene in these paints were 0.23 , $0.73,1.45$, and $1.36 \mathrm{mg} / \mathrm{g}(230,730,1,450$, and $1,360 \mathrm{ppm})$.

Xylene has been detected in distillates of rainbow trout and in carp tissue samples from three rivers not known to be contaminated (Hiatt 1983). The estimated tissue concentrations of $m$ - and $p$-xylene in rainbow trout and carp were 0.05 and $0.12 \mathrm{mg} / \mathrm{kg}$ (ppm), respectively (Hiatt 1983).

Eggs, whether stored or used fresh, contained detectable levels of all three xylene isomers when scrambled (Matiella and Hsieh 1991). The polystyrene packing case in which some of the eggs had been stored also contained detectable levels of the xylene. Actual concentrations of $o-, m-$, and $p$-xylene were 4.0, 7.0, and $3.0 \mathrm{ng} / \mathrm{g}(\mathrm{ppb})$, respectively, in supermarket eggs packed in polystyrene, 3.0. 8.0, and $2.7 \mathrm{ng} / \mathrm{g}$ (ppb), respectively, in fresh eggs not stored, and 2.0, 3.0, and $1.3 \mathrm{ng} / \mathrm{g}$ (ppb), respectively, in fresh eggs stored in polystyrene for 2 weeks.

o-Xylene was detected above $3.72 \mathrm{ppb}$ in 32 of 234 table-ready food items analyzed during a Total Diet Study conducted by the FDA with an average concentration of $8.15 \mathrm{ppb}$ (range 3.72-23.5 ppb) (Heikes et al. 1995). $m$-/p-Xylene was detected in 64 out of the 234 food items with an average concentration of $64 \mathrm{ppb}$ (range 6.20-114 ppb). The concentrations of $o$-xylene in sandwich cookies, margarine, butter, and cake doughnuts measured during this study were $6.31,21.7,12.5$, and $23.5 \mathrm{ppb}$, respectively. The concentrations of $m$ - and $p$-xylene together in these foods were $12.9,114,54.8$, and $35.0 \mathrm{ppb}$, respectively.

The mean and range of $m$ - $/ p$-xylene concentrations were 0.24 and $0.01-0.75 \mathrm{ng} / \mathrm{mL}$, respectively, in 19 whole milk samples, 0.08 and $0.02-0.33 \mathrm{ng} / \mathrm{mL}$, respectively, in eight $2 \%$ milk samples, and 0.27 and 
0.01-0.56 ng/mL, respectively, in five 1\% milk samples collected in Las Vegas, Nevada (Hiatt and Pia 2004). The mean and range of $o$-xylene concentrations were 0.18 and $0.01-0.62 \mathrm{ng} / \mathrm{mL}$, respectively, in the whole milk samples, 0.08 and $0.02-0.29 \mathrm{ng} / \mathrm{mL}$, respectively, in the $2 \%$ milk samples, and 0.21 and $0.01-0.40 \mathrm{ng} / \mathrm{mL}$, respectively, in the $1 \%$ milk samples.

Both $o$ - and $m$-xylene were detected in the aroma concentrates of cured beef (Ramarathnam et al. 1991) as well as in the volatile fraction of raw beef (King et al. 1993). Only o-xylene was detected in cured chicken. None of the xylene isomers were detected in the uncured meat samples. $o_{-}^{-,} m-$, and $p$-Xylene have been detected in the headspace of frankfurter sausages at unspecified concentrations (Chevance and Farmer 1999). Ho et al. (1983) identified $o$-xylene as a volatile flavor compound in fried bacon. o-Xylene has also been identified in the volatiles of fried chicken (Tang et al. 1983). Wu and Liou (1992) measured reported $o_{-}, m-$, and $p$-xylene concentrations of $1.85,0.99$, and $0.72 \mathrm{ppb}$, respectively, in duck meat, 14.91, 4.29, and $5.97 \mathrm{ppb}$, respectively, in duck fat, 1.90, 9.61, and $10.64 \mathrm{ppb}$, respectively, in Cantonese style roasted duck, and 10.29, 18.09, and 21.23 ppb, respectively, in Cantonese style duck gravy.

The concentrations of $o_{-}, \mathrm{m}$-, and $p$-xylene were measured to be $132 \mathrm{ng} / \mathrm{g}(\mathrm{ppb})$, not detected, and $79.4 \mathrm{ng} / \mathrm{g}$ (ppb), respectively, in Korean anchovy; $102 \mathrm{ng} / \mathrm{g}$ (ppb), $378 \mathrm{ng} / \mathrm{g}$ (ppb), and not detected, respectively in Korean big eyed herring; not detected, $124 \mathrm{ng} / \mathrm{g}(\mathrm{ppb})$, and not detected, respectively, in Korean hair tail viscera; and 58.6, 99.9, and $39.0 \mathrm{ng} / \mathrm{g}(\mathrm{ppb})$, respectively in Korean Shrimp pastes (Cha and Cadwallader 1995). Chung (1999) reported measured $o-, m-$, and $p$-xylene concentrations of 31.4, 53.8 , and $36.0 \mu \mathrm{g} / \mathrm{kg}$ (ppb), respectively, in the leg meat of crabs (C. feriatus), 23.6, 40.1, and $2.8 \mu \mathrm{g} / \mathrm{kg}$ (ppb), respectively, in the body meat of the crabs, and 199.7, 329.4, and $234.6 \mu \mathrm{g} / \mathrm{kg}$ (ppb), respectively, in the carapace meat of the crabs. Misharina and Golovnya (1992) also detected xylenes among the volatile components of shrimp and crabmeat. $o$ - and $m$-Xylene have been identified in the volatiles of cooked mussels (Leguen et al. 2000).

Xylene (unspecified isomers) has been identified as a volatile component of Beaufort cheese from the French Alps (Dumont and Adda 1978). o-, $m$-, and $p$-Xylene have been identified as volatile components of Idaho Russet Burbank baked potatoes, chickpea (Cicer arietinum L.) seed, and peaches (Coleman et al. 1981, Rembold et al. 1989, Takeoka et al. 1988). o-Xylene was detected in dry beans, split peas, and lentils at mean concentrations of $9 \mathrm{ppb}(0-25 \mathrm{ppb}), 8 \mathrm{ppb}$, and $3 \mathrm{ppb}$, respectively (Lovegren et al. 1979). Jenkins et al. (2004) found little difference in the mean concentrations of total xylenes in hazelnuts that had been previously treated with xylene compared to control hazelnuts that had not been treated $(0.65-$ 
$1.43 \mathrm{ng} / \mathrm{g}[\mathrm{ppb}]$ compared to $1.49 \mathrm{ng} / \mathrm{g}$ [ppb]). $o^{-}, m_{-}$, and $p$-xylene have all been detected in the volatiles of roasted filberts (Kinlin et al. 1972). The concentration of $p$-xylene in three apricots and two plums ranged from not detected to $2 \mu \mathrm{g} / \mathrm{kg}$ (ppb) while concentrations of this substance ranged from not detected to $9 \mu \mathrm{g} / \mathrm{kg}$ (ppb) in their interspecific hybrid progeny (Gomez et al. 1993).

\subsection{GENERAL POPULATION AND OCCUPATIONAL EXPOSURE}

Because individual xylene isomers are used in large amounts in industrial settings, people who work at or live near these locations may receive a higher exposure to one xylene isomer compared to the other isomers. However, since xylenes are present as a mixture in gasoline and in the solvent components of commonly used commercial products (paint, coatings, etc.), exposure of the general population is expected to be primarily to xylenes as a mixture, and not to the separate xylene isomers.

The California Total Exposure Assessment Methodology (TEAM) Study conducted in 1984 in Los Angeles County (an urban area) and Contra Costa County (a rural area) monitored volatile organic compounds in ambient (outdoor) air, personal air, and breath samples for 188 people (Wallace et al. 1988). (Personal air is defined as air samples which were collected using a sampling vest worn by the participant with the pump and collection cartridge placed close to the breathing level.) In Los Angeles, all three xylene isomers were detected in each air type; higher levels were measured during the winter for all air types and all xylene isomers compared with summer levels. Average concentrations (in $\mu \mathrm{g} / \mathrm{m}^{3}$ [ppb]) are listed in Table 6-8.

Smoking was determined to be the major determinant for the presence of xylene in breath and personal air, with concentrations in the breath of smokers more than double those of nonsmokers. Auto-related situations, such as pumping gasoline and exposure to exhaust, as well as type of employment also contributed significantly to increased concentrations of xylene in breath and personal air (Wallace et al. 1988). A second TEAM study in 1987, of the same Los Angeles families, showed similar trends in relative concentrations of xylene in personal, indoor, and outdoor air (Wallace et al. 1991). Again, outdoor concentrations were lower than indoor concentrations which, in turn, were lower than personal air samples. Mean xylene concentrations (in $\mu \mathrm{g} / \mathrm{m}^{3}$ [ppb]) are listed in Table 6-9.

Studies by Ashley et al. (1995) and Wallace et al. (1987a) supported the argument that xylene exposure is greater for individuals who smoke cigarettes. The reported mean (median) concentrations of $o$-xylene and $m$ - $/ p$-xylene were $0.13 \mathrm{ng} / \mathrm{mL}(0.086 \mathrm{ng} / \mathrm{mL})$ and $0.41 \mathrm{ng} / \mathrm{mL}(0.19 \mathrm{ng} / \mathrm{mL})$, respectively, in the blood 
Table 6-8. Average Personal Air, Outdoor Air, and Breath Concentrations Measured During the 1984 TEAM Study

\begin{tabular}{|c|c|c|c|}
\hline Location & Sample type & $m-/ p$-Xylene (ppb) & o-Xylene (ppb) \\
\hline Los Angeles & Personal air & $28 \quad(6.5)$ & $13(3.0)$ \\
\hline \multirow[t]{2}{*}{ (February) } & Outdoor air & $24 \quad(5.5)$ & 11 (2.5) \\
\hline & Breath & $3.5(0.8)$ & $1.0(0.2)$ \\
\hline Los Angeles & Personal air & $24 \quad(5.5)$ & $7.2(1.7)$ \\
\hline \multirow[t]{2}{*}{ (June) } & Outdoor air & $9.4(2.2)$ & $2.7(0.6)$ \\
\hline & Breath & $2.8(0.6)$ & $0.7(0.2)$ \\
\hline Contra Costa & Personal air & $11 \quad(2.5)$ & $4.4(1.0)$ \\
\hline \multirow[t]{2}{*}{ (June) } & Outdoor air & $2.2(0.5)$ & $0.7(0.2)$ \\
\hline & Breath & $2.5(0.6)$ & $0.6(0.1)$ \\
\hline
\end{tabular}

TEAM Study = The California Total Exposure Assessment Methodology Study

Source: Wallace et al. 1988 
Table 6-9. Average Personal Air, Indoor Air, Outdoor Air, and Breath Concentrations from the 1987 TEAM Study

\begin{tabular}{llcc}
\hline Date & Sample type & $m$-/p-Xylene $(\mathrm{ppb})$ & \multicolumn{2}{c}{ o-Xylene $(\mathrm{ppb})$} \\
\hline February & Personal air & $43(9.9)$ & $16(3.7)$ \\
& Indoor & $30(6.9)$ & $12(2.8)$ \\
& Outdoor & $18(4.2)$ & $6.5(1.5)$ \\
& Breath (median value) & $2.5(0.6)$ & $0.8(0.2)$ \\
& & & \\
July & Personal air & $27(6.2)$ & $9.2(2.1)$ \\
& Indoor & $12(2.8)$ & $4.3(1.0)$ \\
& Outdoor & $7.4(1.7)$ & $2.8(0.6)$ \\
& Breath (median value) & $0.7(0.2)$ & $0.25(0.1)$ \\
\hline
\end{tabular}

TEAM Study = The California Total Exposure Assessment Methodology Study

Source: Wallace et al. 1991 
of 126 nonsmokers and $0.18 \mathrm{ng} / \mathrm{mL}(0.14 \mathrm{ng} / \mathrm{mL})$ and $0.53 \mathrm{mg} / \mathrm{mL}(0.42 \mathrm{mg} / \mathrm{mL})$, respectively, in the blood of 42 smokers (Ashley et al. 1995). The unweighted geometric mean concentrations of $o$-xylene and $m$-/p-xylene were 1.55 and $5.5 \mu \mathrm{g} / \mathrm{m}^{3}$, respectively, in the breath of 200 smokers in New Jersey and California compared to 0.8 and $2.1 \mu \mathrm{g} / \mathrm{m}^{3}$, respectively, in the breath of 322 nonsmokers from these locations (Wallace et al. 1987a). The unweighted geometric mean daytime personal air exposures of $o$-xylene and $m$ - $/ p$-xylene were 7.8 and $22 \mu \mathrm{g} / \mathrm{m}^{3}$, respectively, for the smokers and 6.5 and $16 \mu \mathrm{g} / \mathrm{m}^{3}$, respectively, for the nonsmokers. The weighted geometric mean overnight indoor air concentrations of $o$ - and $\mathrm{m}$-/ $/$-xylene were 5.5 and $14.9 \mu \mathrm{g} / \mathrm{m}^{3}$, respectively, in the homes of the smokers and $4.9 \mu \mathrm{g} / \mathrm{m}^{3}$ and $13.4 \mu \mathrm{g} / \mathrm{m}^{3}$, respectively, in the homes of the nonsmokers. Heavner et al. $(1995,1996)$, however, came to a different conclusion, reporting that there was no significant difference between xylene exposure in smoking and nonsmoking homes. Median exposures reported for $o_{-}^{-}, m-$, and $p$-xylene were 1.51, 3.61, and $1.11 \mu \mathrm{g}$ day $/ \mathrm{m}^{3}$, respectively, at the homes of 15 smokers and $1.63,3.22$, and $1.12 \mu \mathrm{g}$ day $/ \mathrm{m}^{3}$, respectively, at the homes of 35 nonsmokers. It should be noted, however, that Heavner et al. (1995, 1996) studied a relatively small subject population (49 subjects) compared to Ashley et al. (1995) (168 subjects) and Wallace et al. (1987a) (522 subjects).

Individuals are expected to be exposed to xylenes by breathing the air inside homes and buildings since these substances are commonly found in indoor air. Exposure may also arise from ingestion of contaminated drinking water. Common activities identified with increased potential exposure include pumping gasoline, visiting service stations, traveling in a car, painting, scale model building, pesticide use, and smoking (Wallace et al. 1986, 1987b).

Blood levels of $o$-xylene and $m$-/p-xylene were 0.080 and $0.32 \mu \mathrm{g} / \mathrm{L}$, respectively, in 86 commuters from Mexico City, 0.082 and $0.24 \mu \mathrm{g} / \mathrm{L}$, respectively, in 14 commuters from Stamford, Connecticut, 0.20 and $0.44 \mu \mathrm{g} / \mathrm{L}$, respectively, in 26 commuters from Fairbanks, Alaska, 0.045 and $0.14 \mu \mathrm{g} / \mathrm{L}$, respectively, in 19 commuters from Albany, New York, and 0.094 and $0.16 \mu \mathrm{g} / \mathrm{L}$, respectively (Lemire et al. 2004).

Jo and Moon (1999) studied the exposure of South Korean housewives who live near roadside service stations to VOCs. For housewives who lived within $30 \mathrm{~m}$ of a service station, mean $o-, m-$, and $p$-xylene concentrations were 9.8, 7.0, and $12.7 \mu \mathrm{g} / \mathrm{m}^{3}$ (2.3, 1.6, and $\left.2.9 \mathrm{ppb}\right)$, respectively, in indoor air, 6.0, 4.1, and $8.8 \mu \mathrm{g} / \mathrm{m}^{3}(1.4,0.9$, and $2.0 \mathrm{ppb})$, respectively, in outdoor air, and $3.3,3.4$, and $2.4 \mu \mathrm{g} / \mathrm{m}^{3}(0.8,0.8$, and $0.6 \mathrm{ppb}$ ), respectively, in breath samples. For housewives who lived 60-100 $\mathrm{m}$ from a service station, mean $o-, m$-, and $p$-xylene concentrations were $12.2,8.5$, and $15.5 \mu \mathrm{g} / \mathrm{m}^{3}$ (2.8, 2.0, and $\left.3.6 \mathrm{ppb}\right)$, respectively, in indoor air, 3.7, 2.3, and $5.7 \mu \mathrm{g} / \mathrm{m}^{3}(0.9,0.5$, and $1.3 \mathrm{ppb})$, respectively, in outdoor air, and 
4.1, 4.0, and $3.2 \mu \mathrm{g} / \mathrm{m}^{3}(0.9,0.9$, and $0.7 \mathrm{ppb})$, respectively, in breath samples. The mean and range of concentration of xylenes that 25 individuals were exposed to while riding or operating an open sleigh snowmobile were 2.00 and $<0.05-13.0 \mathrm{mg} / \mathrm{m}^{3}(0.5$ and $<0.1-3.0 \mathrm{ppb})$, respectively (Eriksson et al. 2003).

Of 237 consumer products tested for $m$-xylene and 221 products tested for $o$ - and $p$-xylene, 101 products contained $m$-xylene and 93 products contained $o$ - and $p$-xylene at concentrations $>0.1 \%$ weight (EPA 1991b; Sack et al. 1992). The concentrations and percentage of positive detects are listed in Table 6-10.

Based on the estimates of EPA (1983) of median atmospheric concentrations of (see Section 6.4.1) and assuming inhalation of $23 \mathrm{~m}^{3} /$ day by a $70-\mathrm{kg}$ adult, the daily $o$-xylene intakes from air for adults exposed to the median levels in rural, urban, and source-dominated areas would be $0.0001,0.0017$, and $0.0012 \mathrm{mg} / \mathrm{kg} /$ day, respectively. The median $\mathrm{m}$ - and $p$-xylene intakes would be $0.0001,0.0039$, and $0.0024 \mathrm{mg} / \mathrm{kg} /$ day, respectively (EPA 1985a). Assuming a typical ambient air xylene concentration of $4.0 \mathrm{ppb}$, the average daily intake of xylene from air is estimated to be $353 \mu \mathrm{g}$ (HSDB 2007).

General population exposure to xylene can also occur through dermal contact with the many consumer products containing xylene, including cleaning solvents, insecticides, lacquers, paint thinners and removers, and pesticides (EPA 1985a; Fishbein 1985; Gleason et al. 1969). Dermal absorption is reported to be minor following exposure to xylene vapor but may be significant following contact with the liquid (EPA 1985a). The percutaneous absorption rate of $m$-xylene in humans is approximately $2 \mu \mathrm{g} / \mathrm{cm}^{2} /$ minute through the skin of the hands (Engstrom et al. 1977).

Assuming a maximum concentration of total xylenes ( $m-, o_{-}$, and $p$-xylene) $(1.5 \mathrm{mg} / \mathrm{L})$ and a daily intake of $2 \mathrm{~L}$ of drinking water, the adult maximum daily intake for total xylenes through consumption of drinking water is estimated to be $3 \mathrm{mg} /$ day or $0.04 \mathrm{mg} / \mathrm{kg} /$ day (EPA 1985a).

Mean and median concentrations of $o$-xylene detected in 711 blood samples from participants in the Third National Health and Nutrition Examination Survey (NHANES III) were 0.14 and $0.11 \mathrm{ppb}$, respectively (Ashley et al. 1994, 1996). $m$ - and p-Xylene were detected together in 649 blood samples with mean and median concentrations of 0.37 and $0.19 \mathrm{ppb}$, respectively. $o$ - and $p$-Xylene blood levels of 0.094 and $0.16 \mu \mathrm{g} / \mathrm{L}$ (ppb), respectively, were reported for the nonsmoking subset (546 individuals) of participants in NHANES III (Lemire et al. 2004). 
Table 6-10. Concentrations of Xylene in Consumer Products

\begin{tabular}{|c|c|c|c|c|}
\hline \multirow[b]{2}{*}{ Product } & \multicolumn{2}{|c|}{$m$-Xylene } & \multicolumn{2}{|c|}{$o-/ p$-Xylene } \\
\hline & Percent detected & $\begin{array}{l}\text { Concentration of } \\
\text { xylene }\end{array}$ & Percent detected & $\begin{array}{l}\text { Concentration of } \\
\text { xylene }\end{array}$ \\
\hline $\begin{array}{l}\text { Automotive } \\
\text { products }\end{array}$ & 26.7 & 10.6 & 10.0 & 31.0 \\
\hline $\begin{array}{l}\text { Household } \\
\text { cleaners, polishers }\end{array}$ & 33.3 & 1.4 & 0.0 & 0.0 \\
\hline $\begin{array}{l}\text { Paint-related } \\
\text { products }\end{array}$ & 60.3 & 4.2 & 58.2 & 2.8 \\
\hline $\begin{array}{l}\text { Fabric and leather } \\
\text { treatments }\end{array}$ & 0.0 & 0.0 & 33.3 & 0.1 \\
\hline $\begin{array}{l}\text { Oils, greases, and } \\
\text { lubricants }\end{array}$ & 9.3 & 0.2 & 11.9 & 0.2 \\
\hline $\begin{array}{l}\text { Adhesive-related } \\
\text { products }\end{array}$ & 9.1 & 0.2 & 9.1 & 0.2 \\
\hline
\end{tabular}

Source: Sack et al. 1992 
The mean and range of xylene concentrations measured in whole blood specimens collected from 250 hospital patients were 5.2 and $0.5-160 \mathrm{ppm}(5,200$ and 500-160,000 ppb), respectively (Antoine et al. 1986). Mean and median concentrations of $m$-xylene measured in blood samples of 25 individuals living in Italy (including both smokers and non smokers) were 719 and $457 \mathrm{ng} / \mathrm{L}(0.719$ and $0.457 \mathrm{ppb})$, respectively (Perbellini et al. 2002). Mean and median $m$-xylene concentrations in the urine of these individuals were 106 and $96 \mathrm{ng} / \mathrm{L}(0.106$ and $0.096 \mathrm{ppb})$, respectively. The mean and range of xylene concentrations in the blood of the occupants of approximately 57 rural homes in northern Germany were 229 and $71-1,113 \mathrm{ng} / \mathrm{L}$ (0.229 and 0.071-1.113 ppb), respectively, with corresponding indoor air concentrations of 9.29 and 3.64-54.64 ng/L (2.1 and 0.8-12.5 ppb) (Ilgen et al. 2001a). The mean and range of xylene concentrations in the blood of the occupants of approximately 57 urban homes in northern Germany were 262 and $89-1,301 \mathrm{ng} / \mathrm{L}$ (0.262 and 0.089-1.301 ppb), respectively, with corresponding indoor air concentrations of 9.29 and $1.84-87.79 \mathrm{ng} / \mathrm{L}$ (2.1 and 0.4-20.2 ppb), respectively.

Xylenes were detected in eight milk samples collected from mothers at hospitals in Bayonne, New Jersey; Jersey City, New Jersey; Pittsburgh, Pennsylvania; Baton Rouge, Louisiana; and Charleston, West Virginia (EPA 1980a). Concentrations in these samples were not reported.

Occupational exposure to mixed xylenes may occur during their production as well as their end use as industrial solvents. Occupational exposures result from inhalation or dermal exposure and are usually associated with process, storage, or fugitive emissions at petroleum chemical, paint, and plastics plants (Bratveit et al. 2004; Fishbein 1985, 1988). Average daily intake of xylenes from individual occupational exposure sources has not been estimated. Results of a study of solvent exposures in 1981 involving eighty-nine workers in seven plants of three companies applying paints and glues, primarily by spraying, showed that most solvent spraying activities surveyed showed only low-to-moderate exposures to xylene relative to the TLV in the presence of ordinary general room ventilation (Whitehead et al. 1984). The TWA concentrations of xylene for high-aromatic spraying in booths (3.5 ppm), solvent wiping (0.7 ppm), and paint mixing (3.7 ppm) were well below the TLV for xylene (100 ppm) (Whitehead et al. 1984).

The ranges of concentrations of $o$-xylene and $m$ - $/ p$-xylene were $65-1,900 \mu \mathrm{g} / \mathrm{m}^{3}(15-440 \mathrm{ppb})$ and 180 $5,800 \mu \mathrm{g} / \mathrm{m}^{3}$ (41-1,300 ppb), respectively, in the personal air of 24 service station attendants, 2.0 $44 \mu \mathrm{g} / \mathrm{m}^{3}(0.5-10 \mathrm{ppb})$ and $71-120 \mu \mathrm{g} / \mathrm{m}^{3}$ (16-28 ppb), respectively, in the personal air of 6 street vendors, and 16-28 $\mu \mathrm{g} / \mathrm{m}^{3}(3.7-6.5 \mathrm{ppb})$ and $44-80 \mu \mathrm{g} / \mathrm{m}^{3}$ (10-18 ppb), respectively, in the personal air of 7 office workers in Mexico City during their work shifts (Romieu et al. 1999). Mean (range) concentrations of $o$-xylene and $m-/ p$-xylene in the blood of the service station attendants were $0.39(0.16-$ 
$1.2 \mu \mathrm{g} / \mathrm{L})$ and $1.4(0.50-4.7) \mu \mathrm{g} / \mathrm{L}(\mathrm{ppb})$, respectively, at the beginning of the work shift and $0.45(0.15-$ 6.3 ) and $1.3(0.36-16) \mu \mathrm{g} / \mathrm{L}(\mathrm{ppb})$, respectively, at the end of the work shift. Mean (range) concentrations of $o$-xylene and $m$ - $/ p$-xylene in the blood of the street vendors were $0.18(0.13-0.30)$ and $0.75(0.41-1.1)$ $\mu \mathrm{g} / \mathrm{L}$ (ppb), respectively, at the beginning of the work shift and $0.15(0.083-0.20)$ and $0.53(0.25-0.70)$ $\mu \mathrm{g} / \mathrm{L}$ (ppb), respectively, at the end of the work shift. Mean (range) concentrations of $o$-xylene and $\mathrm{m}-/ \mathrm{p}$-xylene in the blood of the street vendors were $0.15(0.081-0.31)$ and $0.55(0.37-0.81) \mu \mathrm{g} / \mathrm{L}(\mathrm{ppb})$, respectively, at the beginning of the work shift and $0.10(0.073-0.21)$ and $0.39(0.19-0.73) \mu \mathrm{g} / \mathrm{L}(\mathrm{ppb})$, respectively, at the end of the work shift.

The ranges of concentrations of xylenes detected in the perimeter air, pump air, and breathing zone air were $<0.001-0.066 \mathrm{ppm}(<1.0-66 \mathrm{ppb})$ at two full service stations in New Jersey, 0.001-0.014 ppm (1.0$4.0 \mathrm{ppb})$ at three self-serve service stations in New York, and $<0.001-0.281 \mathrm{ppm}(<1.0-281 \mathrm{ppb})$ at five self-serve service stations in Connecticut (API 1994).

The American Petroleum Institute (API 1995) studied the exposure of service station attendants and mechanics to fuel components at six stations in New York, three stations in Minnesota, four stations in Arizona, and three stations in Oregon in 1994. During the wintertime, xylene was detected above 0.01$0.30 \mathrm{ppm}$ in only 1 of the 59 short-term exposure cases involving attendants. It was not detected above the detection limit (0.01-0.03 ppm) in 51 long-term exposure cases involving attendants. Xylene was detected above $0.09-0.15 \mathrm{ppm}$ in 7 of 44 short term winter exposure cases involving mechanics with a median (range) concentration of $1 \mathrm{ppm}$ (1-6.4 ppm). It was detected above 0.01-0.02 ppm in 3 out of 44 long-term exposure cases involving mechanics with a median (range) concentration of $1 \mathrm{ppm}$ (1$2.2 \mathrm{ppm}$ ). During the summertime, xylene was detected above $0.09-0.16 \mathrm{ppm}$ in 44 of the 60 short-term exposure cases involving attendants with a median (range) concentration of $0.15 \mathrm{ppm}(0.09-7.70 \mathrm{ppm})$. It was detected above $0.01-0.06 \mathrm{ppm}$ in 52 out of 54 long-term exposure cases involving attendants with a median (range) concentration of $0.05 \mathrm{ppm}(0.01-0.83 \mathrm{ppm})$. Xylene was detected above 0.08-0.16 ppm in 18 of 44 short term summer exposure cases involving mechanics with a median (range) concentration of $0.15 \mathrm{ppm}(0.08-30.0 \mathrm{ppm})$. It was detected above $0.01-0.02 \mathrm{ppm}$ in 35 out of 42 long-term exposure cases involving mechanics with a median (range) concentration of $0.1 \mathrm{ppm}(0.01-1.60 \mathrm{ppm})$.

During a study of exposure to gasoline components during loading and delivery, the mean exposure of tanker drivers to xylene was measured to be $0.8-4 \mathrm{mg} / \mathrm{m}^{3}$ (Hakkola and Saarinen 1996). 
The mean (median, range) concentrations of xylene in the blood of thirteen workers occupied in the production of paints containing xylene and 10 workers occupied in paint spraying were $380 \mu \mathrm{g} / \mathrm{L}$ $(403 \mu \mathrm{g} / \mathrm{L}, 63-715 \mu \mathrm{g} / \mathrm{L})$ and $130 \mu \mathrm{g} / \mathrm{L}(74 \mu \mathrm{g} / \mathrm{L}, 49-308 \mu \mathrm{g} / \mathrm{L})$, respectively, after an 8-hour work shift (Kramer et al. 1999). The mean (median, range) concentrations of xylene in the air during the shifts were $29 \mu \mathrm{g} / \mathrm{L}(30 \mu \mathrm{g} / \mathrm{L}, 5-58 \mu \mathrm{g} / \mathrm{L})$ for the production workers and $8 \mu \mathrm{g} / \mathrm{L}(7 \mu \mathrm{g} / \mathrm{L}, 3-21 \mu \mathrm{g} / \mathrm{L})$ for the spraying workers. The mean (median, range) concentrations of the methylhippuric acid metabolites in urine were $1,221 \mathrm{mg} / \mathrm{L}(1,168 \mathrm{mg} / \mathrm{L}, 194-2,333 \mathrm{mg} / \mathrm{L})$ for the production workers and $485 \mathrm{mg} / \mathrm{L}(397 \mathrm{mg} / \mathrm{L}, 65-$ $1,633 \mathrm{mg} / \mathrm{L}$ ) for the spraying workers. Moen and Hollund (2000) measured xylene concentrations ranging from 0.2 to $0.8 \mathrm{ppm}$ in the air of six car painting garages. Vincent et al. (1994) reported that the mean (range) concentrations of xylenes in personal air during painting operations in an aeronautical workshop were $30.9 \mathrm{mg} / \mathrm{m}^{3}\left(14.3-46.5 \mathrm{mg} / \mathrm{m}^{3}\right)$ after day $1,87.0 \mathrm{mg} / \mathrm{m}^{3}\left(38.7-160.0 \mathrm{mg} / \mathrm{m}^{3}\right)$ after day 2 , and $122.2 \mathrm{mg} / \mathrm{m}^{3}\left(68.3-167.0 \mathrm{mg} / \mathrm{m}^{3}\right)$ after day 3 .

$o$-Xylene and $m$-/ $p$-xylene concentrations in the inhaled air were 5.3 and $15.9 \mu \mathrm{g} / \mathrm{m}^{3}$, respectively, in the inhaled air of individuals who spent less than 3 hours/day in workshops and warehouses compared to 30.4 and $114 \mu \mathrm{g} / \mathrm{m}^{3}$, respectively, for individuals who spent more than 3 hours/day in these places (Hoffmann et al. 2000). The concentrations of $o$-xylene and $m$ - $/ p$-xylene were 5.4 and $16.2 \mu \mathrm{g} / \mathrm{m}^{3}$, respectively, in the inhaled air of individuals who work where no paints or lacquers are used, 12.7 and $38.4 \mu \mathrm{g} / \mathrm{m}^{3}$, respectively, in the inhaled air of individuals who work where paints or lacquers are used occasionally, and 41.8 and $171 \mu \mathrm{g} / \mathrm{m}^{3}$, respectively, in the inhaled air of individuals who work where paints or lacquers are used frequently.

Holz et al. (1995) reported that exposure of workers at a styrene plant to air concentrations of 6.0 $44.1 \mu \mathrm{g} / \mathrm{m}^{3}$ for $o$-xylene and $5.6-64.0 \mu \mathrm{g} / \mathrm{m}^{3}$ for $m$ - and $p$-xylene together did not result in increased breath concentrations compared to non-exposed individuals. $o$-Xylene and $m$ - and $p$-xylene concentrations were 3.4 and $3.7 \mu \mathrm{g} / \mathrm{m}^{3}$, respectively, in the breath of exposed workers after their shift compared to 3.5 and $4.0 \mu \mathrm{g} / \mathrm{m}^{3}$, respectively, in the breath of non-exposed controls. The differences in mean concentrations of the metabolites $o$-methyl hippuric acid and $m$ - and $p$-methyl hippuric acid together in the urine of workers before their shift to mean concentrations in their urine after their shift were 0.6 and $8.1 \mathrm{mg} / \mathrm{g}$ creatinine, respectively, for the exposed workers and 0.2 and $2 \mathrm{mg} / \mathrm{g}$ creatinine, respectively, for the non-exposed controls.

Pedersen and Higgins (1995) reported the vapor concentrations of xylenes that individuals were exposed to while taking part in various activities. Measured vapor concentrations of xylenes were $10.83 \mathrm{ppm}$ 
during varnishing and sealing household wooden doors, $0.92 \mathrm{ppm}$ during activity in a paint mixing booth, $0.069 \mathrm{ppm}$ during automotive break and rotor cleaning, $0.04 \mathrm{ppm}$ during emptying/cleaning a fuel oil holding tank, $0.21 \mathrm{ppm}$ during activity in a printing shop, $1.680 \mathrm{ppm}$ during cleaning firearms, $2.264 \mathrm{ppm}$ during refinishing furniture, and below the detection limit (not specified) during activity in a beauty salon, application of hairspray, activities in an autorepair shop, and activity in a traffic sign painting shop. The concentrations of xylenes measured in various work areas were $0.124 \mathrm{ppm}$ in a paint booth mixing room, $1.100 \mathrm{ppm}$ in a household remodeling bathroom, $0.102 \mathrm{ppm}$ in a household paint stripping room, and below the detection limit (not specified) in a wooden door staining room, a traffic sign painting room, and a traffic sign stripping room.

The average concentrations of xylene vapors that 60 material handling personnel and 51 laboratory personnel were exposed to while working at treatment, storage, and disposal facilities were 1.61 and 0.16 ppm, respectively (Pedersen and Higgins 1995). The average work area vapor concentration at these facilities was $0.47 \mathrm{ppm}$.

The concentrations of xylene in the exhaled breath of shipyard workers commonly exposed to organic solvents ranged from $<1$ to $10.6 \mathrm{ppm}$ (Katsuyama et al. 1998). The mean (range) concentrations of the metabolite methylhippuric acid in the urine of these workers were $0.09 \mathrm{~g} / \mathrm{g}$ creatinine $(0.02-0.40 \mathrm{~g} / \mathrm{g}$ creatinine) before their work shift and $0.22 \mathrm{~g} / \mathrm{g}$ creatinine $(0.02-1.41 \mathrm{~g} / \mathrm{g}$ creatinine) after their work shift.

The mean concentrations of $o$-xylene and $m-/ p$-xylene in the breath of four dry cleaning workers ranged from 4.5 to 9.0 and from 7.6 to $17.7 \mu \mathrm{g} / \mathrm{m}^{3}$ (1.0-2.1 and $\left.1.8-4.1 \mathrm{ppb}\right)$, respectively compared to mean concentrations of 3.7 and $6.5 \mu \mathrm{g} / \mathrm{m}^{3}$ (0.9 and $\left.1.5 \mathrm{ppb}\right)$, respectively, in the breath of their housewives (Jo and Kim 2001). Mean air concentrations measured inside different dry cleaning stores ranged from 16.6 to $85.2 \mu \mathrm{g} / \mathrm{m}^{3}$ (3.8-19.6 ppb) for $o$-xylene and from 33.8 to $161 \mu \mathrm{g} / \mathrm{m}^{3}(7.8-37.0 \mathrm{ppb})$ for $m$-/p-xylene.

The National Occupational Exposure Survey (NOES), conducted by NIOSH from 1980 to 1983, indicated that 1,106,789 workers, including 211,806 women, in 74,063 plants were potentially exposed to mixed xylene in the workplace in 1980 (NIOSH 1990c). An estimated 5,596 workers (including 1,314 women) in 331 plants, 16,863 workers (including 1,194 women) in 1,610 plants, and 1,160 workers (including 545 women) in 178 plants were potentially exposed to $o_{-}, m-$, and $p$-xylene, respectively. The largest numbers of workers exposed to mixed xylene were employed in the machinery (except electrical), special trade contractors, fabricated metal products, and health services industries (as assemblers, janitors, 
cleaners, painting and paint-spraying machine operators, and automobile mechanics). The largest numbers of workers exposed to $o$-xylene were employed in the chemical and allied products industry (as machine operators [not specified], chemical technicians, production inspectors, checkers, and examiners). The largest numbers of workers exposed to $m$-xylene were employed in the electric, gas, and sanitary services and business services industries (as electrical power installers and repairers, supervisors, plumbers, pipe fitters, steam fitters, order clerks, and chemists [except biochemists]). The largest numbers of exposed workers exposed to $p$-xylene were employed in the health services industries (as clinical laboratory technologists and technicians). These estimates were derived from observations of the actual use of mixed xylene and the individual xylene isomers and the use of trade name products known to contain xylene (see Table 6-11 for percentage breakdown).

The NOES database does not contain information on the frequency, level, or duration of exposure of workers to any of the chemicals listed therein. The survey only provides estimates of workers potentially exposed to the chemicals.

\subsection{EXPOSURES OF CHILDREN}

This section focuses on exposures from conception to maturity at 18 years in humans. Differences from adults in susceptibility to hazardous substances are discussed in Section 3.7, Children's Susceptibility.

Children are not small adults. A child's exposure may differ from an adult's exposure in many ways. Children drink more fluids, eat more food, breathe more air per kilogram of body weight, and have a larger skin surface in proportion to their body volume. A child's diet often differs from that of adults. The developing human's source of nutrition changes with age: from placental nourishment to breast milk or formula to the diet of older children who eat more of certain types of foods than adults. A child's behavior and lifestyle also influence exposure. Children crawl on the floor, put things in their mouths, sometimes eat inappropriate things (such as dirt or paint chips), and spend more time outdoors. Children also are closer to the ground, and they do not use the judgment of adults to avoid hazards (NRC 1993).

Children are expected to be exposed to xylenes by the same routes that affect adults. Since xylenes have a low affinity for adsorption onto soil and dust particles and a high volatilization rate, the risk of exposure for small children from ingesting soil or dust is likely to be low. 
Table 6-11. Percentage Breakdown of NIOSH Occupational Exposure Estimates from the NOES Database ${ }^{a}$

\begin{tabular}{lll}
\hline \multirow{2}{*}{ Chemical } & \multicolumn{2}{c}{ NOES } \\
\cline { 2 - 3 } Actual $^{\mathrm{b}}$ & Trade name \\
\hline o-Xylene & $96 \%$ & $4 \%$ \\
-Xylene & $23 \%$ & $77 \%$ \\
$p$-Xylene & $75 \%$ & $25 \%$ \\
Total xylenes & $19 \%$ & $81 \%$ \\
\hline
\end{tabular}

${ }^{\mathrm{a}}$ Derived from NIOSH 1976, 1990c

${ }^{\mathrm{b}}$ Actual observations are surveyor observations in which the surveyor observed the use of the specific agent.

$\mathrm{NIOSH}=$ National Institute for Occupational Safety and Health; NOES = National Occupational Exposure Survey 
During the Minnesota Children's Pesticide Exposure Study (MCPES), xylene was detected in personal, indoor, and outdoor air samples collected from households with children (Adgate et al. 2004a). During the School Health Initiative: Environment, Learning, Disease (SHIELD) study, the concentrations of xylene were measured in the outdoor home air, indoor school air, indoor home air, and personal air of 113 children from two inner-city schools in Minneapolis, Minnesota (Adgate et al. 2004b). Mean and median exposure concentrations reported in these studies were below $7 \mu \mathrm{g} / \mathrm{cm}^{3}$ for both $\mathrm{m}-/ \mathrm{p}$ - and $o$-xylene. The data are listed in Table 6-12.

Sexton et al. (2006) analyzed blood samples collected from 43 inner-city children 3-6 years of age living in Minneapolis, Minnesota. Mean, minimum, and maximum concentrations in 50 blood samples were $0.32,0.09$, and $1.40, \mathrm{ng} / \mathrm{mL}$, respectively, for $m-/ p$-xylene and $0.06,0.02$, and $0.22 \mathrm{ng} / \mathrm{mL}$, respectively, for $o$-xylene.

During a study of the exposure of Danish children to benzene, toluene, and xylenes, the median concentration of xylenes was $18 \mu \mathrm{g} / \mathrm{m}^{3}(4.1 \mathrm{ppb})$ in front door air samplers and $17 \mu \mathrm{g} / \mathrm{m}^{3}(3.9 \mathrm{ppb})$ in personal air samplers of 56 children living in Copenhagen. Median concentrations for 40 Danish children living in rural areas were $2.6 \mu \mathrm{g} / \mathrm{m}^{3}(0.6 \mathrm{ppb})$ in front door air samplers and $13 \mu \mathrm{g} / \mathrm{m}^{3}(3.0 \mathrm{ppb})$ in personal air samplers (Raaschou-Nielsen et al. 1997). The authors concluded that riding in cars and activities involving exposure to gasoline vapors were factors contributing to the exposure of these children to xylenes.

Minoia et al. (1996) studied the exposure of school children living in three Italian towns with 50,000 inhabitants or less (Treviglio-Lombardy; Poggibonsi-Tuscany and Valenza-Piedmont) to xylenes. Mean indoor air concentrations within the children's homes in these three towns were 18.5, 24.1, and $11.7 \mu \mathrm{g} / \mathrm{m}^{3}$ (4.3, 5.6, and $2.7 \mathrm{ppb}$ ), respectively, which correspond to mean inhaled doses of 92.5, 120.5, and $58.5 \mu \mathrm{g}$ /day, respectively. Mean personal air concentrations (referred to as "indoor + outdoor") for children living in Treviglio, Poggibonsi, and Valenza were 20.2, 21.2, and $12.7 \mu \mathrm{g} / \mathrm{m}^{3}(4.7,4.9$, and $2.9 \mathrm{ppb}$ ), respectively, which correspond to inhaled doses of 101.0, 106.0, and $63.5 \mu \mathrm{g} /$ day, respectively. Mean concentrations of xylene measured in urine samples were $316 \mathrm{ng} / \mathrm{L}$ for 144 children living in Treviglio, 112 ng/L for 96 children living in Poggibonsi, and 123 ng/L for 139 children living in Valenza. 
Table 6-12. Personal, Indoor, and Outdoor Air Exposures of Inner-city Children in Minneapolis, Minnesota to Xylenes ${ }^{a}$

\begin{tabular}{|c|c|c|c|c|}
\hline Study & Type of sample & Number of samples ${ }^{b}$ & $m / p$-Xylene $\left(\mu \mathrm{g} / \mathrm{m}^{3}\right)^{\mathrm{c}}$ & $o$-Xylene $\left(\mu \mathrm{g} / \mathrm{m}^{3}\right)^{\mathrm{d}}$ \\
\hline \multicolumn{5}{|c|}{ MCPES $^{\mathrm{e}}$} \\
\hline & Personal & 73 & 6.3 & 2.5 \\
\hline & Home Indoor & $101^{\mathrm{c}}$ & 5.1 & 2.2 \\
\hline & Home Outdoor & $100^{c}$ & 0.5 & 1.5 \\
\hline \multicolumn{5}{|c|}{ SHIELD ${ }^{f}$} \\
\hline & \multicolumn{4}{|l|}{ Winter } \\
\hline & School outdoor & 8 & 2.3 & 0.8 \\
\hline & School indoor & 39 & 2.3 & 0.8 \\
\hline & Personal & 93 & 3.7 & 1.2 \\
\hline & Home indoor & 93 & 3.5 & 1.1 \\
\hline & \multicolumn{4}{|l|}{ Spring } \\
\hline & School outdoor & 10 & 2 & 0.7 \\
\hline & School indoor & 47 & 1.2 & 0.4 \\
\hline & Personal & 88 & 3.3 & 1.1 \\
\hline & Home indoor & 88 & 2.9 & 1 \\
\hline
\end{tabular}

${ }^{a}$ Data are from Adgate et al. 2004a, 2004b.

${ }^{b}$ Xylene isomers were detected in $100 \%$ of all samples unless otherwise specified.

${ }^{c} \mathrm{~m} / \mathrm{p}$-Xylene was detected above $0.5 \mu \mathrm{g} / \mathrm{m}^{3}$ in $99 \%$ of the home outdoor samples.

${ }^{d}$-Xylene was detected above $0.3 \mu \mathrm{g} / \mathrm{m}^{3}$ in $98 \%$ of the home indoor and home outdoor samples.

e $V$ alues reported for MCPES are mean concentrations.

${ }^{f}$ Values reported for SHIELD are median concentrations.

MCPES = Minnesota Children's Pesticide Exposure Study; SHIELD = School Health Initiative: Environment, Learning, Disease 


\subsection{POPULATIONS WITH POTENTIALLY HIGH EXPOSURES}

Among persons not occupationally exposed to xylenes, the highest exposure levels result from smoking and from contact with consumer products containing xylene (Wallace et al. 1988, 1991). Populations living near chemical waste sites where xylene is improperly stored are also likely to be at risk of increased exposure to xylene vapors via inhalation or dermal contact.

Workers in certain occupational groups appear to have the greatest potential for exposure to high concentrations of xylenes. Based on the available case reports of xylene toxicity in humans, painters (or paint industry workers) and laboratory workers appear to be most frequently affected (EPA 1985a). In general, workers involved in the distillation and purification of xylene or employed in industries using xylene as a raw material (e.g., gasoline component) may be at higher risk of exposure (EPA 1985a). The use of xylene in improperly ventilated areas is often the cause for toxic levels of exposure, and increased exposures or breath concentrations have been observed for wood processing plant workers, gas station employees, metal workers, and furniture refinishers.

\subsection{ADEQUACY OF THE DATABASE}

Section 104(i)(5) of CERCLA, as amended, directs the Administrator of ATSDR (in consultation with the Administrator of EPA and agencies and programs of the Public Health Service) to assess whether adequate information on the health effects of xylene is available. Where adequate information is not available, ATSDR, in conjunction with NTP, is required to assure the initiation of a program of research designed to determine the health effects (and techniques for developing methods to determine such health effects) of xylene.

The following categories of possible data needs have been identified by a joint team of scientists from ATSDR, NTP, and EPA. They are defined as substance-specific informational needs that if met would reduce the uncertainties of human health assessment. This definition should not be interpreted to mean that all data needs discussed in this section must be filled. In the future, the identified data needs will be evaluated and prioritized, and a substance-specific research agenda will be proposed. 


\subsubsection{Identification of Data Needs}

Physical and Chemical Properties. The physical and chemical properties of xylene have been well studied, and reliable information is available. On this basis, further studies of the physical-chemical properties of xylene are not essential at the present time.

Production, Import/Export, Use, Release, and Disposal. According to the Emergency Planning and Community Right-to-Know Act of 1986, 42 U.S.C. Section 11023, industries are required to submit substance release and off-site transfer information to the EPA. The TRI, which contains this information for 2004, became available in May of 2006. This database is updated yearly and should provide a list of industrial production facilities and emissions.

Potential for human exposure to xylene is expected to be quite high based on the high volume of production and the widespread domestic and industrial uses of xylene.

Recent estimates of xylene production capacity indicate that over 18 billion pounds of mixed xylene and over 13 billion pounds of xylene isomers may be produced in the United States each year (SRI 2006). Further information on the amounts of xylenes used in various products would be helpful in estimating human exposure to xylenes from consumer products.

Xylenes are widely used in industry as solvents and as precursors of other products (i.e., polyester). Data describing releases of xylenes to the environment (TRI04 2006) and the presence of xylenes in gasoline, paint products, insecticides, and cigarette smoke have been well documented (Wallace et al. 1991). Information on the occurrence of xylenes in food are available and have been included.

Because of its widespread use and release into the environment, xylene has been distributed to most environmental media. It has been detected in air (Wallace et al. 1991), surface water, sediments (Otson et al. 1982b), drinking water (EPA 1985a), and aquatic organisms (Hiatt 1983). Very little data are available that report actual levels of xylenes in soil. Information on the most recent distribution of xylene are needed to estimate exposure.

Incineration is the primary method for disposal of xylene, although information on the disposal methods is not detailed (HSDB 2007). Information on the amount of xylene disposed of by incineration as well as 
the amount of xylene disposed of or abandoned at hazardous waste sites is important for estimating the potential human exposure. Criteria for the disposal of xylenes are currently subject to frequent revision.

Environmental Fate. The environmental fate of xylene has been well studied. Volatilization of xylene is the dominant fate process. Xylene released to surface water primarily volatilizes (Mackay and Leinonen 1975). Xylene also adsorbs to soils and sediments and leaches into groundwater (Gherini et al. 1989). Photooxidation appears to be the most important transformation process in the atmosphere and in surface soils (Anderson et al. 1991; Jori et al. 1986). Biodegradation is likely to be the only significant degradation process for xylene in subsurface soils and aquatic systems (EPA 1984a, 1985a; Haigler et al. 1992). Additional data on the partitioning of xylene released to soil and on longevity and the rates of biotransformation in soils and sediments, are important to further define potential pathways of human exposure.

Bioavailability from Environmental Media. Xylene is absorbed during inhalation (Morley et al. 1970), oral (Abu Al Ragheb et al. 1986; Ogata et al. 1979), and dermal contact (Riihimaki and Pfaffli 1978; Skowronski et al. 1990). Approximately 50\% of the xylene that is inhaled is absorbed into the body (Riihimaki and Savolainen 1980; Wallen et al. 1985), while 90\% of ingested xylene is absorbed (Bray et al. 1949). However, limited information was found in the available literature regarding the bioavailability of xylene from contaminated media such as soil (Turkall et al. 1992) and sediments to which the xylene is sorbed or from contaminated surface waters. This information is needed to estimate human exposure from contaminated environmental media.

Food Chain Bioaccumulation. Xylenes are bioconcentrated in aquatic organisms to a limited extent (Nunes and Benville 1979; Ogata and Miyake 1978). The degree of concentration is believed to be limited by the rapid metabolism and excretion of xylene from some aquatic species. However, additional data on the bioconcentration of xylene by aquatic organisms from contaminated surface waters and sediments would be useful. No information was found in the literature regarding the bioconcentration of xylene in plants or biomagnification of xylene among food chain trophic levels. Although bioconcentration has been predicted for all isomers of xylene because of their tendency to partition into the octanol phase of the octanol-water system, the rapid oxidation of xylene during metabolism seems to preclude bioconcentration in higher animal systems. Thus, biomagnification is not expected to be important for xylene. However, data on the bioaccumulation of xylene in commercially important fish and shellfish are needed since consumption of contaminated fish and shellfish may be a potential source of human exposure. 
Exposure Levels in Environmental Media. Reliable monitoring data for the levels of xylene in contaminated media at hazardous waste sites are needed so that the information obtained on levels of xylene in the environment can be used in combination with the known body burden of xylene to assess the potential risk of adverse health effects in populations living in the vicinity of hazardous waste sites.

Levels of xylene in air and groundwater have been determined (Kolpin et al. 1997; Squillace et al. 1999; EPA 1991a; Wallace et al. 1991). However, there are limited monitoring data on xylene levels in soil and surface waters (EPA 1985a). More monitoring data are needed to better characterize ambient concentrations of xylene in soils and surface water, particularly in the vicinity of hazardous waste sites and petroleum refineries.

The available data allow characterization of human exposure to xylene from most exposure pathways, particularly air (Wallace et al. 1988, 1991). Estimates of human intake of xylene from contaminated air and drinking water have been made based on background levels that have been recorded.

Exposure Levels in Humans. Xylene has been detected in human blood, urine, and exhaled breath. However, exposure associated with living or working near hazardous waste sites and refineries has not been assessed. The most important human exposure sources, workplace and ambient air, are well understood (Fishbein 1985, 1988; Wallace et al. 1988, 1991). Additional monitoring programs involving analysis of human breath or urine are needed to assess the magnitude of exposures and in estimating the average daily dose associated with various sources, particularly for populations in the vicinity of hazardous waste sites.

Several sectors of the work force have the greatest levels of exposure to xylene. Total xylene exposure has been found to be greatest among those employed in the machinery (except electrical), special trade contracting, fabricated metal products, and health services industries (as assemblers, janitors and cleaners, painting and paint-spraying machine operators, and automobile mechanics) (EPA 1985a). More current information on occupational exposure are needed.

This information is necessary for assessing the need to conduct health studies on these populations.

Exposures of Children. Little data are available regarding exposures of children to xylene. Two foreign studies and three studies conducted in the United States have been located. The U.S. studies focus 
primarily on children living in Minnesota. Additional data reporting the exposures of children living in other areas of the United States to xylenes would be helpful.

Child health data needs relating to susceptibility are discussed in Section 3.12.2, Identification of Data Needs: Children's Susceptibility.

Exposure Registries. No exposure registries for xylene were located. This substance is not currently one of the compounds for which a sub-registry has been established in the National Exposure Registry. The substance will be considered in the future when chemical selection is made for subregistries to be established. The information that is amassed in the National Exposure Registry facilitates the epidemiological research needed to assess adverse health outcomes that may be related to exposure to this substance.

\subsubsection{Ongoing Studies}

The Federal Research in Progress (FEDRIP 2006) database and the Federal Research and Development (FED R\&D 2005) database provide additional information obtainable from a few ongoing studies that may fill in some of the data needs identified in Section 6.8.1. These studies are summarized below.

As part of the Third National Health and Nutrition Evaluation Survey (NHANES III), the Environmental Health Laboratory Sciences Division of the National Center for Environmental Health, Centers for Disease Control and Prevention, will be analyzing human blood samples for xylene and other volatile organic compounds. These data will give an indication of the frequency of occurrence and background levels of these compounds in the general population.

Kate Scow (University of California Davis) is studying the relationship between microbial communities and the anaerobic biodegradation of contaminant mixtures in aquifer groundwater. Chemicals of focus will include toluene and xylene. This study will include both microcosm and field studies.

T.Y. Sylva and C.M. Kinoshita (University of Hawaii) is studying the application of bioremediation to hydrocarbon contaminated soils. Laboratory soil biodegradation studies will be performed on organic compounds commonly found in petroleum products (including xylene). 
Alfred Spormann (Stanford University) is studying the microbial degradation of aromatic hydrocarbons under anaerobic conditions. Anaerobic biodegradation studies on BTEX compounds will be examined to gain additional knowledge of microbial metabolism and for evaluating and enhancing intrinsic bioremediation. 


\section{ANALYTICAL METHODS}

The purpose of this chapter is to describe the analytical methods that are available for detecting, measuring, and/or monitoring xylene, its metabolites, and other biomarkers of exposure and effect to xylene. The intent is not to provide an exhaustive list of analytical methods. Rather, the intention is to identify well-established methods that are used as the standard methods of analysis. Many of the analytical methods used for environmental samples are the methods approved by federal agencies and organizations such as EPA and the National Institute for Occupational Safety and Health (NIOSH). Other methods presented in this chapter are those that are approved by groups such as the Association of Official Analytical Chemists (AOAC) and the American Public Health Association (APHA). Additionally, analytical methods are included that modify previously used methods to obtain lower detection limits and/or to improve accuracy and precision.

The analytical methods used to quantify xylene in biological and environmental samples are summarized below. Table 7-1 lists the applicable analytical methods used for determining xylene in biological fluids and tissues. Table 7-2 lists the methods used for determining xylene in environmental samples.

\subsection{BIOLOGICAL MATERIALS}

Extensive commercial, industrial, and domestic use of volatile organic chemicals such as xylene virtually assures that the general population will be exposed to this class of chemicals to some extent. The determination of trace amounts of xylene in biological tissues and fluids has been restricted to only a limited number of analytical methods. These include gas chromatography coupled with mass spectrometry (GC/MS), gas chromatography coupled with hydrogen flame ionization detection (GC/FID), and high-performance liquid chromatography (HPLC).

Xylene can be detected at parts-per-trillion (ppt) levels in whole human blood using a purge and trap apparatus followed by GC/MS; however, this method does not distinguish between $m$ - and $p$-xylene (Ashley et al. 1992). Antifoam agents are frequently used, although a method has been developed that does not require this additive (Cramer et al. 1988). The use of a dynamic headspace purge at room temperature reduces the absolute recoveries of the late eluting compounds. An advantage of this GC/MS technique is that it can be used in conjunction with selected ion monitoring to obtain better sensitivity of target compounds (such as NPL Pollutants) at ppt levels (Cramer et al. 1988). 
Table 7-1. Analytical Methods for Determining Xylene in Biological Materials

\begin{tabular}{|c|c|c|c|c|c|}
\hline $\begin{array}{l}\text { Sample } \\
\text { matrix }\end{array}$ & Preparation method & $\begin{array}{l}\text { Analytical } \\
\text { method }\end{array}$ & $\begin{array}{l}\text { Sample } \\
\text { detection limit }\end{array}$ & $\begin{array}{l}\text { Percent } \\
\text { recovery }\end{array}$ & Reference \\
\hline $\begin{array}{l}\text { Human } \\
\text { blood }\end{array}$ & $\begin{array}{l}\text { Adsorb directly to Amberlite } \\
\text { XAD } 2 \text { resin; extract with } \\
\text { carbon disulfide }\end{array}$ & GC/FID & No data & $\begin{array}{l}\text { 77B98 } \\
\text { (m-xylene) }\end{array}$ & $\begin{array}{l}\text { Norstrom } \\
\text { and } \\
\text { Scheepers } \\
1990\end{array}$ \\
\hline $\begin{array}{l}\text { Human } \\
\text { blood }\end{array}$ & $\begin{array}{l}\text { Purge and trap sample on } \\
\text { Tenax TA trap }\end{array}$ & GC/MS & $\begin{array}{l}1 \mathrm{ng} / \mathrm{mL} \\
\text { (m-xylene) }\end{array}$ & No data & $\begin{array}{l}\text { Cramer et } \\
\text { al. } 1988\end{array}$ \\
\hline \multirow[t]{2}{*}{$\begin{array}{l}\text { Human } \\
\text { blood }\end{array}$} & $\begin{array}{l}\text { Purge and trap sample on } \\
\text { sorbent }\end{array}$ & GC/MS & $5.2 \mathrm{ng} / \mathrm{mL}$ & No data & $\begin{array}{l}\text { Antoine et } \\
\text { al. } 1986\end{array}$ \\
\hline & & & $\begin{array}{l}0.019 \mathrm{ng} / \mathrm{mL} \\
(m-, p-x y l e n e) \\
0.035 \mathrm{ng} / \mathrm{mL} \\
\text { (o-xylene) }\end{array}$ & No data & $\begin{array}{l}\text { Ashley et al. } \\
1992\end{array}$ \\
\hline $\begin{array}{l}\text { Tissues and } \\
\text { body fluids }\end{array}$ & $\begin{array}{l}\text { Saturate sample with sodium } \\
\text { chloride and seal in a vial; } \\
\text { inject into gas chromatograph }\end{array}$ & $\begin{array}{l}\text { GC/FID } \\
\text { and } \\
\text { GC/MS }\end{array}$ & $\begin{array}{l}0.05 \mathrm{mg} / 100 \mathrm{~g} \\
(\mathrm{~m}-, \mathrm{p} \text {-xylene); } \\
0.01 \mathrm{mg} / 100 \mathrm{~g} \\
\text { (o-xylene) }\end{array}$ & No data & $\begin{array}{l}\text { Bellanca et } \\
\text { al. } 1982\end{array}$ \\
\hline Urine & $\begin{array}{l}\text { Derivatize or methylate } \\
\text { sample with } \mathrm{HCl} \text { and } \\
\text { methanol; cool; extract with } \\
\text { chloroform }\end{array}$ & GC/FID & $<0.25 \mathrm{~g} / \mathrm{L}$ & 110.7 ( $m-\mathrm{MHA})$ & $\begin{array}{l}\text { de Carvalho } \\
\text { et al. } 1991\end{array}$ \\
\hline Urine & $\begin{array}{l}\text { Extract sample with THA-OH; } \\
\text { alkylate with isopropyl } \\
\text { bromide; wash with silver } \\
\text { sulfate; dry; redissolve in ethyl } \\
\text { acetate }\end{array}$ & GC/FID & $\begin{array}{l}1 \mathrm{ng}(o-, p- \\
m-\mathrm{MHA})\end{array}$ & $\begin{array}{l}94.2(o-\mathrm{MHA}) \\
96.0(m-\mathrm{MHA}) \\
97.6(p-\mathrm{MHA})\end{array}$ & $\begin{array}{l}\text { Kataoka et } \\
\text { al. } 1991\end{array}$ \\
\hline Urine & $\begin{array}{l}\text { Adsorb to filter paper; extract } \\
\text { with methanol; dilute with } \\
\text { mobile phase or water }\end{array}$ & HPLC & $\begin{array}{l}4 \mathrm{ng}(\mathrm{o}-\mathrm{MHA} \\
\text { and } m-\mathrm{MHA})\end{array}$ & $\begin{array}{l}\text { 99B99.9 } \\
(\text { o-MHA }) \\
97.2 \mathrm{~B} 99.9 \\
(m-\mathrm{MHA})\end{array}$ & Astier 1992 \\
\hline Urine & $\begin{array}{l}\text { Adsorb to Sep-Pack C18 } \\
\text { cartridge; elute with methanol }\end{array}$ & HPLC & $\begin{array}{l}10 \mu \mathrm{g} / \mathrm{mL} \\
(\mathrm{xyl}-\mathrm{m})\end{array}$ & $\begin{array}{l}94.7 \mathrm{~B} 96.1 \\
(\mathrm{xyl}-\mathrm{m})\end{array}$ & $\begin{array}{l}\text { Tanaka et } \\
\text { al. } 1990\end{array}$ \\
\hline Urine & $\begin{array}{l}\text { Acidify with } \mathrm{H}_{2} \mathrm{SO}_{4} \text {; extract } \\
\text { with methyl-t-butyl ether; } \\
\text { concentrate }\end{array}$ & HPLC & $\begin{array}{l}0.1 \mu \mathrm{mol}(o-, p- \\
m-\mathrm{MHA})\end{array}$ & $\begin{array}{l}91 \text { (o-MHA); } \\
107 \text { (m-MHA); } \\
113 \text { (p-MHA) }\end{array}$ & $\begin{array}{l}\text { Tardif et al. } \\
1989\end{array}$ \\
\hline Urine & $\begin{array}{l}\text { Acidify with } \mathrm{HCl} \text {, saturate with } \\
\text { sodium chloride; extract with } \\
\text { ethyl acetate; dry and } \\
\text { redissolve in distilled water }\end{array}$ & HPLC/ UV & $\begin{array}{l}0.2 \mathrm{mg} / \mathrm{mL} \\
\text { (methyl hippuric } \\
\text { acid; method } \\
\text { does not } \\
\text { distinguish } \\
\text { between } p \text { - and } \\
m \text { - isomers) }\end{array}$ & 98 & $\begin{array}{l}\text { NIOSH } 1994 \\
\text { (Method } \\
\text { 8301) }\end{array}$ \\
\hline Urine & $\begin{array}{l}\text { Acidify sample and extract } \\
\text { with ethyl acetate and } \\
\text { methylating solution }\end{array}$ & GC/FID & $5 \mathrm{mg} / \mathrm{L}$ & $\begin{array}{l}81.5(o-\mathrm{MHA}) \\
82.2(m-\mathrm{MHA}) \\
84.8(p-\mathrm{MHA})\end{array}$ & $\begin{array}{l}\text { Caperos } \\
\text { and } \\
\text { Fernandez } \\
1977\end{array}$ \\
\hline
\end{tabular}




\section{Table 7-1. Analytical Methods for Determining Xylene in Biological Materials}

\begin{tabular}{|c|c|c|c|c|c|}
\hline $\begin{array}{l}\text { Sample } \\
\text { matrix }\end{array}$ & Preparation method & $\begin{array}{l}\text { Analytical } \\
\text { method }\end{array}$ & $\begin{array}{l}\text { Sample } \\
\text { detection limit }\end{array}$ & $\begin{array}{l}\text { Percent } \\
\text { recovery }\end{array}$ & Reference \\
\hline Urine & $\begin{array}{l}\text { Adjust } \mathrm{pH} \text { of sample to } 2.0 \text {; } \\
\text { extract with ethylacetate }\end{array}$ & GC/FID & No data & $98(m-\mathrm{MHA})$ & $\begin{array}{l}\text { Engstrom et } \\
\text { al. } 1976\end{array}$ \\
\hline Urine & $\begin{array}{l}\text { Acidify sample with } \mathrm{HCl} \text { and } \\
\text { extract with ethylacetate; add } \\
\text { methanol to ethylacetate } \\
\text { extract; methylate extract with } \\
\text { diazomethane in diethyl ether } \\
\text { solution }\end{array}$ & GC/FID & No data & $\begin{array}{l}88.7 \mathrm{~B} 95 \\
(m-\mathrm{MHA}) \\
79.3 \mathrm{~B} 82 \\
(p-\mathrm{MHA})\end{array}$ & $\begin{array}{l}\text { Morin et al. } \\
1981\end{array}$ \\
\hline Urine & Acidify sample & HPLC & No data & $C V=4.8$ & Astier 1992 \\
\hline Urine & $\begin{array}{l}\text { Acidify sample with } \mathrm{HCl} \\
\text { extract with } \mathrm{n} \text { butyl } \\
\text { chloride:isopropanol }(9: 1)\end{array}$ & HPLC & $\begin{array}{l}0.1 \mathrm{mg} / \mathrm{mL} \\
(m-\mathrm{MHA})\end{array}$ & No data & $\begin{array}{l}\text { Poggi et al. } \\
1982\end{array}$ \\
\hline Urine & $\begin{array}{l}\text { Adjust pH of sample to } 2.0 \text {; } \\
\text { extract with methyl ethyl } \\
\text { ketone; add phenacyl bromide } \\
\text { solution to extract and heat }\end{array}$ & HPLC & $\begin{array}{l}0.02 \mu \mathrm{g} / \mathrm{sample} \\
(m-\mathrm{MHA}) \\
0.02 \mu \mathrm{g} / \mathrm{sample} \\
(p-\mathrm{MHA})\end{array}$ & No data & $\begin{array}{l}\text { Sugihara } \\
\text { and Ogata } \\
1978\end{array}$ \\
\hline Urine & $\begin{array}{l}\text { Add sample specimen to } \\
\text { methanol; centrifuge }\end{array}$ & HPLC & $\begin{array}{l}6 \mathrm{mg} / \mathrm{L}(o-\mathrm{MHA}) ; \\
8 \mathrm{mg} / \mathrm{L} \\
(m-\mathrm{MHA}) \\
8 \mathrm{mg} / \mathrm{L}(p-\mathrm{MHA})\end{array}$ & $\begin{array}{l}102(o-\mathrm{MHA}) \\
102.4(m-\mathrm{MHA}) \\
99.5(p-\mathrm{MHA})\end{array}$ & $\begin{array}{l}\text { Ogata and } \\
\text { Taguchi } \\
1987\end{array}$ \\
\hline Urine & $\begin{array}{l}\text { Acidify sample; extract with } \\
\text { chloroform and concentrate }\end{array}$ & TLC & $\begin{array}{l}6 \mu \mathrm{g} / \mathrm{mL} \\
(m-\mathrm{MHA})\end{array}$ & $100(m-\mathrm{MHA})$ & $\begin{array}{l}\text { Bieniek and } \\
\text { Wilczok } \\
1981\end{array}$ \\
\hline $\begin{array}{l}\text { Exhaled } \\
\text { breath }\end{array}$ & $\begin{array}{l}\text { Trap sample on charcoal } \\
\text { cloth; desorb with carbon } \\
\text { disulfide }\end{array}$ & GC/FID & $\begin{array}{l}0.06 \text { ppm } \\
\text { (m-xylene) }\end{array}$ & 90 & $\begin{array}{l}\text { Glaser and } \\
\text { Arnold 1989; } \\
\text { Glaser et al. } \\
1990\end{array}$ \\
\hline $\begin{array}{l}\text { Exhaled } \\
\text { breath }\end{array}$ & $\begin{array}{l}\text { Sorb to Tenax TA tube; } \\
\text { thermally purge }\end{array}$ & GC/FID & $0.03 \mathrm{ppm}$ & 60 (m-xylene) & $\begin{array}{l}\text { Glaser et al. } \\
1990\end{array}$ \\
\hline $\begin{array}{l}\text { Exhaled } \\
\text { breath }\end{array}$ & $\begin{array}{l}\text { Sorb to Tenax GC tube; dry; } \\
\text { thermally desorb }\end{array}$ & GC/MS & $0.50 \mu \mathrm{g} / \mathrm{m}^{3}$ & No data & $\begin{array}{l}\text { Pellizzari et } \\
\text { al. } 1988\end{array}$ \\
\hline $\begin{array}{l}\text { Whole body } \\
\text { (mice) }\end{array}$ & $\begin{array}{l}\text { Kill mice and inject with } \\
\text { solvent sample; homogenize } \\
\text { sample in liquid nitrogen; } \\
\text { evaporate liquid nitrogen and } \\
\text { extract with carbon disulfide }\end{array}$ & GC/FID & No data & 86 (m-xylene) & $\begin{array}{l}\text { Tsuruta and } \\
\text { Iwasaki } \\
1984\end{array}$ \\
\hline Fish & $\begin{array}{l}\text { Freeze sample; homogenize } \\
\text { in liquid nitrogen; vacuum } \\
\text { distillation }\end{array}$ & $\begin{array}{l}\text { GC/MS } \\
\text { equipped } \\
\text { with fused- } \\
\text { silica } \\
\text { capillary } \\
\text { column } \\
\end{array}$ & No data & No data & Hiatt 1983 \\
\hline
\end{tabular}

$\mathrm{CV}=$ coefficient of variation; GC/FID = gas chromatography/flame ionization detector; GC/MS = gas chromatography/mass spectrometry; $\mathrm{HCl}=$ hydrochloric acid; $\mathrm{HPLC}=$ high performance liquid chromatography; $\mathrm{H}_{2} \mathrm{SO}_{4}=$ sulfuric acid; $\mathrm{MHA}=$ methylhippuric acid; $\mathrm{THA}-\mathrm{OH}=$ tetrahexyl ammonium hydroxide; TLC = thin-layer chromatography; UV = ultra violet; $x y l-m=N$ acetyl-s-xylyl-L-cysteine 


\section{Table 7-2. Analytical Methods for Determining Xylene in Environmental Samples}

\begin{tabular}{|c|c|c|c|c|c|}
\hline $\begin{array}{l}\text { Sample } \\
\text { matrix }\end{array}$ & Preparation method & $\begin{array}{l}\text { Analytical } \\
\text { method }\end{array}$ & $\begin{array}{l}\text { Sample } \\
\text { detection limit }\end{array}$ & Percent recovery & Reference \\
\hline Air & $\begin{array}{l}\text { Collect sample on porous } \\
\text { polymer adsorbent; } \\
\text { thermally desorb }\end{array}$ & GC/FID & $0.1 \mathrm{ppm}$ & No data & Brown 1988b \\
\hline Air & $\begin{array}{l}\text { Sorption to a tube } \\
\text { containing Tenax, } \\
\text { Ambersorb XE 340, and } \\
\text { charcoal; thermally desorb }\end{array}$ & GC/MS & $\begin{array}{l}4.0 \mathrm{ng} / \text { tube } \\
\text { (5B50-L air } \\
\text { sample) }\end{array}$ & $\begin{array}{l}\text { 93B103 (o-xylene); } \\
90.8(p-, m \text {-xylene })\end{array}$ & $\begin{array}{l}\text { Chan et al. } \\
1990\end{array}$ \\
\hline Air & $\begin{array}{l}\text { Draw sample through } \\
\text { copper tubing with a } \\
\text { diaphragm pump }\end{array}$ & GC/PID & $0.3 \mathrm{ppb}$ & No data & $\begin{array}{l}\text { Hester and } \\
\text { Meyer } 1979\end{array}$ \\
\hline Air & $\begin{array}{l}\text { Absorption on Tenax GC } \\
\text { air sampler }\end{array}$ & GC/MS & No data & No data & $\begin{array}{l}\text { Hampton et al. } \\
1982\end{array}$ \\
\hline Air & $\begin{array}{l}\text { Collect on coconut shell } \\
\text { charcoal personal sampler; } \\
\text { desorb with carbon } \\
\text { disulfide }\end{array}$ & GC/FID & $2.6 \mathrm{mg}$ & No data & $\begin{array}{l}\text { NIOSH } 1994 \\
\text { (Method 1501) }\end{array}$ \\
\hline Air & $\begin{array}{l}\text { Pump air sample through } \\
\text { charcoal tubes; extract } \\
\text { charcoal with carbon } \\
\text { disulfide }\end{array}$ & GC/FID & $\begin{array}{l}<0.05 \mathrm{ppm} \\
\text { (o-xylene); } \\
<0.05 \mathrm{ppm} \\
(\text {-xylene })\end{array}$ & $\begin{array}{l}\text { 51B86 (o-xylene); } \\
\text { 51B86 (p-xylene) }\end{array}$ & $\begin{array}{l}\text { Brown 1988a; } \\
\text { Otson et al. } \\
1983\end{array}$ \\
\hline Air & $\begin{array}{l}\text { Collect sample in Tedlar } \\
\text { bags by means of an } \\
\text { automated sequential large } \\
\text { air sampler }\end{array}$ & GC/FID & No data & No data & $\begin{array}{l}\text { Lonneman et } \\
\text { al. } 1974\end{array}$ \\
\hline Air & $\begin{array}{l}\text { Collect air on activated } \\
\text { charcoal; desorb with } \\
\text { carbon disulfide; shake with } \\
75 \% \mathrm{H}_{2} \mathrm{SO}_{4}\end{array}$ & $\begin{array}{l}\text { GC/FID } \\
\text { LC/UV }\end{array}$ & $\begin{array}{l}1 \mu \mathrm{g} / \mu \mathrm{L} \\
\text { No data }\end{array}$ & $\begin{array}{l}\text { 92B100 } \\
92 \mathrm{~B} 104\end{array}$ & $\begin{array}{l}\text { Esposito and } \\
\text { Jacobs } 1977\end{array}$ \\
\hline Air & $\begin{array}{l}\text { Collect sample in } \\
\text { pressurized stainless steel } \\
\text { cannister }\end{array}$ & $\begin{array}{l}\text { GC- } \\
\text { FID/PID }\end{array}$ & $\begin{array}{l}1.3 \mathrm{pg} / \mathrm{sample} \\
\text { (o-xylene) }\end{array}$ & No data & $\begin{array}{l}\text { Nutmagul et al. } \\
1983\end{array}$ \\
\hline Air & $\begin{array}{l}\text { Collect sample in a } \\
\text { pressurized cannister }\end{array}$ & $\begin{array}{l}\text { GC- } \\
\text { FID/ELCD } \\
\text { and } \\
\text { GC/MS }\end{array}$ & $<1 \mathrm{ppm}$ & No data & Pleil et al. 1988 \\
\hline Air & $\begin{array}{l}\text { Collect sample on silica } \\
\text { gel; extract with isopropyl } \\
\text { benzene }\end{array}$ & GC & No data & $>99 \%$ & $\begin{array}{l}\text { Whitman and } \\
\text { Johnston } 1964\end{array}$ \\
\hline $\begin{array}{l}\text { Drinking } \\
\text { water }\end{array}$ & Purge and trap on sorbent & GC/FID & $\begin{array}{l}<1 \mu \mathrm{\mu g} / \mathrm{L} \\
(o-x y l e n e) \\
<1 \mu \mathrm{g} / \mathrm{L} \\
\text { (m-xylene) }\end{array}$ & $\begin{array}{l}75 \text { (o-xylene); } 87 \\
\text { (m-xylene) }\end{array}$ & $\begin{array}{l}\text { Otson and } \\
\text { Williams } 1982\end{array}$ \\
\hline
\end{tabular}




\section{Table 7-2. Analytical Methods for Determining Xylene in Environmental Samples}

\begin{tabular}{|c|c|c|c|c|c|}
\hline $\begin{array}{l}\text { Sample } \\
\text { matrix }\end{array}$ & Preparation method & $\begin{array}{l}\text { Analytical } \\
\text { method }\end{array}$ & $\begin{array}{l}\text { Sample } \\
\text { detection limit }\end{array}$ & Percent recovery & Reference \\
\hline $\begin{array}{l}\text { Drinking } \\
\text { water }\end{array}$ & Extract sample in hexane & GC/FID & $\begin{array}{l}2 \mu \mathrm{g} / \mathrm{L} \\
\text { (o-xylene); } \\
2 \mu \mathrm{g} / \mathrm{L} \\
\text { (m-xylene); } \\
2 \mu \mathrm{g} / \mathrm{L} \\
\text { (p-xylene) }\end{array}$ & $\begin{array}{l}\text { 80B96 (o-xylene); } \\
\text { 80B83 ( } m \text {-xylene); } \\
\text { 78B85 ( } p \text {-xylene })\end{array}$ & $\begin{array}{l}\text { Otson and } \\
\text { Williams } 1981\end{array}$ \\
\hline Water & $\begin{array}{l}\text { Purge and trap; methyl- } \\
\text { silicone-coated packing is } \\
\text { recommended; desorb } \\
\text { thermally }\end{array}$ & GC/MS & $\begin{array}{l}0.06 \mu \mathrm{g} / \mathrm{L} \\
\text { (o-xylene); } \\
0.03 \mu \mathrm{g} / \mathrm{L} \\
(m \text {-xylene); } \\
0.06 \mu \mathrm{g} / \mathrm{L} \\
(p \text {-xylene) }\end{array}$ & $\begin{array}{l}94 \text { (o-xylene); } \\
94 \text { (m-xylene); } \\
97 \text { (p-xylene) }\end{array}$ & $\begin{array}{l}\text { NEMI } 2005 \\
\text { (EPA Method } \\
524.2 \text { ) }\end{array}$ \\
\hline Water & $\begin{array}{l}\text { Purge and trap; desorb } \\
\text { thermally }\end{array}$ & GC/MS & $\begin{array}{l}0.24 \mu \mathrm{g} / \mathrm{L} \\
\text { (o-xylene); } \\
0.48 \mu \mathrm{g} / \mathrm{L} \\
(m \text {-xylene); } \\
0.48 \mu \mathrm{g} / \mathrm{L} \\
(p \text {-xylene) }\end{array}$ & $\begin{array}{l}94 \text { (o-xylene) } \\
100 \text { (m-xylene) } \\
100 \text { (p-xylene) }\end{array}$ & $\begin{array}{l}\text { NEMI } 2005 \\
\text { (ASTM D5790) }\end{array}$ \\
\hline Water & $\begin{array}{l}\text { Purge and trap; desorb } \\
\text { thermally }\end{array}$ & GC/MS & $\begin{array}{l}0.03 \mu \mathrm{g} / \mathrm{L} \\
\text { (o-xylene) }\end{array}$ & 106 (o-xylene) & $\begin{array}{l}\text { NEMI } 2005 \\
\text { (Standard } \\
\text { Methods } \\
6200 \mathrm{~B})\end{array}$ \\
\hline Water & $\begin{array}{l}\text { Purge and trap; desorb } \\
\text { thermally }\end{array}$ & GC/ELCD & $\begin{array}{l}0.02 \mu \mathrm{g} / \mathrm{L} \\
\text { (o-xylene) }\end{array}$ & 68 (o-xylene) & $\begin{array}{l}\text { NEMI } 2005 \\
\text { (Standard } \\
\text { Methods } \\
6200 \mathrm{C} \text { ) }\end{array}$ \\
\hline $\begin{array}{l}\text { Ground- } \\
\text { water }\end{array}$ & $\begin{array}{l}\text { Solid-phase microextration } \\
\text { (methyl-silicone fiber } \\
\text { coated with methyl-silicone } \\
\text { film) }\end{array}$ & GC/FID & $1 \mu \mathrm{g} / \mathrm{L}$ & No data & $\begin{array}{l}\text { Arthur et al. } \\
1992\end{array}$ \\
\hline Water & $\begin{array}{l}\text { Purge and trap; methyl- } \\
\text { silicone-coated packing is } \\
\text { recommended; desorb } \\
\text { thermally }\end{array}$ & $\begin{array}{l}\text { GC- } \\
\text { PC/PID }\end{array}$ & $0.01 \mathrm{~B} 0.05 \mu \mathrm{g} / \mathrm{L}$ & $\begin{array}{l}90 \text { (o-xylene }) \\
90 \text { (m-xylene) } \\
85 \text { ( } p \text {-xylene })\end{array}$ & $\begin{array}{l}\text { APHA } 1992 \\
\text { (equivalent to } \\
\text { EPA Method } \\
503.1 \text { ) }\end{array}$ \\
\hline Water & $\begin{array}{l}\text { Purge and trap; methyl- } \\
\text { silicone-coated packing is } \\
\text { recommended; desorb } \\
\text { thermally }\end{array}$ & $\begin{array}{l}\text { GC- } \\
\text { CC/PID }\end{array}$ & $\begin{array}{l}0.02 \mu \mathrm{g} / \mathrm{L} \\
\text { (o-xylene); } \\
0.01 \mu \mathrm{g} / \mathrm{L} \\
(m \text {-xylene); } \\
0.01 \mu \mathrm{g} / \mathrm{L} \\
(p \text {-xylene) }\end{array}$ & $\begin{array}{l}99 \text { (o-xylene) } \\
100 \text { (m-xylene) } \\
99 \text { (p-xylene) }\end{array}$ & $\begin{array}{l}\text { NEMI } 2005 \\
\text { (EPA Method } \\
502.2 \text { ) }\end{array}$ \\
\hline Soil & $\begin{array}{l}\text { Extract sample with } \\
\text { methanol; centrifuge }\end{array}$ & GC & No data & No data & $\begin{array}{l}\text { Anderson et al. } \\
1991\end{array}$ \\
\hline
\end{tabular}




\section{Table 7-2. Analytical Methods for Determining Xylene in Environmental Samples}

\begin{tabular}{|c|c|c|c|c|c|}
\hline $\begin{array}{l}\text { Sample } \\
\text { matrix }\end{array}$ & Preparation method & $\begin{array}{l}\text { Analytical } \\
\text { method }\end{array}$ & $\begin{array}{l}\text { Sample } \\
\text { detection limit }\end{array}$ & Percent recovery & Reference \\
\hline $\begin{array}{l}\text { Sediment } \\
\text { (clay) }\end{array}$ & $\begin{array}{l}\text { Shake sample with water; } \\
\text { purge and trap on Porapak } \\
\mathrm{N} \text { cartridges; elute with } \\
\mathrm{MeOH}\end{array}$ & $\begin{array}{l}\mathrm{GC} \\
\text { ECD/PID } \\
\text { GC/ELCD }\end{array}$ & $\begin{array}{l}7 \mathrm{ng} / \mathrm{g} \\
1 \mathrm{ng} / \mathrm{g}\end{array}$ & $\begin{array}{l}\text { 70B77 ( } p \text {-xylene); } \\
\text { 68B79 (o-xylene) } \\
\text { No data }\end{array}$ & $\begin{array}{l}\text { Amin and } \\
\text { Narang } 1985\end{array}$ \\
\hline Various & $\begin{array}{l}\text { Direct injection, purge and } \\
\text { trap, or vacuum distillation }\end{array}$ & GC/PID & $\begin{array}{l}0.02 \mu \mathrm{g} / \mathrm{L} \\
(0-x y l e n e) \\
0.01 \mu \mathrm{g} / \mathrm{L} \\
(m-x y l e n e) \\
0.01 \mu \mathrm{g} / \mathrm{L} \\
(p \text {-xylene) }\end{array}$ & $\begin{array}{l}99 \text { (o-xylene) } \\
100 \text { (m-xylene) } \\
99 \text { (p-xylene) }\end{array}$ & $\begin{array}{l}\text { NEMI } 2005 \\
\text { (EPA Method } \\
\text { 8021B) }\end{array}$ \\
\hline Various & $\begin{array}{l}\text { Direct injection, purge and } \\
\text { trap, or vacuum distillation }\end{array}$ & GC/ELCD & No data & No data & $\begin{array}{l}\text { NEMI } 2005 \\
\text { (EPA Method } \\
\text { 8021B) }\end{array}$ \\
\hline Various & $\begin{array}{l}\text { Purge and trap, azeotropic } \\
\text { distillation, vacuum } \\
\text { distillation, head space, or } \\
\text { direct injection }\end{array}$ & GC/MS & NA & $\begin{array}{l}106 \text { (o-xylene) } \\
106 \text { (m-xylene) } \\
97 \text { ( } p \text {-xylene })\end{array}$ & $\begin{array}{l}\text { NEMI } 2005 \\
\text { (EPA Method } \\
\text { 8260B) }\end{array}$ \\
\hline Waste & Extract waste with hexane & GC/MS & No data & No data & $\begin{array}{l}\text { Austern et al. } \\
1975\end{array}$ \\
\hline Waste & $\begin{array}{l}\text { Add sample to a small } \\
\text { volume of ethanol and } \\
\text { dilute with water or raw } \\
\text { wastewater; adjust the pH; } \\
\text { extract with Freon-TF }\end{array}$ & GC/FID & No data & No data & $\begin{array}{l}\text { Austern et al. } \\
1975\end{array}$ \\
\hline
\end{tabular}

$\mathrm{CC}=$ capillary column; ELCD = electron capture detector; FID = flame ionization detector; GC = gas

chromatography; $\mathrm{H}_{2} \mathrm{SO}_{4}=$ sulfuric acid; $\mathrm{MeOH}=$ methanol; $\mathrm{MS}=$ mass spectrometry; LC = liquid chromatography; $\mathrm{PC}=$ packed column; PID = photoionization detector; UV = ultraviolet spectrometry 
To overcome the low recoveries obtained with the purge and trap method, another extraction procedure is recommended that uses Amberlite XAD-2 adsorbent resin present in the blood collection tube when the sampling takes place. This method dispenses with the readsorption of the hydrocarbon from the sampling tube to the polymer and gives recoveries of $77-98 \%$ (Norstrom and Scheepers 1990).

The use of GC/FID followed by a combination of packed and open tubular capillary GC and GC/MS to detect and quantify the isomers of xylene in human tissues and fluids has been reported in the literature. Brain, liver, lung, kidney, and blood samples of individuals who died following occupational exposure to several organic solvents were analyzed using a combination of capillary columns (Bellanca et al. 1982). The sensitivity and resolution of the isomers of xylene were increased, and detection limits of $0.05 \mathrm{mg}$, $0.05 \mathrm{mg}$, and $0.01 \mathrm{mg}$ per 100 grams of sample were obtained for $\mathrm{m-}, \mathrm{O}-$, and $p$-xylene, respectively (Bellanca et al. 1982). Despite this increased resolving power, adequate separation of $m$ - and $p$-xylene was unattainable.

Exposure to xylene may also be indicated by its presence in exhaled breath. Xylene in mainstream breath may be determined by exhaling through a charcoal cloth (Glaser and Arnold 1989); xylene in sidestream breath is trapped using a two-stage Tenax TA sorbent sampler (Glaser et al. 1990) or a Tenax GC cartridge (Pellizzari et al. 1988). The Tenax cartridge is dried over calcium sulfate, and then the xylene is thermally desorbed for GC/MS. Correlations with carbon dioxide measurements were 90 and $60 \%$ for mainstream and sidestream breath, respectively (Glaser et al. 1990), with a quantification limit of $400 \mu \mathrm{g} / \mathrm{m}^{3}$ of $m$-xylene for a 50-L sample (Glaser and Arnold 1989). The detection limit (LOD) was $0.50 \mu \mathrm{g} / \mathrm{m}^{3}$ with a quantification limit 5 times the LOD for a 15-L breath sample (Pellizzari et al. 1988).

In addition to direct measurement of xylene in biological tissues and fluids, it is also possible to determine the concentration of its metabolites in biological fluids. A simple, sensitive, and specific automated HPLC technique was developed for direct and simultaneous quantification of $o-, m-$, and p-methylhippuric acids, the metabolites of $o-, m$-, and p-xylene, respectively (Ogata and Taguchi 1987; Sugihara and Ogata 1978; Tardif et al. 1989). A possible disadvantage of the HPLC technique is that at low concentrations $(<0.6 \mathrm{mg} / \mathrm{L})$ in urine, these methylhippuric acids may not be distinguishable from similar compounds. However, addition of a mobile phase, consisting of mixture of acetonitrile and 1\% phosphoric acid, has been used to distinguish between xylene metabolites and other solvents such as benzene and toluene in the urine (Astier 1992). Use of methanol as a solvent for the urine obviates the need for the customary ethylether extraction step and allows direct urine injection for HPLC (Ogata and Taguchi 1988). N-Acetyl-S-xylyl-L-cysteine, a mercapturic acid, is also a urinary metabolite of xylene 
that may be detected by direct HPLC (Tanaka et al. 1990). The HPLC method recommended by NIOSH (1994) does not distinguish between $p$ - and $m$-methylhippuric acids.

Other techniques that have been successful in quantitatively determining urinary concentrations of metabolites of xylene include GC/FID, GC/MS, and thin layer chromatography (TLC). GC/FID and GC/MS offer the possibility of excellent analytical sensitivity and specificity for urinary metabolites of xylene (Caperos and Fernandez 1977; de Carvalho et al. 1991; Engstrom et al. 1976; Kataoka et al. 1991; Kira 1977; Morin et al. 1981; Poggi et al. 1982). However, most GC analytical methods require the urinary metabolites to be chemically transformed into methyl esters or trimethyl silyl derivatives using ethylacetate or diazomethane. This transformation, however, is problematic and may subsequently cause low reproducibility (Caperos and Fernandez 1977; Engstrom et al. 1976; Morin et al. 1981; Poggi et al. 1982). The methylhippuric acid metabolites of the xylene isomers may be distinguished using an extractive alkylation procedure followed by capillary GC analysis (Kataoka et al. 1991). An extraction method using less toxic reagents (hydrochloric acid with methanol) has been developed (de Carvalho et al. 1991).

A simple and highly reproducible TLC method has been developed for the detection and separation of $m$ or $p$-methylhippuric acid in the urine of individuals exposed to a mixture of volatile organic solvents (Bieniek and Wilczok 1981). However, the authors noted that this analytical technique is time consuming. Furthermore, the developing agent used in this technique ( $p$-dimethylamine benzaldehyde in acetic acid) has the disadvantage that it is irritating to the eyes and mucous membranes. The sensitivity of this method was reported to be $6 \mu \mathrm{g}$ hippuric acid per $1 \mathrm{~mL}$ urine with a recovery of $100 \%$.

When measuring hippuric acids in the urine of workers exposed to xylenes, NIOSH (1994) recommends that a complete spot voiding sample be collected at the end of the shift after 2 days of exposure. As a preservative, a few crystals of thymol should be added to the sample. It should be stored at $4{ }^{\circ} \mathrm{C}$ if analysis is within 1 week. The sample should remain stable for 2 months if it is stored at $-20^{\circ} \mathrm{C}$.

\subsection{ENVIRONMENTAL SAMPLES}

A gas chromatograph equipped with an appropriate detector is the basic analytical instrument used for determining environmental levels of xylene. Precautions in the isolation, collection, and storage of xylene in environmental media are necessary to prevent loss of the volatile xylene compounds to the air. 
The most common method for detecting aromatic hydrocarbons in air is the adsorption of the vapors to either activated charcoal with extraction using carbon disulfide or adsorption to a polymer adsorbent, such as Tenax GC, with thermal desorption. Each method is then followed by injection of the desorbed sample into a gas chromatograph equipped with FID (Brown 1988a, 1988b; NIOSH 1994). The activated charcoal method requires a 12-L air sample, while the polymer adsorbent uses a smaller 5-L sample for determination of the xylene in the sub-parts-per-million range. A GC/MS method has also been developed which uses an adsorbent tube with layers of Tenax, Amberlite, and charcoal (Chan et al. 1990). The use of a molecular sieve to remove water vapor prior to adsorption has been recommended to increase recovery of the hydrocarbons (Whitman and Johnston 1964). A computer-controlled, high-speed GC system has been developed for rapid analysis of volatiles in air (and other media with appropriate vapor generation). The system combines an electrically heated cold-trap inlet (with a vacuum backflushing device on the GC) with a conventional FID. The advantage of the system is that a complete analysis cycle requires only 10 seconds to detect $p$-xylene at a level of 13.4 ppb (Rankin and Sacks 1991).

A differential optical absorption spectrophotometer has also been used to monitor $o$-xylene in air; this method gives a correlation coefficient of approximately 0.66 when compared with standard GC methods (EPA 1991a).

An automated gas chromatograph with photoionization detector (GC/PID) has been developed by Hester and Meyer (1979) to identify gas-phase hydrocarbons (including xylene) for complex systems such as vehicle exhaust gas. The GC/PID method allows for measurement of sub-parts-per-billion level concentrations of air contaminants and does not require trapping or freeze-concentration of samples before analysis. These latter preconcentration steps are usually necessary because of the limited sensitivity of FID techniques commonly used in the analysis of environmental samples. A limitation of the GC/PID technique is that $m$ - and $p$-xylene are detected but not well separated. GC/PID in tandem with FID was used to obtain a more sensitive method to determine xylene levels in the air. A detection limit of $1.3 \times 10^{-12} \mathrm{~g}$ of $o$-xylene per sample was achieved (Nutmagul et al. 1983).

A purge and trap gas chromatographic method involving photoionization detection has been developed by EPA to analyze volatiles in water (APHA 1992; NEMI 2005). A confirmatory analysis by a second analytical column or by GC/MS is advised by EPA. The purge and trap gas chromatographic method can detect the isomers of xylene and has a detection limit for $o_{-}, \mathrm{m}$-, and $p$-xylene of $0.2 \mathrm{ppb}$ (Otson and Williams 1981, 1982; Saunders et al. 1975). A purge and trap method using GC/MS has also been used to detect xylene in waste water (Koe and Tan 1990). 
Emissions of volatile organic compounds from surface waters, including ponds at hazardous waste treatment facilities, may be directly measured by the use of enclosure methods (such as a flux chamber or surface impoundment simulator connected to collection canisters) followed by GC with the effluent split between FID and an electron capture detector. Emission rates of $0.5 \mathrm{mg} / \mathrm{minute} / \mathrm{m}^{2}$ could be measured using the surface impoundment simulator with a precision of 3\% relative standard deviation (Gholson et al. 1991).

GC using both electron capture detection (ECD) and PID has been employed to determine xylene levels in sediment samples (Amin and Narang 1985). The authors indicated that their method involved transfer of samples between containers, and a considerable loss of volatile compounds was obtained.

A procedure has been developed to characterize volatile xylene compounds from fish samples by GC/MS using a fused-silica capillary column (FSCC) and vacuum distillation (Hiatt 1983). The FSCC provides a more attractive approach than packed columns for chromatographic analysis of volatile aromatic organic compounds. An FSCC can be heated to a higher temperature $\left(350{ }^{\circ} \mathrm{C}\right)$ than that recommended for packed column, thereby improving the resolution (in ppb levels) of compounds and reducing column retention times. A physical limitation for compounds that can be detected, however, is that the vapor pressure of the compound must be $>0.78$ torr $\left(\approx 50^{\circ} \mathrm{C}\right)$ in the sample chamber (Hiatt 1983).

\subsection{ADEQUACY OF THE DATABASE}

Section 104(i)(5) of CERCLA, as amended, directs the Administrator of ATSDR (in consultation with the Administrator of EPA and agencies and programs of the Public Health Service) to assess whether adequate information on the health effects of xylene is available. Where adequate information is not available, ATSDR, in conjunction with NTP, is required to assure the initiation of a program of research designed to determine the health effects (and techniques for developing methods to determine such health effects) of xylene.

The following categories of possible data needs have been identified by a joint team of scientists from ATSDR, NTP, and EPA. They are defined as substance-specific informational needs that if met would reduce the uncertainties of human health assessment. This definition should not be interpreted to mean that all data needs discussed in this section must be filled. In the future, the identified data needs will be evaluated and prioritized, and a substance-specific research agenda will be proposed. 


\subsubsection{Identification of Data Needs}

\section{Methods for Determining Biomarkers of Exposure and Effect.}

Exposure. The methods for determining xylene levels in blood and tissue samples and exhaled breath, GC/MS or GC/FID, have sufficient sensitivity to measure xylene levels associated with background levels of exposure as well as xylene levels at which biological effects occur. GC/MS has been employed to detect $O$-xylene at ppm levels in the blood (Ashley et al. 1992; Cramer et al. 1988). However, development of a GC/MS method that incorporates a less rigorously heated purge would be useful. Heated purges currently used in GC/MS have the disadvantage of reducing the absolute recoveries of volatile organic solvents. Better resolution and sensitivity are achievable with the application of a capillary GC/MS column and selection of an appropriate detector or detector combination as an alternative to the packed column approach currently in use. Also, there is a growing need for analytical methods to efficiently separate and quantify trace levels of the isomers of xylene in biological media.

Analytical methods are also available to detect and quantify the xylene metabolites present in the urine which have been correlated with exposure levels (Kawai et al. 1991; Ogata et al. 1979). These methods, HPLC (Astier 1992) and GC (coupled with MS or FID) (de Carvalho et al. 1991; Kataoka et al. 1991; Poggi et al. 1982), have been well characterized with respect to their precision, accuracy, reliability, and specificity and have sufficient sensitivity to measure xylene metabolite levels associated with biological effects. However, these methods may not be sensitive enough to measure metabolite levels associated with background exposure levels.

Currently, no methods are available to quantitatively correlate monitored levels of xylene in tissues with exposure levels or toxic effects in humans, although simultaneous measurement of xylene in exhaled breath and ambient air may prove instrumental in indicating exposure, particularly in the workplace (Glaser et al. 1990). These methods would provide the ability to evaluate possible health effects in humans resulting from exposure to xylene.

Effect. No specific biomarkers of effect have been clearly associated with xylene exposure. Some biological parameters such as hepatic microsomal enzyme activities and central nervous system activity have been tentatively linked with xylene exposure. However, insufficient data exist to adequately assess the analytical methods associated with measurement of these potential biomarkers. 


\section{Methods for Determining Parent Compounds and Degradation Products in Environmental}

Media. Methods for determining xylene and its degradation products in environmental media would help to identify contaminated areas and to determine whether the levels at contaminated sites constitute a concern for human health. Standardized methods are available to detect xylene in air (Brown 1988a, 1988b; Chan et al. 1990; Rankin and Sacks 1991), waste water (Koe and Tan 1990), drinking water (Otson and Williams 1981, 1982), fish (Hiatt 1983), and clay sediments (Amin and Narang 1985). There is growing need for simultaneously achieving lower (less than ppb) detection limits, separating the $m$ - and $p$-isomers of xylene, and obtaining an adequate sample recovery. Such methods would provide useful information for assessing the biological effects of exposure to xylene and for delineating dose-response relationships. A combination of capillary gas chromatography coupled to a multi-detector system, nuclear magnetic resonance (NMR) spectroscopy, and infra-red (IR) spectroscopy would be useful to accurately identify and measure the isomers of xylene in complex environmental systems.

\subsubsection{Ongoing Studies}

The Environmental Health Laboratory Sciences Division of the National Center for Environmental Health, Centers for Disease Control and Prevention, is developing methods for the analysis of xylene and other volatile organic compounds in blood. These methods use purge and trap methodology, highresolution gas chromatography, and magnetic sector mass spectrometry, which give detection limits in the low parts per trillion (ppt) range.

No other ongoing studies concerning the identification of xylene in biological materials or environmental samples were identified. 


\section{REGULATIONS AND ADVISORIES}

The international and national regulations and guidelines regarding xylenes in air, water, and other media are summarized in Table 8-1.

ATSDR has derived inhalation and oral MRLs that apply to mixed xylenes and the individual isomers. An acute-duration inhalation MRL of $2 \mathrm{ppm}$ is based on a minimal LOAEL of $50 \mathrm{ppm}\left(217 \mathrm{mg} / \mathrm{m}^{3}\right)$ for mild objective and subjective respiratory effects and subjective neurological effects in subjects exposed to $m$-xylene vapor for 2 hours (Ernstgard et al. 2002); an uncertainty factor of 30 was applied to the minimal LOAEL. An intermediate-duration inhalation MRL of $0.6 \mathrm{ppm}$ is based on a minimal LOAEL of $50 \mathrm{ppm}$ $\left(217 \mathrm{mg} / \mathrm{m}^{3}\right)$ for a small decrease in the latency of the paw-lick response in male rats exposed to $\mathrm{m}$-xylene vapor 6 hours/day, 5 days/week for 3 months (Korsak et al. 1992); an uncertainty factor of 90 was applied to the human equivalent minimal LOAEL. A chronic-duration inhalation MRL of $0.05 \mathrm{ppm}$ is based on a LOAEL of 14 ppm (geometric mean) for mild subjective respiratory and neurological symptoms in workers exposed to $70 \%$ xylene 8 hours/day, 5 days/week for 1-7 years (Uchida et al. 1993); an uncertainty factor of 100 and modifying factor of 3 was applied to the LOAEL. An acute-duration oral MRL of $1 \mathrm{mg} / \mathrm{kg} /$ day is based on a NOAEL of $125 \mathrm{mg} / \mathrm{kg}$ and a LOAEL of $250 \mathrm{mg} / \mathrm{kg}$ for alteration of visual evoked brain potentials in rats exposed to $p$-xylene for 45 minutes (Dyer et al. 1988); an uncertainty factor of 100 was applied to the NOAEL. An intermediate-duration oral MRL of $0.4 \mathrm{mg} / \mathrm{kg} /$ day is based on a NOAEL of $500 \mathrm{mg} / \mathrm{kg} /$ day (duration-adjusted, $360 \mathrm{mg} / \mathrm{kg} /$ day) and a LOAEL of $1,000 \mathrm{mg} / \mathrm{kg} /$ day (duration-adjusted, $710 \mathrm{mg} / \mathrm{kg} /$ day) for hyperactivity in mice immediately after oral gavage dosing with mixed xylene (plus 17\% ethylbenzene) 5 days/week during weeks 4-51 of a 2-year study (NTP 1986); an uncertainty factor of 100 was applied to the duration-adjusted NOAEL. In addition, a modifying factor of 10 was applied to account for the lack of testing for sensitive neurological effects and lack of developmental and multi-generational data. A chronic-duration oral MRL of $0.2 \mathrm{mg} / \mathrm{kg} /$ day is based on a NOAEL of $250 \mathrm{mg} / \mathrm{kg} /$ day (duration-adjusted, $179 \mathrm{mg} / \mathrm{kg} /$ day) for the lack of any overt neurological toxicity or systemic toxicity in rats that received oral gavage doses of mixed xylene (plus 17\% ethylbenzene) 5 days/week for 2 years (NTP 1986); an uncertainty factor of 100 was applied to the duration-adjusted NOAEL of $179 \mathrm{mg} / \mathrm{kg} /$ day. In addition, a modifying factor of 10 was applied for the lack of testing for sensitive neurological end points and lack of developmental and multigenerational data. Specific details about the MRL derivations are in Appendix A.

EPA (IRIS 2005) has derived an inhalation reference concentration (RfC) for mixed xylenes of $0.1 \mathrm{mg} / \mathrm{m}^{3}$ $(0.02 \mathrm{ppm})$ based on a NOAEL of $50 \mathrm{ppm}\left(217 \mathrm{mg} / \mathrm{m}^{3}\right)$ and a LOAEL of $100 \mathrm{ppm}\left(434 \mathrm{mg} / \mathrm{m}^{3}\right)$ for 
Table 8-1. Regulations and Guidelines Applicable to Xylenes

\begin{tabular}{|c|c|c|c|}
\hline Agency & Description & Information & Reference \\
\hline \multicolumn{4}{|c|}{ INTERNATIONAL } \\
\hline \multicolumn{4}{|c|}{ Guidelines: } \\
\hline IARC & Carcinogenicity classification & Group $3^{a}$ & IARC 2004 \\
\hline \multirow[t]{2}{*}{ WHO } & Air quality guidelines & No data & WHO 2000 \\
\hline & Drinking water quality guidelines & $0.5 \mathrm{mg} / \mathrm{L}^{\mathrm{b}}$ & WHO 2004 \\
\hline \multicolumn{4}{|l|}{ NATIONAL } \\
\hline \multicolumn{4}{|c|}{ Regulations and Guidelines: } \\
\hline \multicolumn{4}{|c|}{ a. Air } \\
\hline \multirow[t]{2}{*}{ ACGIH } & TLV (TWA) & $435 \mathrm{mg} / \mathrm{m}^{3}$ & ACGIH 2004 \\
\hline & STEL & $655 \mathrm{mg} / \mathrm{m}^{3}$ & \\
\hline EPA & $\begin{array}{l}\text { Hazardous air pollutant (isomers and } \\
\text { mixture) }\end{array}$ & Yes & $\begin{array}{l}\text { EPA } 2004 b \\
42 \text { USC } 7412\end{array}$ \\
\hline \multirow[t]{14}{*}{ NAS/NRC } & $A E G L-1^{c}$ & & EPA 2007 \\
\hline & 10,30 , and 60 minutes, 4 and 8 hours & 130 ppm & \\
\hline & AEGL-2 ${ }^{c}$ & & \\
\hline & 10 minutes & $2500 \mathrm{ppm}^{\mathrm{d}}$ & \\
\hline & 30 minutes & $1300 \mathrm{ppm}^{\mathrm{d}}$ & \\
\hline & 60 minutes & $920 \mathrm{ppm}^{\mathrm{d}}$ & \\
\hline & 4 hours & 500 ppm & \\
\hline & 8 hours & 400 ppm & \\
\hline & AEGL-3 ${ }^{c}$ & & \\
\hline & 10 minutes & $7,200 \mathrm{ppm}^{\mathrm{e}}$ & \\
\hline & 30 minutes & $3,600 \mathrm{ppm}^{\mathrm{d}}$ & \\
\hline & 60 minutes & $2,500 \mathrm{ppm}^{\mathrm{d}}$ & \\
\hline & 4 hours & $1,300 \mathrm{ppm}^{\mathrm{d}}$ & \\
\hline & 8 hours & $1,000 \mathrm{ppm}^{\mathrm{d}}$ & \\
\hline \multirow[t]{3}{*}{$\mathrm{NIOSH}$} & REL (15-minute ceiling limit) & $435 \mathrm{mg} / \mathrm{m}^{3}$ & NIOSH 2005 \\
\hline & IDLH & $3,906 \mathrm{mg} / \mathrm{m}^{3}$ & \\
\hline & STEL & $655 \mathrm{mg} / \mathrm{m}^{3}$ & \\
\hline \multirow[t]{3}{*}{ OSHA } & PEL (8-hour TWA) for general industry & $435 \mathrm{mg} / \mathrm{m}^{3}$ & $\begin{array}{l}\text { OSHA 2005c } \\
29 \text { CFR 1910.1000 }\end{array}$ \\
\hline & $\begin{array}{l}\text { PEL (8-hour TWA) for construction } \\
\text { industry }\end{array}$ & $435 \mathrm{mg} / \mathrm{m}^{3}$ & $\begin{array}{l}\text { OSHA 2005b } \\
29 \text { CFR } 1926.55\end{array}$ \\
\hline & PEL (8-hour TWA) for shipyard industry & $435 \mathrm{mg} / \mathrm{m}^{3}$ & $\begin{array}{l}\text { OSHA 2005a } \\
29 \text { CFR 1915.1000 }\end{array}$ \\
\hline \multicolumn{4}{|l|}{ b. Water } \\
\hline EPA & $\begin{array}{l}\text { Designated as hazardous substances in } \\
\text { accordance with Section } 311(b)(2)(A) \text { of } \\
\text { the Clean Water Act }\end{array}$ & Yes & $\begin{array}{l}\text { EPA } 2005 b \\
40 \text { CFR } 116.4\end{array}$ \\
\hline
\end{tabular}


Table 8-1. Regulations and Guidelines Applicable to Xylenes

\begin{tabular}{|c|c|c|c|}
\hline Agency & Description & Information & Reference \\
\hline \multicolumn{4}{|c|}{ NATIONAL (cont) } \\
\hline \multirow[t]{8}{*}{ EPA } & $\begin{array}{l}\text { Drinking water standards and health } \\
\text { advisories }\end{array}$ & & EPA 2004a \\
\hline & 1-day HA (10-kg child) & $40 \mathrm{mg} / \mathrm{L}$ & \\
\hline & 10-day HA (10-kg child) & $40 \mathrm{mg} / \mathrm{L}$ & \\
\hline & DWEL & $7.0 \mathrm{mg} / \mathrm{L}$ & \\
\hline & $\begin{array}{l}\text { National primary drinking water } \\
\text { standards (total xylenes) }\end{array}$ & & EPA 2002b \\
\hline & MCLG & $10 \mathrm{mg} / \mathrm{L}$ & \\
\hline & $\mathrm{MCL}$ & $10 \mathrm{mg} / \mathrm{L}$ & \\
\hline & $\begin{array}{l}\text { Reportable quantities of hazardous } \\
\text { substances designated pursuant to } \\
\text { Section } 311 \text { of the Clean Water Act }\end{array}$ & 100 pounds & $\begin{array}{l}\text { EPA 2005c } \\
40 \text { CFR } 117.3\end{array}$ \\
\hline \multicolumn{4}{|l|}{ c. Food } \\
\hline \multirow[t]{3}{*}{ EPA } & $\begin{array}{l}\text { Exemption from the requirement of a } \\
\text { tolerance }\end{array}$ & & $\begin{array}{l}\text { EPA } 2005 f \\
40 \text { CFR } 180.1025\end{array}$ \\
\hline & Xylenes applied as an emulsion & $\begin{array}{l}\text { Initial concentration not } \\
\text { to exceed } 750 \mathrm{ppm}\end{array}$ & \\
\hline & $\begin{array}{l}\text { Maximum residue of xylenes in potable } \\
\text { water system/return flows of treated } \\
\text { irrigation water into receiving rivers } \\
\text { and streams }\end{array}$ & 10 ppm & \\
\hline FDA & Bottled drinking water & $10 \mathrm{mg} / \mathrm{L}$ & $\begin{array}{l}\text { FDA } 2006 \\
21 \text { CFR } 165.110\end{array}$ \\
\hline \multicolumn{4}{|l|}{ d. Other } \\
\hline \multirow[t]{2}{*}{ ACGIH } & Carcinogenicity classification & $\mathrm{A} 4^{\mathrm{f}}$ & ACGIH 2004 \\
\hline & $\begin{array}{l}\text { Biological exposure indices } \\
\text { Methylhippuric acids in urine (end of } \\
\text { shift) }\end{array}$ & $1.5 \mathrm{~g} / \mathrm{g}$ creatinine & \\
\hline \multirow[t]{5}{*}{ EPA } & Carcinogenicity classification & $\begin{array}{l}\text { Data are inadequate for } \\
\text { an assessment of the } \\
\text { carcinogenic potential }\end{array}$ & IRIS 2005 \\
\hline & Inhalation unit risk & Not applicable & \\
\hline & Oral slope factor & Not applicable & \\
\hline & $\mathrm{RfC}$ & $0.1 \mathrm{mg} / \mathrm{m}^{3}$ & \\
\hline & RfD & $0.2 \mathrm{mg} / \mathrm{kg} / \mathrm{day}$ & \\
\hline
\end{tabular}




\section{Table 8-1. Regulations and Guidelines Applicable to Xylenes}

\begin{tabular}{|c|c|c|c|}
\hline Agency & Description & Information & Reference \\
\hline \multicolumn{4}{|c|}{ NATIONAL (cont) } \\
\hline \multirow[t]{6}{*}{ EPA } & $\begin{array}{l}\text { Superfund, emergency planning, and } \\
\text { community right-to-know }\end{array}$ & & \\
\hline & $\begin{array}{l}\text { Designated CERCLA hazardous } \\
\text { substance }\end{array}$ & & $\begin{array}{l}\text { EPA 2005d } \\
40 \text { CFR } 302.4\end{array}$ \\
\hline & Reportable quantity & & \\
\hline & Xylenes and $p$-xylenes & 100 pounds & \\
\hline & $m$-Xylenes and o-xylenes & 1,000 pounds & \\
\hline & $\begin{array}{l}\text { Effective date of toxic chemical release } \\
\text { reporting }\end{array}$ & 01/01/87 & $\begin{array}{l}\text { EPA } 2005 e \\
40 \text { CFR } 372.65\end{array}$ \\
\hline NTP & Carcinogenicity classification & No data & NTP 2005 \\
\hline
\end{tabular}

${ }^{a}$ Group 3: Not classifiable as to carcinogenicity to humans

${ }^{\mathrm{b}}$ Concentrations of the substance at or below the health-based guideline value may affect the appearance, taste, or odor of the water, leading to consumer complaints.

${ }^{\mathrm{c}} \mathrm{AEGL}-1$ is the airborne concentration of a substance above which it is predicted that the general population, including susceptible individuals, could experience notable discomfort, irritation, or certain asymptomatic nonsensory effects. AEGL-2 is the airborne concentration of a substance above which it is predicted that the general population, including susceptible individuals, could experience irreversible or other serious, long-lasting adverse health effects or an impaired ability to escape. AEGL-3 is the airborne concentration of a substance above which it is predicted that the general population, including susceptible individuals, could experience life-threatening health effects or death. ${ }^{d}$ Values denoted as having safety considerations against the hazard of explosion; the Lower Explosive Limit (LEL) $=9,000 \mathrm{ppm}$ and each value should be $\geq 10 \%$ LEL. Safety considerations against the hazard(s) of explosion(s) must be taken into account.

${ }^{\text {e}}$ Extreme safety considerations against the hazard(s) of explosion(s) must be taken into account.

${ }^{f}$ A4: Not classifiable as a human carcinogen

ACGIH = American Conference of Governmental Industrial Hygienists; $A E G L=$ acute exposure guideline level; CERCLA = Comprehensive Environmetnal Response, Compensation, and Liability Act; CFR = Code of Federal Regulations; DWEL = drinking water equivalent level; EPA = Environmental Protection Agency; FDA = Food and Drug Administration; HA = health advisory; IARC = International Agency for Research on Cancer; IDLH = immediately dangerous to life or health; IRIS = Integrated Risk Informatin System; MCL = maximum contaminant level; MCLG = maximum contaminant level goal; NAS/NRC = National Academy of Sciences/National Research Council; $\mathrm{NIOSH}=$ National Institute for Occupational Safety and Health; NTP = National Toxicology Program; OSHA = Occupational Safety and Health Administration; $P E L=$ permissible exposure limit; REL = recommended exposure limit; $\mathrm{RfC}=$ inhalation reference concentration; $\mathrm{RfD}=$ oral reference dose; $\mathrm{STEL}=$ short-term exposure limit; TLV = threshold limit values; TWA = time-weighted average; USC = United States Code; WHO = World Health Organization 
impaired motor coordination (decreased rotarod performance) in male rats exposed to $m$-xylene vapor 6 hours/day, 5 days/week for 3 months (Korsak et al. 1992); an uncertainty factor of 300 was applied to the NOAEL. EPA (IRIS 2005) has derived an oral reference dose (RfD) for mixed xylenes of $0.2 \mathrm{mg} / \mathrm{kg} /$ day, based on a NOAEL of $250 \mathrm{mg} / \mathrm{kg} /$ day and a LOAEL of $500 \mathrm{mg} / \mathrm{kg} /$ day for dose-related decrease in body weight and increase in mortality in male rats treated by oral gavage 5 days/week for 2 years (NTP 1986); an uncertainty factor of 1,000 was applied to the NOAEL. 
8. REGULATIONS AND ADVISORIES

This page is intentionally blank. 


\section{REFERENCES}

Abdel-Rahman MS, Skowronski GA, Turkall RM. 1993. A comparative study of the kinetic and bioavailability of pure and soil-adsorbed benzene, toluene, and $m$-xylene after dermal exposure. In: Wang RGM, Knaak JB, Maibach HI, eds. Health risk assessment: Dermal and inhalation exposure and absorption of toxicants. Boca Raton: CRC Press, 105-116.

Abdul AS, Gibson TL, Rai DN. 1987. Statistical correlations for predicting the partition coefficient for nonpolar organic contaminants between aquifer organic carbon and water. Hazard Waste Hazard Mater 4:211-222.

Abu Al Ragheb S, Salhab AS, Amr SS. 1986. Suicide by xylene ingestion: A case report and review of literature. Am J Forensic Med Pathol 7(4):327-329.

ACGIH. 1986. Documentation of the threshold limit values and biological exposure indices. 5th ed. Cincinnati, OH: American Conference of Government Industrial Hygenists Inc., 109-110.

ACGIH. 2004. Xylene. In: Threshold limit values for chemical substances and physical agents and biological exposure indices. Cincinnati, $\mathrm{OH}$ : American Conference of Governmental Industrial Hygienists.

Adams JC, Dills RL, Morgan MS, et al. 2005. A physiologically based toxicokinetic model of inhalation exposure to xylenes in Caucasian men. Regul Toxicol Pharmacol 43(2):203-214.

Adey WH, Luckett C, Smith M. 1996. Purification of industrially contaminated groundwaters using controlled ecosystems. Ecolog Eng 7:191-212.

Adgate JL, Church TR, Ryan AD, et al. 2004b. Outdoor, indoor and personal exposure to VOCs in children. Environ Health Perspect 112(14):1386-1392.

Adgate JL, Eberly LE, Stroebel C, et al. 2004a. Personal, indoor, and outdoor VOC exposures in a probability sample of children. J Expo Anal Environ Epidemiol 14:S4-S13.

Adinolfi M. 1985. The development of the human blood-CSF-brain barrier. Dev Med Child Neurol 27:532-537.

Adlercreutz H. 1995. Phytoestrogens: Epidemiology and a possible role in cancer protection. Environ Health Perspect Suppl 103(7):103-112.

Agency for Toxic Substances and Disease Registry. 1989. Decision guide for identifying substancespecific data needs related to toxicological profiles; Notice. Fed Regist 54(174):37618-37634.

Agency for Toxic Substances and Disease Registry. 2005. Toxicological profile for benzene. Atlanta, GA: Agency for Toxic Substances and Disease Registry.

Ahaghotu E, Babu RJ, Chaterjee A, et al. 2005. Effect of methyl substitution of benzene in the percutaneous absorption and skin irritation in hairless rats. Toxicol Lett 159(3):261-271.

*Not cited in text 


\section{REFERENCES}

*AIChE. 1996. m-, o-, $p$-Xylenes. C8H10. In: Physical and thermodynamic properties of pure chemicals. American Institute of Chemical Engineers, Design Institute for Physical Property Data. Philadelphia, PA: Taylor and Francis.

Al-Ghamdi SS, Raftery MJ, Yaqoob MM. 2003a. Acute solvent exposure induced activation of cytochrome P4502E1 causes proximal tubular cell necrosis by oxidative stress. Toxicol in Vitro 17:335341.

Al-Ghamdi SS, Raftery MJ, Yaqoob MM. 2003b. Organic solvent-induced proximal tubular cell toxicity via caspase-3 activation. J Toxicol Clin Toxicol 41(7):941-945.

Al-Ghamdi SS, Raftery MJ, Yaqoob MM. 2004. Organic solvent-induced proximal tubular cell apoptosis via caspase-9 activation. Environ Toxicol Pharmacol 16:147-152.

Altman PL, Dittmer DS. 1974. Biological handbooks: Biology data book. Vol. III. 2nd ed. Bethesda, MD: Federation of American Societies for Experimental Biology, 1987-2008, 2041.

Amin TA, Narang RS. 1985. Determination of volatile organics in sediment at nanogram-per-gram concentrations by gas chromatography. Anal Chem 57:648-651.

Amoozegar A, Warrick AW, Fuller WH. 1986. Movement of selected organic liquids into dry soils. Hazard Waste Hazard Mater 3(1):29-41.

Andersen ME, Krishnan K. 1994. Relating in vitro to in vivo exposures with physiologically based tissue dosimetry and tissue response models. In: Salem H, ed. Animal test alternatives: Refinement, reduction, replacement. New York: Marcel Dekker, Inc., 9-25.

Andersen ME, Clewell HJ, Gargas ML, et al. 1987. Physiologically based pharmacokinetics and the risk assessment process for methylene chloride. Toxicol Appl Pharmacol 87:185-205.

Anderson BE, Zeiger E, Shelby MD, et al. 1990. Chromosome aberration and sister chromatid exchange test results with 42 chemicals. Environ Mol Mutagen 16(18):55-137.

Anderson C, Sundberg K, Groth O. 1986. Animal model for assessment of skin irritancy. Contact Dermatitis 15:143-151.

Anderson TA, Beuchamp JJ, Walton BT. 1991. Organic chemicals in the environment: Fate of volatile and semivolatile organic chemicals in soils: Abiotic versus biotic losses. J Environ Qual 20:420-424.

Andersson K, Fuxe K, Nilsen OG, et al. 1981. Production of discrete changes in dopamine and noradrenaline levels and turnover in various parts of the rat brain following exposure to xylene, ortho-, meta-, and para-xylene, and ethylbenzene. Toxicol Appl Pharmacol 60:535-548.

Ansari EA. 1997. Ocular injury with xylene-a report of two cases. Hum Exp Toxicol 16:273-275.

Antoine SR, Deleon IR, Ildefonso R, et al. 1986. Environmentally significant volatile organic pollutants in human blood. Bull Environ Contam Toxicol 36:364-371.

APHA. 1992. Standard methods for the examination of water and wastewater. 18th ed. Greenberg AE, Clesceri LS, Eaton AD, eds. Washington, DC: American Public Health Association, American Water Works Association, Water Environment Federation, 6-26-6-36, 6-42-6-46, 6-55-6-57. 
API. 1994. A study to characterize air concentrations of methyl tertiary butyl ether (MTBE) at service stations in the Northeast. Washington, DC: American Petroleum Institute.

API. 1995. Service station personnel exposures to oxygenated fuel components - 1994. Washington, DC: American Petroleum Institute.

Arp EW Jr, Wolf PH, Chekoway H. 1983. Lymphocytic leukemia and exposures to benzene and other solvents in the rubber industry. J Occup Med 25:598-602.

Arthur LJ, Curnock DA. 1982. Xylene-induced epilepsy following innocent glue sniffing. Br Med J 284:1787.

Arthur CL, Killam LM, Motlagh S, et al. 1992. Analysis of substituted benzene compounds in groundwater using solid-phase microextraction. Environ Sci and Technol 26:979-983.

Ashley DL, Bonin MA, Cardinali FL, et al. 1994. Blood concentrations of volatile organic compounds in a nonoccupationally exposed US population and in groups with suspected exposure. Clin Chem 40(7):1401-1404.

Ashley DL, Bonin MA, Cardinali FL, et al. 1992. Determining volatile organic compounds in human blood from a large sample production by using purge and trap gas chromatography/mass spectrometry. Anal Chem 64:1021-1029.

Ashley DL, Bonin MA, Hamar B, et al. 1995. Removing the smoking confounder from blood volatile organic compound measurements. Environ Res 71:39-45.

Ashley DL, Bonin MA, Cardinali FL, et al. 1996. Measurement of volatile organic compounds in human blood. Environ Health Perspect 104:871-877.

Askergren A. 1981. Studies on kidney function in subjects exposed to organic solvents: III. Excretion of cells in the urine. Acta Med Scand 210:103-106.

Askergren A. 1982. Organic solvents and kidney function. In: Englund A, Ringen K, Mehlman MY, eds. Advances in modern environmental toxicology: Occupational health hazards of solvents. 2nd ed. Princeton Scientific Publishers Inc., 157-172.

*Askergren A, Allgen L-G, Bergstrom J. 1981a. Studies on kidney function in subjects exposed to organic solvents: II. The effect of desmopressin in a concentration test and the effect of exposure to organic solvents on renal concentrating ability. Acta Med Scand 209:485-488.

Askergren A, Allgen L-G, Karlsson C, et al. 1981b. Studies on kidney function in subjects exposed to organic solvents: I. Excretion of albumin and beta-2-microglobulin in the urine. Acta Med Scand 209:479-483.

Askergren A, Brandt R, Gullquist R, et al. 1981c. Studies on kidney function in subjects exposed to organic solvents: IV. Effect on 51-Cr-EDTA clearance. Acta Med Scand 210:373-376.

Assmuth T, Kalevi K. 1992. Concentrations and toxicological significance of trace organic compounds in municipal solid waste landfill gas. Chemosphere 24(9):1207-1216.

Astier A. 1992. Simultaneous high-performance liquid chromatographic determination of urinary metabolites of benzene, nitrobenzene, toluene, xylene and styrene. J Chromatogr 573:318-322. 
Astrand I. 1982. Work load and uptake of solvents in tissues of man. In: Mehlman MA, ed. Advances in modern environmental toxicology. Vol. 2. Princeton Junction, NJ: Senate Press, 141-152.

Astrand I, Engstrom J, Ovrum P. 1978. Exposure to xylene and ethylbenzene: I. Uptake, distribution and elimination in man. Scand J Work Environ Health 4:185-194.

Atkinson R. 1989. Kinetics and mechanisms of the gas-phase reactions of the hydroxyl radical with organic compounds. J Phys Chem Ref Data 1:227-228.

Atkinson R. 1990. Gas-phase tropospheric chemistry of organic compounds: A review. Atmos Environ 24A:1-41.

Atkinson R, Carter WPL. 1984. Kinetics and mechanisms of gas-phase reactions of ozone with organic compounds under atmospheric conditions. Chem Rev 84:437-470.

Atkinson R, Aschmann SM, Arey J. 1991. Formation of ring-retaining products from the $\mathrm{OH}$ radicalinitiated reactions of $o-, m-$, and $p$-xylene. Int J Chem Kinet 23:77-97.

Atkinson R, Aschmann SM, Fitz DR, et al. 1982. Rate constants for the gas-phase reactions of O3 with selected organics at $296 \mathrm{~K}$. Int J of Chem Kinet 14:13-18.

Atkinson R, Carter WPL, Plum CN, et al. 1984. Kinetics of the gas-phase reactions of NO3 radicals with a series of aromatics at 296+/- 2K. Int J Chem Kinet 16:887-898.

Aurelius MW, Brown KW. 1987. Fate of spilled xylene as influenced by soil moisture content. Water Air Soil Pollut 36:23-31.

Austern BM, Dobbs RA, Cohen JM. 1975. Gas chromatographic determination of selected organic compounds added to wastewater. Environ Sci and Technol 9:588-590.

Backes WL, Sequeira DJ, Cawley GF, et al. 1993. Relationship between hydrocarbon structure and induction of P450: Effects on protein levels and enzyme activities. Xenobiotica 23(12):1353-1366.

Bakke OM, Scheline RR. 1970. Hydroxylation of aromatic hydrocarbons in the rat. Toxicol Appl Pharmacol 16:691-700.

Balogh S, Onayiga R, Edblad D. 1998. Evaluation of an automatic composite sampler for volatile organic compounds in raw wastewater. J Air Waste Manage Assoc 48:271-275.

Balogh T, Tatrai E, Barczai G, et al. 1982. [Study of the embryotoxic effect of xylene mixtures.] Egeszsegtudomany 26:42-48. (Hungarian)

Bandow H, Washida N. 1985. Ring-cleavage reactions of aromatic hydrocarbons studied by FT-IR spectroscopy: II. Photooxidation of $o-, m-$, and $p$-xylenes in the $\mathrm{NO}_{\mathrm{x}}$-air system. Bull Chem Soc Jpn 58:2541-2548.

Barbee GC, Brown KW. 1986. Movement of xylene through unsaturated soils following simulated spills. Water Air Soil Pollut 29:321-331.

Barker JF. 1987. Volatile aromatic and chlorinated organic contaminants in groundwater at six Ontario landfills. Water Pollut Res J Canada 22:33-48. 
Barlow SM, Sullivan FM. 1982. Reproductive hazards of industrial chemicals: An evaluation of animal and human data. London: Academic Press, 1-42.

Barnes DG, Dourson M. 1988. Reference dose (RfD): Description and use in health risk assessments. Regul Toxicol Pharmacol 8:471-486.

Bellanca JA, Davis PL, Donnelly B, et al. 1982. Detection and quantitation of multiple volatile compounds in tissues by GC and GC/MS. J Anal Toxicol 6:238-240.

Beller HR. 2000. Metabolic indicators for detecting in situ anaerobic alkylbenzene degradation. Biodegradation 11:125-139.

Beller HR, Ding WH, Reinhard M. 1995. Byproducts of anaerobic alkylbenzene metabolism useful as indicators of in situ bioremediation. Environ Sci Technol 29:2864-2870.

Berenblum I. 1941. The cocarcinogenic action of croton resin. Cancer Res 1:44-48.

Berger GS. 1994. Epidemiology of endometriosis. In: Berger GS, ed. Endometriosis: Advanced management and surgical techniques. New York, NY: Springer-Verlag.

Bergert KD, Nestler K. 1991. Solvent uptake in relation to physical activity. Sci Total Environ 101:111119.

Bergman K. 1983. Application and results of whole-body autoradiography in distribution studies of organic solvents. CRC Crit Rev Toxicol 12(1):59-118.

*Bernard AM, de Russis R, Normand JC, et al. 1989. Evaluation of the subacute nephrotoxicity of cyclohexane and other industrial solvents in the female Sprague-Dawley rat. Toxicol Lett 45(2-3):271280.

*Bernardelli BC, Gennari MC. 1987. Death caused by ingestion of endosulfan. J Forensic Sci 32:11091112.

Bieniek G, Wilczok T. 1981. Thin-layer chromatography of hippuric and $m$-methylhippuric acid in urine after mixed exposure to toluene and xylene. Br J Ind Med 38:304-306.

Bio/dynamics Inc. 1983. Parental and fetal reproduction toxicity study in rats with mixed xylenes. Submitted to the U.S. Environmental Protection Agency under TSCA Section FYI. FYI00002091.

Blanchard KT, Morris JB. 1994. Effects of $m$-xylene on rat nasal cytochrome P-450 mixed function oxidase activity. Toxicol Lett 70:253-259.

*Bleeker ML, Bolla KI, Agnew J. 1991. Dose-related subclinical neurobehavioral effects of chronic exposure to low levels of organic solvents. Am J Ind Med 19:715-728.

Bonnet P, Raoult G, Gradiski D. 1979. [Lethal concentration 50 of main aromatic hydrocarbons.] Arch Mal Prof 40:805-810. (French)

Borden RC, Black DC, McBleif KV. 2002. MTBE and aromatic hydrocarbons in North Carolina stormwater runoff. Environ Pollut 118:141-152. 
Bos RP, Brouns RME, Van Doorn R, et al. 1981. Nonmutagenicity of toluene, $o-, m-, p$-xylene, $o-$ methylbenzylalcohol and o-methyl benzylsulfate in the Ames assay. Mutat Res 88:273-279.

Boutelet-Bochan H, Huang Y, Juchau MR. 1997. Expression of CYP2E1 during embryogenesis and fetogenesis in human cephalic tissues: Implications for the fetal alcohol syndrome. Biochem Biophys Res Commun 238(2):443-447.

Bouwer EJ. 1989. Biotransformation of aromatics in strip-pit pond. J Environ Eng 115:741-755.

Bowen SE, Hamilton J, Balster RL. 1998. A method for adjusting exposure levels of volatile solvents based on effects on schedule-controlled behavior. Neurotoxicol Teratol 20(2):169-180.

*Bowers DJ, Cannon MS, Jones DH. 1982. Ultrastructural changes in livers of young and aging rats exposed to methylated benzenes. Am J Vet Res 43:679-683.

Braddock JF, McCarthy KA. 1996. Hydrologic microbiological factors affecting persistence and migration of petroleum hydrocarbon spilled in a continuous-permafrost region. Environ Sci Technol 30:2626-2633.

Brasington RD, Thorpe-Swenson AJ. 1991. Systemic sclerosis associated with cutaneous exposure to solvent: Case report and review of the literature. Arthritis Rheum 34:631-633.

Bratveit M, Hollund BE, Moen BE. 2004. Reduced exposure to organic solvents by use of water-based paint systems in car repair shops. Int Arch Occup Environ Health 77(1):31-38.

Bray HG, Humphris BG, Thorpe WV. 1949. Metabolism of derivatives of toluene: 3 . o-, $m$-, and $p$ Xylenes. Biochem J 45:241-244.

Bronstein AC, Currance PL. 1988. Emergency care for hazardous materials exposure. St. Louis, MO: C.V. Mosby Company, 221-222.

Brown RH. 1988a. Method 1- determination of benzene, toluene and xylene in industrial air by charcoal tube, solvent desorption and gas chromatography. IARC Sci Publ (85):225-233.

Brown RH. 1988b. Method 2- determination of benzene, toluene and xylene in industrial air by porous polymer adsorption tube, thermal desorption and gas chromatography. IARC Sci Publ (85):235-242.

Brown SK, Sim MR, Abramson MJ, et al. 1994. Concentrations of volatile organic compounds in indoor air - A review. Indoor Air 4:123-134.

Bruce BW, McMahon PB. 1996. Shallow ground-water quality beneath a major urban center: Denver, Colorado, USA. J Hydrol 186:129-151.

Brzezinski MR, Boutelet-Bochan H, Person RE, et al. 1999. Catalytic activity and quantitation cytochrome P-450 2E1 in prenatal human brain. J Pharmacol Exp Ther 289:1648-1653.

Bushnell, PJ. 1989. Behavioral effects of acute $p$-xylene inhalation in rats: Autoshaping, motor activity, and reversal learning. Neurotoxicol Teratol 10:569-577. 
Calabrese EJ. 1978. Pollutants and high-risk groups: The biological basis of increased human susceptibility to environmental and occupational pollutants. New York, NY: John Wiley and Sons, 4-8.

Cameron GR, Paterson JLH, DeSaram GSW, et al. 1938. The toxicity of some methyl derivatives of benzene with special reference to pseudocumene and heavy coal tar naphtha. J Pathol Bacteriol 46:95107.

Campbell L, Wilson HK, Samuel AM, et al. 1988. Interactions of $m$-xylene and aspirin metabolism in man. Br J Ind Med 45:127-132.

Cannella WJ. 1998. Xylenes and ethylbenzene. In: Kirk-Othmer encyclopedia of chemical technology. John Wiley \& Sons, Inc. http://www.mrw.interscience.wiley.com/kirk/articles/xylecann.a01. February $15,2005$.

*Cannon Laboratories. 1976. Report on rabbit eye irritation. Submitted to the U.S. Environmental Protection Agency under TSCA Section 8D. OTS0206126.

Canter LW, Sabatini DA. 1994. Contamination of public ground water supplies by Superfund sites. Int J Environ Stud 46:35-57.

Caperos JR, Fernandez JG. 1977. Simultaneous determination of toluene and xylene metabolites in urine by gas chromatography. Br J Ind Med 34:229-233.

Carlone MF, Fouts JR. 1974. In vitro metabolism of p-xylene by rabbit lung and liver. Xenobiotica 4:705-715.

Carlsson A. 1981. Distribution and elimination of 14C-xylene in rat. Scand J Work Environ Health 7:51-55.

Carpenter CP, Kinkead ER, Geary DJ, et al. 1975a. Petroleum hydrocarbon toxicity studies: V. Animal and human response to vapors of mixed xylenes. Toxicol Appl Pharmacol 33:543-558.

*Carpenter CP, Kinkead ER, Geary DJ, et al. 1975b. Petroleum hydrocarbon toxicity studies: I. Methodology. Toxicol Appl Pharmacol 32:246-262.

Carpenter SP, Lasker JM, Raucy JL. 1996. Expression, induction, and catalytic activity of the ethanolinducible cytochrome P450 (CYP2E1) in human fetal liver and hepatocytes. Mol Pharmacol 49:260-268.

Casto BC. 1981. Detection of chemical carcinogens and mutagens in hamster cells by enhancement of adenovirus transformation. In: Mishra N, Dunkel V, Mehlman, eds. Advances in modern environmental toxicology. Princeton, NJ: Senate Press, 241-271.

Cha YJ, Cadwallader KR. 1995. Volatile components in salt-fermented fish and shrimp pastes. J Food Sci 60:19-24.

Chan CC, Lin SH, Her GR. 1994. Office worker's exposure to volatile organic compounds while commuting and working in Taipei City. Atmos Environ 28(1-4):2351-2359.

Chan C-C, Nien C-K, Tsai C-Y, et al. 1995. Comparison of tail-pipe emissions from motorcycles and passenger cars. J Air Waste Manage Assoc 45:116-124. 
Chan CC, Vainer L, Martin JW, et al. 1990. Determination of organic contaminants in residential indoor air using an adsorption-thermal desorption technique. J Air Waste Manage Assoc 40:62-67.

Chao J, Lin CT, Chung TH. 1983. Vapor pressure of coal chemicals. J Phys Chem Ref Data 12:10331063.

*Charney W, Lipnowski M, Wugofski L. 1990. Biological exposure index testing: Two case studies. In: Charney W, Schirmer J, eds. Essentials of modern hospital safety. Chelsea, MI: Lewis Publishers, Inc., 273-281.

Chatterjee A, Babu RJ, Ahaghotu E, et al. 2005. The effect of occlusive and unocclusive exposure to xylene and benzene on skin irritation and molecular responses in hairless rats. Arch Toxicol 79(5):294301.

Chen CS, Zolteck J. 1995. Organic priority pollutants in wetland-treated leachates at a landfill in central Florida. Chemosphere 31(6):3455-3464.

Chevance FFV, Farmer LJ. 1999. Identification of a major volatile odor compounds in frankfurters. J Agric Food Chem 47:5151-5160.

Chiou CT, Schmedding DW, Manes M. 1982. Partitioning of organic compounds in octanol-water systems. Environ Sci Technol 16:4-10.

Cho SI, Damokosh AI, Ryan LM, et al. 2001. Effects of exposure to organic solvents on menstrual cycle length. J Occup Environ Med 43(6):565-575.

CHRIS. 1999. m-Xylene, o-xylene, and p-xylene. Chemical Hazards Response Information System. U.S. Coast Guard, U.S. Department of Transportation. http://www.chrismanual.com/. May 23, 2007.

Chung HY. 1999. Volatile components in crabmeats of Charybdis feriatus. J Agric Food Chem 47:2280-2287.

Clapp LW, Talarczyk MR, Park JK, et al. 1994. Performance comparison between activated sludge and fixed film processes for priority pollutant removals. Water Environ Res 66:153-160.

Clewell HJ III, Andersen ME. 1985. Risk assessment extrapolations and physiological modeling. Toxicol Ind Health 1(4):111-131.

Coleman CA, Hull BE, McDougal JN, et al. 2003. The effect of m-xylene on cytotoxicity and cellular antioxidant status in rat dermal equivalents. Toxicol Lett 142:133-142.

Coleman EC, Ho C, Chang SS. 1981. Isolation and identification of volatile compounds from baked potatoes. J Agric Food Chem 29:42-48.

Condie LW, Hill JR, Borzelleca JF. 1988. Oral toxicology studies with xylene isomers and mixed xylenes. Drug Chem Toxicol 11:329-354.

Connor TH, Theiss JC, Hanna HA, et al. 1985. Genotoxicity of organic chemicals frequently found in the air of mobile homes. Toxicol Lett 25:33-40. 


\section{REFERENCES}

Consumer Product Testing. 1976. Primary dermal irritation (rabbit); occular irritation (rabbit). Submitted to the U.S. Environmental Protection Agency under TSCA Section 8D. OTS0206121.

Cramer PH, Boggess KE, Hosenfeld JM, et al. 1988. Determination of organic chemicals in human whole blood: Preliminary method development for volatile organics. Bull Environ Contam Toxicol 40:612-618.

Crofton KM, Lassiter TL, Rebert CS. 1994. Solvent-induced ototoxicity in rats: An atypical selective mid-frequency hearing deficit. Hearing Res 80:25-30.

Daniell W, Stebbins A, Kalman D, et al. 1992. The contributions to solvent uptake by skin and inhalation exposure. Am Ind Hyg Assoc J 53(2):124-129.

Darnall KR, Atkinson R, Pitts JN Jr. 1979. Observation of biacetyl from the reaction of OH radicals with o-xylene: Evidence for ring cleavage. J Phys Chem 83:1943-1946.

David A, Flek J, Frantik E, et al. 1979. Influence of phenobarbital on xylene metabolism in man and rats. Int Arch Occup Environ Health 44:117-125.

Davis NR, Mapleson WW. 1981. Structure and quantification of a physiological model of the distribution of injected agents and inhaled anaesthetics. Br J Anaesth 53:399-405.

Day BJ, DeNicola DB, Marcus CB, et al. 1992. Effect of $p$-xylene on the bioactivation of bromobenzene in rat lung and liver. Fundam Appl Toxicol 19:50-56.

de Carvalho D, Lanchote VL, Bonato PS, et al. 1991. A new derivatization procedure for the analysis of hippuric acid and m-methyl-hippuric acid by gas chromatography. Int Arch Occup Environ Health 63:3337.

De Ceaurriz JC, Micillino JC, Bonnet P, et al. 1981. Sensory irritation caused by various industrial airborne chemicals. Toxicol Lett 9:137-143.

De Ceaurriz J, Desiles JP, Bonnet P, et al. 1983. Concentration-dependent behavioral changes in mice following short-term inhalation exposure to various industrial solvents. Toxicol Appl Pharmacol 67(3):383-389.

De Fré R, Bruynseraede P, Kretzschmar JG. 1994. Air pollution measurements in traffic tunnels. Environ Health Perspect 102(Suppl 4):31-37.

*DeGandarias JM, Echevarria E, Irazusta J, et al. 1993. Brain aminopeptidase activity after subacute xylene exposure. Neurotoxicol and Teratol 15:51-53.

Delzer GC, Zogorski JS, Lopes TJ. 1997. Occurrence of the gasoline oxygenate MTBE and BTEX compounds in municipal stormwater in the United States, 1991-1995. Prepr Ext Abst Div Environ Chem Am Chem Soc 37(1):374-376.

DeMarini DM, Lawrence BK, Brooks HG, et al. 1991. Compatibility of organic solvents with the microscreen prophage-induction assay: Solvent-mutagen interactions. Mutat Res 263:107-113.

Derr RF. 1993. Simulation studies on ethanol metabolism in different human populations with a physiological pharmacokinetic model. J Pharm Sci 82:677-682.

Desi I, Kovacs F, Zahumenszky Z, et al. 1967. Maze learning in rats exposed to xylene intoxication. Psychopharmacologia 11:224-230. 
Desideri PG, Lepri L, Checchini L, et al. 1994. Organic compounds in surface and deep Antarctic snow. Int J Environ Anal Chem 55:33-46.

Desideri PG, Lepri L, Udisti R, et al. 1998. Analysis of organic compounds in Antarctic snow and their origin. Int J Environ Anal Chem 7(3-4):331-351.

DeWulf J, Vanlangenhove H. 1997. Chlorinated C1 and C2-hydrocarbons and monocyclic aromatic hydrocarbons in marine waters: an overview on fate processes, sampling, analysis and measurements. Water Res 31(8):1825-1838.

Dolara P, Lodovici M, Buffoni F, et al. 1982. Variations of some parameters of enzyme induction in chemical workers. Ann Occup Hyg 25(1):27-32.

Dudek B, Gralewicz K, Jakubowski M, et al. 1990. Neurobehavioral effects of experimental exposure to toluene, xylene and their mixture. Pol J Occup Med 3(1):109-116.

Dumont JP, Adda J. 1978. Occurence of sesquiterpenes in mountain cheese volatiles. J Agric Food Chem 26:364-367.

Dutkiewicz T, Tyras H. 1968. Skin absorption of toluene, styrene, and xylene by man. Br J Ind Med 25:243.

Dyer RS, Bercegeay MS, Mayo LM. 1988. Acute exposures to p-xylene and toluene alter visual information processing. Neurotoxicol Teratol 10:147-153.

ECETOC. 1986. Joint assessment of commodity chemicals: No. 6. Xylenes. Brussels, Belgium: European Chemical Industry Ecology and Toxicology Centre.

Eganhouse RP, Cozarelli IM, Scholl MA, et al. 2001. Natural attenuation of volatile organic compounds (VOCs) in the leachate plume of a municipal landfill: Using alkylbenzenes as process probes. Ground Water 39:192-202.

Eganhouse RP, Dorsey TF, Phinney CS, et al. 1996. Processes affecting the fate of monoaromatic hydrocarbons in an aquifer contaminated by crude oil. Environ Sci Technol 30:3304-3312.

Eichmann R, Ketseridis G, Schebeske G, et al. 1980. n-Alkane studies in the troposphere: II. Gas and particulate concentrations in Indian Ocean air. Atmos Environ 14:695-703.

Eichmann R, Neuling P, Ketseridis G, et al. 1979. n-Alkane studies in the troposphere: I. Gas and particulate concentrations in North Atlantic air. Atmos Environ 13:587-599.

Eitzer BD. 1995. Emissions of volatile organic chemicals from municipal solid waste composting facilities. Environ Sci Technol 29:896-902.

Eklund B, Anderson EP, Walker BL, et al. 1998. Characterization of landfill gas composition at the fresh kills municipal solid-waste landfill. Environ Sci Technol 32(32):2233-2237.

Ellenhorn MJ, Barceloux DG, eds. 1988. Xylene. In: Medical toxicology: Diagnosis and treatment of human poisoning. New York, NY: Elsevier, 962-964.

Elovaara E. 1982. Dose-related effects of $m$-xylene inhalation on the xenobiotic metabolism of the rat. Xenobiotica 12:345-352. 


\section{REFERENCES}

Elovaara E, Collan Y, Pfaffli P, et al. 1980. The combined toxicity of technical grade xylene and ethanol in the rat. Xenobiotica 10:435-445.

Elovaara E, Engstrom K, Hayri L, et al. 1989. Metabolism of antipyrine and $m$-xylene in rats after prolonged pretreatment with xylene alone or xylene with ethanol, phenobarbital, or 3-methylcholanthrene. Xenobiotica 19(9):945-960.

Elovaara E, Zitting A, Nickels J, et al. 1987. m-Xylene inhalation destroys cytochrome P-450 in rat lung at low exposure. Arch Toxicol 61:21-26.

Elshahed MS, Gieg LM, McInerney MJ, et al. 2001. Signature metabolites attesting to the in situ attenuation of alkylbenzenes in anaerobic environments. Environ Sci Technol 35:682-689.

Endoh K, Kaneko T, Sato A. 1989. A physiologically based pharmacokinetic model to describe the transfer of organic solvents in the human body: Simulation of kinetic behavior of trichloroethylene using a spreadsheet program. Jpn J Ind Health 31:335-341.

English CW, Loehr RC. 1991. Degradation of organic vapors in unsaturated soils. J Hazard Mater 28:55-64.

Engstrom J, Bjurstrom R. 1978. Exposure to xylene and ethylbenzene: II. Concentration in subcutaneous adipose tissue. Scand J Work Environ Health 4:195-203.

Engstrom K, Husman K, Rantanen J. 1976. Measurement of toluene and xylene metabolites by gas chromatography. Int Arch Occup Environ Health 36:153-160.

Engstrom K, Husman K, Riihimaki V. 1977. Percutaneous absorption of $m$-xylene in man. Int Arch Occup Environ Health 39:181-189.

Engstrom K, Riihimaki V, Laine A. 1984. Urinary disposition of ethylbenzene and $m$-xylene in man following separate and combined exposure. Int Arch Occup Environ Health 54:355-363.

EPA. 1978. Initial report of the TSCA testing committee to the administrator, Environmental Protection Agency. U.S. Enviornmental Protection Agency. EPA5601078001.

EPA. 1980a. Acquisition and chemical analysis of mother's milk for selected toxic substances. Washington, DC: U.S. Environmental Protection Agency, Office of Pesticides and Toxic Substances. EPA5600380029. PB81231029.

EPA. 1980b. Discarded commercial chemical products off-specification species, container residues and split residues thereof. U.S. Environmental Protection Agency. Code of Federal Regulations. 40 CFR 261.33.

EPA. 1981a. Engineering handbook for hazardous waste incineration. Washington, DC: U.S. Environmental Protection Agency. PB81248163.

EPA. 1981b. Hazardous wastes from nonspecific sources. U.S. Environmental Protection Agency. Code of Federal Regulations. 40 CFR 261.31. 
EPA. 1983. Volatile organic chemicals in the atmosphere: An assessment of available data. Research Triangle Park, NC: U.S. Environmental Protection Agency, Office of Research and Development, Environmental Sciences Research Laboratory. EPA600383027a.

EPA. 1984a. Health effects assessment for xylene. Washington, DC: U.S. Environmental Protection Agency, Environmental Criteria and Assessment Office, Office of Health and Environmental Assessment, Office of Research and Development, and Office of Emergency and Remedial Response. EPA540186006.

*EPA. 1984b. Proposed guidelines for carcinogenic risk. U.S. Environmental Protection Agency. Fed Regist 49:46294-46299.

EPA. 1985a. Drinking water criteria document for xylenes. Cincinnati, OH: U.S. Environmental Protection Agency, Office of Health and Environmental Assessment, Environmental Criteria and Assessment Office for the Office of Drinking Water. EPA600X8485. PB86117942.

*EPA. 1985b. Xylene; exemption from the requirement of a tolerance. U.S. Environmental Protection Agency. Code of Federal Regulations. 40 CFR 180.1025.

EPA. 1989. Determination of C2 to C12 ambient air hydrocarbons in 39 U.S. cities, from 1984 through 1986. Project summary. Research Triangle Park, NC: Atmospheric Research and Exposure Assessment Laboratory. EPA600S389058.

EPA. 1990. Interim methods for development of inhalation reference concentrations. Washington, DC: U.S. Environmental Protection Agency, Office of Health and Environmental Assessment, Office of Research and Development, Environmental Criteria and Assessment Office. EPA600890066A.

EPA. 1991a. DOAS urban pollution measurements. Research Triangle Park, NC: U.S. Environmental Protection Agency. EPA600D91044. PB91176966.

EPA. 1991b. Indoor air pollutants from household product sources. Washington, DC: U.S. Environmental Protection Agency. EPA600491025.

*EPA. 1992. Health and safety data reporting: Substances and listed mixtures to which this subpart applies. U.S. Environmental Protection Agency. Code of Federal Regulations. 40 CFR 712.120.

EPA. 1993. Ambient concentration summaries for Clean Air Act. Title III: Hazardous air pollutants. EPA600R94090.

EPA. 1994. Methods for derivation of inhalation reference concentrations and application of inhalation dosimetry. Washington, DC: U.S. Environmental Protection Agency, Office of Health and Environmental Assessment, Office of Research and Development, Environmental Criteria and Assessment Office. EPA600890066F.

*EPA. 1997a. Automated Form R for Windows: User's guide (RY97). Washington, DC: U.S. Environmental Protection Agency, Office of Pollution Prevention and Toxics.

EPA. 1997b. Special report on environmental endocrine disruption: An effects assessment and analysis. Washington, DC: U.S. Environmental Protection Agency, Risk Assessment Forum. EPA630R96012. 
EPA. 2001a. Occurrence of unregulated contaminants in public water systems: A national summary. Washington, DC: U.S. Environmental Protection Agency. EPA815P00005.

EPA. 2001b. Exploration of perinatal pharmacokinetic issues. Washington, DC: U.S. Environmental Protection Agency, Risk Assessment Forum. EPA630R01004. http://cfpub.epa.gov/ncea/raf/ recordisplay.cfm?deid=29420. March 25, 2005.

EPA. 2002a. Inventory update rule. Cas. No. 1330-2-07. Toxic Substance Control Act (TSCA) Inventory Update Database. Washington, DC: U.S. Environmental Protection Agency. http://www.epa.gov/opt/uiru/iur02/index.htm. February 15, 2004.

EPA. 2002b. National primary drinking water regulations. Washington, DC: Office of Ground Water and Drinking Water, U.S. Environmental Protection Agency. EPA816F02013. http://www.epa.gov/safewater/mcl.html. February 15, 2005.

EPA. 2003. Toxicological review of xylenes. Washington, DC: U.S. Environmental Protection Agency EPA635R03001. http://www.epa.gov/iris/toxreviews/0270-tr.pdf. February 15, 2005.

EPA. 2004a. Drinking water standards and health advisories. Washington, DC: Office of Water, U.S. Environmental Protection Agency. EPA822R04005.

http://www.epa.gov/waterscience/drinking/standards/dwstandards.pdf. February 15, 2005.

EPA. 2004b. Hazardous air pollutants. U.S. Environmental Protection Agency. United States Code. 42 USC 7412. http://www.epa.gov/ttn/atw/orig189.html. February 15, 2005.

EPA. 2005a. Acute Exposure Guideline Levels (AEGLs) Washington, DC: Office of Pollution Prevention and Toxic Substances. U.S. Environmental Protection Agency. http://www.epa.gov/oppt/aegl/chemlist.htm. March 28, 2005.

EPA. 2005b. National primary and secondary ambient air quality standards for lead. U.S. Environmental Protection Agency. Code of Federal Regulations. 40 CFR 50.12. http://www.epa.gov/epacfr40/chapt-I.info/chi-toc.htm. February 15, 2005.

EPA. 2005c. Reportable quantities of hazardous substances designated pursuant to Section 311 of the Clean Water Act. U.S. Environmental Protection Agency. Code of Federal Regulations. 40 CFR 117.3. http://www.epa.gov/epacfr40/chapt-I.info/chi-toc.htm. February 16, 2005.

EPA. 2005d. Superfund, emergency planning, and community right-to-know programs. Designation, reportable quantities, and notifications. U.S. Environmental Protection Agency. Code of Federal Regulations. 40 CFR 302.4. http://www.epa.gov/epacfr40/chapt-I.info/chi-toc.htm. February 15, 2005.

EPA. 2005e. Superfund, emergency planning, and community right-to-know programs. Toxic chemical release reporting. U.S. Environmental Protection Agency. Code of Federal Regulations. 40 CFR 372.65. http://www.epa.gov/epacfr40/chapt-I.info/chi-toc.htm. February 16, 2005.

EPA. 2005f. Tolerances and exemptions from tolerances for pesticide chemicals in food. U.S. Environmental Protection Agency. Code of Federal Regulations. 40 CFR 180.1025. http://www.epa.gov/epacfr40/chapt-I.info/chi-toc.htm. March 25, 2005. 
EPA. 2005g. Toxic chemical release inventory reporting forms and instructions: Revised 2004 version. Section 313 of the Emergency Planning and Community Right-to-Know Act (Title III of the Superfund Amendments and Reauthorization Act of 1986). U.S. Environmental Protection Agency. Office of Environmental Information. EPA260B05001.

EPA. 2005h. Xylenes. Modernized STORET system: Regular results by geographic location (stormodb): Characteristic search by CAS number. U.S. Environmental Protection Agency. http://www.epa.gov/storet/dbtop.html. May 24, 2007.

EPA. 2007. Acute exposure guideline levels (AEGLs). Washington, DC: Office of Pollution Prevention and Toxic Substances. U.S. Environmental Protection Agency. http://www.epa.gov/opptintr/aegl/pubs/results85.htm. May 24, 2007.

Epler JL, Rao TK, Geurin MR. 1979. Evaluation of feasibility of mutagenic testing of shale oil products and effluents. Environ Health Perspect 30:179-184.

Eriksson M, Dalhammar G, Borg-Karlson AK. 1999. Aerobic degradation of a hydrocarbon mixture in natural uncontaminated potting soil by indigenous microorganisms at $20^{\circ} \mathrm{C}$ and $6{ }^{\circ} \mathrm{C}$. Appl Microbiol Biotechnol 51:532-535.

Eriksson K, Tjarner D, Marqvardsen, et al. 2003. Exposure to benzene, toluene, xylenes and total hydrocarbons among snowmobile drivers in Sweden. Chemosphere 50:1343-1347.

Ernstgard L, Gullstrand E, Lof A, et al. 2002. Are women more sensitive than men to 2-propanol and mxylene vapours. Occup Environ Med 59(1):759-767.

Ernstgard L, Sjogren B, Warholm M, et al. 2003. Sex differences in the toxicokinetics of inhaled solvent vaporsin humans. Toxicol Appl Pharmacol 193(2):147-157.

Esposito GG, Jacobs BW. 1977. Chromatographic determination of aromatic hydrocarbons in ambient air. Am Ind Hyg Assoc J 38:401-407.

Fang Z, Sonner J, Laster MJ, et al. 1996. Anesthetic and convulsant properties of aromatic compounds and cycloalkanes: Implications for mechanisms of narcosis. Anesth Analg 83(5):1097-1104.

FDA. 2006. Beverages. Bottled water. Washington, DC: U.S. Food and Drug Administration. Code of Federal Regulations. 21 CFR 165.110. http://www.accessdata.fda.gov/scripts/cdrh/cfdocs/cfcfr/CFRSearch.cfm. May 24, 2007.

FED R\&D. 2005. Federal Research and Development summaries. Dialog Information Service, Incorporated.

FEDRIP. 2006. Federal Research in Progress. Dialog Information Service, Incorporated.

Feldt EG. 1986. [Evaluation of the mutagenic hazards of benzene and some of its derivatives.] Gig Sanit (7):21-23. (Russian)

Fellin P, Otson R. 1994. Assessment of the influence of climatic factors on concentration levels of volatile organic compounds (VOCs) in Canadian homes. Atmos Environ 28:3581-3586.

Fishbein L. 1985. An overview of environmental and toxicological aspects of aromatic hydrocarbons: III. Xylene. Sci Total Environ 43:165-183. 
Fishbein L. 1988. Xylenes: Uses, occurrence and exposure. In: Fishbein L, O'Neill IK, eds. Environmental carcinogens: Methods of analysis and exposure measurement. Vol. 10: Benzene and alkylated benzenes. Lyon, France: World Health Organization, International Agency for Research on Cancer, 109-120.

Fisher J, Male D, Bankston L, et al. 1997. Lactational transfer of volatile chemicals in breast milk. Am Ind Hyg Assoc J 58(6):425-431.

Florin I, Rutberg L, Curvall M, et al. 1980. Screening of tobacco smoke constituents for mutagenicity using the Ames' test. Toxicol 18:219-232.

Fomon SJ. 1966. Body composition of the infant: Part I: The male reference infant. In: Falkner F, ed. Human development. Philadelphia, PA:WB Saunders, 239-246.

Fomon SJ, Haschke F, Ziegler EE, et al. 1982. Body composition of reference children from birth to age 10 years. Am J Clin Nutr 35:1169-1175.

Food and Drug Research Labs. 1976a. Primary skin irritation study with rabbits. Submitted to the U.S. Environmental Protection Agency under TSCA Section 8D. OTS0206121.

Food and Drug Research Labs. 1976b. Rabbit eye irritation study. Submitted to the U.S. Environmental Protection Agency under TSCA Section 8D. OTS0200298.

Först C, Stieglitz L, Roth W, et al. 1989a. Determination of volatile organic pollutants in leachate from different landfills. Vom Wasser 72:295-305.

Först C, Stieglitz L, Roth W, et al. 1989b. Quantitative analysis of volatile organic compounds in landfill leachates. Int J Environ Anal Chem 37:287-293.

Fortmann R, Roache N, Chang JCS, et al. 1998. Characterization of emmisions of volatile organic compounds from interior alkyd paint. J Air Waste Manage Assoc 48:931-940.

Foster P, Ferronato C, Jacob V. 1994. Organic compound transfer between gas-phase and raindrops. Fresenius Environ Bull 3:318-323.

Fox DL, Gary M, Jeffries HE. 1984. Organic aerosol formation: OH vs. ozone oxidation pathways. Atlanta, GA: Coordinating Research Council, Inc.

Foy JWD, Schatz RA. 2004. Inhibition of rat respiratory-tract cytochrome P-450 activity after acute low-level m-xylene inhalation: Role in 1-nitronaphthalene toxicity. Inhal Toxicol 16:125-132.

Foy JWD, Silverman DM, Schatz RA. 1996. Low-level m-xylene inhalation alters pulmonary and hepatic cytochrome p-450 activity in the rat. J Toxicol Environ Health 47(2):135-144.

Franchini I, Cavatorta A, Falzoi M, et al. 1983. Early indicators of renal damage in workers exposed to organic solvents. Int Arch Occup Environ Health 52:1-9.

*Fraser MP, Cass GR, Simoneit BRT, et al. 1998a. Air quality model evaluation data for organics. C6C22 nonpolar and semipolar aromatic compounds. Environ Sci Technol 32:1760-1770. 


\section{REFERENCES}

Fraser MP, Cass GR, Simoneit BRT. 1998b. Gas-phase and particle-phase organic compounds emitted from motor vehicle traffic in a Los Angeles roadway tunnel. Environ Sci Technol 32:2051-2060.

Fraser MP, Kleeman MJ, Schauer JJ, et a; 2000. Modeling the atmospheric concentrations of individual gas-phase and particle-phase organic compounds. Environ Sci Technol 34:302-312.

Freundt KJ, Romer KG, Federsel RJ. 1989. Decrease of inhaled toluene, ethyl benzene, $m$-xylene, or mesitylene in rat blood after combined exposure to ethyl acetate. Bull Environ Contam Toxicol 42: 495498.

Fuentes HR, Polzer WL, Smith JL. 1991. Laboratory measurements of diffusion coefficients for trichloroethylene and orthoxylene in undisturbed turf. J Environ Qual 20:215-221.

Furnas DW, Hine CH. 1958. Neurotoxicity of some selected hydrocarbons. Arch Ind Health 18:9-15.

Gabele P. 1997. Exhaust emissions from four-stroke lawn mower engines. J Air Waste Manage Assoc 47:945-952.

Gabele PA. 2000. Emissions from two outboard engines operating on reformulated gasoline containing MTBE. Environ Sci Technol 34:368-372.

Gagnaire F, Langlais C. 2005. Relative ototoxicity of 21 aromatic solvents. Arch Toxicol 79(6):346354.

Gagnaire F, Langlais C, Grossman S, et al. 2006. Ototoxicity in rats exposed to ethylbenzene and to two technical xylene vapours for 13 weeks. Arch Toxicol 81(2):127-143.

Gagnaire F, Marignac B, Langlais C, et al. 2001. Ototoxicity in rats exposed to ortho-, meta- and paraxylene vapours for 13 weeks. Pharmacol Toxicol 89:6-14.

Gamberale F, Annwall G, Hultengren M. 1978. Exposure to xylene and ethylbenzene: III. Effects on central nervous functions. Scand J Work Environ Health 4:204-211.

Gargas ML, Burgess RJ, Voisard DE, et al. 1989. Partition coefficients of low-molecular-weight volatile chemicals in various liquids and tissues. Toxicol Appl Pharmacol 98:87-99.

Gerarde HW. 1959. Toxicological studies on hydrocarbons: III. The biochemorphology of the phenylalkanes and phenylalkenes. Arch Ind Health 19:403-418.

Gerarde HW. 1960. Toxicology and biochemistry of aromatic hydrocarbons. London: Elsevier Publishing Co., 171-180.

Gerin M, Siemiatycki J, Desy M, et al. 1998. Associations between several sites of cancer and occupational exposure to benzene, toluene, xylene, and styrene: Results of a case-control study in Montreal. Am J Ind Med 34:144-156.

Gerner-Smidt P, Friedrich U. 1978. The mutagenic effect of benzene, toluene and xylene studied by the SCE technique. Mutat Res 58:313-316.

Gery MW, Fox DL, Kamens RM, et al. 1987. Investigation of hydroxyl radical reactions with $o$-xylene and $m$-xylene in a continuous stirred tank reactor. Environ Sci Technol 21:339-348. 
Ghantous H, Danielsson BR. 1986. Placental transfer and distribution of toluene, xylene and benzene, and their metabolites during gestation in mice. Biol Res Pregnancy Perinatol 7:98-105.

*Ghantous H, Dencker L, Gabrielsson J, et al. 1990. Accumulation and turnover of metabolites of toluene and xylene in nasal mucosa and olfactory bulb in the mouse. Pharmacol Toxicol 66:87-92.

Gherini SA, Summers KV, Munson RK, et al. 1989. Chemical data for predicting the fate of organic compounds in water: Volume 1, Technical basis: Final report. Palo Alto, CA: Electrical Power Research Institute. EPRI-EA-5818.

Gholson AR, Albritton JR, Jayanty RKM, et al. 1991. Evaluation of an enclosure method for measuring emissions of volatile organic compounds from quiescent liquid surfaces. Environ Sci Technol 25:519-24.

Ghosh TK, Copeland RJ, Parui RN, et al. 1987. Effects of xylene inhalation on fixed-ratio responding in rats. Pharmacol Biochem Behav 27:653-657.

Giger W, Schaffner C. 1981. Groundwater pollution by volatile organic chemicals. Stud Environ Sci 17:517-522.

Ginsberg G, Hattis D, Sonawane B. 2004. Incorporating pharmacokinetic differences between children and adults in assessing children's risk to environmental toxicants. Toxicol Appl Pharmacol 198:164-183.

Giwercman A, Carlsen E, Keiding N, et al. 1993. Evidence for increasing incidence of abnormalities of the human testis: A review. Environ Health Perspect Suppl 101(2):65-71.

Glaser RA, Arnold JE. 1989. Investigation of charcoal cloth as a sorbent for integrated sampling of solvent vapors in mixed-expired breath using a new stainless steel sampler. Am Ind Hyg Assoc J 50:11221.

Glaser RA, Arnold JE, Shulman SA. 1990. Comparison of 3 sampling and analytical methods for measuring meta-xylene in expired air of exposed humans. Am Ind Hyg Assoc J 51:139-150.

Gleason MN, Gosselin RE, Hodge HC, et al. 1969. Clinical toxicology of commercial products: Acute poisoning. 3rd ed. Baltimore, MD: Williams and Wilkins, 227-230.

Goldfrank LR, Kulberg AG, Bresnitz EA. 1990. Hydrocarbons. In: Goldfrank LR, ed. Goldfrank's toxicologic emergencies. Norwalk, CT: Appleton and Lange, 759-768.

Goldie I. 1960. Can xylene (xylol) provoke convulsive seizures. Ind Med Surg 29:33-35.

Gomez E, Ledbetter CA, Hartsell PL. 1993. Volatile compounds in apricot, plum, and their interspecific hybrids. J Agric Food Chem 41:1669-1676.

Gonzalez-Reche LM, Schettgen T, Angerer J. 2003. New approaches to the metabolism of xylenes: Verification of the formation of phenylmercapturic acid metabolites of xylenes. Arch Toxicol 77:80-85.

Gosselin RE, Smith RP, Hodge HC. 1984. Clinical toxicology of commercial products. 5th ed. Baltimore, MD: Williams \& Wilkins, 397-404. 


\section{REFERENCES}

Gossett RW, Brown DA, Young DR. 1983. Predicting the bioaccumulation of organic compounds in marine organisms using octanol/water partition coefficients. Marine Pollut Bull 14:387-392.

Graham LA, Noseworthy L, Fugler D, et al. 2004. Contribution of vehicle emissions from an attached garage to residential indoor air pollution levels. J Air Waste Manage Assoc 54:563-584.

Gralewicz S, Wiaderna D. 2001. Behavior effects following subacute inhalation exposure to m-xylene or trimethylbenzene in the rat a comparative study. Neurotoxicology 22(1):79-89.

Gralewicz S, Wiaderna D, Tomas T. 1995. Development of spontaneous, age-related nonconvulsive seizure electrocortical activity and radial-maze learning after exposure to $\mathrm{m}$-xylene in rats. Int $\mathrm{J}$ Occup Med Environ Health 8(4):347-360.

Green WJ, Lee GF, Jones RA. 1981. Clay-soils permeability and hazardous waste storage. J Water Pollut Control Fed 53:1347-1354.

Greenberg JP, Zimmerman PR. 1984. Nonmethane hydrocarbons in remote tropical, continental and marine atmospheres. J Geophys Res 89:4767-4778.

Griffin RA, Hughes RE, Follmer IR, et al. 1984. Migration of industrial chemicals and soil-waste interactions at Wilsonville, Illinois. In: Land disposal of hazardous waste. Proceedings of the Tenth Annual Research Symposium. Ft. Mitchell, KY: U.S. Environmental Protection Agency, 61-77.

Grosjean D, Fung K. 1984. Hydrocarbons and carbonyls in Los Angeles air. J Air Pollut Control Assoc 34:537-543.

Guisti DM, Conway RA, Lawson CT. 1974. Activated carbon adsorption of petrochemicals. J Water Pollut Control Fed 46:947-965.

Gunasekar PG, Rogers JV, Kabbur MB, et al. 2003. Molecular and histological responses in rat skin exposed to m-xylene. J Biochem Mol Toxicol 17(2):92-94.

Gupta BN, Kumar P, Srivastava AK. 1990. An investigation of the neurobehavioral effects on workers exposed to organic solvents. J Soc Occup Med 40:94-96.

Guzelian PS, Henry CJ, Olin SS, eds. 1992. Similarities and differences between children and adults: Implications for risk assessment. Washington, DC: International Life Sciences Institute Press.

Haddad S, Beliveau M, Tardif R, et al. 2001. A PBPK modeling-based approach to account for interactions in the health risk assessment of chemical mixtures. Toxicol Sci 63:125-131.

Haddad S, Charest-Tardif G, Tardif R, et al. 2000. Validation of a physiological modeling framework for simulating the toxicokinetics of chemicals in mixtures. Toxicol Appl Pharmacol 167:199-209.

Haddad S, Tardif R, Viau C, et al. 1999. A modeling approach to account for toxicokinetic interactions in the calculation of biological hazard index for chemical mixtures. Toxicol Lett 108:303-308.

Haglund U, Lundberg I, Zech L. 1980. Chromosome aberrations and sister chromatid exchanges in Swedish paint industry workers. Scand J Work Environ Health 6:291-298. 


\section{REFERENCES}

Haigler BE, Pettigrew CA, Spain JC. 1992. Biodegradation of mixtures of substituted benzenes by Pseudomonas sp. strain JS150. Appl Environ Microbiol 58:2237-2244.

Hakkola M, Saarinen L. 1996. Exposure of tanker drivers to gasoline and some of its components. Ann Occup Hyg 40(1):1-10.

Hampton CV, Pierson WR, Harvey TM, et al. 1982. Hydrocarbon gases emitted from vehicles on the road: 1. A qualitative gas chromatography/mass spectrometry survey. Environ Sci Technol 16:287-298.

*Hansch C, Leo A. 1979. Substituent constants for correlation analysis in chemistry and biology. New York, NY: John Wiley \& Sons, 232.

Hansch C, Leo A, Hoekman D. 1995. Xylene. Exploring QSAR hydrophobic, electronic, and steric constants. Washington, DC: American Chemical Society, 43.

Harper C, Drew RT, Fouts JR. 1975. Benzene and $p$-xylene: A comparison of inhalation toxicities and in vitro hydroxylations. In: Jollow J, ed. Biological reactive intermediates: Formation, toxicity, and inactivation. Proceedings of an International Conference, Turku, Finland, July 26-27, 1975. New York, NY: Plenum Press, 302-311.

Hass U, Jakobsen BM. 1993. Prenatal toxicity of xylene inhalation in the rat: A teratogenicity and postnatal study. Pharmacol and Toxicol 73:20-23.

Hass U, Lund SP, Simonsen L. 1995. Long-lasting neurobehaviorl effects of prenatal exposure to xylene in rats. Neurotoxicology 16(4):761.

Hass U, Lund SP, Simonsen L. 1997. Long-lasting neurobehavioral effects of prenatal exposure to xylene in rats. Neurotoxicology 18(2):547-552.

Hastings L, Cooper GP, Burg W. 1986. Human sensory response to selected petroleum hydrocarbons. In: MacFarland HN, Holdsworth CE, MacGregor JA, et al. eds. Advances in modern environmental toxicology. Vol. 6. Applied toxicology of petroleum hydrocarbons. Princeton, NJ: Princeton Scientific Publishers, 255-270.

Haworth S, Lawlor T, Mortelmans K, et al. 1983. Salmonella mutagenicity test results for 250 chemicals. Environ Mutagen (Suppl) 1:3-142.

HazDat. 2006. Xylene. HazDat Database: ATSDR's Hazardous Substance Release and Health Effects Database. Atlanta, GA: Agency for Toxic Substances and Disease Registry. www.atsdr.cdc.gov/hazdat.html. July 10, 2006.

Heavner DL, Morgan WT, Ogden MW. 1995. Determination of volatile organic compounds and its apportionment in 49 homes. Environ Int 21:3-21.

Heavner DL, Morgan WT, Ogden MW. 1996. Determination of volatile organic compounds and respirable suspended particulate matter in New Jersey and Pennsylvania homes and workplaces. Environ Int 22:159-183.

Heikes DL, Jensen SR, Fleming-Jones ME. 1995. Purge and trap extraction with GC-MS determination of volatile organic compounds in table-ready foods. J Agric Food Chem 43:2869-2875. 


\section{REFERENCES}

Hejtmankova N, Simanek V, Holcik J, et al. 1979. Antifungal and mutagenic activity of phenolic substances with different alkyl groups: II. A study of the relationship between the biological activity and the constitution of the investigated compounds. Acta Univ Palacki Olomuc Fac Med 90:75-87.

Herman DC, Mayfield CI, Inniss WE. 1991. The relationship between toxicity and bioconcentration of volatile aromatic-hydrocarbons by the alga Selenastrum-capricornutum. Chemosphere 22:665-676.

Hester NE, Meyer RA. 1979. A sensitive technique for measurement of benzene and alkylbenzenes in air. Environ Sci Technol 13:107-109.

Hiatt MH. 1983. Determination of volatile organic compounds in fish samples by vacuum distillation and fused silica capillary gas chromatography/mass spectrometry. Anal Chem 55:506-516.

Hiatt MH, Pia JH. 2004. Screening processed milk for volatile organic compounds using vacuum distillation/gas chromatography/mass spectrometry. Arch Environ Contam Toxicol 46:189-196.

Higgins E, Griest WH, Olerich G. 1983. Applications of Tenax trapping to analysis of gas phase organic compounds. J Assoc Off Anal Chem 66:1074-1083.

Hillefors-Berglund M, Liu Y, von Euler G. 1995. Persistent, specific and dose-dependent effects of toluene exposure on dopamine $\mathrm{D}_{2}$ agonist binding in the rat caudate-putamen. Toxicology 100(1-3):185194.

Hine $\mathrm{CH}$, Zuidema HH. 1970. The toxicological properties of hydrocarbon solvents. Ind Medicine 39:39-44.

Hipolito RN. 1980. Xylene poisoning in laboratory workers: Case reports and discussion. Lab Med 11:593-595.

Ho CT, Lee KN, Jin QZ. 1983. Isolation and identification of volatile flavor compounds in fried bacon. J Agric Food Chem 31:336-342.

Hodge HC, Sterner JH. 1949. Tabulation of toxicity classes. Am Ind Hyg Assoc Q 10:93-96.

Hodgson AT, Rudd AF, Beal D, et al. 2000. Volatile organic compound concentrations and emission rates in new manufactures and site-built houses. Indoor Air 10:178-192.

Hoel DG, Davis DL, Miller AB, et al. 1992. Trends in cancer mortality in 15 industrialized countries, 1969-1986. J Natl Cancer Inst 84(5):313-320.

Hoffmann K, Krause C, Seifert B, et al. 2000. The German Environmental Survey 1990/92 (GERES II) Sources of personal exposure to volatile organic compounds. J Expo Anal Environ Epidemiol 10:115125.

Holmberg PC, Nurminen M. 1980. Congenital defects of the central nervous system and occupational factors during pregnancy, case-referent study. Am J Ind Med 1:167-176.

Holz O, Scherer G, Brodtmeier S, et al. 1995. Determination of low level exposure to volatile aromatic hydrocarbons and genotoxic effects in workers at a styrene plant. Occup Environ Med 52:420-428. 
Honma T, Sudo A, Miyagawa M, et al. 1983. Significant changes in the amounts of neurotransmitter and related substances in rat brain induced by subacute exposure to low levels of toluene and xylene. Ind Health 21:143-151.

HSDB. 2007. Xylene. Hazardous Substances Data Bank. National Library of Medicine. http://toxnet.nlm.nih.gov. May 4, 2007.

Hsieh CC, Chang KH, Kao YS. 1999. Estimating the ozone formation potential of volatile aromatic compounds in vehicle tunnels. Chemosphere 39(9):1433-1444.

Hudak A, Ungvary G. 1978. Embryotoxic effects of benzene and its methyl derivatives: Toluene, xylene. Toxicology 11:55-63.

Hung IF, Liao MH. 1991. Aromatic hydrocarbons in indoor air. J Environ Sci Health A26:487-492.

Hunt MJ, Shafer MB, Barlaz MA, et al. 1997. Anaerobic biodegradation of alkylbenzenes in laboratory microcosms representing ambient conditions. Biorem J 1:53-64.

Hutchins SR. 1991. Optimizing BTEX biodegradation under denitryfying conditions. Environ Tox Chem 10:1437-1448.

Iannuzzi TJ, Huntley SL, Schmidt CW, et al. 1997. Combined sewer overflows (CSOs) as sources of sediment contamination in the lower Passaic River, New Jersey. I. Priority pollutants and inorganic chemicals. Chemosphere 34(2):213-231.

IARC. 2004. Overall evaluations of carcinogenicity to humans: As evaluated in IARC Monographs volumes 1-82 (at total of 900 agents, mixtures and exposures). Lyon, France: International Agency for Research on Cancer. http://www-cie.iarc.fr/monoeval/crthall.html. February 15, 2005.

Ilgen E, Karfich N, Levsen K, et al. 2001a. Aromatic hydrocarbons in the atmospheric environment: Part I. Indoor outdoor sources, the influence of traffic. Atmos Environ 35:1235-1252.

Ilgen E, Levesen K, Angerer J, et al. 2001b. Aromatic hydrocarbons in the atmospheric environment. Part II: Univariate and multivariate analysis and case studies of indoor concentrations. Atmos Environ 35:1253-1264.

Ilgen E, Levsen K, Angerer J, et al. 2001c. Aromatic hydrocarbons in the atmospheric environment. Part III: Personal monitoring. Atmos Environ 35:1265-1279.

Inoue O, Seiji K, Kawai T et al. 1993. Excretion of methylhippuric acids in urine of workers exposed to a xylene mixture: Comparison among three xylene isomers and toluene. Int Arch Occup Environ Health 64:533-539.

IPCS. 1992. Xylene. Poisons information monograph 565. International Programme on Chemical Safety. http://www.inchem.org/documents/pims/xylene.htm. April 07, 2005.

IRIS. 2005. Xylenes. Integrated Risk Information System. Washington, DC: U.S. Environmental Protection Agency. http://www.epa.gov/iris/subst/index.html. February 15, 2004.

Ito T, Yoshitome K, Horike T, et al. 2002. Distribution of inhaled m-xylene in rat brain and its effect on GABAA receptor binding. J Occup Health 44(2):69-75. 
Iyadomi M, Ichiba M, Zhang J, et al. 2000. Evaluation of skin irritants caused by organic solvents by means of the mouse ear thickness measurement method. J Occup Health 42:44-46.

Jajte J, Stetkiewicz J, Wronska-Nofer T. 2003. Combined exposure to m-xylene and ethanol: Oxidative stress in the rat liver. Int J Occup Med Environ Health 16(4):345-350.

*Jakubowski M, Kostrzewski P. 1989. Excretion of methylbenzoic acid in urine as a result of single and combined exposure to $m$-xylene. Pol J Occup Med 2:238-247.

Jang JY, Droz PO. 1997. Ethnic differences in biological monitoring of several organic solvents II. A simulation study with a physiologically based pharmacokinetic model. Int Arch Occup Environ Health 70:41-50.

Jang JY, Droz PO, Berode M. 1997. Ethnic differences in biological monitoring of several organic solvents. I. Human exposure experiment. Int Arch Occup Environ Health 69:343-349.

Jay K, Stieglitz L. 1995. Identification and quantification of volatile organic components in emissions of waste incineration plants. Chemosphere 30(7):1249-1260.

Jenkins J, Runes H, Moate T. 2004. Xylenes in Oregon hazelnuts. Bull Environ Contam Toxicol $72: 1152-1156$.

Jenkins LJ, Jones RA, Siegel J. 1970. Long-term inhalation screening studies of benzene, toluene, oxylene, and cumene on experimental animals. Toxicol Appl Pharmacol 16:818-823.

Jepson GW, McDougal JN. 1997. Physiologically based modeling of nonsteady state dermal absorption of halogenated methanes from an aqueous solution. Toxicol Appl Pharmacol 144:315-324.

Jo WK, Choi SJ. 1996. Vehicle occupants' exposure to aromatic volatile organic compounds while commuting on an urban-suburban route in Korea. J Air Waste Manage Assoc 46:749-754.

Jo WK, Kim SH. 2001. Worker exposure to aromatic volatile organic compounds in dry cleaning. Am Ind Hyg Assoc J 62(4):466-471.

Jo WK, Moon KC. 1999. Housewives' exposure to volatile organic compounds relative to proximity to roadside service stations. Atmos Environ 33:2921-2928.

Jo WK, Kim KY, Park KH, et al. 2003. Comparison of outdoor and indoor mobile source-related volatile organic compounds between low- and high- floor apartments. Environ Res 92:166-171.

Johanson CE. 1980. Permeability and vascularity of the developing brain: Cerebellum vs. cerebral cortex. Brain Res 190:3-16.

Jonai H, Sato M. 1988. Exposure indices for painters exposed to toluene and xylene at low concentrations. Ind Health 26:197-202.

Jori A, Calamari D, Di Domenico A, et al. 1986. Ecotoxicological profile of xylenes: Working party on ecotoxicological profiles of chemicals. Ecotoxicol Environ Safety 11:44-80. 


\section{REFERENCES}

Jovanovic JM, Jovanovic MM, Spasic MJ, et al. 2004. Peripheral nerve conduction study in workers exposed to a mixture of organic solvents in paint and lacquer industry. Croat Med J 45(6):769-774.

Juttner F. 1994. Emission of aromatic hydrocarbons and aldehydes into the water by a pour-stroke outboard motor: Quantitative measurements. Chemosphere 29(2):191-200.

Kaneko T, Endoh K, Sato A. 1991a. Biological monitoring of exposure to organic solvent vapors. I. A physiological simulation model of $m$-xylene pharmacokinetics in man. Yamanashi Med J 6(3):127-135.

Kaneko T, Endoh K, Sato A. 1991b. Biological monitoring of exposure to organic solvent vapors. II. Simulation studies using a physiological pharmacokinetic model for $m$-xylene. Yamanashi Med J 6(3):137-149.

Kaneko T, Horiuchi J, Sato A. 2000. Development of a physiologically based pharmacokinetic model of organic solvent in rats. Pharm Res 42(5):465-470.

Kaneko T, Wang P-Y, Sato A. 1993. Enzyme induction by ethanol consumption affects the pharmacokinetics of inhaled $m$-xylene only at high levels of exposure. Arch Toxicol 67:473-477.

Kaneko T, Wang PY, Tsukada H, et al. 1995. m-Xylene toxicokinetics in phenobarbital-treated rats: Comparison among inhalation exposure, oral administration, and intraperitoneal administration. Toxicol Appl Pharmacol 131:13-20.

Kango RA, Quinn JG. 1989. Adsorption studies of xylenes and ethylbenzene on soil and humic-acid by a purge and trap gas-chromatographic method. Chemosphere 19:1269-1276.

Kao CM, Wang YS. 2001. Field investigation of the natural attenuation and intrinsic biodegradation rates at an underground storage tank site. Environ Geol 40(4-5):622-631.

Karlson U, Frankenberger WT Jr. 1989. Microbial degradation of benzene and toluene in groundwater. Bull Environ Contam Toxicol 43:505-510.

Kataoka H, Manabe K, Nakase S, et al. 1991. Determination of hippuric acid and $o-, m$ - and $p$ methylhippuric acids in urine by capillary gas chromatography. J Pharm Biomed Anal 9:699-704.

Katsuyama H, Tsuchiya G, Saijoh K, et al. 1998. Occupational exposure to low concentrations of organic solvents in shipyards. J Occup Health 40:186-192.

Kawai T, Mizunuma K, Yasugi T, et al. 1991. Urinary methylhippuric acid isomer levels after occupational exposure to a xylene mixture. Int Arch Occup Environ Health 63:69-75.

Kawai T, Yasugi T, Mizunuma K. 1992. Comparative evaluation of urinalysis and blood analysis as means of detecting exposure to organic solvents at low concentrations. Int Arch Occup Environ Health 64:223-234.

Kawata K, Tanabe A, Saito S, et al. 1997. Screening of volatile organic compounds in river sediment. Bull Environ Contam Toxicol 58:893-900.

Keith LH, Garrison AW, Allen FR, et al. 1976. Identification of organic compounds in drinking water from thirteen U.S. cities. In: Keith LH, ed. Identification and analysis of organic pollutants in water. Ann Arbor, MI: Ann Arbor Science Publishers Inc., 329-373. 
Kelly TJ, Muklund R, Spicer CW, et al. 1994. Concentrations and transformations of hazardous air pollutants. Environ Sci Technol 28:378-387.

Kennah HE, Hignet HS, Laux PE, et al. 1989. An objective procedure for quantitating eye irritation based upon changes of corneal thickness. Fundam Appl Toxicol 12:258-268.

Kent B, Spycher N. 1994. Major chemical parameters in groundwater control. Environmental science and pollution control. New York, NY: Dekker M, 479-495.

Kezic S, Janmaat A, Kruse J, et al. 2004. Percutaneous absorption of m-xylene vapour in volunteers during pre-steady and steady state. Toxicol Lett 153:273-282.

Kezic S, Monster AC, Kruse J, et al. 2000. Skin absorption of some vaporous solvents in volunteers. Int Arch Occup Environ Health 73:415-422.

Kezic S, Monster AC, van de Gevel IA, et al. 2001. Dermal absorption of neat liquid solvents on brief exposures in volunteers. Am Ind Hyg Assoc J 62:12-18.

Kilburn KH, Seidman BC, Warshaw R. 1985. Neurobehavioral and respiratory symptoms of formaldehyde and xylene exposure in histology technicians. Arch Env Health 40:229-233.

*Kim SK, Kim YC. 1996. Effect of a single administration of benzene, toluene or $m$-xylene on carboxyhaemoglobin elevation and metabolism of dichloromethane in rats. J Appl Toxicol 16:437-444.

Kim YM, Harrad S, Harrison RM. 2001. Concentrations and sources of VOCs in urban domestic and public microenvironments. Environ Sci Technol 35:997-1004.

King MF, Hamilton BL, Matthews MA, et al. 1993. Isolation and identification of volatiles and condensable material in raw beef with supercritical carbon dioxide extraction. J Agric Food Chem 41:1974-1981.

Kinlin EK, Muralidhara R, Pittet AO, et al. 1972. Volatile components of roasted filberts. J Agric Food Chem 20(5):1021-1028.

Kinney PL, Chillrud SN, Ramstrom S, et al. 2002. Exposures to multiple air toxics in New York City. Environ Health Perspect 110(Suppl. 4):539-546.

Kira S. 1977. Measurement by gas chromatography of urinary hippuric acid and methylhippuric acid as indices of toluene and xylene exposure. Br J Ind Med 34:305-309.

Klaucke DN, Johansen M, Vogt RL. 1982. An outbreak of xylene intoxication in a hospital. Am J Ind Med 3:173-178.

*Klimisch H-J, Pauluhn J, Hollander HW, et al. 1988. Inhalation hazard test: Interlaboratory trial with OECD method 403. Arch Toxicol 61:318-320.

Koe LCC, Tan NC. 1990. GC-MS Identification of gaseous volatiles in wastewater. Environ Monit Assess 15:13-24.

Kolpin DW, Squillace PJ, Zogorski JS, et al. 1997. Pesticides and volatile organic compounds in shallow urban groundwater of the United States. Int Contrib Hydrogeol 21:469-474. 


\section{REFERENCES}

Komori M, Nishio K, Kitada M, et al. 1990. Fetus-specific expression of a form of cytochrome P-450 in human livers. Biochemistry 29:4430-4433.

Korsak Z, Sokal JA, Dedyk A, et al. 1988. Toxic effects of combined exposure to toluene and xylene in animals: I. Acute inhalation study. Pol J Occup Med 1:45-50.

Korsak Z, Sokal JA, Gorny R. 1992. Toxic effects of combined exposure to toluene and $m$-xylene in animals. III. Subchronic inhalation study. Pol J Occup Med Environ Health 5(1):27-33.

Korsak Z, Sokal JA, Swiercz R. 1991. The toxic effects of combined exposure to toluene and $m$-xylene in animals: II. Blood toluene and $m$-xylene during single and combined exposure in rats. Pol J Occup Med 4:377-381.

Korsak Z, Sokal JA, Wasiela T, et al. 1990. Toxic effects of acute exposure to particular xylene isomers in animals. Pol J Occup Med 3:221-226.

Korsak Z, Swiercz R, Jedrychowski R. 1993. Effects of acute combined exposure to n-butyl alcohol and m-xylene. Pol J Occup Med Environ Health 6(1):35-41.

Korsak Z, Wisniewska-Knypl J, Swiercz R. 1994. Toxic effects of subchronic combined exposure to nbutyl alcohol and m-xylene in rats. Int J Occup Med Environ Health 7(2):155-166.

Kostianen R. 1995. Volatile organic compounds in the indoor air of normal and sick houses. Atmos Environ 29:693-702.

Kramer A, Linnert M, Wrbitzky R, et al. 1999. Occupational chronic exposure to organic solvents XVII. Ambient and biological monitoring of workers exposed to xyelens. Int Arch Occup Environ Health $72: 52-55$.

Krishnan K, Andersen ME. 1994. Physiologically based pharmacokinetic modeling in toxicology. In: Hayes AW, ed. Principles and methods of toxicology. 3rd ed. New York, NY: Raven Press, Ltd., 149188.

Krishnan K, Andersen ME, Clewell HJ III, et al. 1994. Physiologically based pharmacokinetic modeling of chemical mixtures. In: Yang RSH, ed. Toxicology of chemical mixtures: Case studies, mechanisms, and novel approaches. San Diego, CA: Academic Press, 399-437.

Kucera J. 1968. Exposure to fat solvents: A possible cause for sacral agenesis in man. J Pediatr 72:857859.

Kumarathasan P, Oyson R, Chu I. 1998. Application of an automated HS-GC method in partition coefficient determination for xylenes and ethylbenzene in rat tissues. Chemosphere 37(1):159-178.

Kurppa K, Husman K. 1982. Car painters' exposure to a mixture of organic solvents: Serum activities of liver enzymes. Scand J Work Environ Health 8:137-140.

Kwok ESC, Atkinson R. 1995. Estimation of hydroxyl radical reaction rate constants for gas-phase organic compounds using a structure-reactivity relationship: An update. Atmos Environ 29(14):16851695. 


\section{REFERENCES}

Kyrklund T, Kjellstrand P, Haglid K. 1987. Brain lipid changes in rats exposed to xylene and toluene. Toxicology 45:123-133.

LaGrone FS. 1991. Potential community exposure to toxic chemicals. Environ Sci Technol 25:366-368.

Lahvis MA, Baehr AL, Baker RJ. 1999. Quantification of aerobic biodegradation and volatilization rates of gasoline hydrocarbons near the water table under natural attenuation conditions. Water Resour Res 35(3):753-765.

Laine A, Savolainen K, Riihimaki V, et al. 1993. Acute effects of $m$-xylene inhalation on body sway, reaction times, and sleep in man. Int Arch Occup Environ Health 65:179-188.

Lapare S, Tardiff R, Brodeur J. 1993. Effect of various exposure scenarios on the biological monitoring of organic solvents in alveolar air. I. Toluene and m-xylene. Int Arch Environ Health 64:569-580.

Lawryk NJ, Weisel CP. 1996. Concentrations of volatile organic compounds in the passenger compartments of automobiles. Environ Sci Technol 30:810-816.

*Lee S-H, Lee SH. 1993. A study on the neurobehavioral effects of occupational exposure to organic solvents in Korean workers. Environ Res 60:227-232.

Leeder JS, Kearns GL. 1997. Pharmcogenetics in pediatrics: Implications for practice. Pediatr Clin North Am 44(1):55-77.

Leguen S, Prost C, Demaimay M. 2000. Critical comparison of three olfactometric methods for the identification of the most potent odorants in cooked mussels (Mytilus edulis). J Agric Food Chem 48:1307-1314.

Lemire S, Ashley D, Olaya P, et al. 2004. Environmental exposure of commuters in Mexico City to volatile organic compounds as assessed by blood concentrations, 1998. Salud Pub Mexico 46(4):32-38.

Leung H-W. 1993. Physiologically-based pharmacokinetic modeling. In: Ballentyne B, Marrs T, Turner P, eds. General and applied toxicology. Vol. 1. New York, NY: Stockton Press, 153-164.

Leung P-L, Harrison RM. 1998. Evaluation of personal exposure to monoaromatic hydrocarbons. Occup Environ Med 55:249-257.

Leung P-L, Harrison RM. 1999. Roadside and in-vehicle concentrations of monoaromatic hydrocarbons. Atmos Environ 33:191-204.

Lewis RJ. 1997. Xylene. In: Hawley's condensed chemical dictionary. 13th ed. New York, NY: John Wiley \& Sons, Inc., 1188.

Lewis RJ. 2000. Xylene. In: Sax's dangerous properties of industrial materials. 10th ed. New York, NY: John Wiley \& Sons Inc., 3702-3703.

Lewis RJ. 2001. Xylene. In: Hawley's condensed chemical dictionary. 14th ed. New York, NY: John Wiley \& Sons, Inc., 1183-1185.

Lide DR. 2005. Xylene. In: CRC handbook of chemistry and physics. 86th ed. Boca Raton, FL: CRC Press, 3-520 to 3-521. 
Liira J, Elovaara E, Raunio H, et al. 1991. Metabolic interaction and disposition of methylethylketone and $m$-xylene in rats at single and repeated inhalation exposures. Xenobiotica 21:53-64.

Liira J, Riihimaki V, Engstrom K, et al. 1988. Coexposure of man to $m$-xylene and methyl ethyl ketone: Kinetics and metabolism. Scand J Work Environ Health 14:322-327.

Lindbohm M-L, Taskinen H, Sallmen M, et al. 1990. Spontaneous abortions among women exposed to organic solvents. Am J Ind Med 17:449-463.

Litton Bionetics. 1978a. Teratology study in rats xylene (final report). Submitted to the U.S.

Environmental Protection Agency under TSCA Section 8D. OTS0205935.

Litton Bionetics. 1978b. Mutagenicity evaluation of xylene. Submitted to the U.S. Environmental Protection Agency under TSCA Section 8D. OTS0205935.

Livingston, AL. 1978. Forage plant estrogens. J Toxicol Environ Health 4:301-324.

Loizou GD, Jones K, Akrill P, et al. 1999. Estimation of the dermal absorption of m-xylene vapor in humans using breath sampling and physiologically based pharmacokinetic analysis. Toxicol Sci 48(2):170-179.

Lonneman WA, Kopczynski SL, Darley PE, et al. 1974. Hydrocarbon composition of urban air pollution. Environ Sci Technol 8:229-236.

Lovegren N, Fisher GS, Legendre MG, et al. 1979. Volatile constituents of dried legumes. J Agric Food Chem 27:851-853.

Lysyj I, Perkins G, Farlow JS. 1980. Trace analysis for aromatic hydrocarbons in natural waters. Environ Int 4:407-416.

MacDonald AJ, Rostami-Hodjegan A, Tucker GT, et al. 2002. Analysis of solvent central nervous system toxicity and ethanol interactions using a human population physiologically based kinetic and dynamic model. Regul Toxicol Pharmacol 35:165-176.

Mackay D, Leinonen PJ. 1975. Rate of evaporation of low-solubility contaminants from water bodies to atmosphere. Environ Sci Technol 9:1178-1180.

Maltoni C, Conti B, Cotti G. 1983. Benzene: A multipotential carcinogen: Results of long-term bioassays performed at the Bologna Institute of Oncology. Am J Ind Med 4:589-630.

Maltoni C, Conti B, Cotti G, et al. 1985. Experimental studies on benzene carcinogenicity at the Bologna Institute of Oncology: Current results and ongoing research. Am J Ind Med 7:415-446.

Marks TA, Ledoux TA, Moore JA. 1982. Teratogenicity of a commercial xylene mixture in the mouse. J Toxicol Environ Health 9:97-105.

Martinez JS, Sala JJG, Vea AM, et al. 1989. Renal tubular acidosis with an elevated anion gap in a glue sniffer: Letter to editor. Human Toxicol 8:139-140.

Matiella JE, Hsieh CY. 1991. Volatile compounds in scrambled eggs. J Food Sci 56:387-390. 
Mattie DR, Bates GD Jr., Jepson GW, et al. 1994. Determination of skin: air partition coefficients for volatile chemicals: Experimental method and applications. Fundam Appl Toxicol 22:51-57.

Mayr U, Butsch A, Schneider S. 1992. Validation of two in vitro test systems for estrogenic activities with zearalenone, phytoestrogens and cereal extracts. Toxicology 74:135-149.

Mayer GJ, Cheng IS, Pau P, et al. 1994. Emissions of air toxics from wastewater treatment plants. Water Environ Res 66:140-144.

McCarroll NE, Keech BJ, Piper CE. 1981a. A microsuspension adaptation of the Bacillus subtilis "rec" assay. Environ Mutagen 3:607-616.

McCarroll NE, Piper CE, Keech BH. 1981b. An E coli microsuspension assay for the detection of DNA damage induced by direct-acting agents and promutagens. Environ Mutagen 3:429-444.

McDougal JN, Jepson GW, Clewell HJ, et al. 1990. Dermal absorption of organic chemical vapors in rats and humans. Fundam Appl Toxicol 14:299-308.

Merian E, Zander M. 1982. Volatile aromatics. In: Hutzinger G, ed. Handbook of environmental chemistry. Vol. 3 (Pt B). Berlin, Germany: Springer, 117-161.

Mill T. 1980. Chemical and photo oxidation. In: Hutzinger O, ed. Handbook of environmental chemistry. Vol. 2. Berlin, Germany: Springer-Verlag, 77-105.

Minoia C, Meroni G, Aprea C, et al. 1996. Environmental and urinary reference values as markers of exposure to hydrocarbons in urban areas. Sci Total Environ 192:163-182.

Mirkova E, Hinkova L, Vassileva L, et al. 1979. Xylene neurotoxicity in pregnant rats and fetuses. Act Nerv Super (Praha) 21:265-268.

Mirkova E, Zaikov C, Antov G, et al. 1983. Prenatal toxicity of xylene. J Hyg Epidemiol Microbiol Immunol 27:337-343.

Misharina TA, Golovnya RV. 1992. Hydrocarbon contaminants of boiled shrimp and crab meat. J High Resolut Chromatogr 15:332-334.

*Mobil Oil Company. 1975. Report on rabbit eye irritation. Submitted to the U.S. Environmental Protection Agency under TSCA Section 8D. OTS0206126.

Moen BE, Hollund BE. 2000. Exposure to organic solvents among car painters in Bergen, Norway. Ann Occup Hyg 44(3):185-189.

Mohamed MF, Kang D, Aneja VP. 2002. Volatile organic compounds in some urban locations in United States. Chemosphere 47:863-882.

Mohtashamipur E, Northpoth K, Woelke U, et al. 1985. Effects of ethylbenzene, toluene, and xylene on the induction of micronuclei in bone marrow polychromatic erythrocytes of mice. Arch Toxicol 58:106109. 
Molnar J, Paksy KA, Naray M. 1986. Changes in the rat's motor behavior during 4-hr inhalation exposure to prenarcotic concentrations of benzene and its derivatives. Acta Physiol Hung 67:349-354.

Morgan DL, Cooper SW, Carlock DL, et al. 1991. Dermal absorption of neat and aqueous volatile organic chemicals in the Fischer 344 rat. Environ Res 55:51-63.

*Morikawa K, Fukuoka M, Kakiuchi M, et al. 1990. Detrusor hyperreflexia induced by intravesical instillation of xylene in conscious rats. Jpn J Pharmacol 52:587-595.

Morin M, Chambon P, Chambon R, et al. 1981. Measurement of exposure to xylenes by separate determination of $m$ - and $p$ - methyl hippuric-acid in urine. J Chromatogr 210:346-349.

Moriwaki H, Tsujimoto Y, Shimizu M, et al. 2005. Influence of sodium benzoate on the metabolism of o-xylene in the rat. Xenobiotica 35(5):487-497.

Morley R, Eccleston DW, Douglas CP, et al. 1970. Xylene poisoning: A report on one fatal case and two cases of recovery after prolonged unconsciousness. Br Med J 3:442-443.

Morozzi G, De Bartolomeo A, Fabiani R, et al. 1999. Induction of DNA damage and apoptosis by volatile organic compounds and by benzene metabolites. Ig Mod 112:1503-1513.

Morselli PL, Franco-Morselli R, Bossi L. 1980. Clinical pharmacokinetics in newborns and infants: Age-related differences and therapeutic implications. Clin Pharmacokin 5:485-527.

Morvai V, Hudak A, Ungvary G, et al. 1976. ECG changes in benzene, toluene and xylene poisoned rats. Acta Med Acad Sci Hung 33:275-286.

Morvai V, Ungvary G, Herrmann HJ, et al. 1987. Effects of quantitative undernourishment, ethanol and xylene on coronary microvessels of rats. Acta Morphol Hung 35:199-206.

Moser VC, Coggeshall EM, Balster RL. 1985. Effects of xylene isomers on operant responding and motor performance in mice. Toxicol Appl Pharmacol 80:293-298.

Moszczynski P, Lisiewicz J. 1983. Occupational exposure to benzene, toluene and xylene and the T lymphocyte functions. J Clin Hematol Oncol 13:37-41.

Moszczynski P, Lisiewicz J. 1984a. Occupational exposure to benzene, toluene and xylene and the T lymphocyte functions. Haematologia 17:449-453.

*Moszczynski P, Lisiewicz J. 1984b. [Cytochemical and immunological tests of those exposed to paintand-varnish organic solvents: IV. Activity of nonspecific esterase in lymphocytes.] Med Pr 35:273-278. (Polish)

Muralidhara, Krishnakumari MK. 1980. Mammalian toxicity of aromex and xylene used in pesticidal formulations. Indian J Exp Biol 18:1148-1151.

Mushrush GW, Mose DG, Sullivan KT. 1994. Soil vapor and groundwater analysis from a recent oil spill. Bull Environ Contam Toxicol 52:31-38.

*Nakajima T, Sato A. 1979a. Enhanced activity of liver drug-metabolizing enzymes for aromatic and chlorinated hydrocarbons following food deprivation. Toxicol Appl Pharm 50:549-556. 
Nakajima T, Sato A. 1979b. [Metabolic antagonism among benzene, toluene and $m$-xylene in vitro.] Jpn J Ind Health 21:546-547. (Japanese)

Nakamura S, Oda Y, Shimada T, et al. 1987. SOS-inducing activity of chemical carcinogens and mutagens in Salmonella typhimurium TA1535/pSK1002: Examination with 151 chemicals. Mutat Res 192:239-246.

Narvaez J, Song CD. 2003. Letter to the editor. Cancer 22(5):495.

NAS/NRC. 1989. Report of the oversight committee. In: Biologic markers in reproductive toxicology. Washington, DC: National Academy of Sciences, National Research Council, National Academy Press, $15-35$.

Nathwani JS, Phillips CR. 1977. Adsorption-desorption of selected hydrocarbons in crude oil in soils. Chemosphere 4:157-162.

NCI. 1985. Monograph on human exposure to chemicals in the workplace: Xylene. Bethesda, MD: National Cancer Institute, Division of Cancer Etiology. PB86155124.

Nelson KW, Ege JF Jr, Ross M, et al. 1943. Sensory response to certain industrial solvent vapors. J Ind Hyg Toxicol 25:282-285.

NEMI. 2005. Xylene. National Environmental Methods Index. U.S. Environmental Protection Agency. U.S. Geological Survey. http://www.nemi.gov. April 17, 2005.

Nersesian W, Booth H, Hoxie D, et al. 1985. Illness in office attributed to xylene [Letter]. Occup Health Saf 54:88.

NIOSH. 1975. Criteria for a recommended standard--occupational exposure to xylene. Rockville, MD: U.S. Department of Health, Education, and Welfare, Public Health Service, Center for Disease Control, National Institute for Occupational Safety and Health. NIOSH 75-168. PB246702.

NIOSH. 1976. National occupational hazard survey (1970). Cincinnati, OH: National Institute for Occupational Safety and Health, Department of Health and Human Services.

NIOSH. 1981. p-Xylene: Development of a biological standard for the industrial worker. Cincinnati, OH: National Institute for Occupational Safety and Health. PB82152844.

NIOSH. 1990a. Indoor air quality and work environment study. Volume 2: Results of indoor air environmental monitoring. Cincinnati, OH: U.S. Department of Health and Human Services, Hazards Evaluation and Technical Assistance Branch, National Institute for Occupational Safety and Health. PB92103183.

*NIOSH. 1990b. NIOSH pocket guide to chemical hazards. Washington, DC: U.S. Department of Health and Human Services, Public Health Service, Centers for Disease Control, National Institute for Occupational Safety and Health. DHHS (NIOSH) Publ. No. 90-117.

NIOSH. 1990c. National occupational exposure survey (1980-83). Cincinnati, OH: National Institute for Occupational Safety and Health, Department of Health and Human Services. 
NIOSH. 1994. NIOSH manual of analytical methods. 4th ed. U.S. Department of Health and Human Services. Methods 1501 and 8301.

NIOSH. 2005. Xylenes. NIOSH pocket guide to chemical hazards. Atlanta, GA: National Institute for Occupational Safety and Health, Centers for Disease Control and Prevention. http://www.cdc.gov/niosh/npg/npgdname.html. February 15, 2004.

Norstrom A, Scheepers PTJ. 1990. Determination of n-decane and $m$-xylene in blood using amberlite XAD-2. Chemosphere 21:459-466.

Norstrom A, Andersson B, Aringer L, et al. 1988. Determination of specific mercapturic acids in human urine after experimental exposure to toluene or o-xylene. In: Bartsch H, Hemminki K, O'Neill IK, eds. Methods for detecting DNA damaging agents in humans: Applications in cancer epidemiology and prevention; Symposium on detection of DNA-damaging agents in man. Lyon, France: World Health Organization, International Agency for Research on Cancer. IARC Sci Publ (89):232-234.

Norstrom A, Andersson B, Levin JO, et al. 1989. Biological monitoring of $o$-xylene after experimental exposure in man: Determination of urinary excretion products. Chemosphere 18:1513-1523.

NRC. 1980. Drinking water and health. Vol. 3. Washington, DC: National Research Council, National Academy Press, 178-181, 231-261.

NRC. 1993. National Research Council. Pesticides in the diets of infants and children. Washington, DC: National Academy Press.

NTP. 1986. National Toxicology Program technical report on the toxicology and carcinogenesis studies of xylenes (mixed) (60\% m-xylene, 14\% p-xylene, 9\% o-xylene, and 17\% ethylbenzene) (CAS No. 133020-7) in F344/N rats and B6C3F1 mice (gavage studies). Research Triangle Park, NC: U.S. Department of Health and Human Services, Public Health Service, National Institutes of Health, National Toxicology Program. NTP TR 327. NIH Publication No. 87-2583.

NTP. 2005. Report on carcinogens. 11th ed. Research Triangle Park, NC: U.S. Department of Health and Human Services, Public Health Service, National Toxicology Program. http://ntpserver.niehs.nih.gov/ntp/roc/toc11.html. February 15, 2004.

Nunes P, Benville PJ. 1979. Uptake and depuration of petroleum hydrocarbons in the manila clam, tapes semidecussata reeve. Bull Environ Contam Toxicol 21:719-726.

Nutmagul W, Cronn DR, Hill HJ. 1983. Photoionization/flame-ionization detection of atmospheric hydrocarbons after capillary gas chromatography. Anal Chem 55:2160-2164.

Nylen P. 1996. Differing non-additive alterations in different parts of the nervous system of the rat. Food Chem Toxicol 34:1121-1123.

Nylen P, Hagman M. 1994. Function of the auditory and visual systems, and of peripheral nerve, in rats after long-term combined exposure to n-hexane and methylated benzene derivatives. II. Xylene. Pharmacol and Toxicol 74:124-129.

Nylen P, Ebendal T, Eriksdotter-Nilsson M, et al. 1989. Testicular atrophy and loss of nerve growth factor-immunoreactive germ cell line in rats exposed to $n$-hexane and a protective effect of simultaneous exposure to toluene or xylene. Arch Toxicol 63: 296-307. 
Odermatt JR. 1994. Relative proportions of aromatic compounds (BTEX) in a gasoline impacted aquifer in San Diego County, California. Hydrocarbon Contam Soils Groundwater 4:33-45.

Ogata M, Miyake Y. 1978. Disappearance of aromatic hydrocarbons and organic sulfur compounds from fish flesh reared in crude oil suspension. Water Res 12:1041-1044.

Ogata M, Taguchi T. 1987. Quantitation of urinary metabolites of toluene, xylene, styrene, ethylbenzene, benzene and phenol by automated high performance liquid chromatography. Int Arch Occup Environ Health 59:263-272.

Ogata M, Taguchi T. 1988. Simultaneous determination of urinary creatinine and metabolites of toluene, xylene, styrene, ethylbenzene and phenol by automated high performance liquid chromatography. Int Arch Occup Environ Health 61:131-140.

Ogata M, Tomokuni K, Takatsuka Y. 1970. Urinary excretion of hippuric acid and $m$ - or $p$ methylhippuric acid in the urine of persons exposed to vapours of toluene and $m$ - or $p$-xylene as a test of exposure. Br J Ind Med 27:43-50.

Ogata M, Yamasaki Y, Meguro T, et al. 1979. Quantitation of urinary o-xylene metabolites in rats and human beings by high-performance liquid chromatography. Ind Health 17:123-125.

Ogata M, Yamazaki Y, Sugihara R, et al. 1980. Quantitation of urinary o-xylene metabolites of rats and human beings by high performance liquid chromatography. Int Arch Occup Environ Health 46:127-139.

Olson BA, Gamberale F, Iregren A. 1985. Coexposure to toluene and $p$-xylene in man: Central nervous functions. Br J Ind Med 42:117-122.

O'Neil MJ, Smith A, Heckelman PE, et al, eds. 2001. The Merck index. An encyclopedia of chemicals, drugs, and biologicals. 13th ed. Whitehouse Station, NJ: Merck \& Co, Inc., 1799.

OSHA. 2005a. Air contaminants. Occupational safety and health standards for shipyard employment. Occupational Safety and Health Administration. Code of Federal Regulations. 29 CFR 1915.1000. http://www.osha.gov/comp-links.html. February 15, 2005.

OSHA. 2005b. Gases, vapors, fumes, dusts, and mists. Safety and health regulations for construction. Occupational Safety and Health Administration. Code of Federal Regulations. 29 CFR 1926.55, Appendix A. http://www.osha.gov/comp-links.html. February 15, 2005.

OSHA. 2005c. Limits for air contaminants. Occupational safety and health standards. Occupational Safety and Health Administration. Code of Federal Regulations. 29 CFR 1910.1000. http://www.osha.gov/comp-links.html. February 15, 2005.

Otson R, Williams DT. 1981. Evaluation of a liquid-liquid extraction technique for water pollutants. J Chromatogr 212:187-197.

Otson R, Williams DT. 1982. Headspace chromatographic determination of water pollutants. Anal Chem 54:942-946.

*Otson R, Williams DT, Biggs DC. 1982a. Relationships between raw water quality, treatment, and occurrence of organics in Canadian potable water. Bull Environ Contam Toxicol 28:396-403. 


\section{REFERENCES}

Otson R, Williams DT, Bothwell PD. 1982b. Volatile organic compounds in water at thirty Canadian potable water treatment facilities. J Assoc Off Anal Chem 65:1370-1374.

Otson R, Williams DT, Bothwell PD. 1983. Charcoal tube technique for simultaneous determination of selected organics. Am Ind Hyg Assoc J 44:489-494.

Owen GM, Brozek J. 1966. Influence of age, sex and nutrition on body composition during childhood and adolescence. In: Falkner F, ed. Human development. Philadelphia, PA: WB Saunders, 222-238.

Padilla SS, Lyerly DP. 1989. Effects of $p$-xylene inhalation on axonal transport in the rat retinal ganglion cells. Toxicol Appl Pharmacol 101:390-398.

Palmer KT, Rycroft RJG. 1993. Occupational airborne contact urticaria due to xylene. Contact Dermatitis 28:44.

Pap M, Varga C. 1987. Sister-chromatid exchanges in peripheral lymphocytes of workers occupationally exposed to xylenes. Mutat Res 187:223-225.

Park SH, Schatz RA. 1999. Effect of low-level short-term o-xylene inhalation of benzo[a]pyrene (BaP) metabolism and BaP-DNA adduct formation in rat liver and lung microsomes. J Toxicol Environ Health A 58(5):299-312.

Park SH, AuCoin TA, Silverman DM, et al. 1994. Time-dependent effects of o-xylene on rat lung and liver microsomal membrane structure and function. J Toxicol Environ Health 43(4):469-481.

Patel JM, Harper C, Drew RT. 1978. The biotransformation of $p$-xylene to a toxic aldehyde. Drug Metab Dispos 6:368-374.

Patel JM, Harper C, Gupta BN, et al. 1979. Changes in serum enzymes after inhalation exposure of $p$ xylene. Bull Environ Contam Toxicol 21:17-24.

Pedersen BA, Higgins GM. 1995. Evaluation of chemical exposures in the hazardous waste industry. J Air Waste Manage Assoc 45:89-94.

Pelekis M, Gephart LA, Lerman SE. 2001. Physiological-model-based derivation of the adult and child pharmacokinetic intraspecies uncertainty factors for volatile organic compounds. Regul Toxicol Pharmacol 33:12-20.

Pellizzari ED, Wallace LA, Gordon SM. 1992. Elimination kinetics of volatile organics in humans using breath measurements. J Expos Anal Environ Epidemiol 2:341-355.

Pellizzari ED, Zweidinger RA, Sheldon LS. 1988. Determination of benzene, toluene, and xylene in breath samples by gas chromatography/mass spectrometry. IARC Sci Publ (85):267-279.

Perbellini L, Pasini F, Romani S, et al. 2002. Anaylsis of benzene, toluene, ethylbenzene and m-xylene in biological samples from the general population. J Chromatogr B Analyt Technol Biomed Life Sci 778:199-210.

*Pezzagno G, Imbriani M, Ghittori S, et al. 1988. Urinary concentration, environmental concentration, and respiratory uptake of some solvents: Effect of the work load. Am Ind Hyg Assoc J 49:546-52. 
Pierce CH, Dills RL, Silvey GW, et al. 1996. Partition coefficients between human blood or adipose tissue and air for aromatic solvents. Scand J Work Environ Health 22:112-118.

Pleil JD, Oliver KD, McClenny WA. 1988. Ambient air analyses using nonspecific flame ionization and electron capture detection compared to specific detection by mass spectrometry. J Air Pollut Control Assoc 38:1006-1010.

Poggi G, Giusiani M, Palagi U, et al. 1982. High-performance liquid chromatography for the quantitative determination of the urinary metabolites of toluene, xylene, and styrene. Int Arch Occup Environ Health 50:25-31.

Pool BL, Lin PZ. 1982. Mutagenicity testing in the Salmonella typhimurium assay of phenolic compounds and phenolic fractions obtained from smokehouse smoke condensates. Food Chem Toxicol 20:383-391.

Poulin P, Krishnan K. 1996a. A tissue composition-based algorithm for predicting tissue: Air partition coefficients of organic chemicals. Toxicol Appl Pharmacol 136:126-130.

Poulin P, Krishnan K. 1996b. A mechanistic algorithm for predicting blood: Air partition coefficients of organic chemicals with the consideration of reversible binding in hemoglobin. Toxicol Appl Pharmacol 136:131-137.

Pound AW. 1970. Induced cell proliferation and the initiation of skin tumor formation in mice by ultraviolet light. Pathology 2:269-275.

Pound AW, Withers HR. 1963. The influence of some irritant chemicals and scarification on tumor initiation by urethane in mice. Br J Cancer 17:460-470.

Pratt GC, Palmer K, Wu CY, et al. 2000. An assessment of air toxics in Minnesota. Environ Health Perspect 108(9):815-825.

Pryor GT, Rebert CS, Howd RA. 1987. Hearing loss in rats caused by inhalation of mixed xylene and styrene. J Appl Toxicol 7:55-61.

Pyykko K. 1980. Effects of methylbenzenes on microsomal enzymes in rat liver, kidney and lung. Biochim Biophys Acta 633:1-9.

Quigley CJ, Corsi RL. 1995. Emissions of VOCs from a municipal sewer. J Air Waste Manage Assoc 45:395-403.

Raaschou-Nielsen O, Lohse C, Thomsen BL, et al. 1997. Ambient air levels and the exposure of children to benzene, toluene, and xylenes in Denmark. Environ Res 75(2):149-159.

Ramarathnam N, Rubin LJ, Diosady LL. 1991. Studies on meat flavor. 2. A quantitative investigation of the volatile carbonyls and hydrocarbons in uncured and cured beef and chicken. J Agric Food Chem 39:1839-1847.

Ramsey JC, Andersen ME. 1984. A physiologically based description of the inhalation pharmacokinetics of styrene in rats and humans. Toxicol Appl Pharmacol 73:159-175. 
Rank J. 1985. Xylene induced feeding and drinking behavior and central adrenergic receptor binding. Neurobehav Toxicol Teratol 7:421-426.

Rankin C, Sacks R. 1991. Computer-controlled, high speed, repetitive gas chromatography system. LCGC 9:428, 430, 432-434.

Ransley DL. 1984. Xylenes and ethylbenzene. In: Grayson M, ed. Kirk-Othmer encyclopedia of chemical technology. Vol. 24, 3rd ed. New York, NY: John Wiley \& Sons, 709-744.

Rasheed A, Hines RN, McCarver-May DG. 1997. Variation in induction of human placental CYP2E1: Possible role in susceptibility to fetal alcohol syndrome? Toxicol Appl Pharmacol 144:396-400.

Rathbun RE. 1998. Transport, behavior, and fate of volatile organic compounds in streams. In: Transport, behavior, and fate of volatile organic compounds in streams. Washington, DC: U.S. Geological Survey Professional Paper 1589.

Rebert CS, Schwartz RW, Svendsgaard DJ, et al. 1995. Combined effect of paired solvents on the rat's auditory system. Toxicology 105:345-354.

Recchia G, Perbellini L, Prati GF, et al. 1985. [Coma due to accidental ingestion of xylene: Treatment with charcoal hemoperfusion.] Med Lav 76:67-73. (Italian)

Reisch MS. 1992. Top 50 chemicals production stagnated last year. Chem Eng News 70:16-22.

Rembold H, Wallner P, Nitz S, et al. 1989. Volatile components of chickpea (Cicer arietinum L.) seed. J Agric Food Chem 37:659-662.

Reusser DE, Istok JD, Beller HR, et al. 2002. In situ transformation of deuterated toluene and xylene to benzylsuccinic acid analogues in BTEX-contamination aquifers. Environ Sci Technol 36:4127-4134.

Richer C-L, Chakrabarti S, Senecal-Quevillon M, et al. 1993. Cytogenetic effects of low-level exposure to toluene, xylene and their mixture on human blood lymphocytes. Int Arch Occup Environ Health 64:581-585.

Riediker M, Williams R, Devlin R, et al. 2003. Exposure to particulate matter, volatile organic compounds, and other sir pollutants inside patrol cars. Environ Sci Technol 37:2084-2093.

*Riihimaki V. 1979a. Conjugation and urinary excretion of toluene and $m$-xylene metabolites in a man. Scand J Work Environ Health 5:135-142.

Riihimaki V. 1979b. Percutaneous absorption of $m$-xylene from a mixture of $m$-xylene and isobutyl alcohol in man. Scand J Work Environ Health 5:143-150.

Riihimaki V, Hanninen O. 1987. Xylenes. In: Browning E. Toxicity and metabolism of industrial solvents. Vol. 1. Amsterdam: Elsevier Science Publishers B.V., 64-84.

Riihimaki V, Pfaffli P. 1978. Percutaneous absorption of solvent vapors in man. Scand J Work Environ Health 4:73-85.

Riihimaki V, Savolainen K. 1980. Human exposure to $m$-xylene: Kinetics and acute effects on the central nervous system. Ann Occup Hyg 23:411-422. 
Riihimaki V, Laine A, Savolainen K, et al. 1982a. Acute solvent-ethanol interactions with special references to xylene. Scand J Work Environ Health 8:77-79.

Riihimaki V, Pfaffli P, Savolainen K. 1979a. Kinetics of $m$-xylene in man: Influence of intermittent physical exercise and changing environmental concentrations on kinetics. Scand J Work Environ Health 5:232-248.

Riihimaki V, Pfaffli P, Savolainen K, et al. 1979b. Kinetics of $m$-xylene in man: General features of absorption, distribution, biotransformation and excretion in repetitive inhalation exposure. Scand J Work Environ Health 5:217-231.

Riihimaki V, Savolainen K, Pfaffli P, et al. 1982b. Metabolic interaction between $m$-xylene and ethanol. Arch Toxicol 49:253-263.

Roberts JM, Fehsenfeld FC, Liu SC, et al. 1984. Measurements of aromatic hydrocarbon ratios and NOx concentrations in the rural troposphere: Observation of air mass photochemical aging and NOx removal. Atmos Environ 18:2421-2432.

Roberts FP, Lucas EG, Marsden CD, et al. 1988. Near-pure xylene causing reversible neuropsychiatric disturbance [Letter]. Lancet 2(8605):273.

Rogers JV, Gunasekar PG, Garrett CM, et al. 2001. Dermal exposure to m-xylene leads to increasing oxidative species and low molecular weight DNA levels in rat skin. J Biochem Mol Toxicol 15(4):228230.

Romer KG, Federsel RJ, Freundt KJ. 1986. Rise of inhaled toluene, ethyl benzene, $m$-xylene, or mesitylene in rat blood after treatment with ethanol. Bull Environ Contam Toxicol 37:874-876.

Romieu I, Ramirez M, Meneses F, et al. 1999. Environmental exposure to volatile organic compounds among workers in Mexico City assessed by personal monitors and blood concentrations. Environ Health Perspect 107:511-515.

Rosen MB, Crofton KM, Chernoff N. 1986. Postnatal evaluation of prenatal exposure to $p$-xylene in the rat. Toxicol Lett 34:223-229.

Rosengren LE, Kjellstrand P, Aurell A, et al. 1986. Irreversible effects of xylene on the brain after long term exposure: A quantitative study of DNA and the glial cell marker proteins S-100 and GFA. Neurotoxicology 7:121-136.

Roy WR. 1994. Groundwater contamination form municipal landfills in the USA. In: Adriano DC, ed. Contamination of groundwaters: Case studies. Northwood, UK: Scientific Review, 411-446.

RTECS. 2007. Xylene. Registry of Toxic Effects on Chemical Substances. National Institute of Occupational Safety and Health. MDL Information Systems, Inc. May 14, 2007.

*Rumsey TS, Cabell CA, Bond J. 1969. Effects of an organic phosphorus systemic insecticide on reproductive performance in rats. Am J Vet Res 30: 2209-2214. 


\section{REFERENCES}

Rydzynski K, Korsak Z, Jedlinska U, et al. 1992. The toxic effects of combined exposure to toluene and $m$-xylene in animals. IV. Liver ultrastructure after subchronic inhalatory exposure. Polish J Occup Med Environ Health 5(1):35-42.

Sack TM, Steel DH, Hammerstrom K, et al. 1992. A survey of household products for volatile organiccompounds. Atmos Environ A Gen Top 26:1063-1070.

Saillenfait AM, Gallissot F, Morel G, et al. 2003. Developmental toxicities of ethylbenzene, ortho-, meta-, para-xylene and technical xylenes in rats following inhalation exposure. Food Chem Toxicol 41:415-429.

Sanborn HR, Malins DC. 1980. The disposition of aromatic hydrocarbons in adult spot shrimp (Pandalus piatyceros) and the formation of metabolites of naphthalene in adult and larval spot shrimp. Xenobiotica 10:193-200.

Sandmeyer EE. 1981. Aromatic hydrocarbons. In: Clayton GD, Clayton FE, eds. Patty's industrial hygiene and toxicology. Vol. 2B. 3rd ed. New York, NY: John Wiley \& Sons, 3253-3431.

Sanemasa I, Araki M, Deguchi T, et al. 1982. Solubility measurements of benzene and the alkylbenzenes in water by making use of solute vapor. Bull Chem Soc Jpn 55:1054-1062.

Sato A. 1988. Toxicokinetics of benzene, toluene and xylenes. IARC Sci Publ (85):47-64.

Sato A, Nakajima T. 1979. Partition coefficients of some aromatic hydrocarbons and ketones in water, blood and oil. Br J Ind Med 36:231-234.

Sauer TJ, Sackett WM, Jeffrey LM. 1978. Volatile liquid hydrocarbons in the surface coastal waters of the Gulf of Mexico. Marine Chem 7:1-16.

Saunders RA, Blachly CH, Kovacina TA, et al. 1975. Identification of volatile organic contaminants in Washington, D.C. municipal water. Water Res 9:1143-1145.

Savolainen K. 1980. Combined effects of xylene and alcohol on the central nervous system. Acta Pharmacol Toxicol 46:366-372.

Savolainen K, Linnavuo M. 1979. Effects of $m$-xylene on human equilibrium measured with a quantitative method. Acta Pharmacol Toxicol 44:315-318.

Savolainen H, Pfaffli P. 1980. Dose-dependent neurochemical changes during short-term inhalation exposure to $m$-xylene. Arch Toxicol 45:117-122.

Savolainen H, Seppalainen AM. 1979. Biochemical and physiological effects of organic solvents on rat axon membranes isolated by a new technique. Neurotoxicology 1:467-477.

Savolainen K, Riihimaki V. 1981a. An early sign of xylene effect on human equilibrium. Acta Pharmacol Toxicol 48:279-283.

Savolainen K, Riihimaki V. 1981b. Xylene and alcohol involvement of the human equilibrium system. Acta Pharmacol Toxicol 49:447-451. 
Savolainen H, Pfaffli P, Helojoki M, et al. 1979a. Neurochemical and behavioral effects of long-term intermittent inhalation. Acta Pharmacol Toxicol 44:200-207.

Savolainen H, Vainio H, Helojoki M, et al. 1978. Biochemical and toxicological effects of short-term, intermittent xylene inhalation exposure and combined ethanol intake. Arch Toxicol 41:195-205.

Savolainen K, Kekoni J, Riihimaki V, et al. 1984. Immediate effects of $m$-xylene on the human central nervous system. Arch Toxicol 7(Suppl):412-417.

Savolainen K, Riihimaki V, Laine A. 1982a. Biphasic effects of inhaled solvents on human equilibrium. Acta Pharmacol Toxicol 51:237-242.

Savolainen K, Riihimaki V, Laine A, et al. 1982b. Short-term exposure of human subjects to $m$-xylene and 1,1,1-trichloroethane. Arch Toxicol 5(Suppl):96-99.

Savolainen K, Riihimaki V, Linnoila M. 1979b. Effects of short-term xylene exposure on psychophysiological functions in man. Int Arch Occup Environ Health 44:201-211.

Savolainen K, Riihimaki V, Luukkonen R, et al. 1985a. Changes in the sense of balance correlate with concentrations of $m$-xylene. Br J Ind Med 42:765-769.

*Savolainen K, Riihimaki V, Muona O, et al. 1985b. Conversely exposure-related effects between atmospheric $m$-xylene concentrations and human body sense of balance. Acta Pharmacol Toxicol 57:6771.

Savolainen K, Riihimaki V, Seppalainen AM, et al. 1980a. Effects of short-term m-xylene exposure and physical exercise on the central nervous system. Int Arch Occup Environ Health 45:105-121.

Savolainen K, Riihimaki V, Vaheri E, et al. 1980b. Effects of xylene and alcohol on vestibular and visual functions in man. Scand J Work Environ Health 6:94-103.

Schauer J, Kleeman MJ, Cass GR, et al. 1999. Measurement of emissions from air pollution sources. 2. C1 through C29 organic compounds from medium duty diesel trucks. Environ Sci Technol 33:15781587.

Schauer JJ, Kleeman MJ, Cass GR, et al. 2001. Measurement of emissions from air pollution sources 3 C1-C29 organic compounds for fireplace combustion of wood. Environ Sci Technol 35:1716-1728.

Schauer JJ, Kleeman MJ, Cass GR, et al. 2002. Measurement of emissions from air pollution sources. $\mathrm{C}_{1}-\mathrm{C}_{32}$ organic compounds from gasoline-powered motor vehicles. Environ Sci Technol 36:1169-1180.

*Sedivec V, Flek J. 1976a. Exposure test for xylenes. Int Arch Occup Environ Health 37:219-232.

Sedivec V, Flek J. 1976b. The absorption, metabolism, and excretion of xylenes in man. Int Arch Occup Environ Health 37:205-217.

Seidenberg JM, Anderson DG, Becker RA. 1986. Validation of an in vivo developmental toxicity screen in the mouse. Teratog Carcinog Mutagen 6:361-374.

Seifert B, Abraham H-J. 1982. Indoor air concentrations of benzene and some other aromatic hydrocarbons. Ecotoxicol Environ Saf 6:190-192. 


\section{REFERENCES}

Seip HM, Alstad J, Carlberg GE, et al. 1986. Measurement of mobility of organic compounds in soils. Sci Total Environ 50:87-101.

Selgrade MJ, Daniels MJ, Jaskot RH, et al. 1993. Enhanced mortality and liver damage in virus-infected mice exposed to $p$-xylene. J Toxicol Environ Health 40:129-144.

Senczuk W, Orlowski J. 1978. Absorption of $m$-xylene vapours through the respiratory track and excretion. Br J Ind Med 35:50-55.

Seppalainen AM, Laine A, Salmi T, et al. 1989. Changes induced by short-term xylene exposure in human evoked potentials. Int Arch Environ Health 61:443-449.

Seppalainen AM, Laine A, Salmi T, et al. 1991. Electroencephalographic findings during experimental human exposure to meta-xylene. Arch Environ Health 46:16-24.

Seppalainen AM, Salmi T, Savolainen K, et al. 1983. Visual evoked potentials in short-term exposure of human subjects to $m$ - xylene and 1,1,1-trichloroethane. Appl Behav Pharmacol Toxicol 1983:349-352.

*Seppalainen AM, Savolainen K, Kovala T. 1981. Changes induced by xylene and alcohol in human evoked potentials. Electroencephalogr Clin Neurophysiol 51:148-155.

Setchell BP, Waites GMH. 1975. The blood-testis barrier. In: Creep RO, Astwood EB, Geiger SR, eds. Handbook of physiology: Endocrinology V. Washington, DC: American Physiological Society.

Sevcik P, Hep A, Peslova M. 1992. Intravenous xylene poisoning. Intensive Care Med 18:377-378.

Sexton K, Adgate JL, Fredrickson AL, et al. 2006. Using biologic markers in blood to assess exposure to multiple environmental chemicals for inner-city children 3-6 years of age. Environ Health Perspect 114(3):453-459.

Shepson PB, Edney EO, Corse EW. 1984. Ring fragmentation reactions on the photooxidations of toluene and $o$-xylene. J Phys Chem 88:4122-4126.

Shields HC, Fleischer DM, Weschler CJ. 1996. Comparisons among VOCs measured in three types of U.S. commercial buildings with different occupant densities. Indoor Air 6:2-17.

Shimizu H, Suzuki Y, Takemura N, et al. 1985. The results of microbial mutation test for forty-three industrial chemicals. Jpn J Ind Health 27:400-419.

Silverman DM, Schatz RA. 1991. Pulmonary microsomal alterations following short-term low-level inhalation of para-xylene in rats. Toxicology 65:271-281.

Simmons JE, Allis JW, Grose EC, et al. 1991. Assessment of the hepatotoxicity of acute and short-term exposure to inhaled $p$-xylene in F-344 rats. J Toxicol Environ Health 32:295-306.

Singh HB, Salas LJ, Cantrell Bk, et al. 1985. Distribution of aromatic hydrocarbons in the ambient air. Atmos Environ 11:1911-1919.

Singh M. 2006. Written communication (August 29, 2006) to Margaret Fransen, Syracuse Research Corporation, regarding ortho-xylene.

Skender LJ, Karacic V, Bosner B. 1993. A selection of biological indicators in occupational exposure to toluene and xylene. Arch Ind Hyg Toxicol 44:27-33. 
Skowronski GA, Turkall RM, Kadry AR, et al. 1990. Effects of soil on the dermal bioavailability of $m$ xylene in male rats. Environ Res 51:182-193.

Smith BR, Plummer JL, Wolf CR, et al. 1982. $p$-Xylene metabolism by rabbit lung and liver and its relationship to the selective destruction of pulmonary cytochrome P-450. J Pharmacol Exp Ther 223:736742.

Smolik R, Grzybek-Hryncewicz K, Lange A, et al. 1973. Serum complement level in workers exposed to benzene, toluene and xylene. Int Arch Arbeitsmed 31:243-247.

Smyth HJ, Carpenter CP, Weil CS, et al. 1962. Range-finding toxicity data: List VI. Am Ind Hyg Assoc J 23:95-107.

Sollinger S, Levsen K, Wunsch G. 1994. Indoor pollution by organic emissions from textile floor coverings: Climate test chamber studies under static conditions. Atmos Environ 28:2369-2378.

Sovik AK, Alfnes E, Breedveld GD, et al. 2002. Transport and degradation of toluene and o-xylene in an unsaturated soil with dipping sedimentary layers. J Environ Qual 31:1809-1823.

Spicer CW, Buxton BE, Holdren MW, et al. 1996. Variability of hazardous air pollutants in an urban area. Atmos Environ 30:3443-3456.

Spirtas R, Stewart PA, Lee JS, et al. 1991. Retrospective cohort mortality study of workers at an aircraft maintenance facility. I. Epidemiological results. Br J Ind Med 48:515-530.

Squillace PJ, Moran MJ, Lapman WW, et al. 1999. Volatile organic compounds in untreated ambient groundwater of the United States, 1985-1995. Environ Sci Technol 33(23):4176-4187.

SRI. 2006. Directory of chemical producers: United States of America. Menlo Park, CA: SRI Consulting.

Stefaniak AB, Breysse PN, Murray MPM, et al. 2000. An evaluation of employee exposure to volatile organic compounds in three photocopy centers. Environ Res 83:162-173.

Stewart PA, Lee JS, Marano DE, et al. 1991. Retrospective cohort mortality study of workers at an aircraft maintenance facility. II. Exposures and their assessment. Br J Ind Med 48:531-537.

Stickney JA, Roberts AE, Silverman DM, et al. 1989. The effect of $m$-xylene on rat lung benzo[a]pyrene metabolism and microsomal membrane lipids: Comparison with p-xylene. Toxicology 58:155-165.

Stickney JA, Silverman DM, Schatz RA. 1991. Role of isozyme-specific inhibition of cytochromeP450IIB1 activity in meta-xylene-induced alterations in rat pulmonary benzo(a)pyrene metabolism. Xenobiotica 21:641-649.

Storm DL. 1994. Chemical monitoring of California's public drinking water sources: Public exposures and health impacts. In: Wang RGM, ed. Water contamination and health: Integration of exposure assessment, toxicology, and risk assessment. New York: Marcel Dekker, Inc., 67-124.

Stutz DR, Janusz SJ. 1988. Hazardous materials injuries: A handbook for pre-hospital care. 2nd ed. Beltsville, MD: Bradford Communications Corporation, 298-299. 
Sugihara R, Ogata M. 1978. Quantitation of urinary $m$ - and $p$-methylhippuric acids as indices of $m$ - and p-xylene exposure. Int Arch Occup Environ Health 41:281-286.

Swann RL, Laskowski DA, McCall PJ, et al. 1983. A rapid method for the estimation of the environmental parameters octanol/water partition coefficient, soil sorption constant, water to air ration, and water solubility. Residue Rev 85:17-28.

Sweet CW, Vermette SJ. 1992. Toxic volatile organic compounds in urban air in Illinois. Environ Sci Technol 26:165-173.

Swiercz R, Lorsak Z, Rydzynski K. 1995. Kinetics of n-butyl alcohol and m-xylene in blood during single and combined inhalation exposure in rats. Int J Occup Med Environ Health 8(4):361-365.

Szybalski W. 1958. Special microbiological systems: II. Observations on chemical mutagenesis in microorganisms. Ann NY Acad Sci 76:465-489.

Takagi H, Washida N, Akimoto H, et al. 1980. Photooxidation of $o$-xylene in the $\mathrm{NO}-\mathrm{H}_{2} \mathrm{O}$-air system. J Phys Chem 84:478-483.

Tahti H. 1992. The neurotoxicity of organic-solvents, studied with in vitro models. Altern Lab Anim 20:290-296.

Takeoka GR, Flath RA, Guntert M, et al. 1988. Nectarine volatiles: Vacuum steam distillation versus headspace sampling. J Agric Food Chem 36:553-560.

Takeuchi A, Kawai T, Zhang ZW, et al. 2002. Toluene, xylenes and xylene isomers in urine as biological indicators of low-level exposure to each solvent; a comparative study. Int Arch Occup Environ Health 75:387-393.

Tanaka M, Shimizu M, Tsujimoto Y. 1990. High-performance liquid chromatographic determination of urinary mercapturic acids in rat. J Chromatogr 526:289-292.

Tang J, Jin QZ, Shen G-H, et al. 1983. Isolation and identification of volatile compounds from fried chicken. J Agric Food Chem 31:1287-1292.

Tansel B, Eyma RR. 1999. Volatile organic contaminant emissions from wastewater treatment plants during secondary treatments. Water Air Soil Pollut 112:315-325.

Tardif R, Charest-Tardif G. 1999. The importance of measured end-points in demonstrating the occurrence of interactions: A case study with methylchloroform and m-xylene. Toxicol Sci 49:312-317.

Tardif R, Brodeur J, Plaa GL. 1989. Simultaneous high-performance liquid chromatographic analysis of hippuric acid and ortho-, meta-, and para-methylhippuric acids in urine. J Anal Toxicol 13:313-316.

Tardif R, Charest-Tardif G, Brodeur J. 1996. Comparison of the influence of binary mixtures versus a ternary mixture of inhaled aromatic hydrocarbons on their blood kinetics in the rat. Arch Toxicol 70:405413.

Tardif R, Charest-Tarif G, Brodeur J, et al. 1997. Physiologically based pharmacokinetic modeling of a ternary mixture of alkyl benzenes in rats and humans. Toxicol Appl Pharmacol 144:120-134. 
Tardif R, Lapar S, Charest-Tardif G, et al. 1995. Physiologically-based pharmacokinetic modeling of a mixture of toluene and xylene in humans. Risk Anal 15(3):335-342.

Tardif R, Lapare S, Krishnan K, et al. 1993a. Physiological based modeling of the toxicokinetic interaction between toluene and $m$-xylene in the rat. Toxicol Appl Pharmacol 120:266-273.

Tardif R, Lapare S, Krishnan K, et al. 1993b. A descriptive and mechanistic study of the interaction between toluene and xylene in humans. Int Arch Occup Environ Health, 65:S135-S137.

Tardif R, Lapare S, Plaa GL, et al. 1991. Effect of simultaneous exposure to toluene and xylene on their respective biological exposure indices in humans. Int Arch Occup Environ Health 63:279-284.

Tardif R, Plaa GL, Brodeur J. 1992. Influence of various mixtures of inhaled toluene and xylene on the biological monitoring of exposure to these solvents in rats. Can J Physiol Pharmacol 70:385-393.

Tardif R, Sato A, Lapare S, et al. 1994. Ethanol induced modification of $m$-xylene toxicokinetics in humans. Occup Environ Med 51:187-191.

Taskinen H, Anttila A, Lindbohm ML, et al. 1989. Spontaneous abortions and congenital malformations among the wives of men occupationally exposed to organic solvents. Scand J Work Environ Health $15: 345-352$.

Taskinen H, Kyyronen P, Hemminki K, et al. 1994. Laboratory work and pregnancy outcome. J Occup Med 36(3):311-319.

Tassaneeyakul W, Birkett DJ, Edwards JW, et al. 1996. Human cytochrome P450 isoform specificity in the regioselective metabolism of toluene and o-, m- and p-xylene. J Pharmacol Exp Ther 276(1):101-108.

Tatrai E, Ungvary G. 1980. Changes induced by $o$-xylene inhalations in the rat liver. Acta Med Acad Sci Hung 37:211-216.

Tatrai E, Ungvary G, Cseh IR, et al. 1981. The effects of long-term inhalation of ortho-xylene on the liver. Ind Enviv Xenobiotica, Proceedings of International Conference, Prague, Czechoslovakia, May 2730, 1980. New York, NY: Springer-Verlag, 293-300.

Teschke K, Ahrens W, Andersen A, et al. 1999. Occupational exposure to chemical and biological agents in the nonproduction departments of pulp, paper, and paper product mills: An international study. Am Ind Hyg Assoc J 60:73-83.

Thierrin J, David GB, Barber C. 1995. A ground-water tracer test with deuterated compounds for monitoring in situ biodegradation and retardation of aromatic hydrocarbons. Ground Water 33(3):469475.

Thomas K, Colborn T. 1992. Organochlorine endocrine disruptors in human tissue. In: Colborn T, Clement $\mathrm{C}$, eds. Chemically induced alterations in sexual and functional development: The wildlife/human connection. Princeton, NJ: Princeton Scientific Publishing, 365-394.

Thrall KD, Gies RA, Muniz J, et al. 2002. Route-of-entry and brain tissue partition coefficients for common superfund contaminants. J Toxicol Environ Health A 65(24):2075-2086. 


\section{REFERENCES}

Thrall KD, Poet TS, Corley RA, et al. 2000. A real-time in-vivo method for studying the percutaneous absorption of volatile chemicals. Int J Occup Environ Health 6(2):96-103.

Thrall KD, Woodstock AD. 2003. Evaluation of the dermal bioavailability of aqueous xylene in F344 rats and human volunteers. J Toxicol Environ Health A 66:1267-1281.

Toftgard R, Gustafsson J-A. 1980. Biotransformation of organic solvents: A review. Scand J Work Environ Health 6:1-18.

Toftgard R, Nilsen OG. 1981. Induction of cytochrome P-450 in rat liver after inhalation of aromatic organic solvents. In: Industrial and environmental xenobiotics: Metabolism and pharmacokinetics of organic chemicals and metals: Proceedings of International Conference, Prague, Czechoslovakia, May 27-30, 1980. New York, NY: Springer-Verlag, 307-317.

Toftgard R, Nilsen OG. 1982. Effects of xylene and xylene isomers on cytochrome P-450 and in vitro enzymatic activities in rat liver, kidney, and lung. Toxicology 23:197-212.

Toftgard R, Nilsen OG, Gustafsson J-A. 1981. Changes in rat liver microsomal cytochrome P-450 and enzymatic activities after the inhalation of n-hexane, xylene, methyl ethyl ketone and methylchloroform for four weeks. Scand J Work Environ Health 7:31-37.

Tran NK, Steinberg SM, Johnson BJ. 2000. Volatile aromatic hydrocarbons and dicarboxylic acid concentrations in air at an urban site in the southwestern US. Atmos Environ 34:1845-1852.

TRI04. 2006. TRI explorer: Providing access to EPA's toxics release inventory data. Washington, DC: Office of Information Analysis and Access. Office of Environmental Information. U.S. Environmental Protection Agency. Toxics Release Inventory. http://www.epa.gov/triexplorer/. July 10, 2006.

Triebig G, Barocka A, Erbguth F, et al. 1992a. Neurotoxicity of solvent mixtures in spray painters. II. Neurologic, psychological, and neuroradiologic findings. Int Arch Occup Environ Health 64:361-372.

Triebig G, Schaller KH, Weltle D. 1992b. Neurotoxicity of solvent mixtures in spray painters. I. Study, design, workplace exposure, and questionnaire. Int Arch Occup Environ Health 64(5):353-359.

Trost B, Stutz J, Platt U. 1997. UV-absorption cross sections of a series of monocylic aromatic compounds. Atmos Environ 31(23):3999-4008.

Tsao CW, Song HG, Bartha AR. 1998. Metabolism of benzene, toluene, and xylene hydrocarbons in soil. Appl Environ Microbiol 64:4924-4929.

Tsuruta H. 1982. Percutaneous absorption of organic solvents: III. On the penetration rates. Ind Health 20:335-345.

Tsuruta H, Iwasaki K. 1984. A procedure for determining volatile solvents in mouse whole body. Ind Health 22:219-222.

Tuazon EC, McLeod H, Atkinson R, et al. 1986. $\alpha$-Dicarbonyl yields from the NOx-air photooxidation of a series of aromatic hydrocarbons in air. Environ Sci Technol 20:383-393.

Turkall RM, Skowronski GA, Kadry ARM, et al. 1992. Sex-differences in the bioavailability of soiladsorbed meta-xylene in orally exposed rats. Toxicol Lett 63:57-67. 


\section{REFERENCES}

Uchida Y, Nakatsuka H, Ukai H, et al. 1993. Symptoms and signs in workers exposed predominantly to xylenes. Int Arch Occup Environ Health 64:597-605.

Ungvary G. 1985. The possible contributions of industrial chemicals (organic solvents) to the incidence of congenital defects caused by teratogenic drugs and consumer goods-an experimental study. Prog Clin Biol Res 160:295-300.

Ungvary G. 1990. The effect of xylene exposure on the liver. Acta Morphol Hung 38:245-258.

Ungvary G, Tatrai E. 1985. On the embryotoxic effects of benzene and its allkyl derivatives in mice, rats, and rabbits. Arch Toxicol (Supplement) 8:425-430.

Ungvary G, Cseh J, Manyai S, et al. 1980a. Enzyme induction by o-xylene inhalation. Acta Med Acad Sci Hung 37:115-120.

Ungvary G, Tatrai E, Hudak A, et al. 1980b. Studies on the embryotoxic effects of ortho-, meta- and para-xylene. Toxicology 18:61-74.

Ungvary G, Varga B, Horvath E, et al. 1981. Study on the role of maternal sex steroid production and metabolism in the embryotoxicity of para-xylene. Toxicology 19:263-268.

USDOC. 2005. U.S. Trade quick reference tables. U.S. Department of Commerce, Bureau of the Census, Foreign Trade Division. http://www.ita.doc.gov/td/industry/otea/Trade-Detail/. January 23, 2007.

USITC. 1991. Synthetic organic chemicals: United States production and sales, 1990. Washington, DC: U.S. International Trade Commission. USITC Publication 2470.

Vaidyanathan A, Foy JWD, Schats RA. 2003. Inhibition of rat respiratory-tract cytochrome P-450 isozymes following inhalation of m-xylene: Possible role of metabolites. J Toxicol Environ Health A 66(12):1133-1143.

van Doorn R, Bos RP, Brouns RME, et al. 1980. Effect of toluene and xylenes on liver glutathione and their urinary excretion as mercapturic acids in the rat. Arch Toxicol 43:293-304.

VCCEP. 2006. Report of the peer consultation meeting on xylenes. Erlanger, KY: American Chemistry Council Benzene, Toluene, and Xylenes Consortium for the Volunary Children's Chemical Evaluation Program. http://www.tera.org/peer/vccep. October 01, 2006.

Verschueren K. 2001. Xylene. Handbook of environmental data on organic chemicals. New York, NY: John Wiley \& Sons, Inc., 2189-2195.

Vieira I, Sonnier M, Cresteil T. 1996. Developmental expression of CYP2E1 in the human liver: Hypermethylation control of gene expression during the neonatal period. Eur J Biochem 238:476-483.

Vincent R, Poirot P, Subra I, et al. 1994. Occupational exposure to organic solvents during paint stripping and painting operations in the aeronautical industry. Int Arch Occup Environ Health 65:377380. 
Vodickova L, Frantik E, Vodickova A. 1995. Neutrotropic effects and blood levels of solvents at combined exposures: Binary mixtures of toluene, o-xylene and acetone in rats and mice. Cent Eur J Public Health 3(2):57-64.

Von Burg R. 1982. Toxicology updates: Xylene. J Appl Toxicol 2:269-271.

Wallace L, Nelson W, Ziegenfus R, et al. 1991. The Los Angeles team study: Personal exposures, indoor-outdoor air concentrations, and breath concentrations of 25 volatile organic compounds. J Exposure Anal Environ Epidemiol 1:157-142.

Wallace L, Pellizzari E, Hartwell T, et al. 1986. Concentrations of 20 volatile organic compounds in the air and drinking water of 350 residents of New Jersey compared with concentrations in their exhaled breath. J Occup Med 28:603-608.

Wallace L, Pellizzari E, Hartwell T, et al. 1987a. Exposures to benzene and other volatile compounds from active and passive smoking. Arch Environ Health 42:272-279.

Wallace L, Pellizzari E, Hartwell T, et al. 1987b. The TEAM study: Personal exposures to toxic substances in air, drinking water, and breath of 400 residents of New Jersey, North Carolina, and North Dakota. Environ Res 43:290-307.

Wallace L, Pellizzari E, Hartwell T, et al. 1988. The California team study: Breath concentrations and personal exposures to 26 volatile compounds in air and drinking water of 188 residents of Los Angeles, Antioch, and Pittsburg, CA. Atmos Environ 22:2141-2163.

Wallen M, Holm S, Byfalt Nordqvist M. 1985. Coexposure to toluene and $p$-xylene in man: Uptake and elimination. Br J Ind Med 42:111-116.

Wang W, Liszewski M, Buchmiller R. 1995. Occurrence of active and inactive herbicide ingredients at selected sites in Iowa. Water Air Soil Pollut 83:21-35.

Washington WJ, Murthy RC, Doye A, et al. 1983. Induction of morphologically abnormal sperm in rats exposed to o-xylene. Arch Androl 11:233-237.

Weidemeier TH, Swanson MA, Wilson JT, et al. 1995. Patterns of intrinsic bioremediation at two U.S. Air Force Bases. In: Hinchee RE, eds. Intrinsic bioremediation. Columbus, OH: Battelle Press, 31-51.

Weschler CJ, Shields HC, Rainer D. 1990. Concentrations of volatile organic compounds at a building with health and comfort complaints. Am Ind Hyg Assoc J 51:261-268.

West JR, Smith HW, Chasis H. 1948. Glomerular filtration rate, effective renal blood flow, and maximal tubular excretory capacity in infancy. J Pediatr 32:10-18.

Whitehead LW, Ball GL, Fine LJ, et al. 1984. Solvent vapor exposures in booth spray painting and spray glueing, and associated operations. Am Ind Hyg Assoc J 45:767-772.

Whitman NE, Johnston AE. 1964. Sampling and analysis of aromatic vapors in air: A gas-liquid chromatographic method. Am Ind Hyg Assoc J 25:464-469.

WHO. 2000. Air quality guidelines. 2nd ed. Geneva, Switzerland: World Health Organization. http://www.euro.who.int/air/Activities/20050104_1. February 15, 2005. 
WHO. 2004. Guidelines for drinking-water quality. 3rd ed. Geneva, Switzerland: World Health Organization. http://www.who.int/water_sanitation_health/dwq/gdwq3/en/. February 15, 2005.

Widdowson EM, Dickerson JWT. 1964. Chemical composition of the body. In: Comar CL, Bronner F, eds. Mineral metabolism: An advanced treatise. Volume II: The elements Part A. New York: Academic Press.

Wilcosky TC, Checkoway H, Marshall EG, et al. 1984. Cancer mortality and solvent exposures in the rubber industry. Am Ind Hyg Assoc J 45:809-811.

Williams DT, Nestmann ER, LeBel GL, et al. 1982. Determination of mutagenic potential and organic contaminants of Great Lakes drinking water. Chemosphere 11:263-276.

Wilson JT, Kampbell DH, Armstrong J. 1994. Natural bioreclamation of alkylbenzenes (BTEX) from a gasoline spill in methanogenic groundwater. In: Hinchee RE, ed. Hydrocarbon bioremediation. Boca Raton, FL: Lewis Publishers, 210-218.

Wilson SC, Jones KC. 1996. The fate and behavior of volatile aromatic hydrocarbons in sewage sludgeamended soil. In: Schnoor J, Doi J, eds. Volatile organic compounds in the environment. West Conshahocken, PA: American Society for Testing and Materials, 119-123.

Wilson SC, Alcock RE, Sewart AP, et al. 1997. Organic chemicals in the environment: Persistence of organic contaminants in sewage sludge-amended soil: A field experiment. J Environ Qual 26:1467-1477.

Wimolwattanapun S, Ghosh TK, Mookherjee S, et al. 1987. Effect of inhalation of xylene on intracranial self-stimulation behavior in rat. Neuropharmacology 26:1629-1632.

Windham GC, Shusterman D, Swan SH, et al. 1991. Exposure to organic solvents and adverse pregnancy outcome. Am J Ind Med 20:241-259.

Wisniewska-Knypl JM, Wronska-Nofer T, Jajte J, et al. 1989. The effect of combined exposures to ethanol and xylene on rat hepatic microsomal monooxygenase activities. Alcohol 6:347-352.

Wolf MA, Rowe VK, McCollister DD, et al. 1956. Toxicological studies of certain alkylated benzenes and benzene: Experiments on laboratory animals. Arch Ind Health 14:387-398.

Wolfe GW. 1988a. Subchronic toxicity study in rats with $m$-xylene. Rockville MD: Dynamac Corporation.

Wolfe GW. 1988b. Subchronic toxicity study in rats with $p$-xylene. Rockville MD: Dynamac Corporation.

Wronska-Nofer T, Rosin J, Bartosz G. 1991. Interaction of ethanol and xylene in their effects on erythrocytes and other hematological parameters in the rat. J Appl Toxicol 11:289-292.

Wu CM, Liou SE. 1992. Volatile components of water-boiled duck meat and Cantonese style roasted duck. J Agric Food Chem 40:838-841.

Yalkowsky SH, He Y. 1992. Handbook of aqueous solubility data. New York, NY: CRC Press, 490493. 
Zabiegala B, Namiesnik J, Przyk E, et al. 1999. Changes in concentration levels of selected VOCs in newly erected and remodeled buildings in Gdansk. Chemosphere 39(12):2035-2046.

Ziegler EE, Edwards BB, Jensen RL, et al. 1978. Absorption and retention of lead by infants. Pediatr Res 12:29-34.

Zhong B, Baozhen, Tang, et al. 1980. A comparative study of the cytogenetic effects of benzene, toluene and xylene. Hereditas 2(2): 29-31.

Zielinska B, Fung KK. 1994. The composition and concentration of hydrocarbons in the range of C2 to C18 emitted from motor vehicles. Sci Total Environ 146/147:281-288.

Zielinska B, Fujita E, Sagebiel J, et al. 1998. Arizona hazardous air pollutants monitoring program. J Air Waste Manage Assoc 48:1038-1050.

Zimmermann R, Blumenstock M, Heger HJ, et al. 2001. Emission of nonchlorinated and chlorinated aromatics in the flue gas of incineration plants during and after transient disturbances of combustion conditions: Delayed emission effects. Environ Sci Technol 35:1019-1030. 
9. REFERENCES

This page is intentionally blank. 


\section{GLOSSARY}

Absorption-The taking up of liquids by solids, or of gases by solids or liquids.

Acute Exposure-Exposure to a chemical for a duration of 14 days or less, as specified in the Toxicological Profiles.

Adsorption-The adhesion in an extremely thin layer of molecules (as of gases, solutes, or liquids) to the surfaces of solid bodies or liquids with which they are in contact.

Adsorption Coefficient $\left(\mathbf{K}_{\mathbf{o c}}\right)$ - The ratio of the amount of a chemical adsorbed per unit weight of organic carbon in the soil or sediment to the concentration of the chemical in solution at equilibrium.

Adsorption Ratio (Kd) - The amount of a chemical adsorbed by sediment or soil (i.e., the solid phase) divided by the amount of chemical in the solution phase, which is in equilibrium with the solid phase, at a fixed solid/solution ratio. It is generally expressed in micrograms of chemical sorbed per gram of soil or sediment.

Benchmark Dose (BMD) - Usually defined as the lower confidence limit on the dose that produces a specified magnitude of changes in a specified adverse response. For example, a BMD 10 would be the dose at the $95 \%$ lower confidence limit on a $10 \%$ response, and the benchmark response (BMR) would be $10 \%$. The BMD is determined by modeling the dose response curve in the region of the dose response relationship where biologically observable data are feasible.

Benchmark Dose Model-A statistical dose-response model applied to either experimental toxicological or epidemiological data to calculate a BMD.

Bioconcentration Factor (BCF) - The quotient of the concentration of a chemical in aquatic organisms at a specific time or during a discrete time period of exposure divided by the concentration in the surrounding water at the same time or during the same period.

Biomarkers-Broadly defined as indicators signaling events in biologic systems or samples. They have been classified as markers of exposure, markers of effect, and markers of susceptibility.

Cancer Effect Level (CEL) - The lowest dose of chemical in a study, or group of studies, that produces significant increases in the incidence of cancer (or tumors) between the exposed population and its appropriate control.

Carcinogen-A chemical capable of inducing cancer.

Case-Control Study - A type of epidemiological study that examines the relationship between a particular outcome (disease or condition) and a variety of potential causative agents (such as toxic chemicals). In a case-controlled study, a group of people with a specified and well-defined outcome is identified and compared to a similar group of people without outcome.

Case Report-Describes a single individual with a particular disease or exposure. These may suggest some potential topics for scientific research, but are not actual research studies.

Case Series-Describes the experience of a small number of individuals with the same disease or exposure. These may suggest potential topics for scientific research, but are not actual research studies. 
Ceiling Value-A concentration of a substance that should not be exceeded, even instantaneously.

Chronic Exposure-Exposure to a chemical for 365 days or more, as specified in the Toxicological Profiles.

Cohort Study-A type of epidemiological study of a specific group or groups of people who have had a common insult (e.g., exposure to an agent suspected of causing disease or a common disease) and are followed forward from exposure to outcome. At least one exposed group is compared to one unexposed group.

Cross-sectional Study-A type of epidemiological study of a group or groups of people that examines the relationship between exposure and outcome to a chemical or to chemicals at one point in time.

Data Needs-Substance-specific informational needs that if met would reduce the uncertainties of human health assessment.

Developmental Toxicity-The occurrence of adverse effects on the developing organism that may result from exposure to a chemical prior to conception (either parent), during prenatal development, or postnatally to the time of sexual maturation. Adverse developmental effects may be detected at any point in the life span of the organism.

Dose-Response Relationship-The quantitative relationship between the amount of exposure to a toxicant and the incidence of the adverse effects.

Embryotoxicity and Fetotoxicity-Any toxic effect on the conceptus as a result of prenatal exposure to a chemical; the distinguishing feature between the two terms is the stage of development during which the insult occurs. The terms, as used here, include malformations and variations, altered growth, and in utero death.

Environmental Protection Agency (EPA) Health Advisory-An estimate of acceptable drinking water levels for a chemical substance based on health effects information. A health advisory is not a legally enforceable federal standard, but serves as technical guidance to assist federal, state, and local officials.

Epidemiology-Refers to the investigation of factors that determine the frequency and distribution of disease or other health-related conditions within a defined human population during a specified period.

Genotoxicity-A specific adverse effect on the genome of living cells that, upon the duplication of affected cells, can be expressed as a mutagenic, clastogenic, or carcinogenic event because of specific alteration of the molecular structure of the genome.

Half-life-A measure of rate for the time required to eliminate one half of a quantity of a chemical from the body or environmental media.

Immediately Dangerous to Life or Health (IDLH) - The maximum environmental concentration of a contaminant from which one could escape within 30 minutes without any escape-impairing symptoms or irreversible health effects.

Immunologic Toxicity-The occurrence of adverse effects on the immune system that may result from exposure to environmental agents such as chemicals. 
Immunological Effects-Functional changes in the immune response.

Incidence-The ratio of individuals in a population who develop a specified condition to the total number of individuals in that population who could have developed that condition in a specified time period.

Intermediate Exposure-Exposure to a chemical for a duration of 15-364 days, as specified in the Toxicological Profiles.

In Vitro-Isolated from the living organism and artificially maintained, as in a test tube.

In Vivo-Occurring within the living organism.

Lethal Concentration $(\mathbf{L O})\left(\mathbf{L C}_{\mathbf{L O}}\right)$ - The lowest concentration of a chemical in air that has been reported to have caused death in humans or animals.

Lethal Concentration $\mathbf{( 5 0 )}_{(}\left(\mathbf{L C}_{50}\right)$ - A calculated concentration of a chemical in air to which exposure for a specific length of time is expected to cause death in $50 \%$ of a defined experimental animal population.

Lethal Dose $(\mathbf{L O})\left(\mathbf{L D}_{\mathbf{L o}}\right)$ - The lowest dose of a chemical introduced by a route other than inhalation that has been reported to have caused death in humans or animals.

Lethal Dose $\mathbf{( 5 0 )}_{(\mathbf{L})}\left(\mathbf{L D}_{\mathbf{5 0}}\right)$ - The dose of a chemical that has been calculated to cause death in $50 \%$ of a defined experimental animal population.

Lethal Time $\mathbf{( 5 0 )}_{(\mathbf{S 0}}\left(\mathbf{L T}_{50}\right)$-A calculated period of time within which a specific concentration of a chemical is expected to cause death in $50 \%$ of a defined experimental animal population.

Lowest-Observed-Adverse-Effect Level (LOAEL) - The lowest exposure level of chemical in a study, or group of studies, that produces statistically or biologically significant increases in frequency or severity of adverse effects between the exposed population and its appropriate control.

Lymphoreticular Effects-Represent morphological effects involving lymphatic tissues such as the lymph nodes, spleen, and thymus.

Malformations-Permanent structural changes that may adversely affect survival, development, or function.

Minimal Risk Level (MRL) — An estimate of daily human exposure to a hazardous substance that is likely to be without an appreciable risk of adverse noncancer health effects over a specified route and duration of exposure.

Modifying Factor (MF) - A value (greater than zero) that is applied to the derivation of a Minimal Risk Level (MRL) to reflect additional concerns about the database that are not covered by the uncertainty factors. The default value for a MF is 1 .

Morbidity—State of being diseased; morbidity rate is the incidence or prevalence of disease in a specific population.

Mortality-Death; mortality rate is a measure of the number of deaths in a population during a specified interval of time. 
Mutagen-A substance that causes mutations. A mutation is a change in the DNA sequence of a cell's DNA. Mutations can lead to birth defects, miscarriages, or cancer.

Necropsy-The gross examination of the organs and tissues of a dead body to determine the cause of death or pathological conditions.

Neurotoxicity-The occurrence of adverse effects on the nervous system following exposure to a chemical.

No-Observed-Adverse-Effect Level (NOAEL) - The dose of a chemical at which there were no statistically or biologically significant increases in frequency or severity of adverse effects seen between the exposed population and its appropriate control. Effects may be produced at this dose, but they are not considered to be adverse.

Octanol-Water Partition Coefficient $\left(\mathbf{K}_{\mathbf{o w}}\right)$ - The equilibrium ratio of the concentrations of a chemical in $n$-octanol and water, in dilute solution.

Odds Ratio (OR) - A means of measuring the association between an exposure (such as toxic substances and a disease or condition) that represents the best estimate of relative risk (risk as a ratio of the incidence among subjects exposed to a particular risk factor divided by the incidence among subjects who were not exposed to the risk factor). An OR of greater than 1 is considered to indicate greater risk of disease in the exposed group compared to the unexposed group.

Organophosphate or Organophosphorus Compound-A phosphorus-containing organic compound and especially a pesticide that acts by inhibiting cholinesterase.

Permissible Exposure Limit (PEL) - An Occupational Safety and Health Administration (OSHA) allowable exposure level in workplace air averaged over an 8-hour shift of a 40-hour workweek.

Pesticide-General classification of chemicals specifically developed and produced for use in the control of agricultural and public health pests.

Pharmacokinetics - The dynamic behavior of a material in the body, used to predict the fate (disposition) of an exogenous substance in an organism. Utilizing computational techniques, it provides the means of studying the absorption, distribution, metabolism, and excretion of chemicals by the body.

Pharmacokinetic Model-A set of equations that can be used to describe the time course of a parent chemical or metabolite in an animal system. There are two types of pharmacokinetic models: data-based and physiologically-based. A data-based model divides the animal system into a series of compartments, which, in general, do not represent real, identifiable anatomic regions of the body, whereas the physiologically-based model compartments represent real anatomic regions of the body.

Physiologically Based Pharmacodynamic (PBPD) Model-A type of physiologically based doseresponse model that quantitatively describes the relationship between target tissue dose and toxic end points. These models advance the importance of physiologically based models in that they clearly describe the biological effect (response) produced by the system following exposure to an exogenous substance.

Physiologically Based Pharmacokinetic (PBPK) Model-Comprised of a series of compartments representing organs or tissue groups with realistic weights and blood flows. These models require a 
variety of physiological information: tissue volumes, blood flow rates to tissues, cardiac output, alveolar ventilation rates, and possibly membrane permeabilities. The models also utilize biochemical information, such as air/blood partition coefficients, and metabolic parameters. PBPK models are also called biologically based tissue dosimetry models.

Prevalence-The number of cases of a disease or condition in a population at one point in time.

Prospective Study - A type of cohort study in which the pertinent observations are made on events occurring after the start of the study. A group is followed over time.

$\mathbf{q}_{1}{ }^{*}$-The upper-bound estimate of the low-dose slope of the dose-response curve as determined by the multistage procedure. The $\mathrm{q}_{1} *$ can be used to calculate an estimate of carcinogenic potency, the incremental excess cancer risk per unit of exposure (usually $\mu \mathrm{g} / \mathrm{L}$ for water, $\mathrm{mg} / \mathrm{kg} / \mathrm{day}$ for food, and $\mu \mathrm{g} / \mathrm{m}^{3}$ for air).

Recommended Exposure Limit (REL) —A National Institute for Occupational Safety and Health (NIOSH) time-weighted average (TWA) concentration for up to a 10-hour workday during a 40-hour workweek.

Reference Concentration (RfC) — An estimate (with uncertainty spanning perhaps an order of magnitude) of a continuous inhalation exposure to the human population (including sensitive subgroups) that is likely to be without an appreciable risk of deleterious noncancer health effects during a lifetime. The inhalation reference concentration is for continuous inhalation exposures and is appropriately expressed in units of $\mathrm{mg} / \mathrm{m}^{3}$ or $\mathrm{ppm}$.

Reference Dose (RfD)-An estimate (with uncertainty spanning perhaps an order of magnitude) of the daily exposure of the human population to a potential hazard that is likely to be without risk of deleterious effects during a lifetime. The RfD is operationally derived from the no-observed-adverse-effect level (NOAEL, from animal and human studies) by a consistent application of uncertainty factors that reflect various types of data used to estimate RfDs and an additional modifying factor, which is based on a professional judgment of the entire database on the chemical. The RfDs are not applicable to nonthreshold effects such as cancer.

Reportable Quantity (RQ) - The quantity of a hazardous substance that is considered reportable under the Comprehensive Environmental Response, Compensation, and Liability Act (CERCLA). Reportable quantities are (1) 1 pound or greater or (2) for selected substances, an amount established by regulation either under CERCLA or under Section 311 of the Clean Water Act. Quantities are measured over a 24-hour period.

Reproductive Toxicity-The occurrence of adverse effects on the reproductive system that may result from exposure to a chemical. The toxicity may be directed to the reproductive organs and/or the related endocrine system. The manifestation of such toxicity may be noted as alterations in sexual behavior, fertility, pregnancy outcomes, or modifications in other functions that are dependent on the integrity of this system.

Retrospective Study - A type of cohort study based on a group of persons known to have been exposed at some time in the past. Data are collected from routinely recorded events, up to the time the study is undertaken. Retrospective studies are limited to causal factors that can be ascertained from existing records and/or examining survivors of the cohort.

Risk-The possibility or chance that some adverse effect will result from a given exposure to a chemical. 
Risk Factor-An aspect of personal behavior or lifestyle, an environmental exposure, or an inborn or inherited characteristic that is associated with an increased occurrence of disease or other health-related event or condition.

Risk Ratio-The ratio of the risk among persons with specific risk factors compared to the risk among persons without risk factors. A risk ratio greater than 1 indicates greater risk of disease in the exposed group compared to the unexposed group.

Short-Term Exposure Limit (STEL) - The American Conference of Governmental Industrial Hygienists (ACGIH) maximum concentration to which workers can be exposed for up to 15 minutes continually. No more than four excursions are allowed per day, and there must be at least 60 minutes between exposure periods. The daily Threshold Limit Value-Time Weighted Average (TLV-TWA) may not be exceeded.

Standardized Mortality Ratio (SMR) — A ratio of the observed number of deaths and the expected number of deaths in a specific standard population.

Target Organ Toxicity-This term covers a broad range of adverse effects on target organs or physiological systems (e.g., renal, cardiovascular) extending from those arising through a single limited exposure to those assumed over a lifetime of exposure to a chemical.

Teratogen-A chemical that causes structural defects that affect the development of an organism.

Threshold Limit Value (TLV)—An American Conference of Governmental Industrial Hygienists (ACGIH) concentration of a substance to which most workers can be exposed without adverse effect. The TLV may be expressed as a Time Weighted Average (TWA), as a Short-Term Exposure Limit (STEL), or as a ceiling limit (CL).

Time-Weighted Average (TWA) - An allowable exposure concentration averaged over a normal 8-hour workday or 40-hour workweek.

Toxic Dose $\left.\mathbf{( 5 0}_{\mathbf{5 0}} \mathbf{T}_{\mathbf{5 0}}\right)$ - A calculated dose of a chemical, introduced by a route other than inhalation, which is expected to cause a specific toxic effect in $50 \%$ of a defined experimental animal population.

Toxicokinetic - The absorption, distribution, and elimination of toxic compounds in the living organism.

Uncertainty Factor (UF) - A factor used in operationally deriving the Minimal Risk Level (MRL) or Reference Dose (RfD) or Reference Concentration (RfC) from experimental data. UFs are intended to account for (1) the variation in sensitivity among the members of the human population, (2) the uncertainty in extrapolating animal data to the case of human, (3) the uncertainty in extrapolating from data obtained in a study that is of less than lifetime exposure, and (4) the uncertainty in using lowestobserved-adverse-effect level (LOAEL) data rather than no-observed-adverse-effect level (NOAEL) data. A default for each individual UF is 10; if complete certainty in data exists, a value of 1 can be used; however, a reduced UF of 3 may be used on a case-by-case basis, 3 being the approximate logarithmic average of 10 and 1 .

Xenobiotic-Any chemical that is foreign to the biological system. 


\section{APPENDIX A. ATSDR MINIMAL RISK LEVELS AND WORKSHEETS}

The Comprehensive Environmental Response, Compensation, and Liability Act (CERCLA) [42 U.S.C. 9601 et seq.], as amended by the Superfund Amendments and Reauthorization Act (SARA) [Pub. L. 99499], requires that the Agency for Toxic Substances and Disease Registry (ATSDR) develop jointly with the U.S. Environmental Protection Agency (EPA), in order of priority, a list of hazardous substances most commonly found at facilities on the CERCLA National Priorities List (NPL); prepare toxicological profiles for each substance included on the priority list of hazardous substances; and assure the initiation of a research program to fill identified data needs associated with the substances.

The toxicological profiles include an examination, summary, and interpretation of available toxicological information and epidemiologic evaluations of a hazardous substance. During the development of toxicological profiles, Minimal Risk Levels (MRLs) are derived when reliable and sufficient data exist to identify the target organ(s) of effect or the most sensitive health effect(s) for a specific duration for a given route of exposure. An MRL is an estimate of the daily human exposure to a hazardous substance that is likely to be without appreciable risk of adverse noncancer health effects over a specified duration of exposure. MRLs are based on noncancer health effects only and are not based on a consideration of cancer effects. These substance-specific estimates, which are intended to serve as screening levels, are used by ATSDR health assessors to identify contaminants and potential health effects that may be of concern at hazardous waste sites. It is important to note that MRLs are not intended to define clean-up or action levels.

MRLs are derived for hazardous substances using the no-observed-adverse-effect level/uncertainty factor approach. They are below levels that might cause adverse health effects in the people most sensitive to such chemical-induced effects. MRLs are derived for acute (1-14 days), intermediate (15-364 days), and chronic (365 days and longer) durations and for the oral and inhalation routes of exposure. Currently, MRLs for the dermal route of exposure are not derived because ATSDR has not yet identified a method suitable for this route of exposure. MRLs are generally based on the most sensitive chemical-induced end point considered to be of relevance to humans. Serious health effects (such as irreparable damage to the liver or kidneys, or birth defects) are not used as a basis for establishing MRLs. Exposure to a level above the MRL does not mean that adverse health effects will occur.

MRLs are intended only to serve as a screening tool to help public health professionals decide where to look more closely. They may also be viewed as a mechanism to identify those hazardous waste sites that 
are not expected to cause adverse health effects. Most MRLs contain a degree of uncertainty because of the lack of precise toxicological information on the people who might be most sensitive (e.g., infants, elderly, nutritionally or immunologically compromised) to the effects of hazardous substances. ATSDR uses a conservative (i.e., protective) approach to address this uncertainty consistent with the public health principle of prevention. Although human data are preferred, MRLs often must be based on animal studies because relevant human studies are lacking. In the absence of evidence to the contrary, ATSDR assumes that humans are more sensitive to the effects of hazardous substance than animals and that certain persons may be particularly sensitive. Thus, the resulting MRL may be as much as 100 -fold below levels that have been shown to be nontoxic in laboratory animals.

Proposed MRLs undergo a rigorous review process: Health Effects/MRL Workgroup reviews within the Division of Toxicology and Environmental Medicine, expert panel peer reviews, and agency-wide MRL Workgroup reviews, with participation from other federal agencies and comments from the public. They are subject to change as new information becomes available concomitant with updating the toxicological profiles. Thus, MRLs in the most recent toxicological profiles supersede previously published levels. For additional information regarding MRLs, please contact the Division of Toxicology and Environmental Medicine, Agency for Toxic Substances and Disease Registry, 1600 Clifton Road NE, Mailstop F-32, Atlanta, Georgia 30333. 


\section{MINIMAL RISK LEVEL (MRL) WORKSHEET}

$\begin{array}{ll}\text { Chemical Name: } & \text { Xylenes (mixed xylenes, ortho-, meta-, and para-xylenes) } \\ \text { CAS Numbers: } & 1330-20-7,95-47-6,108-38-3,106-42-3 \\ \text { Date: } & \text { August 2007 } \\ \text { Profile Status: } & \text { Final } \\ \text { Route: } & \text { [X] Inhalation [ ] Oral } \\ \text { Duration: } & \text { [X] Acute [ ] Intermediate [ ] Chronic } \\ \text { Graph Key: } & 49 \\ \text { Species: } & \text { Human }\end{array}$

Minimal Risk Level: 2 [ ] mg/kg/day [X] ppm

[Note: A single acute-duration inhalation MRL has been derived based on data for $m$-xylene that applies to mixed xylenes and all of the individual isomers. The justification for deriving a common value is that the isomers have similar toxicokinetic properties and elicit similar toxicological effects, with no isomer consistently exhibiting the greatest potency, depending on the end point. Further discussion of this rationale is presented in Section 3.2.]

Reference: Ernstgard L, Gullstrand E, Lof A, et al. 2002. Are women more sensitive than men to 2-propanol and $m$-xylene vapors? Occup Environ Med 59:759-767.

Experimental design: Fifty-six healthy volunteers ( 28 per sex) between the ages of 20 and 49 years were exposed to $50 \mathrm{ppm}\left(200 \mathrm{mg} / \mathrm{m}^{3}\right) \mathrm{m}$-xylene, clean air (controls), or $150 \mathrm{ppm}$ 2-propanol in a dynamic exposure chamber for 2 hours. Each subject experienced the three treatments. Sessions were separated by intervals of 2 weeks. The experiment was initially designed to be balanced with two men and two women exposed on each occasion with all six possible orders of treatment represented. (Schedule changes by the volunteers resulted in imbalances in treatment orders, but ANOVA analysis indicated that the imbalance had no significant effect on the results.) Subjects rated the level of perceived discomfort using a visual analog scale $(0-100 \mathrm{~mm})$ in a questionnaire with 10 questions during exposure $(3,60$, and 118 minutes from the start of exposure), and post-exposure (140 and 350 minutes from onset). Items included discomfort in eyes, nose, throat, or airways; breathing difficulty; solvent smell; headache; fatigue; nausea; dizziness; and feeling of intoxication. Pulmonary function measurements were conducted via spirometer prior to exposure, immediately after exposure, and 3 hours post-exposure. These included vital capacity (VC), forced vital capacity (FVC), forced expiratory volume in one second $\left(\mathrm{FEV}_{1}\right)$, peak expiratory flow (PEF), and forced expiratory flow in 25,50 , or $75 \%$ of $\mathrm{FVC}\left(\mathrm{FEF}_{25}, \mathrm{FEF}_{50}\right.$, $\mathrm{FEF}_{75}$ ). Nasal swelling was assessed by acoustic rhinometry before, immediately after, and 3 hours after the end of exposure. Nasal lavages obtained before and 3 hours after the end of exposure were evaluated for markers of inflammation (lysozyme, albumin, myeloperoxidase, and eosinophilic cationic protein). Eye blinking was measured throughout exposure by electromyography and color vision was assessed before, immediately after, and 3 hours post-exposure.

Effect noted in study and corresponding doses: Statistically significant increases, compared to airexposure, in the average rating of self-reported symptoms resulting from exposure to $m$-xylene at $50 \mathrm{ppm}$ were observed for discomfort in the eyes and nose, detection of solvent smell, and feeling of intoxication in both sexes after 60 and 118 minutes, in discomfort in the throat or airways in women after 60 minutes, in breathing difficulty and nausea in men at 118 minutes and women at both timepoints, in headache and fatigue in men at both timepoints, and in dizziness in men at 118 minutes. All of the statistically significant increases in the subjective ratings were minimal except for detection of solvent smell, which was moderate. 
Statistically significant minimal changes, compared to air-exposure, were observed in objective measures of respiratory function (percent change from pre-exposure measurements) in women, but not men, 3 hours after the end of the exposure to $m$-xylene: FVC reduced by $2.81 \%$ (compared to reduced by $0.06 \%$ for air exposure, $\mathrm{p}<0.01$ ), $\mathrm{FEV}_{1} / \mathrm{FVC}$ increased by $1.09 \%$ (compared to decreased by $0.34 \%$ for air exposure, $\mathrm{p}<0.03$ ), and $\mathrm{FEF}_{75}$ increased by $3.32 \%$ (compared to decreased by $5.53 \%$ for air exposure, $\mathrm{p}<0.04$ ). No statistically significant change was observed immediately after exposure. Irrespective of exposure condition, there were diurnal changes in lung function $\left(\mathrm{FVC}\right.$ and $\left.\mathrm{FEV}_{1}\right)$ that were more pronounced in women than in men. There were no solvent-related changes in nasal volume or cross-sectional area, but ANOVA revealed a sex-dependent decrease in nasal volume over time (greater in women) irrespective of exposure condition. Exposure to $m$-xylene did not induce significant adverse changes in other parameters.

Dose and end point used for MRL derivation:

\section{[ ] NOAEL [X] LOAEL}

The minimal LOAEL of $50 \mathrm{ppm} m$-xylene is for slight respiratory effects (reduced forced vital capacity, increased discomfort in throat and airways in females, and breathing difficulty in both sexes) and subjective symptoms of neurotoxicity (headache, dizziness, a feeling of intoxication). The LOAEL is minimal because the magnitude of the changes was small. The data were not suitable for benchmark dose analysis because a single exposure level was tested.

Uncertainty Factors used in MRL derivation: 30

[X] 3 for use of a minimal LOAEL

[ ] 10 for extrapolation from animals to humans

[X] 10 for human variability

Was a conversion factor used from $\mathrm{ppm}$ in food or water to a $\mathrm{mg} /$ body weight dose? Not applicable.

If an inhalation study in animals, list conversion factors used in determining human equivalent dose: Not applicable.

Was a conversion used from intermittent to continuous exposure? Not applicable.

Other additional studies or pertinent information that lend support to this MRL: The database for acuteduration inhalation exposure to xylenes includes a number of studies in humans and laboratory animals. Studies in humans identify neurological and respiratory effects as the most sensitive end points for acuteduration inhalation exposure. Effects observed in humans at 50-690 ppm included irritation of the respiratory tract (breathing difficulty, discomfort in nose and throat, reduced forced air capacity), neurotoxicity (dizziness, headache, impaired short-term memory, increase in reaction times), and eye irritation (Carpenter et al. 1975a; Dudek et al. 1990; Gamberale et al. 1978; Nelson et al. 1943; NIOSH 1981). Other neurological effects in humans included altered visual evoked potentials following repeated exposure to $200 \mathrm{ppm} m$-xylene and impaired body balance following a single 4-hour exposure at $400 \mathrm{ppm}$ (Savolainen et al. 1984; Seppalainen et al. 1989).

Acute-duration neurological effects in animals were observed at concentrations of $113 \mathrm{ppm}$ and higher. These included transiently decreased operant responses in rats repeatedly exposed to $113 \mathrm{ppm}$ mixed xylene (Ghosh et al. 1987), altered responses to electric shock in rats and mice exposed once to 230$320 \mathrm{ppm} o$-xylene (Vodickova et al. 1995), and decreased axonal transport in rats repeatedly exposed to 800 ppm mixed xylene (Padilla and Lyerly 1989). Effects related to motor incoordination were observed 
following exposures to mixed xylenes or individual isomers in the range of 1,010-1,982 ppm (Carpenter et al. 1975a; De Ceaurriz et al. 1983; Korsak et al. 1988, 1990, 1993). Sensory-related changes (altered visual- or auditory-evoked potentials or hearing losses) occurred at exposures in the range of 1,4001,600 ppm p-xylene (Crofton et al. 1994; Dyer et al. 1988; Pryor et al. 1987; Rebert et al. 1995). Other neurological effects included disturbances in brain catecholamine or dopamine following repeated exposure to mixed xylene or individual isomers at 2,000 ppm (Andersson et al. 1981), narcosis in rats at $\geq 1,940 \mathrm{ppm}$ (Molnar et al. 1986), and ataxia and seizures in cats exposed to 9,500 ppm mixed xylene (Carpenter et al. 1975a).

The lowest effect levels for other end points affected by acute-duration inhalation exposure in animals were higher than the lowest LOAEL for sensitive end points in humans. Rat developmental effects (reduced fetal body weight and delayed ossification) were observed at xylene exposures in the range of 350-2000 ppm (Saillenfait et al. 2003; Ungvary et al. 1980b); maternal body weight effects were observed at $700 \mathrm{ppm}$ (Ungvary et al. 1980b). Acute-duration respiratory effects in animals include decreases in lung surfactant following exposure to mixed xylenes or individual isomers at 1,000 ppm or higher (Elovaara et al. 1987; Patel et al. 1978; Toftgard and Nilsen 1982) and decreased respiratory rates in mice briefly exposed to $m$ - or $o$-xylene at concentrations of 1,361-2,700 ppm (De Ceaurriz et al. 1981; Korsak et al. 1990, 1991, 1993). Increased mortality has been reported in rodents repeatedly exposed to at $700 \mathrm{ppm}$ (Ungvary et al. 1980b) or once to $\geq 2,010 \mathrm{ppm}$ (Bonnet et al. 1979; Cameron et al. 1938; Carpenter et al. 1975a; Harper et al. 1975; Hine and Zuidema 1970).

Respiratory and neurological effects are selected as co-critical effects of acute-duration inhalation exposure since they occurred at the lowest tested exposure level of $50 \mathrm{ppm}$. Ernstgard et al. (2002) was selected as the principal study for acute-duration inhalation exposure because it provides the lowest LOAEL for the co-critical effects.

Agency Contacts (Chemical Managers): Mike Fay, Ph.D.; John F. Risher, Ph.D.; Jewell D. Wilson, Ph.D. 


\section{MINIMAL RISK LEVEL (MRL) WORKSHEET}

$\begin{array}{ll}\text { Chemical Name: } & \text { Xylenes (mixed xylenes, ortho-, meta-, and para-xylenes) } \\ \text { CAS Number: } & 1330-20-7,95-47-6,108-38-3,106-42-3 \\ \text { Date: } & \text { August 2007 } \\ \text { Profile Status: } & \text { Final } \\ \text { Route: } & \text { [X] Inhalation [ ] Oral } \\ \text { Duration: } & \text { [ ] Acute [X] Intermediate [ ] Chronic } \\ \text { Graph Key: } & 137 \\ \text { Species: } & \text { Rat }\end{array}$

Minimal Risk Level: 0.6 [ ] mg/kg/day [X] ppm

[Note: A single intermediate-duration inhalation MRL has been derived based on data for $m$-xylene that applies to mixed xylenes and all of the individual isomers. The justification for deriving a common value is that the isomers have similar toxicokinetic properties and elicit similar toxicological effects, with no isomer consistently exhibiting the greatest potency, depending on the end point. Further discussion of this rationale is presented in Section 3.2.]

Reference: Korsak Z, Wisniewska-Knypl J, Swiercz R. 1994. Toxic effects of subchronic combined exposure to n-butyl alcohol and m-xylene in rats. Int J Occup Med Environ Health 7:155-166.

Experimental design: Groups of 12-24 male Wistar rats were exposed to $m$-xylene at 0,50 , or $100 \mathrm{ppm}$, for 6 hours/day, 5 days/week for 3 months. Before the start of the study and at the end of each month of exposure, rats were examined for motor coordination (rotarod performance). The level of analgesia was tested at termination in the paw-lick response to hot-plate test at $54{ }^{\circ} \mathrm{C}$.

Effect noted in study and corresponding doses: A dose-related increase in the failure rate on the rotarod performance test was observed. The change at $100 \mathrm{ppm}$ was significantly different $(\mathrm{p} \leq 0.05)$ from controls. There was no change in severity over time. Increased sensitivity to pain was indicated by a significant decrease $(\mathrm{p} \leq 0.05)$ compared to controls in the latency of the paw-lick response at 50 and $100 \mathrm{ppm}: 12.2 \pm 3.1,8.7 \pm 3.8$, and $8.6 \pm 2.7$ seconds, respectively, for control, 50, and $100 \mathrm{ppm}$. The $50 \mathrm{ppm}$ concentration of $m$-xylene is a LOAEL and serves at the basis for the intermediate inhalation MRL for xylenes.

Dose and end point used for MRL derivation:

\section{[ ] NOAEL [X] LOAEL}

The low concentration of $50 \mathrm{ppm}$ was a minimal LOAEL for a statistically significant decrease in the mean latency of the paw-lick response. The LOAEL is considered minimal because the effect was subtle, differing from the control by $<29 \%$, and did not significantly increase in severity with dose. The data were not suitable for benchmark dose analysis because the response was essentially a plateau.

Uncertainty Factors used in MRL derivation: 90

[X] 3 for use of a minimal LOAEL

[X] 3 for extrapolation from animals to humans using dosimetric adjustment

[X] 10 for human variability

Was a conversion factor used from ppm in food or water to a $\mathrm{mg} /$ body weight dose? Not applicable. 
If an inhalation study in animals, list conversion factors used in determining human equivalent dose: The minimal rat LOAEL was converted to a human equivalent following the EPA (1994) dosimetric equation for an extra-respiratory effect from an inhaled category 3 gas: minimal $\mathrm{LOAEL}_{(\mathrm{HEC})}=$ minimal rat LOAEL $\times \lambda_{\mathrm{a}} / \lambda_{\mathrm{h}}$, where $\lambda_{\mathrm{a}}$ and $\lambda_{\mathrm{h}}$ are blood:air partition coefficients for rats and humans, respectively. Reported blood:air partition coefficients for $m$-xylene are 39.9 for Wistar rats and 26.4 for humans (Sato and Nakajima 1979). Since the rat value is higher than the human, the default value of 1 was used for $\lambda_{\mathrm{a}} / \lambda_{\mathrm{h}}$ (EPA 1994). The minimal LOAEL $\mathrm{HEC}_{\text {is }}$ is therefore $50 \mathrm{ppm}$.

Was a conversion used from intermittent to continuous exposure? No. The rapid clearance of xylene from the body does not justify such a conversion.

Other additional studies or pertinent information that lend support to this MRL: The database for intermediate-duration inhalation to xylenes includes one controlled-exposure study in humans and several animal bioassays. Effects in humans exposed to 100-150 ppm p-xylene 1-7.5 hours/day, 5 days/week for 4 weeks included increased reporting of subjective symptoms of irritation of the nose and throat (NIOSH 1981). This study found no significant alterations in objective measures of neurological function (electroencephalography, tests of motor activity, and cognitive performance) and no alterations in pulmonary function in human subjects, but the study was limited in that some group sizes were small $(\mathrm{n}=2)$. Animal toxicity bioassays that tested at concentrations below $100 \mathrm{ppm}$ reported no clinical signs of neurotoxicity or no adverse effects on brain weight (Hillefors-Berglund et al. 1995; Jenkins et al. 1970). In a special neurobehavioral assay, a LOAEL of $50 \mathrm{ppm}$ was identified for decreased latency of the paw-lick response in rats exposed to $m$-xylene for 3 months (Korsak et al. 1994); impaired rotarod performance was noted in this study at $100 \mathrm{ppm}$, but not at $50 \mathrm{ppm}$. Other intermediate-duration studies also reported neurobehavioral effects (impaired rotarod performance, passive avoidance learning) following exposure to $100 \mathrm{ppm} m$-xylene for 4 weeks to 6 months (Gralewicz and Wiaderna 2001; Gralewicz et al. 1995; Korsak et al. 1994); some neurological impairment persisted for 5-9 weeks after exposure (Gralewicz and Wiaderna 2001). Other effects included distribution of astroglia cells in the brain of Mongolian gerbils exposed to $160 \mathrm{ppm}$ mixed xylene for 4 months (Rosengren et al. 1986), delayed maxillary ossification and impaired rotarod performance in rats gestationally exposed to $200 \mathrm{ppm}$ technical-grade xylene for 6 hours/day (Hass and Jakobsen 1993), and impaired motor coordination and spatial orientation following gestational exposure at 500 ppm (Hass et al. 1995, 1997). Neurological effects at higher concentrations included tremors in dogs exposed to $780 \mathrm{ppm} o$-xylene for 6 hours/day, 5 days/week for 6 weeks (Jenkins et al. 1970), alterations in brain neurotransmitters following continuous exposure to $800 \mathrm{ppm}$ mixed xylenes for 30 days (Honma et al. 1983), and auditory effects in rats (midrange hearing loss, death of hair cells of the Cochlea, or decreases in auditory brainstem responses) following exposure to $\geq 800 \mathrm{ppm}$ mixed xylenes or $900 \mathrm{ppm} p$-xylene for 13 weeks (Gagnaire et al. 2001; Nylen and Hagman 1994; Pryor et al. 1987).

Non-neurological effects were observed at higher concentrations. Cardiovascular effects (increased thickness of coronary microvessels) were observed in rats exposed to $230 \mathrm{ppm}$ mixed xylenes 6 hours/day, 5 days/week for 4 weeks (Morvai et al. 1987). Hepatic effects (increased liver weight) were observed at a LOAEL of $600 \mathrm{ppm}$ in rats discontinuously exposed to mixed xylenes for 4 weeks (Toftgard et al. 1981). In rats exposed gestationally to mixed xylenes or $o$-xylene, a LOAEL of $500 \mathrm{ppm}$ was identified for decreased fetal body weights in the absence of maternal toxicity (Bio/dynamics 1983). LOAELs for adult body weight effects were $1,000 \mathrm{ppm}$ or higher (Tatrai et al. 1981). Increased deaths among squirrel monkeys and rats were noted following discontinuous intermediate-duration exposure to 780 ppm o-xylene (Jenkins et al. 1970), but no systemic effects were noted in rats or dogs exposed to 810 ppm mixed xylenes (Carpenter et al. 1975a).

Agency Contacts (Chemical Managers): Mike Fay, Ph.D.; John F. Risher, Ph.D.; Jewell D. Wilson, Ph.D. 


\section{MINIMAL RISK LEVEL (MRL) WORKSHEET}

$\begin{array}{ll}\text { Chemical Name: } & \text { Xylenes (mixed xylenes, ortho-, meta-, and para-xylenes) } \\ \text { CAS Number: } & 1330-20-7,95-47-6,108-38-3,106-42-3 \\ \text { Date: } & \text { August 2007 } \\ \text { Profile Status: } & \text { Final } \\ \text { Route: } & \text { [X] Inhalation [ ] Oral } \\ \text { Duration: } & \text { [ ] Acute [ ] Intermediate [X] Chronic } \\ \text { Graph Key: } & 150 \\ \text { Species: } & \text { Human }\end{array}$

Minimal Risk Level: $0.05 \quad$ [ ] mg/kg/day $[\mathrm{X}] \mathrm{ppm}$

[Note: A single chronic-duration inhalation MRL has been derived based on data for mixed xylene that applies to mixed xylenes and all of the individual isomers. The justification for deriving a common value is that the isomers have similar toxicokinetic properties and elicit similar toxicological effects, with no isomer consistently exhibiting the greatest potency, depending on the end point. Further discussion of this rationale is presented in Section 3.2.]

Reference: Uchida Y, Nakatsuka H, Ukai H, et al. 1993. Symptoms and signs in workers exposed predominantly to xylenes. Int Arch Occup Environ Health 64:597-605.

Experimental design: 175 workers (107 men, 68 women) were exposed to mixed xylenes in Chinese factories during the production of rubber boots or plastic coated wire, or in printing work. Two hundred forty-one nonexposed workers (116 men, 125 women) were recruited from the same or other factories as a comparison population. Exposures, measured with a diffusive sampler, indicated that xylenes accounted for $>70 \%$ total exposure, with $\mathrm{m}$-xylene accounting for $50 \%$ of the xylene exposure, followed by $p$ - and $o$-xylenes. Toluene exposure and ethylbenzene exposure were about 1 and $3 \mathrm{ppm}$, respectively, with no benzene exposure. Subjects were evaluated for subjective symptoms in a questionnaire and also examined for objective parameters (serum biochemistry, hematology, and urinalysis). Exposures were corroborated by measurements of xylene metabolites in urine.

Effect noted in study and corresponding doses: The TWA (arithmetical mean) for xylenes was $21 \mathrm{ppm}$ for an average of 7 years (geometric mean $14 \mathrm{ppm}$ ). One man was exposed to $175 \mathrm{ppm}$. Subjective symptoms included increased prevalence of anxiety, forgetfulness, inability to concentrate, eye and nasal irritation, dizziness, and sore throats. Exposure and urinary metabolites are further described in Inoue et al. (1993). The findings of hematology (red blood cell, platelet and white blood cell counts, hemoglobin), serum biochemistry (total protein, albumin, aspartate aminotransferase, alanine aminotranferase gammaGTP, alkaline phosphatase, leucine aminopeptidase, lactate dehydrogenase, amylase, BUN, creatinine), and urinalysis were normal, indicating no liver or kidney effects. The geometric mean of $14 \mathrm{ppm}$, a LOAEL for subjective effects, is used as the basis of the chronic-duration MRL.

Dose and end point used for MRL derivation:

\section{[ ] NOAEL [X] LOAEL}

The LOAEL of $14 \mathrm{ppm}$, the TWA geometric mean, was for subjective symptoms of neurotoxicity (anxiety, forgetfulness, floating sensation) and respiratory toxicity (nasal irritation and sore throat) and eye irritation. The geometric mean was chosen over the arithmetical mean because it is a better representation of central tendency. The data were not suitable for benchmark dose analysis because a single average exposure level was reported. 
Uncertainty Factors used in MRL derivation:

[X] 10 for use of a LOAEL

[ ] 10 for extrapolation from animals to humans

[X] 10 for human variability

\section{Modifying Factors used in MRL derivation:}

[X] 3 to account for the lack of supporting studies evaluating the chronic neurotoxicity of xylene.

Was a conversion factor used from $\mathrm{ppm}$ in food or water to a $\mathrm{mg} /$ body weight dose? No.

If an inhalation study in animals, list conversion factors used in determining human equivalent dose: Not applicable.

Was a conversion used from intermittent to continuous exposure? No. The rapid clearance of xylene from the body does not justify such a conversion.

Other additional studies or pertinent information that lend support to this MRL: Although no other chronic-duration inhalation data are available for humans, the database for shorter duration exposures supports the findings of Uchida et al. (1993). Neurotoxicity (dizziness, headache, impaired short-term memory, increased reaction times), irritation of the respiratory tract (breathing difficulty, discomfort in nose and throat, reduced forced air capacity), and eye irritation have been reported in humans acutely exposed to $\geq 50 \mathrm{ppm} m$-xylene or mixed xylenes or $\geq 100 \mathrm{ppm} p$-xylene (Carpenter et al. 1975a; Dudek et al. 1990; Ernstgard et al. 2002; Gamberale et al. 1978; Nelson et al. 1943; NIOSH 1981). Repeated intermediate-duration exposure to $\geq 100 \mathrm{ppm} p$-xylene also increased the reporting of subjective symptoms for irritation of the nose and throat (NIOSH 1981).

Studies in animals also confirm that the nervous system is a sensitive target of inhalation exposure to xylene; respiratory function has not been assessed in animals at low concentrations. The most sensitive effects observed in acute-duration studies involved impaired neurological performance (operant responses) in rats exposed to $113 \mathrm{ppm}$ mixed xylenes (Ghosh et al. 1987) and altered responses to electric shock in rats and mice exposed to 230-320 ppm o-xylene (Vodickova et al. 1995). The most sensitive effects in intermediate-duration studies were for increased sensitivity to pain in rats exposed to $50 \mathrm{ppm}$ $m$-xylene (Korsak et al. 1994) and impairments in rotarod performance and passive avoidance learning in rats exposed to $\geq 100 \mathrm{ppm} m$-xylene (Gralewicz and Wiaderna 2001; Gralewicz et al. 1995; Korsak et al. 1994). In addition, rats exposed during gestation to $\geq 160 \mathrm{ppm}$ mixed xylene exhibited neurological impairments after birth (degraded rotarod performance, motor coordination, and spatial orientation) (Hass and Jakobsen 1993; Hass et al. 1995; Hass et al. 1997). Conversely, the most sensitive non-neurological effects in inhalation assays in animals were developmental effects in rats acutely-exposed at $350 \mathrm{ppm}$ (Ungvary et al. 1980b) and cardiovascular effects in rats exposed for an intermediate duration at $230 \mathrm{ppm}$ (Morvai et al. 1987).

Acute exposure of volunteers to $50 \mathrm{ppm}$ xylene and $50 \mathrm{ppm}$ toluene appeared to reduce the neurological effects of xylenes (Dudek et al. 1990). Therefore, it is unlikely that the low concentration of toluene $(1 \mathrm{ppm})$ reported in the Uchida et al. (1993) contributed to the observed effects.

The only other chronic-duration inhalation study was for rats exposed to $1,096 \mathrm{ppm} o$-xylene 8 hours/day, 7 days/week for 1 year (Tatrai et al. 1981). Hepatic changes included increases in liver weight and microsomal activity and proliferating endoplasmic reticulum, but no histopathological lesions. Body weight was reduced by $11 \%$ in exposed rats. Calculation of a chronic-duration inhalation MRL from this 
rat study would not be appropriate since the study did not examine neurological effects, which are the most critical end points for inhalation exposure to xylene.

Agency Contacts (Chemical Managers): Mike Fay, Ph.D.; John F. Risher, Ph.D.; Jewell D. Wilson, Ph.D. 


\section{MINIMAL RISK LEVEL (MRL) WORKSHEET}

$\begin{array}{ll}\text { Chemical Name: } & \text { Xylenes (mixed xylenes, ortho-, meta-, and para-xylenes) } \\ \text { CAS Number: } & 1330-20-7,95-47-6,108-38-3,106-42-3 \\ \text { Date: } & \text { August 2007 } \\ \text { Profile Status: } & \text { Final } \\ \text { Route: } & \text { [ ] Inhalation [X] Oral } \\ \text { Duration: } & {[X] \text { Acute [ ] Intermediate [ ] Chronic }} \\ \text { Graph Key: } & 20 \\ \text { Species: } & \text { Rat }\end{array}$

Minimal Risk Level: $1 \quad$ [X] mg/kg/day [ ] ppm

[Note: A single acute-duration oral MRL has been derived based on data for $p$-xylene that applies to mixed xylenes and all of the individual isomers. The justification for deriving a common value is that the isomers have similar toxicokinetic properties and elicit similar toxicological effects, with no isomer consistently exhibiting the greatest potency, depending on the end point. Further discussion of this rationale is presented in Section 3.2.]

Reference: Dyer RS, Bercegeay MS, Mayo LM. 1988. Acute exposures to p-xylene and toluene alter visual information processing. Neurotoxicol Teratol 10:147-153.

Experimental design: Male Long-Evans rats had electrodes implanted in their skulls and were allowed to recover from surgery for 1 week prior to exposure and testing for visual processing. Two different experiments were conducted. $p$-Xylene (99.8\% pure) was diluted in corn oil and administered by gavage at $1 \mathrm{~mL} / \mathrm{kg}$. Groups of 10-11 rats received single doses of $p$-xylene at doses of $0,500,1,000$, or $2,000 \mathrm{mg} / \mathrm{kg}$ in corn oil and were tested 75 minutes later. Based on the results of this experiment, groups of 14-16 rats were dosed at 0,125 , or $250 \mathrm{mg} / \mathrm{kg}$ and tested 45 minutes later. The eyes of exposed rats were treated with topical atropine ( $1 \%$ in saline) 10 minutes prior to visual testing, which took place in a rectangular box with three mirrored sides. A trial consisted of the response to a single strobe-generated flash. Trials were presented at $0.3 \mathrm{~Hz}$ and a total of 128 trials were averaged for each flash-evoked potential. The latencies and amplitudes of the P1, N1, P2, N2, P3, and N3 waveforms were determined in the first experiment and the N3 waveform measured in the second experiment.

Effect noted in study and corresponding doses: Forty-five minutes after administration of $250 \mathrm{mg} / \mathrm{kg}$, the amplitude of the N3 peak was decreased by $47 \%$ (statistically significant). At higher doses $(500,1,000$, and 2,000 mg/kg), the N3 peak was not observed until 75 minutes after dosing. At 2,000 mg/kg, mild sedation and hypothermia $\left(1^{\circ} \mathrm{C}\right)$ were observed. Increased latencies were observed for the P1, N1, and $\mathrm{P} 2$ peaks, but this effect was attributed to the hypothermia. Effects on the N3 amplitude were still apparent 8 hours post dosing. By 16 hours post dosing, recovery was complete. Amphetamines $(0.6,1.2$, or $2.5 \mathrm{mg} / \mathrm{kg}$ ) produced effects on the $\mathrm{N} 3$ amplitude similar to $p$-xylene. The study authors suggested that the effects of xylene may be secondary to changes in arousal or excitability. No effects were noted at $125 \mathrm{mg} / \mathrm{kg} /$ day, and this NOAEL serves as the basis for the acute oral MRL for xylenes.

Dose and end point used for MRL derivation:

\section{[X] NOAEL [ ] LOAEL}

The NOAEL of $125 \mathrm{mg} / \mathrm{kg} /$ day was for alteration of visual evoked potentials. The data were not suitable for benchmark dose analysis because the responses at $250 \mathrm{mg} / \mathrm{kg} /$ day (reduction in N3 peak amplitude) and $\geq 500 \mathrm{mg} / \mathrm{kg} /$ day (delay in N3 peak appearance) were not comparable. 
Uncertainty Factors used in MRL derivation:

[ ] 10 for use of a LOAEL

[X] 10 for extrapolation from animals to humans

[X] 10 for human variability

Was a conversion factor used from $\mathrm{ppm}$ in food or water to a $\mathrm{mg} /$ body weight dose? No.

If an inhalation study in animals, list conversion factors used in determining human equivalent dose: Not applicable.

Was a conversion used from intermittent to continuous exposure? Not applicable.

Other additional studies or pertinent information that lend support to this MRL: Evaluation of the limited database for acute-duration oral exposure to xylenes suggests that neurotoxicity represents the most sensitive end point in laboratory animals; no quantitative human data are available. Effects observed in acute-duration, single-dose oral studies included altered visually evoked potentials in rats receiving $250 \mathrm{mg} / \mathrm{kg} p$-xylene (Dyer et al. 1988) and reduced pulmonary microsomal activity in rats receiving $1,000 \mathrm{mg} / \mathrm{kg}$ p-xylene (Patel et al. 1978). In rats exposed 5 days/week for 2 weeks, a significant loss of Cochlear hair cells responsible for detecting midrange frequencies was observed following gavage dosing with $900 \mathrm{mg} / \mathrm{kg} / \mathrm{day} p$-xylene but not $\mathrm{m}$ - or $o$-xylene (Gagnaire and Langlais 2005). Reduced body weight gain was observed in rats following repeated dosing for 13 weeks with $1,000 \mathrm{mg} / \mathrm{kg}$ mixed xylenes or $2,000 \mathrm{mg} / \mathrm{kg} /$ day $o$ - or $p$-xylene (Condie et al. 1988; NTP 1986). Repeated exposure to mixed xylenes at $2,000 \mathrm{mg} / \mathrm{kg} /$ day resulted in impaired respiration (shallow and/or labored breathing) in rats and mice and increased mortality (NTP 1986). At $2,060 \mathrm{mg} / \mathrm{kg} /$ day, developmental toxicity (cleft palate) was observed in mice gestationally exposed to mixed xylenes (Marks et al. 1982). Serious neurological effects (coma, incoordination, prostration, decreased hindleg movement) were observed in rats that received single oral gavage doses of $\geq 4,000 \mathrm{mg} / \mathrm{kg}$ mixed xylenes (Muralidhara and Krishnakumari 1980; NTP 1986).

The database for inhalation studies on xylene supports neurotoxicity as the sensitive target following short- or long-term exposure. Neurotoxicity was manifest as self-reported symptoms of headache, dizziness, and a feeling of intoxication in human subjects acutely-exposed to $50 \mathrm{ppm} m$-xylene (Ernstgard et al. 2002) and decreased operant responses in rats exposed to $113 \mathrm{ppm}$ mixed xylene (Ghosh et al. 1987). Conversely, the most sensitive non-neurological effects in animals were developmental effects in rats exposed at $350 \mathrm{ppm}$ (Ungvary et al. 1980b).

Neurotoxicity was selected as the critical effect for acute-duration oral exposure to xylene because it was observed at the lowest exposure level and demonstrated dose-related increases in severity. The study by Dyer et al. (1988) was selected as the principal study, since it provides the lowest LOAEL for the critical effect.

Agency Contacts (Chemical Managers): Mike Fay, Ph.D.; John F. Risher, Ph.D.; Jewell D. Wilson, Ph.D. 


\section{MINIMAL RISK LEVEL (MRL) WORKSHEET}

$\begin{array}{ll}\text { Chemical Name: } & \text { Xylenes (mixed xylenes, ortho-, meta-, and para-xylenes) } \\ \text { CAS Number: } & 1330-20-7,95-47-6,108-38-3,106-42-3 \\ \text { Date: } & \text { August 2007 } \\ \text { Profile Status: } & \text { Final } \\ \text { Route: } & \text { [ ] Inhalation [X] Oral } \\ \text { Duration: } & \text { [ ] Acute [X] Intermediate [ ] Chronic } \\ \text { Graph Key: } & 39 \\ \text { Species: } & \text { Mouse }\end{array}$

Minimal Risk Level: $0.4 \quad[\mathrm{X}] \mathrm{mg} / \mathrm{kg} / \mathrm{day} \quad[\mathrm{j}$ ppm

[Note: A single intermediate-duration oral MRL has been derived based on data for mixed xylenes that applies to mixed xylenes and all of the individual isomers. The justification for deriving a common value is that the isomers have similar toxicokinetic properties and elicit similar toxicological effects, with no isomer consistently exhibiting the greatest potency, depending on the end point. Further discussion of this rationale is presented in Section 3.2.]

Reference: NTP. 1986. National Toxicology Program technical report on the toxicology and carcinogenesis studies of xylenes (mixed) (60\% m-xylene, 14\% p-xylene, $9 \% o$-xylene, and 17\% ethylbenzene) (CAS No. 1330-20-7) in F344/N rats and $\mathrm{B}^{2} \mathrm{C} 3 \mathrm{~F}_{1}$ mice (gavage studies). Research Triangle Park, NC: U.S. Department of Health and Human Services, Public Health Service, National Institutes of Health, National Toxicology Program. NTP TR 327. NIH Publication No. 87-2583.

Experimental design: $\mathrm{B}_{6 \mathrm{C} 3 \mathrm{~F}_{1}}$ mice $(50 / \mathrm{sex} /$ dose) were administered 500 or $1,000 \mathrm{mg} / \mathrm{kg} /$ day mixed xylenes (9.1\% o-, $60.2 \% \mathrm{~m}$-, and $13.6 \% \mathrm{p}$-xylene, and $17 \%$ ethylbenzene) in corn oil by gavage, 5 days/week for 103 weeks. Mice were observed twice daily for mortality and clinical signs; clinical signs were recorded daily for 16 months and monthly thereafter. Body weights were recorded weekly for the first 12 weeks and once a month thereafter. Animals in a moribund condition and those surviving to 103 weeks were sacrificed humanely. All animals were subjected to gross necropsy at termination; all gross lesions and a full range of tissues in each animal were examined for histopathology.

Effect noted in study and corresponding doses: There were no significant treatment-related effects of mixed xylene on body weight, survival, or the incidence of neoplastic or non-neoplastic lesions in mice receiving doses as high as $1,000 \mathrm{mg} / \mathrm{kg} / \mathrm{day}, 5$ days/week for 104 weeks. The only effect of treatment with mixed xylenes was hyperactivity in high-dose male and female mice, consistently observed during the half-hour period following administration in weeks 4-103. Hyperactivity observed from week 4 to week 51 is considered an overt neurological effect of intermediate-duration exposure to mixed xylene.

Dose and end point used for MRL derivation:

\section{[X] NOAEL [ ] LOAEL}

The NOAEL of $500 \mathrm{mg} / \mathrm{kg} /$ day for hyperactivity in male and female mice was adjusted for intermittent exposure (5 days $/ 7$ days), resulting in a duration-adjusted NOAEL of $360 \mathrm{mg} / \mathrm{kg} / \mathrm{day}$. The data were not suitable for benchmark dose analysis because the incidence of hyperactivity at the LOAEL was $100 \%$ and provides no information as to the shape of the low end of dose-response curve. 
Uncertainty Factors used in MRL derivation:

[ ] 10 for use of a LOAEL

[X] 10 for extrapolation from animals to humans

[X] 10 for human variability

\section{Modifying Factor used in MRL derivation:}

[X ] 10 for the lack of testing for sensitive neurological end points and lack of developmental and multi-generational data.

Was a conversion factor used from $\mathrm{ppm}$ in food or water to a $\mathrm{mg} /$ body weight dose? No.

If an inhalation study in animals, list conversion factors used in determining human equivalent dose: Not applicable.

Was a conversion used from intermittent to continuous exposure? Yes. The mouse NOAEL of $500 \mathrm{mg} / \mathrm{kg}$ was multiplied by 5 days/7 days, resulting in a duration-adjusted mouse NOAEL of $360 \mathrm{mg} / \mathrm{kg} / \mathrm{day}$.

Other additional studies or pertinent information that lend support to this MRL: The intermediateduration oral toxicity of xylenes has been investigated in several oral gavage bioassays in rodents exposed to mixed xylene (Condie et al. 1988; NTP 1986), m-xylene (Elovaara et al. 1989; Wolfe 1988a), and p-xylene (Wolfe 1988b). The observed effects include increases in minimal chronic nephropathy and$17-37 \%$ increases in relative hepatic weight in rats exposed to $\geq 750 \mathrm{mg} / \mathrm{kg} /$ day mixed xylene (Condie et al. 1988); 27-46.3\% increases in serum transaminase levels in rats exposed to $750-1,500 \mathrm{mg} / \mathrm{kg} / \mathrm{day}$ mixed or $m$-xylene (Condie et al. 1988; Elovaara et al. 1989; Wolfe 1988a); reduced levels of cytochrome $\mathrm{P}-450$ in the lung in rats exposed to $800 \mathrm{mg} / \mathrm{kg} /$ day $\mathrm{m}$-xylene (Elovaara et al. 1989); 15-25\% decreases in body weight gain in rats exposed to $800-1,000 \mathrm{mg} / \mathrm{kg} /$ day mixed, $m$-, or $p$-xylene (NTP 1986; Wolfe 1988a, 1988b); hyperactivity or increased aggressiveness subsequent to dosing with mixed xylene in rats at $1,500 \mathrm{mg} / \mathrm{kg} /$ day or mice at $1,000 \mathrm{mg} / \mathrm{kg} /$ day (Condie et al. 1988; NTP 1986); and 11\% increased relative spleen weight, $16 \%$ increased relative kidney weight, and increased hematological effects (mild polycythemia and leukocytosis) in female rats dosed with $1,500 \mathrm{mg} / \mathrm{kg} / \mathrm{day}$ mixed xylene (Condie et al. 1988). No hepatic effects were noted in F344 rats given mixed xylene at doses as high as $1,000 \mathrm{mg} / \mathrm{kg} /$ day or $\mathrm{B} 6 \mathrm{C} 3 \mathrm{~F}_{1}$ mice dosed at $\leq 2,000 \mathrm{mg} / \mathrm{kg} /$ day 5 days $/$ week for 13 weeks (NTP 1986) or in Sprague-Dawley rats dosed with $p$-xylene at $800 \mathrm{mg} / \mathrm{kg} /$ day for 90 days (Wolfe 1988b). No renal effects were observed in rats or mice exposed to mixed xylene in studies by NTP (1986) or in rats exposed to $m$ - or $p$-xylene (Wolfe 1988a, 1988b). The lack of hepatic or renal effects in some studies may be related to strain differences, exposure differences (discontinuous vs. continuous), or isomer specificities.

A limitation of standard intermediate-duration oral bioassays for xylene is that no testing was conducted for sensitive neurological effects. The only overt neurological effect of long-term exposure to xylene was hyperactivity noted in male and female mice immediately after dosing with $1,000 \mathrm{mg} / \mathrm{kg}(710 \mathrm{mg} / \mathrm{kg} / \mathrm{day}$, duration adjusted) mixed xylene beginning at week 4 of the 2-year NTP (1986) bioassay; hyperactivity was not observed at $500 \mathrm{mg} / \mathrm{kg}(360 \mathrm{mg} / \mathrm{kg} /$ day, duration adjusted). Neurotoxicity (hyperactivity) observed during the first year of that study (week 4-51) is selected as the critical effect of intermediateduration exposure because it was observed at the lowest LOAEL $(710 \mathrm{mg} / \mathrm{kg} / \mathrm{day}$, duration-adjusted).

Selection of neurotoxicity as the critical effect is consistent with the inhalation-exposure database. The most sensitive effects from inhalation exposure to xylenes were neurotoxicity: for the acute duration, self-reported symptoms of headache, dizziness, and feeling of intoxication in human subjects exposed to 
$50 \mathrm{ppm} m$-xylene (Ernstgard et al. 2002); for the intermediate duration, increased sensitivity to pain in rats exposed to $50 \mathrm{ppm} m$-xylene (Korsak et al. 1994); and for the chronic duration, increased reporting of symptoms of anxiety, forgetfulness, and a floating sensation in workers exposed to $14 \mathrm{ppm}$ mixed xylene (Uchida et al. 1993). The most sensitive acute inhalation effect in animals was impairment of operant responses observed following exposure to $\geq 113 \mathrm{ppm} m$-xylene (Ghosh et al. 1987). Conversely, the most sensitive LOAELs for non-neurological effects in inhalation studies were for developmental effects in rats following acute exposure at $\geq 350 \mathrm{ppm}$ (Ungvary et al. 1980b) or for cardiovascular effects in rats following intermediate exposure at $\geq 230 \mathrm{ppm}$ (Morvai et al. 1987).

The NTP (1986) study is selected as the principal study for intermediate-duration exposure to xylene because it provides the lowest adverse effect level, a LOAEL of $710 \mathrm{mg} / \mathrm{kg} / \mathrm{day}$, and a NOAEL of $500 \mathrm{mg} / \mathrm{kg}(360 \mathrm{mg} / \mathrm{kg} /$ day, duration adjusted) for the critical effect.

Agency Contacts (Chemical Managers): Mike Fay, Ph.D.; John F. Risher, Ph.D.; Jewell D. Wilson, Ph.D. 


\section{MINIMAL RISK LEVEL (MRL) WORKSHEET}

$\begin{array}{ll}\text { Chemical Name: } & \text { Xylenes (mixed xylenes, ortho-, meta-, and para-xylenes) } \\ \text { CAS Number: } & 1330-20-7,95-47-6,108-38-3,106-42-3 \\ \text { Date: } & \text { August 2007 } \\ \text { Profile Status: } & \text { Final } \\ \text { Route: } & \text { [ ] Inhalation [X] Oral } \\ \text { Duration: } & \text { [ ] Acute [ ] Intermediate [X] Chronic } \\ \text { Graph Key: } & 47 \\ \text { Species: } & \text { Rat }\end{array}$

Minimal Risk Level: $0.2 \quad[\mathrm{X}] \mathrm{mg} / \mathrm{kg} / \mathrm{day} \quad[$ ] ppm

[Note: A single chronic-duration oral MRL has been derived based on data for mixed xylenes that applies to mixed xylenes and all of the individual isomers. The justification for deriving a common value is that the isomers have similar toxicokinetic properties and elicit similar toxicological effects, with no isomer consistently exhibiting the greatest potency, depending on the end point. Further discussion of this rationale is presented in Section 3.2.]

Reference: NTP. 1986. National Toxicology Program technical report on the toxicology and carcinogenesis studies of xylenes (mixed) (60\% m-xylene, 14\% p-xylene, $9 \% o$-xylene, and 17\% ethylbenzene) (CAS No. 1330-20-7) in F344/N rats and $\mathrm{B}^{2} \mathrm{C} 3 \mathrm{~F}_{1}$ mice (gavage studies). Research Triangle Park, NC: U.S. Department of Health and Human Services, Public Health Service, National Institutes of Health, National Toxicology Program. NTP TR 327. NIH Publication No. 87-2583.

Experimental design: $\mathrm{F} 344 / \mathrm{N}$ rats (50/sex/dose) were administered 250 or $500 \mathrm{mg} / \mathrm{kg} /$ day mixed xylenes (9.1\% o-, $60.2 \% \mathrm{~m}$-, and $13.6 \% \mathrm{p}$-xylene, and 17\% ethylbenzene) in corn oil by gavage, 5 days/week for 103 weeks. Rats were observed twice daily for mortality and clinical signs; clinical signs were recorded daily for 16 months and monthly thereafter. Body weights were recorded weekly for the first 12 weeks and once a month thereafter. Animals in a moribund condition and those surviving to 103 weeks were sacrificed humanely. All animals were subjected to gross necropsy at termination; all gross lesions and a full range of tissues in each animal were examined for histopathology.

Effect noted in study and corresponding doses: No adverse effects were noted in male or female rats treated at the low dose of $250 \mathrm{mg} / \mathrm{kg} /$ day. At $500 \mathrm{mg} / \mathrm{kg}$, body weights were $5-8 \%$ lower in male rats after week 59, but the differences from controls were not biologically significant. In addition, survival at termination was significantly lower compared to controls in male rats treated at $500 \mathrm{mg} / \mathrm{kg} / \mathrm{day}$. No other treatment-related non-neoplastic or neoplastic effects were observed in male or female rats.

Dose and end point used for MRL derivation:

\section{[X] NOAEL [ ] LOAEL}

The MRL is based on a NOAEL of $250 \mathrm{mg} / \mathrm{kg}$. Although no histopathology was observed in any organ, including the nervous system, in rats exposed to $500 \mathrm{mg} / \mathrm{kg}$, this dose was not used as the basis for MRL derivation because survival was decreased in male rats at this dose level. Therefore, the next lowest dose level was used as the basis for the MRL. The NOAEL of $250 \mathrm{mg} / \mathrm{kg}$ was adjusted for intermittent exposure (5 days/7 days), resulting in a duration-adjusted NOAEL of $179 \mathrm{mg} / \mathrm{kg} / \mathrm{day}$. The data were not suitable for benchmark dose analysis because of the lack of measurable adverse effects aside from lethality. 
Uncertainty Factors used in MRL derivation:

[ ] 10 for use of a LOAEL

[X] 10 for extrapolation from animals to humans

[X] 10 for human variability

\section{Modifying Factor used in MRL derivation:}

[X] 10 for the lack of testing for sensitive neurological end points and lack of developmental and multi-generational data.

Was a conversion factor used from $\mathrm{ppm}$ in food or water to a $\mathrm{mg} /$ body weight dose? No.

If an inhalation study in animals, list conversion factors used in determining human equivalent dose: Not applicable.

Was a conversion used from intermittent to continuous exposure? Yes. The NOAEL of $250 \mathrm{mg} / \mathrm{kg} / \mathrm{day}$ was multiplied by 5 days $/ 7$ days, resulting in a duration-adjusted NOAEL of $179 \mathrm{mg} / \mathrm{kg} /$ day.

Other additional studies or pertinent information that lend support to this MRL: In the absence of data associating health effects in humans with chronic-duration oral exposure to xylenes, the animal bioassays provide a minimal basis for deriving an MRL for humans chronically exposed to xylenes. The parallel NTP (1986) oral gavage study in $\mathrm{B}^{6} \mathrm{C}_{3} \mathrm{~F}_{1}$ mice reported no significant treatment-related effects of mixed xylene on body weight, survival, or the incidence of neoplastic or non-neoplastic lesions in mice receiving doses as high as $1,000 \mathrm{mg} / \mathrm{kg} / \mathrm{day}, 5$ days/week for 104 weeks. The only effect of treatment with mixed xylenes was hyperactivity in high-dose male and female mice, consistently observed during the half-hour period following administration in weeks 4-103.

Histopathology in specific target organs was not observed in rats or mice exposed to mixed xylene for 2 years (NTP 1986). Limitations of these studies include the lack of organ weight data, and the lack of hematology, urinalysis, or clinical chemistry data that might identify target organs for xylene. The lack of neurobehavioral analysis is also a deficiency, given that neurotoxicity was the most sensitive effect following acute-, intermediate-, or chronic-duration inhalation exposure, as well as acute-duration oral exposure to xylenes (Dyer et al. 1988; Ernstgard et al. 2002; Korsak et al. 1994; Uchida et al. 1993). The sensitive neurotoxic effects in animals were revealed by specialized neurobehavioral or neurophysiological tests and would not be apparent in standard toxicity assays.

Agency Contacts (Chemical Managers): Mike Fay, Ph.D.; John F. Risher, Ph.D.; Jewell D. Wilson, Ph.D. 
APPENDIX A

This page is intentionally blank. 


\section{APPENDIX B. USER'S GUIDE}

\section{Chapter 1}

\section{Public Health Statement}

This chapter of the profile is a health effects summary written in non-technical language. Its intended audience is the general public, especially people living in the vicinity of a hazardous waste site or chemical release. If the Public Health Statement were removed from the rest of the document, it would still communicate to the lay public essential information about the chemical.

The major headings in the Public Health Statement are useful to find specific topics of concern. The topics are written in a question and answer format. The answer to each question includes a sentence that will direct the reader to chapters in the profile that will provide more information on the given topic.

\section{Chapter 2}

\section{Relevance to Public Health}

This chapter provides a health effects summary based on evaluations of existing toxicologic, epidemiologic, and toxicokinetic information. This summary is designed to present interpretive, weightof-evidence discussions for human health end points by addressing the following questions:

1. What effects are known to occur in humans?

2. What effects observed in animals are likely to be of concern to humans?

3. What exposure conditions are likely to be of concern to humans, especially around hazardous waste sites?

The chapter covers end points in the same order that they appear within the Discussion of Health Effects by Route of Exposure section, by route (inhalation, oral, and dermal) and within route by effect. Human data are presented first, then animal data. Both are organized by duration (acute, intermediate, chronic). In vitro data and data from parenteral routes (intramuscular, intravenous, subcutaneous, etc.) are also considered in this chapter.

The carcinogenic potential of the profiled substance is qualitatively evaluated, when appropriate, using existing toxicokinetic, genotoxic, and carcinogenic data. ATSDR does not currently assess cancer potency or perform cancer risk assessments. Minimal Risk Levels (MRLs) for noncancer end points (if derived) and the end points from which they were derived are indicated and discussed.

Limitations to existing scientific literature that prevent a satisfactory evaluation of the relevance to public health are identified in the Chapter 3 Data Needs section.

\section{Interpretation of Minimal Risk Levels}

Where sufficient toxicologic information is available, ATSDR has derived MRLs for inhalation and oral routes of entry at each duration of exposure (acute, intermediate, and chronic). These MRLs are not meant to support regulatory action, but to acquaint health professionals with exposure levels at which adverse health effects are not expected to occur in humans. 
MRLs should help physicians and public health officials determine the safety of a community living near a chemical emission, given the concentration of a contaminant in air or the estimated daily dose in water. MRLs are based largely on toxicological studies in animals and on reports of human occupational exposure.

MRL users should be familiar with the toxicologic information on which the number is based. Chapter 2, "Relevance to Public Health," contains basic information known about the substance. Other sections such as Chapter 3 Section 3.9, "Interactions with Other Substances," and Section 3.10, "Populations that are Unusually Susceptible" provide important supplemental information.

MRL users should also understand the MRL derivation methodology. MRLs are derived using a modified version of the risk assessment methodology that the Environmental Protection Agency (EPA) provides (Barnes and Dourson 1988) to determine reference doses (RfDs) for lifetime exposure.

To derive an MRL, ATSDR generally selects the most sensitive end point which, in its best judgement, represents the most sensitive human health effect for a given exposure route and duration. ATSDR cannot make this judgement or derive an MRL unless information (quantitative or qualitative) is available for all potential systemic, neurological, and developmental effects. If this information and reliable quantitative data on the chosen end point are available, ATSDR derives an MRL using the most sensitive species (when information from multiple species is available) with the highest no-observed-adverse-effect level (NOAEL) that does not exceed any adverse effect levels. When a NOAEL is not available, a lowest-observed-adverse-effect level (LOAEL) can be used to derive an MRL, and an uncertainty factor (UF) of 10 must be employed. Additional uncertainty factors of 10 must be used both for human variability to protect sensitive subpopulations (people who are most susceptible to the health effects caused by the substance) and for interspecies variability (extrapolation from animals to humans). In deriving an MRL, these individual uncertainty factors are multiplied together. The product is then divided into the inhalation concentration or oral dosage selected from the study. Uncertainty factors used in developing a substance-specific MRL are provided in the footnotes of the levels of significant exposure (LSE) tables.

\section{Chapter 3}

\section{Health Effects}

\section{Tables and Figures for Levels of Significant Exposure (LSE)}

Tables and figures are used to summarize health effects and illustrate graphically levels of exposure associated with those effects. These levels cover health effects observed at increasing dose concentrations and durations, differences in response by species, MRLs to humans for noncancer end points, and EPA's estimated range associated with an upper- bound individual lifetime cancer risk of 1 in 10,000 to 1 in 10,000,000. Use the LSE tables and figures for a quick review of the health effects and to locate data for a specific exposure scenario. The LSE tables and figures should always be used in conjunction with the text. All entries in these tables and figures represent studies that provide reliable, quantitative estimates of NOAELs, LOAELs, or Cancer Effect Levels (CELs).

The legends presented below demonstrate the application of these tables and figures. Representative examples of LSE Table 3-1 and Figure 3-1 are shown. The numbers in the left column of the legends correspond to the numbers in the example table and figure. 


\section{LEGEND}

\section{See Sample LSE Table 3-1 (page B-6)}

(1) Route of Exposure. One of the first considerations when reviewing the toxicity of a substance using these tables and figures should be the relevant and appropriate route of exposure. Typically when sufficient data exist, three LSE tables and two LSE figures are presented in the document. The three LSE tables present data on the three principal routes of exposure, i.e., inhalation, oral, and dermal (LSE Tables 3-1, 3-2, and 3-3, respectively). LSE figures are limited to the inhalation (LSE Figure 3-1) and oral (LSE Figure 3-2) routes. Not all substances will have data on each route of exposure and will not, therefore, have all five of the tables and figures.

(2) Exposure Period. Three exposure periods - acute (less than 15 days), intermediate (15364 days), and chronic (365 days or more) - are presented within each relevant route of exposure. In this example, an inhalation study of intermediate exposure duration is reported. For quick reference to health effects occurring from a known length of exposure, locate the applicable exposure period within the LSE table and figure.

(3) Health Effect. The major categories of health effects included in LSE tables and figures are death, systemic, immunological, neurological, developmental, reproductive, and cancer. NOAELs and LOAELs can be reported in the tables and figures for all effects but cancer. Systemic effects are further defined in the "System" column of the LSE table (see key number 18).

(4) Key to Figure. Each key number in the LSE table links study information to one or more data points using the same key number in the corresponding LSE figure. In this example, the study represented by key number 18 has been used to derive a NOAEL and a Less Serious LOAEL (also see the two "18r" data points in sample Figure 3-1).

(5) Species. The test species, whether animal or human, are identified in this column. Chapter 2, "Relevance to Public Health," covers the relevance of animal data to human toxicity and Section 3.4, "Toxicokinetics," contains any available information on comparative toxicokinetics. Although NOAELs and LOAELs are species specific, the levels are extrapolated to equivalent human doses to derive an MRL.

(6) Exposure Frequency/Duration. The duration of the study and the weekly and daily exposure regimens are provided in this column. This permits comparison of NOAELs and LOAELs from different studies. In this case (key number 18), rats were exposed to "Chemical x" via inhalation for 6 hours/day, 5 days/week, for 13 weeks. For a more complete review of the dosing regimen, refer to the appropriate sections of the text or the original reference paper (i.e., Nitschke et al. 1981).

(7) System. This column further defines the systemic effects. These systems include respiratory, cardiovascular, gastrointestinal, hematological, musculoskeletal, hepatic, renal, and dermal/ocular. "Other" refers to any systemic effect (e.g., a decrease in body weight) not covered in these systems. In the example of key number 18, one systemic effect (respiratory) was investigated.

(8) NOAEL. A NOAEL is the highest exposure level at which no harmful effects were seen in the organ system studied. Key number 18 reports a NOAEL of 3 ppm for the respiratory system, which was used to derive an intermediate exposure, inhalation MRL of $0.005 \mathrm{ppm}$ (see footnote "b"). 
(9) LOAEL. A LOAEL is the lowest dose used in the study that caused a harmful health effect. LOAELs have been classified into "Less Serious" and "Serious" effects. These distinctions help readers identify the levels of exposure at which adverse health effects first appear and the gradation of effects with increasing dose. A brief description of the specific end point used to quantify the adverse effect accompanies the LOAEL. The respiratory effect reported in key number 18 (hyperplasia) is a Less Serious LOAEL of $10 \mathrm{ppm}$. MRLs are not derived from Serious LOAELs.

(10) Reference. The complete reference citation is given in Chapter 9 of the profile.

(11) CEL. A CEL is the lowest exposure level associated with the onset of carcinogenesis in experimental or epidemiologic studies. CELs are always considered serious effects. The LSE tables and figures do not contain NOAELs for cancer, but the text may report doses not causing measurable cancer increases.

(12) Footnotes. Explanations of abbreviations or reference notes for data in the LSE tables are found in the footnotes. Footnote "b" indicates that the NOAEL of 3 ppm in key number 18 was used to derive an MRL of $0.005 \mathrm{ppm}$.

\section{LEGEND}

\section{See Sample Figure 3-1 (page B-7)}

LSE figures graphically illustrate the data presented in the corresponding LSE tables. Figures help the reader quickly compare health effects according to exposure concentrations for particular exposure periods.

(13) Exposure Period. The same exposure periods appear as in the LSE table. In this example, health effects observed within the acute and intermediate exposure periods are illustrated.

(14) Health Effect. These are the categories of health effects for which reliable quantitative data exists. The same health effects appear in the LSE table.

(15) Levels of Exposure. Concentrations or doses for each health effect in the LSE tables are graphically displayed in the LSE figures. Exposure concentration or dose is measured on the log scale " $\mathrm{y}$ " axis. Inhalation exposure is reported in $\mathrm{mg} / \mathrm{m}^{3}$ or $\mathrm{ppm}$ and oral exposure is reported in $\mathrm{mg} / \mathrm{kg} / \mathrm{day}$.

(16) NOAEL. In this example, the open circle designated $18 \mathrm{r}$ identifies a NOAEL critical end point in the rat upon which an intermediate inhalation exposure MRL is based. The key number 18 corresponds to the entry in the LSE table. The dashed descending arrow indicates the extrapolation from the exposure level of $3 \mathrm{ppm}$ (see entry 18 in the table) to the MRL of $0.005 \mathrm{ppm}$ (see footnote "b" in the LSE table).

(17) CEL. Key number $38 \mathrm{~m}$ is one of three studies for which CELs were derived. The diamond symbol refers to a CEL for the test species-mouse. The number 38 corresponds to the entry in the LSE table. 
(18) Estimated Upper-Bound Human Cancer Risk Levels. This is the range associated with the upperbound for lifetime cancer risk of 1 in 10,000 to 1 in 10,000,000. These risk levels are derived from the EPA's Human Health Assessment Group's upper-bound estimates of the slope of the cancer dose response curve at low dose levels $\left(\mathrm{q}_{1}{ }^{*}\right)$.

(19) Key to LSE Figure. The Key explains the abbreviations and symbols used in the figure. 


\section{SAMPLE}

$1 \rightarrow \quad$ Table 3-1. Levels of Significant Exposure to [Chemical x] - Inhalation

\begin{tabular}{|c|c|c|c|c|c|c|c|c|c|}
\hline & & \multirow[b]{2}{*}{$\begin{array}{l}\text { Key to } \\
\text { figure }^{a}\end{array}$} & \multirow{2}{*}{\multicolumn{2}{|c|}{$\begin{array}{c}\text { Exposure } \\
\text { frequency/ } \\
\text { Species duration }\end{array}$}} & \multirow[b]{2}{*}{ System } & \multirow[b]{2}{*}{$\begin{array}{l}\text { NOAEL } \\
(\mathrm{ppm})\end{array}$} & \multicolumn{2}{|l|}{ LOAEL (effect) } & \multirow[b]{2}{*}{ Reference } \\
\hline & & & & & & & $\begin{array}{l}\text { Less serious } \\
(\mathrm{ppm})\end{array}$ & Serious (ppm) & \\
\hline \multirow[t]{2}{*}{2} & $\rightarrow$ & \multicolumn{8}{|c|}{ INTERMEDIATE EXPOSURE } \\
\hline & & & 5 & 6 & 7 & 8 & 9 & & 10 \\
\hline 3 & $\rightarrow$ & Systemic & $\downarrow$ & $\downarrow$ & $\downarrow$ & $\downarrow$ & $\downarrow$ & & $\downarrow$ \\
\hline \multirow{7}{*}{\multicolumn{2}{|c|}{4}} & 18 & Rat & $\begin{array}{l}13 \mathrm{wk} \\
5 \mathrm{~d} / \mathrm{wk} \\
6 \mathrm{hr} / \mathrm{d}\end{array}$ & Resp & $3^{b}$ & 10 (hyperplasia) & & Nitschke et al. 1981 \\
\hline & & CHRONIC & XPOSURE & & & & & & \\
\hline & & Cancer & & & & & 11 & & \\
\hline & & & & & & & $\downarrow$ & & \\
\hline & & 38 & Rat & $\begin{array}{l}18 \mathrm{mo} \\
5 \mathrm{~d} / \mathrm{wk} \\
7 \mathrm{hr} / \mathrm{d}\end{array}$ & & & 20 & $\begin{array}{l}\text { (CEL, multiple } \\
\text { organs) }\end{array}$ & Wong et al. 1982 \\
\hline & & 39 & Rat & $\begin{array}{l}89-104 \mathrm{wk} \\
5 \mathrm{~d} / \mathrm{wk} \\
6 \mathrm{hr} / \mathrm{d}\end{array}$ & & & 10 & $\begin{array}{l}\text { (CEL, lung tumors, } \\
\text { nasal tumors) }\end{array}$ & NTP 1982 \\
\hline & & 40 & Mouse & $\begin{array}{l}79-103 \mathrm{wk} \\
5 \mathrm{~d} / \mathrm{wk} \\
6 \mathrm{hr} / \mathrm{d}\end{array}$ & & & 10 & $\begin{array}{l}\text { (CEL, lung tumors, } \\
\text { hemangiosarcomas) }\end{array}$ & NTP 1982 \\
\hline
\end{tabular}




\section{SAMPLE}

13

Figure 3-1. Levels of Significant Exposure to [Chemical X] - Inhalation
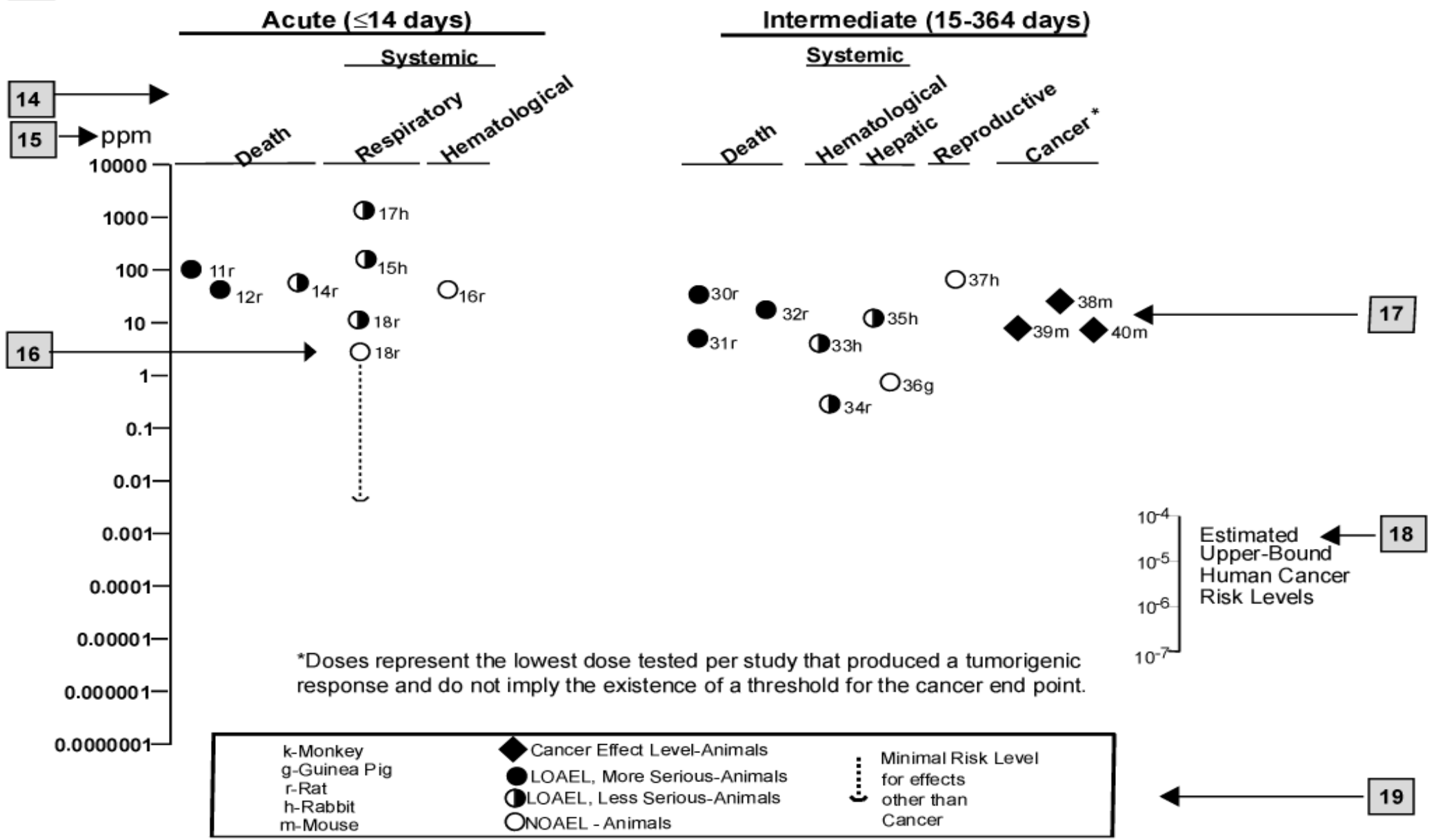
APPENDIX B

This page is intentionally blank. 


\section{APPENDIX C. ACRONYMS, ABBREVIATIONS, AND SYMBOLS}

\begin{tabular}{|c|c|}
\hline ACGIH & American Conference of Governmental Industrial Hygienists \\
\hline ACOEM & American College of Occupational and Environmental Medicine \\
\hline ADI & acceptable daily intake \\
\hline ADME & absorption, distribution, metabolism, and excretion \\
\hline AED & atomic emission detection \\
\hline AFID & alkali flame ionization detector \\
\hline AFOSH & Air Force Office of Safety and Health \\
\hline ALT & alanine aminotransferase \\
\hline AML & acute myeloid leukemia \\
\hline AOAC & Association of Official Analytical Chemists \\
\hline AOEC & Association of Occupational and Environmental Clinics \\
\hline AP & alkaline phosphatase \\
\hline APHA & American Public Health Association \\
\hline AST & aspartate aminotransferase \\
\hline atm & atmosphere \\
\hline ATSDR & Agency for Toxic Substances and Disease Registry \\
\hline AWQC & Ambient Water Quality Criteria \\
\hline BAT & best available technology \\
\hline $\mathrm{BCF}$ & bioconcentration factor \\
\hline BEI & Biological Exposure Index \\
\hline BMD & benchmark dose \\
\hline BMR & benchmark response \\
\hline BSC & Board of Scientific Counselors \\
\hline $\mathrm{C}$ & centigrade \\
\hline CAA & Clean Air Act \\
\hline CAG & Cancer Assessment Group of the U.S. Environmental Protection Agency \\
\hline CAS & Chemical Abstract Services \\
\hline $\mathrm{CDC}$ & Centers for Disease Control and Prevention \\
\hline CEL & cancer effect level \\
\hline CELDS & Computer-Environmental Legislative Data System \\
\hline CERCLA & Comprehensive Environmental Response, Compensation, and Liability Act \\
\hline CFR & Code of Federal Regulations \\
\hline $\mathrm{Ci}$ & curie \\
\hline CI & confidence interval \\
\hline CL & ceiling limit value \\
\hline CLP & Contract Laboratory Program \\
\hline $\mathrm{cm}$ & centimeter \\
\hline CML & chronic myeloid leukemia \\
\hline CPSC & Consumer Products Safety Commission \\
\hline CWA & Clean Water Act \\
\hline DHEW & Department of Health, Education, and Welfare \\
\hline DHHS & Department of Health and Human Services \\
\hline DNA & deoxyribonucleic acid \\
\hline DOD & Department of Defense \\
\hline DOE & Department of Energy \\
\hline DOL & Department of Labor \\
\hline DOT & Department of Transportation \\
\hline DOT/UN/ & Department of Transportation/United Nations/ \\
\hline NA/IMCO & North America/Intergovernmental Maritime Dangerous Goods Code \\
\hline
\end{tabular}




\begin{tabular}{|c|c|}
\hline DWEL & drinking water exposure level \\
\hline ECD & electron capture detection \\
\hline ECG/EKG & electrocardiogram \\
\hline EEG & electroencephalogram \\
\hline EEGL & Emergency Exposure Guidance Level \\
\hline EPA & Environmental Protection Agency \\
\hline $\mathrm{F}$ & Fahrenheit \\
\hline $\mathrm{F}_{1}$ & first-filial generation \\
\hline FAO & Food and Agricultural Organization of the United Nations \\
\hline FDA & Food and Drug Administration \\
\hline FEMA & Federal Emergency Management Agency \\
\hline FIFRA & Federal Insecticide, Fungicide, and Rodenticide Act \\
\hline FPD & flame photometric detection \\
\hline fpm & feet per minute \\
\hline FR & Federal Register \\
\hline FSH & follicle stimulating hormone \\
\hline g & gram \\
\hline GC & gas chromatography \\
\hline gd & gestational day \\
\hline GLC & gas liquid chromatography \\
\hline GPC & gel permeation chromatography \\
\hline HPLC & high-performance liquid chromatography \\
\hline HRGC & high resolution gas chromatography \\
\hline HSDB & Hazardous Substance Data Bank \\
\hline IARC & International Agency for Research on Cancer \\
\hline IDLH & immediately dangerous to life and health \\
\hline ILO & International Labor Organization \\
\hline IRIS & Integrated Risk Information System \\
\hline $\mathrm{Kd}$ & adsorption ratio \\
\hline $\mathrm{kg}$ & kilogram \\
\hline $\mathrm{kkg}$ & metric ton \\
\hline $\mathrm{K}_{\mathrm{oc}}$ & organic carbon partition coefficient \\
\hline $\mathrm{K}_{\mathrm{ow}}$ & octanol-water partition coefficient \\
\hline $\mathrm{L}$ & liter \\
\hline $\mathrm{LC}$ & liquid chromatography \\
\hline $\mathrm{LC}_{50}$ & lethal concentration, $50 \%$ kill \\
\hline $\mathrm{LC}_{\mathrm{Lo}}$ & lethal concentration, low \\
\hline $\mathrm{LD}_{50}$ & lethal dose, $50 \%$ kill \\
\hline $\mathrm{LD}_{\mathrm{Lo}}$ & lethal dose, low \\
\hline LDH & lactic dehydrogenase \\
\hline $\mathrm{LH}$ & luteinizing hormone \\
\hline LOAEL & lowest-observed-adverse-effect level \\
\hline LSE & Levels of Significant Exposure \\
\hline $\mathrm{LT}_{50}$ & lethal time, $50 \%$ kill \\
\hline $\mathrm{m}$ & meter \\
\hline MA & trans,trans-muconic acid \\
\hline MAL & maximum allowable level \\
\hline $\mathrm{mCi}$ & millicurie \\
\hline MCL & maximum contaminant level \\
\hline MCLG & maximum contaminant level goal \\
\hline MF & modifying factor \\
\hline
\end{tabular}




\begin{tabular}{|c|c|}
\hline MFO & mixed function oxidase \\
\hline $\mathrm{mg}$ & milligram \\
\hline $\mathrm{mL}$ & milliliter \\
\hline $\mathrm{mm}$ & millimeter \\
\hline $\mathrm{mmHg}$ & millimeters of mercury \\
\hline mmol & millimole \\
\hline mppcf & millions of particles per cubic foot \\
\hline MRL & Minimal Risk Level \\
\hline MS & mass spectrometry \\
\hline NAAQS & National Ambient Air Quality Standard \\
\hline NAS & National Academy of Science \\
\hline NATICH & National Air Toxics Information Clearinghouse \\
\hline NATO & North Atlantic Treaty Organization \\
\hline NCE & normochromatic erythrocytes \\
\hline $\mathrm{NCEH}$ & National Center for Environmental Health \\
\hline NCI & National Cancer Institute \\
\hline ND & not detected \\
\hline NFPA & National Fire Protection Association \\
\hline ng & nanogram \\
\hline NHANES & National Health and Nutrition Examination Survey \\
\hline NIEHS & National Institute of Environmental Health Sciences \\
\hline NIOSH & National Institute for Occupational Safety and Health \\
\hline NIOSHTIC & NIOSH's Computerized Information Retrieval System \\
\hline NLM & National Library of Medicine \\
\hline $\mathrm{nm}$ & nanometer \\
\hline nmol & nanomole \\
\hline NOAEL & no-observed-adverse-effect level \\
\hline NOES & National Occupational Exposure Survey \\
\hline NOHS & National Occupational Hazard Survey \\
\hline NPD & nitrogen phosphorus detection \\
\hline NPDES & National Pollutant Discharge Elimination System \\
\hline NPL & National Priorities List \\
\hline NR & not reported \\
\hline NRC & National Research Council \\
\hline NS & not specified \\
\hline NSPS & New Source Performance Standards \\
\hline NTIS & National Technical Information Service \\
\hline NTP & National Toxicology Program \\
\hline ODW & Office of Drinking Water, EPA \\
\hline OERR & Office of Emergency and Remedial Response, EPA \\
\hline OHM/TADS & Oil and Hazardous Materials/Technical Assistance Data System \\
\hline OPP & Office of Pesticide Programs, EPA \\
\hline OPPT & Office of Pollution Prevention and Toxics, EPA \\
\hline OPPTS & Office of Prevention, Pesticides and Toxic Substances, EPA \\
\hline OR & odds ratio \\
\hline OSHA & Occupational Safety and Health Administration \\
\hline OSW & Office of Solid Waste, EPA \\
\hline OTS & Office of Toxic Substances \\
\hline OW & Office of Water \\
\hline OWRS & Office of Water Regulations and Standards, EPA \\
\hline PAH & polycyclic aromatic hydrocarbon \\
\hline
\end{tabular}




$\begin{array}{ll}\text { PBPD } & \text { physiologically based pharmacodynamic } \\ \text { PBPK } & \text { physiologically based pharmacokinetic } \\ \text { PCE } & \text { polychromatic erythrocytes } \\ \text { PEL } & \text { permissible exposure limit } \\ \text { pg } & \text { picogram } \\ \text { PHS } & \text { Public Health Service } \\ \text { PID } & \text { photo ionization detector } \\ \text { pmol } & \text { picomole } \\ \text { PMR } & \text { proportionate mortality ratio } \\ \text { ppb } & \text { parts per billion } \\ \text { ppm } & \text { parts per million } \\ \text { ppt } & \text { parts per trillion } \\ \text { PSNS } & \text { pretreatment standards for new sources } \\ \text { RBC } & \text { red blood cell } \\ \text { REL } & \text { recommended exposure level/limit } \\ \text { RfC } & \text { reference concentration } \\ \text { RfD } & \text { reference dose } \\ \text { RNA } & \text { ribonucleic acid } \\ \text { RQ } & \text { reportable quantity } \\ \text { RTECS } & \text { Registry of Toxic Effects of Chemical Substances } \\ \text { SARA } & \text { Superfund Amendments and Reauthorization Act } \\ \text { SCE } & \text { sister chromatid exchange } \\ \text { SGOT } & \text { serum glutamic oxaloacetic transaminase } \\ \text { SGPT } & \text { serum glutamic pyruvic transaminase } \\ \text { SIC } & \text { standard industrial classification } \\ \text { SIM } & \text { selected ion monitoring } \\ \text { SMCL } & \text { secondary maximum contaminant level } \\ \text { SMR } & \text { standardized mortality ratio } \\ \text { SNARL } & \text { suggested no adverse response level } \\ \text { SPEGL } & \text { Short-Term Public Emergency Guidance Level } \\ \text { STEL } & \text { short term exposure limit } \\ \text { STORET } & \text { Storage and Retrieval } \\ \text { TD } & \text { toxic dose, 50\% specific toxic effect } \\ \text { TLV } & \text { threshold limit value } \\ \text { TOC } & \text { total organic carbon } \\ \text { TPQ } & \text { threshold planning quantity } \\ \text { TRI } & \text { Toxics Release Inventory } \\ \text { TSCA } & \text { Toxic Substances Control Act } \\ \text { TWA } & \text { time-weighted average } \\ \text { UF } & \text { uncertainty factor } \\ \text { U.S. } & \text { United States } \\ \text { USDA } & \text { United States Department of Agriculture } \\ \text { USGS } & \text { United States Geological Survey } \\ \text { VOC } & \text { volatile organic compound } \\ \text { WBC } & \text { white blood cell } \\ \text { WHO } & \text { World Health Organization } \\ & \end{array}$




$\begin{array}{ll}> & \text { greater than } \\ \geq & \text { greater than or equal to } \\ = & \text { equal to } \\ < & \text { less than } \\ \leq & \text { less than or equal to } \\ \% & \text { percent } \\ \alpha & \text { alpha } \\ \beta & \text { beta } \\ \gamma & \text { gamma } \\ \delta & \text { delta } \\ \mu \mathrm{m} & \text { micrometer } \\ \mu \mathrm{g} & \text { microgram } \\ \mathrm{q}_{1}{ }^{*} & \text { cancer slope factor } \\ - & \text { negative } \\ + & \text { positive } \\ (+) & \text { weakly positive result } \\ (-) & \text { weakly negative result }\end{array}$


APPENDIX C

This page is intentionally blank. 


\section{APPENDIX D. INDEX}

absorbed dose $12,123,126,129,134,163$

acetylcholine .75

adipose tissue

$123,126,128,134,139,143,153,171$

adsorbed $124,126,131,136,137,182,216$

adsorption..... $12,124,193,203,217,249,267$

aerobic 220

alanine aminotransferase (see ALT) 68,104

ALT (see alanine aminotransferase) 70 ambient air $225,243,256,269$ anaerobic $220,221,257,258$

aspartate aminotransferase (see AST) .68

AST (see aspartate aminotransferase) 70

bioaccumulation 218,255

bioavailability 255

bioconcentration factor 203, 218

biodegradation.

biomarker

$11,203,219,220,221,222,257,258$

blood cell count

$162,163,164,181,184,259,269$

body weight effects

67,113

breast milk

$12,13,14,18,21,71,105,106,109,156,160$

cancer

$148,160,184,249$

carcinogen $5,6,14,25,82,108,117,159,177$

carcinogenic

$6,14,17,27,108,273$

carcinogenicity

cardiovascular

$173,175,178,274$

cardiovascular effects.

$66,102,113,173$

cholinesterase..... $66,102,113,173$

chromosomal aberrations 116,117

clearance $120,121,122$

cognitive function 181

death. $6,27,29,30,80,83,109,155,157,178,274$

deoxyribonucleic acid (see DNA)

dermal effects. .77

developmental effects

30, 105, 115

DNA (see deoxyribonucleic acid)

$7,18,79,80,81,108,116,169,179,183$

dopamine....

endocrine.

18,77

endocrine effects

$71,105,109,157,158$

erythema

$71,105,158$

fetal tissue

$14,114,116$

fetus.

$128,153,160,184$

gastrointestinal effects

general population. $77,114,117,118,119,120,121,122,156,157,163,178$

genotoxic.... $7,81,158,160,161,175,176$

genotoxicity. $7,81,158,160,161,175,176$

groundwater

half-life

$5,11,12,162,205,239,257,259,274$

$27,121,173$

hematological effects

$121,122,175,178$

$2,3,11,173,203,215,217,219,220,234,235,255,256,257$

$11,113,124,126,134,137,162,217,218,220,221,227$

$24,67,103,113$ 
hepatic effects

hydrolysis

hydroxyl radical

immune system

immunological

immunological effects

$\mathrm{K}_{\text {ow }}$

$\mathrm{LD}_{50}$

leukemia

lymphoreticular

metabolic effects

micronuclei

milk

musculoskeletal effects

neurobehavioral

neurological effects

neurotransmitter.

non-Hodgkin's lymphoma .

norepinephrine .

nuclear.

octanol-water partition coefficient

ocular effects

odds ratio.

partition coefficients

pharmacodynamic.

pharmacokinetic

photolysis

placenta

rate constant

renal effects

reproductive effects

respiratory effects.

retention

salivation

sequestered

solubility

systemic effects

thyroid

toxicokinetic

tremors

tumors

vapor pressure

volatility

volatilization
$13,24,68,69,103,113,169$ 203, 219

$155,203,218$ .72, 106

$27,72,106,114,115,116,168,179$

$72,106,179$

$28,152,187,218$

$82,83,109$

$67,82,117$

$72,106,115,116$

.72

121

$150,153,237,245$

68,103

$14,20,75,80,81,152,157,158,160,177,179,180,183$

$13,15,18,19,20,21,22,23,25,72,73,74,75$,

76, 77, 78, 106, 107, 116, 176, 177, 180, 183, 271

77

.82

.75

270

126,218

$71,105,115$

.78

$21,28,126,139,143,144,152,153$

138,151

$28,131,134,138,139,140,151,159,161,166$

219

$7,128,153,178$

$139,143,147,218,220,221$

$13,24,70,71,104,113,114,181$

$14,78,107,108,116$

$13,16,18,19,30,64,65,78,83,109,113,176,177,271$

$76,123,134,153,162,167,217,268$

106

12

$152,153,154,181$

$21,30,83,109,175$

$27,166,182,183$

117,168

$206,217,268$

203

$11,12,203,206,217,219,221,222,232,235,249$ 
